\title{
THE LAWS OF HEREDITY
}

ARCHDALL REID

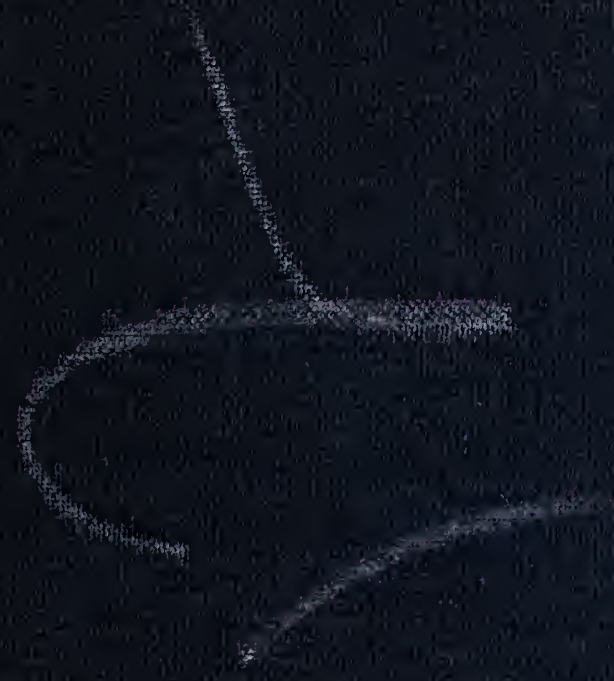



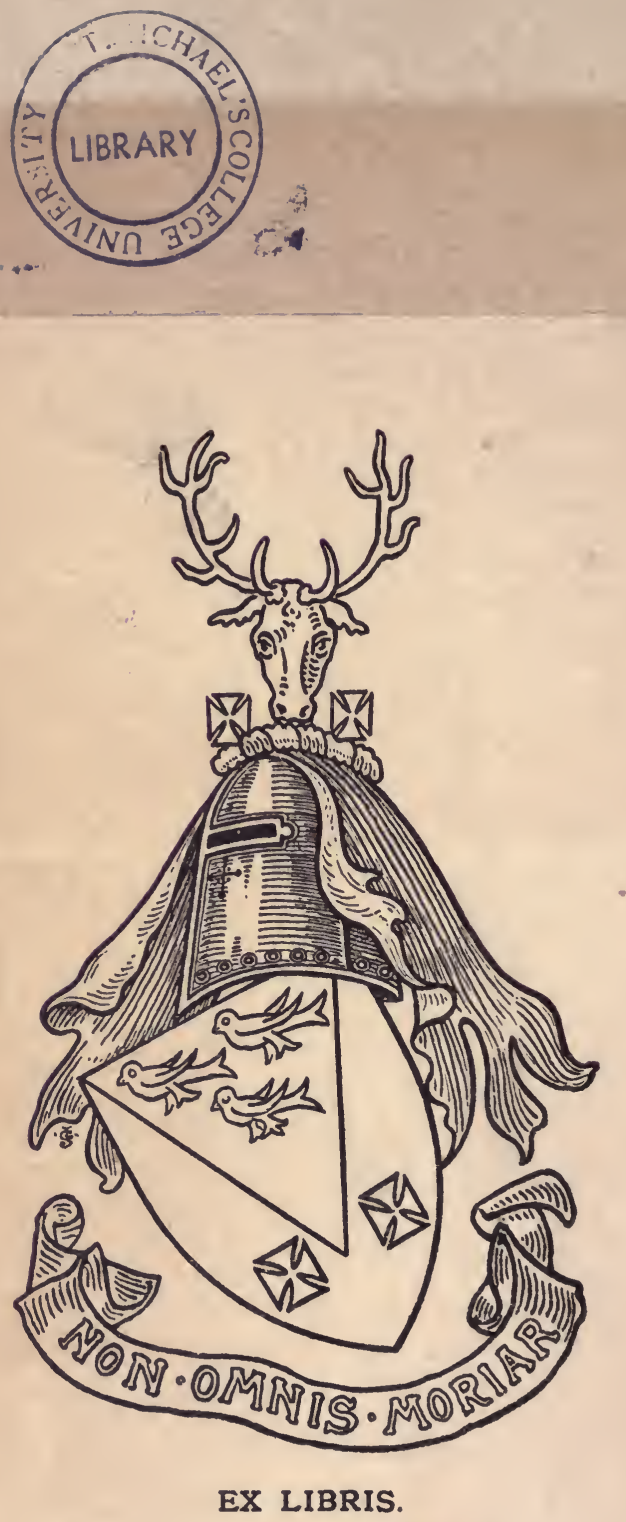

Bertram (r. X. Tainole,

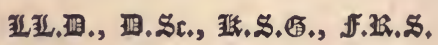



mously long and uncompromisingly stiff, yet it does not seem to help us very materially in coming to a conclusion on the laws with which it deals. Unquestionably the? most interesting and the most novel portion of the book is that which deals with the relation of modern views as to the rôle of bacteria in disease with the question of Natural Selection. That they are a potent agent in selection no one can doubt and if anyone does Dr Reid's pages may be commended to his attention. That there are other selective agencies at work no person can doubt who examines the facts of Nature and existence for himself with open eyes. But what we have always maintained is that having got so far upon the road and having conceded, if you like, that Natural Selection exists-which all scientific men do not concede-and that it is a potent sieve-if a sieve can be called potent-that even then we have only started upon the path at the end of which lies the explanation of variation and of inheritance That variations occur everybody knows, that Natural Selection may tend to perpetuate or to eliminate them we may admit, but what causes the variations to appear? That is the real heart of the question. The Lamarckians have an answer which is at any rate arguable. Dr Reid will have none of it, and in our opinion rather underrates the influence of Lamarckian opinion at the present day. Weismann also had or has an answer, but his solution of the difficulty only pushes the question one stage further back and leaves it there unanswered. Until some solution of that mystery has been arrived at, if indeed it ever is arrived at or is arrivable at, we may learn what laws govern heredity, if one may still be allowed to use the misleading nomenclature generally applied to what are called Natural Laws, one may come to a conclusion as to Mendel and his views or de Vries and his, but we shall remain all the time on the threshold only and not within the recesses of the temple of Nature's secrets. 
Digitized by the Internet Archive in 2007 with funding from Microsoft Corporation • 
THE LAWS OF HEREDITY 


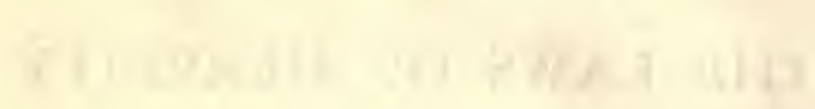




\title{
THE
}

\section{LAWS OF HEREDITY}

\author{
BY \\ G. ARCHDALL REID \\ M.B., F.R.S.E.
}

WITH A

DIAGRAMMATIC REPRESENTATION

BY

HERBERT HALL TURNER

SAVILIAN PROFESSOR OF ASTRONOMY, OXFORD

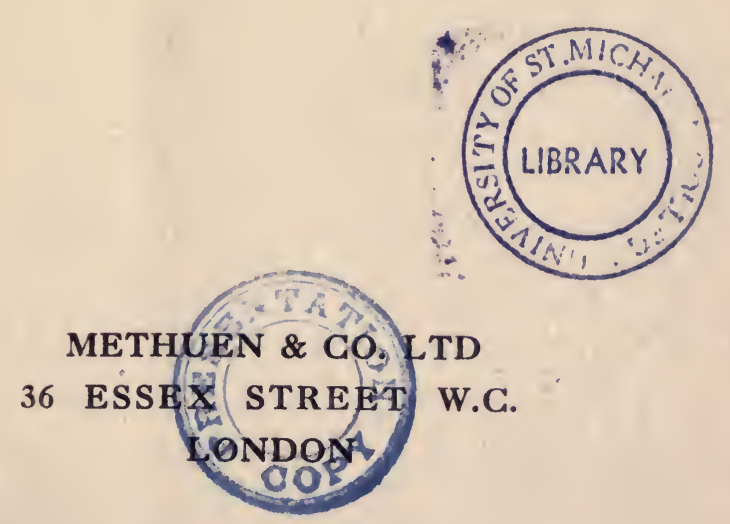



TO THE

\section{MEMORY}

$\mathrm{OF}$

\section{THOMAS HENRY HUXLEY}

A GREAT EXPONENT OF SCIENTIFIC METHOD 



\section{P R E F A C E}

WHE present volume covers a wider area than my last: The Principles of Heredity. No problems can be named of greater importance to the community than those of Heredity. I have tried, therefore, to make the argument as plain as possible to the general reader. And, since facts which we are able to observe and verify for ourselves, are more impressive and valuable intellectually than those we are obliged to accept at second hand, I have used, whenever possible, material that is familiar to every one.

Some biologists may regard Chapter III., which deals with scientific method, as unnecessary and even as impertinent. But every writer hopes to gain wide assent to his reasoning; and, there are, apparently, amongst thinkers about living beings, such fundamental differences of opinion as to what constitutes evidence, what logical proof, and what science, that no one can hope to secure wide assent unless he first lay down the principles that have guided his own thinking and obtains assent to them. If the history of biology be considered, I believe it will be seen that many of the controversies, in which its students have engaged, arose and persisted merely because the disputants, holding what were really divergent views as to the nature of science, failed to reach a preliminary agreement with respect to their basic assumptions. As far as my powers permitted, I have followed the rules of procedure that have been enunciated by every authority who has both thought and written about the subject. When these rules have been disregarded, the writers, however right they may be able to prove themselves ultimately, have never expressed their dissent in reasoned statements; they have merely implied it in their methods of work.

If, then, the reader disagrees with commonly accepted opinions, if, for example, he especially values evidence not solely because it is authentic and relevant but because it has been gathered in some particular way, or if he thinks that we should not use all the 
authentic evidence available, or that facts newly asserted are of greater value than truths long familiar and disputed by no one, or that it is beneath the dignity or not within the mission of science to furnish proof, or that knowledge derived from a simple enumeration of instances is superior to knowledge which is founded on a discovery of causes, or that we should found hypotheses on the exception rather than the rule, or that it is right to uphold contradictories, if in short he has individual and original views as to what constitutes proof and what science, then I hope he will endeavour to formulate his reasons clearly. Unless he does so, his opinions, no matter how correct they may be, are apt to bear the appearance of prejudices.

Professor H. H. Turner has contributed an Appendix in which some of the main arguments of the text are thrown into diagrammatic form. No part of the work is likely to prove so interesting and convincing as this Appendix. I have, besides, to record my great indebtedness to him for much very valuable advice.

It is impossible to express adequately my deep sense of obligation to Dr H. B. Donkin. Not only has he been a sort of reference library, but he has read and re-read the whole of the rough and the more finished manuscript, and the former has grown into the latter largely under his guidance.

Other helpers, whom I take this opportunity of thanking most heartily, are Miss J. Ritter, Mr A. D. Darbishire, Mr. P. C. Glubb, and $\mathrm{Mr} \mathrm{D}$. Waudby.

While writing the volume, I have received great kindness and encouragement from Dr Alfred Russel Wallace, Sir William Thiselton-Dyer, and Sir E. Ray Lankester. 


\section{CONTENTS}

CHAPTER I

THE CHARACTERS OF LIVING BEINGS

CHAPTER II

THE METHOD OF DEVELOPMENT .

CHAPTER III

THE METHOD OF SCIENCE

CHAPTER IV

THE LAMARCKIAN DOCTRINE

CHAPTER V

VARIABILITY

CHAPTER VI

RETROGRESSION

107

CHAPTER VII

MENDEL'S LAWS

CHAPTER VIII

THE MUTATION THEORY

\section{CHAPTER IX}

THE FUNCTION OF SEX

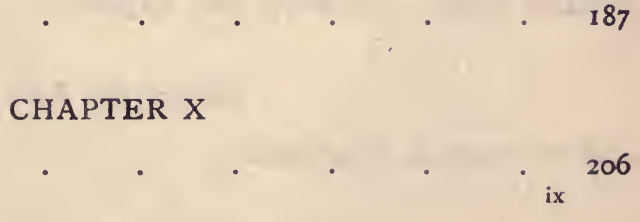


CHAPTER XI

HUMAN DISEASES

CHAPTER XII

ACQUIRED IMMUNITY 237

CHAPTER XIII

THE PRESENT EVOLUTION OF MAN 256

CHAPTER XIV

EPIDEMIC AND ENDEMIC DISEASE 266

CHAPTER XV

ALCOHOL

ALcohot

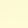
284

CHAPTER XVI THE EVOLUTION AGAINST NARCOTICS 298

CHAPTER XVII IDEALISM AND COMMON SENSE . 324

CHAPTER XVIII NECESSARY TRUTH 338

CHAPTER XIX

THE RELATION OF MIND TO BODY 356

CHAPTER XX

REFLEX ACTION, INSTINCT, AND REASON 367

\section{CHAPTER XXI}


CHAPTER XXII

CHAPTER XXIII

PHYSICAL DETERIORATION AND MICROBIC DISEASE

CHAPTER XXIV

INTEMPERANCE AND INSANITY 456

\section{CHAPTER XXV}

EDUCATION .

APPENDIX .

GLOSSARY .

INDEX 
With Earth's first Clay They did the Last Man knead, And there of the Last Harvest sow'd the Seed :

And the first Morning of Creation wrote What the Last Dawn of Reckoning shall read. 


\section{THE LAWS OF HEREDITY}

\section{CHAPTER I}

\section{THE CHARACTERS OF LIVING BEINGS}

The cell-Conjugation of cells-The germ-plasm-Hereditary tendencies or potentialities-The stimuli under which living beings develop-Nutriment, injury, use, etc.-The evolution of useful reactions to stimuli-Almost all living structures are capable to some extent of repairing injuries, but not nearly all are capable of growing under the stimulus of use-The real meaning of the terms inborn, acquired, and inheritable-The confusion and waste of labour which has resulted from a use of inaccurate and misleading terms.

I. HHE material basis of all known life is the living 'cell.' A cell is a mass, usually very minute, of a jelly-like substance known as protoplasm. The lowest plants and animals are single cells. Higher living beings are compounded of two or more, it may be billions of cells, most of which are adherent together, though some-for example, the blood-cells-may move freely within the organism. Cells multiply by self-division, the mother-cell distributing itself between the daughter-cells. In the case of unicellular or single-cell organisms the daughter-cells separate, but in higher types they remain together. As a consequence a man, for example, is an organised colony or community, a family or tribe or race of cells, all of which have descended from a common cell-ancestor, the fertilized ovum or egg. Speaking in general terms, the descendants of a unicellular organism closely resemble their ancestor. Each individual is able to continue the species by self-division, and each performs all the functions necessary to existence, such as the procurement of food. But the descendants of the fertilized ovum, though they remain together, indeed because they remain together, break up into many types. Thus, in a man there are skin-cells, bone-cells, nerve-cells, various kinds of gland-cells, and others. In other words, the members of this community are specialized in form and function. Each has a special shape and structure whereby it is adapted to perform some 
particular duty. None are fitted to perform all the functions of life, and none, therefore, can long maintain a separate existence. Thus a skin or muscle cell parted from the rest of the community quickly perishes. Even the duty of continuing the race is delegated to a particular set of cells, the germ-cells, which do not otherwise share in the labours of the community.

2. Germ-cells derived from a female body (e.g. a woman) are termed ova; whereas those derived from a male body are termed sperms. Sperms and ova differ in appearance, but we have no reason to suppose that they are not equivalent as 'bearers of heredity.' They are not male and female ; only the bodies, the cell-communities which they inhabit, are male and female. A sperm from a male body unites with an ovum from a female body. The single cell thus formed is termed the fertilized ovum. The fertilized ovum, dividing and redividing many times, builds up, by means of the descendants thus arising, a new cell-community, a new 'organism,' a new body, a new 'individual,' a human being, for instance.

3. That fertilization must precede reproduction is a rule almost universal amongst multicellular species. Without fertilization their ova and sperms perish, leaving no descendants. But fertilization is not quite universal. Some species consist only of females. They are parthenogenetic, that is, the virgin females produce offspring. Without union with another germ-cell, the ovum becomes the ancestor of a new cell-community. More common, especially in the vegetable kingdom, is self-fertilization. Here the individual is both male and female (hermaphrodite) and produces both sperms (pollen grains) and ova (ovules), which unite with one another. But cross-fertilization is by far the most common. It is secured in most animals by the device of sex, in plants more commonly by the ripening at different periods of the sperms and ova of the same plant, or by other devices. Neither cross- nor self-fertilization, then, is necessary to reproduction. But the fact that fertilization, especially cross-fertilization, the mingling of the qualities of two separate cell-communities to secure which nature has evolved sex, is so widespread is evidence that it must possess some very important function, some very important advantage. Darwin found that plants, which normally reproduced by crossing, gave origin to weakly offspring if artificially self-fertilized. It was argued, consequently, that the function of cross-fertilization was to invigorate or rejuvenate. But animals and plants which are parthenogenetic, or which normally fertilize their own ovules, are vigorous. Clearly, then, though crossing is necessary to the well- 
being of species which are normally cross-fertilized, it is not a necessary antecedent of vigour, and cannot have arisen as a means to that end. At present we are hardly in a position to discuss this subject. Later, when we have collected a large array of facts, we shall be able to deal with it to greater advantage.

4. The multicellular individual, then, consists of various kinds of cells (each of which corresponds to a unicellular organism) which perform different functions, each kind its own special function. Some of these cells are germ-cells, the rest are body or 'somatic' cells. The latter, which are usually much the more numerous, provide the former with shelter and nutrition, much as worker bees shelter and nourish queens and drones. They never unite, never 'conjugate,' in the way that sperms and ova do. Only the germs are marriageable; and, as we have just seen, in the great majority of animals and plants they observe the degrees of consanguinity very strictly, and do not unite except with members of another cell-community, and then only to found a new colony of cells, an offspring.

5. In each sperm and ovum is a dot, the nucleus. The essential feature of the union of sperm and ovum is now believed to be the union, the intimate mixture, of their nuclei, so that the two nuclei become one. Under high powers of the microscope there may be seen within the nucleus specks and threads of a substance known as chromatin. When the fertilized ovum and its descendant cells divide into daughter-cells, the chromatin, which grows with the cells, displays remarkable movements, and is distributed, apparently with great quantitative equality, between the daughters, thus forming their nuclei. Now this chromatin, contained in the germ-cells, is believed with some reason to be the germ-plasm, the 'bearer of heredity,' the substance that carries the 'hereditary tendencies,' ${ }^{1}$ which direct development, and thus determine the kind of individuals that shall arise from the germ-cells. A man differs greatly from an elephant because the germ-plasms of the

'I fear the expression 'hereditary tendency' is somewhat clumsy. I use it for lack of a better and imply nothing mystical. I mean no more by it than those potentialities for development which are carried by germ-cells. These potentialities differ, of course, with every species, and indeed with every individual. Thus the hereditary tendencies of a man are those which enable him to develop from a human germ into a human being. He does not develop into a rabbit or a tree, because the hereditary tendencies of human germs are such that rabbits and trees cannot arise from them. From a developmental point of view the germplasm is compounded of structures carrying hereditary tendencies. By this, however, I do not mean that definite structures in the germ-plasm carry definite tendencies, each structure a tendency. I know nothing about that. 
two species are very different. He differs yet more from a plant, because the germ-plasms are yet more different. One man resembles another because all human germ-plasm is much alike, but every man differs somewhat from every other, because the germplasm contained in no two germ-cells is exactly similar. A species undergoes evolution when, and only when, its germ-plasm undergoes change. When, by careful breeding, we improve our domestic animals and plants, the alteration is, in essence, always a germinal change.

6. It is now believed by almost every one who has an adequate acquaintance with the facts that the organic world, the world of plants and animals, arose by processes of evolution. Even people who still deny the whole truth admit, and have always admitted, a large part of it. Thus all men believe that the various branches of the human species had origin in a common stem-Adam and Eve, heathen deities, a species of lower animals, it matters not which. But human races have since diverged widely in size, shape, colour, and many other traits. Moreover, the direction of the change has always been such that every race has become particularly well-fitted to the surroundings in which it has long dwelt. Thus Englishmen flourish better in England than Negroes, ${ }^{1}$ who, on the other hand, are better adapted to conditions of life prevalent in West Africa. The reality of evolution, therefore, is not in dispute. Only the extent of it is disputed, and only, as a rule, by people whose knowledge is correspondingly limited.

7. We need not pin our faith to the hypothesis that the chromatin is the germ-plasm; we need not even suppose that the germ-plasm is contained wholly or even mainly in the nucleus. Nor need we accept any theories as to the composition of it. But some such substance there must be in the germ-cell, some substance which is the germ-plasm, the bearer of heredity; and probability points to it being the chromatin of the nucleus. One very important point to which we shall have to refer again and yet again should be noted. Since the offspring of the same parents invariably differ more or less among themselves, it follows that, though the quantitative division of the chromatin is apparently exact, the qualitative division may not be so. The daughter-cells may receive similar quantities; but it is possible that they do not receive exactly similar kinds of germ-plasm.

8. The whole of the child, therefore, is derived from a single cell, the fertilized ovum, which in turn was derived wholly from 
two germ-cells, one from each parent, and these again were each derived by single lines of descent (i.e. lines in which no conjugation occurred) from the fertilized ovum whence each parent sprang. Never in all the generations between fertilized ovum and fertilized ovum does conjugation take place. The somatic cells of the parent, therefore, as far as we know, contribute no living elements to the child; they merely provide temporary shelter and nutriment. The child, therefore, does not, as is popularly supposed, resemble his parent because his several parts are derived from similar parts of the parent-his head from his parent's head, his hands from his parent's hands, and so forth; he resembles him only because the germ-plasm which directed his development was a split-off portion of the germ-plasm which directed the development of the parent. The egg produces the fowl, but the fowl as a whole does not produce the egg-only one cell from the fowl, the fertilized ovum, produces it.

9. The germ-cells are, in a real sense, immortal. Saving accidents, they divide perpetually, and at intervals conjugate, and there is no dead body. In like manner the germ-plasm is potentially immortal. It grows and divides into separate portions, but does not die unless killed or starved. Each fertilized ovum builds, with mortal cell-descendants, a temporary dwelling, the body, around its potentially immortal descendants, the germs, which hand on to succeeding generations their all-important trust, the germ-plasm. Thus there is 'continuity of the germ-plasm.' This conception of continuity is one of the main pillars of the modern science of heredity. It is of very recent origin. Darwin, for example, believed that the somatic cells emitted minute living representatives, 'gemmules,' as he termed them, one of which from each cell found its way to each germ and re-constituted the germplasm. The germ-plasm, therefore, was supposed to be formed afresh in each germ, when, of course, there could be no continuity; and the various parts of the child would be derived from corresponding parts of the parent. It is now known, however, that the information on which Darwin relied was founded on a mistaken interpretation of the facts. Contrary to what he supposed, the evidence is massive that parental 'acquirements' are never 'transmitted 'to offspring.

IO. Under the constant direction of its hereditary tendencies the fertilized ovum proliferates, and the cell-community thus originated grows (develops) through the continued but regulated multiplication of cells, and so takes shape as an 'individual,' 
animal or plant. Nutriment supplies the materials for all this increase. But more than material is needed. Neither growth nor any other form of 'vital' activity ever occurs except as a reaction to some appropriate kind of stimulus. This capacity to assimilate nutriment, and to react in certain other peculiar ways to certain definite stimuli, are the marks of a living being. There are, then, three necessary factors, in the absence of any of which growth cannot occur in living beings. The first is tendency (capacity, potentiality) to grow, which determines the kind of development that shall occur. The second is stimulus, which awakens the tendency. The third is nutriment, which supplies the materials for growth, the clay and the straw out of which the bricks are made. Hereditary tendencies are internal factors traceable ultimately to the germ-plasm, but both nutriment and stimuli proceed from the world external to the growing structure. Usually not only the kind of growth, but the extent of it, is determined by the hereditary tendencies. Thus, no matter how abundant the supply of nutriment and stimulus, neither mouse nor elephant, for example, can increase beyond a certain size, which, within narrow limits, is definite for the species. In other words, stimuli, which are capable of exciting growth in one period of life (when the animal is young) cease to be capable of exciting it at another (when the animal is adult), no matter how large the supply of nutriment. The limits of growth, however, are much less rigidly drawn in plants, some of which, indeed, seem capable of endless growth, as when propagated by cuttings. It should be noted that nutriment supplies, not only the materials for growth, but acts-sometimes, not always-as stimulus as well. Thus female bees develop in one way (becoming queens) if one kind of food is supplied, and in another way (becoming workers) if they receive a different kind of food. ${ }^{1}$

${ }^{1}$ May I beg the reader to pay especial attention to this paragraph. There is, I believe, nothing in it disputable; but a great deal that, in effect, is disputed hinges on it. The points to which I wish particularly to call attention are these : (I) An individual can develop only within limits which are predetermined by 'hereditary tendencies' that have their roots in his germ-plasm. Thus a man could not develop a human limb, nor increase the size of his muscles by use, nor heal a wound of the limb by a scar unless his germ-plasm was of a kind that enabled him to respond to the right stimuli in the right way. (2) An individual makes no growth, develops in no way, unless he is stimulated to develop in that way by the right stimulus, which may be nutriment, or use, or injury, or something else. (3) Nutriment furnishes the materials (the clay and the straw) for all growth. It also, as I say, acts, apparently, as the stimulus for some growth. In other words some growth occurs, apparently, mevely because the individual absorbs nutriment: here nutriment acts both as material for growth and as stimulus to it. But in other cases growth occurs only under the stimulus of use 
I I. Living beings develop mainly under the influence of three distinct kinds of stimuli-nutriment, use, and injury. To take the example of the human being: up to the time of birth the infant develops wholly, or at least principally, under the stimulus of nutriment. After birth some of his structures continue to grow under this stimulus, for example his hair, his teeth, his external ears, and his organs of reproduction. But, as regards other of his structures, though nutriment continues to supply the materials for growth, it ceases to supply the stimulus. Thus, no matter how well the child is fed, the muscles of his limbs do not develop unless they are used. It is a noticeable fact that structures-and those structures only-which grow under the influence of use, tend to atrophy when disused. Thus disuse, or lessened use, diminishes the size of the muscles in a human limb, while under similar conditions the external ears and the reproductive organs persist unchanged. Since the development which occurs under the stimulus of use is always a mere extension-though often a most important extension-of that which occurs under the stimulus of nutriment, it is frequently difficult to distinguish structures which owe their growth wholly to nutriment from those which owe it in part to use. But the tendency of the latter class to atrophy when disused supplies us with a rough criterion. ${ }^{1}$ Lastly, if the individual be injured, as by a cut, the wound supplies the stimulus for the growth which occurs during the process of healing.

I2. Other stimuli to growth besides nutriment, use, and injury, exist, though we need not pause to discuss them at length. Thus all organs develop within a certain range of temperature, and best or injury; here nutriment furnishes only the materials. The reader will see later that, for the sake of convenience, I include under 'nutriment' a number of other stimuli, but that I sharply distinguish from it use and injury. He will find that the reason why $I$ include a number of stimuli under the heading of nutriment, but exclude from that category use and injury, is because biologists are accustomed to term all characters which arise under the stimulus of use and injury ' acquirements,' whereas all other characters are termed by them 'inborn' or 'innate.' The effect of distinguishing characters by means of the stimuli under which they arise, rather than by the words 'innate ' or 'acquired,' will be to make us take a view of heredity and evolution, especially mental heredity and evolution, immensely different, but I think, demonstrably more correct and comprehensive than the view hitherto accepted.

${ }^{1}$ The criterion is very rough, however. If a structure atrophies under disuse then, as far as I am aware, we may be sure it has grown under the influence of use. But it is not true that all structures which have grown under the influence of use atrophy when disused. Thus the bones of the limbs grow under the influence of use, but they do not atrophy like the muscles, at least to the same extent, when disused. Apparently this is due to the fact that bones are not to the same extent composed of living cells. 
only within narrow limits which are not the same for every species. Some organisms develop markedly diverse characters at different temperatures. Light is essential to the development of very many species, especially of plants. Certain plants develop in one way in the water, and in another way on the land. A brine shrimp (Artemia) develops one form in brackish water and another in water that is salter. Within the animal body the 'internal' secretion of various glands exercises an important influence on the development of many organs. For the purposes of our discussion, however, all these stimuli, and others besides, may be bracketed with nutriment, use, or injury; they are, in effect, in many cases at least, conditions under which nutriment and use act as stimuli, or which, when in excess, produce injury.

I3. All the characters which it is possible for an individual under any circumstances to possess are traceable ultimately to an inter-action between the environment and hereditary tendencies or potentialities which have their roots in the germ-plasm. Because the germ-plasm of a dog differs from that of a man, because the hereditary tendencies are different, the two animals develop into very different individuals, and would so develop though placed under identical conditions as regards nutriment, use, and injury. On the other hand, if, were it possible, two human germs were exactly alike in all their potentialities, differences of nutriment, use, and injury would cause corresponding differences between the individuals that arise from them. The human arm first develops under the stimulus of the nutriment that reaches it from the world external to it, because of an antecedent potentiality in the germplasm. For the same reason it continues to grow under the influence of use, or, when it is damaged, under the stimulus of injury. Stimulus, is the invariable antecedent to all growth.

I4. Since no character can be used or injured until it exists, the power of growing under the stimulus of nutriment must have been the very first product of evolution, the first peculiarity by which living beings were distinguished from mere chemical compounds. Possibly the earliest living beings were homogeneous throughout, consisting altogether of a primitive kind of germ-plasm, there being no cell-body as distinguished from the nucleus, no 'cyto-plasm' properly so called, in which case the minutest living fragment was perhaps capable of continuing the species. Probably also each organism was capable of indefinite growth, or at least of an amount of growth which was limited only by the supply of nutriment. The individual, however, can never have been large; 
for, with very primitive organization, the passage of food from the periphery to the centre through living and assimilating tissue cannot have been great. Very possibly, too, these lowly organisms were so loosely compacted that they easily fell to pieces under shocks from the environment. Every fragment, then, constituted a member of the species. Doubtless definite shape and size, as well as definite differentiation of structure within the individual and particular methods of reproduction which resulted therefrom, such as periodic self-division, were products of prolonged evolution. Probably the lowest living beings which we are capable of distinguishing as living, even under the highest powers of the microscope, are products, as regards size and in other ways, of ages of evolution. So far, of course, all is pure conjecture ; but it is something more than conjecture that early evolution must have consisted of such changes in the germ-plasm, such changes in the hereditary tendencies, that each species came to respond, by growing in its own particular way, to the stimulus of nutriment, and so evolved its own peculiar characteristics.

15. We can guess only vaguely at the stage of evolution at which the power of responding by growth to the influence of injury, and so repairing damaged structures, first appeared. If the primitive living beings were capable of indefinite growth, but not of spontaneous self-division, then this continued growth after fragmentation in itself constituted a sort of repair of injury. Probably, therefore, the power of responding to the stimulus of injury was evolved concurrently with that of responding to the stimulus of nutriment and was originally part of and indistinguishable from it. Even in higher types, unicellular and multicellular, which have evolved heterogeneous structures and are of more or less definite shapes and sizes, the underlying resemblance remains; nutriment stimulates the individual to produce the characters of the species, injury stimulates him to reproduce them more or less perfectly when they are lost or damaged.

I6. This power of reproducing lost or damaged parts, of growing under the stimulus of injury, varies greatly in different species and in different structures of the same species. Since it is a product of evolution, since it is one of the means by which organisms are adapted to the environment, its degree of development in any species or structure is proportionate to its utility. Relatively speaking, the higher animals, which have very complex organizations and lead very active lives, possess it in small measure. As a rule they replace important losses of tissue only by scars. It is 
advantageous to a deer, for example, to be able to heal a wound, but the conditions of its life are such that it would very rarely have time or opportunity to reproduce a lost limb. In effect the power to do so would be useless. The stag, however, habitually reproduces its lost antlers. They are very heavy structures which are useful to him at the season when he contends with his rivals; but burdensome, and therefore, not only useless but worse than useless, at other times. He sheds them, it is true, in the absence of injury, but the whole process closely resembles loss and reproduction through injury, and demonstrates the difficulty of drawing a sharply dividing line between nutriment and injury as stimuli.

17. Some plants which sprang originally from germ-cells multiply indefinitely by budding. Each bud is regarded by botanists as a 'person,' but a person in this sense is not quite the same as an 'individual' which takes origin in a germ. Buds, even when growing separated from the parent, are, in a real sense, portions of the individual that rose from the germ. If we amputate the end of a twig the injury may or may not heal as a scar, but the production of buds is commonly increased. Are they to be regarded as developing under the stimulus of nutriment, or of injury? Here again we see the close connexion between the power of responding to the stimulus of nutriment and that of responding to the stimulus of injury. Injury, indeed, may be regarded as one of the conditions under which the stimulus of nutrition comes into action. It awakens a power which has slumbered.

I8. Some animals lose living and active parts by injury almost as regularly and replace them almost as readily as the stag his antlers or the bear his winter coat. Thus in some newts the external gills are much exposed and liable to be bitten off by members of the same species. They are readily replaced, whereas internal parts of the same animal show small powers of regeneration: they merely heal. ${ }^{1}$ Lizards which frequently escape from enemies with the loss of the tail are able to reproduce it; but they cannot reproduce a limb, the loss of which usually involves capture and death. The power of regeneration ${ }^{2}$ possessed by fishes is apparently no greater than, if as great, as that possessed by mammals. Some worms, much exposed to injury, are able to

1 Weismann, Theory of Evolution, vol. ii. pp. 12-13.

2 The term regeneration is usually limited to a more or less perfect replacement of the lost part or tissues by similar structures; whereas in mere healing a scar, which may differ greatly from the lost part, is developed. The two processes differ in degree, not in kind, and are united by gradations. Regeneration is very perfect healing; healing is imperfect regeneration. 
reproduce the whole individual from a fragment. Great powers of regeneration are the rule in plants or parts of plants which are exposed to injury from animals, for example in grasses.

I9. The power of responding to the stimulus of injury, then, is clearly allied to and is derived from the power of responding to the stimulus of nutriment. The germ-plasm of every type of animal and plant has so changed from the primitive kind that under the stimulus of nutriment the individual tends to develop the characteristics which evolution conferred on his immediate predecessors; it has also so changed that wherever the power of regeneration is useful, and to the extent to which it is useful, injury stimulates to the replacement or repair of lost or damaged parts. Like the power of growing under the stimulus of nutriment, the power of repairing injury is always an adaptation. The kind of repair that normally occurs is ever the most useful and extensive possible under the conditions.

20. The characters which develop under the stimulus of injury are interesting and important, but vastly more interesting, both from the theoretical and the practical standpoint, are those which develop under the stimulus of use. If we study almost any work on the sister subjects, heredity and evolution, published more than a decade ago, and a great many published since, we frequently meet an entirely unwarrantable assumption-the assumption that all structures and organs in all types of living beings tend to grow and develop if used. For example, the celebrated Lamarck, the predecessor and in a way almost the peer of Darwin, who during the nineteenth century exerted an immense influence on biological thought, founded his theory of racial change on the supposition that all structures and qualities are capable of increase under the influence of use and decrease under that of disuse. The same idea bulks largely in the writings of Spencer, Lewes, Romanes, Cope, and a multitude of others.

2I. In man, as we have seen, certain structures grow under the influence of use, but the amount even of this growth (i.e. multiplication of cells) is by no means strictly proportionate to the amount of use, and is always rigidly limited. Different men respond in different degrees to the stimulus of use, but even a 'natural ' athlete can increase the size of his muscles only up to a certain point, after which cell-multiplication no longer occurs, no matter how much he uses his muscles. All growth due to use occurs mainly during early life; in old age it practically ceases in most structures though they are still used. Lastly, the growth of 
some structures, for example, the hair, teeth, nose, external ears and reproductive organs, is apparently not influenced in the slightest degree by use. It is plain, therefore, that the power of growing under the influence of use, unlike that of responding to injury, is not a property common to all or nearly all living structures. It is present only in some structures, and in them not equally at all periods of life.

22. If we continue to study the matter closely we make another discovery and a very important one; we find that this power, like that of repairing injury, is present only in structures where it is useful, to the extent to which it is useful, and during the time it is useful. In man, for example, it long persists in the muscles of the limbs and in the heart and kidneys, which even in advanced age hypertrophy under increased strain. But no structures are more used than the joints. With the rest of the limbs they grow under the influence of use during youth, after which the power of so growing, which would then be useless, indeed injurious, departs from them. The tongue again is a structure which is immensely used. We do not know whether it grows in part under the stimulus of use or only under that of nutriment; but, in any case, growth ceases when adult life is attained; for no amount of use, apparently, can add anything to it subsequently. Manifestly, therefore, the power of growing under the stimulus of use is also an adaptation. It is not a necessary property of all living protoplasm, but a thing which has been implanted in some structures by evolution.

23. Moreover we have massive evidence that it is a late and a high product of evolution. It does not date back to the origin of life like the faculties' of growing in response to the stimuli of nutriment and injury. It is observable only in the higher organisms, and in its greatest development only in the highest. Thus a human infant is born very immature and helpless; it owes most of its subsequent growth, its advance towards maturity, mainly to use. A young foal is born better developed; it owes less to use and more to the stimulus of nutriment. There is evidence that amphibians (e.g. frog) and fish owe little or nothing to use; they seem to develop perfectly, or at least very well, when so rigidly confined that use has no great scope to act as a stimulus. Most insects seem to grow wholly under the stimulus of nutriment (and injury). Certainly use plays no part in bringing about the changes which occur during their metamorphoses. Lower in the animal scale there is even less probability that use plays any part in develop- 
ment. Some plants grow tougher and more firmly rooted when exposed to strong winds; but throughout the vegetable kingdom the faculty, if present at all, is developed only in the slightest degree. At present, however, we need not labour this question of the evolution of the power of growing in response to use. We shall be in possession of much more definite and decisive evidence, especially when we consider mind.

24. In brief the notion that the power of growing, especially of growing adaptively, under the stimulus of use, is present in all living tissues, is merely a vague guess founded on popular observation of our familiar human development. There is no veil like the veil of familiarity. Never yet has this question been made the subject of close and accurate investigation. Since some human structures develop in this way, it has been assumed that all structures in all animals and plants can, and do, so develop. Like many other popular guesses it has been accepted quite without question even by thinkers in science. Vast systems of philosophy have been built on it and acclaimed as the highest products of human wisdom. But it is very doubtful whether any other error in the whole range of biology, indeed of scientific thought, has been productive of more confusion, futile discussion and practical mischief. My language is strong, but the reader will find it fully justified. ${ }^{1}$

25. Obviously, then, powers of developing in response to definite stimuli are, in the case of all species, delicate adjustments to the environment. If any individual varies from the ancestry so as to lose this adjustment, this power of responding by growth in definite ways to definite stimuli, he fails to develop the right characters to the right extent and perishes. Every type of plant and animal, the lichen, the beetle, and the man, for example, fits its own niche in nature as the hand does the glove; but it fits only because its germ-plasm has been so fashioned by evolution that every normal individual grows into adaptation to his environment in response to stimuli.

26. Speaking generally, all growth is either immediately adaptive or is a provision for future adaptation. Thus human germ-cells are adapted by their structures and capacities for existence in the testes and ovaries. Under the stimulus of nutriment they develop into embryos and thus are able to fit the next environment, the uterus. Here they continue to develop till they grow into fitness for life in the mother's arms. Thereafter, use and injury come into play; and, with some characters developing under

${ }^{1}$ See $\mathrm{N}$ chapter iv. 
one stimulus, some under another, and some under a third, and all developing in proportion, the infant gradually becomes adapted to the environments of the child and the adult. If he be injured, development begins by stoppage of the blood-flow and continues till growth is complete and a scar forms. Since every one is injured at one time or another, without this healing life would be impossible under conditions normal for the species. As we have just seen, growth under the stimulus of use is also a part of normal development. The human being cannot become a normal adult, fit for the struggle of existence, without it.

27. It is clear then that all characters that it is possible for the individual to develop are equally rooted, as it were, in the germplasm. Manifestly no character is more closely or less closely connected with it than any other. But the traits which develop under the stimulus of nutriment have two characteristics which have so impressed students of heredity that attention has been especially concentrated on them.

28. First, since no individual can develop without food as material for growth, the stimulus of nutriment is always present; and, therefore, if the individual lives long enough, all the characters that arise under its influence inevitably appear unless prevented by injury. ${ }^{1}$ On the other hand, characters, which arose in the parent under the stimulus of injury or use, may not arise in the child, for similar stimuli may not be received. Thus the only effect of injury which inevitably appears in the human being is the navel. Other scars are not reproduced unless, as is unlikely, exactly similar injuries are received by parent and child. In every normal child and adult certain effects of use (e.g. the muscular development of the limbs) are always present, but though in practice we recognize them as effects of use (for the physical and mental exercises we give our children are nothing other than conscious attempts to develop them in this way), yet in theory they are seldom recognized as such. Like other people, students of heredity tend to think in compartments, and in their discussions they have usually argued as if reactions to use were limited to such exceptional and trivial developments as the extra

1 This statement is not quite correct. Thus, though male characters are latent in the female and vice versa, and though they arise under the stimulus of nutriment, they do not develop. The individual has two sets of sexual characters, the development of one of which is a bar to the development of the other. But, apart from such cases of alternative reproduction in which the development of one character prevents the development of another, the statement that characters, which arise in response to the stimulus of nutriment inevitably appear is true. 
growth of the blacksmith's arm which results from the nature of his employment, or the thickening of the skin in the palm of the hand which results from rough labour.

29. Second, since nothing can be used or injured before it exists, characters that develop under the stimulus of nutriment are always the first to reappear. They are the earliest products both of racial evolution and individual development. Therefore all characters that arise through use or injury are 'modifications' of those that arose under the stimulus of nutriment. But this creates no real distinction, for all growth, whether in the embryo, fœtus, infant, child, or adult, whether arising in response to nutriment, injury, or use, is a modification, due to cell-multiplication, of previous growth. Nevertheless, since changes due to the stimulus of nutriment arise gradually and insensibly, whereas those due to injury are usually impressed in a very obvious way on pre-existing characters, and those recognized as due to use are also so impressed, the first have seldom been recognized as 'modifications,' while the second and third have been so recognized. At any rate, the names 'modification' and 'acquirement' have been especially applied to the effects of injury and use. That is, it has been implied that these stimuli alter the individual in such a way as to make him different from what, if I may use the expression, nature intended him to be. Obviously, however, the reasoning here is superficial and inexact. The error arises because the thinking is in terms, not of the germplasm which carries the hereditary potentialities of individual, but of the individual himself, who only develops such of his traits as he happens to be stimulated to develop. As I say all characters are equally rooted in the germ-plasm, all are equally products of evolution, and all arise equally in response to stimuli. They are all ready to arise, and they all arise inevitably if the right stimuli be forthcoming. Whether they do, or do not arise, depends, not on the nature of the individual, but on that of the environment. It is as 'natural' for him to develop a scar or a callosity under the right conditions as to develop a beard or a hand. In any case he fulfils his nature.

30. Half a century of controversy has resulted from an insufficient appreciation of the fact that the characters which develop under the stimulus of injury and use are as closely related to the germ-plasm, are as natural to the individual, as those which develop under the stimulus of nutriment. Biologists are accustomed to divide all the characters of living beings into those which are 'inborn' and those which are 'acquired,' and while all biologists 
maintain that 'inborn' characters are 'germinal' and tend to be transmitted to offspring, most of them insist that 'acquirements' are not germinal and are never inherited. If we consider these words carefully it becomes clear that by an innate or inborn character they imply one which has developed under the stimulus of nutriment, whereas by an acquirement they imply one which has developed under the stimulus of use or injury; when they speak of an innate character as inherited they imply that in the parent and child alike it developed under the stimulus of nutriment; and when, as is still sometimes the case, they allege the inheritance of a parental acquirement, they imply that a character which was developed under the influence of use or injury in the parent, is, in the child, TRANSFERRED to a different category and developed under the stimulus of nutriment.

3I. But now, bearing in mind the foregoing paragraphs-bearing in mind that all evolution consists in a germinal change, that a germinal change consists in an altered potentiality to produce characters, that obviously no characters can arise unless the potentiality to develop them is present in the germ-plasm and unless it is awakened by fitting stimuli, that all characters which are potentially present arise with equal certainty if fitting stimuli be applied, that all growth is a modification of pre-existing characters, that no characters are derived from similar characters in the parent (e.g. the child's hand from the parent's hand) but all take origin in the fertilized ovum, bearing in mind also that the evolution of the higher animals has consisted mainly in the evolution of a power of developing 'acquirements' under the stimulus of use, and that the mass of characters developed under this stimulus is quite as essential a part of that 'normal' development whereby the maturity of the individual is attained and his survival secured as the characters developed under the stimulus of nutriment-bearing in mind all this, let the reader ask himself in what respects characters that develop in response to the stimulus of nutriment are more germinal, inborn, and hereditary than those which arise in response to other stimuli. I believe he will be forced to conclude that these expressions are erroneous in that they altogether fail to indicate, in that they obscure rather than reveal, the true distinctions between classes of characters. They have come into use only because biologists, though speaking constantly of germinal characters, have as constantly thought in terms, not of the germplasm, but of the individual. Manifestly no characters are really more germinal, inborn, or inheritable than any others. All are 
germinal, inborn and inheritable in exactly the same sense. ${ }^{1}$ The true distinction between the different classes of characters are indicated when they are described as arising in response to the different classes of stimuli. If the reader still thinks otherwiseas he will constantly be tempted to do if he keeps his attention fixed, not on the germ-plasm, but on the individual-let him endeavour to formulate a clear idea as to what makes one character more inborn than another. He will certainly fail. Moreover, if he tries to think of a character of the multicellular individual which is more innate and inheritable than any other, he will succeed only in recalling characters which appear more regularly than others merely because the stimulus which evokes the former is received more certainly than that which evokes the latter.

32. Occasionally what are known as identical twins are born. They are supposed to be derived from a single fertilized ovum. They are extremely alike, presumably because their germ-plasms were similar. It is conceivable that the germ-plasms might be exactly similar; in which case, under precisely similar stimuli, they would develop into individuals precisely similar. But if from some cause, for example a twisting of the umbilical cord, one twin received an inferior supply of nutriment, they would differ. Everyone would then agree that the difference, though one of 'innate' characters, would be acquired ; but it would puzzle anyone to indicate the twin that made the differentiating acquirement.

33. Only the germ-plasm, its structures, and hereditary tendencies, are really inborn and inheritable; for they really pass from ancestral to descendant germ-cells. The germ-cell is a unicellular organism which, like other unicellular types, divides itself between its offspring, which in turn divide themselves in like manner. The daughter-cells and their descendants, therefore, inherit the structures and qualities of the parent cell in a sense quite different from and much more real than that in which a cellcommunity ' inherits' the structures and qualities of the community from whence it is derived. ${ }^{2}$

${ }^{1}$ The only conceivable exception is a temporary change, for example, an injury (e.g. a cut) considered apart from the reaction by which it is repaireda reaction which, however, begins at once, or almost at once, by coagulation of blood, etc. Even this exception is more apparent than real, for an injury derives its characteristics not only from the agent which inflicts it, but also from the structure which receives it, which in turn derived its characters from the egg.

${ }^{2}$ It may be asked "If the germ-cell, the fertilized ovum, divides itself between its daughter-cells, which, as well as their descendant cells, repeat the process, how does it happen that all the cell-descendants are not close copies of one another and of the fertilized ovum whence the community sprang ? Why do some of these 
34. The names we use do not greatly matter if they serve to indicate the truth; an erroneous term, provided it is obviously erroneous, may convey a right impression. But the words inborn, acquired, and inheritable, appear-when we think of individuals, instead of the germ-plasm as, very naturally, we tend to do-so obviously correct that their use has been, and is still productive of endless confusion and controversy. ${ }^{1}$ For example, it was formerly believed that parental acquirements were transmissible to offspring: in other words it was maintained in effect that a character (e.g. a scar) which the parent was able to acquire in a certain way (as a reaction to injury), because a long course of evolution had rendered such acquisition possible to the members of his race, tended to be reproduced by the child in a different category of characters and in a way (as a reaction to nutriment) in which no member of his race had ever acquired it before, and with which, therefore, evolution had nothing to do. An actual miracle was supposed to happen, the miraculous nature of which was concealed under a misuse of terms.

descendants become skin-cells, others muscle-cells, and so on ? What brings about this differentiation amongst the cells of the community ?" Two answers to this question are conceivable. Though the division of the cells is apparently equal quantitatively, it does not follow that it is equal qualitatively. Equal amounts of germ-plasm may pass into daughter-cells; but the kind of germplasm which passes into one daughter may not be quite similar to that which passes into the other daughter. Obviously if this differentiation were accentuated in succeeding cell-divisions it would account for the fact that skin or bone-cells, for example, are so dissimilar, not only in appearance but more especially in functions, from germ-cells. That there may be a good deal of truth in this hypothesis is rendered probable by the fact that not only do the body (somatic) cells differ qualitatively from the germ-cells, but the germ-cells differ in the same way amongst themselves; for they give rise to cell-communities (children) which may present marked differences. The second conceivable explanation is that the appearances and qualities which cells develop depend on their environments. According to this hypothesis, a skin-cell assumes the appearance and qualities of its kind, not because its germ-plasm ('idio-plasm ') differs from that of a germor muscle-cell, but because it is differently situated, and therefore differently stimulated. That there may be much truth in this suggestion also is proved by the fact that fragments of many cell-communities, for instance fragments "of begonia leaf, which apparently contain no germ-cells, are capable of giving rise to an entire 'person,' an entire cell-community, which contains every kind of cell, including germ-cells. Possibly the true explanation lies in a combination of these hypotheses. In other words, it is possible that cells, like individuals, differ amongst themselves partly because they differ in germ-plasm, and partly because they differ in environments.

1 In the misuse of them I have sinned as deeply as anyone-see, for example, The Present Evolution of Man, Alcoholism, and The Principles of Heredity. In the latter work, however, I made some endeavour to make the situation clear. See note, p. 249, and especially the second edition, Appendix A. 
35. Most biologists now reject this, the Lamarckian hypothesis, on the ground that it is against the weight of evidence. It is not realized, however, that here we have a question not of the transmission but of the transmutation of characters; for the child is not supposed to reproduce that which the parent developed (an effect of use or injury), but something vastly different (a response to the stimulus of nutriment). As a fact the evidence against the Lamarckian hypothesis is very conclusive. Characters which evolution has fitted parents to develop under the stimulus of use and injury do not tend to be automatically transmuted in the offspring into characters which develop under the stimulus of nutriment. Such a change would be as intrinsically improbable as a change of eyes into ears. But the language used has raised a false issue, has diverted attention from the real significance of responses to use and injury, and of the evolution which enables the individual to develop them. The belief, reached after long and heated controversy, that 'acquirements' are not 'inherited,' has caused nearly all students of heredity to think of them as mere accidents which have no importance except in so far as they obscure the outlook and render the discovery of the truth difficult. Hence, for example, the notion that the acquirements of the blacksmith-which comprise among other things almost his entire muscular development since infancy-are limited to the extra development which results from the nature of his labour. We shall see how mistaken is this view, and how great a gap in biological thought and study has resulted in consequencc. In effect it has shorn the study of heredity and evolution not only of one of its most important branches, but of its main title to practical utility as well. ${ }^{1}$

36. Nevertheless, since these terms, innate, acquired, and inheritable are firmly established in the literature of heredity, their use is now in many ways convenient. For the sake of clear thinking it is necessary, however, to bear their real meanings carefully in mind-to remember always, when speaking of multicellular organisms, that by an inborn character is meant one which develops under the stimulus of nutriment, by an acquirement one which develops under the stimulus of use or injury, and by inheritance the reproduction, as a nutritional character, by the child of a parental character of any sort. We shall thus avoid that confusion of thought which is the common accompaniment of misleading terminology. "Men believe that their reason rules over words;

1 See chapters $\mathrm{xx} .-\mathrm{xxv}$. 
but it is also the case that words react, and in their turn use their influence on the intellect." 1

37. Though the terms innate and acquired are misleading when used to compare or contrast the characters (which have developed under different stimuli) of the same individual, they are quite accurate when used to compare the characters of different individuals. Thus when we say that an individual is innately like or unlike another we imply, in effect, that the likeness or unlikeness is due to a germinal similarity or dissimilarity. On the other hand when we say that the individuals agree or differ in their acquirements, we imply that the stimuli under which they developed have been similar or dissimilar in kind or degree. In the latter case the individuals, as compared to each other, have eaten more, or less, or different kinds of food, taken more, or less, or different kinds of exercise, or received more, or less, or different kinds of injury.

${ }^{1}$ Bacon, Novum Organum, i. 59. 


\section{CHAPTER II}

\section{THE METHOD OF DEVELOPMENT ${ }^{1}$}

The adaptation of the individual-The method of adaptation-VariationsModifications-Progression-Retrogression-The recapitulation of the lifehistory-The opinions of embryologists.

38. T NDIVIDUALS who survive and have offspring, especially if they have a full quota of offspring, must necessarily have been well fitted from first to last, from ovum to adult, to their surroundings. The changes in the individual during development, especially in the higher animals, are very great, but, speaking generally, they are always adaptive. Thus the larva of the dragon-fly which inhabits the water is structurally fitted for a life in it, and undergoes an adaptive change when, as perfect insect, it migrates to land. Thus again the human embryo in the womb is fitted to an environment enormously different to that in which life is possible to the adult. All, or almost all, the changes it undergoes during development are adaptive also. Offspring, to survive in the same environments, must closely resemble their parents. This necessary resemblance is secured by development along similar lines. Step by step, from germ to adult, the child treads in the developmental footsteps of the parent. When, as occasionally happens, the recapitulation of the parental development is not fairly close, the child is a 'monster,' out of harmony with its environment, and so inevitably perishes.

39. Though every normal child resembles its parent as a whole, it invariably differs in details, which, though innumerable, are minute as compared to the likenesses. Thus, though the normal offspring of a human being is always another human being, it is never an exactly similar one. These differences, when innate (i.e. germinal) are known as variations, and must be carefully distinguished from differences which result merely from an unequal play of stimuli (nutrition, use, injury, etc.) on parent and child. The latter are acquired differences or modifications, terms which, as we have seen, are also used, though incorrectly, to distinguish 
nutritional characters from those which result from other stimuli. ${ }^{1}$ Small variations which grade into one another are termed 'continuous': large variations ('sports,' abnormalities, congenital deformities, 'mutations') are termed 'discontinuous.' ${ }^{2}$ All variations, all differences between parent and child due to differences in germ-plasms, may be placed in one or other of two categories : either they are progressive, or they are retrogressive. On progressive variations is founded progressive evolution, as when the wing of a species of bird undergoes increase in size or power. On retrogressive variations is founded retrogressive evolution, as when the wing, owing to lessened utility, undergoes subsequent decrease. $^{3}$ An extra digit on the hand of a child of normal parents is an example of progressive variation; a missing digit, if founded on a germinal peculiarity, not on a mutilation, is an example of retrogressive variation. Of course any given variation may be compound, consisting of both progressive and retrogressive variations. Thus a congenitally malformed digit may display both additions and subtractions when compared to the structure in the parent.

40. Obviously, since the child treads in the developmental footsteps of the parent, since, like the parent, it is in turn germ, embryo, fœtus, infant, child, youth, and adult, a progressive variation implies a complete recapitulation of the parental development (as regards the particular structure or character that has varied), plus an additional step; whereas a retrogressive variation implies an incomplete recapitulation, one or more of the steps of the parental development being omitted. The line of thought on which we are now engaged is so very essential to a clear understanding, both of evolution and heredity, that it will be well worth the reader's time to close this volume for a space, and to endeavour to think of a variation which is neither progressive nor retrogressive, but something else. He will find it impossible. If he then tries to

1 The reader must constantly bear in mind the real, or at least the more useful distinction between variations and acquired differences. Obviously, to take an example, there is, between two men one of whom is 'by nature' lean, who cannot get fat no matter what he eats, and the other who is thin merely because he is starved, a difference more radical, more innate than that between a fat, well-fed man and one who has been starved, but would get fat if afforded the opportunity.

2 See $\S$ 249, 286.

' Sir Ray Lankester has objected to the expression 'retrogressive evolution' on the ground that retrogression implies an exact and orderly reversal of the antecedent progression. But if we term the sum of progressive variations 'progression,' it appears right to term the sum of retrogressive variations ' retrogression.' At any rate I cannot find a better term and, at least, my meaning is clear. 
imagine a progressive variation which is not a prolongation, a step added to a complete recapitulation of the parental development, or a retrogressive variation which is not an abbreviation, an incomplete recapitulation of the parental development, he will fail again.

4I. Amongst typical unicellular organisms there can, of course, be no recapitulation of the kind that occurs amongst higher types ; the individual divides into two daughter-cells which resemble itself and which separate; there is no development of a cell-community, and consequently no recapitulation of the process of developing one. But with multicellular organisms from first to last recapitulation must have been the sole method of development. It is the only method by which the cell-community, the individual, as distinguished from the single cell, can grow into the likeness of the parent community, and so become adapted to the environment in which it finds itself. Starting from the same point, it must follow the same road to reach the same goal. The typical unicellular organism, on the other hand, treads no developmental footsteps ; it remains stationary at the starting-point. If the child of a man, for example, did not recapitulate the development of his parent he would be a 'monster.' Monsters are known to us, but, outside mythology, not monsters who survive and have offspring. Development of the cell-community, otherwise than by recapitulation of the parental development, is indeed not entirely inconceivable; for we can imagine that an elephant, for instance, may give birth to a mouse, or a human being to an acorn. But it never happens. It matters not in the least whether we accept the popular doctrine that the higher types were specially and divinely created as highly organized cell-communities, much like their latest descendants, or whether we hold the scientific theory that they were derived originally from lowly unicellular forms of which the germcell is the modern representative. In any case there must have been recapitulation of the parent in every generation. ${ }^{1}$

42. But, though, in the case of individuals who themselves survive and have offspring, the recapitulation of the parental development invariably occurs, it is not necessarily-indeed it never is-quite complete nor accurate. The child varies from the parent in every stage of development-as embryo, as fotus, and

${ }^{1}$ The argument is not affected by the occurrence of what is known as alternation of generations, by the budding of offspring from parents which have arisen from germ-cells, nor even by the fact that parents and offspring sometimes develop differently when the conditions under which development occurs are different. Under similar conditions they would have developed along similar lines. See $\$ 92$. 
as infant, as well as in the adult stage. Progressive variations, occurring at the end of the development, add to the parental total of development : retrogressive variations subtract from it; both kinds, occurring during development-interpolated into it-render it inaccurate, and, in the process of many generations, very inaccurate.

43. But, if the child recapitulates the development of the parent, the latter also recapitulated that of the grandparent, who in turn recapitulated that of the great-grandparent, and so on up to the first multicellular ancestor. Consider, now, the simplest conceivable case of progressive evolution followed by retrogression. Imagine a line of individuals A, B . . . L, M, in whom a structure undergoes uninterrupted increase in a single direction-as it were in a straight line-by successive progressive variations occurring at the end of the development of each successive individual. Suppose the structure began in $\mathrm{B}$ as a variation from $\mathrm{A}$, and underwent evolution by successive steps in $\mathrm{C}, \mathrm{D}, \ldots$ till it reached its culmination in M. Suppose also that it began to retrogress in $\mathrm{N}$, and that it continued to do so uninterruptedly till it quite disappeared in $\mathrm{Z}$.

44. Consider first the progression. Clearly, since each individual down to $M$ recapitulates the development of his parent and makes in addition another step, the development of B must consist in a recapitulation of $A$, followed by his own variation. $C$, again, since he recapitulates $B$, must first recapitulate $A$, then proceed to $B$ 's variation, and then to his own. $D$ in turn must recapitulate $A$, then the variations in order of $\mathrm{B}$ and $\mathrm{C}$, and then, and then only, proceed to his own variation. M, the last of the race in whom a progressive variation occurs, must found his variation on a recapitulation of $A$, plus a recapitulation in orderly succession of the variations of all the intervening ancestors. In other. words the development of the structure in $M$ is a recapitulation of its evolution in the race. In no other way that can be conceived could the structure have been evolved. Apply this reasoning to every structure and character in the body, and we perceive that the theory that every individual in his own development climbs his own genealogical tree must necessarily be true. Given the unquestionable fact that the child recapitulates the development of the parent, any method of development other than by a recapitulation of the life-history of the race is, not only impossible, but actually unthinkable. One truth necessarily involves the other.

45. But to say that development is a recapitulation of the lifehistory is one thing. To say that in every instance it presents a 
complete and accurate history is quite another thing. On the contrary, since variations occur in every stage of development, in every structure, and in every generation, since like other features of the germ-plasm these variations may be persistent, since, therefore, they tend to accumulate during the passage of generations, it is certain that in no single species and even in no organ or structure is recapitulation complete or accurate. The structure we have just considered reached its culmination in $\mathrm{M}$, and began to retrogress in N. Now, obviously, since development is by recapitulation, $\mathrm{N}$ cannot retrogress except by an act of incomplete recapitulation; that is by failing to reproduce the variation by means of which $M$ advanced a step beyond $\mathrm{L}$. Obviously again in that case $\mathrm{N}$ reverts to $\mathrm{L}$; in effect he is $\mathrm{L}$; and $\mathrm{M}$, unless his variation becomes latent, a contingency we shall consider later, ${ }^{1}$ disappears from the history. Of course, N's act of retrogression may be larger, and may carry him back to $\mathrm{K}$ or some remote ancestor, say F or E. O's retrogressive variation will carry him yet farther. $Z$, in whom retrogression is complete, will, in effect, be A, in whom the structure had not even its beginnings. In all M's descendants, therefore, the recapitulation will be incomplete. Even if the structure be evolved again in some of Z's descendants by a fresh series of progressive variations, it will still be incomplete. A chapter of the life-history will be missing even though one like it be added subsequently.

46. For the sake of clear thinking it must be noted that when we allude to individuals as persisting or disappearing from the life-history, we are using a mere figure of speech. As we have seen, the structures of offspring are not derived from the structures of their progenitors. In reality we are considering alterations in the germ-plasm, which, though capable of change, is continuous and potentially immortal. Germ-cells are unicellular organisms, with unicellular germinal descendants. Multicellular individuals, as we have seen, are merely episodes in the unending career of the germplasm, dwellings which it periodically builds about itself and from which it departs as they tend to become outworn. To be precise, then, the alterations in the germ-plasm are such that individuals recapitulate, with more or less accuracy, the evolution of the race of individuals that spring from the slowly changing germ-cells.

47. The case we have imagined-uninterrupted progression followed by uninterrupted retrogression-is purely ideal. Really in nature progression tends to alternate irregularly with retrogression. Thus, though the hand of every child tends to vary

\footnotetext{
1 See $§$ I 86-9; see also chapter vii.
} 
somewhat from that of his parent, the human hand has not altered appreciably during thousands of years. Even when progression or retrogression ensues as a whole, individual members of the race may reverse the process temporarily. Thus $\mathrm{B}, \mathrm{C}, \mathrm{D}$ may exhibit progression ; $E$ retrogression, through reversion to $C$; while $F$ may resume the progression and so resemble $\mathrm{D}$.

48. Moreover, structures possess breadth and thickness as well as length. Their progressions and retrogressions, therefore, are in three dimensions, and are founded on a number of variations, internal and external, quantitative and qualitative, which is practically unlimited. Each of these variations may be independent of all others; even single cells may vary independently; so that while progression is occurring in some respects, retrogression may be occurring in others. The apparent result, if we think of the structure as a whole, is chaos. But we are able to avoid the seeming confusion if we think of each variation separately. We then perceive that it must be progressive or retrogressive, that it must consist in a prolongation or an abbreviation of the life-history of the part as presented by the parent:

49. Lastly, as we have already noted, offspring vary from their parents, not only at the end of development, but during the course of it-not only as adults, but also as embryos. Not only do they add sentences (progressive variations), or omit sentences (retrogressive variations) from the end of the life-history as related by the parent, but they interpolate, or omit, or alter sentences in the body of the work. These interpolated progressive and retrogressive variations, like those occurring at the end of development, may or may not be inherited ; that is, they may or may not represent persistent changes in the germ-plasm. But many of them are persistent. They accumulate, and in the course of ages so alter the life-history, especially in its earlier parts, which have longest been exposed to change, that it may, and usually does, become unrecognizable. Nevertheless it ever remains a real history, a real recapitulation, though in part a recapitulation of past error, of additions and subtractions, and of interpolations which are compounded of both additions and subtractions. As a result, the human embryo, for example, differs so greatly from its prototypes of the life-history, that we cannot, with any degree of accuracy, trace the early ancestry of our race by watching the development of the individual. At first it is unicellular; then it faintly resembles very low multicellular types; next it reproduces in succession, more and more clearly, higher and higher types till it 
presents the appearance of an ordinary human being. But manifestly the additions and subtractions have been vast. It possesses, for instance, a placenta, an organ by which it is attached to the mother, through which it is nourished, and which at one time is larger than the embryo itself; but which, of course, could not have been present in its prototypes. On the other hand, the prototypes were capable of maintaining an independent existence by means of functionally active organs, which the embryo, fitted as it is only for a passive parasitic life within the uterus, has lost or almost lost. Nevertheless the life-history unfolded by the child is just as real, just as complete, and probably more accurate than any written chronicle that attempts to describe the whole past of a race.

"There is a history in all men's lives

Figuring the nature of the times deceased."

50. The history is not told in words, but in graphic signs, in mimicry. It was begun by the first multicellular ancestor, and then consisted of a single word or sentence, a single cell-division. Each succeeding generation copied it, and many generations added sentences. But the copying was never exact. Therefore, as the history lengthened it became legendary and even mythical in its earlier parts-the parts which have been longest and most often copied and emended, and are therefore most altered. Doubtless, as in written histories, almost every embryonic legend and myth is founded on fact; but it is as hard in the one case as in the other to dig the truth from the concreted mass of fiction. In some instances the earlier history is not represented even by legend. It is entirely lost, as in the case of plants which have ceased to propagate by seeds, and increase only by means of detached portions of the adult individual, such as buds and suckers. In other cases, as among the medusæ, whole volumes in the middle of the history have been gradually shortened till they have quite disappeared. ${ }^{1}$ In all cases, if the individual lives to complete the story, the last chapters are usually very full, and in the main exact-more full and exact than any written history can be. Thus the offspring of the human being, however shortened and altered its development, however vague its representation of its remote ancestors, is always another human being who reproduces, as a rule with wonderful completeness, all the immensely complex details that go to the making of a man. But even here, as in a

1 "In the history of the Hydroidæ any phase, planuloid, polypoid, or medusoid, may be absent." Dr Strethill Wright, quoted by Darwin; Animals and Plants, vol. ii. p. 364 . 
written history of a recent generation, small details, unimportant variations of the parent from the grandparent, are often omitted. Nature is careful to store in the graphic record which is the developing body of the individual, only those facts that are important and significant, only those variations which have played a real part in the evolution of the race. When writing her history she concerns herself, not with individuals, but with populations; she preserves, not the transient details of individual lives, the small variations which appear in the parent and are lost in the child, but only those great and enduring general movements by which whole races or sections of races have differentiated from the ancestral type. And her aim is strictly practical. Her history of ancestors is told for the benefit of descendants. Therefore even great general movements, when they grow remote, are eliminated from the record, or are replaced by legend and myth if they interfere with the smooth tenor of the narrative-if they interfere with the quick development of the child along the shortest and simplest lines.

5 I. Hitherto the doctrine of recapitulation has been accepted by scientific men on the evidence of observed likenesses between the embryos of the higher and the adults of the lower animals. It has not yet been realized that, given the undisputed facts that the organic world arose by evolution, and that the child recapitulates the parental development, any method of development other than that by the recapitulation of the life-history is literally inconceivable. When Darwin, succeeding where Lamarck, Spencer, and others had failed, converted a sceptical world to a belief in evolution, these vague likenesses were held to furnish the strongest proof in existence of his contention. Recently, however, some embryologists, deceived by the frequent unlikenesses, have denied the recapitulation of the life-history. According to them the resemblances are due to a "sort of memory" possessed by the embryo, a suggestion which may possess poetical merits. As a fact, we should know the doctrine of recapitulation as true even if an embryo resembling a lower type had never been seen, and it had been ascertained merely that the embryos of different generations resembled one another as much as the adult individuals. The function of an embryologist is not to furnish evidence for, or against, a necessary truth; but to ascertain to what extent this or that type of animal or plant recapitulates its evolution with completeness and accuracy. It is no excuse to declare that only complete and accurate recapitulation has been denied. No one, 
having even an elementary acquaintance with the facts, has alleged that recapitulation is ever other than incomplete and inaccurate. Thus no one has maintained that at one stage of human development the embryo is a water-breathing animal, with all its structures and faculties perfect, or that the ancient prototypes of the embryo swam about dragging a placenta behind them.

52. Summing up the foregoing, the question I wish to place before the reader is this: If offspring recapitulate closely but not exactly the parental development, if progression always implies a complete recapitulation plus an addition, and retrogression an incomplete recapitulation (with a step or steps subtracted), if through all the ages offspring have always recapitulated the main features of parental development, is it possible, or even thinkable, that individual development can be anything other than a recapitulation of the life-history - not an exact recapitulation, but one with additions and subtractions?. 


\section{CHAPTER III}

\section{THE METHOD OF SCIENCE}

Biological sects-Facts patent to our senses-Facts obscured to them-The function of laboratory methods of inquiry-The methods by which the facts of the various sciences are observed-' Exact Methods'-The nature of science-The scientific value of facts-The mental processes by means of which facts are classified - Hypotheses and theories-The necessity for testing thinking-The method by which it is done-Induction and deduction-Laboratory methods when used as means of discovery and as tests for thinking-The common neglect of testsThe essence of the experimental method-The value of controversy-The distinction between facts and theories-The legitimate and illegitimate uses of deduction-The contrasts between physical, chemical, and biological theoriesThe value of experiment in these sciences-Laws of nature-Deduction an essential part of the mental processes of rational beings.

\section{3. $7 \mathrm{HE}$ reasoning by which we have demonstrated that the development of the individual is a recapitulation of the evolution of the race, is in some measure de-} ductive. The facts from which we started our thinking have all been gathered by simple observation. Thus the fact that offspring tend to recapitulate with variations the parental development has been simply observed. The language used by some biological workers indicates an opinion that deduction is illegitimate, or at least unsafe. ${ }^{1}$ Others maintain, in effect, that materials furnished by experiment, or some such aid to observation, affords the only safe basis or test for reasoning. ${ }^{2}$ The points thus raised, involv-

${ }^{1}$ The statement that deduction-any and every sort of deduction-is con . sidered illegitimate by any section of scientific workers may be received with incredulity by people who are aware of the part it has played in the creation of science. An acquaintance with medical literature and thought would dispel any doubt. For instance, three years ago there was published a large work (The Food Factor in Disease, by Francis Hare, M.D. ; Longmans, Green and Co.), the preface of which actually consists in an apology for the use of the method-an apology for testing thinking. The book, an exceptionally original, thoughtful, and able one, failed, I understand, completely.

2 "The recognition that only by experimental methods can we hope to place the study of Zoology on a footing with the sciences of chemistry and physics is a comparatively new conception, and one that is by no means admitted as yet by all zoologists. I do not wish to disparage those studies that deal with the descriptive and the historical problems of biology. They also afford a wide field for activity, and the more familiar we become with the structure and modes of development 
ing, as they do, questions as to what constitutes valid thinking and what reliable evidence, are so fundamentally important that it is necessary to consider them at some length.

54. Students of heredity and evolution who think about, as well as record, the facts they observe, are divided into schools, some of which overlap; for example, the selectionist, the mutationist, the Mendelian, and the biometric. If these divisions indicated merely associations of men who adopt different methods of research, then, since a division of labour is advantageous when dealing with a subject so large and difficult, the existence of schools would be, not only harmless, but useful. Differences of opinion could then be settled as they arose by a comparison and sifting of evidence. But unfortunately the separation is some-

of animals, so much the better can we apply the experimental method. In fact, many of the problems of biology only become known to us as the result of direct observation. The wider, therefore, our general information, the greater the opportunity for experimentation.

"It is undoubtedly true that many zoologists who have spent their lives in acquiring a broad knowledge of the facts of their science fail to make use of their information by testing the very problems that their work suggests. This is owing, no doubt, to their exclusive interest in the observational and descriptive sides of biology, but also in part, I think, to the fact that the experimental method has not been sufficiently recognized by zoologists as the most important tool of research that scientists employ. (T. H. Morgan, Experimental Zoology, p. 3.)

"The essence of the experimental method consists in requiring that every suggestion (or hypothesis) be put to the test of experiment before it is admitted to a scientific status. From this point of view the value of a hypothesis is to be judged, not by its plausibility, but by whether it meets the test of experiment." (Op. cit., p. 6.)

"It is sometimes said that nature has already carried out innumerable and wonderful experiments, and that we can never hope to excel her in this power. Is it not better, therefore, to examine patiently and reverently what she has done, and in this way learn how her processes have been carried out? Let us not be blinded by rhetorical questions of this kind. No doubt nature has carried out prodigious experiments; but we can never be certain that we know how she has obtained her results until we repeat the process ourselves. What would the chemist or the physicist say if he were told that nature has already carried out experiments on a much greater scale than he can hope to accomplish, and that he should drop his experimental methods and study his physics in a thunderstorm and his chemistry in an eruption." (Op. cit., p. 8.)

"If the truth must be told, the experimental method was given up for a long time by the majority of specialists themselves in favour of the controversial, and, indeed, this tendency has by no means yet died out among the habits of some professed evolutionists. On the other hand, during the past fifteen to twenty years, a few scattered workers have diligently applied themselves to the study of the facts of variation and inheritance, with results which already more than justify the anticipation in which their work was begun-namely, that by such methods alone can any real progress in our knowledge of the processes of evolution be brought about." (R. H. Lock, Variations, Heredity, and Evolution, p. 3.) 
times more radical. Exactly as in religion, it depends largely on the fact that the schools, or some of them, value, or seem to their opponents to value, evidence, not in proportion as it is authentic and relevant, but in proportion as it has been gathered by this or that process of inquiry.

55. Now, of course, both the methods of thinking and the evidence on which the opinions of any school are based may be altogether wrong, and, therefore, their opponents may be right in refusing to use them. But, in that case, the reasons for rejection should be set out plainly and precisely. It is not enough to ignore evidence, or stigmatize thinking as 'deductive,' or 'philosophic,' or 'obsolete.' Epithets are very unconvincing to opponents. A reasoned statement ought to justify what in its absence has the appearance of mere prejudice. Otherwise, in the lack of common criteria for facts and methods of thinking, differences of opinion tend to become as irremovable and irrational as those of religious sects. Scientific men, no matter how open-minded, are sure, of course, to differ more or less in opinion, for they differ in knowledge and reflective power; but sectarian differences arise only when evidence and modes of thought are accepted or rejected on improper grounds.

56. In the present chapter I shall discuss the question as to what kinds of evidence and processes of thought are permissible in science. Like other men, I belong, more or less exclusively, to a particular school; but, very naturally, like them, I believe I am not a member of a sect. I recognize, however, the possibility that I, not the people with whom I disagree, may be prejudiced; at any rate $\mathrm{I}$ recognize that my opinions may appear as prejudices to opponents, unless I first demonstrate that the facts and thinking on which they are founded are such as it is right to use. Hereafter, since I shall not have begged the question by merely assuming that certain classes of facts and processes of thought are particularly right or particularly wrong, but shall have stated explicitly the grounds for my convictions, the reader, if he differs from me, will at least have it in his power to indicate exactly and easily where and how I am mistaken. If he finds I am wrong in the present chapter, I am sure he will be wise not to proceed further; for it is not possible that reasoning that is based on initial inaccuracies of fact or thought can contain anything of value. However, I do not believe he will think me mistaken. He may wonder, indeed, why I have taken the trouble to write the chapter. I am tolerably sure that all of it will seem obviously true and previously 
known to him; but, if he has the patience to read the rest of the book, he will find that it was very necessary. In numbers of recent publications the rules of ordinary scientific procedure have been broken, and the breach, so far from being admitted as a fault, has been claimed, explicitly or implicitly, as a merit. Even a cursory consideration of scientific controversies renders it evident that the fundamental source of almost every disagreement has been a neglect, on one side or the other, to conform in practice with that which in theory is regarded as a truism. Therefore, if we can reach a preliminary agreement as to the materials for thought and modes of thought which it is legitimate to employ, more than half of the causes of disagreement will be eliminated at a stroke. At this stage, for lack of illustrations with which to point the argument, the discussion will not be very complete. For example, I shall find it impossible to demonstrate that masses of valuable evidence, bearing on various great problems of heredity, have frequently been ignored without valid excuse. But throughout the remainder of the work I shall indicate illustrations as they arise.

57. Some facts-by far the greater number of facts known to us-are patent to our senses; that is to say, we have only to look, feel, hear, taste, smell, or invoke the muscular sense, and we become aware of them. It is the function of our senses to supply us with facts, and without them we could not maintain existence. But other facts are of such a nature, or are so obscured by the settings in which they occur, that they cannot be directly observed by our senses, or inferred from the evidence supplied by them with any degree of certainty. We have, then, to resort to experiment, biometry, or some such 'laboratory' method, to render, if possible, these obscured facts as certainly known as those which are patent from the first. When used for purposes of discovery, a laboratory method is nothing other than a means of eliminating obscuring conditions and so making hidden facts perceptible, or at least capable of being inferred with greater certainty. "The object of experiment is to eliminate unessential conditions in the phenomena ; when complete knowledge of the essential conditions can be obtained by observation, experiment is unnecessary." 1 "Experiment only has an advantage over observation, in so far as it is capable of supplementing the usual deficiencies of the latter." 2

58. The ascertained facts of some sciences, for example, systematic zoology, botany, anatomy, and geology, are almost all

1 Welton, Manual of Logic, vol. ii. p. II 5.

${ }^{2}$ Lotze, Logic, Eng. Trans., vol. ii. p. 40. 
patent. If we are possessed of normal faculties, we have only to observe in the right places and at the right times and we are able at once to note them. Experiment would be useless and absurd, indeed impossible, here. Thus we could not by experiment ascertain that a species of butterfly was winged and contained several varieties. There could have been no such sciences as zoology and botany had we depended on experiment. Therefore their students have relied almost exclusively on simple observation. The right times and places in which to observe may be hard to find, but, once found, the phenomena can be noted.

59. In other sciences, for example, physics and chemistry, many, indeed most of the facts are obscured. Thus we cannot by our unaided senses discover the composition of water or alcohol, or the way in which gases behave under pressure. Therefore physicists and chemists have been obliged to rely very largely on experiment.

6o. The study of heredity, which draws its materials from every science that deals with life, stands midway. Here the laboratory cannot create a science; it can only help to create one. Most of what we know about life is patent to our senses and can be simply observed. Especially is this the case with regard to facts of structure, for example, the shape, size, and anatomical relations of the parts of an animal. But some facts, especially those relating to function (e.g. the function of the thyroid gland), are obscured, and to discover them, if it be possible, we must resort to a laboratory method. Therefore, if we say, or imply that facts supplied by simple observation are useless to the students of heredity, we declare, in effect, that most facts relating to structure and many relating to function are useless-we declare, in effect, that when studying heredity we must not draw our facts from systematic zoology, botany, comparative anatomy, embryology, palæontology, indeed most sciences that deal with life.

6I. In contradistinction to simple observation, the laboratory methods of inquiry have sometimes been termed 'exact'; but, very obviously, the term, implying as it does that simple observation is necessarily less exact, is erroneous. They are especially exact on particular occasions only. For example, we do not need them to make us entirely sure that men resemble apes physically more than they do other animals, that the offspring of human beings are human, that every generation follows closely but not exactly in the developmental footsteps of the one that precedes it, that the forms of life which have inhabited the earth have changed with the geological epochs, that stags have antlers and elephants have tusks, 
that Englishmen are on the average fairer than negroes and taller than African pigmies, and so on as regards millions of facts.

62. On the other hand, we cannot without the experiment of weighing discover the precise weight of a stag, nor without biometry the exact degree in which Englishmen are on the average fairer than negroes and taller than pigmies. In a sense, of course, the statement, for example, that Englishmen are on the average exactly so many inches and fractions of an inch taller than pigmies is more precise than the bare statement that they are taller; but only in a sense. For the one statement is not truer, but only contains more of truth than the other. That is, it is more detailed. Obviously, whether or not the additional details are needed in any given inquiry, depends on circumstances. They are not always needed.

63. Beyond question, biological facts discovered in the laboratory are often very valuable-as valuable as any other verified facts, and on particular occasions more valuable because designedly discovered with a view to fill gaps which prevent the linking together of facts already known. There is, however, nothing especially magical, scientific, or accurate in data obscured to our senses till revealed by a laboratory inquiry. Such an inquiry can do no more than render them as patent, but no more patent than the majority of facts on which our knowledge of living beings is based. The latter class of facts, indeed, are usually more capable of easy verification than any discovered in the laboratory. In noting them we have only to guard against errors of observation, not also against errors in experimenting. If the reader will think over the evidence on which I shall draw for the purposes of the present volume, I believe he will conclude that, if any of it bears a doubtful aspect to his mind, it is that large mass which has been furnished by laboratory inquiry; for, while some of the latter is controverted, and all of it must be accepted by most people at second hand, nearly all the rest is indisputably true, as he will know from his own experience of life.

64. Sometimes it is said that we must make biology an exact science by imitating the methods of physicists and chemists-by which is meant that we must experiment, or compile statistics. It is right that we should do both when necessary; but, plainly, physics and chemistry are exact sciences, not because the facts on which they are based are obscured and have to be laboriously discovered by laboratory methods, but because they deal with data that are capable of being exactly measured, and because physicists and chemists have had both the means and the will to test their 
thinking exactly.1 Mathematics, which is not an experimental science, is exact for similar reasons. The study of heredity is not, and cannot be, exact in the same sense, for no two individuals, nor even two of their cells, are ever precisely alike. When we unite a given quantity of oxygen with a given quantity of hydrogen, the result, which can always be predicted with absolute confidence, is ever a definite quantity of water of a definite composition. But, when two germ-cells are united, it is impossible to foresee the exact nature of the product. Doubtless, when dealing, not with individuals, but with populations, biometry, the statistical method of investigating the phenomena of life, does in some cases enable us to forecast the future with considerable accuracy; but this accuracy is infinitely less comprehensive than that obtained in physics and chemistry where predictions are true, not only as regards averages, but also as regards particular instances. It is conceivable, though, owing to the complexity of the conditions, not at all probable, that biologists will one day be able to predict with the same accuracy and confidence as physicists and chemists; but it is hard to understand how this goal will be the sooner attained by ignoring everything that is patent to our senses. Physicists and chemists, as rational beings, would be happy if their facts were patent, and they were thus spared some of the labours of the laboratory. It is their good fortune, not their merit, that they are able to measure and test exactly.

65. In brief terms, sciences are experimental or non-experimental in proportion as the facts on which they are founded are, or are not, obscured. They are exact or inexact in proportion as they are, or are not, capable of being founded on precise measurements. Some sciences are both experimental and exact; but the two circumstances have no necessary relation. Dogs run and wag their tails. But they do not run because they wag their tails. Neither are sciences exact because they are experimental. In science, as in the ordinary affairs of life, a source of error as common as or more common than the use of inaccurate data, is a neglect to use the whole of the facts available. Certainly the use of all the evidence is, through the exposure of discrepancies, one of the most effective means of ascertaining whether any of it is inaccurate or has been misinterpreted.

66. Science is founded on facts. But collections of facts do not by themselves constitute science. They are only the raw materials out of which it is manufactured. Science consists in the 
classification, the systematization of facts. When we classify facts, we indicate their relations one to another; in other words, we interpret them in terms of one another. Therefore, not only observation, but thought, is necessary. "The classification of facts, the recognition of their sequences and relative significance, is the function of science." 1 "The goal of science is clear-it is nothing short of the complete interpretation of the universe." 2 "There is no way to gain a knowledge of the universe except through the gateway of the scientific method. The hard, stony path of classifying facts and reasoning upon them is the only way to ascertain truth." 3 "We must never forget that it is principles, not phenomena-the interpretation, not the knowledge of factsthat are the objects of inquiry." 4 "The world only becomes intelligible when it is conceived as a systematic unity, the elements of which are throughout in necessary relation to each other. The attainment of knowledge is nothing but the more thorough and complete determination of these relations. Hence, every element of reality is understood just to the extent to which its necessary relations to other elements are grasped. No doubt reality as presented to us is infinitely complex, and consists of various phenomena which resemble each other generally, yet differ indefinitely in detail. But every postulate of knowledge compels us to think every variation and every detail, even the smallest, as so determined by conditions that, under the circumstances, it could not possibly be other than it is." 5 "Scientific thought does not mean thoughts about subjects with long names. There are no scientific subjects. The subject of science is the human universe ; that is to say, everything that has been or may be related to man." 6

67. As there are no scientific subjects, so there are no scientific facts. There are only facts which have, or have not, been used in science-which have or have not been systematically classified. We have only to think of our houses and friends, of our general surroundings, to recall countless facts quite as indisputably true as any that have been incorporated into science, but which, owing to our deficiencies, are not as yet facts for science; for though each of us seeks to classify these everyday facts in such a way as will enable him to move in comfort and security in his world, yet we cannot link them together in such categories as will enable man-

${ }^{1}$ Pearson, Grammar of Science, ed. 1900, p. 6.

${ }^{2}$ Op. cit., p. I4.

s op. cit., p. I7.

${ }^{4}$ Herschel, Natural Philosophy, § Io.

5 Welton, Manual of Logic, vol. ii. p. 188.

${ }^{6}$ Clifford, Essays, p. 86. 
kind in general to interpret nature in that systematic way that constitutes science.

68. The scientific value to us of a fact depends, first, on our power to verify it, to make ourselves and other people sure that it is a fact; and, second, on our power to classify it, to recognize its relations to other bodies of verified evidence. All classification is, in essence, a process of reasoning, of interpreting. If, armed with a note-book, I went into the world and jotted down accurate statements concerning the individual animals and plants I happened to meet, my collection of facts would not constitute science. But if, with their help, I were able to classify living beings into species, orders, genera, and kingdoms, my facts would become, what they were not before, foundations of science. During the process of classification, as I passed from individuals to varieties, and from the latter to species, and so on, my descriptions would become more and more brief, simple, and generalized. Instead of describing many individuals I would describe a single variety; instead of describing many varieties, I would describe a single species, and so on. But, since a knowledge of species involves a knowledge of varieties, which, in turn, involves a knowledge of individuals, each step in this simplification, this classification, would involve the manipulation of larger and more complex masses of facts, and, therefore, more complex thinking. But, as long as my facts were properly verified and classified, neither they nor the processes of thought involved would grow any the less, or more, scientific for being more complex. If, from the data I had gathered, I were able to formulate a theory of evolution, I should classify an immense body of facts in a new way that would involve very farreaching and complex thought.

69. Obviously, however, the complexity of the mental processes involved in classification does not depend wholly, or even mainly, on the number of facts to be classified; for, however numerous the facts, their relations may be apparent on the surface. In many cases, we have only to discover the facts, and they fall, by themselves as it were, into the right categories. Thus in anatomy, though the mass of facts is large, and the discovery of some of them may be difficult, it is comparatively easy to describe the bones, ligaments, nerves, and the rest, and state their anatomical relations. It is different with such a study as heredity. Here the relations between the facts are not easy to trace, and the mental processes involved in classification are correspondingly complex and subtle. Thus, suppose a strong and athletic man, who has 
good health and takes plenty of exercise and nourishing food, has a son resembling himself, then what is the relation between the facts? To what shall we attribute the likeness between father and son? Is the son strong and healthy merely because he was 'born' with the characteristics with which the father was 'born,' and because he has lived like him? Or has the life led by the father altered the germ-plasm and improved the child's heritage? Or are both factors to be taken into account? Obviously, our task in ascertaining the relations between the facts is harder here than in anatomy. We are obliged to draw a 'mediate' inference. The immense number of erroneous and discarded hypotheses with which the past of heredity is littered is evidence of the difficulty found by its students in drawing correct inferences. ${ }^{1}$

70. An inference is a hypothesis. But at first it is only a working hypothesis, a mere guess. Before we can be sure it is true, before we can convert it into an established theory, we must test the thinking by which it was reached, and so ascertain whether it is correct. Now there is one way, and one way only, by which the truth of hypotheses can be tested. As we test facts by appealing to facts, so we must test thoughts by appealing to thoughts-to inferences. We must proceed by two steps. First, we must make a rigorous deductive inference of consequences. Next, we must ascertain by an appeal to reality in the world around us whether these predicted consequences actually do occur. ${ }^{2}$ For example, when families dwell under unhealthy conditions, as in the slums of great cities, the parents tend to be sickly and the children puny. In this case

1 The necessity of adopting diverse methods when observing and classifying the facts of the various sciences is hardly realized by some students. Occasionally one hears an anatomist, zoologist, or botanist, a 'systematist,' lament the rage for speculation and declare that the function of science is merely to observe and classify facts-by which is meant of course the kind of observing and classifying that is usually adequate for the purposes of the systematic sciences. But, obviously, such observing would be of little use to the chemist, and such classifying, when applied to a subject so abstruse as heredity, would totally fail to establish the relations between the facts. The difficulty of simply observing compels the chemist to experiment; the difficulty of classifying compels the student of heredity to speculation, which, however, when founded on strictly ascertained facts and rigorously tested by a deductive inference of consequences, is not unscientific, but merely an especially strenuous effort to think accurately-to classify correctly. See $\$ \S 8$ I9 et seq.

2 " Every hypothesis is an attempt to find meaning in observed phenomena, to constitute reality in a rational way. It follows that the fundamental condition of a valid hypothesis is that it should explain and give meaning to the facts of observation. And it can only do this if it embraces those facts in that systematic whole which is the one form under which it is possible to think the universe. This 
we have a choice between two hypotheses, two inferences, two statements of what may be the relations between the phenomena. First, we may infer that parental ill-health tends to injure the germ-plasm, and, therefore, that the children are innately puny, innately incapable of becoming robust. If this hypothesis is true, then it follows as a necessary consequence that, if the children continue to dwell in the unhealthy environment, the weakened germplasm will be still more injured and the grandchildren will be still more puny, and therefore that the race will degenerate step by step. At any rate, if it can be shown that races become innately degenerate under such conditions, the hypothesis may be regarded as a theory the truth of which has been established. Here,

(a) The facts are that the children of unhealthy parents are very frequently puny.

(b) The hypothesis, the induction, is that parental ill-health tends to injure the germ-plasm, and so render the children and their descendants innately puny.

(c) The deduced consequences are that families, classes, and races, exposed to unhealthy conditions, tend to become degenerate.

(d) The appeal to reality is the ascertaining whether populations exposed to unhealthy conditions actually do become degenerate.

By this means we test our thinking, our hypothesis. Second, we may infer that the puniness of the children is due, not to the deterioration of the germ-plasm, but merely to inferior development resulting from the unhealthy conditions to which the children themselves are exposed. That is, we suppose that the puniness is not innate. If this second hypothesis is true, the race will not grow degenerate through being exposed to unhealthy conditions. On the contrary it will become more resistant to those conditions through the weeding out of the naturally less resistant individuals. Having drawn these two contradictory inferences, we have, as I say, to ascertain which of them is true by appealing to reality in the world around us. ${ }^{1}$

general condition, then, may be considered as involving three subordinate conditions :-

(I) That the hypothesis be self-consistent, and in harmony with all the other laws included in the conceived system of reality.

(2) That it furnish a basis for rigorous deductive inference of consequences.

(3) That these inferred consequences be in agreement with reality.

Of these conditions the first two are applicable to the formation of every hypothesis, no matter how provisional a character it may have; the third is a condition of the acceptance of a hypothesis as true." (Welton, Manual of Logic, vol. ii. pp. 95-6.)

${ }^{1}$ See $\$ 733$. 
7I. It is obligatory that we make this deductive appeal to reality. When it is done, certain facts-all we find it possible to employ in this way-are used as the basis of the hypothesis. Next other facts -all the rest which are relevant and known to us-are used, in as many ways as possible, to test the truth of the hypothesis. The latter class of facts are usually much the more numerous. In brief, we found the hypothesis on certain relevant facts and prove it by means of the rest. In this way only, especially when we are engaged in the elucidation of some obscure and difficult matter, is it possible to use all the facts. If we do not proceed thus-first formulating our hypothesis, next making a rigorous deductive inference of consequences, and lastly appealing to reality for confirmation-our thinking is mere guessing. A hypothesis is quite valueless to science unless it permits a deductive appeal to reality, and quite unproved until it has been found to stand that test. "The sole condition to which we need conform in framing any hypothesis is that we both have and exercise the power of inferring deductively from the hypothesis to the particular results, which are to be compared to known facts." 1 No scientific worker, that was not a mere observer, has ever done great work who did not so test his hypotheses; no hypothesis has ever been permanently included in science but has been so tested. "If it be an advantage for the discoverer of truth that he be ingenious and fertile in inventing hypotheses which may connect the phenomena of nature, it is indispensably requisite that he be diligent and careful in comparing his hypotheses with the facts, and ready to abandon his invention as soon as it appears that it does not agree with the course of actual occurrences. This constant comparison of his own conceptions and supposition with observed facts under all aspects forms the leading employment of the discoverer; this candid and simple love of truth, which makes him willing to suppress the most favourite production of his own ingenuity as soon as it appears to be at variance with realities, constitutes the first characteristic of his temper. He must have neither the blindness which cannot, nor the obstinacy which will not, perceive the discrepancy of his fancies and his facts. He must allow no indolence, or partial views, or self-complacency, or delight in seeming demonstration, to make him tenacious of the schemes which he devises, any further than they are confirmed by their accordance with nature. The framing of hypotheses is, for the inquirer after truth, not the end, but the beginning of his work. Each of his

${ }^{1}$ Jevons, Principles of Science, p. 265. 
systems is invented, not that he may admire it and follow it in all its consistent consequences, but that he may make it the occasion of a course of active experiment and observation. And if the results of this process contradict his fundamental assumptions, however ingenious, however symmetrical, however elegant his system may be, he rejects it without hesitation. He allows no natural yearnings for the offspring of his own mind to draw him aside from the higher duty of loyalty to his sovereign, Truth: to her he not only gives his affections and his wishes, but strenuous labour and scrupulous minuteness of attention." 1

72. In science, therefore, we must not only use words that express our meanings unmistakably, ${ }^{2}$ we must not only verify our facts, but we must always test our thinking deductively "by observing the present state of the world, by assiduously studying the history of past ages, by sifting the evidence of facts, by carefully combining and contrasting those which are authentic, by generalizing with judgment and diffidence, by perpetually bringing the theory we have constructed to the test of new facts, by correcting or altogether abandoning it, according as the new facts prove it to be partially or fundamentally unsound." 3 All this is axiomatic, and has been axiomatic since men began to think scientifically. Therefore it would be difficult to conceive anything more absurd than the statement that we must eschew deduction and rely wholly on induction. "All inductive reasoning is but the inverse application of deductive reasoning. Being in possession of certain particular facts or events expressed in propositions, we imagine some more general proposition expressing the existence of a law or cause ; and deducing particular results of that supposed general proposition, we observe whether they agree with the facts in question." 4 Induction and deduction "differ only as the road by which we ascend from a valley to a mountain does from that by which we descend from the mountain to the valley, which is no difference of road, but only a difference in the going." 5 Induction is that process by which we infer a generalization from a consideration of particular facts or from a consideration of inferior inductions, each of which is then used as a fact. Thus it is an induction which has been drawn a thousand times that parental ill-health results in innately enfeebled offspring. It is also a common induction that it has no such effect on offspring. Deduction is the process

${ }^{1}$ Whewell, Novum Organum Renovatum, pp. 80-1.

2 See chapter $\mathbf{i}$.

3 Macaulay.

4 Jevons, Principles of Scrence, p. 265.

' Port Royal Logic, p. 314. 
by which we test the truth of our inductions. Obviously, we cannot test the truth of either of the foregoing inductions, nor decide between them, unless we appeal deductively back again to the facts of the world around us. Experiment is one of the ways in which we may test an inference. After we have considered a collection of facts and drawn an induction, we say to ourselves, "If our hypothesis is true, if we have thought correctly, such and such consequences must follow "; and, if possible, we experiment to ascertain if they do follow. If they do actually follow, then there is a high probability that our hypothesis is correct, and that probability is raised to a higher degree by every new and successful appeal to reality. On the other hand, a valid appeal, experimental or other, if unfavourable, immediately and conclusively demonstrates the falsity of the hypothesis, which therefore must be abandoned or emended. "Thus Kepler records that he advanced nineteen hypotheses which he afterwards disproved, before he arrived at the true statement of the laws of planetary motion." 1 "A similar spirit was shown by Newton in respect to his hypothesis that the moon is retained in her orbit by the force of gravity. From this hypothesis he calculated that the moon ought to be deflected from the tangent of its orbit something more than fifteen feet every minute. But the apparent deflection was only thirteen feet. This discrepancy, comparatively small though it was, Newton accepted as a disproof of his hypothesis, and 'laid aside at that time any further thoughts of this matter.' But some fifteen years later the distance of the moon from the earth had been more exactly ascertained, and Newton repeated his calculations, working with these new values. The agreement between the calculated and the normal deflection was then seen to be remarkably precise, and the hypothesis became an established theory." 2

73. Unless an experiment is entirely aimless, entirely a product of unscientific thought, it is always an outcome of deduction. Even when it is used, not to test thinking, but merely to elicit fresh information, it is still an outcome of deduction; for it is deduction which leads us to suppose that our experiment will have useful results. It follows that there are two ways in which we may use experiment (or any other laboratory method). First we may use it as a deductive test of previous thinking, in which it tends to secure great accuracy in thinking. Second, we may use it as a means to reveal an obscured fact, when, of course, it tends to secure accuracy in observing. But, in the latter case, if we base

${ }^{1}$ Welton, Manual of Logic, vol. ii. p. 66.

${ }^{2}$ Op. cit., vol. ii. p. 88. 
thinking on the result of the experiment, in other words, if we reason, not towards the experiment, but from it, then our thinking is not tested by it. We get information which may be very accurate and very valuable ; but we get no more than this information. The subsequent thinking, if any, may, or may not, be accurate. To discover its accuracy we have to use tests which that particular experiment does not furnish. Now it is a very common delusion that, because experiment when used as a test tends to render thinking accurate, therefore all thinking connected with experiment tends to be.accurate. Consequently a neglect to test thinking founded on experiment, combined with an assumption that such thinking is necessarily accurate, is very frequent. A hypothesis, whether resulting from induction or deduction, which has stood a valid experimental test, has stood a single test so nearly conclusive that it can be made more conclusive only by continued testing by other appeals to reality. But a hypothesis which is merely founded on obscured facts revealed by experiment has no special claims to accuracy. For example, if I, having a notion that Brown wishes to enter my house surreptitiously, give him the opportunity and entrap him, my hypothesis is tested by experiment. It is then very nearly conclusively proved; the only doubt remaining being due to the fact that at the time that I supposed Brown wished to enter my house he might not yet have thought of it. But if, on the fact revealed by the experiment, I build the notion that he came to steal the spoons, I must test my new hypothesis by a fresh appeal to reality. It matters not, however, whether I discover the spoons on him by a formal experiment (i.e. by searching him), or whether I simply observe them sticking out of his pocket. In either case the appeal to reality, if successful, is equally decisive. In everyday life it is often not worth while to test our hypotheses, but, however much science occupies our thoughts, our thinking is never scientific unless we do so.

74. The two functions of experiment, testing and discovery, are sharply distinct. Nevertheless they are often confused by people who claim for hypotheses founded on experiment the high scientific status of theories tested by experiment. We shall see that while many important biological hypotheses have been founded on experiment, there is hardly an example of one which has been tested by it, or which it is practicable to test by it. Moreover, we must ever keep in mind the manifest truth that the field of biological experiment is limited. A suitable experiment, whether to test thinking or reveal facts, cannot always be devised. Thus, in the case we have just considered 
as to whether unhealthy conditions are causes of racial degeneration or of evolution, no" suitable or practicable experiment can be thought of. ${ }^{1}$ Hundreds or thousands of experiments have been performed, it is true, but with the most inconclusive results. ${ }^{2}$ Here, before we are able to reach a valid conclusion, we must appeal to a far wider area of reality, which includes not only the experimental data, but very much more besides. ${ }^{3}$

75. In biology, then, the essence of the experimental method does not consist " in requiring every suggestion (or hypothesis) to be put to the test of experiment before it is admitted to the scientific status"-an impossible task in many cases and foolish in more; but only in requiring that every suggestion which can usefully be so tested shall be so tested. Since such suggestions are relatively very few, exclusive reliance on experiment would result in a classification of facts so imperfect as to be unworthy of the name of science. The statement, "No doubt nature has carried out prodigious experiments ; but we can never be certain that we know how she has obtained her results until we can repeat the process for ourselves," 4 implies a lack of insight or a singular unawareness of known facts. For example, every instance of an individual who has offspring during or after suffering from a general disease is a very stringent experiment carried out by nature and not to be bettered by man. The accumulated result of millions or billions of such experiments, continued for thousands of years on hundreds of successive generations, are-because the instances are so numerous, obvious, and confirmatory of one another, because we are able successfully to employ every known method of induction, and because we can test the thinking thoroughly by deduction-every whit as easy to infer with certainity as if they had been undertaken by the most careful experimental worker. ${ }^{5}$ "Controversy " ${ }^{6}$ implies criticism. To abandon it would be to abandon the critical method, which, in turn, would mean the rash and unquestioning acceptance or ignoring of hypotheses. Biological disputes are comparatively seldom due to disagreements about facts. Almost always the opponents of a hypothesis suppose that its authors have drawn wrong inferences, and their objections are attempts to test the latter by demonstrating that they are not in harmony with the conceived system of reality. Fortunately, however, the labours of experimental workers have tended, on the whole, to increase, rather than diminish, controversy. The experimental method is

1 See $\S$ I 35 .

${ }^{4}$ See $\S 53$ (footnote).
2 See §§ I 32-4, I40-4, I60.

5 See chapter xiii.
${ }^{3}$ See $\$ \S 145$ et seq.

6 See $\$ 53$ (footnote). 
not new in biology. That which, amid such a wealth of patent facts, is very new, is exclusive reliance on obscured facts and a confusing of experimental discovery with experimental testing. "What would the physicist or chemist say if he were told" that he must not use patent facts, for example those of astronomy, when inventing or testing his theories? An experiment, or any other laboratory inquiry, is a mode of observing, not of thinking. It is sometimes an appeal to reality which results from deductive thinking, but unless correctly thought out, not necessarily a decisive appeal. It is valuable as testing our thinking or as making patent a fact previously obscured ; but often we are able to appeal to evidence patent from the first, and an obscured fact, newly discovered, is not necessarily more valuable to us than a patent fact that has long been ignored or insufficiently used. An observer is quite as likely to found illegitimate thinking on the results of experiment as on any other data. Indeed, thinking founded on experiment has often been deplorably reckless and inaccurate.

76. The function of deduction is not limited to the testing of inductions. "If the hypothesis is true, it will generally be possible to infer deductively from it facts which have not been before explained or which have even been unobserved. . . As Whewell says, "When the hypothesis of itself, and without adjustment for the purpose, gives us the rule and reason of a class of facts not contemplated in its construction, we have a criterion of its reality, which has never yet been produced in favour of falsehood.' 1 The history of science is full of such extension and prediction. Thus, for example, the discovery of Neptune was predicted by deductive reasoning from the principle of gravitation." 2 Again, the universe is a unity in which every phenomenon is related, however remotely, to every other. A brief yet comprehensive statement, in which the relations of a number of phenomena are summarized, is termed by us a law; and it is the aim of science not only to express the relations of phenomena in laws, but to indicate the relations of these laws-to weld them into larger syntheses, more comprehensive interpretations. ${ }^{3}$ In this process of welding deduction plays an

1 Novum Organum Renovatum, p. 90.

2 Welton, Manual of Logic, vol. ii. p. 100.

${ }^{3}$ What is a natural 'law'? It is commonly described as an "observed uniformity," "the statement of a general truth ; that is, a truth that holds good universally in that science, as contrasted with a particular truth, which holds good in some cases only" (Welton, op. cit., vol. i. p. I I). But this definition is too wide (see $\S 586$, footnote). The flowers of the common primrose are yellow; mammals have heads; birds are bi-pedal. These are observed uniformities, 
essential part. Moreover, here again it is a means of testing ; for we cannot combine incompatible, that is true and false, deductions. At any rate, the attempt to combine them tends to sift the true from the false. First we gather facts, as it were, into bundles by

each of which includes a considerable range of phenomena ; but they are scarcely what we understand by 'laws of nature.' A law is rather a description of a uniformity in the way in which bodies affect one another; or, in other words, a description of the way in which they behave under uniform conditions. Material bodies have properties or qualities which bring them into relation with each other. From uniform relations, consequent on the possession of qualities of like nature, uniform results follow. Thus, the statement that all material bodies attract each other directly as their mass and inversely as the square of their distance is unmistakably a law. A law is, in fact, a theory; it describes a uniformity in terms of causation (see $\S 586$ ); therefore it describes a uniformity in the sequence of phenomena. It is a peculiarity of a natural law in the strict scientific sense that we are able to deduce consequences from it, and so are able not only to link up with it, and thereby 'explain' phenomena not directly mentioned in it, but we are able also in this way to test the truth of it. At any rate, a law is valuable to us as a means of systematizing our facts in proportion as we are able to make deductive inferences of consequences from it. No consequences worth mentioning can be deduced from the uniformities that common primroses are yellow, mammals have heads, and birds are bi-pedal. Here we do not describe ways in which bodies affect one another. But any number of consequences can be deduced from the law of gravitation. Here we do describe a way in which bodies are affected by each other. We do not know why material bodies attract each other, nor why they attract each other in the degrees that they do. But, if the law of gravitation is a correct statement, if material bodies do attract each other in the degrees stated in it, then, since contradictories cannot both be true, given certain conditions, certain consequences must follow. Thus, given a planet moving in accordance with the laws of motion with a certain momentum, at a certain distance from the sun, then it must circle in a certain path round the sun ; given the moon at a certain distance from the earth, then the tides of the ocean must follow its course ; given lack of support, then stones must fall to the ground ; given a fluid condition, then the surface of a body (e.g. water) must conform to the general contour of the surface of the earth at the latitude in which it is situated ; and so on. "Those ideas that hold good throughout the widest domains of research and that supplement the greatest amount of experience are the most scientific" (Mach, Principles of Mechanics, p. 49c). Those sciences (e.g. physics and mathematics) which are the most completely welded together by laws and deductions from them, are the most completely scientific, the most completely interpretative, the most completely intelligible. The fewer the laws necessary for this process of welding the better ; for then the welding is the more perfect since all the more is included within the range of each law. Those sciences (e.g. anatomy) that are the least deductive, that are the least interpretative, are the least completely scientific (see \$ 819 et seq.). If the reader devotes a moment's consideration to the subject, he will perceive that nearly all thinking about heredity and evolution, like much of the thinking in mathematics and physics, but unlike all thinking in the 'systematic' sciences (e.g. zoology and botany), consists in attempts to explain the facts, or, in other words, to link them together in chains of causation. When we link facts in this way, we join them by inferences. An inference is a hypothesis. Hypotheses can be proved only by deductive appeals to reality. A law is a hypothesis that has been fully established by such appeals. All the 
inductions; next we ascertain by deduction whether the separate inductions are in harmony with each other and with reality in general; and, lastly, treating each induction as a fact, we bind them together in a wider induction "To the Deductive Method, thus characterized in its three constituent parts, Induction, Ratiocination, and Verification, the human mind is indebted for its most conspicuous triumphs in the investigation of nature." 1 "At any step of Induction . . . the inductive proposition is a Theory with regard to the Facts it includes, while it is to be looked upon as a Fact with respect to the higher generalizations in which it is included. In any other sense ... the opposition of Fact and Theory is untenable, and leads to endless perplexity and debate. Is it a Fact or a Theory that the planet Mars revolves in an ellipse about the Sun? To Kepler, employed in endeavouring to combine the separate observations by the conception of an Ellipse, it is a Theory; to Newton, engaged in inferring the law of force from a knowledge of the elliptical motion, it is a Fact. There are . . . no special attributes of Theory and Fact which distinguish them from one another. Facts are phenomena apprehended by the aid of conceptions and mental acts as Theories also are. We commonly call our observations Facts, when we apply, without effort or consciousness, conceptions perfectly familiar to us, while we speak of Theories when we have previously contemplated the Facts and the connecting Conceptions separately, and have made the conception by a conscious mental act." 2

established inferences of mathematics and physics have been proved thus. It follows that the aim of the student of heredity and evolution is to discover laws and so convert biology into a deductive science. If he proceeds according to the method by which alone interpretative science has been created hitherto, he will begin by collecting and verifying relevant facts; on these facts he will found inferences; and these hypotheses he will endeavour to test in every direction possible by the only means possible-by deductive appeals to reality. This last step will not only establish his hypotheses if true, or disprove them if untrue, but it will also bring within their range numbers of facts which would not otherwise have been thought of in connection with them. The history of such biological work as has involved thinking about the sequences of phenomena-about cause and effect-indicates very clearly that almost every controversy has arisen because the ordinary rules of logical and scientific method were neglected. Facts have been incorrectly described, or they have not been verified, or hypotheses incapable of proof have been formulated, or hypotheses capable of proof have not been tested, or opponents have ignored the proofs. Owing largely to the care with which facts are verified and hypotheses tested, and the readiness with which proofs are accepted, controversies are relatively rare amongst the students of physics, and rarer still amongst mathematicians. (See Mill, Logic, Bk. III., from which a passage is quoted as footnote to $\S 352$. See also chapters xviii. and xxv. of the present work.)

${ }^{1}$ See Mill, Logic, III. ii. 3. ${ }^{2}$ Whewell, Novum Organum Renovatum, p. I I6. 
77. In biology, especially in medicine, this process of combining inductions is occasionally stigmatized as 'deduction.' Apparently it is held that any inference which unites two or more inductions in a larger synthesis ought to be reached, no matter if impossible, by a single untested inference from particular phenomena. This curious opposition between fact and theory is well illustrated by the common declaration that this or that inference, of the truth of which the writer is convinced, "is a fact, not a theory"; by which is meant only that in the opinion of the writer the inference is incontrovertible, or, in other words, that it is a theory, not a hypothesis.

78. When, as in mechanics and physics generally, nearly all our accurate knowledge has been gathered in the laboratory, it is often possible to test a new induction by a deductive appeal to other laboratory results which have already been achieved or are possible of achievement. It is largely by such appeals that the facts, inductions, and deductions of physics have been welded into a science. ${ }^{1}$ Moreover, since most of the facts are obscured, the field of laboratory discovery is limitless, and, therefore, one experimental achievement constantly suggests another, with the result that new facts are continually discovered and new inductions and deductions formulated.

79. It is different in most branches of biology, So much is already known, or can be ascertained by simple observation, that the opportunities of the laboratory are greatly restricted. If we depend solely on it, if we close our eyes to the universe outside its walls, we may, indeed, reach many valuable facts and inductions. But there will always be the danger that we have wasted our time-that the facts have already been reached, or could be reached more quickly, easily, and certainly by simple

${ }^{1}$ It must be borne in mind, however, that physicists have, especially in astronomy, made a very considerable deductive appeal to the material supplied by simple observation. "The use of a scientific instrument does not make an observation into an experiment, unless the instrument modifies the object which is being observed. Thus we invariably speak of observing with a telescope or a microscope" (Welton, Manual of Logrc, p. II6). "The attraction of the sun accounted for the motions of the planets; the attraction of the planets was the cause of the motions of the satellites. But this being assumed, the perturbations and the motions of the nodes and aphelia only made it requisite to extend the attraction of the sun to the satellites and that of the planets to each other :the tides, the spheroidal form of the earth, the precession, still required nothing more than that the moon and sun should attract the parts of the earth, and that these should attract each other-so that all the suppositions resolved themselves into the single one, of the universal gravitation of all matter." (Whewell, Novum Organum Renovatum, p. 92.) 
observation. ${ }^{1}$ In any case, since the inductions reached through the laboratory are so few and widely separated; since, in effect, there is no system of reality to which appeal can be made, or at most only a very fragmentary system, every induction must remain isolated and untested. Under such conditions, the only kind of deduction possible is one which expands the induction without testing it or linking it up with any other, which cannot be tested itself, and which, therefore, is valueless and illegitimate. ${ }^{2}$ We shall see that, almost without exception, every important biological induction based on laboratory work-important in the sense of having attracted wide attention-has been expanded in this way, and that it is the deduction, not the induction, on which stress is laid and which is regarded as the Theory. Moreover, not only have untested inductions often been expanded by untested deductions, but, as often, the latter have been regarded as proved simply and solely because the phenomena on which they are remotely based were, like those of physics and chemistry, observed in the laboratory. ${ }^{3}$ It is true that we are exhorted to use experiment as a test, but, especially when the larger problems of heredity are under consideration, the advice is rarely heeded even by those who utter it. In effect, biologists have used the laboratory only as a means of discovery.

8o. The theories (laws) of physicists and chemists have usually stood the test of time, not because their facts were experimentally observed, nor even because their data were capable of being exactly measured, but chiefly because, though the facts were difficult to gather, the thinking founded on them was in many cases relatively simple, and, more especially, because it was in all cases very rigorously tested. The formulation of a physical or chemical law often consists in little more than a bare summary of the facts discovered in an experiment or series of experiments, a summary almost as easy to make as a classification of the facts of anatomy. In other words the relations of the facts are comparatively easy to discern. Compare, for example, Boyle's law of the

1 See $\$ 225$ et seq.

2 "The deductions which Bacon abolished were from premises hastily snatched up or arbitrarily assumed. The principles were neither established by legitimate canons of experimental inquiry, nor the results tested by that indispensable element of a rational Deductive Method, verification by specific experience. Between the primitive method of deduction and that which I have attempted to characterize, there is all the difference which exists between the Aristotelian physics and the Newtonian theory of the heavens." (J. S. Mill, Logic, III. xiii. 7.)

${ }^{3}$ See $\$ \S 208$ et seq., see also chapters vii. and viii. 
behaviour of gases under pressure with Darwin's theory of natural selection. In the former case much thought was needed to gather the data ; but they could be exactly measured, and, once obtained, a single induction sufficed to enable the discoverer to perceive his law; and, though a great deal of testing was still necessary, yet the means of performing it were not hard to think of. In the latter case, the facts lay patent but multitudinous under the eyes of every man. Individuals, as regards some of their characters at any rate, could be measured with exactness; but the measurements of no child agreed with those of its parents. The difficulty lay, therefore, not so much in observing facts as in interpreting themin tracing hidden analogies, in seeing likenesses amid differences, in separating the relevant from the irrelevant, essentials from nonessentials, in brushing aside the veil of familiarity, in discarding superstitions, in devising tests. Boyle's difficulties, however great, were not of the same order as Darwin's. They lay mainly in discovering the facts, all of which were obscured. Darwin's difficulties lay in tracking his way through the enormous and confusing maze of patent facts that spread and grew around him.

81. Obviously biology does not lend itself to experiment so readily as physics and chemistry. Apart from the circumstance that many of its facts are patent, it is too complex. The first interpretation of a physical or chemical experiment is often accepted without cavil, and is comparatively rarely overthrown. But seldom is the first interpretation of a biological experiment accepted without hot controversy, and it is quite usual for the original interpretation to be overthrown and replaced by another, and another, and yet another. These difficulties of interpretation indicate the difficulties of satisfying the conditions which make experiment useful-the difficulty of so eliminating irrelevant complications, that in the case of each experiment only one interpretation of the result is possible, or at least reasonable.

82. The notion that deduction is necessarily illegitimate and dangerous is demonstrably incorrect. That opinion has never guided successful students in their efforts to interpret nature. It is illegitimate only when we start with an unproved assumption, or when it is used merely to expand untested inductions by thinking which cannot itself be tested by an appeal to reality. Rightly used, it is our prime means of securing accuracy when thinking about sequences of events. Physicists have constantly used it, and with magnificent 
results. ${ }^{1}$ Mathematics, the most exact of all sciences, is almost purely deductive. Many of the more important biological generalizations could not have been confirmed or even reached without the employment of deduction. ${ }^{2}$ Deduction is as essential a part of human mental operations as induction. We seldom use the word 'therefore' except when our thinking is deductive. All calculation is deduction. Our every-day thinking-that incessant play of thought, that associating, disassociating, comparing, discriminating, inferring, in which we are incessantly engaged-is largely, indeed mainly, deductive. We gather facts from the world around us and by processes of induction reach thousands or tens of thousands of conclusions. But, like the parts of a scattered machine, the universe our mind constructs for us would have no meaning, no unity, and our knowledge little utility, unless these inductions were linked together, and amplified, and multiplied a thousand-fold by deduction. It is hardly possible, even for a young child, to become aware of a sight, sound, odour, taste, or tactile sensation without, consciously or unconsciously, recalling facts and generalizations previously formulated, and linking the new experience to them by deductive thinking. Nature drives us ceaselessly to the acquirement of skill in deduction, without which we would be lost in a chaos. Our intelligence is due to it. The scientific imagination is usually but another name for its daring yet restrained and careful employment. Not a single monument of the power of human thought, embodied in the literature of the past, but is full of it. No great man of action or thought, from statesman and general to traveller and scientific worker, has existed but was a master of it. Not even the simplest tool was ever thought of, made, or used but through the employment of it ; indeed the whole history of invention and the application of invention is but a chapter in the history of deductive thought. Only by means of it, by appealing from our inductions back again to the facts, can we, especially when some complex matter is under consideration, take the whole of the evidence into account. As a mode of thinking it is an adaptation, the highest product of

1 “ Newton's comprehension of logical method was perfect; no hypothesis was entertained unless it was definite in conditions, and admitted of unquestionable deductive reasoning, and the value of each hypothesis was entirely decided by the comparison of its consequences with facts." (Jevons, Principles of Science, p. 583.)

2 For example the theories of evolution and natural selection, both of which, moreover, were founded on facts that had been collected mainly by simple observation. See $\$$ 350-I. 
mental evolution, to the use of which we are continually driven by the most imperious of our instincts. ${ }^{1}$ Lacking it we should be imbeciles of that not very uncommon kind that has a considerable memory for facts but cannot learn to link them together except in a very rudimentary way. The only people by whom it is not habitually used are actual idiots and imbeciles of a low type. I use the words advisedly and literally in reference to phenomena which may easily be observed-in reference to idiots and imbeciles who are medically certified and placed under restraint as such. ${ }^{2}$ One of the main causes of the mental inferiority of the lower animals is that, like imbeciles, they are unable to employ it. Scientific deduction, like scientific induction, differs from our common use of the process only in the greater precautions we take to make it accurate. It would be strange if a process, so useful and necessary in the ordinary affairs of life, should become valueless and dangerous the moment we endeavoured to use it with care, and if men, to become scientific, must cease to be rational.

83. All the foregoing is, I take it, quite indisputably true. At any rate, all that I have said about the right method of scientific inquiry is indisputably true. No one will maintain that facts gathered in one way are on that account more valuable than equally authenticated facts gathered in another way, or that it is a rational proceeding to found untested judgments on fragmentary evidence when completer evidence, by which the judgments may be tested, is available-to use, for example, only data gathered by one laboratory method (e.g. experiment) when relevant data gathered by another laboratory method (e.g. biometry) or by simple observation is at hand. It may be said that no one has ever maintained that authentic and relevant facts should be ignored or that hypotheses should be left untested. No one, indeed, would venture to do so openly and formally. But theory and practice are not identical, and such astonishing dicta as "The day of vague and inaccurate thinking is past; we must, like physicists and chemists, rely on experiment," "The problems of heredity can only be solved in the laboratory and breeding-pen," are not uncommon, and have meant in practice, as we shall see in numerous instances, nothing other than an ignoring of relevant data combined with a neglect to test thinking. Untested hypotheses-hypotheses which are merely founded on laboratory work, but are taken as proved by it-are, in fact, exceedingly common in biology. ${ }^{3}$ At

1 See $\$ 666$.

${ }^{3}$ See, for example, chapters iv. to $\mathrm{x}$. 
present, however, I desire merely, but as strongly as possible, to fix the reader's attention on the theory of scientific inquiry and obtain his assent to it. He must bear in mind that the method of work on which I insist is no new thing. It is the method which has been proclaimed as the only right one by every authority who has both thought about and expressed himself on the subject; it is the one which has been followed by every great scientific worker; it is the one by which the truth of every law of nature known to us has been established. At the risk of tiresome reiteration, this method of work may be indicated as follows :When, as in the study of heredity and evolution, we wish to establish the relations between facts that occur in sequences (i.e. when we wish to discover chains of cause and effect), we must formulate hypotheses. But hypotheses remain mere guesses until they have been tested and found to be true. They can be tested only by making deductive inferences of consequences from them and then appealing to reality to ascertain if these consequences actually follow. If a hypothesis enables us to predict correctly in this way, it is in all probability true ; and it is the more certainly true (and useful) in proportion to the number of different correct predictions it enables us to make-in proportion as it accords with the whole universe of relevant truth. Moreover, such deductive appeals to reality not only enable us to test hypotheses and so convert them into laws, they not only enable us to bring within the range of each real law masses of data which had not been used for the corresponding hypothesis, but they enable us to perceive the relations between the several laws, and so, by linking the laws together, to advance towards that harmony of knowledge which is one of the principal aims of science.

84. To return to the problem of recapitulation-the facts are that the offspring of each successive generation resemble their parents closely but not exactly, and that, apart from the differential play of stimuli, this imcomplete resemblance is due to a recapitulation with variations of the parental development. The induction is that the development of the individual is a recapitulation, with additions and omissions, of the evolution of the race. ${ }^{1}$

1 The thinking here is somewhat complex, and complex thinking is often denounced as 'deductive,' especially by medical writers. But when we bear in mind that individual progression depends on a complete recapitulation (as regards the parts under consideration) of the parental development plus an additional step, and retrogression on an incomplete recapitulation, and that racial progressions and retrogressions are nothing other than the sums of progressions and retrogressions made by individuals during the evolution of the race, the thinking 
The deduced consequences are that developing individuals probably present traces of ancestral forms. The appeal to reality is the search for such traces. A successful appeal ends in the discovery of them and the proof of the induction. It is agreed on all hands that they do actually occur.

85. Hitherto, biologists have always founded the notion that development is a recapitulation of evolution on the evidence supplied by embryos. Adopting the method of 'induction from simple enumeration,' they have concluded that the developing young of living beings often resemble organisms lower in the scale of life than the adult type, and thence have deduced the further conclusion that the resemblance is due to a descent from lower forms. That which, rightly used, should have furnished tests, has been made the material on which was built the hypothesis which has remained, therefore, a much controverted working hypothesis. I have endeavoured to establish it as a theory, an invariable law, founded on facts of causation and tested by an appeal to reality. "It cannot be too often repeated, that we ought never to depend on frequency of occurrance, wherever it is possible to have recourse to facts of causation." 1 "The notion of cause" is "the root of the whole theory of Induction." 2 The facts, all of which are patent, and the reasoning, some of which is deductive, are before the reader. If now, bearing in mind the undisputed truth that offspring recapitulate (with variations) parental development, he is able to conceive individual development, as anything other than a recapitulation, however altered, of the life-history of the race, he is capable of an intellectual feat which is quite certainly beyond my powers.

is seen to be obviously inductive. To reach the notion that development is a recapitulation of evolution we merely sum up the persistent variations of the successive generations.

${ }^{1}$ Fowler, Inductive Logic, pp. 270-1.

2 J. S. Mill, Logic, III. v. 2. If the reader disagrees with the notion that we create better science when we link together our material by facts of causation than when we simply enumerate likenesses and differences, it would be well if he now broke the continuity of this work and, before proceeding further, read chapters xviii. and xxv. Meanwhile, I should be glad if he took this problem of recapitulation as a test case and asked himself which is the better science, the disputable and often disputed guess that is founded on a simple enumeration of the likenesses between embryos and lower forms of life, or the indisputable truth that is demonstrated when, starting with the familiar generalisation that offspring recapitulate the parental development, we establish a chain of causation, and so reach the necessary conclusion that the development of the individual is a brief and inaccurate résumé of the evolution of the race? 


\section{THE LAMARCKIAN DOCTRINE}

Adaptation-The main problem of heredity-Theories of evolution-The Lamarckian hypothesis-The Darwinian hypothesis-The incompatibility of the two-Evidence against the former-The most decisive proof of all-Correlation-Diseases-Maternal impressions-Telegony.

86. 10 the student of heredity and evolution, who not only gathers facts but strives to interpret them by ascertaining their causal relations one to another, the cardinal fact of life may be said to be the close adaptation of every species to its environment, and the equally close co-adaptation of the organs, tissues, cells, and functions of the normal individual to one another. Thus the normal man, the normal ant, and the normal oak are closely fitted during all stages of development to the conditions of their surroundings, and could exist in no other. Within plant and animal bodies, with rare and uncertain exceptions, every structure and function, no matter how vast their multitude and complexity nor how great the changes undergone during development, works in exquisite co-adaptation with all its neighbours. Hardly one of all these structures, whether useful in preserving the individual life, or sexually ornamental and therefore useful in preserving the species, whether functionally active or vestigial, but serves, or has served, or will serve during the life of the individual the great purpose of adaptation, the great purpose of enabling the individual to survive and transmit his germ-plasm to offspring. Nearly one and all, their existence has or had no significance other than utility to the individual or to the race. So surely as we learn much about a structure, almost so surely do we discover its function, its utility in the past or the present of the race, or the past or the present or the future of the individual. It is impossible to use language too emphatic on the necessity to the student of heredity and evolution of bearing constantly in mind this all-important truth of adaptation. Its wonderful completeness has been denied, but only, I think, when people, even scientific men, have founded their thinking on exceptions instead of the rule, and even then 
only when they have assumed that structures have no utility if they have been unable to detect the utility. Such exceptions are extremely rare in very well-known and understood types, and correspondingly more frequent in plants and animals less familiar to us. We may find them in plants, insects, and molluscs, but we have only to devote a moment's thought to the closely coordinated structures and functions of such a being as man to perceive how very exceptional is the exception to the rule of utility. ${ }^{1}$

87. Adaptation must be the anchor about which the student's thoughts for ever swing, the touchstone by which he must test every theory of racial change and individual variation. The normal individual is a bundle of adaptations. His growth, his development, is nothing other than a process of individual adaptation. Evolution is nothing other than a process of racial adaptation. A theory of evolution is, or should be, nothing more than an attempt to explain, or, if we prefer, summarize in brief terms the processes by which adaptation is achieved. A theory of heredity seeks to account for likenesses and differences between parents and offspring; if it fails to be compatible with a theory of evolution which correctly interprets the facts in terms of adaptation, it is sure to be erroneous. Many theories of heredity and evolution have gone, or are going to the limbo of discarded hypotheses, merely because they do not accord with the truth that species are closely adapted by all or nearly all their structures and functions to the environment. The plain fact that living beings are able to exist is proof of adaptation. Many students of heredity and evolution, especially those belonging to the experimental school, will think that I put the case too strongly, but I shall justify every statement up to the hilt.

88. To be adapted to the environment in which he is produced, the child must resemble the parent. The resemblance is due to his recapitulation of the parental development. Presumably the recapitulation occurs because the germ-plasm in the fertilized ovum whence the child sprang resembled (because derived from) the germ-plasm of the fertilized ovum whence the parent sprangbecause the two portions of germ-plasm had similar hereditary tendencies which were awakened by stimuli similar in kind and degree. Thus half the task of the student of heredity is easily accomplished. He is able-in a way, superficial perhaps, but valid as far as we know-to account for the resemblances. Of 
course, if we choose, we may seek to pry deeper and speculate about the composition, the 'architecture,' of the germ-plasm, and the hidden springs of its behaviour. But, in the almost total lack of verifiable evidence, we should be reduced to mere valueless guessing-valueless because incapable of being tested. We know, however, that there must be a germ-plasm, a substance that is the bearer of heredity, in the germ-cell, and that in the case of every species of living beings it must possess definite hereditary tendencies which differ from those of every other species, and we have very strong reasons for supposing that it is derived by direct and real descent from the germ-plasm of preceding germ-cells. On these tolerably sure foundations we are able to build a very great deal.

89. It is more difficult to account for the differences in detail between parent and child than for the resemblances in mass. Some of these differences are readily explained by the fact that the stimuli under which parent and child develop are never exactly similar. The nutriment, use, and injury which awakened the hereditary tendencies of the parent are sure to have differed somewhat from those which awakened the tendencies of the child. These account for the acquired differences. The real difficulty arises when we seek to account for 'variations,' for those innate differences which are founded, not on differences in stimuli, but in germ-plasms. We have to answer the question, "How does it happen that the child 'varies' from the parent?" Or more precisely, "How does it happen that the germ-plasm of the child differs from that of the parent?" If the problem is extremely complex and difficult, it is also very important. Indeed it is perhaps the main problem of heredity. If we solve it correctly, we shall have discovered not only the correct interpretation of many of the phenomena of heredity, but, since all racial change is founded on the variations of individuals, of evolution also. In this matter, just because the problem is so complex and difficult, we must, linking together a number of principles reached by induction, consider heredity and evolution together. Variations, taken by themselves, are capable of a number of interpretations, any one of which, as far as we know, may, or may not, be correct; taken in conjunction with the facts of adaptation it becomes evident that only one interpretation can possibly be true.

90. Before beginning our investigations we must make up our minds to do some very careful thinking. I do not say this because we are about to deal with exceptionally abstruse matters, nor 
because I think the reader's task will be especially difficult, but because we are now coming on very debatable ground where hardly two scientific men think alike, and because this question of the causation of variations lies at the very heart of our subject. Unless we have clear ideas concerning it, we shall have misty ideas concerning cognate subjects. Accordingly, as we form one or other opinion, we shall take one or other of several widely divergent views of heredity, of evolution, of all the problems of life. We shall have to deal with large and complex bodies of evidence, a thing which cannot be done without using correspondingly complex processes of thought, though unfortunately attempts to shirk the necessity have been common enough. I think we have materials that are amply sufficient to enable us to come to a definite conclusion. I believe also that the problem is still regarded as debatable only because men, instead of considering the whole mass of evidence and so testing their thinking, have often founded their opinions on isolated bodies of facts. Often, displaying a superstitious and pedantic reverence for facts collected by this or that method of inquiry, they have ignored massive and indisputable evidence, collected in other ways. A simplicity of view has often been achieved in this manner, but at the expense of breadth and depth of view, and, therefore, of correctness, clearness, and comprehensiveness of perception. In other words, the phenomena have not been viewed in their complete setting.

9I. Species arose and were adapted to their environments either by miracle or by natural processes. If by miracle, then three methods of creation are conceivable. (I) All species, extinct or persisting, may have been called into being simultaneously, or nearly so, in full possession of the characters which they have ever since possessed. If this, the popular hypothesis, be true, there can have been no evolution, or very little, and, therefore, since untold numbers of species still persist, the environments can have undergone no important alterations. The entirely conclusive evidence furnished by geology proves, however, that both species and environments have undergone vast though gradual changes. (2) Unchanging species and varieties may have been created, not simultaneously, but successively during a period which extended over many millions of years, and in a way that exactly mimicked evolution, and apparently was designed to delude us into a belief in it. Probably hardly anyone, whether acquainted or not with the facts, now accepts this solution, which logically should impel us to believe that our domesticated breeds of plants and animals 
are special creations. (3) There may have been evolution, but evolution miraculously directed. This belief implies that species changed in adaptation to a changing environment, not because the environment, acting on them, caused the change, but because a Power, in a sense outside the environment, moulded them into adaptation to it without using it as an instrument. It is, however, a rule, as good in science as in the ordinary affairs of life, not to appeal to the supernaturnal until natural explanations have been exhausted.

92. A variety of the belief in miracle has sometimes been advocated by men of science-chiefly botanists. God and miracle are not mentioned, but we are told that species are fitted to their environments by means of an inherent adaptive growth-force; that is, we are asked to believe that offspring-not merely surviving offspring, but offspring in general-tend to vary from their parents in such a way as to be better fitted to the environment, and that thus evolution results. If we examine the evidence on which the supporters of this, the 'bathmic,' hypothesis rely, we find no very strenuous attempt to distinguish between variations and modifications-between inherent alterations dependent on alterations in the germ-plasm, and changes that result merely from a differential play of stimuli. Many of the examples quoted by them appear to be nothing other than what $\mathrm{Mr} \mathrm{A}$. Bacot has termed 'répertoire patterns.' 1 Thus the plant Berberis vulgaris bears leaves in a moist and spines in a dry atmosphere. Hippuris has two forms, one terrestrial and the other aquatic, which may be converted, the one into the other, by changing the environment, the leaves under the water growing long and undulating, those in the air short and erect. It has been assumed by the supporters of the bathmic theory that all these differences are germinal-that the individual that develops in the one way in the one situation, differs innately to the extent of his differences from the individual that develops in the other way in the other situation. But, as a fact, we have no reason to suppose that they are other than acquired. They furnish, however, very interesting evidence that nature may fit a species for existence in two separate environments, in each of which it is capable of flourishing, but in one of which, under one set of stimuli, it develops characters different from those which it develops in the other environment under another set of stimuli. Presumably, such species have evolved under conditions which rendered this capacity for alternative development useful because the one environment

\footnotetext{
${ }^{1}$ Nature, Jan. 3oth, 1908, p. 294.
} 
was liable to be changed into the other. The evidence in favour of the existence of an adaptive growth-force, then, is very equivocal. On the other hand, if it be true, as massive evidence demonstrates, that organs and structures undergo progressive evolution (i.e. become larger and more complex) only when they are endowed with a considerable degree of utility (i.e. selection value), and retrogression only when they lack utility, the bathmic doctrine is certainly untrue, and we are able to explain changes in structure or function, or increase or decrease in them without mysticism. Normal growth, after all, is only a form of adaptation; it occurs only when it fits the species more closely to the environment; and it is adaptation, not increase in size or complexity, that is the main problem. We do not explain why a force is adaptive or render it less mysterious by terming it an adaptive growth-force. Moreover, the fact that breeders are able to modify plant and animal types in innumerable directions is evidence of some value that variations occur, not in one or two or a few directions, but all round the specific mean like bullet-marks round a bull's-eye. We shall see how strongly this latter hypothesis is confirmed by the facts of human evolution against disease.

93. Rejecting miracle then, there remain only two scientific hypotheses which are intelligible, at any rate at first sight-the Lamarckian doctrine of adaptation through the transmitted effects of use, disuse, and injury, and the Darwinian doctrine of natural selection. Doubtless, in a way, the Lamarckian doctrine, curiously combined with a belief in special creations, has been a part of popular superstition since prehistoric times. Even now "the man in the street" is firmly convinced that parental 'acquirements' are readily transmuted into 'inborn' traits in the child. But Lamarck first of all, or at least most consistently, gave scientific form to the popular belief by allying it to a theory of evolution. His speculations attracted little attention, even amongst students, till Darwin and Wallace formulated their theory of Natural Selection. Subsequently, for a score or more of years, scientific thinkers, including Darwin himself, believed that the organic world arose partly through the 'transmission' of acquirements and partly through the natural selection of favourable variations. During the last twenty years, however, scientific opinion has veered to a conviction that Darwin and Wallace, the most modest of men, worked better than they supposed-or rather than Darwin supposed, for Wallace has long and consistently repudiated the Lamarckian doctrine. It is now generally believed that acquirements are never 'transmitted,' and, 
therefore, that evolution proceeds entirely on lines of natural selection. This emended doctrine has received the name of 'Neo-Darwinian.' Often, however, it is termed simply the 'Darwinian,' though, as we see, Darwin did not hold it in its entirety.

94. The question as to whether acquirements are, or are not, transmitted has been the subject of probably the most lengthened and ardently debated of biological controversies. Prolonged investigation, in the course of which the plant and animal kingdoms were ransacked, failed to reveal a single convincing and unmistakable instance of the transmission of an acquirement. But though the Lamarckian doctrine is abandoned by the majority of serious students, it is worth while to devote some space to it, partly because it is still an article of popular belief, partly because the study of it will enable us to contrast it with the Darwinian theory and so achieve a better understanding of what is meant by Natural Selection, and partly because we shall thus early have an opportunity to demonstrate the importance of testing every speculation by a rigorous deductive appeal to reality.

95. The Lamarckian doctrine supposes that parental 'acquirements' tend to be 'inherited,' in however small a degree, by offspring. That is, it supposes that useful characters, which evolution enables the parent to acquire as a response to use or injury, tend in the child to betransmuted into less adaptive characters, which develop in response to the stimulus of nutriment. In the face of common experience its scientific adherents did not maintain that the whole of the parental acquirement was commonly 'inherited.' Thus, if the tails of a pair of dogs were amputated, it was not thought probable that the offspring would be born tailless, though it was believed that this sometimes happened. It was thought merely that some portion of the parent's loss tended to be 'inherited,' and therefore, that if the mutilation were repeated during successive generations, a race with diminished tails and ultimately a tailless race would result. Again, it was not believed probable that, if a man increased the size of his muscles by exercise, all of the increase would be 'inherited,' but only some of it. In fact, it was maintained that acquirements were transmitted, as a rule, only 'faintly' and 'fitfully.' 1

96. Obviously the Lamarckian hypothesis professes to be a theory both of heredity and evolution, the latter being an induction from the former. As a theory of heredity it seeks to account for

${ }^{1}$ See Romanes, Darwin and after Darwin, vol. ii. p. 152. 
variations by supposing, in effect, that each parental acquirement tends to alter the germ-plasm in such a particular and remarkable manner that the 'acquirement' is reproduced by the child, in whole or part, as an 'inborn' trait. As a theory of racial change, it supposes that progression is due mainly to use and to such injuries and nutritive changes as result in increase of size (or function), whereas retrogression is due principally to disuse and to such injuries or nutritive changes as result in decrease of size or function, for example from mutilations or starvation.

97. Now, whether acquirements be, or be not, transmitted, we know that children often vary from their parents (differ innately at birth from what their parents were at birth) quite apart from such 'transmission.' Thus the child of normal parents may be born with an extra digit, a character which is never acquired (i.e. developed) by the parent in response to the stimulus of injury or use. The problem which we have to solve, then, is not whether the Lamarckian theory of heredity correctly accounts for the occurrence of all variations, but only whether it correctly accounts for the occurrence of any variations. Similarly many characters are present in every species the evolution of which cannot conceivably have been due to the 'transmitted' effects of use, injury, or any other stimuli, for example nearly all the characters of plants, and in animals, such characters as brilliant colour markings, the external ears, the reproductive organs, and feathers and hairs. It follows, therefore, that while the Lamarckian doctrine may afford a partial explanation of the occurrence of variations and of evolution, it cannot afford a complete explanation.

98. The unicellular organism "is a part and usually a half of the parent." Its structures, therefore, are derived from similar structures in the parent; that is, there is real inheritance. The multicellular organism, on the other hand, is derived from a single cell, which, after dwelling as a parasite in the parent cell-community, leaves its home to become the ancestor of a new community. The parts of this new cell-community, therefore, are not derived from similar parts of the parent; that is, there is no real inheritance of the characters of the first community by the second. The two resemble one another for a totally different reason-because their development has been directed by similar germ-plasms, the hereditary tendencies of which have been awakened by similar stimuli. It is evident, therefore, that the problem of the alleged transmission of acquirements is not the same in both cases. Indeed, 
the question of the transmission of acquirements as presented by multicellular organisms has no parallel, and can have none amongst unicellular types. This distinction is clear to most students of heredity, who, as a rule, maintain that parental acquirements may be inherited by unicellular, but not by multicellular plants and animals. Occasionally, however, some writers, chiefly medical men, attempt to argue from the one question to the other; but the reasoning is obviously invalid. On the other hand, multicellular organisms present a problem which, while totally distinct from the question of the 'transmission' of their acquirements, is a real parallel, though seldom or never recognized as such, to the problem of the 'transmission' of acquirements as presented by unicellular types. We shall consider it in its proper place. ${ }^{1}$ At present we must confine ourselves to the much debated 'transmission,' which Lamarck maintained was the source of the evolution of the higher organisms.

99. A cell-community, a multicellular plant or animal, is compounded of germ-cells and somatic cells. The Lamarckian doctrine supposes that when the somatic cells acquire characters under the influence of use or injury, these acquirements so affect the germplasm contained in the germ-cells that the cell-communities which spring from them tend to reproduce the acquirements of the somatic cells, not as 'acquirements,' not as direct effects of use or injury, but as nutritional characters. Thus, when the muscle cells of a man, for example, increase in number and power as a result of use, the germ-cells dwelling within the cell-community are supposed to be affected in such a remarkable and particular way that the children which arise from the latter tend to develop larger and more efficient muscles without the aid of use. Speaking metaphorically, the acquirements made by Brown and Jones (the somatic cells) are supposed to so affect Robinson and White (the germcells) that the descendants of the latter are characterized by traits which look like those which Brown and Jones 'acquired,' but which, since they are 'inborn,' are really very different. Obviously this power of transmuting acquirements-if it exists-is a very wonderful thing, which must have arisen de novo amongst the higher types ; for, as we have just seen, nothing like it occurs, or can occur, amongst unicellular forms, which separate and do not form cellcommunities, and so cannot influence one another in this way. A large and complex organism, such as man, may make a million different acquirements, each of which is distinct from every other; 
and therefore, if the Lamarckian hypothesis be true, are differently recorded in the germ-cells.

I00. How did this amazing power arise? What is the machinery for it? If we exclude miracle, the only conceivable causal agent is Natural Selection-at any rate I can conceive no other, and I do not believe the reader can. But Natural Selection is concerned only in shaping adaptations. It must be shown, therefore, that this power of transmuting acquirements is generally useful to species; for it is inconceivable that Natural Selection would or could call into existence so wonderful and complex a power if the result injured the race and stultified itself. Obviously it must not be assumed, as is always done by the adherents of Lamarck, that the existence of this power is 'natural' and inevitable-so natural and inevitable that it is necessary rather to disprove than to prove the actuality of the transmission.

IOI. It is argued sometimes that the multicellular individual is not really multicellular; but that protoplasmic bridges connect the cells and convert the whole into a sort of gigantic unicellular organism. Presumably, on similar grounds, we should regard Siamese twins, not as two individuals, but as one. But, even if we agree that the multicellular is really unicellular, the difficulties to be surmounted by the Lamarckian theorist are not diminished. It still remains true that the parts of the child are not derived from similar parts of the parent, but, on the contrary, from a minute portion of him (the germ-cell). We gain nothing by calling this portion a portion instead of a separate cell ; for it is just as hard to understand how this portion can be influenced with percision in millions of definite different directions by changes occurring elsewhere. Herbert Spencer sought to overcome the difficulty by conceiving the living body as a kind of crystal, the nature of which was liable to be so radically changed by the action of the environment that detached portions of it tended to assume the new, not the old shape. A chip from a crystal, it is true, grows in a suitable medium into the likeness of the parent crystal ; but there the analogy ends. The crystal does not spontaneously enit portions of itself that serve as starting-points for daughter crystals, which vary from the parent. It is not heterogeneous in structure. It is not a storehouse of active energy. It does not alter profoundly and continuously in shape during growth as the embryo does, nor with the seasons like plants or the stag when he sheds his antlers, nor from moment to moment like a man when he moves. It does not consist of a fluctuating stream of matter which enters 
as nourishment and is subsequently exchanged for other matter. It is not a focus of chemical activity. It grows only on the surface. In short, the analogy is strained and false.

102. The Darwinian hypothesis, at any rate as at first enunciated, was not a theory of heredity, but only one of evolution. It did not seek to account for variations, but took them for granted and built on that foundation. Indeed, the only theory of heredity formulated by Darwin, that of Pangenesis, was designed more to satisfy the claims of the Lamarckian conception of evolution than of the one which has received his name. We shall see, however, if we submit the theory of Natural Selection to a rigorous deductive inference of consequences, and appeal to reality for confirmation, that one particular theory of heredity, and that one only, fits it, whereas all the others are incompatible with it.

I03. The theory of Natural Selection is founded mainly on two undisputed inductions and two deductions from them. The inductions are $(a)$ that offspring, while resembling parents on the whole, always vary from them somewhat in every character, being perhaps superior in some characters and inferior in others; and $(b)$ that in every species the number of individuals that come into being exceeds, sometimes very greatly, the number of those who survive and have offspring. The deductions are (a) that, as a general rule, the individuals selected by nature for survival are, on the whole, better adapted to the environment than those selected for elimination; and $(b)$ that in this way the 'specific mean,' the racial average, is raised, and consequently evolution results. Unlike the doctrine of the transmission of acquirements, the theory of Natural Selection, as held by its modern followers, really aspires to account for all adaptations.

104. The main contrast between the Lamarckian and the Darwinian hypotheses arises from the fact that the former attributes progressive evolution to influences that act beneficially on the individual, whereas the latter attributes it to influences that kill or weaken, and so on the average prevent the unfittest, the most affected, from having offspring, or at any rate their full quota of offspring. Until very recently all students of heredity were zoologists or botanists, or men who drew their facts from materials collected by them. Even now it is difficult to persuade such workers that useful evidence may be drawn from other sources. When, therefore, they thought of elimination, their minds turned naturally to quick or sudden death, the only forms of elimination familiar to us in wild nature. They conceived the 
animal as growing strong and active through contending with rivals, escaping from enemies, or pursuing prey; or else as perishing in the fullness of his vigour. They imagined the plant as overwhelmed by the storm, or as emerging more hardy and firmly rooted from the struggle. In brief they conceived the same agent as eliminating the weak and as strengthening the strong. They had no difficulty, therefore, in believing that a hypothesis which attributed evolution to the transmission of acquirements was compatible with one which attributed it to the survival of the fittest. They had thought of no facts by which they could test this opinion. Consequently for many years scientific men were adherents of both doctrines, as a few still are.

I05. But agencies which, while strengthening the survivors, destroy the weak by violent deaths, are not the only causes of elimination. Some agencies, for example many human diseases, both destroy the weak and weaken the strong. Apparently they are never other than harmful, at any rate they are never beneficial. Almost every individual of many human races is exposed to one or more agencies of this sort. If, then, the Lamarckian doctrine is true, such agencies should be causes of continuous racial deterioration. On the other hand, if that doctrine is false and the Darwinian doctrine true, if the acquirements of the parent end with the parent and are not inherited by the child, then such agencies should, through the raising of the racial average in every generation by the survival of the fittest, be causes of protective evolution. Here the two theories are in conflict; we see plainly that they are incompatible; if the one gives a true account of racial change, the other does not; for it is impossible that a race can both deteriorate and improve in the same characters at the same time. Therefore we are able to test them by means of crucial instances; that is, we are able to appeal to reality for guidance. The fact that every race (e.g. English and Negro) that has been exposed to any disease (e.g. tuberculosis or malaria) has undergone no discoverable deterioration, but has become resistant to the disease, has evolved against it, precisely in proportion to the length and severity of its past affliction, is decisive evidence against the Lamarckian and in favour of the Darwinian doctrine.

106. It is true that some human diseases (e.g. measles) leave the individual who recovers from them with an increased power of resisting subsequent attacks, and that generations that have suffered from these maladies are on that account proportionately 
resistant. But it can be shown that the resisting power which the individual develops is radically different in kind from that which the race evolves. ${ }^{1}$. The latter power, therefore, cannot have arisen through the inheritance of acquirements. In any case, the fact remains that many diseases and other conditions (e.g. tuberculosis, slum-life, and alcoholism), which confer increased resisting power on the race, not only confer none on the individual, but actually tend to destroy any that he may possess. ${ }^{2}$ Here the race evolves in a direction precisely contrary to the direction taken by the acquirements of the individual.

I07. Another point of contrast, important but seldom noted, between the two hypotheses, is the differing complexity of the racial change which would necessarily result on the one hand from natural selection, and on the other from the transmission of acquirements.

108. The natural inference from the theory of Selection, the theory which attributes the evolution of a character, not to the amount of use to which it is subjected but to its utility (selection value), is that progressive evolution proceeds on lines of comparative simplicity. We have abundant evidence that structures that do not assist appreciably in the preservation of life or in the struggle for offspring, for example the wings of a species of bird to which flight has become useless, or the special features of our prize breeds of plants and animals, tend to deteriorate when no longer selected. It is a reasonable inference, therefore, that a certain stringency of selection is necessary for progression, that a lower degree of stringency suffices for the maintenance of a structure, and that a still lower degree of stringency, or, even more, complete cessation of selection, is followed by retrogression. These inferences are borne out by what we observe in nature. For instance, as we shall see later, the human race is undergoing progression in a few particulars. $^{3}$ The mass of human characters, however, are stationary or nearly so. They were evolved during different but overlapping periods of a long-extended past, and their progression ceased when further increase was not of utility. No matter how much used or useful, they are merely maintained at a certain standard of efficiency. Thus apparently, the human hand, heart, lungs, liver, and many other characters have changed little, if at all, during thousands of years. Lastly, many human structures, having quite lost utility, have become vestigial, presumably through cessation of selection. The germ-plasm has so altered that they

${ }^{1}$ See $\S 432$.

2 See $\$ 435$.

${ }^{3}$ See chapter xiii. 
cannot now develop in the individual under any form of stimulusneither under nutriment, nor use, nor injury.

I09. On the other hand, were the Lamarckian doctrine true, every structure which was much used (e.g. the lower limbs of man, and, in particular, the joints) would increase in size proportionately to the amount of use-with results disastrous to the co-adaption of structures; every structure which was little used (e.g. organs of generation), or was not 'used' (e.g. hair, bones of skull), or was disused, would degenerate, and structures which were much exposed to injury (e.g. wings of butterflies) would tend to deteriorate. Evolution and degeneration would thus proceed on lines as complex as the individual and with extraordinary results. Here, then, again, if acquirements were transmissible, since species would be affected as wholes, since almost every individual would alter in the same direction, natural selection would have no scope. The species would drift helplessly out of harmony with the environment. In point of fact, therefore, the Lamarckian hypotheses of heredity and evolution, when closely examined (i.e. when we make rigorous deductive inferences of consequences), are found not to be theories of adaptation at all ; and they are always, not merely sometimes, incompatible with the Darwinian hypothesis.

IIO. It is obvious at a glance, that, if actual injuries and growths (e.g. scars), which arose in response to injuries, were transmuted into characters which arose inevitably (because developed under the stimulus of nutriment), in the absence of injury the result would be, not adjustive, but distinctly harmful. Similarly, characters which develop under the stimulus of use are particularly useful when so developed. They possess a quality of usefulness which is lacking in those developed under the stimulus of nutriment-a kind of unconscious"intelligence, as it were, which, within the limits of possible development, enables the individual to grow just the right characters to just the right extent. Thus the muscles of the athlete, whose need is greater, are larger and more efficient than those of the ordinary man; but they diminish when he goes out of training. The arms of the sailor tend to develop more in proportion than his legs, whereas the reverse is the case with the soldier. The heart and |kidneys develop beyond the ordinary in disease when life depends on a response to an increased strain thrown on them. The skin thickens and hardens when and where such changes are useful. When the need is over, all use-developments tend to disappear, and the organism is 
relieved of unnecessary burdens. The capacity to develop under the stimulus of use, therefore, renders the individual more adaptable than he would otherwise be. In fact, he is doubly adapted to the environment : first by the evolution of his race, which fits him to the environment common to all the members of his species, and second by peculiarities in his own development, which arise under the stimulus of use and injury and fit him to his own special surroundings. ${ }^{1}$

I I I. Yet another quality of special usefulness is conferred by the capacity to develop under the strain of use. The only organisms which possess this power to any considerable extent are the more complex animals. Thus, a man is compounded of thousands of structures, all of which must be closely co-adapted, or the individual as a whole lacks efficiency. The greater the complexity, the more necessary but difficult is nature's task of maintaining co-adaptation. By the evolution of the power of growing under the strain of use the difficulty is in great measure surmounted, for each structure thus endowed develops in proportion to the strain placed on it. The size and strength of the human heart, for example, is, in general terms, always in proportion to the needs of the individual, be he healthy or diseased, big or small-especially in early life, when the power of developing under the strain is greatest. On the other hand, the magnitude of such structures as the teeth, the ears, the nose, the hair, and the reproductive organs, all of which are wholly 'inborn,' is often comparatively disproportionate as compared with associated parts. ${ }^{2}$

I12. It follows that if a character which developed in the

${ }^{1}$ See chapter xxi.

2 Much has been written on the 'correlation' of parts and qualities. The word has been used to describe at least three distinct classes of phenomena. First, there is the infrequently observed but much discussed tendency of parts, which have apparently no close functional connection, to vary together in a non-adaptive way - in a way that has not resulted directly from evolution, but is seemingly a kind of by-product of it. Thus "white cats which have blue eyes are almost always deaf" (Darwin, Animals and Plants, vol. ii. p. 322) ; "multiplicity of horns in sheep is generally accompanied by great length and coarseness of the fleece" (Youatt on Sheep, pp. 142-69, quoted by Darwin). "White terriers suffer most fron distemper, white chickens from a parasitic worm in their trachea, white pigs from scorching in the sun, and white cattle from flies" (Vernon, Variations in Plants and Animals, p. 85). A normal individual (e.g. man) is a bundle of adaptations. He consists of a thousand or ten thousand parts, each of which is co-adapted, not merely to a single other, but to many others, and must have been so co-adapted during all the shifts and changes of evolution. It is difficult to understand how this perpetual co-adaptation in all directions can have been secured unless each part has been independently variable. Only so can the species have been sufficiently ductile to the action of Natural Selection. We 
parent under the stimulus of use were 'inherited' by the child (i.e. were developed in the latter under the stimulus of nutriment), a great part of its utility would be lost, for the character would then develop, not in proportion to the needs of the individual, but, within limits, in proportion to the supply of nutriment. The alleged power of transferring characters due to injury and use from their own category to another, therefore, is clearly not an adaptation. If it exists at all it must have been evolved, not through Natural Selection, but through miracle-through a miracle which was designed, not to benefit but to injure the species.

I13. Formerly, as noted already, the magnitude of the useacquirements made by the higher animals was not realized by biologists. Indeed it is very seldom realized even now. The study of them is still the most neglected department of biology. In fact only exceptional acquirements were and are regarded as acquirements. Ordinary acquirements, those made by every normal individual who reaches maturity, were thought to be inborn traits. For instance, the truth that almost the whole growth of the infant's limbs after birth is due to use was undreamed of. This failure to realize the magnitude and importance of the use-acquirements has led to a failure to note the evolution of the 'inborn' power of making them. The use-acquirement was regarded, not as an adaptation, a product of evolution, but as a kind of accident which might befall any structure, and which, being transmitted, might result in the evolution of inborn characters. Its magnitude was tacitly assumed to be so small that the great part it plays in co-adapting, in correlating, the parts of the individual, and otherwise fitting him to the environment was not thought of. On the other hand it was believed that the transmission of use-acquirements

are compelled to assume, therefore, in accordance with the facts as far as observed, that this non-adaptive kind of correlation is very rare-rare, that is, as compared to the total number of the characters of the normal individual. Second, there is that kind of correlation which results directly from Natural Selection, and which secures the proportionate development of those characters which develop under the stimulus of nutrition. For example, a large infant has usually, not only a large body, but, correlated with it, proportionately large limbs and head. Third, there is that kind of correlation which results from the proportionate development of associated structures under the strain of use. Since this kind of correlation is superimposed on and perfects the correlation of parts which have developed under the stimulus of nutrition, it is usually the closest of all. Thus the correlation in size between the muscles of the human adult arm is obviously closer than that between ear and head. Correlation in the second and third instances is only another name for co-adaptation, and evidence is hardly needed to establish the fact that living beings tend to have their parts so adjusted as to render them capable of existence. 
did much in the course of generations to bring about co-adaptation and correlation. Thus Herbert Spencer maintained that without the transmission of use-acquirements the evolution of the higher animals would have been impossible. He instanced the weighty horns of the elk, pointing out that their usefulness to the individual depended on the co-adaptation of a thousand parts in head, neck, trunk, and limbs, and insisted that, since it is unlikely that all of them can ever have varied favourably together in any individual and practically impossible that they can have so varied in a succession of individuals, their co-adaptation was explainable only on the hypothesis that acquirements tend to become inborn. Obviously, his reasoning was founded, first on a belief that all characters grow under the stimulus of use, and second on an insufficient appreciation of the magnitude of use-acquirements, which, as in other cases, brought about co-adaptation between the horns of the elk and the structures associated with them.

I I . More recently some biologists, more particularly Professors Lloyd Morgan, Osborn, and Mark Baldwin, while admitting that use-acquirements are, in all probability, not transmissible, have argued that their presence, by acting as a screen behind which progression could occur, may conduce indirectly to evolution of a kind which closely mimics that which would result from the inheritance of them. Thus suppose drowning were a stringent selective cause of human elimination, then those individuals who learned to swim quickest and best would survive in the greatest numbers. If any of their offspring varied in such a way that they were able, in some measure, to swim 'naturally' (i.e. without learning), they would be at an advantage, and would survive in larger numbers than contemporaries who had more to learn. In this way a human race that, like many lower animals, could swim instinctively, would ultimately be established. In other words, without any inheritance of acquirements, characters, which in the ancestors developed under the influence of use, would in the descendants develop under the influence of nutriment. But the evidence that this view, which has not been tested by an appeal to reality, though the test is possible, is mistaken is overwhelming. Characters developed under the influence of use are, as we see, very much more adaptive than those developed under the stimulus of nutriment. Therefore, though there is unlimited evidence of the former replacing the latter, there is none to the contrary. Thus the helplessness, the immaturity of the human being, before he has made his useacquirements, demonstrates to what an extent the power of 
developing under the influence of nutriment has been displaced in him by that of developing under the stimulus of use. For example, the ability to run and swim instinctively is so general amongst the lower mammalia that there can be little doubt that the ancestors of man formerly possessed, but subsequently lost, this power. ${ }^{1}$

I I 5. Here, indeed, we have the strongest evidence of all against the Lamarckian hypothesis. When we find it impossible to formulate a probable hypothesis as to how acquirements can be transmuted into inborn characters, or to conceive by what process, short of miracle, the power of thus transmuting them can have been evolved, it becomes necessary to ask for very strong and unequivocal evidence of the alleged transmutation. When we ascertain by a rigorous deductive inference of consequences that the hypothesis is apparently incompatible with the fact that life exists and is adapted to its surroundings, our reasons for rejecting it are greatly strengthened. The only doubt that remains is as to whether, in spite of all our testing, we have reasoned correctly. But when we find that the whole course of evolution has been such that, so far from 'inborn' characters tending to replace 'acquirements,' the reverse has constantly happened;; when we find that the human being, for example, has lost so much that was inborn in his remote ancestors, that he has become incapable of reaching maturity except under the stimulus of use, all doubt vanishes. The appeal to reality then furnishes decisive proof. Having regard to the facts, a supposition directly contrary to the Lamarckian hypothesis (viz., a supposition that inborn characters tend to be transmuted into acquirements) may perhaps be maintained with some appearance of probability. But a supposition that acquirements tend to be transmuted into innate characters is very obviously in conflict with what has occurred in nature. ${ }^{2}$

I I6. Though the Lamarckian doctrine is abandoned by nearly

1 See $\$ 673$.

${ }^{2}$ Most biologists, I think, reject, or rather believe that they reject, the I.amarckian doctrine on grounds furnished by experiment. I have heard (so very odd, occasionally, are the notions of scientific men concerning science) all other evidence and reasoning, all attempts to establish a chain of causation and make biology, like physics and mathematics, a deductive science, denounced as ' weak.' But it appears to me that the experimental evidence is strongly in favour of the, at any rate, 'faint and fitful' transmission of acquirements, a transmission which, if sufficiently general, would inevitably lead to racial change. We cannot disprove a statement that an event sometimes happens by proving that sometimes it does not happen. One positive example outweighs a hundred negative instances, and Brown Séquard, Romanes, and numbers of other observers have recorded, or thought they recorded, many. To this day French observers, especially French medical men, frequently announce such experiments as the 
all students of heredity and evolution, a belief in it still lingers among a section of medical men-mainly because the distinction between 'innate' and 'acquired' characters is not drawn by them with sufficient clearness. ${ }^{1}$ Gout, hæmophilia, syphilis, mutilations, maternal impressions, and telegony are cases in point. Gout is an acquirement, an injury which often results from dietetic errors. It occurs most commonly amongst people who have exceptional opportunities to eat and drink to excess. Some people are innately more liable to contract it than others-just as they may be innately taller, or darker, or different in any other way. Their germ-plasm is such that they develop the disease more readily under the fit stimuli. There is even such a thing as "poor man's gout." Their children tend, of course, to reproduce the parental peculiarity when placed, as they usually are, under similar conditions. All this, however, affords no support to the contention that parental excess, or parental gout resulting from excess, increases the liability of offspring to the disease. The well-to-do offspring of poor Irish peasants have been found

excision of the stomachs of dogs, and declare that the stomachs of the offspring are, on the average, smaller than the normal. Really, I suppose most biologists think these observers mistaken, because, consciously or unconsciously, they have become convinced, actually on deductive grounds, that it is impossible and incredible that acquirements can be transmitted. Some distinguished supporters of the Lamarckian hypothesis still exist, however (see, for example, Mr Francis Darwin's Presidential Address to the British Association, 1908; Professor G. Henslow's book, The Heredity of Acquired Characters in Plants, and Professor Marcus Hartog's and Dr Mercier's articles in the Contemporary Review, September, November, and December 1908). They argue very rightly from the experimental evidence, insist even more rightly that the fact that we are unable to conceive how acquirements can be transmitted is not proof that they are not transmitted, and declare finally that they must be transmitted because (according to them) it is impossible to conceive how plants and animals have evolved into adaptation to their surroundings without such transmission. I have tried in vain, however, (see, for example, the Contemporary Review, October 1908) to persuade them to realize that it is not the transmission of acquirements, but the transmutation of useful into less useful or actually injurious characters that is really the subject of discussion, or to persuade them to consider the truth that, while nature furnishes no clear evidence that inborn traits have replaced acquirements, she furnishes evidence, perfectly clear and immensely massive, that the contrary has happened on an enormous scale.

1 " In much of the evidence on causation terms were used without fixed meaning, in different senses by different witnesses, or in more than one sense by a single witness. This confusion has been no less noticeable in the medical than in the general evidence given on the important question of 'Heredity,' both before the Commission and at other inquiries of a cognate kind; and it seems to us to be desirable that this subject, which forms an important part of physiology, should be more specially emphasized than it now is in the medical curriculum." (Report of the Royal Commission on the Care and Control of the Feeble-Minded, 1908, p. 180.) 
to be, on the average, as liable to gout as the scions of the aristocracy.

I I7. In hæmophilia the blood lacks coagulating power ; therefore the flow from a wound in a 'bleeder,' as a sufferer from the disease is colloquially termed, is difficult to staunch. The condition is always an innate, a nutritional character, and, as such, is reproduced by offspring. Often, however, it is spoken of as 'acquired' by the parent, and inherited by the child.

II8. On the other hand, syphilis is due to a microbe that multiplies in the body, and may pass from the parent to the germcell, embryo, or fœetus, precisely as it may pass to an adult individual. It is no more inherited than a bullet which pierces a mother and enters her fotus. The microbe elaborates a poison, a 'toxin,' which likewise passes into the child with the nutrient fluids, and so may injure it. This injury also is never inherited; it is always an acquirement. ${ }^{1}$

II9. Medical men see innumerable mutilations. Sometimesvery rarely indeed - a child is born with a deformity which bears some resemblance to the parental mutilation. Thereupon the inheritance of the latter is alleged, the possibility of coincidence being ignored. The evidence is of the same nature and not intrinsically superior to that which causes some people to believe in prophetic dreams.

I20. Deformities, mutilations, and the like are so common as to be observed frequently by every one. If a pregnant woman observes one, and bears a normal child, no notice is taken of the fact; but if the child is deformed she is apt to attribute the misfortune to a maternal impression. Medical men have thought it worth while to collect and publish many instances of such happenings. But "Dr William Hunter, in the last century, told my father that during many years every woman in a large lying-in hospital was asked before her confinement whether anything had especially affected her mind, and the answer was written down; and it so happened that in no instance could a coincidence be detected between the woman's answer and any abnormal structure; but when she knew the nature of the structure she frequently suggested a fresh cause." 2

I2I. Telegony is the term applied to the alleged influence of a previous sire. Thus if a woman bears two children, the first to a

${ }^{1}$ For a more detailed discussion of the evidence furnished by syphilis, see \$§ 4 I6-8.

2 Darwin, Animals and Plants, vol. ii. pp. 252-3. 
dark and the second to a fair husband, then, if the latter child happens to be darker than its father, she is apt to attribute the variation to the influence of her first husband. Though still widely credited by animal breeders, telegony has been disproved experimentally, at any rate as far as negative evidence can disprove anything, by Professor Cossar Ewart. ${ }^{1}$

122. Both maternal impressions and telegony are interesting, chiefly as indications of the curious looseness of thought and expression which occasionally prevails in biological discussion. They were often quoted in support of the Lamarckian doctrine; but, plainly, they have no bearing on it. In neither case is it alleged that the mother transmits her acquirement to offspring. In the one case she receives a mental impression, but the child is supposed to develop something entirely different, a physical abnormality. In the other she receives a physical impression, and the child is said to develop a physical character, but not the character the mother acquired. Thus a negress who has borne a child to a white man, and who subsequently bears an exceptionally fair child to a negro, has not herself become fair. In brief, the alleged phenomena do not belong to the category of transmitted acquirements. They are marvels even more marvellous.

123. As long as we think superficially the Lamarckian hypothesis presents an appearance of fascinating simplicity and obviousness. Since the multicellular organism is a 'unit' for most purposes of life, it is natural to regard it as a unit from the standpoint of heredity-to think of it as if it were unicellular, to suppose in effect that the parts of the child are derived from similar parts of the parent, and, therefore, that when a character is acquired by the parent, the same character reappears in, is inherited by, the child. If, in addition, we fail to observe that the complex higher animals fit their environments mainly because they grow into adaptation to them through the development of use-acquirements, it is possible to use this very fact of adaptation in an argument intended to demonstrate the inadequacy of Natural Selection as a cause of evolution. But when we delve beneath the surface and think with precision in terms of the germ-plasm instead of vaguely in terms of the individual, the simplicity and obviousness vanish, and the doctrine becomes almost unthinkable. We see that it depends on a number of assumptions which, to say the least, are wildly improbable. Manifestly, when an acquirement is said to be transmitted, no real inheritance can occur; for a character that is

${ }^{1}$ See The Penycuik Experiments (London: Adam and Chas. Black, I899). 
developed by the parent under the influence of use or injury is profoundly different from one which is developed by the child under the stimulus of nutriment. The doctrine assumes that all structures in all living beings are capable of growth under the stimulus of use, and that the amount of this growth is comparatively trivial; whereas the truth is that only some structures in some living beings can grow in this way, and in some types the amount of growth so made is by far the major part of the total normally achieved. It pretends to account for adaptive variations, but in almost every instance a character transferred from the category of those produced by use or injury to that of those produced under the stimulus of nutriment would become less adaptive, and often positively harmful. It professes to account for evolution, but, were it true, every species would drift swiftly out of harmony with the surroundings, and therefore to destruction. Except miracle, we can conceive no means through which the power of transferring characters from one category to another can have originated. Lastly, there is massive evidence not only that have inborn traits not replaced acquirements, but that the contrary has happened.

124. The Lamarckian hypothesis is dead as an accepted interpretation of the facts. Probably none of the younger students of heredity accept, or will ever again accept it. Indeed, so far as I am able to judge, nobody, even if he happens to be a professional biologist, now holds it unless he is unaware of, or ignores much that has been published of recent years on the subject. At any rate, while such arguments as those which were used, for example, by Spencer about the horns of the elk are still advanced, the reasoning which has demonstrated their invalidity is invariably ignored. 


\section{CHAPTER V}

\section{VARIABILITY}

Variations as distinguished from modifications-Spontaneous variations as distinguished from those due to the direct action of the environment-Evidence of the existence of the latter-Discussion of the evidence-Variability in unicellular and multicellular organisms.-The insusceptibility of the germ-plasm to direct action of the environment-The fallacies of experimental evidenceVariation in new environments-Nearly all variations are spontaneous-The cause of spontaneous variations-The three fundamental laws of heredity.

125. T TE dismiss, then, the Lamarckian doctrine. The only intelligible theory of evolution remaining is that of Natural Selection. Our task, then, is to ascertain, on the one hand, whether this doctrine accords with all the facts of adaptation-is contradicted by none-and, on the other hand, to discover the theory of heredity which accords with it. Since we are able to account for innate likenesses between parents and offspring, ${ }^{1}$ we have, in effect, to study the origin of innate differences, of variations.

I26. The reader may be reminded that a variation-in the sense in which we use the word, which is that of common scientific usage-is a difference between parents and offspring, due to a difference in germ-plasms. It is not a 'modification,' a difference caused by an unequal play of stimuli on parent and child. While biologists still believed in the transmission of acquirements, the term 'variation' was used to indicate any difference, whether germinal or due to an unequal play of stimuli. But, when the Lamarckian theory was being discarded, and the importance of distinguishing between the two kinds of differences was realized, a tendency to limit the term to differences founded on changes in the germ-plasm became general. Even now, however, the word is sometimes given unlike meanings by various writers. Thus some authors, still using it in its old sense, distinguish by means of an adjective between "blastogenetic, genetic, or germinal variations" (i.e. variations properly so called) and "somatogenic variations" (i.e. modifications). Others imply that any change in the child ${ }^{1}$ See $\$ 88$. 
which arises from a change in the germ-cell is a variation. But it is conceivable, indeed, as we shall see, it is certain, that the germcell may be affected (e.g. by nutrient fluids or by poisons), and the child which springs from it altered, while the hereditary potentialities of the germ-plasm, which passes on to the descendants, remain unchanged. ${ }^{1}$ In such cases the alteration in the child is just as much a 'modification,' and just as little transmissible, as if the agency which produced it had acted, not on the germ-cell, but on its cell-descendants - that is on the tissues of the child. Evidently, then, if we accept the modern meaning, and the word variation is to have a signification sufficiently clear and precise to make it valuable in science, it should be limited to those changes in the germ-plasm which mark real alterations of the hereditary tendencies ; thus it should not be applied to those temporary alterations in the well-being of the germ-plasm which result from changes in nutriment or from the presence of toxins and the like-alterations which may result in modifications in the child that springs from the germ-cell in which they have occurred, but are not reproduced by the descendants. It is in this precise sense we shall use the word.

127. Either variations are 'spontaneous' or else they result from changes in the hereditary tendencies due to the direct action of the environment on the germ-plasm. By the term spontaneous, it is not intended to imply that variations ever arise without antecedent cause, but merely that the cause or causes of them are inherent in the germ-plasm itself. Thus, if the germ-plasm gained or lost qualities as a consequence of the normal nuclear activity, growth, and change which precede cell-multiplication, or if, owing to inherent causes, the division of it between the daughter-cells were qualitatively inexact, the variations thus resulting would be spontaneous. Again, when offspring have two parents, a father and a mother, ${ }^{2}$ the variations from one, or other, or both parents that may result from that mingling of germ-plasms which occurs in the conjunction of sperm and ovum, are to be reckoned as spontaneous, for they do not arise from the action of the environment external to the germ-plasm. By variations which result from the action of the environment are meant those variations which arise through the direct and immediate action of its surroundings on the germ-plasm. In multicellular organisms, especially warmblooded organisms, the cells of the soma and the substances (food, toxins, etc.) contained in the nutrient fluids constitute the main effective part of the environment of the germ-cell and its contained

1 See $\S \S 132$ and 136.

2 Some offspring have only one parent, see $\$ 233$. 
plasm. I use the words 'direct' and 'immediate' because it is conceivable that spontaneous variations are due indirectly and remotely to the action of the environment, which, through Natural Selection in the past, may have so dealt with the germ-plasm of the species as to create a tendency to spontaneous variability. ${ }^{1}$

I28. Normal offspring resemble their parents on the whol, but differ from them in detail. We have to decide whether these variations in details are spontaneous or caused by the direct infuence of the environment, or, if by both, in what proportion, as a general rule, the two kinds of variations occur. Now the offspring of the same parents always vary, not only from both the parents, but among themselves. Thus even 'identical' twins are never absolutely alike, and the members of a litter of dogs, pigs, or kittens often differ greatly in size, shape, colour, strength, activity, mental disposition, length and texture of hair, and every other character. These differences cannot be due to the direct action of the environment, for the germ-cells, embryos, and fotuses existed from first to last under conditions that were practically identical. They must, therefore, be spontaneous. At any rate, I, for one, cannot believe that such small differences of environment as may have existed before birth can result in one puppy being big and black and another small and brown, in one being rough and the other smooth-haired, or in one resembling the father, another the mother, and a third a remote ancestor, and so on. Moreover, it has been observed that, when the parents are the same, differences between members of the same litter are just as great as those between members of successive litters. It follows, then, that some variations at least are spontaneous, and our task in the present chapter is to ascertain, not whether ALL variations are due to the direct action of the environment, but whether ANI variations are due to it.

I29. With the intention of proving that variations are due to the direct action of the environment a vast amount of evidence has been accumulated by biologists. A little of it is fairly conclusive, much is inconclusive, and much has no bearing on the point at issue.

I 30. Zymotic diseases-malaria, tuberculosis, typhoid, dysentery, cholera, diphtheria, and the like-are produced by different species of unicellular organisms. Bacteriologists are able to increase or decrease the virulence of many diseases by altering artificially the environment of the microbes. Thus small-pox, when removed to 
the calf, becomes cow-pox; returned to man, it is that very different and much milder complaint, vaccinia. Rabies, passed through a succession of monkeys, grows milder in type for man; passed through dogs, more virulent; through rabbits, still more virulent. When diseases are passed from one species of animal to another, they are generally comparatively mild for the second species at first, but tend to become more virulent to it the longer they afflict it, till a maximum of virulence is reached. Therefore animals that are affected by microbes that have inhabited their own, or a nearly allied species, usually suffer more severely than when the microbes are derived from a more alien source. The organisms of diphtheria and tuberculosis may be gradually 'attenuated' by cultivation in artificial media, those of anthrax quite quickly by heat. Many more instances of the same nature have been recorded.

131. Medical men have published voluminous statistics proving that diseased and intemperate people often have children degenerate or defective physically or mentally, and that slum-bred children are, on the average, inferior in physique to the offspring of rural folk; and they have assumed that these filial defects are innate and due to the parental ill-health.

132. In his Presidential Address to the Zoological Section of the British Association, ${ }^{1}$ Professor J. Cossar Ewart maintained that " there is a considerable amount of evidence in support of the view that changes in any part of the body or soma which affect the general welfare influence the germ-cells."

"It may first be asked," he added, "Does disease, in as far as it reduces the general vigour or interferes with the nutrition of the germ-cells, act as a cause of variation? I recently received a number of blue-rock pigeons from India infected with a blood parasite (Halteridium), not unlike the organism now so generally associated with malaria. In some pigeons the parasites were very few in number, in others they were extremely numerous. The eggs of a pair of these Indian birds with numerous parasites in the blood proved infertile. Eggs of a hen-bird with numerous parasites fertilized by a male with few parasites proved fertile, but the young died before ready to leave the nest. An old Indian bird, however, with comparatively few parasites, mated with a half-bred English turbit, produced a single bird. The half-bred turbit has reddish wings and shoulders, but is otherwise white. The young bird by the Indian blue-rock is of a reddish colour all over, but in make not unlike the cross-bred turbit hen.

1 See Nature, vol._lxiv., I901. 
"Some time before the second pair of eggs was laid, the parasites had completely disappeared from the Indian bird, and he looked as if he had quite recovered from his long journey as well as from the fever. In due time a pair of young were hatched from the second eggs, and as they approached maturity it became more and more evident that they would eventually present all the distinctive points of the wild rock-pigeon. The striking difference between the first birds of the second nest might, however, be due, not to the malaria parasities, but to the change of habitat.

"Against this view, however, is the fact that another Indian bird, infected to about the same extent as the mate of the half-bred turbit, counted for little when mated with a second halfbred turbit; while two Indian birds in which extremely few parasites were found at once produced blue-rock-like birds when bred-one with a fantail and the other with a tumbler.

"Another possible explanation of the difference between the bird of the first and the birds of the second nest is that the germ-cells were for a time infected by the minute protozoon Halteridium in very much the same way as the germ-cells of ticks are infected by the parasite of Texas fever. But of this there is no evidence, for even in the half-grown birds hatched by the pure-bred malarious Indian rocks the most careful examination failed to detect any parasites in the blood. In all probability Halteridium can only be conveyed from one pigeon to another by Culex or some other gnat.

"These results from pigeons suffering from malaria seem to indicate that the germ-cells are liable to be influenced by fevers and other forms of disease that for the time being diminish the vitality of the parents. Further experiments may show that the germ-cells are influenced in different ways by different diseases.

"Sometimes the germ-cells suffer from the direct action of their immediate environment, from disturbance in or around the germ glands. If, for example, inflammation by the ducts or other channels reaches the germ-glands, the vitality of the germ-cells may be considerably diminished; if serious or prolonged, the germ-cells may be as effectively sterilized as are the bacteria of milk by boiling."

I33. Obviously Professor Ewart assumes that if offspring alter in consequence of an alteration in the germ-cell, the change is necessarily a variation. His experiments on fertilization are interesting. He found "that when a well-matured rabbit doe is 
prematurely (2.e. some time before ovulation is due) fertilized by a buck of different strain, the young take after the sire; when the fertilization takes place at the usual time, some of the young resemble the buck, some the doe, while some present new characters, or reproduce, more or less accurately, one or more of the ancestors. When, however, the mating is delayed about thirty hours beyond the normal time, all the young, as a rule, resemble the doe. It may be inferred that in mammals, as in echinoderms, the characters of the offspring are related to the conditions of the germ-cells at the moment of conjugation, the offspring resulting from the union of equally ripe germ-cells differing from the offspring developed from the conjugation of ripe and unripe germ-cells, and still more from the union of over-ripe germ cells." 1

134. A species of butterfly is bright coloured in Germany; but of a darker hue in Italy. Weismann reared the Italian variety in a low and the German in a high temperature. The former became lighter, but not so light as the German variety; the latter became darker, but not so dark as the Italian type. Like Professor Ewart, he does not appear to have ascertained whether the alterations were reproduced by subsequent generations. ${ }^{2}$ Hoffman sowed wild plants very thickly in pots. After several generations they produced double flowers never observed before. Pictet altered the colour of butterflies by transferring the caterpillars from the normal food plant to another. He found that each kind of abnormal food impressed characteristic effects on offspring. These effects persisted and increased for some generations, but eventually the race became adapted to the new food, and thereupon returned to the primitive type." "Dr Bachman states that he has seen turkeys raised from the eggs of the wild species lose their metallic tints and become spotted with white in the third generation." $4 \mathrm{Mr}$ Hewitt "found he could not breed wild ducks true for more than five or six generations, as they proved so much less beautiful. The white colour round the neck of the mallard became broader and more irregular, and white feathers appeared in the ducklings' wings." 5 Metzger cultivated in Europe a tall variety of maize brought from the warmer parts of America. "During the first year the parents were twelve feet high, and a few seeds were perfected; . . . in the second generation the plants were from nine to ten feet in height and ripened their seeds better. ... Some of the seeds had even

${ }^{1}$ Loc. cit., p. 482.

2 The Germ-plasm, p. 389.

${ }^{3}$ L'Influence de l'Alimentation et de l'Humidite sur la Variation des Papillons.

${ }^{4}$ Animals and Plants, vol. ii. p. 250.

5 Op. cit., vol. i. p. 340 . 
become yellow, and in their now rounded form they approached the common Europeon maize. In the third generation nearly all resemblance to the original and very distinct American parent was lost. In the sixth generation the maize perfectly resembled a European variety." 1 "Clayton allowed six bean plants to grow in a spot where they would catch all the sunshine of the day, while six other similar plants were protected by a boarding which effectually screened off the sun. When freshly gathered in October, the weight of the beans and the pods of the exposed plants was to that of the protected as 99:29, whilst the weight of the day beans was as $16: 5$. The next year the weight of the fresh beans and pods obtained from the sunshine-grown seeds of the previous year, was half as much again as in the case of the plants from shadegrown seeds, in spite of the fact that all of the plants were now grown in sunshine and under precisely similar conditions. In the fourth year plants with an exclusively shady ancestry produced flowers but failed to mature fruit." ${ }^{2}$ European dogs are said to deteriorate steadily in India, and English horses in the Falklands.

I35. It is unnecessary to multiply examples. Obviously some of this evidence is capable of an interpretation different from that given by its observers. For example, the medical statistics are wholly inconclusive. Not only diseased and intemperate, but healthy and temperate people have defective offspring; whereas diseased and intemperate people often have normal children. It has not been shown that the proportion of defective offspring is greater in the one case than in the other. ${ }^{3}$ Even if that were done it would still have to be shown that the parents were not themselves defective before they became drunken or diseased; for it is roundly asserted that people naturally (i.e. innately) defective, mentally or physically, are especially prone to intemperance and disease, and, of course, the offspring of such people tend, in any case, to inherit their defects. ${ }^{4}$ Next it would have to be proved that the defects of the children were true variations, true alterations of the hereditary tendencies reproducible by descendants, not merely modifications caused by temporary injury (due to the health or habits of the parents) to the germ-cell, embryo, or foetus. In the case of slum-bred children, also, it has not been shown that the

1 Op. cit., vol. i. p. 340.

2 Vernon, Variations in Animals and Plants, p. 247.

${ }^{3}$ See $\S \S 776-7$.

" See, for example, Branthwaite, The British Journal of Inebriety, January 1908. 
defects are innate-that the defects would not develop equally in country children were they transferred early in life to the slums. ${ }^{1}$ At present the reader may be inclined to regard these criticisms as frivolous and overstrained, but he will find later there is ample warrant for them.

136. Much the same objections may be taken to Professor Ewart's inferences. Evidently his birds acquired the disease in their native habitat, and, since so many or all of them had the malady, it is doubtless very prevalent there. Evidently, again, since the disease resembles malaria, since it travelled from India in the birds, and since so many birds were suffering from it, it is a malady of long duration. If, then, it is a cause of sterility, we are left to wonder how, under the conditions, the race has persisted in India. But granting that it is a cause of sterility, it does not follow that it is a cause of variations. As a fact Ewart's experiments prove only that, like other cells, the germs, and even the germ-plasm may be enfeebled by toxins; they do not prove that the hereditary tendencies are thereby altered. His deduction might have been, but was not, tested by showing that the socalled variations were transmissible to descendants. Ewart himself supplies clear evidence that the germ-plasm was not altered, for one of his Indian birds, which, when diseased, had offspring resembling the English parent, after recovery had offspring resembling itself. Evidently the germ-plasm that remained in this bird was capable of recovering from its enfeeblement, and we have no reason to suppose that the germ-plasm which passed from him and entered a healthy female body was less capable of recovery. It certainly fertilized the ovum, and the fact that the offspring resembled the English parent is no proof that succeeding generations would not have reproduced the Indian characters. Professor Ewart has, indeed, furnished warrant for believing that the condition of the germ-plasm at the time of conjugation determines to some extent the characters of the offspring, but no warrant for supposing it fixes the characters of subsequent descendants. All that is demonstrated is that enfeeblement of germ-plasm may render latent for a generation the characters of the parent whence it is derived. The force of this objection is even better seen in the experiments by which he influenced the characters of rabbits. In fact, his observations seem rather to elucidate the conditions under which latency occurs than the conditions under which variations arise. It has long been known that sexual characters

1 See $\$$ 732-5. 
are transmissible in a state of latency through parents of the opposite sex, ${ }^{\mathbf{1}}$ and the Mendelian experiments demonstrate that latency occurs in characters other than the sexual. ${ }^{2}$

I37. The bacteriological evidence is important and deserves to be considered at length. Every unicellular organism is a germcell, just as every germ-cell is in effect a unicellular organism. In the last analysis, therefore, the problems of heredity presented by them are similar. In other words, if we think in terms of the germ-plasm, not in terms of the cell-community, the problems of heredity presented by unicellular organisms and germ-cells are similar. We noted that the question as to whether the acquirements of unicellular organisms are, or are not, inheritable is totally distinct from the problem of the transmission of acquirements amongst unicellular types. ${ }^{3}$ Its real counterpart amongst the problems of multicellular life is the question whether or not changes caused in the germ-cells by the direct action of their environments are inherited by descendant germ-cells. Obviously this is a very different problem from the question whether the particular changes caused by the environment in the somatic cells are inherited by the descendants of the germ-cells, whether the acquirements of Brown and Jones are inherited by the descendants of Robinson and White. Now unquestionably a unicellular organism or a germ-cell may be injured or benefited by its environment. The problem we have to solve, then, is whether such changes tend to be inherited by the descendant cells. In other words we have to discover whether they necessarily or usually imply alterations (i.e. variations) in the germ-plasm.

I38. Of all unicellular organisms the disease-producing (pathogenic) microbes have been most closely studied, and of all their characters their powers of offence and defence have claimed the most attention. When they enter a living body, a man for instance, a struggle ensues between them and the cells of the host. They, or at least some species of them, secrete toxins, soluble poisons which defend them from the cells, which in turn secrete substances (enzemes) poisonous to the microbes. ${ }^{4}$ If only a few microbes enter the body they are destroyed. Thus it has been found experimentally that if less than sixteen thousand virulent anthrax bacilli be introduced into a normal young rabbit, they all perish. A greater number multiply and cause disease in a suspectible individual, presumably because they so poison the cells of the body that the latter are unable to poison them. 
If recovery occurs, the cells of the body gradually become accustomed to the toxins, which are digested by them. ${ }^{1}$ They obtain the upper hand; the individual acquires immunity; the microbes are destroyed; and, as a consequence, the sufferer is no longer infective to his fellows.

I39. A human race undergoes evolution against such a disease as measles or diphtheria when it becomes more resistant. It becomes more resistant when successive generations become, through Natural Selection, more capable of reacting to the toxins and so 'acquiring' immunity. The germ-plasm becomes such that under the stimulus of nutriment individuals develop, who, if they experience the disease, are able to develop further and 'acquire' actual immunity. Therefore, while the power of acquiring immunity is an 'inborn' character, the immunity itself is a 'use-acquirement.' That is to say, the individual who acquires immunity becomes used to the disease (develops under its stimulus), just as the muscles of the athlete become used to endure fatigue, or the skin of the palm to endure rough labour. The process of evolution is a long and a slow one, extending over many generations. The final effect, as we see, is not the creation of a race the individuals of which resist infection (i.e. are innately immune), but the creation of a race the individuals of which tend to recover from infection (i.e. acquire immunity). This is not true of all diseases, but it is true of the majority. ${ }^{2}$ We shall study the subject at length in a subsequent chapter. Meanwhile the points on which I wish to lay stress are (I) that human races become adapted through Natural Selection to the presence of diseases, and (2) that in the case of many diseases this adaptation consists in a swift reaction to infection - a reaction so swift that the cells of an affected individual begin to produce their enzymes as soon (within a few minutes) as the microbes make their presence felt. This production of enzymes is a step on the road to immunity-but only a step, for after the enzymes disappear more or less completely from the body, the immunity remains. Here we have an instance of the high organism making a pre-eminently advantageous useacquirement in spite of the fact that its cells are continually bathed in and injured by the toxins.

I40. By skilful management it is possible to alter artificially the characters of unicellular species. Thus certain saprophytic organisms (which normally obtain their nutriment from dead organic matter) may, under favourable conditions, be gradually 
'trained' to attack living beings; that is, they may be trained to produce toxins so protective to themselves, and, therefore, so injurious to the cells of the host, that they are able to live and multiply in the dangerous environment till the host dies or acquires immunity. This training, this conversion of a saprophytic into a parasitic type, always involves placing the microbes in contact with living cells. For example, streptococci, which are normally saprophytic, may be rendered very virulent (i.e. capable of maintaining existence in living tissues) by passage through a series of animal hosts, but cannot otherwise be rendered virulent. On the other hand, purely parasitic organisms may be trained to saprophytic habits of life. If placed in a suitable non-living medium (e.g. broth) where they are no longer persecuted by the cells, they slowly lose their toxins, and, while becoming more capable of existing in that particular medium, become less capable of maintaining themselves in the living body. In either case the change, since it increases in successive generations till fitness to the new environment is achieved, is both germinal and adaptive. So close is the adjustment to the environment that, usually, each microbic disease is limited to a single species of animal. Thus most human diseases are peculiar to man. When more than one species is attacked by the same disease, as is often brought about experimentally, the microbes tend to separate into varieties or races, each of which is most virulent (i.e. best adapted to, best protected in) the species it has made its host. Each parasitic species has its peculiar toxin, a means of defence which is always the same for the same species, but which differs from that of every other species, as may be judged from the symptoms of the maladies they produce. Thus, streptococci always produce the same symptoms, the same toxins, when they become parasitic. The existence of the toxin, a very complex chemical substance, implies a remarkable producing apparatus. The evolution from saprophyte to parasite is mainly the evolution of this apparatus. Unless we assume that the microbes of disease, highly specialized organisms, were miraculously created to afflict the higher species, we must assume that they were all evolved from saprophytic types.

14I. Now we reach the problem as to the mode by which saprophytic species become parasitic. It is probable that all unicellular species, even the saprophytes, have some sort of toxins by means of which they dissolve and digest their food or protect themselves from other unicellular organisms, and which, like the saliva of snakes, is capable of undergoing evolution till it 
becomes poisonous to other forms of life. ${ }^{1}$ Saprophytes cannot, under normal circumstances, contend against the living cells of multicellular types; but that the toxins are strong in at least some of their species is proved by the fact that the organisms, for example, which cause putrefaction in dead bodies may, under favourable conditions, infect and by poisoning destroy men and other animals. ${ }^{2}$ Unicellular organisms multiply very quickly, as may be judged by the rapidity with which an epidemic of infectious disease may sweep a large and populous country. Many generations elapse, therefore, even under the most favourable experimental conditions, before a saprophytic species becomes truly parasitic. Now, in this passage from an environment to which they are adapted to another which at first is very unfavourable', do the microbes make useful 'acquirements' which are 'transmitted' and accumulated in descendants? Or do they alter through the Natural Selection of favourable spontaneous variations? The former is the accepted hypothesis. Indeed, bacteriologists do not seem aware that there is an alternative. Reasoning from the analogy of the human being, they suppose, in effect, that microbes are able to make advantageous acquirements under the stimulus of use, and, further, that, being unicellular, they are capable of transmitting these acquirements to offspring, so that the latter start development where the parents finished.

I42. But it is necessary to exercise a little scientific imagination. The power of making use-acquirements is apparently a late product of evolution, limited to the higher species, in which alone it is perceptible. At any rate we have no right to assume thus easily that lowly microbes possess it. Moreover, since the microbes of disease begin by multiplying and flourishing greatly in an infected person, manifestly they have at first much the best of the struggle, and would keep it did they grow stronger under the stimulus of use. Indeed, it is evident that, if microbes not only made use-acquirements but transmitted them, then, since their multiplication is so rapid, their virulence would soon become so exalted that the

1 The evolution of a toxin implies the power, not only of producing it, but of resisting it. Thus the cells of the poison gland of the snake are immune to the venom, the cells of the alimentary canal are resistant to the digestive ferments, the body-cells to the poisons which destroy the microbes. So, also, the microbes are resistant to their own toxins.

${ }^{2}$ It is said that the carnivora are particularly immune to septicæmia. If this is true, it is an interesting example of special evolution. Owing to the nature of their food and the wounds they are apt to receive in pursuit of it, carnivora are particularly exposed to infection. 
higher species, which can make but cannot transmit acquirements, could not contend against them and would soon become extinct. Again, the human being is capable of making only such useacquirements as evolution has fitted his race to develop. The microbes, while they are becoming parasitic, are in an entirely new and unfavourable environment, in which they are exposed to poisoning and enfeeblement through the enzymes secreted by the cells of the host. Evolution has not adapted them to make the right acquirements, any more than it has adapted human beings to make acquirements fitting them for flight or for life under water. Under the conditions, what sort of acquirements could they make which would enable them to produce stronger and stronger and, therefore, different toxins, and so protect themselves from the cells ?

I43. It is conceivable that the toxins might in some way be enfeebled by injury to the producing apparatus and that this injury might be transmitted and so accumulated in subsequent generations till the microbes perished; but to believe that they are ever strengthened, that the producing apparatus of the individual is improved by the accidental effects resulting from the direct action of the environment, is to believe in a coincidence, a 'fluke' so remarkable that it amounts to miracle-a miracle that must have happened to every species of pathogenic microbe during its transition from saprophyte to parasite, and which the human operator is able to initiate at will by placing saprophytic species in contact with living cells, a miracle as marvellous as if chance blows of a hammer on pieces of metal had produced and fitted together a delicate and elaborate machine.

I44. In brief, if saprophytic microbes, while becoming parasites, are in any way affected by their new environments-and doubtless they are affected-then, since they neither cause the extinction of the species they attack nor themselves become extinct, it is certain that their acquirements are not transmitted. The notion that, like the higher animals, they are able to make use-acquirements, is a mere guess, unsupported by any evidence, but opposed by much. Even were the guess correct, then, since the higher organisms do not make acquirements which evolution has not fitted them to develop, it is hard to understand how unicellular organisms could react in a way that enabled them to meet contingencies never before experienced by the race. The analogy between them and the higher animals, therefore, is strained and inaccurate at all points.

145. Consider, now, the contrary hypothesis, that saprophytic 
species become adapted to a parasitic existence through the Natural Selection of spontaneous variations. Doubtless unicellular organisms, like the higher types, vary spontaneously. If that be admitted, then, presumably, in the struggle with the cells, the microbes with the weaker defensive powers, the weaker toxins, or the weaker powers of producing toxins under unfavourable conditions, tend to perish, while those with the stronger powers tend to survive. In that case evolution must follow till the saprophytic race becomes adapted to a parasitic habit of life, after which it would be maintained in adaptation by the same process of selection. The facts that microbic species evolve virulence only when in a position to be selected by living cells, that this virulence and their other germinal changes adapt them precisely to the particular species of animal they attack, and that they tend invariably to lose the adaptation (i.e. undergo retrogression) when no longer in a position to be so selected, that these changes in both directions are continuous and so gradual that many generations must come and go before they are completed, and that they proceed no further than enables the species to persist in the new environment, are so highly significant that we have no alternative but to attribute them, like the adaptive changes of the higher species, to selection on the one hand or cessation of it on the other. They are too adaptive to be due to any other cause. They fit the many and diverse species of unicellular parasites too closely to their environments to be results of mere coincidence, of mere accidental effects, produced by the direct action of the environment.

146. The belief that the increasing virulence of saprophytic organisms, when they are becoming parasitic, results from the direct action of the environment on their germ-plasm, is one of those 'obvious' inferences which, when all the evidence is taken into account, are found to rest on a series of impossible assumptions. Here, as always when considering any complex and difficult problem, we cannot link together all the evidence unless we make a rigorous deductive inference of consequences and follow it by an appeal to reality. The generalization that microbic species change adaptively when placed under right conditions in changed environments is really 'obvious'; it is an 'immediate' inference. The generalization that they change because their germ-plasm is directly affected by the environment is not really obvious, and must be tested. When the test is made, when we do not shirk the hard thinking necessary, it is seen to be obviously inaccurate.

147. Selection affords an explanation of the adaptive changes 
of microbic species at once probable and in accord with all we know of nature. Studying these changes, it becomes evident that Natural Selection is operative only under certain conditions. The germ-plasm must be variable; but it must be spontaneously variable, not variable under the direct action of the environment. Spontaneous variations, occurring all round the specific mean, afford materials for Natural Selection and tend to enable the species to meet all contingencies. But variations caused by the direct action of the environment have an opposite effect. Under conditions nearly the same for all the individuals exposed to them, they cannot occur all round the specific mean, but in one general direction only. Therefore they cause a drift, which may or may not be adaptive, but which, since a new environment is usually unfavourable and therefore injurious, can be adaptive only as a most unlikely coincidence. Even if such a drift were favourable at first, the accumulation, the exaggeration of it during succeeding generations would be sure ultimately to ruin the co-adaption of parts and functions and so render it unfavourable; for there is no imaginable reason to suppose that if such a drift occurred in ancestors it would not continue in descendants. Virulence is not the only adaptation found in the microbe. The whole organism is compounded of adaptations, every one of which is co-adapted to all the others. Except by the theory of evolution through Natural Selection it is as hard to account for any of them as it is to account for virulence. As we see, Natural Selection cannot control a drift in which every individual participates. Only in one way can it be prevented-by the germ-plasm becoming highly insusceptible, highly resistant to the direct action of the environment. We are driven, then, to the conclusion that the germ-plasm is both spontaneously variable and highly resistant to the direct action of the environment. In other words, we must believe that in any species that is not undergoing extinction, spontaneous variations greatly preponderate over those which are caused by the direct action of the environment.

I48. The evidence is conclusive that the germ-plasm of all persistent species possesses a high power of resisting enforced change. For example, some-probably all-human diseases are of great antiquity. Descriptions of them written two or three thousands of years ago are accurate for the present day. During all that time, though exposed to enzymes and other potent influences, the microbes, minute atoms of naked protoplasm, have not altered appreciably. Fitted closely to a stable environ- 
ment, the human being, they have been stable. They have not drifted.

149. There is nothing especially magical or mysterious about this germinal power of resisting enforced change. There is abundant evidence that it exists. If it did not exist no species could persist. But, when we say that it exists, we do not imply that the germ-plasm is incapable of being injured, incapable of enfeeblement, or death, or alteration of its hereditary tendencies. We imply merely that such enforced alteration of the germ-plasm of a species that has been fitted by evolution to its environment is usually an injury, and that the evidence is that when the injury is severe enough to cause alteration of the hereditary tendencies, it is usually severe enough to cause the death of the germ-plasm also. In other words, we imply that the hereditary tendencies are implanted in the germ-plasm as firmly, or almost as firmly as life itself. Indeed collectively they are the life; because of them the individual (and the germ-plasm) absorbs nutriment, excretes waste-material, grows, multiplies, and so forth.

I50. The power to resist enforced change is essential to existence. Presumably, therefore, it is, like other essential characters, a product of evolution. It is, at least, probable that just as individual unicellular organisms vary as regards other particulars, so they vary as regards the power of their germ-plasms to resist enforced change. If this power be weak the germ-plasm tends to be destroyed or altered by the influences to which it is inevitably exposed. The descendants of the altered individuals, if there be any, inheriting the weak resisting power, tend to be still more changed and ultimately to be eliminated. But, if the resisting power be great, and if individuals vary as regards it, while at the same time spontaneous variations occur in all directions, the race tends to survive; for in that case there is no inevitable drift; natural selection has scope, and the resisting power tends to grow stronger with selection. The conclusion we reach, then, is that Natural Selection has established and maintains the high insusceptibility of the germ-plasm to the direct action of the environment. ${ }^{1}$

15 I. Turn, now, to multicellular animals and plants. Not only

1 Interesting evidence of the stability of the germ-plasm of unicellular organisms is furnished by Dr Pearl in his study of Paramocium caudatum. These organisms multiply for many generations without conjugation. But at intervals conjugant individuals appear. "Dr Pearl grew Paramœcia under much variety of environment, and found that the non-conjugant type was highly correlated with the environment and the conjugant type singularly little affected by the 
the germ-cell but every cell of the community is a unicellular organism. We may take the human being, as the type best known to us, for an example. Consider first his somatic celis. From the moment the fertilized ovum begins to proliferate to the death of the aged man, strong influences-want, plenty, disease, health, hardship, alcohol, and so forth-play upon the cells; yet each cell comes true to its type. Thus all sorts of stimulants and irritants may act on the skin-cells and change them greatly; but, when the cause of change is removed, the hereditary tendencies are found to be unaltered, for the young cells develop into skin-cells of quite the old kind. I have transferred clippings from an old man's skin to the scalded and denuded arm of a young woman, and they have grown with all the vigour of youth. If a lymphatic gland be diseased (e.g. tuberculous) for a score of years, and recovery then occurs, the cells are still typical of their kind. Disease enfeebles cells, but recovery from disease is evidence of the stability of their hereditary tendencies. Were the latter easily altered, there could be no recovery, and every attack of measles or chicken-pox, maladies in which toxins are abundant and in which the enfeeblement of the cells is shown by the disturbance of function, would be fatal. Consider the cells which line the alimentary canal and the extraordinary variety of influences to which they are subjected. Life could not exist if the germ-plasm (idio-plasm) were not resistant. Evidently, then, as a condition essential to the survival of the individual and the persistence of the species, somatic cells hold their hereditary tendencies with extraordinary tenacity-with a tenacity as great as that displayed by the microbes of disease.

152. Further and very decisive evidence is afforded by the extreme stability of plants when propagated by slips and suckers. Centuries may elapse, the plant, continuously so propagated, may environment. The whole inquiry was, of course, undertaken to illustrate Weismann's position, that while acquired characters are not inherited, the environment can influence inheritance when one cell is both soma and germ. In biology it has become almost axiomatic to assume that the Protozoa can inherit acquired characters owing to this identity, while in the Metazoa the acquired character of the soma is at the very least not usually inherited. Dr Pearl brings out the all-important point that the gamete in Paramœcium is not, like the nonconjugant cell, markedly influenced by the environment" (Professor Karl Pearson, Nature, Oct. 18, I906, p. 609). Mr H. S. Jennings has also published observations on Paramcecium. He says nothing concerning conjugant individuals, terms all new characters variations, and concludes, "In order that it may be inherited (by more than one of the progeny), a characteristic must be the result of such a modification of the mother cell as will cause it to behave in a certain way at reproduction." Apparently he means that spontaneous variations tend to be transmitted, but not acquirements. 
be grown in all the quarters of the globe, yet usually no appreciable change is observable. Even when a change is seen it is generally a mere 'modification,' for the plant, if restored to its ancestral environment, resumes the original form. 'Bud variations' occur, it is true, but they are comparatively very few, and we have little reason to believe that they are due to the direct action of the environment. Thus, when a nectarine appears on a peach tree, it is more likely that the change in the branch that bore it was spontaneous than that it was caused by direct action; for the latter would imply that the environment of that one branch differed amazingly from the environments of the other branches.

I 53. The germ-plasm of the microbe is very complex; but more complex to an almost infinite degree is the germ-plasm of such a being as man. The descendant cells of the fertilized ovum are not mere copies of that ancestor, but members of a vast community in which there is great diversity of form and function, and in which every cell takes its appointed place and does its predestined work. Presumably the orderly complexity of the body, with its immense ramifications of large and small blood-vessels, lymphatics and nerves, its organized groups of muscle, blood, gland, and nerve cells, its skin, bone, and cartilage, indicates a corresponding complexity of the germ-plasm. Yet, though this germ-plasm is constantly exposed to all sorts of potent influences (e.g.toxins), and has been so exposed for thousands of generations, so great is its stability that all this great complex and even many developmental processes which date back to millions of years before the evolution of the vertebrate type are reproduced with unfailing accuracy by every normal individual. Quite apart from the fact that Natural Selection would have no scope if the hereditary tendencies drifted this way or that at the mercy of the environment, it is evident that no complex type could maintain the regular development and the co-adaptation of its parts, unless the germ-plasm were endowed with very great resisting power.

I 54. Amongst multicellular organisms selection occurs in every stage of development, amongst germ-cells and embryos as amongst adults. Germ-cells, like unicellular organisms, are fitted to their environments by characters which depend on the hereditary tendencies carried by the germ-plasm. As is proved by the variations of the individuals that develop from them, they vary amongst themselves. They are in competition with one another, some being better adapted for survival and the successful performance of their functions than their fellows; some having more and 
some less resistant germ-plasm. Granting that species have arisen through Natural Selection, then the hereditary potentialities which enable the germ-cells to develop into higher individuals (embryos and adults) are of course due to selection occurring amongst the latter; but the characters that fit them to their own environments, for example the locomotory tail of the spermatozoon, are equally of course due to selection occurring amongst themselves. The selection that maintains the stability of the germ-plasm, therefore, falls first of all on the germs which cannot survive as cells if the germ-plasm is much altered, or develop into higher organisms if it is more than a little altered. Evolution is never perfect; it is probable, therefore, that many, perhaps very many, germs vary unfavourably in this particular and perish, and that to this circumstance is due the fact that evidence of germinal alteration resulting from the direct action of the environment is rarely found amongst adult individuals.

I 55. Germ-cells are very numerous amongst the higher animals, though the number of possible offspring is small. Thus, the germcells of such an organism as man exceed a million-fold the number of his children. This superabundance, like all other characters of the species, is, presumably, an adaptation. According to accepted doctrine it serves to ensure fertilization. But nature is very parsimonious of her materials, and when we remember the perfection of her adaptations (e.g. the eye, the ear, or the digestive organs), the device of providing a vast multitude of cells in order to secure the fertilization of a very few seems so unnecessarily wasteful and clumsy as to awaken a suspicion of the correctness of the interpretation. Such a device may be necessary when the germ-cells are scattered broadcast in the environment and meet by chance, as in the case of some plants, but hardly when they enter a special receptacle in the body of the female. It seems not improbable, therefore, that the function of the apparent superabundance is to provide materials for a selection which serves, above all, to maintain the resisting power of the germ-plasm. At any rate material in which such a selection can occur is provided as abundantly by the higher animals as by the lower.

I 56. Leaving aside the question as to how precisely the high insusceptibility of the germ-plasm has been evolved, there can, as I say, be no doubt of its existence. But though the germ-plasm possesses high powers of resisting enforced change, though death usually accompanies such change, it does not follow that conditions cannot be found in which the germ-plasm is altered and yet not destroyed. Thus, Clayton's beans progressively 
deteriorated when placed in the shade, as do European dogs in India and horses in the Falklands. But in all these cases the change, when clearly germinal, when it is transmitted and so increases from generation to generation, is ever a deterioration, not an adaptation. The germ-plasm in those cells that develop into offspring is altered and injured, but not sufficiently to cause its death or even to prevent the development of the individual. Now, obviously, the deterioration cannot continue, generation after generation, indefinitely. If not checked, the race must perish eventually. But there are plants that dwell in the shade, there are native races of dogs in India, and some varieties of horses flourish in climates severer than that of the Falklands, and so on. They are said to have become 'acclimatized,' to have reached an 'equilibrium'-meaningless terms unless it be explained what they imply. They really imply adaptation, an essential feature of which consists in the germ-plasm becoming insusceptible to the direct action of the environment. Thus if, amongst Clayton's beans, there had been any germs the plasm of which had so varied as to be unaffected by shade, then, since he obtained seed in the first three generations, he could have established by Natural Selection a race as capable of existing away from the influence of direct sunshine as mosses and ferns. He failed because, instead of proceeding step by step, he made the conditions too rigorous. When captive, wild animals are sterile, though still displaying sexual desires; it is possible, in some cases at least, that the germ-plasm in their germ-cells is so altered as to be incapable of directing development.

157. We observed that medical men are in the habit of declaring that parental diseases and intemperance usually result in filial degeneration, and we noted that this inference could not possibly be correct, for otherwise the human race would have long ago become extinct; whereas, on the contrary, it has everywhere undergone protective evolution. It is possible, nay probable, that disease and intemperance are sometimes, though rarely-i.e. under exceptional conditions or in exceptional germ-cells-causes of variations; but, in view of their long-continued prevalence, which has made them quite as 'normal' a part of the environment as the dangers to which other types of animals and plants are exposed, in view also of the fact that in every case protective racial evolution, not deterioration, has occurred, it is hard to understand how they can be constant or even frequent causes of the variations which are seen in children and adults. Nevertheless, suppose we 
admit for the moment that the medical inference is true for the present day, and that disease and drink act on the human race as the climate of India is said to act on recently imported European dogs. It is not denied, indeed it is a matter of common observation, that diseased or drunken parents frequently have perfectly normal offspring. Here, at once, we see Natural Selection at work on the germ-cells. These normal individuals, whose germ-plasm was insusceptible, are those that continue the race. If, then, the germplasm of the race is not now, after thousands of years of selection, as a general rule insusceptible, it is in process of becoming so. Possibly the American maize mentioned by Metzger furnishes another instance of this kind of selection.

I58. The conclusion we reach, then, is that, though variations may result from the direct action of the environment, such variations are, in effect, always injuries, and are of rare occurrence in individuals who survive and have offspring. Adaptation (i.e. evolution) depends almost exclusively on spontaneous variations. These do not imply damage to the germ-plasm, but are products of its vital activity. Occurring in vast abundance all round the specific and parental means, they supply the sole material for Natural Selection.

I59. We conceive the germ-plasm, then, as living and active, closely adjusted to its environment, growing, dividing, varying, capable of being destroyed and injured, but resisting death and injury, and within limits capable of repairing damage and returning to its original state-as behaving exactly as a living individual does. Plenty of biologists think of it as drifting like a dead thing at the mercy of circumstances, incapable of making vital reactions, capable of undergoing all sorts of radical alterations and yet of surviving. But never yet have I met anyone holding this opinion who was willing to make a rigorous deductive inference of consequences, and explain how it happens that races exposed to unfavourable conditions undergo, not deterioration, but adaptation. As well might it be maintained that if a man goes continually down hill he will ultimately arrive at the top.

I60. The problem of the causation of variations is, both theoretically and practically, one of the most important of all biological problems. A discussion prolonged but singularly futile, and founded mainly on experiments and observations, innumerable but peculiarly irrelevant, has raged about it. From such experiments as those of Clayton on beans, men have inferred that all 
variations are caused by the direct action of the environment. As a fact it is impossible to solve the problem experimentally. An experiment cannot be made unless the animal or plant used for the purpose is removed from its normal environment to one very different; and the question is, not whether it is possible to devise conditions in which the germ-plasm, though not destroyed outright, is injured beyond recovery, but whether, under the conditions in which species maintain their characters or undergo adaptive change, variations commonly arise through the direct action of the environment. In other words, the question is whether variations are normally spontaneous or due to enforced alteration. In the search for experimental data and the 'obvious inferences' so often drawn from them, the whole problem of adaptation has been actually, though not ostensibly, ignored. Men have failed to make the obligatory deductive inference of consequences followed by an appeal to reality. They have not asked themselves how the multitudinous and delicate adaptations and co-adaptations of animals and plants could have arisen if species had drifted at the mercy of the environment, nor troubled their heads with such notorious facts as that human beings persist and for ages have persisted in lands where every individual is saturated with the poisons of malaria or other virulent diseases. That is to say they have not taken the whole of the facts into consideration. If the reader's thinking, also, is not to be mere guessing, he must follow the procedure which has led to the creation of all science that is more than a mere catalogue of likenesses and differences, ${ }^{1}$ and, bearing in mind that species become adapted to their surroundings, ask himself to what the adaptation is due, to a vital reaction resulting from the Natural Selection of favourable spontaneous variations, or to a helpless drift resulting from germinal changes caused by the direct action of the environment. In other words, he must ask himself which of these contradictory hypotheses can be conceived as being in accord with the facts of existence.

I6I. Some biologists have argued that influences (e.g. abundant nutriment) from the environment acting on the germ-plasm tend to cause variations all round the specific mean. But if the germplasm contained in the germ-cells of the species is everywhere very similar-as it must be, since the individuals of the species resemble one another-then a force acting on it, if it causes any change, must cause everywhere much the same change and therefore a general drift in this or that direction. It is only when

${ }^{1}$ See $\S \S 8$ Ig et seq. 
the germ-plasm in the different cells is dissimilar (i.e. has varied spontaneously) that the changes, if any, can be dissimilar. But, if it be admitted that germ-cells vary spontaneously, it is unnecessary to appeal to the action of the environment to explain facts which are already sufficiently explained. In science " neither more nor more onerous causes are to be assumed than are necessary to account for the phenomena."

162. The apparently well authenticated fact that species (e.g. wild plants) removed to a new environment (e.g. cultivated garden) tend, though healthy and prolific, to display, especially after the lapse of several generations, greater variability than in the ancestral habitats, has also been advanced in support of the hypothesis that variations are commonly caused by the direct action of the environment. Here again we are asked to believe that an influence (i.e. the sum of the new influences in the new environment, which presumably is much the same for all or nearly all the germ-cells) causes variations all round the specific mean. But Natural Selection not only adapts species to changing environments but keeps adapted species stable in stable environments. Speaking generally, natural environments are very stable-so stable that all distinct species are very old, and some have persisted without appreciable change for enormous epochs of time. During the entire historical era, though natural varieties may have arisen, not a single species is known to have altered appreciably as a whole. Variability is not lacking, since man is able to create varieties very swiftly by artificial selection. Obviously, any considerable variation in a species, already closely adapted by thousands of years of selection to its environment, is almost sure to be disadvantageous. A superior tendency to vary, itself a variation, is, therefore, disadvantageous, and is eliminated in an environment to which the species is already well adapted. But it is advantageous in a new environment to which the species must become adapted, and not unfavourable, or not so unfavourable in a new environment (e.g. garden) to which the species is adapted, but where the old causes of elimination do not act. In brief terms the characters which adapt species to their environments include a right degree of variability; that is, the degree of variability displayed by every species is itself an adaptation. When monsters, for example, perish, it is not only unfavourable variations which are eliminated, but also a tendency to vary to an extent too great to produce fitness to the parental environment. It follows, then, that the fact that species tend to become more variable in a new environment is 
evidence, not that the germ-plasm is directly affected by its surroundings, but that Natural Selection, which controls and which formerly limited the amount of variability, no longer does so to the same extent.

163. The foregoing leads us to a very important problem. Though environments as a whole are very stable, yet they are seldom if ever absolutely stable in every particular for prolonged periods. One or other factor amongst the complex of which they are compounded tends always to be undergoing change. New factors of elimination appear, or old factors become more stringent or less stringent. Thus, during the last few centuries many new and fatal diseases have appeared amongst the human inhabitants of the Western Hemisphere; while in the Eastern world the increase of population has accentuated the stringency of selection by old-established diseases at the same time that elimination by war, famine, and wild beasts has diminished. Species change in adaptation to changing environments, and thus all forms of life known to us have arisen. The fact that they have undergone evolution is proof that their environments have undergone change. Man, for example, is not fitted for the same environment as that in which his pre-human ancestors existed, nor would the latter be fitted for his present surroundings. ${ }^{1}$ The evolution of a species is founded on the variations of its individuals. Were offspring exact reproductions of parents, the race would persist only if it were well adapted to a perfectly stable environment. A right degree of spontaneous variability therefore is an essential condition of persistence. Presumably, therefore, it is an adaptation-with insusceptibility to the direct action of the environment, the most useful and important of all adaptations; as much an adaptation as hands, eyes, lungs, or any other of the functioning characters of living types. It is not, as is often implied, a chance property of germcells, comparable, for example, to the colour of a dead leaf. And, if it is an adaptation, then, reasoning by analogy, it has, like all other adaptations, been established and maintained by Natural Selection.

I64. We need not attempt to discuss at length the question as to how variability began in living beings. Like all other problems relating to the beginnings of life, its origins are involved in obscurity. Variations of some sort must have preceded Natural Selection, if only for a moment, for otherwise the latter could have had no material with which to work. But, probably, even these 
earliest variations were spontaneous, for it is at least unlikely that the multiplication of the primitive living beings involved exact quantitative and qualitative division. Doubtless early variations, however caused, were seized upon by Natural Selection, which, as a necessary antecedent to all else, established the insusceptibility of the germ-plasm and regulated the tendency to vary in all directions about the specific mean. In other words, though Natural Selection cannot have been the original source of variations, it has established variability as an adaptation and ensures its constant occurrence, and in each species has regulated the amount of it according to the needs of the time. At any rate, however spontaneous variability originally arose, it certainly exists and presents all the signs of an adaptation controlled by Natural Selection.

165. Striking evidence that spontaneous variability is a strictly regulated adaptation, and that germinal insusceptibility to the direct action of the environment is also an adaptation, is furnished by four well-authenticated facts. First, plants propagated asexually (by slips, suckers, etc.) vary very little as long as they are so propagated, no matter how numerous the pseudo-generations, nor how diverse the influences to which they are exposed and by which they may be modified; wherefore gardeners, whenever possible, propagate valuable varieties by means of cuttings. ${ }^{1}$ This indicates that variations are, at least, rare amongst somatic cells, for, if they were common, plants propagated asexually would be changeful. Second, offspring arising by seminal generations (i.e. from germ-cells) always vary from their parents. This indicates that variations occur normally within the limits of the germ-tract, that is in those lines of cells by which the germ-cells of the individual descend from the fertilized ovum whence he sprang. Third, the offspring of a plant reared from its seed vary no more and no less, apparently, when the seed is gathered at the end of the first season, than when it is gathered after many seasons during the course of which the plant has been propagated asexually. In other words, the lengthening of the germ-tract by any number of pseudo-generations (i.e. slips and suckers) does not appear to increase the number and magnitude of variations. This indicates that variations do not arise all along the germ-tract, but once for all in some particular part of it ; for, if they arose all along the tract, the lengthening of it would tend to influence their number and magnitude. Fourth, 'identical' twins, which originate from a 
single fertilized ovum, are closely alike, while those which arise from different ova (even in the case of peas in the same pod) differ as much, or almost as much, as offspring born at successive births or seasons. This indicates that the part of the germ-tract at which variations normally arise is the last, or one of the last, cell-divisions that precede the ripening of the germ-cells; for if variations occurred earlier, not only identical twins, but whole batches of offspring would tend to be identical, especially when the reproduction was parthenogenetic.

I66. The following diagram may help to elucidate the argument.

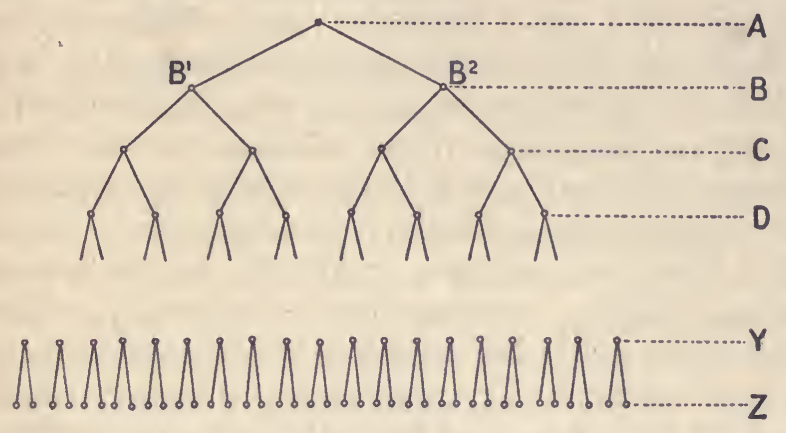

167. Suppose $A$ is a fertilized ovum from which the cells of the germ-tract B, C, D ... Y, Z, are derived. $Z$ represents the descendant germs. Granting that variations arise at a particular point in the tract, then, if they arise at $A, B^{1}$ will differ from $B^{2}$, as will their descendants; but all the descendants of $B^{1}$ will be alike, as will be those of $B^{2}$. If variations arise at $B$, there will be four batches of identical descendants. If at $\mathrm{C}$, eight. And so on. But if the point at which variations arise is at $Y$, there will be no identical germs, except (especially in the case of complex organisms) as a very unlikely coincidence. On the other hand if variations arose all along the germ-tract, then, though all the germs would tend to differ, the lengthening of the germ tracts, as by pseudo-generations, would tend to increase variability, which, as we see, does not happen.

I68. Now, the evidence is that all these laws (laws in the scientific sense of uniformities in the sequences of events), unlike the laws of physics, have exceptions. Thus somatic cells sometimes vary spontaneously, as when a branch bearing nectarines appears on a peach tree. Again, when a cancer appears in the tissues of a man, the variation seems frequently to be caused, 
in some measure at least, by the environment, for such tumours are seen, especially in irritated tissues. Germ-cells, or cells of the germ-tract, appear also to be affected sometimes by the direct action of the environment, as in the case of Clayton's beans and European dogs in India. But, as we have insisted, evolution is never perfect; the 'laws' created by it have ever exceptions; for it is only by eliminating the exceptions that nature creates and maintains uniformities amongst living beings. The fact that these exceptions occur is in accordance, therefore, with all we know concerning the adaptations of living beings.

r69. The advantage of the practical limitation of the occurrence of variations to a particular part of the germ-tract is obvious. If variations occurred amongst somatic cells, they would tend to ruin co-adaptation among the cells that varied (and their descendants) and the other somatic cells (and their descendants) - a co-adaptation which has been achieved only by prolonged evolution. A cancer which results, seemingly, from a variation in a somatic cell (a variation, like other variations, inheritable by the descendants of the cell) is an example. Moreover, such variations, even if favourable to the individual, would be useless to the species, for, save in the event of the Lamarckian hypothesis being true, they would not be reproduced by the offspring of the individual. On the other hand, germinal variations, while affording the necessary materials for Natural Selection, do not affect the well-being of the community of which the germs are members, for here the germs have no functions. Again, if variations occurred all along the germ-tract, they would accumulate, and offspring would tend to vary, not in the right degree, but in proportion to the length of the tract and in proportion to the duration of time that elasped between the fertilizing of the ovum from which the parent arises and the production of his offspring; that is, the offspring earliest produced would tend to vary less than those produced later. Lastly, if the point at which variations normally occur were situated, not at the end of the germ-tract, but earlier in it, then, in proportion as the point was near the beginning and distant from the end of the tract, larger and larger batches of germ-cells would, as I say, tend to be identical. By limiting normal variability to the end of the germtract, nature ensures, on the one hand, that all germ-cells shall vary from one another, and, on the other, secures control over variations, which are then soonest put to the test of fitness to the environment.

I70. It may be thought that I have carried deductive thinking too far in the preceding paragraphs. But the facts on which I 
have relied are, I believe, not disputed, and I find it hard-as I think the reader will-to conceive interpretations of them other than those I have suggested. Germinal insusceptibility to the direct action of the environment and a right degree of variability are just as much adaptations, essential to the survival of the species, as any of the structures and functions of the individual. Presumably, therefore, they are just as much under the control of Natural Selection. The limitation of variations to a particular part of the germ-tract would not appear a task more difficult for Natural Selection than the evolution of such wonderfully complex and delicate structures as the eye and ear.

I7r. We are often told that the 'cause' or 'origin' of spontaneous variations is unknown. If it be meant that the molecular processes in the germ-plasm which result in variations are unknown, the statement is true. In that sense we do not know the cause of any kind of variations, not even of those which clearly result from the direct action of the environment. But in another sense it is untrue-as untrue as if, knowing that a certain variation was caused by a certain influence in the environment, we declared that the origin of it was unknown. The fertilized ovum has two classes of cell-descendants, both of which vary spontaneously, the somatic cells and the germ-cells. The former do not vary away from type ; nevertheless they vary in a regular manner from the fertilized ovum and amongst themselves, some becoming skin-cells, others muscle-cells, and so on. We suppose we know something very definite about the origin of these alterations when we declare that they result from evolution and are under the control of Natural Selection. Precisely the same declaration may be made about the less regular but equally constant variations of germ-cells. A germ-cell which has not varied, has varied in that it has departed from a type which, in effect, always varies. People who insist that we do not know the cause of variations, and that the word 'spontaneous' is nothing other than a cloak for ignorance, are unaware of the sense in which that word is used, or they have not considered the evidence, or they are the kind of people who insist on experimental evidence and are unaware of the impossibility of devising experiments which would furnish it.

I72. We reach, then, three principal laws of heredity-three summaries of facts. (a) The germ-plasm is highly insusceptible to the direct action of the environment; $(b)$ the vast majority of variations-all those on which evolution is founded-are 
spontaneous; (c) the development of the multicellular organism is a recapitulation-however inaccurate and incomplete-of the evolution of the race. All these three characters, insusceptibility, regulated variability, and recapitulation are due to Natural Selection-or miracle. If the reader, bearing in mind the whole of the evidence, not merely isolated items, will think the matter out carefully, I believe he will find there is no escape from the conclusion that all species must possess the first two characters or perish, and that the third also must be present in all multicellular types. He will be driven to conclude, also, that they are not mere chance characters, but products of evolution which Natural Selection wonderfully and beautifully regulates, which form the foundation of all other adaptations, and are, therefore, the most universal and important of all of them. The non-recognition of them as adaptations, necessary and inevitable adaptations, which underlies so much of modern thought and research, attests better than anything else the failure, fatal to a right comprehension of life, to realize that all normal living beings are, speaking practically, nothing other than bundles of adaptations. 


\section{CHAPTER VI}

\section{RETROGRESSION}

Evidence of retrogression-Theories of retrogression-Conditions under which retrogression occurs-The speed of retrogression-The difficulty of recognizing the identity of retrogression and reversion-Latent characters-The retrogression of variations-The magnitude of the part played by retrogression-Retrogression in the developing individual-Reversed selection-'Ancestral units '-The ' contributions of ancestors '-The law of ancestral inheritance-Biometry.

173. 1 HOUGH, speaking practically, the doctrine of evolution is accepted by all who know the facts, that of evolution by Natural Selection has not as yet received such general assent. A vague terminology, and a belief that nutritional characters are peculiarly 'innate' and that 'acquirements' are not products of evolution, combined with the obviousness of the Lamarckian doctrine and the difficulty of explaining co-adaptation when the magnitude of 'use-acquirements' is not realized, still inclines some biologists to a faith that the 'inheritance' of acquirements accounts, in part at least, for evolution. Others, because they are unable to perceive the utility of some characters in types so far removed from the human that our knowledge of their dangers and activities is very imperfect, have supposed that such characters have no utility, that they are not adaptations, and, therefore, that they do not owe their evolution to Natural Selection, which, consequently, does not play an exclusive, but, on the contrary, only a more or less minor part in the causation of specific change. ${ }^{1}$ Yet others, because a few species, when the environment is changed, display quite suddenly considerable adaptive alterations, pin their faith to that circumlocution for miracle, an 'adaptive growth-force.' 2

I74. But perhaps the main reason against a full acceptance of the Darwinian doctrine-that, at any rate, which has influenced most biologists in the past-has been drawn from the retrogression of useless parts and organs. In all complex types occur traces of organs which formerly were useful and of considerable relative 
magnitude, but which are now shrunken to mere vestiges. Thus in the ostrich the wings are no longer capable of sustaining the animal in flight. In the apteryx they are quite functionless and so rudimentary as to be hidden in the feathers of the body. In the extinct dinornis they had, apparently, disappeared altogether. In most snakes no traces remain of limbs, but in the python the hinder pair are still represented by vestiges. In some species of whales, also, the hinder pair have disappeared ; in others the small remains of them are buried in the body. Man has numerous vestiges of structures which formerly were functional, for example, the hairy covering of his body.

I75. Lamarckians have attributed the retrogression of useless parts to the transmitted effects of disuse. But man, for example, never 'used' the hair of his head more than that of his body; nevertheless the former persists, whereas the latter has become vestigial. However, it is unnecessary to discuss the Lamarckian doctrine here. The reasons which caused us to reject it as an interpretation of progression are valid against it as an interpretation of retrogression.

I76. At first Darwinians attributed retrogression to reversed selection, "the selection which effects not increase of an organ, but decrease of it." They argued that useless structures are worse than useless, they are burdensome; therefore nature secures their disappearance by eliminating the most burdened individuals. But the evidence is very ample that selection is a cause of racial change only when it is stringent, and a hairy man is not really burdened to an appreciable extent as compared with less hairy men; at any rate, not to such an extent as to diminish his chances of survival. Again, pythons and whales, which have vestiges of hind limbs, and the apteryx, which has vestiges of fore limbs, are hardly at a disadvantage as compared to species in which retrogression has been complete. A fortiori, individuals of the same species which have vestiges a trifle smaller than their fellows, have on that account no greater chance of survival. Osborn was very right when he declared in reference to this question, "If acquired variations are transmitted, there is some unknown principle in heredity; if they are not transmitted, there must be some unknown factor in evolution."

I77. Next, Darwinians argued that the struggle for nutriment is very severe, and that useless structures absorbed nutriment and so increased elimination. But useless structures, being inert especially when composed of a stable tissue like bone, absort 
very little nutriment. Moreover, it is not the total amount of nutriment absorbed by the vestiges that is in question, but only the difference in the amounts consumed in the vestigial structures of the individual that survives and the one that perishes. It requires faith of a really magnificent order to believe, for instance, that the daily addition or subtraction of a grain or less of nutriment can have influenced the survival rate of the Greenland whale.

I78. Next, it was asserted that the parts of the individual struggle amongst themselves for nutriment, and the parts most used being most stimulated received more than their share, and as a consequence flourish at the expense of the others, which atrophy. This hypothesis, however, is merely a particular application of the Lamarckian doctrine.

I79. In his hypothesis of Germinal Selection, Weismann has very ingeniously transferred the struggle for nutriment from the individual to the germ-plasm. He supposes that the parts of the individual are represented in the germ-plasm by 'determinants,' which multiply like cells, so that descendant determinants are present in descendant germ-cells. A weak determinant produces a minus (retrogressive) variation. Being weak, it is vanquished in the struggle for food by stonger determinants, and so grows weaker, and transmits its weakness to offspring and descendants. If the part it represents be useful, the individual who has the minus variation tends to perish through Natural Selection, the race being continued by the individuals in which the determinant is stronger, and the part therefore better developed. If it is useless, he tends to survive, and the process of weakening continues with added force in successive generations, till at length the determinant perishes and the part disappears. In brief, Weisman 1 supposes that the determinants of useless parts are not helped by selection, whereas the determinants of useful parts are helped by it; and, therefore, that the former tend to be vanquished by the latter in the struggle for nutriment.

I80. Weismann's hypothesis consists of two portions-an induction and a deduction, the latter being an expansion of the former. (a) Mere cessation of selection is followed by retrogression, (b) because the determinants of useless parts tend to be starved. It is possible to agree with the first, which is founded on observed facts and can be tested, without assenting to the second, which is neither founded on observed facts nor has been tested. According to this hypothesis, lack of nutriment is a cause 
of retrogressive variations, and abundance of nutriment a cause of progressive variations. In other words, it is supposed that variations are not always spontaneous, but are, very often at least, caused by the direct action of the environment-a notion which, in the main, Weismann himself has strenuously controverted. But, if determinants-supposing they exist as discrete elements in the germ-plasm-compete for nourishment, the competition should be sharpest and most destructive when nutriment is least abundant, and non-existent when it is superabundant. In that case species which possess very abundant nutritive supplies (e.g. internal parasites, unicellular and multicellular) should display little or no retrogression, whereas species, the nutritive supply of which is so small that they are engaged in an endless struggle for it, in which many perish (e.g. most, if not all fishes, reptiles, birds, and mammals) should display little progression and much retrogression. The exact opposite is the truth. The microbes of syphilis remain microscopical though their determinants have absolutely soaked in nutriment for thousands of years, and of all known organisms the multicellular pasasites display the greatest amount of retrogression. Some of them are little more than egg-cases. Fishes, reptiles, birds, and mammals, on the other hand, are typical examples of progression. In neither case has the inevitable drift ensued which would necessarily have followed were this hypothesis true-a drift which would tend to abolish Natural Selection, and therefore adaptation.

I8I. Of all the splendid services rendered by Weismann to biology none is more valuable than his long and successful struggle to overthrow the Lamarckian doctrine. Since merely useless parts tend to become vestigial and disappear, his success involves the corollary that simple cessation of selection (i.e. cessation of utility), which may, or may not, imply cessation of use, is followed by retrogression, which in turn implies that to prevent retrogression a certain stringency of selection is necessary, while to cause progression a still greater degree of stringency is required. The retrogression of old-established parts is very slow. Thus the great changes in whales and snakes since their limbs began to retrogress indicates an enormous lapse of time. Doubtless, also, geological epochs must pass before Greenland whales and pythons lose even the small vestiges of hind limbs which they still possess. On the other hand, newly acquired characters tend to disappear very rapidly on cessation of selection, as may be noted in the case of the special traits of our prize breeds. Absolutely new 
characters (i.e. variations) tend to disappear in the next generation (e.g. birth-marks, moles, etc.). ${ }^{1}$

I82. Since, then, the tendency to retrogression is greater in the case of new characters than of old-established traits, the stringency of selection by which the former are maintained, or by which fresh progression may be caused in them, is proportionately intense. ${ }^{2}$ In other words, offspring frequently display progressive variations in the case of old-established characters, whereas in the case of new traits the great mass of variations is retrogressive. Thus, if we try to improve the long-established characters of a comparatively old race, for example ordinary horses, our task is much less difficult than if we operate on the new characters which have recently been evolved under stringent selection, for example the special traits of race-horses. ${ }^{3}$ In the latter case the tendency to retrogression is sometimes so great that our utmost efforts may do no more than prevent retrogression. In other words, stringent selection no longer causes progression.

I83. Since even old-established characters retrogress on cessation of selection, it follows that the frequency, or the magnitude, or both, of retrogressive variations tends to be greater than that of progressive variations, and that the difference is most considerable in the case of newly evolved characters. The fact that retrogressive variations tend to exceed progressive variations in magnitude is well shown by the fact that while many generations of selection may be needed to evolve a prize breed, the offspring of a pure-bred prize pair may in a single generation display a loss of the special characters of the parents and revert to the ancestral type. This is not uncommon in the case of prize animals, but it is even more

1 Certain variations tend, apparently, to possess a greater degree of stability than others. We shall deal with them when we discuss Mendelism and the now popular Mutation theory of evolution. See chapters vii., viii., and ix.

${ }^{2}$ Some Mutationists maintain that antiquity (i.e. long-continued selection) has nothing to do with the stability of characters. They insist that all true variations are absolutely permanent from the first; whereas 'fluctuating variations' depend, not on change in the hereditary tendencies, but on differences of nutrition and the like-on differences in stimuli. According to them evolution is founded entirely on the former class of variations. We shall consider this view also later.

3 “ Two years ago thirty-two yearlings were sold for 51,250' guineas. These thirty-two yearlings are represented by two winners of five races, Florio Rubatino and La Reine, who have contributed $£^{2000}$ to the total cost; and there is not, so far as can be known, a single one of the thirty with any prospect of making a racehorse." (Quoted from the Times, December 27th, 1897, by Sir Walter Gilbey, Race-horses, p. 6.) 
common with cultivated plants. ${ }^{1}$ Amongst the latter, owing to the fact that offspring are more numerous, especially when it is possible to propagate a favourable individual by means of slips from which seed may be obtained, selection has been more stringent and progression correspondingly rapid.

184. Now a character (e.g. eye, limb, digestive system) which is old-established is one the usefulness of which has been tested by thousands of generations of continued selection. The permanent usefulness of more recently evolved characters (e.g. special features of prize-breeds) has been less thoroughly tested. They have favoured survival, but have arisen under conditions which, speaking comparatively, may be only temporary. Absolutely new characters (i.e. variations) have not been tested at all, or have been tested for one life only. The fact that characters retrogress with a speed which is inversely proportionate to the length of time during which their usefulness has been proved at once raises a suspicion that we are on the track of a very beautiful and very

1 "If a considerable number of improved cattle, sheep, or other animals of the same race were allowed to breed freely together, with no selection, but with no change in their conditions of life, there can be no doubt that after a score or a hundred generations they would be very far from excellent of their kind" (Darwin, Animals and Plants, vol. ii. p. 265). "When selection is suspended, rapid deterioration (from the fancier's standpoint) is the inevitable result. If, e.g., a number of pigeons, good specimens of a distinct breed, are isolated and left unmolested for a few years, they rapidly degenerate, i.e. they lose their show points (be they beaks, frills, ruffs, or metallic tints) and reassume the more fixed characters " (Ewart, Presidential Address to Zoological Section of the British Association, 190I). In I8IO-I4 Lady Monk and Lord Gambier collected some plants of the wild heart's-ease and so began the cultivation of the modern pansy. Twenty years after, " a book entirely devoted to this flower was published, and 400 named varieties were on sale" (Animals and Plants, vol. i. p. 392). Half a century later Darwin wrote, "Cultivators speak of this or that kind as being? remarkably constant and true ; by this they do not mean, as in other cases, that the kind transmits its characters by seed, but that the individual plant does not change much under culture. The principle of inheritance, however, does hold good to a certain extent, even with fleeting varieties of the Heartsease, for to gain good sorts it is indispensable to sow the seeds of good sorts. Nevertheless in almost every large seed-bed a few almost wild seedlings will appear through reversion" (op. cit., p. 393). At the present time the seed of the pansy " is of such a quality and is saved in so many distinct colours that for all ordinary purposes the trouble of striking cuttings and keeping stocks in pots all the winter through is mere waste of time and pot room" (The Culture of Vegetables and Flowers from Seeds and Roots, issued by Messrs Sutton \& Co., p. 195). Messrs Sutton give examples of flowers the varieties of which have been recently fixed by selection carried through many generations. Indeed, any number of similar examples might be given. Mendelian experimenters, however, explain the stability of old-established characters otherwise than by continued selection. We shall discuss their hypothesis presently. 
important adaptation. But before discussing this matter it will be well to inquire more closely into the exact nature of progressive and retrogressive variations on which all racial change depends.

I85. We have seen that, since the child recapitulates, with variations, the development of the parent, he must necessarily recapitulate, with inaccuracies, with additions and subtractions, the lifehistory of the race. Whence it follows that a progressive variation implies a complete recapitulation (as regards the character affected) of the life-history as presented by the parent, plus an addition; whereas a retrogressive variation implies an incomplete recapitulation of the parental development, and therefore a reversion to the stage of development reached by some progenitor more remote than the parent. There is really no escape from these conclusions ; for granting the undisputed fact that the child recapitulates the development of the parent, other conclusions are actually, literally, unthinkable.

I86. The reader, however, must be very careful not to confuse latency with retrogression. Latency implies, not the loss of a capacity to develop in this or that direction, but only its inactivity. We shall see later ${ }^{1}$ that it occurs when two rival hereditary tendencies are present in the germ-plasm, one of which becomes dormant while the other directs the development of the individual. Thus colour-blindness and hæmophilia, which are commonly restricted to males, tend to be transmitted in a latent condition through daughters to grandsons. All the sexual characters, primary and secondary, are latent in individuals of the opposite sex. Thus the daughters of heavily bearded men tend to have heavily bearded sons. Male aphides are absent during the summer months, and reproduction, therefore, is parthenogenetic, but at the end of the warm season males are produced, which fertilize the winter eggs. Amongst honey bees also the reproduction of drones is parthenogenetic. Male characters, therefore, are latent in the germ-plasm of the females. "It is well known that a large number of female birds, such as fowls, various pheasants, partridges, pea-hens, ducks, etc., when old or diseased or when operated on, assume many or all of the secondary male characters of their species. In the case of the hen pheasant this has been observed to occur far more frequently during certain years than during others. A duck ten years old has been known to assume both the perfect winter and summer plumage of the drake. Waterton gives a curious case of a hen which had ceased laying, and had assumed the plumage, voice, spurs, and warlike disposition of the cock;

$$
\text { }{ }^{1} \text { See chapter vii. }
$$


when opposed to an enemy, she would erect her hackles and show fight. Thus every character, even to the instinct and manner of fighting, must have lain dormant in this hen as long as her ovaria continued to act. The females of two kinds of deer, when old, have been known to acquire horns; and as Hunter has remarked, we see something of an analogous nature in the human species." 1 C. E. Walker injected an emulsion of testes into hens, thus supplying the necessary stimuli, and caused them to develop the combs, wattles, and warlike disposition of cocks. ${ }^{2}$ Professor Giard, Geoffrey Smith and others have shown that when the testes of crabs are destroyed by parasites (Rhizocephala) the males may develop all the characters of females, including ovaries. In some cases the animal becomes a perfect hermaphrodite. ${ }^{3} \mathrm{Mr} \mathrm{J}$. H. Orton has demonstrated that the molluscs Crepidula fornicata and Calyptrcea chinensis are males at first but become females later. ${ }^{4}$

I87. In addition to the temporary latency typically seen in the case of the sexual characters, there is a more permanent form. "Besides visible changes which it [the germ-cell] undergoes, we must believe that it is crowded with invisible characters proper to both sexes, to both the right and the left sides of the body, and to a long line of male and female ancestors separated by hundreds and even thousands of generations from the present time; and these characters, like those written on paper in invisible ink, lie ready to be evolved whenever the organism is disturbed by certain known or unknown conditions." 5 Thus individuals derived from domesticated breeds of pigeons or fowls may reproduce the characters of extremely remote wild ancestors. In cross-breeding between domesticated varieties the re-appearance of hitherto latent ancestral characters is so common that Darwin declared "we must conclude that a tendency to this peculiar form of transmission is an integral part of the general law of inheritance." 6

I 88. There is a good deal of evidence that latency is influenced directly, in a measure at least, by the environment. Thus Ewart rendered latent at will the male or female characters of rabbits. Amongst bees the female characters develop fully only under certain conditions of environment in which, apparently, nutriment

1 Animals and Plants, vol. ii. p. 26.

2 The Infuence of the Testes upon the Secondary Sexual Characters of Fowls, 1908.

${ }^{3}$ Fauna und Flora des Golfes von Neapel, Monograph, 29; Rhizocephala, Geoffrey Smith, Berlin, I906.

- Proc. Roy. Soc., B., vol. 1xxxi. pp. 68-84.

- Animals and Plants, vol. ii. pp. 35-6.

'Loc. cit., vol. ii. p. 3 I. 
plays the chief part. The same is the case with aphides, which produce males only when the weather grows cold and nutriment less abundant. In true retrogression, on the other hand, there is not mere latency, but absolute loss. A hereditary tendency is eliminated from the germ-plasm. If the character which was lost was a new variation, it can recur in the race only through another variation, of like nature; if it has arisen through evolution, through the piling up of progressive variations during a succession of generations, it can recur only through a similar process of selection. It is conceivable, of course, that no characters are ever completely lost, but that all apparently lost characters become latent. But this implies that, though the germ-plasm may gain, it cannot lose ; that the only true kind of variations are progressive variations; and that while it is possible for the individual to vary so that he completes the parental development and adds a step, it is impossible for him to vary so as not to complete it, except by making a character latent. It implies that all the variations of the two parents, the four grandparents, the eight great-grandparents, of all the billions of ancestors, are represented in the germ-plasm; that nothing, not even a minute variation in a hair, that ever appeared during the immensely long and varied life-history, has ever been lost; and therefore, that a human embryo, for instance, has latent not only all the physical and mental characters of the many types which preceded him, but all the variations of these characters which occurred in all his ancestors. The germplasm in a microscopical germ-cell is doubtless a very complex entity, but this hypothesis would endow it with a greater number of hereditary tendencies than it has chemical atoms. In truth we have no choice but to believe that hereditary tendencies may not only become latent, but that they may be entirely lost. Indeed, when we remember how numerous have been the variations and the ancestors of every individual, and how immense the changes during the life-history, it is evident that the proportion of characters which have persisted, either in a patent or in a latent condition, must be infinitely small when compared to those which have been altogether lost-which have retrogressed absolutely.

189. A complete discussion of latent characters may be postponed with advantage. We need note only that when a character, for example the gorgeous plumage of Gallus bankiva, the wild ancestor of our domestic poultry, becomes latent, then (since retrogression and reversion are identical) subsequent retrogression, which eliminates the more sober colours of the 
domestic bird, and so permits the reappearance of the ancestral traits, may present every appearance of progression. Obviously, the domestic characters which were new to the race and which rendered latent more ancient traits, result from progression, and their disappearance and the consequent reappearance of the latent wild characters is an act of retrogression as well as reversion. It may happen, of course, as normally occurs in the case of the sexual characters, that the traits of the domestic fowls are not eliminated when the wild plumage reappears, but replace the latter as dormant characters, and therefore that they may reappear in descendants, and even alternate with the wild traits as the sexual characters alternate with one another; but the fact that it is sometimes extremely difficult to breed out hitherto latent ancestral characters when once they have reappeared, seems to indicate that their recrudescence may occasionally be due to a loss of the newer domestic characters, not merely to the latency of the latter. ${ }^{1}$

190. It will be worth the reader's time to try to imagine an act of retrogression which is not also an act of reversion. To take an extreme example: suppose a child is born lacking a limb. Experience shows that a deficiency so great occurring in a single generation usually indicates latency rather than loss. But suppose it is an instance of real retrogression-i.e. of the total and permanent loss of a complex hereditary tendency from the germ-plasm-then clearly the child has reverted to that enormously remote ancestor in whom the limb did not exist. It is not maintained of course that there ever was a period during the life-history when three perfect limbs were possessed by the race while the fourth was lacking. But since every part is independently variable, it is necessary in this connection to think of every part separately.

191. Not only is the limb independently variable, but each smallest part of it possesses the same power. Suppose a child comes into existence with a malformed limb. This variation may be compounded of any number of smaller variations, some of which

i' " I may here add a remark made by Mr Wicking, who has had more experience than any other person in England in breeding pigeons of various colours, namely, that when a blue, or a chequered bird, having black wing bars, once appears in any race and is allowed to breed, these characters are so strongly transmitted that it is extremely difficult to eradicate them " (Animals and Plants, vol. i. p. 210). On the other hand if, for example, albino mice be crossed with black-and-white Japanese waltzing mice, the ancestral grey colour appears, but if the mongrels be inter-bred, the albino and the black-and-white types reappear. In this case the albino and the black-and-white colour become merely dormant when the grey reappears. Medelians will dispute the inference, but it can be substantiated (see chapter vii.). 
are progressions and others retrogressions. If we think of each of the component variations separately, and, if necessary, analyse them, we see that every progression must be built on the development as presented by the parent, must consist of an addition after a complete recapitulation (as far as that character is concerned) of the life-history as presented by the parent; whereas every retrogression must consist of a subtraction from the complete recapitulation-a reversion. Think how we will, puzzle how we may, we must always arrive at the conclusion that retrogression is identical with reversion.

I92. There are, then, two kinds of 'reversion.' (I) The individual may revert ancestor-wards through retrogressionthrough the total loss of a developmental potentiality from the germ-plasm. (2) He may reproduce a dormant ancestral trait. The latter, in appearence at least, is also reversion, even though nothing is lost to the germ-plasm-even though the more modern trait becomes in its turn merely dormant. Most biologists limit the term reversion to the more or less glaring reproduction of a latent ancestral trait, but only, I think, because they have not as yet thought of retrogression in connection with reversion.

193. The reason why retrogression seldom presents the appearance of reversion, and why, therefore, there has been a general failure to recognize the relations between the two, arises from the fact that, since the parts of the individual are independently variable, retrogressive and progressive variations are usually closely intermingled, with the result that the nature of the former is masked. They cannot be clearly discerned as reversions. Moreover, in oldestablished types especially, the majority of variations tend to be of very small magnitude. Sometimes, however, retrogression is extensive and affects some conspicuous character or a number of characters together, and then, as when a prize breed loses its special traits, we are able to recognize its nature. In by far the great majority of instances, however, retrogression is not obviously identical with reversion. Not sight, but thought, convinces us of the identity. Thus, while the wing of a bird, whose latest descendants have lost the power of flight, was evolving from the reptilian fore-limb, much was lost as well as gained. Consequently when the wing became useless, for example in the apteryx, and retrogression en masse set in, there was no return to the reptilian limb, for the major part of the latter had already undergone retrogression during its change to an organ of flight.

194. Every individual varies from his parent in an almost 
infinite number of ways. A proportion of his variations are progressive, and of them the great majority are useless, since they do not make the child superior to, that is, more adapted to the environment, than the parent. They are small redundancies which do not affect the survival of the individual, but which, if reproduced by subsequent generations, would accumulate and ultimately overburden the race. We may note many of them in any human being, but we cannot by mere observation form any conception of their number; for nearly all of them are too obscure and minute to be recognizable as redundancies, and most of them are situated internally. But, when we are able to note one (e.g. a mole) clearly in a parent, we are almost sure to find it lacking when we examine the child. Natural Selection has played no direct part in its elimination; yet it has ceased to burden the race. To put the matter in another way: no individual is absolutely perfect in every particular, and many of his imperfections are due to progressive variations. These redundancies are not as a rule reproduced, for though they occur in every generation, the human race, for example, has not altered appreciably in shape for thousands of years. That is, they do not accumulate. They disappear, but, very obviously, not through selection. Selection, indeed, could not reach them, for singly they do not affect survival, and they never occur en masse in one individual and not at all in another. The evident fact is that the useless progressive variations of one generation tend to be planed away by the retrogressive variations of the next. It is otherwise with useful progressions. They tend to be preserved by selection, even though, paradoxical as it may sound, they are in many cases, when taken separately, beyond the reach of it. In a future chapter we shall study the very admirable device by which the elimination of useless and preservation of useful progressive variations is achieved without a separate elimination of individuals for each separate kind of variation. ${ }^{1}$ Meanwhile the point to be noted is that selection plays no direct part in the retrogression of the mass of progressive variations.

195. Every multicellular species has descended from unicellular organisms. During the long course of evolution it has undergone innumerable changes of form. Many structures, which once were useful and for that reason underwent progression, subsequently became useless and retrogressed utterly, or to such an extent that hardly a trace of them remains in the abbreviated life-history that is narrated in the development of the latest descendants. Other

${ }^{1}$ See chapter ix. 
parts, as their functions and anatomical relations changed, have been so greatly and continually modified that, like a much-mended garment, the original structures have nearly disappeared and have been replaced or almost replaced by new material. Others again have been so much less modified that the careful observer is able to gather a good deal concerning the life-history. In yet others, generally the more modern structures, for example the antlers of the stag, the life-history is plain : only variations which were never incorporated into the life-history of the race have vanished; the structures develop in the individual on much the same lines as they were evolved in the race. In every species, therefore, we find evidence of an immense amount of retrogression. Consider, for example, how much man must have lost since he became a vertebrate, and how much more in the even longer antecedent period.

196. Retrogression, then, has been as much a part of evolution, of adaptation, as progression. It has planed away useless and burdensome structures. It has straightened, simplified, and abbreviated recapitulation; the life-history is not told in all its details; the narrative is limited to particulars essential to its development; and thus development is rendered possible during the brief lifetime of the individual. If a man, for example, recapitulated all the changes undergone by his ancestors, he would require, not,years, but ages for development. Lastly, retrogression, combined with some progression, has rendered possible the existence of the developing individual in environments vastly different from those inhabited by its prototypes of the ancestry. The human embryo, for instance, dwells within its mother's uterus. Its prototypes had an independent existence. Were they, with their structures and faculties complete, placed in the uterus, they would perish just as surely as the embryo would were it placed in the ancestral environments.

197. Within the uterus the embryo leads a protected vegetative life. It has no such active struggle for existence as the adult. The now useless power, which depends on structures and faculties, of fighting actively for itself, as did its prototypes, who lived in a world where nutriment was scarce and enemies plentiful, has been lost through retrogression. The sole business of the embryo is to fit itself as quickly and as thoroughly by development as its nature permits for the battle which occurs later. Quick development implies quick recapitulation, which in turn implies abbreviated recapitulation. In all the higher animals the length of the period of gestation is always proportionate to, and therefore presumably dependent on, 
the amount and complexity of this intra-uterine preparation. Even after birth the young of the higher animals are protected to give time for still more development, still more recapitulation, particularly that development which results physically from use and mentally from that synonym for use, experience. Such young animals, owing to the decay (precisely similar to that observable in the embryo) of faculties, especially instincts, present in the ancestry, are incapable of maintaining independent existence. In brief, whenever the active struggle is abolished, the structures and faculties by which it was maintained tend to disappear, except in so far as they serve as foundations on which are reared characters that are useful later in the battle of life.

198. Animals comparatively low in the scale of life, many insects for instance, do not protect their young after birth. Moreover, birth occurs at an early period of development-that is, when offspring have recapitulated nothing or comparatively little of the life-history. But very complex and rapid development occurs during the quiescent period in the egg-case. To take the familiar example of the butterfly-the caterpillar when he emerges has already undergone development in which he has recapitulated a vast phase of the evolution of his race. Next, for a long period, during which an active life is led, very few structural alterations, except increase in size, occur. Probably during this active time the environment, speaking relatively, very nearly resembles the ancestral environments, and the caterpillar his remote ancestors who developed no further. Doubtless he differs from them, but not to the extent that the embryo in the egg-case differs from the ancestors it represents. The structures and faculties have not undergone the same amount of retrogression; the life-history is recapitulated in greater detail. The function of this active period is the accumulation of nutriment which furnishes material both for the growth of the caterpillar and for his subsequent developments. Next comes another period of quiescence in the chrysalis, which again affords opportunity for great retrogression and, therefore, for the later phases of the life-history to be very rapidly retold. In this period occur changes which swiftly recapitulate the evolution that added an aerial stage to the development of a hitherto purely terrestrial insect. In the last, the active butterfly stage, however long it may endure, there is again complete, or almost complete cessation of structural alteration.

199. The quiescent periods of development in the egg-case and the chrysalis are strictly homologous to the period of intra-uterine 
development in the higher animals. In them is achieved the same end-the fore-shortening of long and changeful periods of the lifehistory. All, or almost all rapid recapitulation occurs during periods of quiescence when the individual is protected or concealed, that is when he is least exposed to selection. Better than anything else development indicates the extent to which the organism is a bundle of adaptations. Thus the embryo of the butterfly develops fast while it is stationary in its egg-case, where it remains till food is abundant; the active caterpillar, fitted to its environment by all its structures and instincts, stores nutriment in its tissues against a time when, did it continue active, it would be very disadvantageously situated while changing into the adult insect. The human infant is born to a mother who is fitted to tend just such a developing being at just such a stage of its development. Were its development different, did it recapitulate the lifehistory in fuller detail, she could neither bear nor tend it. In both the caterpillar and the human being the abbreviations of the lifehistory, though happening at different periods of life, occur at precisely the most useful periods. After the period of reproduction of offspring the fitness of the individual to the environment declines-swiftly in the lower animals, more slowly in animals that tend their young. For obvious reasons, Natural Selection can no longer preserve it. There is no further recapitulation. There is nothing to recapitulate.

200. Plants lead a life more quiescent than most animals. Speaking generally, the seed drops to the ground, and the young plant emerges from it into an environment which hardly changes again during the lifetime of the individual, but which, since the higher plants have evolved from lower forms, must have changed greatly for the species. In them, therefore, retrogression has so abbreviated a major portion of the life-history that no one merely examining the embryo of an oak, for example, would gather more than a hint of the immensely long and changeful process by which the species underwent evolution.

20I. We see then how great a part retrogression plays in evolution and by what varied devices nature assists its operations. It swiftly eliminates the useless progressive variations, the redundancies, which occur in vast numbers in every individual. More slowly, in proportion to their antiquity, it rids the species of characters which have lost their utility. It abbreviates and simplifies development. It wars against progression, but great progression would be impossible without it. It furnishes a half of 
the sum of adaptation, leaving only the other half to the direct action of Natural Selection. Yet all these great effects result solely because in every structure of every species the tendency to vary retrogressively is so much stronger than the tendency to vary progressively that retrogression is checked only by selection. Obviously this tendency of retrogressive variations to preponderate over progressive variations is highly adaptive-as adaptive as spontaneous variability itself. The question then arises whether it is an actual adaptation or merely something.which has arisen accidentally during the course of evolution. We saw that the regulated variability which distinguishes all forms of life was, beyond reasonable doubt, maintained, and controlled by Natural Selection. The tendency to retrogression is only one of the ways in which variability is regulated. We have, therefore, every reason to suppose it is no chance accompaniment of life, but an adaptation ranking in universality and importance with spontaneous variability, insusceptibility, and recapitulation. In other words, we have reason to believe that it is a $L A W$ that has resulted from the selection of germ-plasms which, while tending to vary all round the specific mean, yet tended, on the whole, to vary retrogressively.

202. The problem of retrogression cannot be approached by any of the laboratory methods and therefore tends to be ranked by some minds among the questions of 'philosophy.' Moreover, the very simplicity of the device by which nature achieves such great results is apt to awaken incredulity. I can only ask the reader, while bearing in mind the facts of adaptation and also the fact that both useless progressive variations and characters that have become useless tend to disappear in the absence of direct selection, to try here again to conceive interpretations other than those I have suggested. Judging from my own experience, I think he will fail.

203. We conclude, then, that the great mass of retrogression we observe in nature is not due directly to Natural Selection-that is to reversed selection. Since, as a rule, environments change gradually, it occurs under conditions which afford no scope to reversed selection. It is not to be supposed, for example, that the apteryx suddenly abandoned habits of flight. While the structures and instincts which especially?fitted it for a terrestrial life were undergoing progressive evolution, its wings were still helpful in sustaining existence. But they became continuously less useful, were less and less maintained by selection, and so at last underwent retrogression. Sometimes, however, we may observe 
what appears to be clear evidence that reversed selection has caused real retrogression. Thus certain blind crabs inhabiting caverns have lost their eyes, but not the stalks on which the eyes were carried; since the eye is a more ancient organ than the stalk, it should, on cessation of selection, have been more persistent; yet it disappeared first. Evidently something more than mere cessation of selection was at work. In utter darkness the eye, a prominent and vulnerable organ, would be not only useless, but worse than useless; and therefore reverted individuals would be actively selected for survival, and the organ would be more rapidly eliminated than could happen under cessation of selection. In other cave-dwelling animals, which, as we suppose, are not descended from stalk-eyed ancestors, the rudimentary eye is covered by skin. Presumably in this case the partial retrogression has resulted from mere cessation of selection, the eye being protected from injury and therefore from reversed selection by the progression which resulted in the growth of the skin. Here, as ever, nature has followed the line of least resistance.

204. But though reversed selection is seldom a cause of retrogression, it has nevertheless a very important function. Reversed selection implies reversed progression of the race, which, in turn, implies reversed development of the individual. When, therefore, we see a structure better developed in the immature individual than in the adult, we may be sure that the diminution in the adult is due to reversed selection. Thus the remote ancestor of the modern horse, the Hipparion, had three functional toes. The embryo of the horse also has three toes of considerable size. But the two outer toes in each limb, which in the embryo are nearly as well developed as in the Hipparion, degenerate partially during development, so that the horse is born with only one functional toe. "Occasionally a foal is born with two hoofs on one or more of its limbs ; at very long intervals a foal appears with three hoofs on one or more of its limbs." Now, when the outer toe persists in the individual, we have plainly, in a real sense, an arrest of development. The toes remain in the embryonic, the ancestral condition ; the whole life-history is not recapitulated. But in the normal horse there is no arrest of development; the recapitulation is carried out to its fullest extent, though in the later stages in a reversed direction. The toes, which were useful to the ancestors, are harmless to the embryo, but would be harmful to the adult, and, therefore, have been eliminated by reversed selection in the latter before retrogression has eliminated them in the 
former. Still better examples are seen in the disappearance in later life of structures which are useful to the developing individual, but which have no place in the scheme of adaptations of which the adult is compacted. It is because of "selection which affects not increase of an organ but decrease of it," that the human being loses the placenta, the frog the special structures of the tadpole, and many insects their larval organs. To sum up, cessation of selection causes real retrogression, a real loss to the race (i.e. to the germ-plasm) of ancestral characters; reversed selection, on the other hand, though it sometimes collaborates with cessation of selection to produce real loss, causes, as a rule, like ordinary selection, not loss but gain to the germ-plasm, to which it adds a hereditary tendency. It does not abbreviate; on the contrary it lengthens the life-history. Speaking generally, it causes the loss in the later stages of development of characters which have developed earlier. That is its special, its more common function.

205. In the present work we have accepted Weismann's theory of the continuity of the germ-plasm. That theory fits the facts so well that it has very few opponents at the present day. It supposes that the germ-plasm is not formed afresh in every germcell, but, by means of cell-divisions, is handed on by germ-cells to descendant germ-cells. The process is a continuation of that which occurred in the unicellular ancestors. The germ-plasm varies and, since it is alive, its chemical constituents (carbon, oxygen, nitrogen, etc.) change in a constant stream. Nevertheless from age to age it is continuous in the same sense as the human body is continuous from day to day. ${ }^{1}$ In addition we have supposed,

${ }^{1}$ As far as I am able to judge, this, in essence, is the form in which Weismann wishes us to accept his theory. He writes in his most recent work, "The expression (ancestral plasm), however, has been very frequently misunderstood, as if it were intended to mean that the ids retained unchanged for all time the characters of their respective ancestors; and I have even been credited with supposing that our own ids still consist of the determinant-complexes of our fish-like or amœba-like ancestors. But in reality no id exactly or completely corresponds to the type, that is to the whole being of any one of the ancestors in whose germ-plasm it was formerly contained, for each of the ancestors had many ids in his germ-plasm, and his entire constitution was not determined by any one of them alone, but by the co-operation of them all. . . . Thus, according to our view, the germ-plasm consists of ids, each of which contains all the determinants of the whole ontogeny, but usually in individually different quality" (The Evolution Theory, Eng. Trans., vol. ii. p. 38). Weismann's conception of the germ-plasm, then, appears to be of a substance containing discrete particles which he terms ids, any one of which is capable of directing development. The ids differ from one another, but only in so far as is implied by the variations of individuals. As noted by him, the term 'ancestral' has resulted in confusion; it is inaccurate and misleading. The conception of the germ-plasm 
what none will dispute, that the germ-plasm is the bearer of hereditary tendencies or potentialities, and that according to the nature of the latter is the nature of the individual which springs from the germ-cell. Further, we have supposed, what very few will dispute, and what indeed is implied in the theory of continuity, that the germ-cell receives no living elements from the somatic cells, but only shelter and nutrition. It is a unicellular organism living in the midst of a community which is the multicellular organism. From our point of view, therefore, individuals, for example men, are nothing more than dwellings which the germplasm builds about its germinal descendants. It follows-but here we are contradicted by the language of all biological literature - that the child inherits nothing from his parent. He resembles his parent only because he mimics the development of the latter, only because his development was directed by a split-off portion of the germ-plasm that directed the development of his parent. Yet again, we have insisted that it is quite inconceivable that the development of the multicellular individual can be other than a recapitulation, more or less altered, of the life-history of his race.

206. If we are right, then, necessarily, such progressive variations of the ancestors as have not been lost through retrogression, and therefore are reproduced in development, appear in the order in which they occurred during evolution, the sole exception being interpolated variations. In a general sense, therefore, successive generations of ancestors are represented (mimicked) during development in order, beginning with the unicellular organism which is represented. by the germ-cell, and ending with such progressive variations of the preceding generations as are reproduced by the last generation. We must think in terms of the germ-plasm, however, and bear in mind that, since retrogression is constantly at work, even in characters that on the whole are progressive ; since, while some characters have undergone

which I have endeavoured to place before the reader is that of a substance which contains, or is, in some way, associated with the hereditary tendencies which direct development. I suppose that the germ-plasms which direct the development of any two individuals differ qualitatively somewhat. Whether or not the germ.

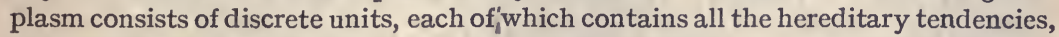
and one of which wholly or principally directs development, is a question I do not discuss: I know of no facts bearing on the matter and no means of testing the thinking. But, as will be seen, I do very strongly protest against the hypothesis that the germ-plasm consists of discrete particles each of which represents a particular ancestor. That hypothesis, I think, is not only not warranted by valid evidence, but is negatived by the high probability that the germ-plasm is continuous in a real sense from generation to generation. 
progression, others have undergone retrogression or have remained stationary, and since variations have been interpolated, no generation of ancestors is ever reproduced exactly by descendants. Therefore the statement that ancestors are represented in orderly succession (in the order in which they came into existence) is true in a general and vague sense only.

207. Apparently, however, many biologists believe, not that the germ-plasm undergoes such a change during evolution that development is in a general sense a recapitulation of the lifehistory, not that the successive generations of ancestors are vaguely mimicked in turn during development, but that each ancestor adds to the germ-plasm a 'unit' or 'contribution' which may control, or may assist in controlling, development from start to finish, from germ-cell to adult.

208. The best known of the hypotheses which are founded on the notion of ancestral contributions is Sir Francis Galton's 'Law of Ancestral Inheritance.' This hypothesis, or modifications of it, which differ in detail but are similar in principle, have been so widely accepted that it is necessary to discuss it somewhat at length. Galton analysed statistical data concerning stature, eye-colour, artistic faculty, and health, collected from about one hundred and fifty families of human beings, and extending over three or more generations. Next, he analysed statistics concerning the colour of Bassett hounds, a race of dwarf blood-hounds originated by Sir Everett Millais some twenty years previously. He concluded, "The two parents contribute between them on the average one-half (or 0.5 ) of the total heritage of the offspring; the four grandparents one-fourth (or 0.5$)^{2}$; the eight great-grandparents one-eighth (or 0.5$)^{3}$, and so on. Thus the sum of the ancestral contributions is expressed by the series $(0.5)+(0.5)^{2}+(0.5)^{3}$, etc., which, being equal to unity, accounts for the whole heritage." 1

209. Now obviously Galton's statistics do not furnish evidence that ancestors contribute units to the germ-plasm. That deduction, if it is in his mind, is drawn from the observation that offspring may, in this or that character, resemble ancestors more than they do parents. Probably it was reached before the statistics were collected. Galton merely noted certain resemblances between the individuals of three or four generations, and calculated that on the average offspring resemble each parent to the extent of onequarter of their total characters, each grandparent to the extent

1 The Average Contribution of each Several Ancestor to the Total Heritage of the Offspring, Proc. Roy. Soc. London, vol. Ixi. pp. 40 I-r 3 . 
of one-sixteenth, and so on. He concluded further, that what was true of the characters investigated was true of all characters, that what was true of the generations investigated was true of all generations, and seemingly also that the resemblances were due to 'contributions' to the germ-plasm. The notion that ancestors 'contribute' to the heritage of the child (i.e. add definite and apparently discrete 'units' to the germ-plasm) is founded on, but not tested by his facts.

210. The principal difficulty in discussing the Law of Ancestral Inheritance arises from the fact that since the nature of a 'contribution' is not defined, the hypothesis is not 'definite in conditions.' Conceivably, the word implies a variation which occurred in the germ-plasm at the time that an ancestor existeda variation which may or may not blend with other variations occurring in the same and in preceding and succeeding generations. Or 'contributions' may be regarded as discrete units which represent separate ancestors in the germ-plasm and have been added to it by them. Since biometricians, as a rule, hold with Weismann that the germ-plasm is continuous and that inheritance is usually blended, it seems correct to conclude that what they mean by their word 'contribution' is a blend of the variations occurring in a generation of ancestors. On the other hand the language used appears to indicate clearly that what they mean is that the germplasm is compounded of discrete units, each of which was somehow contributed by a separate ancestor-contributed by the soma to the germ-plasm, by the dwelling to the inhabitant. Probably such an interpretation would be repudiated. Nevertheless, it would certainly seem to be implied that discrete units representing ancestors do in some way come into existence in the germ-plasm and influence the development of the individual from start to finish.

2II. "We seem to inherit bit by bit, this element from one progenitor, that from another... while the separate bits are themselves liable to some small change during the process of transmission. Inheritance may therefore be described as largely if not wholly 'particulate,' and as such it will be treated in these pages. Though this word is good English and accurately expresses its own meaning, the application now made of it will be better understood through an illustration. Thus, many of the modern buildings in Italy are historically known to have been built out of the pillaged structures of older days. Here we may observe a column or a lintel serving the same purpose for a second time, and perhaps bearing an inscription that testifies to its origin, 
while as to the other stones, though the mason may have chipped them here and there, and altered their shapes a little, few, if any, came direct from the quarry. This simile gives a rude but true idea of the exact meaning of Particulate Inheritance, namely, that each piece of the new structure is derived from a corresponding piece of some older one, as a lintel was derived from a lintel, a column from a column, a piece of wall from a piece of wall.

212. "I will pursue this rough simile just one step further, which is as much as it will bear. Suppose we were building a house with second-hand materials carted from a dealer's yard, we should often find considerable portions of the same old houses to be still grouped together. Materials derived from various structures might have been moved and much shuffled together in the yard, yet pieces from the same source would frequently remain in juxtaposition and it may be entangled. They would lie side by side ready to be carted away at the same time and to be re-erected together anew. So in the process of transmission by inheritance, elements derived from the same ancestor are apt to appear in large groups, just as they had clung together in the pre-embryonic stage, as perhaps they did. They form what is expressed by the words 'traits'-traits of feature and character-that is to say continuous features and not isolated points.

213. "We appear, then, to be severally built up out of a host of minute particles of whose nature we know nothing, any one of which may be derived from any one progenitor, but which are usually transmitted in aggregates, considerable groups being derived from the same progenitor. It would seem that while the embryo is developing itself, the particles more or less qualified for each new post wait as it were in competition, to obtain it. Also that the particle that succeeds, must owe its success partly to accident of position and partly to being better qualified than any equally well placed competitor to gain a lodgment. Thus the step by step development of the embryo cannot fail to be influenced by an incalculable number of small and mostly unknown circumstances." 1

214. Many biologists evidently do mean by 'contribution' a unit added to the germ-plasm. For example, we are told, "The Law of Ancestral Inheritance proves that all ancestors, however remote, are able to leave the impress of their individuality upon the sex-cells in diminishing proportion according to their remoteness. Such a fact can only be accounted for by assuming the ${ }^{1}$ Galton, Natural Inheritance, pp. 7-9. 
existence in the germ-plasm of definite units carrying definite characters, and the regular halving in the average strength or amount of such characters during the reducing division of the nuclear matter of the sex-cells which precedes each act of reproduction." 1

2I 5. Apparently, then, each ancestor is supposed to contribute a unit to the germ-plasm, which, since the contributions taken together amount to unity, is thus totally compounded of ancestral units-two large units from the parents, four units each a quarter as large from the grandparents, and so on. But each ancestor's unit is also totally compounded of units from his ancestors, whose units in turn are totally compounded of units from their ancestors, and so on in endless recession. Now, imagine a line of individuals, $A, B, \ldots$ $Y, Z$. Then $Z$ will have a unit from $A$. He will also have one from $B$ (which will contain a contribution from $A$ ). Likewise, he will have one from $C$ (which will contain a contribution from $A$ and one from $B$ [which will contain one from A]). Yet again $Z$ will have a unit from $D$ (which will contain a contribution from $A$ and one from $B$ [which will contain one from $A$ ] and another from $C$ [which will contain a contribution from $A$ and one from $B$ \{which will contain a contribution from A\}])-and so on. Obviously A's unit will be repeated many times over, in almost infinite subdivision, in all the contributions of his descendants. So, also, as regards B's unit and the units of the other ancestors, especially the more remote. Really, however, ancestral contributions have not this ideal simplicity of composition; for every individual is of almost infinitely long descent, and if of a sexually dimorphic species, has two parents, four grandparents, eight great-grandparents, and so on.

216. At first sight a law of ancestral contributions seems simple : we suppose merely that each ancestor adds something representing himself to the germ-plasm. If, however, we make the necessary rigorous deductive inference of consequences, it becomes almost unthinkable. It is opposed, in language at any rate, to the notion that the ancestors of the germ-cells are, not 'individuals,' but the cells of the germ-tract, which merely dwell within the individuals. Notwithstanding all its appearance of mathematical precision, it is so vaguely expressed that I doubt if any two people understand it alike. $^{2}$ I describe it as I understand it, or as far as I am able to

1 Vernon, Variations in Animals and Plants, pp. I34-5.

2 "This law, that the mean Characters of the offspring can be calculated with the more exactness, the more extensive our knowledge of the corresponding characters 
understand it. Presumably a 'contribution' to the heritage of a descendant means more than mere resemblance; otherwise it is difficult to understand why a word so apt to mislead is used when the unequivocal word 'resemblance,' which occurs readily to the mind, is sufficient to describe the phenomenon. An ancestral contribution which is halved and quartered and so forth would certainly seem to imply a discrete unit representing an ancestor in the germ-plasm ; and apparently since every ancestor's heritage is compounded from those of his predecessors, it would appear also that the contribution of every ancestor is represented in the contribution of every successor. To say the least, it is hard to believe that machinery for this perpetual and complicated division can exist. ${ }^{1}$

of the ancestry, may be termed the Law of Ancestral Heredity" (Mr Udney Yule, "Mendel's Laws and their Probable Relations to Intra-racial Heredity," The New Philologist, 1902, p. 202. "The degree to which a parental character affects offspring depends not only upon its development in the individual parent, but on its degree of development in the ancestors of that parent "(Prof. W. F. R. Weldon). Mr Yule and Professor Weldon avoid the use of the word contribution. As formulated by them the Law expresses what would appear an obvious truth; but it is then widely different from the Law as formulated by Galton and accepted by Mr Vernon.

I I have reasoned from what appears to me the plain meaning of Galton's words. Here is another and apparently very similar interpretation : "A man may receive a quarter of his hereditary characters from each parent, and a sixteenth from each grandparent, but all except a! very minute portion of these characters are common to all men, they being, in fact, the characters proper to the species, Homo sapiens, as such. Instead of a quarter of a unit from each parent a man receives only a hundredth or a thousandth of a unit of characters peculiar to the parents as such, all the rest being the characters common to all the members of the race. Even this minute fraction of a unit does not in any way represent characters acquired by the parent during his lifetime, but is itself built up of proportions of peculiar characters received from his parents, grandparents, and other ancestors in accordance with the law of heredity" (Vernon, Variations in Animals and Plants, pp. 146-7). Presumably Dr Vernon does not intend to imply that a man receives a quarter of his bulk, his mass, from each parent. Extension and weight are derived wholly from nutriment. We must go back, therefore, as always in the study of heredity, to the germ-plasm, or rather to its hereditary potentialities. If I understand $\mathrm{Dr}$ Vernon aright, he accepts the theory of discrete ancestral units which are halved and quartered and so on in the germ-plasm, but thinks that all ancestral units, at any rate all units that have come into existence since man evolved, are much alike-i.e. he thinks that, apart from the potency due to bulk, units differ only in comparatively insignificant details ; just as men are alike in being men, but differ inasmuch as they vary somewhat from oneanother. The hand is a character " proper to the species, Homo Sapiens, as such." Is it then contended that the child derives one-quarter of his tendency to develop that organ under the stimuli of nutrition and use from each parent, one-sixteenth from each grandparent, and so on ? The theory of Ancestral Inheritance assumes that the influence of an ancestor eleven generations back is on the average more than a billion times weaker than that of parent. But the character of a particular, 
217. The hypothesis that ancestral contributions are halved in every generation is a deduction from Galton's (and other) statistics, and from the circumstance that a 'reduction' division occurs in the germ-tract. So much has been made of the reduction division that it is necessary to consider it. It commonly happens that when cells are about to multiply by self-division the 'chromatin' of each nucleus gathers into 'rods' or masses termed chromosomes, the number of which is definite for each species. "Thus in man we find 32 , in a mouse 24, in a donkey 36, in a cockroach 32, in Ascaris megalocephala, var. univalens, 2 ; var. bivalens, 4 , and so on in different organisms." 1 In ordinary cell division the chromosomes split, each half from each chromosome forming a new chromosome which passes to one of the daughter cells. The division of the cells thus extends to a minute division of the structures within them. In this way the normal number of the chromosomes is preserved. But when the cells of the germ-tract are about to produce actual germs fit for conjugation, the chromosomes do not split. In the case of sperms, half of them pass as entire bodies to each daughter cell, which thus receives only half the normal number of chromosomes. This is the 'reduction' division. A second division in which the chromosomes split, results in four cells, each of which, possessing half the usual number of chromosomes, becomes a spermatozoon. In the case of the ovum, four cells are not formed; when the reduction division occurs, one-half of the total number of chromosomes are cast out of the cell in a 'polar' body, leaving almost the whole of the cell-body to the remaining chromosomes. At the next division the reduced number of chromosomes split as in ordinary celldivision; but one-half of the product is again cast out of the cell as a second polar body, leaving the ripened ovum, like the ripened sperm, with only one-half of the normal number of chromosomes. Therefore the divisions which result in four cells in the case of the sperms, result only in one in the case of the ova.

2I8. An obvious interpretation of these phenomena is that reduction implies nothing more than a preparation for conjugation; for, if the number of chromosomes were not reduced to half, they would be doubled in each generation when sperm united with ovum,

for example albino, ancestor more remote than the eleventh may be reproduced by offspring to the exclusion of the character of more recent ancestors. His ' unit,' therefore, must be strong enough to direct development. Must we assume, therefore, that the germ-plasm is compounded of millions or billions of units, the more modern of which are millions or billions of times more potent than is necessary for the reproduction of a character ?

${ }^{1}$ C. E. Walker, The Essentials of Cytology, p. 2 I. 
and this process would continue indefinitely. An obvious interpretation of the occurrence of polar bodies is that they are abortive cells-abortive because the ovum has retained almost the whole of the cell-body and the contained food, for lack of which the polar bodies perish. Neither of these interpretations can be tested; they are, from the nature of the case, merely working hypotheses. In science guesses should be avoided if possible; but, at any rate, the guess that the extension of the polar bodies is a mere detail in the preparation of the ovum for conjugation is at least as likely to be correct as the guess that reduction implies a halving of ancestral contributions. Moreover, when unicellular organisms unite, mixing their nuclei but not their cytoplasm, as happens in some cases, there is no extrusion and death of polar bodies. The notion that polar bodies are merely cells which perish from lack of cytoplasm and nutriment is supported, besides, by the fact that, before disintegrating, their chromosomes usually multiply by splitting as in ordinary cell-division. Probably, therefore, extrusion and death are not essential parts of the process, but only the means by which a double or quadruple supply of nutriment is conferred on the ovum wherewith to start the new cell-community. However, as we see, reduction has been held to indicate a halving of ancestral contributions-an enormous but untestable deduction.

219. On the other hand, suppose by an 'ancestral contribution' is meant, not a unit representing an ancestor in the germ-plasm and added to it by him, but a variation which has occurred in it at the time he came into existence. Then, since each individual, on the average, contributes a quarter of the heritage of his child, we must suppose that he varied from his parents to the extent of onequarter of the sum of his characters. His parents did the same, and so does the child. Therefore, on the average, the child varies to the extent of three-quarters from its great-grandparents. But were this true of all characters, so rapid would be the rate of racial change, that a human being, for example, would become nonhuman in three generations. If it be argued that the variations of the individual from his parent do not imply variations from remote progenitors, then how are the several 'contributions' to be distinguished from one another? How is it known that, on the average, each parent contributes a quarter, each great-grandparent a sixteenth, and so on?

220. The immense rate of change indicated by Galton's statistics is due apparently to the fact that he investigated only 
variable characters in very variable species. He himself terms the chosen characters 'moderately exceptional.' 1 Bassett hounds were established very recently, and display the variability of new types. In many characters human beings display the variability which follows cessation of selection. The reproduction of coatcolour in the lower animals, and of eye-colour in men, tends to be 'alternative.' 2 Probably human stature has never been very rigidly fixed by Natural Selection, and variability has been influenced, in England at any rate, by a great intermixture of races. Artistic faculty and health are not only very variable, but develop under the influence of an environment which, as regards these characters, is very varied. ${ }^{3}$

22r. Not until ancient, stable, non-exceptional characters, for example heart, lungs, and the like, have been investigated shall we be in a position to formulate or perceive the impossibility of formulating a numerical Law of Ancestral Heredity (i.e. to indicate the average rate of reversion). When that is done, I think it will be admitted that variability, including the tendency to reversion, is an adaptation regulated by Natural Selection, and, therefore, that the degree of variability differs with the stringency of Natural Selection in every species and character. I think, also, that it will then be admitted that the true Law of Ancestral Heredity is formulated when we declare that, In any character the tendency to retrogression (i.e. reversion to ancestors) is proportionate, on the average, to the speed of the antecedent progression.

222. The Law of Ancestral Inheritance is an example of the biometric and statistical method of inquiry. As a method biometry is, of course, like experiment, excellent. It enables the patient worker to gather many obscured truths which, but for it, would lie beyond our reach. But it is extraordinarily slow and laborious, and, like experiment, has its limitations as a means of discovery and as a test of thinking. So many biological facts are patent, and so much biological thinking can be tested by reference to this patent evidence that, apparently, it has been difficult to find subjects suitable for biometric inquiry, except when the patent evidence is ignored. Occasionally, therefore, such inquiry has been directed to the discovery of matters already known, or which can be made known at a thousandth part of the labour involved and with an even greater degree of certainty. Inferences founded on materials furnished by biometry require to be tested as carefully as any other; but, as in experiment, the two functions of discovery and testing
${ }^{1}$ Natural Inheritance, p. I.
${ }^{2}$ See $\$$ 239, 277, 278.
${ }^{3}$ See chapter xxii. 
are, apparently, frequently confused, and thoughts are claimed as accurate merely because they are based on, not tested by, statistical data.

223. In the Law of Ancestral Inheritance we have an instance of an induction expanded, but not tested by a deduction, the latter being regarded as the theory. Here the induction is that, on the average, offspring resemble ancestors (at any rate in certain characters and within three or four generations) in the stated degrees. The deduction-the really tremendous expansion of the induction-is that ancestors contribute in the stated degrees to the heritages of descendants. Though the universe is a unity, the facts and 'laws' of which are in harmony with one another, and it is the mission of science not only to interpret the facts in terms of the laws but also to demonstrate the relations between the latter, no appeal is made to any conceived system of reality. The axiom that every induction must be used as a basis for a rigorous deductive inference of consequences is disregarded. In other words the correctness of the thinking, as distinguished from the facts, is left untested.

224. The law evidently bears on the theory of the Continuity of the Germ-plasm, which supposes that the parents and ancestors of the individual contribute nothing to the child, and on the theory of Recapitulation, which supposes that ancestors are represented during development, not en masse, but serially; but no attempt is made to show that it accords with these hypotheses, or, if it does not, in what respects they are wrong. It remains as apart from anything else that is known or that has been surmised by science concerning living beings as a miracle.

225. The Law of Filial Regression furnishes another instance of biometric work. It has been ascertained that when a group of parents differs in any particular from the mean or average (mediocrity) of their race, the offspring on the average differ less, grandchildren still less, and so on. In other words, if a group (or individual) in any generation differs from the racial mean, its (or his) predecessors and successors will, as a rule, be found less different. "Thus take fathers of stature 72 ", the mean height of their sons is $708^{\prime \prime}$, or we have a regression towards the mean of the general population. . . . The father with a great excess of the character contributes sons with an excess, but a less excess of it; the father with a great defect of the character contributes sons with a defect, but less defect of it. The general result is a sensible stability of type and variation from generation to generation. . . . Now a man is not only the product of his father, but of all his 
past ancestry, and unless very careful selection has taken place, the mean of that ancestry is probably not far from that of the general population." 1 The induction here is that there exists a tendency for exceptional groups to return towards mediocrity unless Natural Selection interferes. The deduction is that on cessation of selection the race or species tends to remain stable. But when we appeal to reality the evidence is massive that cessasion of selection results in retrogression. Here again no attempt is made to demonstrate that the Laws of Regression and Retrogression are in accord, or, if not in accord, in what particular the latter is wrong. The truth appears to be that, though regression results in a levelling up to the specific mean as well as a levelling down to it, yet, in a degree that varies in different species and characters according to the rapidity of the antecedent progression, the tendency to level down is somewhat greater than the tendency to level up. The point aimed at is not the specific mean, but below it. Consequently this point tends continually to fall unless rendered stable or raised by selection. Therefore regression is but the first phase of retrogression. As a rule, however, the latter proceeds so slowly, by such imperceptible gradations, that its detection is far beyond the reach of the laboratory, which can examine, at most, only a few generations.

226. Formerly some men of science maintained that Natural Selection had no real existence, or at least had not been demonstrated. Natural Selection implies a selective mortality, which our acquaintance with the lives of wild plants and animals is not sufficiently intimate to prove. It was shown, however, that stringent and unmistakable selection occurs amongst all races of human beings, the only 'wild' types which we are in a position to observe with the requisite degree of thoroughness. ${ }^{2}$ In England, for example, we are being selected by tuberculosis and other maladies; in Africa, malaria plays the same part; in India dysentery, and so forth. The immediate result is that individuals weak against any lethal disease are weeded out wherever it is present. The remote result is that every race is resistant to every disease in proportion to the length and severity of its past experience of it. Therefore the evidence of Natural Selection followed by evolution is plain and

1 Pearson, Grammar of Science (Ed. 1900), p. 456.

${ }^{2}$ See The Present Evolution of Man, 1896. The common notion that man is not a 'wild' or natural type is, of course, nonsense. He is not under the care of and has not been artificially selected by any other type. Civilization has altered the conditions under which he lives, and therefore the incidence of selection and the direction of evolution, but that is all. It has not altered the 'laws' of heredity for him. 
unmistakable. Besides the data which each person is able to verify for himself, and which therefore is especially valuable scientifically to him, a vast volume of very precise and detailed statistical information relating to almost every human race and disease has been published by all the Departments of Public Health in both hemispheres. Presumably, if selection followed by evolution occurs amongst men, it occurs amongst plants and lower animals. At any rate, if it occurs amongst men, the inference that it occurs and has occurred amongst other species is at least as likely to be true as if drawn from a demonstration of its occurrence amongst some other type of animal or plant.

227. Such, however, has been the passion for obscured facts that this patent evidence has been ignored. Laborious laboratory inquiries which extended over several years have been undertaken to demonstrate the actual occurrence of Natural Selection. Doubtless they satisfied the workers, but they were declared by opponents to be invalid on the ground that natural conditions were not accurately reproduced. Even by adherents of Natural Selection they are declared to be "at present not absolutely convincing." 1 Of all the statistical evidence, the best is thought to be that collected by Bumpus in America. ${ }^{2}$ After a severe snowstorm one hundred and thirty-six stricken sparrows were gathered, of which seventy-two revived, while sixty-four perished. Bumpus, after making careful measurements, concluded that "Natural Selection is most destructive to those birds which have departed most from the ideal type, and its activity raises the general standard of excellence by favouring those birds which approach the structural ideal." What precisely is the structural ideal is of course a matter of opinion. It is at least possible that the birds which survived possessed a superior power of resisting cold and hardship, and that this power is not correlated to any of the dimensions-length of head, beak, and so forth-that Bumpus was able to measure. Compare the volume, precision, and relevancy of this evidence with that concerning disease gathered by simple observation, and more especially with that stored by Departments of Public Health. Compare also the relative difficulty of demonstrating the connection between cause and effect in the two instances.

228. Biometricians have also sought to demonstrate that human mental 'ability' and powers of resisting disease are 'inheritable.' They admit that the human species has arisen by evolution from

1 Vernon, Variations in Animals and Plants, p. 337.

${ }^{2}$ Biological Lectures, p. 2 I I. 
a lower type, and that the offspring of human beings are also human; that is to say, that human beings tend to reproduce parental characteristics. It is likewise a patent truth that man has more intellectual 'ability' than any lower animal, and another that some human races, those that have suffered most, are more resistant to disease than others. At least, all these truths, however ignored in the laboratory, are universally believed. Again, it is a patent truth that a species or race is merely an aggregate of related individuals, whence it follows that if one generation of a species or race tends to transmit its characters to the next, then individuals must tend to transmit their characters also. Consequently it is inconceivable that human ability and powers of resisting disease can have been evolved unless the paternal characteristics, including variations, in these respects tended to be inherited. Given the evolution, the general tendency to inherit degrees of capacity is a necessary corollary which hardly needs biometric confirmation. Here, as always, we create much better science when we deduce one truth as a necessary corollary from another (i.e. when we link the two truths together by a chain of causation) than when we state them separately as isolated facts or hypotheses. ${ }^{1}$ This unnecessary isolation of hypotheses is a principal feature and fault of nearly all laboratory work. Science describes nature; and nature is a unity in which nothing is isolated. Moreover we have no means of estimating the ability of individuals except by noting the degree of intelligence observable, and, as we shall see later, intelligence depends largely on mental training, on education, which differs greatly in different classes and races and cannot be estimated in the laboratory. We have no means of estimating resisting power to disease except by observing the degree of resistance displayed under conditions of food, general health, intensity of exposure to disease, and the like, all of which may vary with time and place and are also outside the range of the laboratory.

229. Similarly biometricians have sought to demonstrate that individual degrees of fertility in men and horses tend to be transmissible. But how, unless we presuppose some recent and extraordinary change in nature, is the contrary conceivable? Manifestly the relative fertility of species (groups of related individuals) is an adaptation, a product of evolution, which could not have arisen unless the variations, the peculiarities, of individuals had tended to be inherited by offspring. The degree of fertility ${ }^{1}$ See $\$ \S 75$ (footnote), 352 (footnote). 
differs in different species, but always bears a close relation to the number of offspring which it is possible to rear. Plants and low animals, whose characters are developed wholly or almost wholly under the stimulus of nutriment, do not protect nor train their young, which are able to fend for themselves from the first. Therefore their offspring may number millions. The physical and mental characters of the higher animals are developed largely under the stimulus of use; the individual is born more or less helpless, and must be protected and trained during development. Therefore the number of offspring that can be reared is very small compared to that in the lower types. Everywhere we find an adaptation as regards fertility which would have been impossible unless individual peculiarities in fertility had been transmissible and had thus afforded materials for Natural Selection. It is said sometimes that, throughout nature, intelligence and fertility are in inverse ratio to each other. This is true; but not for the reason alleged-that they are fundamentally antagonistic to one another. It is true only because intelligence has to be trained. ${ }^{1}$ The greater the intelligence achievable by an animal, the more helpless it is at birth and the more prolonged and strenuous must be its preliminary training by its parents. Therefore, since intelligent animals are able to protect and train only a few offspring, parsimonious nature has rigorously limited the number of the latter. In the case of man, as proved in the laboratory and more massively by RegistrarGenerals and the officials thatcorrespond to them in foreign countries, there are considerable differences in fecundity - in the number of offspring actually produced as distinct from the number which might be produced-between races and classes. This may indicate differences in fertility; but before the question can be decided, we must pass, as in the cases of 'ability' and resisting power to disease, outside the laboratory and inquire into a multitude of circumstances, such as the customs of the people, average age at marriage, religion, morality, sanitation and general health, physiological knowledge, desire for large or small families, and the like. Unless these are considered, every deduction concerning human fertility which expands an induction that was reached through a statistical consideration of fecundity is illegitimate.

230. Numerous biometric studies of variability have been published. In each case various characters in a number of individuals (the more the better) of the same race have been measured and the dimensions tabulated. In this way a very considerable 
knowledge of the variability of certain characters in certain races has been obtained-that is, a knowledge of the range within the limits of which variations normally occur, the relative frequency of each degree of deviation from the mode (the most common type), and the degree in which the deviations of various characters tend to be correlated with one another. Such knowledge is gathered only at great expense of time and patience. It can, as a rule, include only the variations of the fittest (the survivors), and never more than a very few measurements in a very few animals and plants. It may, of course, like knowledge of any other facts, prove very useful. But curiously little effort has been made to use it, to interpret it, to link it up with laws, with uniformities of causation. Apparently the result, such as it is, of each inquiry has been held to justify the labour. As far as I am able to judge, however, the biometric evidence concerning variability is valuable chiefly for the light it sheds on, and the support it affords to the hypothesis that variations are both 'spontaneous' and under the control of Natural Selection. It demonstrates that variations tend to occur about equally about the specific mean, the plus and minus variations of every dimension of a character being of nearly equal frequency and magnitude; material thus being afforded which enables Natural Selection to meet every contingency. Again, while all characters vary, large variations which tend to ruin the co-adaptation of the parts of the organism are rarer than smaller variations and most rare in the case of characters in which the co-adaptation to the parts needs to"be very close ; thus the squirrel's head and forefoot vary less widely in proportion as regards length than the tail. Yet again, when characters are functionally correlated, the variations tend to be correlated also; thus if the length of a bone in a man's arm is greater or less than the average, it is more likely that the other bones in his arm will be correspondingly long than that his teeth, nose, or beard will be so. If the Bathmic, or the Lamarckian doctrine, or the hypotheses that variations are commonly caused by the direct action of the germ's environment were any of them true, variations would not be grouped about the specific mean, but would all be in one direction or at least would strongly preponderate in it. If variability were a 'fundamental' property of living beings, uncontrollable by Natural Selection, all characters should be equally variable. At any rate small variations should not so considerably and universally preponderate in closely, as compared to less closely, co-adapted parts. This last hypothesis, however, is even more decisively negatived by the fact that the 
lengthening of the germ-tract, the increase in the number of cellgenerations which occurs when plants are propagated by slips, does not result in an appreciable difference in the number and range of variations, as it would if variability were a fundamental character beyond the control of natural selection, and occurring, therefore, all along the germ-tract.

23I. To sum up: the biometric method, as employed by many of its exponents, suffers from the same defect as the experimental method. Very often no facts other than those revealed by a particular inquiry are taken into account, though many may be available; and, therefore, inferences from the evidence are left not only isolated, but untested. The science created depends altogether on a simple enumeration of instances, not on a knowledge of causes. "But in estimating probabilities, it is not a matter of indifference from which of these two sources we derive our assurance. The probability of events as calculated from their mere frequency in past experience affords a less secure basis for practical guidance than their probability as deduced from an equally accurate knowledge of the frequency of occurrence of their causes." 1 Not seldom in biometric inquiries-as in the case of those inquiries about disease and fertility which we have just examined-several scores or hundreds of observers and thinkers are employed for years in ascertaining, with a much lesser degree of certainty, that which a single thinker may deduce in two minutes from known and admitted truths. "These are but samples of the errors frequently committed by men who, having made themselves familiar with the difficult formulæ which algebra affords for the estimation of chances under suppositions of a complex character, like better to employ those formulæ in computing what are the probabilities to a person half informed about a case, than to look out for means of being better informed. Before applying the doctrine of chances to any scientific purpose, the foundation must be laid for an evaluation of the chances, by possessing ourselves of the utmost attainable amount of positive knowledge." 2 Experimental observers, whose evidence we shall consider next, have formulated hypotheses which have seemed fundamentally wrong to biometricians. The latter, or some of them, were induced to employ their method mainly, I believe, in order to demonstrate the erroneousness of the conclusions reached by their opponents. But, if these conclusions are mistaken, and I think there are entirely conclusive grounds for believing that ${ }^{1}$ Mill, Logic, III. xviii. 4

2 Op. cit., III. xviii. 4 . 
they are mistaken, the truth would have been all the more thoroughly proved had a completer use been made of the biometric data-had the biometric conclusions (of which the principal is the hypothesis that evolution is founded on small variations, "fluctuations' as they are called, not on mutations or sports) been linked up with and tested by conclusions which are disputed by nobody and are founded on facts, also undisputed, gathered by simple observation. 


\section{CHAPTER VII}

\section{MENDEL'S LAWS}

Sexual dimorphism-Cross-fertilization-Parthenogenesis-Self-fertilizationThe effect of conjugation-Sex an adaptation-Sexual characters-Alternative and blended inheritance-Theories of the function of sex-The theory of continuous evolution-The theory of discontinuous evolution-The Mendelian theoryDominance and recessiveness-Unit segregation and gametic purity-Exceptions to the Mendelian doctrine-Latency, temporary and permanent-Compound allelomorphs-Interpretations of Mendelian phenomena.

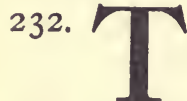

HE higher animals are all dimorphic, that is, each species is divided into two groups which differ widely in physical and mental characters. In the one group are the males; in the other, the females. Obviously, the sexual differentiation is connected with reproduction. The mental peculiarities of the two sexes are such as to impel to a series of actions which eventually result in a union of sperm with ovum under conditions advantageous to the development of the future cell-community, to which end also the physical characteristics of the parents are fitted. In all species the physical and mental sexual characters furnish beautiful examples of co-adaptation.

233. Reproduction through the union of germs from two distinct individuals is so familiar that we are apt to accept it as 'natural' and so dismiss it from our thoughts. Conjugation, however, is not a necessary antecedent of reproduction. Even in the higher animals it occurs only once-when sperm and ovum conjugate-during the production of the millions or the billions of the cell-community. The somatic cells never conjugate, and the germ-cells only when a new cell-community is about to be produced, and then, in most cases, only with a cell from another body. In the case of some plants and animals, for instance Cypris reptans reproduction is apparently entirely parthenogenetic, no males having ever been observed. Since these organisms retain their sexual organs, they are, of course, descended from types in which reproduction was, at least, sometimes preceded by conjugation. In aphides reproduction is parthenogenetic during warm weather when food is plentiful, but the winter eggs are fertilized. Queen 
and worker bees are derived from fertilized, drones from unfertilized eggs. There are thus gradations between complete parthenogenesis and conjugation occurring at the start of every cellcommunity. The reproduction of unicellular forms has been imperfectly studied. Possibly in some, or many, or most cases conjugation never occurs. Even where it has been noted there are always long intervals of asexual multiplication. In paramœcium, for example, at lengthy but seemingly more or less fixed intervals, conjugant individuals appear which differ somewhat from the rest of the population in appearance. They correspond to the germcells of multicellular organisms, and their descendants, which intervene between one act of conjugation and the next, to the generations of the cell-community. ${ }^{1}$

234. The ovum is usually a large and passive cell, more or less laden with nutriment for the earliest needs of the new cell-community. The sperm is small and mobile, and is thus enabled to reach and penetrate the ovum. But, as already noted, ${ }^{2}$ we have every reason to believe that these differences of size, shape, and function imply nothing as far as the germ-plasm is concerned. Apparently the germ-plasm used in fertilization is situated wholly, or almost wholly in the nuclei of the sex-cells, and the nuclei of sperm and ovum seem equivalent as bearers of heredity. In most cases, conjugating sperms and ova are derived from distinct cellcommunities of the same variety. Conjugation between the sexcells of distinct species, and in a lesser degree of distinct varieties, is hindered or prevented by physical or physiological incapacity, ${ }^{3}$ and, in the case of the higher animals, by mental disinclination as well. On the other hand, nature has elaborated many devices to prevent conjugation between the germs of the same individual.

235. In most animal species only sperms or ova are produced by the same individual. Some low animals, however, for example snails and earth-worms, and many plants are hermaphrodite, possessing male and female organs of generation, and producing both sperms and ova. In such cases, self-fertilization is prevented by the ripening of the sperms and ova at different periods, or in other ways. The prevention is usually less perfect in the case of plants than of animals, so that in the former case self-fertiliza-

1 See $\S \mathrm{r}$.

2 See $\S 2$.

${ }^{3}$ Physical disability prevents, for example, a very large dog mating with a very small one. Physiological disability renders mating sterile. In the latter case the sperms do not fertilize the ova, or, as is probable in some cases, the embryos do not develop beyond a very early stage. 
tion may more often alternate with cross-fertilization. Some plants, and some plant-like marine animals are normally selffertilized. ${ }^{1}$ Thus there are connecting links between constant cross- and constant self-fertilization.

236. The normal effect of conjugation, then, is to mix, with a degree of intimacy which is in dispute, two germ-plasms which on the whole resemble one another somewhat closely, but which differ in details. The difference is greatest when individuals of different species cross, and least when there is self-fertilization. Indeed, self-fertilization is an approach to parthenogenesis in which there is no mixture. Another approach is the alternation of parthenogenesis with conjugation as in aphides. Plainly, then, conjugation is not an essential part of reproduction, which may and does occur without it. Indeed it is precisely the parthenogenetic types that reproduce most rapidly. But it is an accompaniment of reproduction so nearly universal that we have reason to believe that it must possess some extremely important function. Presumably it is an adaptation which, like other adaptations, has been created and maintained by Natural Selection-an adaptation so vastly useful that, to attain it, most multicellular species have become dimorphic and have evolved all the manifold physical and mental peculiarities of sex, including the keenest and fiercest of instincts, so useful that to possess it the possible number of offspring has been halved, since two individuals instead of one are necessary to the production of each child. ${ }^{2}$ Thus great advantages are lost for the sake of what is probably a greater.

237. The function of sex (of conjugation, of the mixing of germ-plasms) has been, especially of recent years, one of the most widely and acrimoniously debated of all the problems of heredity. Too often in these discussions has the great probability that sex is an adaptation, and, therefore, like other adaptations, a product of Natural Selection, been ignored. Sex is so widespread and is served by so many and such diverse physical and mental characters, that to doubt that it has utility, to suppose it is a mere chance accompaniment of life, is the very madness of scepticism. No theory of sex is likely to be true that assigns to it a non-adaptive function. What, then, is the function of sex? In what way is it useful? How does it serve to adapt species to their environments? Moreover, how is it that some types are cross-fertilized, others self-fertilized, and others parthenogenetic, while yet others exhibit gradations between cross-fertilization, occurring regu-

${ }^{1}$ Origin of Species, p. 123.

${ }^{2}$ Sce $\$ 336$. 
larly at the genesis of every cell-community, and complete parthenogenesis?

238. Before we attempt to solve the problem of sex we must study for a space the phenomena of 'inheritance.' When reproduction is parthenogenetic, offspring reproduce parental chäracters with variations. When two parents are concerned in reproduction the process is more complicated. In sexually dimorphic species the two parents resemble one another closely in some particulars, for example in blood, muscles, glands, heart, lungs, liver, nerves, and the like. In others, the sexual characters, they differ much more widely. The child, being male or female, develops only one set of the sexual characters. The other set, however, is not wholly absent. Its items are merely rudimentary or latent. The male breasts in man are examples of rudimentary organs. Of latency there is unlimited evidence: for example, female birds have been known in old age to develop the plumage of the opposite sex; elderly women occasionally develop to some extent beards and moustaches; hæmophilia and colour-blindness, which are usually male characters, are transmitted through daughters to grandsons, and the good milking qualities of cows through sons to granddaughters; the female characters of bees are apparently transmitted through the drones, and the male characters through the queens; doubtless, however, both sets of characters are transmitted through both sexes. The male characters of aphides are transmitted through a long series of pathenogenetic females. This form of reproduction, in which one only of two possible characters, or sets of characters, is developed, is termed 'alternative.'

239. The development (not inheritance, for both sets are 'inherited') of the sexual characters, then, is alternative. But the term 'sexual' must be given a wide meaning. It applies not only to those primary differences of shape and function which are directly concerned in reproduction, but also to those secondary peculiarities of shape, colour, scent, and the like which, being mostly on the surface of the body, and therefore observable, serve as sexual attractions, as well as to the other physical and mental peculiarities which distinguish the sexes. Most of the sexual characters tend to hang together in sets, a male set and a female set; but some characters, which are sexual in the sense that their function, in whole or part, is to attract the opposite sex, occur in both sexes; for example in human beings the mane on the scalp and the coloration of the hair, skin, and eyes. But even in the case of those 
characters which are common to both sexes and which may have other functions besides sexual attraction, development tends to be alternative. Thus the child of parents, one of whom has blue eyes and the other hazel, usually has blue eyes or hazel eyes, never eyes that are a blend between blue and hazel. So too, in a lesser degree, children sometimes reproduce the colour and texture of hair of one parent, and to a still lesser degree the colour of the skin.

240. Obviously the alternative development of the sexual characters is an adaptation, a product of evolution. It is a device by which cross-fertilization is secured. Though evolution has caused most of the sexual characters to hang together in sets, yet the separation of male and female characters is not always strictly preserved. Occasionally a character, usually distinctive of one sex, is found in an individual of the opposite sex. Thus, a man may possess well-developed female breasts, or a woman a full beard. Sometimes the male and female characters may be found so intermixed, as for example in the so-called human hermaphrodites, ${ }^{1}$ that an individual of one sex may present every external appearance of belonging to the other. Or the male and female characters, instead of being inherited alternatively, may blend in varying degrees-as when a woman is partially bearded or has a masculine pelvis.

24I. Selection, natural and sexual, however, must always have tended to eliminate these abnormalities and so preserve the purity of the sexual characters, their alternative development, and their aggregation in distinct sets. Selection is, of course, more stringent as regards the primary sexual characters, which are the more essential part of the machinery of reproduction, than as regards the secondary characters. Consequently blending or displacement from the set in which they are normally found is rarer in the case of the former than the latter. Thus men may have female breasts and women beards, but never, as far as I am aware, have testes been found associated with a uterus. By artificial selection man has more than once succeeded in transferring secondary sexual characters from one sex to the other. Thus formerly only the female Polish fowl had the domed skull and cerebral hernia which now characterizes both sexes, ${ }^{2}$ and the male Sebright bantam has the

${ }^{1}$ A real hermaphrodite is an individual in whom both sets of sexual characters, the male and the female, are patent; for example, snails and most flowering plants. A 'so-called' hermaphrodite is an individual in whom the male and female characters have blended, or one in whom some male and some female characters are patent.

2 Animals and Plants, vol. i. p. 269. 
same hackles, saddle, and stickle feathers as the female. "Consequently the tail is short and truncate as in a hen."

242. Manifestly the differentiation and the alternative development of the sexual characters is not in itself an end. A male has patent one set of characters and a female another, and males and females are reproduced alternatively, not because the individual is thereby brought into closer adaptation to the general environment and his or her life more surely preserved, but to afford facilities for mixing the germ-plasm, a process which, for some reason, we have yet to discuss and if possible discover is of advantage to the species. Therefore it is likely that the sexual characters have come into existence because the non-sexual characters are, in some way, beneficially affected by the intermixture of the parental germ-plasms. When, therefore, we have discovered how the nonsexual traits are thus usefully affected by conjugation, we shall have discovered the function of sex.

243. Formerly, because, in the higher animals, young individuals come into existence only after acts of conjugation, the 'obvious inference' was drawn that the function of sex is to invigorate, revitalize, or rejuvenate aged and debilitated germ-plasms. But, as indicated by Weismann, it is difficult to understand how the union of two outworn and enfeebled things can result in rejuvenescence. Moreover, parthenogenetic species, and plants which are propagated by slips, retain their vitality perfectly.

244. Next Weismann, followed by the majority of biologists, believed that the special function of the intermixture of somewhat dissimilar germ-plasms which occurs in conjugation is to provide the spontaneous variations which are the materials on which Natural Selection works, $\mathrm{He}$ concluded consequently that parthenogenetic species vary little if at all, and, therefore, are incapable of adapting themselves to changes in the environment. He supposed that they secured survival, not by close adaptation but only by a very rapid rate of reproduction. But rapid reproduction is an adaptation which can have arisen only through the Natural Selection of favourable variations. Therefore variations must have occurred even after conjugation was abandoned. Besides, it has been proved directly that he was mistaken. Variations occur in abundance when reproduction is parthenogenetic. Thus Weismann himself discovered evidence of variations occurring in the absence of sexual reproduction, ${ }^{1}$ and as a result of a biometric inquiry conducted on the parthenogenetic Daphnia

\footnotetext{
1 The Germ-Plasm, Eng. Trans., pp. 344-6.
} 
magna, Dr E. Warren found " on measuring the offspring it was obvious that the children of the same brood exhibited considerable variability." 1 A more extended inquiry on Aphides led him to suppose, however, that variability in asexual reproduction, though considerable, is less than when offspring have two parents. We shall see that this relative paucity of variations is due, probably, to a comparative lack of retrogressive variations. Lastly, it is a well-established fact that parthenogenetic species, for example dandelions and hawkweeds, are particularly rich in varieties, a fact implying abundant variations. ${ }^{2}$

245. Variability, then, can occur apart from conjugation. We saw that the evidence is strong that spontaneous variability is an adaptation controlled and maintained by Natural Selection, on which account every species and organ tends to be variable in the right degree. ${ }^{3}$ The least variable types and structures are those which are closely adapted to the environment by stringent selection. The more variable types and structures are those which are less closely adapted and in which therefore variability is encouraged by Natural Selection, or those which are not stringently selected for the reason that very close adaptation is not necessary. Thus men and garden plants are exceptionally variable because they are so circumstanced that individuals that have varied largely, for example in colour, have survived in considerable numbers and have transmitted their increased variability to descendants. Thus again the squirrel's tail is more variable than its head and forefoot because there is a greater need for close adaptation in the latter. Since, then, spontaneous variations occur in parthenogenetic reproduction, the materials are present for the work of Natural Selection, and that agency is able to increase or decrease the variability of the species to any useful extent. Bi-parental reproduction is not necessary for the purpose; and it is, at the very least, most improbable that species can have been burdened with sex to produce that which was already present, and which, apparently, is kept within bounds only by selection. Undoubtedly,

1 Proc. Roy. Soc. London, 1899, vol. 1xii. p. I54.

2 See De Vries, Species and Varieties, pp. 59-6r. "Thousands of forms (of dandelion and hawkweed) may be cultivated side by side in the Botanical Gardens and exhibit slight but undoubted differentiating features, and reproduce themselves truly." I follow De Vries in supposing that dandelions and hawkweeds are parthenogenetic. I know nothing of the matter personally. There seems, however, to be some doubt (see Bateson, Mendel's Principles of Heredity, p. 247). At any rate it appears clear that these plants, if they do conjugate, do so comparatively rarely.

${ }^{3}$ See $\$$ I63. 
therefore, Professer Karl Pearson is right in declaring that "Variability is not a product of bi-parental inheritance ; . . . whatever be the physiological function of sex in evolution, it is not the production of greater variability." 1

246. At the present day I think most biologists who have given any thought to the question believe that the function of sex is (through the mixing of germ-plasms) to mix the parental qualities. There are, however, great differences of opinion as to the nature and effect of this mixing. Suppose a man with black eyes and six digits on each hand mates with a woman with blue eyes and the normal number of digits; then experience demonstrates that each of the descendants of such a pair will have black eyes or blue eyes (or perhaps grey or hazel eyes, as in some ancestor): never, or hardly ever, eyes that are intermediate; and six digits or five digits, not five and a half digits. But one or more of the children may develop the father's eyes in combination with the mother's hands, or the mother's eyes with the father's hands. In such a child the parental characters are mixed; but they are mixed as black and blue marbles in a bag, not as black and blue colours are blended on a painter's palate. On the other hand, the offspring of a white man and a negress actually does blend the parental skin colours; the descendants are not black or white, but of a colour that is more or less intermediate.

247. Here, then, we have two distinct types of 'inheritance,' the alternative and the blended, both of which undoubtedly occur. Since in the first type the father's or the mother's character is reproduced, not a blend, and since the grandchild may have patent the character which was latent in the parents, the resemblance to the method by which the sexual characters are 'transmitted' is evident, the main difference being due to the fact that whereas the various sexual characters tend strongly to hang together in distinct patent or latent sets, the non-sexual characters, the reproduction of which is alternative, tend to mix and so form new combinations. Now the question we shall have to decide is whether the function

1 Grammar of Science, pp. 473-4, ed. 1900. In my work, The Principles of Hevedity (p. 49), I controverted Professor Pearson's statement, and pointed out that a mulatto, for instance, the offspring of a white man and black woman, varies from both parents in a definite way. I must now confess that I seem to have mistaken Pearson's meaning. Conjugation is certainly a cause of some variations, in that the offspring when they blend parental characters vary from both'parents. But variations and variability are different things. A parthenogenetic species may be as variable or more variable than one the reproduction of which is bi-parental. 
of sex is to mix non-sexual parental characters as colours are blended, or to mix them as marbles are mixed. What, then, is the rule in nature? Is blended 'inheritance' the normal for nonsexual characters and alternative 'inheritance' abnormal? Or is alternative 'inheritance' the normal? Or are they both normal in the sense that nature has evolved both as adaptations-in the sense that both have utility? This question is being very sharply debated at the present time. Formerly it was universally believed -by Darwin for example-that offspring tend, as a rule, to blend parental characters, and this view is now upheld by most biologists, and in particular by the biometric or statistical school. The experimental or 'Mendelian' school, on the other hand, insist that the non-sexual characters of parents are, as a rule, not blended in offspring. 1

248. Naturally these divergent views of heredity have led to, or are associated with, equally divergent views of evolution. Speaking generally, biometricians maintain that variation and evolution are both continuous; in other words, they suppose that nature causes evolution by gradually and continuously raising the specific mean through the selection of 'normal' or 'fluctuating' variations.

249. On the other hand, experimental workers, who are usually 'Mendelians' and 'Mutationists,' insist that there are two distinct kinds of variations, the continuous and the discontinuous. The former, they maintain, fluctuate about a mean to which they tend to return so strongly in succeeding generations, that they cannot be made the foundation of permanent racial divergence by any stringency of selection. In other words, they suppose that, owing

${ }^{1}$ It is interesting to note how the different methods by which the rival schools pursue their inquiries have tended to lead to divergent theories of heredity. We shall see presently that blended 'inheritance' is the rule when parents differ comparatively little, that is, when their differences are of degree rather than of kind ; and that alternative 'inheritance' is the rule when parental differences are greater, that is when they are of kind rather than of degree. The statistician dealing with aggregates of individuals, and noting that even when parental differences are great, offspring tend to ' regress ' towards the parental and specific mean, is impressed by the fact that extreme plus and minus deviations from the average type of the race are rare and are connected by lesser variations. He is inclined, therefore, to lay stress on the truth that the vast majority of variations are ' continuous,' and to believe that offspring tend, as a rule, to blend parental characters. The experimental worker, on the other hand, deals with individuals more than with aggregates, and naturally chooses as materials for study sharply contrasted parental qualities, the inheritance of which can be more easily observed in offspring than mere shades of difference. He tends, therefore, to lay stress on ' discontinuous ' variations, and to believe that alternative inheritance is the rule. 
to the tendency to reversion, or rather regression, these fluctuating variations cannot be accentuated or piled on one another by selection continued through any number of generations. According to them evolution is founded solely on discontinuous variations ('mutations'), which, though they may not always differ from continuous variations in magnitude, yet differ totally in kind and in that they are absolutely stable. ${ }^{1}$ It is thought that, if an individual in whom one of these discontinuous variations appears has mated with an individual who has it not, then a proportion of the descendants inherit it and a proportion do not; there is rarely a blend. If it is unfavourable, the descendants that are of the new type are eliminated, and it disappears from the race; if it is favourable, the ancestral type may be eliminated in the struggle for existence and the new type established in its place; if the variation is neither favourable nor unfavourable, the two types exist side by side. According to the Mendelian and mutationist view, therefore, evolution does not proceed continuously (along a smooth incline, as it were), but by a series of steps of varying but usually considerable magnitude. The theory of evolution by discontinuous variations finds its strongest advocate in Professor Hugo de Vries, the distinguished Dutch botanist. His opinions differ, however, in some very important particulars from those of the more extreme Mendelians. ${ }^{2}$

250. The theory of continuous evolution (that is, evolution founded on ordinary 'fluctuating' variations) is sometimes termed the 'selection' theory, that of discontinuous evolution the 'mutation' theory. Both theories, however, are doctrines of evolution by the selection of favourable variations. They differ in that they attribute evolution to the piling up of different classes of variations. It is true, however, that mutationists often lay much less stress on selection and adaptation than selectionists.

25 I. Sir Francis Galton, who may be said to have founded the biometric school, distinguishes three kinds of inheritance: (I) Particulate, or inheritance "bit by bit, this element from one progenitor that from another," 3 as when black and white poultry are crossed and the offspring have in varying degrees some feathers black or barred, and others white. (2) Exclusive, when the character of only one parent is reproduced to the total exclusion of the other, as when the plumage of the offspring of black and

${ }^{1}$ Doubtless the opinion that discontinuous variations are absolutely stable is not held by all mutationists. Apparently, however, it is held by many of them. See $\$ 285$.

${ }^{2}$ See $\$ 282$.

${ }^{3}$ Natural Inheritance, p. 7 . 
white fowls is wholly black or wholly white. Particulate inheritance may be regarded as exclusive inheritance occurring in patches. (3) Blended inheritance, as in the skin colour of a mulatto. Blended inheritance may be regarded as particulate inheritance occurring in a very fine mosaic. Galton thinks, "There are probably no heritages that perfectly blend or that absolutely exclude one another, but all heritages have a tendency in one or the other direction, and the tendency is often a strong one." 1 There can be no doubt, however, that small progressive variations (fluctuations) present in one parent, but absent in the other, tend to disappear absolutely in the offspring. In such cases, therefore, there is no blending. ${ }^{2}$

252. The greater part of modern experimental work follows the lines laid down by Gregor Mendel, a monk who became Abbot of Brinn, and who about the middle of the last century crossed many varieties of the edible pea-Pisum sativum. Peas are normally self-fertilized, the pollen-grains and ovules of the same flower uniting. But the plants have conspicuous flowers, which, presumably, underwent evolution because they attracted insects and so secured cross-fertilization. We must suppose, therefore, that peas have descended from ancestors that interbred. If the anthers of a flower be amputated before the pollen is ripe, and pollen from another plant be conveyed to the pistil, it is still possible to secure cross-fertilization.

253. Mendel worked with a number of strongly contrasted varieties - 'tall' and 'dwarf' plants, for example. From the mongrel seeds, thus obtained, only tall plants developed. They were allowed to self-fertilize themselves, as were their descendants. About one-quarter of the offspring-the grandchildren of the original cross-developed as dwarfs and three-quarters as tall plants. No plants were intermediate. Thereafter the dwarfs continued to breed 'true' as long as the experiments were continued, producing no tall plants in succeeding generations. It seemed evident that the race was purged of the influence of the tall variety. On the average, a similar number of tall plants behaved in like manner, producing only tall descendants. Their race, also, had become 'pure.' But the remaining plants (fifty per cent. of the whole) behaved like their cross-bred parents, producing tall and dwarf offspring in the old proportion, the dwarfs being all pure, and the tall plants pure and impure. This process, also, continued until the experiments ceased.

$$
{ }^{1} \text { op. cit, p. } 12 .
$$


254. Mendel supposed that the 'tall' and 'dwarf' characters were represented by units in the substance we now call germplasm. He thought that each pollen grain and unfertilized ovule (gamete, or germ-cell) contained one unit for stature, and therefore that each fertilized ovule (zygote) contained two. ${ }^{1}$ One only of these units directed development, and the character which resulted from its influence he termed the 'dominant.' The latent character was termed the ' recessive.'

255. He supposed further that before the development of the germ-cells of the mongrel plant which sprang from the zygote, the units multiplied and separated, so that in each of its pollen grains and ovules only one unit was present, a dominant unit or a recessive unit. When the plant flowered and self-fertilization again took place, it was a matter of chance whether these units paired with their own kind in the zygote, or with units of the opposite type. If a dominant unit met another dominant the result was a 'pure' dominant plant, which never had, nor.could have a recessive descendant so long as self-fertilization continued. In like manner, if a recessive unit met another recessive the line became exclusively recessive. But, if a dominant met a recessive, the resulting plant was an 'impure' dominant, which looked like the pure dominant, but was really different in that it was a hybrid having descendants of the three types, pure dominants, recessives, and impure dominants. Every plant which displayed the recessive character was pure of course, for otherwise the dominant element would have been patent.

256. If the reader will shake together in a bag a hundred black and a hundred white buttons, representing dominant and recessive units respectively, and then withdraw them at hazard in pairs, he will find that, on the average, half the buttons will be paired with their own kind and half with the opposite kind. He will then have approximately twenty-five black pairs representing pure dominants, twenty - five white pairs representing recessives, and fifty black-and-white pairs representing impure dominants. The proportion of pure dominants, recessives, and impure dominants, therefore, is supposed to be regulated by the law of chance.

257. It was found that peas possessed several Mendelian characters besides length of stem, for example colour of cotyledons (yellow or green), colour of seed skins (brownish or white), colour of flowers (purple or white), shape of ripe pods( inflated or constricted), colour of unripe pods (green or yellow), position of flowers (axial or terminal), and shape of seeds (round or wrinkled).

1 See Bateson, Mendel's Principles of Heredity, pp. 338-9. 
One of each of these characters is always dominant over its alternative, for example yellow in the cotyledons is dominant over green, purple in flowers over white, round shape over wrinkled shape in seeds.

258. But, if a variety has one character which is dominant over its alternative in another variety, it does not follow that the rest of its characters are also dominant. One or more characters in the second variety may be dominant. Consequently, if two varieties are crossed, the first mongrel generation (all impure dominants) may exhibit some of the characters of one variety and some of the other.

259. Moreover, if a variety having a certain set of Mendelian characters is crossed with a variety having another set, then the descendants are apt to exhibit the characters in neze combinations even after they have become 'pure.' Thus, if a variety with inflated pods, wrinkled seeds, and green cotyledons be crossed with one having constricted pods, round seeds, and yellow cotyledons, the 'pure' descendants may exhibit every combination of the alternative characters, for example contracted pods with round seeds and yellow cotyledons, or inflated pods with wrinkled seeds and yellow cotyledons. It appears, therefore, that unlike sexual characters, the alternative or Mendelian characters do not necessarily hang together in sets, but are 'inherited' independently of one another. When, therefore, we study heredity in terms of the Mendelian theory, we must think, as always, not of the individual as a whole, but of his characters separately. Obviously, the hypothesis that characters are represented in the germ-plasm by units, which pair in the fertilized ovum but separate in its germinal descendants, is an inference, not an observed fact. These units cannot be seen in the germ-plasm, or, if seen, recognized. It is this inference which is especially the Mendelian hypothesis and on which stress is laid. ${ }^{1}$

260. The points to be especially noted when studying the Mendelian theory are (I) the alternative units are supposed not to blend or unite in the fertilized ovum (zygote) with their 'opposite numbers,' but to remain distinct and ultimately to segregate, so that the germ-cells (gametes) of the individual that arises from the zygote contain each only one unit for each character; hence the terms ' unit segregation' and 'gametic purity.' (2) The different

1 "This purity of the germ-cells and their inability to transmit both of the antagonistic characters is the central fact proved by Mendel's work. We thus reach the conception of unit characters existing in antagonistic pairs." (First Report to the Evolution Committee of the Royal Society, by Miss Sanders and Mr Bateson, p. I26, 1902.) 
units of a set are supposed not to hang together but to be inherited independently of one another so that new combinations may be formed. They have been termed 'allelomorphs,' alternative inheritable units, each of which in one set of allelomorphs is capable of displacing its opposite number in another set. (3) One unit of every pair tends to be dominant over its alternative, so that when both are present in a zygote the individual which springs from it displays the dominant character only. Such, at any rate, were the chief points of the Mendelian theory as originally propounded, and as held very recently.

26I. More extended study, however, has rendered it evident that in all points the Mendelian doctrine needs more or less qualification. Dominance is seldom if ever complete. As regards any Mendelian character, the hybrids of the first generation (all impure dominants) usually resemble the dominant parent more nearly than the recessive; but the influence of the latter may be observed, at least very often. "Even in the pea it is not the case that the heterozygote [mongrel] always shows the dominant allelomorph as clearly and in the same intensity as the pure dominant, and, speaking generally heterozygotes, though in numerous instances readily referable to one or other of the allelomorphic types, exhibit these types in a more or less modified form." 1 Frequently indeed a combination of parental qualities amounting to particulate, mosaic, or even blended inheritance is observable in offspring. Thus a cross between black and white plumage in poultry may result in barred offspring, as often happens when the white Leghorn fowl is crossed with the black Cochin $;^{2}$ or in a minute patchwork, as in the case of the blue Andalusian fowl which results from crossing a black bird with a 'splashed' white; or there may be complete blending, as in the offspring of a white man and negress. ${ }^{3}$

262. Sometimes the usually recessive character may be dominant. ${ }^{4}$ Again, instead of one type being dominant over the other, the character of neither parent is reproduced, but a common ancestral form reappears. For example, when albino mice are crossed with black-and-white Japanese waltzing mice "a grey

1 First Report to Evolution Committee, p. 129.

2 Davenport, Inheritance in Poultry, p. 40.

3 Adherents of the Mendelian doctrine sometimes maintain that blended inheritance is nothing other than a fine mosaic. Doubtless they are right; but I imagine their opponents mean nothing more by blended inheritance than that it is a very fine mosaic-a mosaic as fine, perhaps, as the mixture of colours in a painter's brush. All blending, for example a mixture of alcohol and water, is a mosaic, even if one of chemical molecules.

4 First Report to Evolution Committee, p. 120. 
house-mouse resembling in size, colour, and wildness the wild house-mouse" 1 is produced; when yellow-grey Belgian rabbits are crossed with white (albino) Angoras the offspring have "wild-grey coats indistinguishable from those of the wild-grey." 2 When $\mathrm{Mr}$ Hurst crossed Black Hamburgh with Buff Cochin poultry, "in the first plumage the two sexes are quite distinct. The cockerels had golden-brown hackles, red-brown saddles, black and brown tails and wings, and buff-brown breasts regularly spangled with black. The pullets were as dull and sober in colour as the cockerels were brilliant, being black intermixed with light and dark brown, with darker tails and heads and spangled breasts. It seems rather curious that a cross between two breeds, each of which has normally similar plumage in both sexes, should produce offspring in which the two sexes are so distinctly differentiated." 3 Among domesticated pigeons the plumage of the wild blue-rock, and among poultry that of the ancestral Gallus bankiva may reappear when domesticated breeds are crossed. When the horse is crossed with the Burchell zebra, which has a few broad stripes, the offspring present the narrower and more numerous stripes of a remote ancestor. ${ }^{4}$ Similar instances of 'reversion' are common amongst plants ; for example, if a jonquil be crossed with a daffodil, Narcissus odorus results. ${ }^{5}$

263. Dominance may be influenced by the environment, as in the case of Ewart's pigeons, whose offspring reproduced the characters of the English parent when the Indian parent was ill, but those of the latter when its health was good. ${ }^{6}$

264. Dominance may be affected by the sex of the parent. Thus, when white Leghorn and Indian Game poultry are crossed, "all male cross-breds and female cross-breds from Indian Game mother almost always have the ground colour white, but female cross-breds from white Leghorn mothers have the ground colour more or less dingy-brownish white." 7 "Booting (feathered foot) is always dominant when the feathered form is the mother, no matter what the race." 8 "The canary has two forms which are both in a sense albino, the yellow and the cinnamon. When green (viz. non-

\footnotetext{
${ }^{1}$ First Report to Evolution Committee, p. 145.

2 C. C. Hurst, Experimental Studies on Heredity in Rabbits, p. 301.

${ }^{3}$ Second Report to Evolution Committee, p. 135 .

4 Ewart, The Penicuick Experiments, p. 92.

${ }^{5}$ Vernon, Variations in Animals and Plants, p. I69.

${ }^{6}$ See $\S 132$.

7 First Report of Evolution Committee, p. 99.

${ }^{8}$ Davenport, Inheritance in Poultry, p. 86.
} 
albino) females are crossed with cinnamon males, both greens and cinnamons are invariably female. When cinnamon females are crossed with green males the young are always green." 1 The instance "most familiar amongst animals is perhaps that of the Mule (Mare and Jackass) and the Hinny (She-ass and Stallion) and amongst plants the hybrids of Digitalis." 2

265. Dominance may be influenced by race. "While long tail and crest feathers are dominant in poultry, long hair (equally due to the prolonged life of the follicle) is recessive in mammals (Castle, I903, 1905; pp. 64-7, 73-4; Hurst, 1904). White is usually recessive to pigment in flowers and mammals, but is usually dominant to pigment in poultry." 3 "Yellow (beak and foot colour) of the white Leghorn dominates over black of the Minorca, but yellow of the dark Brahma is dominated by the Minorca." 4

266. Dominance may be affected by the idiosyncrasies of the parent. "In all cases as yet investigated (in poultry) individuals differ greatly in the results they give, and we can only suppose that these differences represent different proportions in the number of effective dominant and recessive germs which they produce." 5

267. Lastly, in some cases it is difficult to say which of a pair of characters is dominant, since both are patent in about equal numbers in the first mongrel generation, as in the case of a sixth digit in man or a fifth toe in poultry. Even when a character is usually strongly dominant over its alternative, recessive individuals sometimes appear in the first mongrel generation. ${ }^{6}$

268. To sum up: it is very evident that "Dominance is a matter of degree, not of kind;" " Dominance is a phenomenon presenting various degrees of intensity." 8

269. We have next to consider the hypothesis of gametic purity -a hypothesis which implies that the pure dominants and recessives which spring from impure dominants of the first and succeeding mongrel generations, extracted pure dominants and recessives as they are called, inherit only one kind of unit out of each pair of alternative units, and therefore (as regards each separate character) are in effect derived from only one of the crossed ancestral lines.

${ }^{1}$ Second Report to Evolution Committee, p. 128.

${ }^{2}$ First Report to Evolution Committee, p. I 32.

${ }^{3}$ Davenport, Inheritance in Poultry, p. 132.

4 Ibid., p. 87.

5 First Report to Evolution Committee, p. 106.

- First Report to Evolution Committee, pp. I I 9 et seq.

7 Inheritance in Poultry, p. 85 .

${ }^{8}$ First Report to Evolution Committee, p. 126. 
In order to understand the ensuing discussion with ease the reader is advised to familiarize himself with the following : (I) According to the Mendelian hypothesis from a cross-bred individual, if selffertilized or mated with another impure dominant, should arise pure dominants, recessives, and impure dominants in the average proportion of one pure dominant and one recessive to two impure dominants, this proportion being due to the circumstance that the dominant and recessive units should be present in the germ-cells of the cross-bred in about equal numbers. If the three kinds of offspring depart much from the stated proportions, it is evident that something besides pure chance is at work. The impure dominants of the next and succeeding generations should behave in a similar manner. (2) Since an impure dominant produces an equal number of dominant and recessive gametes, therefore, if such an individual of any generation be crossed with a pure dominant (which produces only dominant gametes), half the offspring, on the average, should be pure, and half impure dominants. If crossed with a recessive half the offspring should be impure dominants and half recessives. (3) Pure extracted dominants, mated together, should produce only pure dominants, extracted recessives only recessives, and extracted recessives crossed with pure extracted dominants only impure dominants in the first cross. (4) With respect to each separate character, then, pure extracted dominants and recessives are not, from the Mendelian point of view, hybrids at all ; they are believed to be gametically pure, having reverted wholly to one or other of the types that were crossed, the other type being quite eliminated from them. They should exhibit, therefore, no tincture of the alternative characters.

270. In all points, however, the doctrine of unit segregation and gametic purity needs qualification. Thus, when Mendel crossed round with wrinkled peas, he obtained in the second mongrel generation-when the proportion of dominants (pure and impure) to recessives should have been as three to one-in one case fortythree dominants to two recessives, and in another fourteen dominants to fifteen recessives; and, when he crossed yellow with green peas, he got in one case twenty dominants to nineteen recessives, and in another thirty-two dominants to one recessive. ${ }^{1}$ In some cases extracted recessives (presumably pure) when crossed with pure recessives gave dominant offspring instead of only recessives. In one such case ninety-four dominants and a single recessive resulted. ${ }^{2}$ In other cases only pure dominants producing only

1 First Report to Evolution Committee, p. 128.

2 Op. cit., p. 79. 
pure dominant offspring resulted in the first hybrid generation. ${ }^{1}$ Millardet's 'false hybrids' are celebrated. He "found that when certain varieties, especially of strawberry, are crossed together, (I) the cross-breds may precisely reproduce the maternal type, without any indication of the paternal characters ; 2 in other cases the cross-bred individuals may show either the maternal characters pure (save in one case the colour of fruits) or the paternal characters pure. Seeds from plants thus exclusively reproducing one parental type themselves gave plants again exclusively of that type. To such forms he gives the name 'faux hybrides' or 'hybrides sans croisement." " 2 Other similar cases might be mentioned. ${ }^{3}$

27I. All these facts are inconsistent with the Mendelian doctrine. For example, if unit segregation and gametic purity are realities, how is it possible (I) that only pure dominants, having only dominant descendants, can occur in the first hybrid generation, or (2) that recessives can ever produce dominant offspring and descendants? We may indeed adopt the far-fetched hypothesis that in the first case recessive units or gametes were not produced, or perished, leaving only dominants to continue the race, and in the second the more probable conjecture that the recessives were in reality impure dominants in which the recessive units had temporarily become dominants.

272. But another explanation not only inherently more probable, but supported by evidence that appears massive and conclusive, is possible. We saw that the development, not inheritance, of the sexual characters is alternative. In every individual, for example man or woman, one of each pair of sexual characters is as a rule entirely dominant over its alternative. The alternative, however, is present in a latent condition, that is every individual is an impure dominant. Thus, in man beardedface is dominant over smooth-face, whereas the latter is dominant in a woman; but the man transmits the latent female character to his daughters and the woman the latent male character to her sons. Here the inheritance, so-called, is alternative in a completer sense than in the case of Mendelian characters ; for each character is in turn dominant over its alternative. But in some cases male characters may become latent for prolonged periods, as in aphides during the summer. Female characters are seemingly, permanently recessive in the ova of the queen bee, for unfertilized eggs produce

1 Op. cit., p. 80.

op. cit., p. I54.

Op. cit., p. 81. See also Bateson, Mendel's Principles of Heredity, chapter xiv. 
only drones; while, presumably, male characters are permanently latent in such species as propagate only by parthenogenesis.

273. Now the presumption is that the inheritance of the Mendelian characters is of the same type as that of the sexual characters, and therefore that, instead of segregation and gametic purity, what really occurs is patency and latency. If that be the case the recessive character, which is temporarily latent in the 'impure' dominant, is permanently latent in a line of 'pure' dominants, whereas, on the other hand, the dominant is permanently latent in the recessives-permanently latent, that is, until the conditions which bring about such latency are disturbed in much the same way as when the conditions of latency of the female characters in the ova of the bee are disturbed by fertilization, or those which bring about the latency of the male characters in the summer ova of aphides are disturbed by the advent of winter. If this be true, the independent inheritance of characters on which Mendelians insist is a myth. There is only independent development, independent patency and latency. When, therefore, one variety is crossed with another and the descendants show the varietal characters in new combinations, all the alternative characters of both varieties are present, but only a half of them are patent, and this half includes characters from both species.

274. Evidence more direct than that furnished by analogy is not lacking. Contrary to what was formerly believed, the 'extracted ' pure dominants and recessives of the second and succeeding generations are rarely without traces of the alternative characters. They are in truth real mongrels (not only as regards the whole individual, but as regards each separate character) in which one or more of the characters of one of the crossed varieties predominates more or less, the degree of this predominance varying from an absolute, or almost absolute completeness that approaches the commonly complete dominance of the sexual characters, to a blend intermediate between the crossed varieties. Thus in the case of poultry, "very frequently, if not always, the character that has once been crossed has been affected by its opposite with which it was mated and whose place it has taken in the hybrid. It may be extracted, therefrom, to use in a new combination, but it will be found altered. This we have seen to be true for almost every character sufficiently studied-for the comb form, the nostril form,

1 I made this suggestion in the second edition of The Principles of Heredity (Appendix B); I find, however, I was anticipated by Professor T. H. Morgan (Science, xxii., 1905). 
cerebral hernia, crest, muff, tail length, vulture hock, foot-feathering, foot-colour, earlobe, and both general and special plumage colour. Everywhere unit characters are changed by hybridizing." 1 Clearly, then, as is sometimes the case with the sexual characters, especially the less stringently selected secondary sexual characters (e.g. in human 'hermaphrodites'), the allelomorph, which is supposed by Mendelians to be absent, very often, indeed almost always makes its presence felt. We must assume, therefore, that before separating-if they do separate-the alternative units-if there are any units-infect one another (i.e. blend) so as to form hybrid units; or else we must assume that they do not blend, but remain together, and that one (but not always the same one) dominates more or less completely over the other. In any case, "construed in the strictest sense the doctrine of gametic purity is untenable." 2

275. Now ample evidence exists that allelomorphs sometimes combine to form compound allelomorphs. When two varieties are crossed, one or other form may dominate, or the type may blend, or the ancestral type may appear. When this, the first mongrel generation, is self-fertilized or bred together, it sometimes happens that there is no return in the offspring to the grandparents (the types that were crossed). On the contrary the descendants break up into two or more forms distinct from the grandparents, one or more of which may breed true. For example, if the Stanley sweet-pea, which has dark maroon petals (standards and wings), be crossed with the Giant White, the offspring are Giant Purple Invincibles with maroon standards and blue wings. "These firstcrosses, self-fertilized, gave Giant White, Giant Purple (without blue wings), Mars (a well-known red variety), Her Majesty (a full magenta, well-known), and a form like Her Majesty, but flaked with white. One plant of each was saved and its self-fertilized seed sown. Mars and Her Majesty came true. The Giant White was tested, and it came true also. The Her Majesty flaked with white, however, gave Whites, Her Majesty, and Her Majesty flaked white again. The Giant Purple gave Giant White, Her Majesty, Giant Purples, and two plants of a streaky cream colour. ... We are then led to the conclusion that the allelomorph transmitting the coloration of Stanley is compound, and that it can be broken up into simpler and possibly component elements." ${ }^{3}$

1 Inheritance in Poultry, p. 80.

2 Castle and Allen, The Heredity of Albinism. Proceedings of the American Academy of Arts and Sciences, vol. xxxviii. No. 21, p. 615.

${ }^{3}$ First Report to Evolution Committee, pp. 142-3. See also Bateson, Mendel's Principles of Heredity,, pp. 60 et seq. 
276. It is admitted, then, that two or more dissimilar allelomorphs sometimes unite permanently to form compound allelomorphs which are transmitted to descendants as units. In the case quoted above, the component characters are, I believe, supposed to have been more or less patent, and therefore the Stanley presented an appearance different from any of the types from which it was obtained by cross-breeding. But, if compound allelomorphs in which each component unit is patent may occur, it is not unreasonable to suppose that allelomorphs, supposing they exist, may sometimes combine to form more or less permanent compounds in which one (or more) of the components is patent and the other (or others) latent. That this has happened at least very frequently there can be no possible doubt. We see it in aphides, in which the allelomorphs for the male characters are latent all the summer. Every case of the reappearance of a latent ancestral character (at any rate in a pure-bred race) ${ }^{1}$ is an instance of it.

277. If we cross a Japanese waltzing mouse with an albino mouse, then the ancestral wild-grey colour reappears in a large proportion of the offspring. It is supposed, doubtless correctly, by Mendelians, that the special characters of the two domestic breeds arose originally as mutations from the wild type. Since it is very improbable that two animals varied discontinuously in the same way and at the same time and place, the mutants (as the individuals that underwent mutation are termed) were crossed with the wild type. $^{2}$ The reproduction was Mendelian, and artificial selection established a race of 'pure' recessives amongst the descendants -Japanese waltzing mice in the one case and albinos in the other. According to the Mendelian doctrine of unit segregation and gametic purity the grey colour should have been quite absent (not latent) from both varieties. ${ }^{3}$ It was only latent, however. It follows that in this case there was no unit segregation nor gametic purity. What really occurred was, not the elimination of the 'grey allelomorph' but the formation of a compound

1 See $\S 280$.

2 “Mendel's discovery, it will be understood, applies only to the manner of transmission of a character already existing. It makes no suggestion as to the manner in which such a character came into existence. The facts, however, leave no room for doubt that at least one character of each pair of simple allelomorphs has arisen discontinuously. The fact that the gametes of the cross transmit each member of the pair pure, is as strong an indication as can be desired of the discontinuity between them." First Report to Evolution Committee, p. I5 I. See §290.

3 See $\$ 255$. 
allelomorph in which the grey was permanently latent. It would appear, therefore, that the essential features of Mendelian inheritance are, not segregration and gametic purity, but the permanent dominance of one alternative character and the permanent latency of the other.

278. Any number of similar instances might be quoted. Thus when $\mathrm{Mr}$ A. D. Darbishire crossed fawn-and-white Japanese waltzing mice with albinos, the descendants exhibited a number of colours which he classified as yellow, fawn colour, grey (pale and dark, the colours of the wild mouse), black, lilac (pale and bluegrey), and chocolate. ${ }^{1}$ In this as in many other instances (e.g. Stanley sweet-pea, quoted above) the allelomorphs from both the crossed varieties were evidently highly compound, several latent characters being revealed. The appearance of the black coloration is particularly interesting. It has appeared also when pigmented guinea-pigs and rabbits were crossed with an albino variety. Thus Mr C. C. Hurst crossed Belgian rabbits which have yellowgrey fur with angoras which are albinos. ${ }^{2}$ Both varieties had been pure-bred for at least eight and probably many more generations. The offspring all displayed "grey coats like that of the common wild rabbit." Bred together the mongrels produced wild greys, albinos, Dutch-marked, and black offspring. The three latter types bred true when mated with their own kind. They were pure recessives. The greys were pure and impure dominants, with albino, black, or Dutch markings as the latent element. There was never a return to the yellow-grey Belgian coloration. A further series of experiments proved that the black coloration as well as the grey and Dutch markings had been introduced by the various albinos of the original cross. Now albinism is a typical recessive character which, according to the doctrine of unit segregration, should always be pure. Yet we see that in mice, rabbits, and guinea-pigs it may be associated with latent grey, black, or Dutch coloration. Plants furnish evidence similar in kind, and even greater in quantity. Thus if two whiteflowered varieties of garden plants be crossed the offspring may have coloured flowers.

279. The reproduction of ancestral traits seems to afford evidence that is decisive in favour of the hypothesis of alternate

1 "On the Result of Crossing Japanese Waltzing with Albino Mice." Biometrika, Jan. I904.

"Experimental Studies on Heredity." Linnean Society's Journal, Zoology, vol. xxix. 
latency and patency, and fatal to the hypothesis of segregation. Nevertheless, Professor Cuénot has attempted to surmount the difficulty. ${ }^{1}$ He supposes that colour depends, not on a single unit or hereditary tendency, but on two, a colour factor and a colour determiner. According to him, if the one or the other be absent, the individual is an albino. As a fact, it is the factor that is supposed to be absent from the albino, which, it appears, usually carries one determiner and may carry more. According to the latest views the factor is always the same for all the colours of a species, but the determiners (which Bateson has surmised are ferments ${ }^{2}$ ) may be as many as there are differently coloured varieties. $^{3} \quad$ Colours (i.e. ferments) vary in dominance, the ancestral colour, for example 'wild-grey' in rabbits and mice, often being the most dominant of all. Consequently, if an albino rabbit or mouse be crossed with some coloured domestic variety (e.g. Belgian rabbit or Japanese waltzing mouse), then, since the albino races seem always to carry the grey determiner, and the coloured variety carries the factor, the offspring are all grey. If more determiners are carried by the albino, the descendants are not only grey and white, but other colours as well. In this way the reappearance of ancestral traits when varieties are crossed is accounted for in terms, not of latency, but of segregation.

280. Cuénot's hypothesis of factors and determiners has been used to interpret, not only the reproduction of ancestral colours but also that of all other ancestral peculiarities, for example shape and instinct. Presumably these, also, are due to ferments. But, since both Cuénot's hypothesis and the hypothesis of segregation, which it is intended to support, are merely founded on, not tested by experiment, the repetition of the explanation in any number of instances does not prove them to be true. Apparently, however, mere repetition is all their adherents consider necessary. The facts fit the hypothesis of simple latency and patency at least equally well. Indeed they fit the latter better; for, if we hold it, we have no exceptions, such as those I have summarized in paragraphs $27 \mathrm{I}$ and 284 , to explain away. Here then we have two rival hypotheses, two interpretations of the same facts. Both of them remain simple guesses, utterly valueless to science, unless they can be tested. "In such a case it is necessary to look for some instance which can be

1 La loi de Mendel et l'hérédrté de la pigmentation chez les souris (2me note). Arch. de Zool., exp. et gén (4), Notes et revue, pp. xxxiii.-xli.

2 Mendel's Principles of Heredity, pp. 226-9.

${ }^{3}$ The Progress of Genetics since the Rediscovery of Mendel's Papers, by W. Bateson. 
explained on one only of these rival hypotheses; . . . such a test case is called a Crucial Instance." 1 "One single circumstance, which admits of one explanation only, is more decisive than a hundred others which agree in all points with one's own hypothesis, but are equally well explained on an opposite hypothesis." 2 Now, as regards the rival hypotheses of segregation and latency, any number of cases have been recorded which furnish quite decisive crucial instances; but, though the experimental school has piled one untested interpretation on another, though they have explained alternative reproduction by segregation, segregation by factors and determiners, and determiners by ferments, never yet has any appeal been made to them. Ancestral traits may be awakened experimentally by crossing domesticated varieties; but these are not the only occasions when they appear. Their reproduction by pure-bred individuals is far from uncommon. "Thus we see that, in purely bred races [of pigeons] of every kind known in Europe, blue birds occasionally appear having all the marks which characterize $C$. livia." 3 The following is a very striking instance of the reappearance of long latent ancestral characters in an individual which not only reproduced them, but had previously reproduced those of her own variety as well. Several like instances have been recorded. "Mr Hewitt possessed an excellent Sebright gold-laced bantam hen, which, as she became old, grew diseased in her ovaria, and assumed male characters. In this breed the males resemble females in all respects except in their combs, wattles, spurs, and instincts; hence it might have been expected that the diseased hen would have assumed only those masculine characters which are proper to the breed; but she acquired, in addition, wellarched tail sickle-feathers quite a foot in length, saddle-feathers on the loins, and hackles on the neck-ornaments which, as $\mathrm{Mr}$ Hewitt remarks, 'would be held as abominable in this breed.' The Sebright bantam is known to have originated about the year 1800 from a cross between a common bantam and a Polish fowl, recrossed by a hen-tailed bantam, and carefully selected; hence there can hardly be a doubt that the sickle feathers and hackles which appeared in the old hen were derived from the Polish fowl or common bantam; and we thus see that not only certain masculine characters proper to the Sebright bantam, but other masculine characters derived from the first progenitors of the breed, removed

1 Welton, Manual of Logic, vol. ii. p. 102.

Ueberweg, Logic, Eng. Trans., p. 5 I 3.

3 Animals and Plants, vol. i. p. 206. 
by a period of above sixty years, were lying latent in this hen-bird, ready to be evolved as soon as her ovaria became diseased." 1 Here there can have occurred no reunion of previously divorced factors and determiners. The only conceivable interpretation of the facts is that the ancestral characters were latent: and if they are latent in the pure-bred varieties, we have no right to assume that they segregate in the mongrel. "Entia non sunt multiplicanda prater necessitatem."

281. Very obviously Cuénot and his followers have not taken all the evidence into account. His hypothesis merely complicates the problem and confuses the issues by introducing the entirely gratuitous assumption that the production of characters depends, not on a single unit or hereditary tendency in the germ-plasm, but on two, a factor and a determiner. It must be understood that there is no real evidence in favour of this hypothesis. It was invented only because without some such supposition the reproduction of ancestral traits rendered the hypothesis of segregation untenable. It is unnecessary, therefore, to consider what have been euphoniously termed the 'later refinements' of the Mendelian hypothesis, such as negative 'elements' in which apparently 'absence' constitutes the alternative allelomorph. As I say the reproduction of ancestral traits by pure-bred races constitutes decisive proof that the Mendelian phenomena are due to alternate patency and latency, not to segregation.

282. De Vries offers yet another explanation of Mendelian phenomena. $^{2}$ According to him a new species differs from the parent stock by the addition of a fresh progressive character (a mutation) to the sum-total of ancestral characters. A new variety, on the other hand, differs from the parent stock in that it has latent or patent a character which is in the opposite condition in the parent stock. When, therefore, species cross they tend to be more or less sterile, for the character is unpaired; or if the union is fertile there tends to be more or less of blending. When varieties cross with the parent type the patent character is dominant over the latent character, and there is Mendelian inheritance, with segregation of patent and latent units in succeeding generations. But, if this theory be correct, the albino rabbit must have been latent in the yellow-grey, the yellow-grey in the albino, and the black, wild-grey, and Dutch colorations in both. The ancestors of both races, therefore, must have been all these colours in turn

1 Animals and Plants, vol. ii. p. 29.

- Species and Varieties. Their Origin by Mutation. Lectures IX and X. 
at one time or another, which is very improbable. Again, since the inheritance of polydactylism in man and the lower animals is alternative, Professor de Vries' theory involves the corollary that mammals have descended from ancestors which possessed six digits, one of which has become latent, which also, to say the least, is very improbable.

283. Taking all the facts into consideration, I think there can be no doubt that reproduction of at least many mutations is alternative from the first. The only matter open to doubt (and even that, as we see, is not open) is whether the alternation involves alternative inheritance (unit segregation and gametic purity) or merely alternative development (alternative patency and latency). Great stress is often laid on the frequency with which alternative characters are reproduced in the second mongrel generation in what are known as the Mendelian proportions. Thus, if round and wrinkled peas are crossed, the average proportions in the second mongrel generation are three round peas to one wrinkled; if rose comb is crossed with pea comb, the proportions are nine walnut combs, three rose, three pea, and one single. These proportions are certainly very interesting and remarkable. Beyond doubt they suggest segregation rather than patency and latency. But suggestion is not the same thing as demonstration. The latter must depend on surer evidence, such as that furnished by purebred varieties. Here, as I say, we have positive testimony that the Mendelian phenomena do not indicate segregation.

284. To sum up: we have a choice between two possible explanations of Mendelian phenomena. On the one hand is the orthodox Mendelian doctrine of dominance (patency and latency) in the first hybrid generation, and segregation and gametic purity in the pure dominants and recessives of succeeding generations; on the other hand is the theory of temporary patency and latency in the first generation and more perfect and permanent patency and latency in the pure dominants and recessives of succeeding generations. I am not aware of any fact in the whole range of Mendelian literature that contradicts the second theory. But the first is contradicted by the facts (I) that pure dominants and recessives may occur in the first hybrid generation; (2) that apparently pure dominant races may produce recessives, and recessive races dominants; (3) that dominants and recessives may occur in altogether disproportionate numbers in any generation succeeding the first; (4) that extracted dominants and recessives nearly always bear traces of their hybrid origin (i.e. display some degree of 
patency of the latent allelomorph); and (5) that in a very great number of cases (i.e. in pure-bred varieties) it has been actually proved that pure dominants and recessives carry the alternative unit in a latent condition. Moreover, the analogy of the sexual characters points clearly to latency rather than to segregation. 


\section{CHAPTER VIII}

\section{THE MUTATION THEORY}

The nature of mutations-The impossibility of distinguishing between fluctuations and mutations by means of experiment-The alleged immutability of mutations-Our power of studying smaller variations-Human inter-varietal crossings-Latent characters are common when domestic varieties cross ; they are rare when natural varieties cross-The effects of Natural and Artificial selection are not identical-Objections to the mutation theory-The distinction between inheritance and reproduction-The modes in which mutations are inherited and reproduced.

285. TT THETHER due to unit segregation and gametic purity, or to patency and latency of the components of compound allelomorphs, there can be no doubt of the actual occurrence of the Mendelian phenomena. We must endeavour, therefore, to estimate the part played by them in nature. The more extreme section of the experimental school insists that all those differences (even minute differences) between parents and children which we have defined as variations (innate or germinal differences, differences in hereditary tendencies) are mutations, that the inheritance of all mutations is Mendelian, and that mutations are permanent changes which "selection alone can eliminate," whereas 'continuous,' ' normal,' or 'fluctuating 'variations are mere modifications due to changes in the action of the environment. Consequently, according to them, all evolution, at any rate all permanent and considerable evolution, is founded solely on mutations. It follows if this hypothesis be true, that the stability of racial characters is due, not to their long-continued selection, but to the circumstance that they have arisen through the accumulation of mutations, all of which were stable from the first. ${ }^{1}$

1 " Doubtless some of the so-called fluctuations are in reality small mutations, whilst others are due to environmental influence. The difficulty of distinguishing between the two is very great. The simultaneous existence of small mutations and large fluctuations leads to the disguising of the former by the latter. Only careful and laborious analysis will avail us here, and such analysis is precisely what is at present lacking. The position is roughly as follows. Of the inheritance of mutations there is no doubt. Of the transmission of fluctuations there is no very strong evidence. It is therefore reasonable to regard the mutation as the main, if not the only basis of evolution. And the great service which Mendel has 
286. The word mutation is one of the loose, ill-defined terms so common in biology and the bane of it. Thus, to Mr Punnett it implies a variation, large or small, which is stable because the inheritance of it is Mendelian. To Professor de Vries it implies a 'sport,' 1 a change " of wide amplitude," 2 the inheritance of which

rendered to this branch of philosophy is the demonstration of the fact that the mutation when once it has arisen is not likely to be swamped by interbreeding with the normal form, provided that it is not injurious to the species. We now recognize discontinuity in inheritance as well as in variation. The new character which arises as a mutation has its representative in the gamete. Once it has arisen selection alone can eliminate it. Mendel's discovery then has led us to materially alter our ideas of the evolutionary process. The small fluctuating variations are not the materials on which selection works. Such fluctuations are often due to conditions of the environment, to nutrition, correlation of organs, and the like. There is no indisputable evidence that they can be worked up and fixed as a specific character" (Mr R. C. Punnett, Mendelism, ed. ii., pp. 72-3). "Modification of characters by selection, when sharply alternative conditions (i.e. mutations) are not present in the stock, is an exceedingly difficult and slow process, and its results of questionable permanency. Even in so-called 'improved' breeds, which are supposed to have been produced by this process, it is more probable that the result obtained represents the summation of a series of mutations rather than that of ordinary fluctuating variations. For mutations are permanent; variations are transitory. A moment's reflection will indicate the probable reason. Variations which are distributed symmetrically about a modal condition, so as to produce when graphically expressed a frequency of error curve, represent the result of a number of causes acting independently of each other. These causes are principally external, consisting in varying conditions of food supply, temperature, density, moisture, light, etc. These conditions alter from generation to generation, and so do effects dependent upon them. Mutations, on the other hand, have an internal origin, in the hereditary substance itself. They are relatively independent of the environment, being affected only by such causes as affect the nature of the hereditary substance itself, one of which apparently is cross breeding" (Professor M. E. Castle, "The Mutation Theory of Organic Evolution, from the Standpoint of Animal Breeding." Science, April 7, 1905). "Lop-eared rabbits have ears two or three times as long and as wide as those of ordinary rabbits. A cross between lop-eared rabbits and ordinary ones produces offspring with ears of intermediate size, which sometimes stand erect and sometimes lop. The ear characters which were so distinct in the parents have in this case lost their identity in the offspring, and apparently cannot be recovered again in the original condition, for the offspring transmit to their young the blended character rather than the extreme conditions found in their respective parents" (Castle, "Recent Discoveries in Heredity." Popular Science Monthly, pp. 194-5). Professor Castle refutes himself in a very interesting way. Probably no one will deny that the lop-eared condition has an "internal origin in the hereditary substance itself." Certainly it is quite permanent in the race and has no appearance of depending on "varying conditions of food-supply, temperature, density, moisture, light," etc. But it blends with the ordinary ear. Therefore it cannot have resulted from a mutation the inheritance of which was Mendelian; for if it blends now, it must have blended formerly. Therefore it must have resulted from the selection of fluctuations the inheritance of which was blended.

${ }^{1}$ Species and Varieties, p. 191.

Op. cit., p. 7I5. 
is not alternative, which tends to be stable because it involves the acquisition of a new unit ${ }^{1}$ by the germ-plasm, but which is necessarily stable only when the individual possessing it mates with a similar individual. ${ }^{2}$

287. A little thought renders it evident that none of the problems raised by the Mendelian and Mutation hypotheses can be solved on the data furnished by experiment alone. Experimental evidence lacks minuteness and precision. It is, besides, far too fragmentary. Thus experiments which cover only a few generations are absurdly inadequate to decide the questions whether or not mutations are permanent and whether evolution can or cannot be founded on the continued selection of fluctuations. If, however, we turn to the more ample and conclusive evidence afforded by the life-history of species, we see at once that of the two deductions that evolution is founded on mutations and that mutations are permanent, one must be wrong, for, with mere cessation of selection, every character (e.g. eyes and limbs) tends to retrogress till it disappears. The statement that mutations are immutable is a very good example of the mistaken judgments which tend to follow too exclusive a reliance on the meagre results obtainable by a single method of inquiry. The observer is constantly under the temptation to draw inferences larger than are warranted by the scanty data at his command. It is interesting to note that, since mutations may be small, what Darwin and his followers call variations (changes "which have an internal origin in the hereditary substance itself"), some members of the experimental school term mutations; and what the former call acquirements (due to "varying conditions of food-supply, temperature, density, moisture, light," etc.), the latter term variations. Except that we are told that mutations do not blend or retrogress, nothing more is done than to change the names and conduct the argument on that basis.

288. Again, the question as to whether the larger variations (i.e. the mutations of de Vries) are more permanent than smaller variations (i.e. fluctuations and the small mutations of Punnett), and whether the inheritance of small mutations tends to be alternative, cannot be decided by the mere experimental observer. He has no special knowledge of the lesser variations, for the study

1 "We may assume that these units are represented in the hereditary substance of the cell-nucleus by definite bodies of too small a size to be seen, but constituting together the chromosomes." Op. cit., p. 306.

op. cit., Lecture IX. 
of which his method is unsuited. The whole literature of experimental inquiry may be ransacked and not a single observation on the smaller variations will be found. "For almost always the points which tell are too fine to be dealt with in our analysis." 1 Probably domesticated poultry, owing to the number and diversity of the varieties and the ease with which they may be bred, and the offspring compared with the parents and with one another, have been the subject of more exhaustive experimental study than any other type. The method of study in this case, as in all others, has always been to cross the more dissimilar varieties-a rumpless fowl with one possessing a rump, a long-tailed fowl with a shorttailed one, a white individual with a black, and so on. The following is a fairly complete list of the characters studied :-

Comb form.

Nostril form.

Cerebral hernia.

Crest.

Muff.

Beard.

Frizzling.
Uropygium.

Tail length.

Vulture hock.

Booting.

Extra toe.

Colour of mandible and foot.

Iris colour.
Ear-lobe colour.

General plumage colour.

Colour of hackles. Wing bars.

Shafting.

Body lacing.

Pencilling. ${ }^{2}$

Obviously we do not study the smaller variations, the 'fluctuations,' when we cross any one of these characters with its opposite. They are great differences which have arisen as sports or been established by generations of careful selection, not those small variations which commonly distinguish mating individuals when the breeding is intra-varietal.

289. Our power of observing small differences is proportionate to our familiarity with the object of study. Thus the shepherd is able to distinguish between the individuals of his flock and even to note a good deal when he sees a strange flock; but the ordinary observer can hardly distinguish one variety of sheep from another unless they display some glaring differences of size, shape, or colour. To the Englishman landing in China the natives seem as much alike as peas-as in fact they are. He becomes trained in time to distinguish Chinamen, but not peas. But from earliest infancy we are forced to observe very closely the human beings among whom we are reared. Indeed it is a main business of our lives to do so.

1 Bateson, Mendel's Principles of Heredity, p. vii.

${ }^{2}$ Quoted from Inheritance in Poultry, by Mr C. B. Davenport, p. 8I. 
So acute does our observation become that when we have once seen and noted a face we are usually able to distinguish it again even after the passage of months or years. Here our power of discrimination far surpasses our power of expression in words. Nevertheless, so confusing is the effect of modifications and spontaneous variations that, even in the members of our own families, we are unable to note clearly the effect produced by the crossing of parental differences, though these are often considerable. No experimental observer, no matter to what an extent a recluse and a student, is as familiar with any type of animal or plant as he is with human beings, for, at least, he is always more intimate with himself, a human being, than with anything else. Much less, therefore, is his power of observing the inheritance of small differences in other types. When, therefore, relying on experimental evidence, he states that the inheritance of the smaller mutations, like that of 'sports,' is alternative, or when he declares that all true variations are mutations, and therefore extremely stable, he is guessing. $\mathrm{He}$ has, as I say, no data on which to found a judgment. Unless we, also, are content merely to guess, we must test his hypotheses by the ordinary scientific procedure of making a rigorous deductive inference of consequences followed by an appeal to nature, to ascertain whether these consequences accord with reality. The reader must bear in mind that the experimental evidence is not neglected when more is added to it, and the thinking not the less likely to be correct because we use our best endeavours to ensure its accuracy. I do not think it will be possible to contest a single fact of this added evidence which I shall adduce; and, if it is incontestible, then, as far as I am able to judge, the conclusions we shall reach follow as necessary consequences.

290. When we cross two varieties of fowls or any other domestic animals or plants, then, as regards the differences we are able to note-the larger differences-the development is often alternative. There is usually some blending observable both in the first and in the succeeding hybrid generations; but it is not the conspicuous feature. The conspicuous feature is alternative reproduction; the resemblance to one parent type is much closer than to the other; there is seldom such blending that both the opposing parental characters are equally, or nearly equally represented. Now races of men differ among themselves in size, shape, colour, and so on, almost as much as do varieties of poultry. If human varieties cross, and almost every human variety has crossed repeatedly with almost every other, the inheritance of 
parental differences is never alternative. As regards a few features-I can think only of eye-colour-the characters of a dark race may dominate over those of a light-coloured one; but the dominance is permanent; it is transmitted to all the members of all succeeding generations that breed together. Therefore the reproduction is not Mendelian. But as regards the great majority of characters the blending is perfect and often nearly equal, and as such is transmitted to the latest descendants. A race of permanent and patent mongrels is formed. If three or four races are crossed together, as has happened in South America, each contributes its quota in perpetuity to the blend. It is a common belief amongst experimental workers that "a fusion of characters is rather a rare phenomenon. Human skin-colour is the one striking case." But really that is very wrong. Fusion is very much the general rule, not the exception. ${ }^{1}$ The fact that the gametes of the cross blend "is as strong an indication as can be desired of the "continuity' between them." 2 When the negro, for example, crosses with a white race, the offspring, the mulatto, is conspicuously a blend in all except eye-colour. His hair, it is true, more closely resembles in texture the African than the Caucasian

1 Strange as it may appear to people who have travelled in Asia, Africa, America, Australasia, or Polynesia, experimental workers, or some of them, express doubts as to whether human races really blend. "It would be extremely interesting to students of genetics to learn upon what evidence Dr Archdall Reid bases his positive statement that there is no segregation in the case of the Mulatto" (Mr R. H. Lock, Nature, Oct. I7th I907; see also Bateson, Mendel's Principles of Heredity, pp. 208-9). "The evidence on which I base my assertion that there is no segregation in the mulatto is that of my own eyes. Mulattos vary amongst themselves, but the blend is usually very obvious and is reproduced in subsequent generations when the breeding is inter se. With every infusion of European blood the negro type-skin colour, hair texture, shape of features, and the like-grows fainter until at length the ' touch of the tar brush' is hardly, if at all, perceptible; and this blending, as far as I am aware, occurs not only in all crossed human varieties, but in other natural varieties as well. There may be exceptions, in fact I believe there are; but blending appears to be the rule in the vast majority of instances" (G. Archdall Reid, Nature, Oct. 3ist 1907). "If Dr Archdall Reid will produce authenticated pedigrees showing the repeated crossing of the mulatto with pure white blood and pure black blood respectively, together with a detailed account of all the offspring produced, he will make a very substantial contribution to our knowledge of heredity in the human race, and one which will be examined with very great interest by Mendelians" (Mr R. H. Lock, Nature, Nov. I4th). "Since Mr Lock is probably the only human being who doubts the blending of the black and white races in Mulattos and their descendants, it would be well if he, rather than I, undertook the collection of pedigrees. He would feel himself on the track of a great discovery which would enlighten even Mulattos, whereas I should feel I was wasting time" (G. Archdall Reid, Nature, Nov. 2 Ist).

¿ See footnote, § 277 . 
type, but proof of blending is furnished by the fact that continued infusion of white blood in succeeding generations results in a gradual change of type till at last the hair loses all traces of woolliness. In these later generations also the colour of the eye may suddenly be changed to a lighter hue. This last fact affords another crucial instance which demonstrates that Mendelian inheritance implies, not segregation, but latency. The inheritance of eye-colour is alternative in ordinary intra-varietal breeding. But when a white race is crossed with a black the eye-colour of the former becomes permanently latent, or, at least, as long as the mongrels breed together. But the latency is weakened by successive infusions of white blood, till at length the descendants may have blue or hazel eyes. If there were segregation of units accompanied by the destruction or the non-reproduction of those for light colour, the latter could never reappear, no matter how often the infusion of white blood were repeated.

29r. In addition to manifesting nearly complete general blending, human races when crossed differ from crossed varieties of domestic plants and animals in another, an equally remarkable, and a very important way. Never in human crossings is a latent ancestral character brought to light, whereas the reappearance of such characters, dormant perhaps for many generations, is one of the most conspicuous results of the crossing of domestic varieties. Now what is the significance of these very remarkable differences of inheritance? How is it that in the one case blended inheritance is the rule, whilst in the other alternative reproduction combined with a frequent development of hitherto latent characters is general? If we are able to unvavel this mystery, I think we shall be able to solve those problems of heredity which, of recent years, have especially occupied the attention of experimental workers, and indeed of most biologists.

292. The majority of experimental observers claim that the inheritance of mutations is alternative. If we substitute 'development' for 'inheritance,' the evidence that they are right seems clear, at least as regards many mutations. In man occur polydactylism, hypophylangia, alkaptonuria (a dark-coloured condition of the urine), hæmophilia, colour-blindness, deaf-mutism, and other large variations, ${ }^{1}$ all of which may be classed as mutations, and the development of which is alternative. Again, the majority of experimental observers declare that a mutation is properly defined as a large discontinuous variation, one which is not 
connected by intermediate links with smaller variations. Doubtless in this also they are right. The mutations which have been discovered in man all display this peculiarity. Yet again they claim that domestic varieties of plants and animals have arisen, not through the fixation of fluctuating variations by continued selection, but through the artificial selection of mutations. Here, also, there is good reason to believe that they are right, at least in large measure. Breeders of plants and animals are no more able than experimental workers to distinguish the smaller differences between mating individuals. Moreover, they are in haste to get good results. Of necessity they have selected large mutations rather than small fluctuations. ${ }^{1}$ This, however, has not always been the case. For example, when evolving speed in the American trotting horse, a favourable mutation, involving as it would a co-adaptive change in a thousand structures-that is a thousand co-ordinated mutations-would be an exceedingly rare, indeed, an almost impossible occurrence. Therefore the breeder must depend on fluctuations, which in this case, though imperceptible to the eye, can be measured by the test of speed. It is admitted that the inheritance of fluctuations is blended, and that, as regards them, offspring display a strong tendency to return to the ancestral type. For this reason, according to Mendelians, do trotting horses tend to retrogress unless carefully selected, and when crossed with inferior types to transmit blended characters to descendants.

293. Experiments in crossing have been limited in great measure to domestic plants and animals. Wild types are less accessible, more difficult to manipulate, and often more or less sterile when crossed. When, however, the cross is fertile, the hybrid, like man, usually blends the parental characters and transmits the blend to its descendants. In hardly a single instance has the crossing of natural varieties revealed a latent ancestral character. ${ }^{2}$ Darwin noted long ago that "Gärtner further states that reversions rarely occur with hybrid plants raised from species which have not long been cultivated, whilst, with those which have

1 " He [Man] often begins his selection by some half-monstrous form, or at least by some modification prominent enough to catch the eye or to be plainly useful to him." Origin of Species, 6th ed., p. 60.

${ }^{2}$ I know of only one to which my attention was called by Sir William ThiseltonDyer. When Kalanchee flammea was crossed at Kew with $K$. Bentii, the hybrid displayed characters which can hardly have been other than ancestral traits which have remained latent in one or other species (see Morphological Notes, by William Thiselton-Dyer, K.C.M.G., F.R.S., Annals of Botany, vol. xvii. No. lxvi., March I903, pp. 435-4I. 
been long cultivated, they are of frequent occurrence. This conclusion explains a curious discrepancy: Max Wichura, who worked exclusively on willows which had not been subjected to culture, never saw an instance of reversion; and he goes so far as to suspect the careful Gärtner had not sufficiently protected his hybrids from the pollen of the parent species. Naudin, on the other hand, who chiefly experimented on cucurbitaceous and other cultivated plants, insists more strenuously than any other author on the tendency to reversion in all hybrids. The conclusion that the condition of the parent-species, as affected by culture, is one of the proximate causes leading to reversion, agrees well with the converse case of domesticated animals and cultivated plants being liable to reversion when they become feral; for in both cases the organization or constitution must be disturbed, though in a very different way." 1

294. We are driven then to the conclusion that Natural and Artificial selection are essentially unlike. Nature and man do not select the same class of characters. Man, perforce, chooses mutations. Nature, with finer powers of discrimination and with unlimited time and material at her disposal, chooses, perhaps with very rare exceptions, fluctuating variations. In no other way is it possible to account for the striking differences revealed when natural and artificial varieties are crossed. It is known that man tends to select mutations, and that the reproduction of mutations tends to be alternative. It is clear that when reproduction is alternative, characters tend to become latent, and when latent to become patent in cross-breeding. If, then, as experimental workers suppose, nature choosesmutations, how does it happen that naturalvarieties, when crossed, not only blend very often, but hardly ever reveal latent characters ? ${ }^{2}$

295. The fact that natural varieties often blend their characters

1 Animals and Plants, vol. ii. pp. 24-5.

2 Since latent characters are in effect found only in domesticated varieties, it follows that Darwin, who drew the materials on which his conclusion was founded wholly from artificial varieties, formulated too wide an inference when he declared that, "besides the visible changes which it [the germ] undergoes, we must believe that it is crowded with invisible characters, proper to both sexes, and to both the right and left side of the body, and to a long line of male and female ancestors separated by hundreds or even thousands of generations from the present time ; and that these characters, like those written on paper with invisible ink, lie ready to be evolved whenever the organization is disturbed by certain known or unknown conditions" (Animals and Plants, vol. ii. pp. 35-6). It follows, also, that Professor de Vries is mistaken in defining a variety as a type which has patent or latent a character which is in the opposite condition in the parent species. A type which carries a latent character is always a mongrel ; for it is only when varieties possessing alternative characters cross, that latency occurs. Thus, all sexually dimorphic 
when crossed is decisive proof that they have not arisen by mutations, the reproduction of which is alternative, for had they so arisen the cross-breds would also display alternative reproduction. As we see, artificial varieties, which, admittedly, have arisen by mutations, display, not only alternative reproduction, but latent characters in abundance. It does not follow, however, that all characters which alternate in cross-breeding have arisen by mutation. So far as has been observed, reproduction is alternative only when characters are sharply contrasted; and sharp contrasts may arise as well by the piling up of fluctuations occurring during many generations as by single mutations. It would seem, however, that alternative reproduction is much more the rule when the contrast has been reached by mutation than when it has resulted from the accentuation of fluctuations.

296. On the other hand, if it be contended that mutations occurring in wild nature have been preserved, not because their reproduction was alternative, but because the mutants were sterile with the parent type but fertile inter se, then it must be shown that mutations, not only useful but similar in kind, occur in such numbers that physiological isolation ${ }^{1}$ without extinction is possible. The fact that human races are fertile when crossed is proof that physiological isolation can have played no part in their differentiation. Unless, then, heredity is different in plants and lower animals from what it is in man, we must believe that the natural varieties of the former were similarly inter-fertile at their origin, but that, in many of the cases which have been examined, differentiation is now physiologically greater than in the case of human races. Doubtless most natural varieties are more ancient than those of man; for few species can have so quickly invaded diverse environments, where selection differed, as the human being, who is omnivorous, and therefore not dependent for nutriment on

types are mongrels (in a real sense) between male and female. Again, the human races of North-Western Europe, where travel and interbreeding have been so common, and which in the past has been so often invaded by alien conquerors, are mongrels between several races, as is shown by the fact that, unlike all other animal races, they have variously coloured eyes. Yet, again, the albino rabbit and mouse are mongrels between the white and the ancestral grey types. In this connexion it matters not, of course, whether a variety is composed of many individuals, or of only a single individual who has mutated from the parent type. De Vries states that latency is of universal occurrence (Species and Varieties, p. 222), but he gives no evidence. He mentions instances of retrogression in plants, and assumes (p. 631) without further proof that it implies, not total loss, but latency. He assumes, in effect, that while the germ-plasm may gain characters, it cannot lose them, see § I88.

1 See $\S 332$. 
special plants and animals, or on soil or climate, and who, owing to his mental powers, is otherwise very adaptable.

297. The sole warrant for the belief that fluctuating variations cannot be made more stable by continued selection is furnished by the fact that recently evolved domestic varieties, such as the American trotting-horse, tend to retrogress rapidly when no longer selected. We have already discussed this question in part $;^{1}$ presently we shall see how sex is instrumental in determining the rapid retrogression of characters of recent origin. The sole warrant for the hypothesis that the organic world has arisen by the selection of mutations is furnished by the fact that some mutations have been observed in wild nature, and that mutations appear exceptionally stable. Doubtless, however, they are common enough. In man, the only natural species with which we are at all intimately acquainted, they are very common, and often the same mutation is repeated in many individuals. The medical profession owes its prosperity largely to them: they fill our hospitals; surgeons are busy every day rectifying such mutations as hare-lip and clubfoot. But never yet has a mutation been recorded-neither in man, nor in lower animals, nor in plants-that gave its possessors an advantage in the struggle for existence so overpowering that it enabled them to supplant the ancestral type. And hardly a mutation has been recorded which enabled its possessors to persist alongside the parent variety, at any rate when the reproduction was bi-parental. ${ }^{2}$

298. Mutationists declare that the general failure to detect

1 See $\S$ I 82.

2 The most widely discussed mutations are those of Enothera lamarckiana, an evening primrose, investigated by de Vries. Several species of evening primroses were brought to Europe from America during the seventeenth and eighteenth centuries. It is not known when, if ever, $O$. lamarckiana was introduced. It was first observed by Lamarck at the beginning of the last century in a garden at Paris, and at once recognized as a type new to science. The fact that this 'stately;' 'beautiful,' and noticeable plant was previously unknown to botanists, the circumstances under which it was discovered, and the fact that it is not known to grow wild anywhere in America, render it likely that it originated as a garden variety in Europe, possibly in the very garden in which I.amarck found it. Most probably, like so many other cultivated varieties, it is a mongrel, possessing an unknown number of latent characters. At any rate it is a descendant of a wild American plant, which, for more than a century, had been cultivated in Europe, and which, presumably owing to changes in selection due to the great changes in its environment and possibly to crossing, had become highly variable. The patch of plants in which de Vries discovered his mutants from O. lamarckiana had escaped from cultivation and was growing wild in a disused potato field. More mutations occurred after he had altered the environment yet again by re. moving specimens to his garden, where he cultivated the plants on a large scale. 
favourable discontinuous variations in wild nature is due solely to difficulties of observation. But there are no such difficulties in observing the neglected human species which we are so very capable of observing. It is not really beneath the dignity of science to utilize familiar facts. Mankind has spread over the whole habitable globe; it inhabits very diverse environments; it is numbered in millions, and has hundreds, if not thousands of stable varieties. Some races such as the Esquimaux and the Tierra-del-Fuegians, are apparently as 'pure' as natural races of lower animals; others are simple mongrels; yet others are compound mongrels. Some mongrel human races are very ancient; others are comparatively new. ${ }^{1}$ Racial changes of a permanent sort there have

The mutants, which were of at least seven different types, were comparatively few in number in the field. Most of the types were weakly and perished. In the field two types " have held their own during the last sixteen years, and probably more, without, however, being able to increase their numbers to any noticeable extent. Others perish as soon as they make their appearance, or a few individuals are allowed to bloom, and probably leave no progeny. But perhaps the circumstances may change, or the whole strain may be dispersed and spread to new localities with different conditions. Some of the latter may be found favourable to the robust gigas or vubrinervis, which requires a dryer air, with rainfall in the spring-time and sunshine during summer " (Species and Varieties, p. 574). Mutations are comparatively few, changes of climate rare, and the dispersal of most plants, except by human agency, slow. The chance of these mutations surviving in nature seems, therefore, somewhat remote. It is significant that de Vries " made experiments with some hundred species that grow wild in Holland. . . . No real mutability could be discovered" (p. 520) in the native plants growing in their ancestral environment. Or, to express the fact in other and probably more correct terms, these natural varieties revealed no latent characters. To account for the fact that $O$. lamarckiana mutated frequently while the native Dutch plants mutated not at all, the hypothesis was formulated that in the lifehistories of species periods of great mutability alternate with periods of stability. " Of course this mutable state must have had a beginning, as it must sometimes come to an end. It is to be considered as a period within the lifetime of the species, and probably it is only a small part of it" (p. 29). Manifestly this deduction, like that of colour-factors, is a pure guess, invented to bolster up another hypothesis which was found not to agree with reality. We may predict with entire confidence that every variety which has evolved under artificial selection and has been frequently crossed is an 'eversporting variety;' whereas every species which has evolved under Natural Selection is stable.

1 " In the case of a problem like that of man, complicated as it is by the fact that he has ' crossed more often than any other animal,' and further rendered intractable by the circumstance that he is not amenable to experiment, a great difficulty arises in discovering which are the actual allelomorphs concerned" (Mr R. H. Lock, Nature, I 7 th Oct. I907). " How can the fact that human races have crossed more often than any other animal complicate the problem ? My statement implied, not that every race is a chaotic mixture of types, nor even that there are no pure types, but only that we have here a very large and varied mass of material on which to found our judgments " (G. Archdall Reid, Nature, Oct. 3ist 1907). 
been in plenty. In some places, as in parts of New Guinea, where war is nearly perpetual and inter-marriages between tribes comparatively rare, the inhabitants of almost every district, like the land shells of Samoa ${ }^{1}$ display stable characters which clearly differentiate them from their neighbours. Even in Great Britain we have still these local races, as in the Yorkshire dales and Highland glens. So surely as a human race separates into two or more sections between which interbreeding is restricted, just so surely does differentiation set in. The section that remains in the ancestral environment continues comparatively stable; the section that migrates undergoes swifter but always continuous and steady adaptive change. Evidently there has been no lack of such variations as contribute to stable differentiation.

299. If, then, evolution is due solely to mutations, how does it happen that no favourable human mutations have been notedneither large observable mutations, nor those which render mating physiologically sterile? The latter certainly have not occurred, for differentiated human races are perfectly fertile when crossed. The former as certainly have not occurred; for written human history stretches into thousands of years, and men are fond of recording wonders, and none of this kind have been recorded. I think no mutationist, in face of this massive evidence, will venture to uphold the thesis that human differentiation has resulted from mutations. And, if not human differentiation, why the differentiation of other natural species and varieties? As I say, we have no reason to suppose that heredity in the animal man differs from heredity in lower animals and in plants.

300. In a few-speaking comparatively, a very few-instances, mutations, occurring chiefly in self-fertilized plants, have been known to persist without artificial aid for several generations. ${ }^{2}$ In every such case the mutation has been comparatively unimportant as regards the function of the part that has undergone change. A leaf or a flower has altered its shape or colour or texture, a flowerstem has grown longer or shorter, the wing of a butterfly has grown darker, and so forth. But all organs are not on the surface of the body ; their co-adaptations, therefore, are not always so few and simple as those of leaves and flowers. Consider a mutation

${ }^{1}$ See $\$ 33^{2}$.

2 The only mutation, human or other, if mutation it can be called, known to have been persistent under natural conditions for a considerable length of time, of which I am aware, is the Hapsburg lip, an ungainly feature of the reigning house of Austria. I imagine it would have disappeared long ago if the faces it marred had not belonged to an inbred imperial family. 
occurring within that immensely complex and delicately adjusted machine, a higher animal. To be useful the mutation must not only be an improvement in itself, but it must be correlated to a thousand readjustments-a thousand co-adapted mutations in other parts of the machine. Otherwise it will be positively harmful. ${ }^{1}$ Indeed, the principal difficulty to be surmounted by the supporters of the mutation hypothesis is not adaptation to the environment but co-adaptation within the organism. Suppose, however, we cede the point, and agree that the miracle may happen and a thousand co-adapted mutations may occur in a single individual. Then, either the reproduction will be blended, or it will be alternative. If it be blended, the mutation will be swamped unless more miracles happen and many other individuals mutate in the same way and at the same time and place. If it be alternative, the co-adaptation will be lost in succeeding generations through mixture with the characters of individuals who have not mutated; for Mendelian observers insist, and very rightly, that the alternative characters are reproduced independently of one another, and that all the characters of the same individual are not necessarily patent or dormant together.

30I. It must be noted, morever, that, not only are the parts of a living being co-adapted, but that different species of animals and plants are also co-adapted to one another. Thus the plants which herbivorous animals are capable of using as food possess great powers of multiplying themselves and of reacting to the stimulus of injury, of regenerating lost parts. Thus also the powers of pursuit possessed by carnivora are closely related to the powers of escape possessed by their prey. If evolution were by mutation, then a mutation, increasing the speed of one species, could only be met by a mutation occurring at the same time and place, increasing the speed of the other. For example, if wild dogs suddenly mutated in this way, and the various animals they hunt did not, the inevitable extinction of the latter would soon be followed by that of the former.

302. It is possible to account for the co-adaptation of parts and species to one another on the theory that evolution results from the continued selection of constantly occurring small fluctuations-from the constant and steady change of species in adaptation to changes in their surroundings; but how is it possible to account for it on the theory of large and, speaking comparatively,

${ }^{1}$ De Vries (Darwin and Modern Science, p. 68) insists that correlated mutations are common. But correlation is not the same thing as co-adaptation. 
very rare mutations? No real attempt has been made to surmount all these difficulties. Merely because some mutations have been observed it has been assumed that all evolution proceeds by steps and not up a smooth incline. Merely because some mutations, the inheritance of which is alternative, are comparatively stable, it has been assumed that all mutations are absolutely stable. Merely because fluctuations cannot be fixed by a short course of experimental selection, it is assumed that they cannot be fixed by the age-long selection of nature. Merely because varieties which have been evolved under artificial selection display when crossed alternative reproduction in widely divergent characters, it has been thought that all inheritance is alternative. All these inferences are illegitimate expansions of single inductions-illegitimate, because there has been no rigorous deductive inference of consequences, and no appeal to reality for confirmation.

303. It is not to be maintained, of course, that no mutation has ever been selected by nature. It is maintained merely that species are so accurately adapted to their environments, including one another, and that their structures-nervous, circulatory, alimentary, bony, muscular, glandular, etc.-are so closely co-adapted that a favourable mutation must be an extraordinarily rare thing-so rare that as factors in evolution mutations are negligible. We are told sometimes that, whilst Natural Selection may account for the survival of the fittest, it does not account for the arrival of the fittest. This jingle of words implies, of course, that fluctuations are too small to have selection value. ${ }^{1}$ But we shall see in later chapters, when we study the recent evolution of man, who, as I say, is the wild type we are best able to observe, that fluctuations have so real a survival value that, according as men fluctuate favourably or unfavourably-solely on that account-they survive and see their grandchildren, or perish in youth. The arrival of the fittest, owing to the necessary co-adaption of parts and species, is an immensely greater difficulty for the mutationist than for the selectionist

304. We have noted that living beings are bundles of adaptations. No phenomenon of life is more universal than the occurrence of fluctuations. Presumably, therefore, fluctuating variability is an adaptation, and a highly useful character. It has a function. Mutationists-at any rate many of them-admit, for example in the case of trotting-horses, that fluctuations are transmissible to some extent, and may be the sources of some temporary evolution. But we are asked to believe that opulent but parsimonious nature, 
that prolific creator of wonderful adaptations, has so contrived that directly this evolution becomes of importance as a factor in survival it is brought to a standstill; the species must wait for that rarest of phenomena, an adaptive mutation.

305. To sum up, the Mendelian doctrine-the notion that inheritance is alternative, that allelomorphs meet in the zygote but separate in the descendant gametes-is founded on experiment. It has not been tested by experiment. Experimental tests reveal the existence of blending when natural varieties are crossed, and of latent characters when artificial varieties are crossed, and, therefore, in both cases negative the hypothesis of segregation. The attempt to explain away latent traits by the hypothesis of factors and determiners is founded on a pure guess which is negatived by their occurrence in pure-bred varieties. The mutation hypothesis can hardly even be said to be founded on experiment; for most mutations have been simply observed. In any case the notion that evolution depends solely or mainly on mutations neither has, nor can be tested experimentally; for we can never be sure that we have correctly reproduced 'natural' conditions, nor can we continue our experiments long enough. But both the Mendelian and Mutation hypotheses may be tested by the ordinary and necessary scientific procedure of making a rigorous deductive inference of consequences and appealing to reality for confirmation. In neither case have their supporters applied the test. Both hypotheses, therefore, are "awful examples" of all that scientific thinking should not be.

306. The reader perceives that the traits which mainly distinguish the experimental biological school from all other schools in all other branches of science are its self-imposed restrictions in observing and thinking-restrictions which render it impossible for the experimental worker to prove his hypotheses to the satisfaction of other people, or for others to disprove them to his satisfaction. With the exception of data furnished by some few human abnormalities and the like, almost the whole of the facts used have been drawn from experiment, and none of them have been used to test the thinking. We read occasionally in the literature of that school such pronouncements as, "Evolution became the exercising ground of essayists. . . . Genetic experiment was first undertaken, as we have seen, in the hope that it would elucidate the problem of species. ... The time has now come when appeals for the vigorous prosecution of this method should rather be based on other grounds." 1 "The time is not ripe for the discussion of the origin

${ }^{1}$ Bateson, Mendel's Principles of Heredity, pp. 3-4. 
of species. With faith in evolution unshaken-if indeed the word faith can be used in application to that which is certain-we look on the manner and causation of adapted differentiation as still wholly mysterious." 1 The "essayists" were such men as Darwin, Wallace, Huxley, Ray Lankester, and Thiselton-Dyer in England, Weismann and Osborn abroad. Some of them did not themselves experiment ; but it is possible to think correctly, even about experiments, without first having personally controlled the breeding operations of rabbits and guinea-pigs. Moreover, the quantity of data already in hand to which Darwin's work called attention was so large as to occupy more than sufficiently the time of the most industrious thinker who was capable of dealing with complex material. The method of the essayists was not, as might be gathered from the words I have quoted, to neglect experimental work. Some of them constantly experimented. On the contrary, their method was to use, not only the experimental, but all the materials at their command, and to test their thinking in every way possible. Therefore their writings necessarily took the form of "essays." It is only when we use isolated fragments of evidence and guess about it that we are able in biology to avoid-if that be a merit-even the appearance of reasoning.

307. The writings in which the Mendelian and mutation hypotheses are formulated are as much essays as those which are denounced as such. They are distinguished from the latter only by very novel conceptions concerning the nature of science and of logical proof. It would not be surprising were a section of the general public, which has only the vaguest ideas as to what science is and how it has been created, deluded into the belief that experiment is an invariable accompaniment of special accuracy in scientific observation and thought. For experiment is an instrument which has been employed almost solely by men of science. But that a whole school of biologists should entertain that notion, apparently in complete unawareness that the question as to what constitutes evidence and what proof has been elaborately discussed by some of the greatest thinkers of the past, is certainly very remarkable. It is, perhaps, conceivable that experimental workers are right in neglecting patent facts and tests for thinking; but I do not think I can be wrong or prejudiced when $I$ insist that so great a departure from established scientific usage should be vindicated by at least some formal attempt at justification. But never yet has this been done. As matters

1 Bateson, Darwin and Modern Science, p. 99. 
stand, appeals that we shall vigorously prosecute the experimental method and abstain from controversy mean little more than that we shall ignore all material gathered otherwise than by experiment, and abstain from testing the correctness of guesses-that we shall permit biology to become a tumbling-ground for irresponsible whimsies. Declarations that the time is not ripe for the discussion of the origin of species, and that the manner and causation of adapted differentiation are still wholly mysterious, imply nothing more than that it has been found impossible to bring the Mendelian and mutation hypotheses into accord with the fact that living beings exist and are fitted to their surroundings. The denial (based on the supposed inutility of structures, the uses of which have not been ascertained) that living beings are, in a wonderfully complete degree, adaptational forms, has precisely the same origin. 


\section{CHAPTER IX}

\section{THE FUNCTION OF SEX}

Most Mendelian characters are sexual characters-The parallel between Mendelian and sexual characters-It has been said that the inheritance of sexual characters is Mendelian-The truth is that the inheritance of Mendelian characters is sexual-The function of sex if (I) the Mendelian hypothesis is true, (2) if the mutation hypothesis is true, (3) if evolution is founded on fluctuations and inheritance is blended.

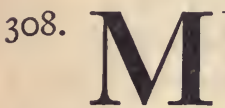

UTATIONS and Mendelian reproduction are realities. If, then, the organism is a bundle of adaptations, and evolution builds on fluctuating variations which blend, how is their presence to be accounted for? We have considered several great problems of heredity and evolution, for example the nature of the distinction between classes of characters, the mode in which the individual develops, the mode in which species evolve, the causation of variations, and the like. Obviously the mutation hypothesis is concerned with none of these except evolution. But evolution is merely another name for the adaptation which results from progression and retrogression; and, as we have seen, it is not believable that mutations, which occur only rarely and which, speaking practically, are never adaptive, can have furnished the materials for the multitudinous, the perpetually changing, the infinitely complex and delicate adjustments of living beings. We are driven, then, to the conclusion that they are nothing other than abnormalities, extreme variations which arise only because evolution (and therefore the regulation of variations) is never perfect-just that class of variations which nature checks by the weeding out of the exceptionally variable individuals, but which human breeders encourage and preserve, that class on which surgeons expend the resources of their art to eliminate in the individual but to perpetuate in the race.

309. We have to delve deeper before we can account for the Mendelian phenomena. Experimental workers assume that in Mendel's laws they have acquired a master-key to all or most of the problems of heredity. But, if we consider the question closely, it becomes evident that Mendel's laws are concerned with nothing 
more than the effect or function of conjugation. ${ }^{1}$ Manifestly they have no bearing on such problems as progression, retrogression, recapitulation, variability, the causation of variations, the distinction between characters, and the rest we have discussed. They merely attempt to describe the way in which parental characters are distributed amongst offspring and descendants under conditions of conjugation. To parthenogenesis they do not apply; for here, since no allelomorphs meet, none can be dominant or recessive, none can segregate. In fact, they apply to no problem that is presented in common by parthenogenetic and conjugating species. At any rate I can think of none; and though I have inquired industriously amongst the adherents of "the new science of genetics," I have quite failed to secure enlightenment. ${ }^{2}$

3I0. It has been said, indeed, that since "the rule that all organisms pass through a sexual cycle at some period of their existence has extremely few exceptions," ${ }^{3}$ all problems of biology are problems of conjugation. It would be as reasonable to declare that, since all organisms die, therefore all problems of biology are problems of death. Such a statement is merely equivalent to a declaration that the universe is a unity, the facts and laws of which are related. Again, it has been said, "It is the claim of the Mendelians that they have discovered in certain cases some of the fundamental characters of an organism-the units of hereditary transmission, which are represented in the reproductive cells by definite entities known as allelomorphs." 4 But, obviously, if there is no segregation, but merely patency and latency, the discovery has not been made. In this instance, also, it would be well if the reader paused and asked himself, not whether Mendelism solves any problems, but whether it has a conceivable bearing on any problem save that of conjugation-of sex.

3II. We saw that individuals of sexually dimorphic species have two sets of sexual characters, one of which is patent and the other latent, one of which is dominant and the other

1 Of course every effect is not necessarily a function. A function is that which bestows utility. One effect of sex is to halve the possible number of offspring. But no one will believe that this halving is the function of sex. Besides, nature is able by the selection of fertility to increase the number of offspring produced by a pair up to any useful limit, and so obviate this effect. On the other hand, if, as Mendelians suppose, bi-parental reproduction mixes parental characters as marbles are mixed, but leaves them otherwise unchanged, it would seem we have no alternative but to suppose that such mixing is both the effect and the actual function of sex. I think it is impossible to imagine any other function.

${ }^{2}$ See, for example, Nature, 3rd Oct. 1907 to Jan. 23 rd 1908.

3 R. H. Lock, Nature, Nov. I4th, 1907. ${ }^{4}$ Op. cit., Nov. I4th, 1907 . 
recessive. Every individual is in fact an 'impure dominant.' The non-sexual characters, on the other hand, are all patent. When the breeding is intra-varietal, as it normally is in nature, mating individuals (apart from their sexual characters) differ, with rare exceptions, only to the extent of fluctuations, the inheritance of which, admittedly, is blended. The exceptions are mutations which, like the sexual characters, tend not to blend, but to become patent or latent in descendants. We saw also that when natural varieties, which have arisen through the selection of fluctuations, cross, they tend to blend their characters; but when artificial varieties, which have arisen through the selection of mutations, cross, the reproduction of the characters in which they differ tends to be alternative. Evidently, then, the mode in which mutations are reproduced by offspring and descendants presents a close parallel to the mode in which the sexual characters are reproduced.

312. The parallelism between the two sets of phenomena, the sexual and the Mendelian, grows clearer the more we study it. We have already noted many of the likenesses, but it will be useful to bring them together. (a) Of necessity some of the sexual characters, especially the primary characters, are developed in distinct sets-a male set and a female set-which are kept separate by selection. Most of the Mendelian characters, on the other hand, are developed indifferently by both sexes. But many characters the functions of which are sexual (in that they serve as attractions), though not exclusively sexual, are reproduced in the latter way; for example, colour of eyes, and, to some extent, colour of skin and hair in the intra-varietal matings of man, and colour of plumage and hair in the inter-varietal matings of many birds and mammals. (b) Sometimes characters peculiar to one sex are transferred to the other, as in the cases of the bony cranial protuberance of the Polish fowl ${ }^{1}$ and the plumage of the Sebright bantam. Some Mendelian characters are transmitted sexually, as colour-blindness and hæmophilia in man. They would be termed sexual, not Mendelian, were they more common. Deaf-mutism appears to furnish a further connecting link, for the condition tends to affect the males in some families and the females in others. ${ }^{2}$ Moreover, the dominance and recessiveness, the patency and latency of Mendelian characters is sometimes affected by the sex of the parents, the offspring of reciprocal crosses being unlike. ${ }^{3}$

1 Animals and Plants, vol. i., p. 269.

2 C. J. Bond, British Medical Journal, Oct. 28 th 1905.

${ }^{3}$ See $\S 264$; see also Bateson, Mendel's Laws of Heredity, chapter x. 
3I3. (c) The alternative development-the alternative patency and latency - of sexual characters is more perfect than that of most Mendelian characters, but this is only what might have been expected; for it has been secured by selection, just as the less perfect, or rather the different alternation in aphides, and bees has been similarly secured. (d) When Mendelian characters are crossed, one of each pair is usually the dominant in the first hybrid generation. Thus colour is usually dominant over white in mammals and flowers, whereas the contrary is the case in poultry. In the case of sexual characters one or other alternative dominates in any individual. But sometimes the offspring of a single pair are wholly of one sex, a phenomenon not very rare in human families ; whilst, on the other hand, in the case of some Mendelian alternatives, both types tend to appear in the first hybrid generation-for example, normal and extra toes in poultry. (e) As might have been expected also, the sexual characters show a lesser tendency to blend than Mendelian characters; but some degree of blending is not uncommon in the former, as when women have masculine pelves and partially bearded faces. $(f)$ The crossing of varieties may bring to light long dormant Mendelian characters-for example, wild-grey in mice and rabbits; it may also reveal strictly sexual characters which have long been latent; for example, broodiness and sexual peculiarities of plumage in fowls; and sometimes the lost sexual characters may reappear in connection with traits properly belonging to the opposite sex. ${ }^{1} \quad(g)$ As Ewart ${ }^{2}$ and others have shown, the dominance of both sexual and non-sexual alternative characters is capable of being influenced by the environment.

314. (h) If the characters of crossed varieties blend or are lost, there is a very general tendency to sterility, as is the case when natural varieties interbreed; but if they alternate so that the characters are unchanged, as when artificial varieties cross, fertility is perfect. "We know no Mendelian case in which fertility is impaired." 3 We have here a very clear indication of the parallelism between sexual and Mendelian characters, for under such conditions, as in mules, sexual characters also tend to blend or be lost with consequent sterility. (i) Mendelian characters which segregate in later generations are sometimes blended in the first hybrid generationfor example, in the case of Andalusian fowls; or there may be particulate inheritance in the first generation and more complete

${ }^{3}$ First Report of Evolution Committee, p. 148. 
separation subsequently. ${ }^{1}$ In like manner, when varieties are crossed, a pseudo-hermaphroditism is not uncommon, that is, the inheritance of male and female characters is then particulate, the separation into male and female sets is not preserved; there is on the contrary Mendelian independence of characters. ( $j$ ) As we have seen, there is no real segregation of Mendelian any more than of sexual characters. There is only patency and latency. (k) Sometimes both alternative Mendelian characters, for example, extra toe and normal foot in poultry, appear, like the sexual characters, in the different individuals of the first hybrid generation, and would continue to appear in each succeeding generation were they like the sexual characters crossed in each generation. (l) In other instances, the Mendelian traits are long latent, like the sexual characters in aphides; but like them reappear at length, as in cases of reversion in pure-bred varieties. ${ }^{2}(\mathrm{~m})$ In another peculiarity, and that a very striking and important one, Mendelian and sexual characters display a suggestive resemblance. Both are traits in which, unlike fluctuations, the mating individuals are strongly contrasted; for the Mendelian characters are usually mutations or characters which originated by mutation in ancestors.

315. Plainly, the parallel between sexual and Mendelian reproduction is very close. It is so close that experimental workers, though they have not by any means perceived all the likenesses, have surmised that the inheritance of sexual characters accords, in many instances at least, with Mendel's laws. ${ }^{3}$ Indeed, they have supposed that the inheritance of sex is merely an example of Mendelian inheritance, ${ }^{4}$ and that continued investigation will eliminate any apparent contradictions and anomalies. But another interpretation of the facts is conceivable. Examine any list of Mendelian characters, for example those of poultry ${ }^{5}$ or peas. ${ }^{6} \mathrm{~A}$ glance shows that, as a rule, the majority of the items in such a list are sexual in the sense that, as attractions or otherwise, they are concerned with reproduction. It is possible, then, that, so far from it being a fact that the reproduction of sexual characters is an example of reproduction of Mendelian characters, the reverse may be the case.

3I6. The question we are now in a position to ask ourselves is, Which mode of reproduction, the sexual or the Mendelian, contains the other? Which is a variety of the other? In deciding

1 Inheritance in Poultry, p. 85.

2 See $\S 280$.

${ }^{3}$ See Bateson, Mendel's Laws of Heredity, chapter x.

4 See Castle, Heredity of Sex.

5 See $\$ 288$.

6 See $\$ 257$. 
this problem, we must bear in mind that sexual reproduction is almost universal; whereas, to judge by facts that have been actually observed, not merely imagined, Mendelian reproduction is one of the rarest things in nature. Speaking practically, it occurs only when mutations are reproduced; and all the evidence indicates that mutations occur relatively seldom, and, even then, are very seldom preserved, except when the selection is artificial. Mendelian reproduction, therefore, is, in effect, unknown in normal-that is, intra-varietal-breeding. Natural varieties very rarely cross, and only rarely display Mendelian traits when man crosses them. Such traits are common only when artificial varieties, in which mutations have been accumulated by man, are crossed by man. They seem common to experimental workers only because the materials for thought to which they have limited themselves have been obtained almost exclusively in this latter way. I think, therefore, that no one who has been accustomed to found his judgments, not on the exception but the rule, will hesitate to conclude that Mendelian reproduction is a variety of sexual reproduction, and that the converse is as absolutely untrue as if we had declared that mammals are a variety of dogs.

317. If we found our judgments on verified evidence alone, the conclusion seems irresistible that Mendelian 'inheritance' is a human creation, and that the right interpretation of the facts appears to be, that nature treats mutations, when man interferes and presents them to her, as sexual characters. She is not a conscious selecting agency, and they resemble the sexual characters and differ from the fluctuations which she normally meets, in that they constitute wide differences between mating individuals. But her treatment of them is more or less uncertain; hence, as compared to sexual traits, their imperfect alternation and their tendency to blend in greater or.lesser degree. It appears, then, that Mendelian characters are nothing other than non-sexual or semi-sexual characters abnormally reproduced in the mode that sexual characters are normally reproduced. It follows that experimental workers have been engaged in investigating, not heredity in general, not even the function of sex, but only certain anomalies of sexual reproduction which occur under conditions of artificial selection and crossing. They are the unhappy victims of a vast practical joke unconsciously played by the human breeder.

"The earth hath bubbles, as the water has,

And these are of them." 1 
They furnish examples of the disasters which tend to befall us when we limit the area whence we draw the materials for thought, and fail to test our thinking in every way possible, when, in fact, our attitude is sectarian, not scientific.

318. The question of the relation between sexual and Mendelian characters cannot, however, be disposed of in this summary fashion. At any rate, since the reader may still consider it a matter of opinion whether the inheritance of sexual characters is Mendelian or the reproduction of Mendelian characters sexual, it ought not to be so disposed of. It is necessary to make the usual deductive inference of consequences, and then to compare these consequences with reality. It is an indisputable truth that living beings exist and have undergone evolution. Does the hypothesis that the inheritance of characters, including the sexual, is Mendelian accord with this truth, or is it incompatible with it? Or, on the contrary, does the hypothesis that inheritance tends to be blended, and that Mendelian traits are abnormalities of sexual reproduction introduced by man, accord with it? In brief, we have now to investigate the function of conjugation, of sex.

319. If the Mendelian theory is true, the effect and, therefore, presumably, the function of conjugation is to mix parental characters as marbles are mixed, not to blend them as colours are blended. Now suppose, for example, that a tall man mates with a short woman and offspring are born to them. This means that both parents have been so well adapted to the environment, and their characters so well co-adapted to one another, that they have been enabled to survive till they were mature. But if characters are transmitted independently of one another, the 'tall' and 'short' characters of the parents will be mixed in the offspring. This is not what happens in nature. It is not even what happens when artificial varieties are crossed by man; for only a very few of the characters (e.g. colour of plumage and extra toe) of such varieties have been established by him through the selection of mutations. But it is what is implied in the Mendelian doctrine. It must be remembered that tallness and shortness in human beings and other animals depend, not on a single character like internodal length in the pea, but on a multitude of structures, all of which are supposed to be inherited independently of one another. ${ }^{1}$ Moreover, relative tallness and shortness are not necessarily fluctuations, for there are stable tall and short human races

1 Bateson, Mendel's Principles of Heredity, p. 209. 
which, as it happens, are able to cross successfully. On the Mendelian theory, however, the effect of bi-parental reproduction should be calamitous; and one is left to wonder how it happened that this form of reproduction replaced parthenogenesis, in which such a disastrous mingling of incongruous elements would, at least, not occur. Moreover, self-fertilization is not uncommon, and is a product of evolution. Here, since, almost invariably, only like characters meet, there can be, speaking practically, no mixing of unlike allelomorphs, and we must suppose that conjugation - that sex, which has burdened living beings with so many characters-has no function whatever. It is surely not surprising, then, that Mendelians, conscious of the tangle, should feel moved to declare that they "look on the manner and causation of adapted differentiation as still wholly mysterious," and that "we can profitably reject, as I believe, much of the theory of Natural Selection, and more especially the idea that adaptations have arisen because of their usefulness." 1

320. On the other hand, if the mutation theory as conceived by Professor de Vries is true, that is, if the variations on which progressive evolution is founded are large discontinuous mutations, and if mutants tend to be sterile when crossed with the parent type, or if fertile, to have offspring which blend the parental characters, then, again, one is left to wonder at the extraordinary function assigned by implication to sex. When reproduction is parthenogenetic, useful mutations, if they occur, have an excellent chance of surviving. But when reproduction is bi-parental, they can very rarely, if ever, escape being lost through sterility, or swamped through blending. According to this hypothesis, therefore, it would appear that the function of sex is to render nature powerless to produce adaptive changes. And yet we must suppose sex to have been evolved by nature. In this case also how shall we account for the occurrence of self-fertilization?

321. Examine now the theory that evolution proceeds through the constant selection of 'continuous,' 'normal,' or 'fluctuating' variations, the inheritance of which is blended. . As we have seen,

${ }^{1}$ See $\S 306$.

'The term 'blended,' with the meaning given it in the present work, is not altogether correct; but it is convenient. It is used in contradistinction to 'alternative ' and includes all shades of blending from an equal mixture of parental characters to an exclusive reproduction of the character of one parent with total loss of the character of the other. Exclusive inheritance in this sense differs from dominance in that the former implies the complete loss of the character of one parent, whereas the latter implies only its latency. To the Mendelian 
every species which is sexually dimorphic, possesses two kinds of characters. In some characters parents resemble one another closely; that is, they differ normally only to the extent of fluctuations. The inheritance of these characters is blended. In other characters parents differ so widely that, did we not know them to be male and female, we should suppose them to belong to different species. The inheritance of these larger differences is apparently alternative. Really it is blended; only the reproduction is alternative; for since parents transmit their characters through children of the opposite sex to grandchildren of the same sex, we know that, though every individual possesses only one set of non-sexual characters, he possesses two sets of sexual characters, one of which is patent and the other latent. If, then, similar characters blend (e.g. the ears of one parent with the ears of the other, and so on), there can be no doubt, in the case of the sexual traits, that the patent male characters of the father blend with the latent male characters of the mother, while the patent female characters of the latter blend with the latent female characters of the former. The child, however, presents a deceptive appearance of inheriting only one set of characters in which there is no blending. Abnormally, the patent sexual traits of the one sex blend with those of the other as in many of the so-called hermaphrodites of sexually dimorphic species. Except in the case of mutants, which in nature only very rarely survive and have offspring, all the larger differences between mating individuals are sexual. But in some characters mating individuals do not differ widely. Thus the coloration of the male and female may be similar. The inheritance of such characters is admittedly blended. In parthenogenesis there is neither latency nor, of course, blending. Latency and blending are phenomena of bi-parental reproduction only.

322. The only real exception to blended inheritance that I can think of occurs when a mutation first arises. If the mutant (the individual that has mutated) be crossed with a normal individual the mutation may be diminished, in which case the inheritance is blended; or it may be quite eliminated, leaving no representative in the germ-plasm, in which case there occurs that extreme form of 'blended' inheritance which we have termed exclusive; or the mutation may be present in the offspring unchanged by conjugaexclusive inheritance is identical with gametic purity, but, as we have seen, it is certain that gametic purity does not occur when 'inheritance' is alternative. In such cases we have only alternative development. The term prepotent as applied to a parent, implies that his or her character predominates in the blend in any degree up to an exclusive inheritance. To the Mendelian prepotence implies dominance. 
tion in a patent or latent condition, in which case there is no blending in that generation. In subsequent generations there is blending if descendants of the mutants interbreed, for they will have the character in a patent (dominant) or latent (recessive) condition. It is not to be supposed, however, that mutations are reproduced unaltered in offspring and descendants. Presumably, like other characters, they are subject to fluctuating variations, of which the general trend is retrogressive. They disappear, therefore, in time by sudden mutation, or probably more usually by gradual retrogression, unless preserved by selection. We have no reason to suppose that they are less liable to retrogression when latent than when patent. No doubt, numbers of latent characters have persisted through many generations in domestic species; but, speaking comparatively, all domestic species are of recent origin, and the retrogression of some useless patent characters (e.g. limbs when they have become useless) is very slow. Nature is parsimonious of her materials; it is improbable, therefore, that the germ-plasm is burdened for ever with useless dormant characters. At any rate, there is not an iota of evidence that it is so burdened. An interesting point in connection with latent traits is the reappearance in offspring of numerous narrow, rather faint stripes when the horse, which has no patent stripes, is crossed with the Burchell zebra, which has a few broad ones. This is the most ancient latent character known to me. It is commonly believed that the narrow stripes are due to reversion to a common ancestor. This may or may not be true; but it does not follow that the dormant trait is carried by both species. Dormant traits are practically unknown in natural varieties such as the zebra; and horses, even when pure bred, sometimes display traces of stripes. It is probable, therefore, that the horse is the carrier of them, that they were rendered dormant in the ancestry by the artificial selection of mutations, and that they have grown faint through retrogression.

323. Offspring vary 'spontaneously' from their parents in two ways. First, they blend parental characters, and so differ from both parents; or, if there is exclusive inheritance, they differ from one parent. Second, they differ from both parents otherwise than by blending. With this latter sort of spontaneous variations we have already dealt. ${ }^{1}$ We have to consider the variations which result from blending. Suppose, now, to choose a homely example, a man with an exceptionally broad nose mates with a woman with an exceptionally high nose. Then, if the result be 
a blend, the offspring approach the ordinary specific type more nearly than either parent; that is, there is retrogression or 'regression' as regards the progressive variations of both parents -a retrogression towards the specific mean. Again, suppose there is exclusive inheritance, so that the peculiarity of one parent is entirely lost. We noted that this very often happened in the case of those numerous small redundancies, those small progressive variations which offspring invariably display. ${ }^{1}$ Here, again, there is return to the specific type and retrogression. When offspring inherit exclusively from the progressive parent, there is, of course, no retrogression; but this, as proved by the general tendency to retrogression, happens comparatively infrequently, and, in any case, there is no progression.

324. Blended inheritance, therefore, nearly always implies retrogression when parents differ. The characters in which they differ tend to be lost, wholly or partially, within the limits of the difference. It never implies progression. When progression occurs, it is due, not to blending, not to the intermixture of parental germ-plasms, but to that spontaneous variability which is present in all forms of life, including parthenogenetic types. Notwithstanding all that has been written on the subject, there is absolutely no evidence that sex is ever a source of progressive variations. Whenever we are able to trace its influence clearly, blending (i.e. retrogression) is what we observe. It is true that cross-breeding may reveal latent characters in a domestic species. But this apparent progression is never real. Indeed, it frequently implies retrogression. ${ }^{2}$ Again, when varieties are crossed and their constitution 'broken,' as it has been termed, the increased variability which has been said to occur in descendants may well be due to alteration, through retrogression, of that tendency to vary only within narrow limits, which was imposed by Natural Selection on both varieties after they became adapted to their surroundings - to a return to that condition of wider variability which was imposed on them while they were becoming adapted. Varietal crossing, however, is a rare phenomenon in nature. It is one of the abnormalities which especially distinguish artificial breeding.

325. The function of sex, therefore, is to bring about retrogression. But this retrogression does not occur at haphazard. On the contrary, sex, a principal source of retrogression, does its work with as much discrimination as Natural Selection, 
the sole source of progression. For example, to choose another homely instance, suppose a human race inhabits a region where malaria is prevalent, but where tuberculosis and measles are absent. Then offspring will vary from their parents, favourably and unfavourably, with respect to all three diseases. In other words, they will, as compared to the parents, be more resistant or less resistant to each disease-more or less able to survive if exposed to it. It does not follow, however, that an individual who has varied favourably as regards malaria, will also have varied favourably as regards tuberculosis, measles, or any other disease. Since malaria is present, those who have varied unfavourably in relation to it will be eliminated, while those that have varied favourably will be preserved. Like will mate with like, and therefore little or no retrogression caused by blending will occur to check progression caused by Natural Selection. On the other hand, the progressive variations with respect to tuberculosis and measles will be mere useless redundancies. Since there will be no elimination of those that vary unfavourably, unlike individuals will mate. The result will be retrogression. ${ }^{1}$ Apply this reasoning to all the characters of the individual, and the function of sex becomes apparent. Natural Selection preserves or causes progression in useful characters by eliminating the individuals that do not possess them in sufficient degree. On the other hand, sex, a product of evolution through Natural Selection, automatically eliminates useless characters, but without eliminating the individuals that possess them.

326. The fact that races undergo protective evolution only

1 When two dwarf breeds of dogs are crossed the offspring tend to be larger than either parent. This would seem to indicate progression as a result of crossing. But such breeds have resulted from the artificial selection of mutations. In them, presumably, the larger size is latent. Again, inbred artificial varieties tend to be delicate ; and Darwin noted that, when two such varieties are crossed, the result is often an increase of health and vigour. It seems not unreasonable to suppose that the apparent progression is really due to retrogression. Nature eliminates unfavourable variations in all directions in evolving varieties. But man, devoting his attention exclusively to one or two points (e.g. speed in race-horses, size, shape, and colour in flowers), neglects or cannot perceive others which, though unfavourable, are not eliminated by selection because the individuals are protected, nor by conjugation because the individuals, being inbred, are much alike. Crossing unites unlike individuals and so eliminates the unfavourable redundancies; for example, it tends to remove the traits that cause 'delicacy' and so to restore the pristine vigour. It performs that which it is the function of conjugation to perform. If this interpretation be mistaken we have no alternative but the hypothesis of rejuvenescence. It should be noted that the traits that man consciously selects are usually mutations. But those which he unconsciously selects (e.g. 'delicacy') are usually fluctuations. 
against the diseases to which they are exposed is, I conceive, clear proof that the organic world has not arisen by mutations which are permanent and which therefore nothing but selection can eliminate. We shall see in the next section of the present volume how great that evolution has been, how closely it adjusts each variety to its special environment, and how laden it is with momentous consequences. Now, since all races evolve against all the diseases to which they are exposed, it is evident that favourable variations against every disease occur in every race. Unless disease miraculously evokes these variations, they are spontaneous, and therefore, unlike the evolution which results from their selection, not dependent on the presence of the disease. Either they are fluctuations which retrogress in the absence of selection, or they are mutations which, it is said, only selection can eliminate. If experimental workers are right and they are mutations, then, when a variation which increases the power of resisting a disease occurs in a country in which the disease is not present, it will not be eliminated by selection, for it will be a harmless character. Since such variations are spontaneous they will occur as abundantly when the disease is absent as when it is present. If, then, evolution is by mutation, how does it happen that only races long exposed to a disease are resistant to it, so that whole races tend to die out when exposed to new diseases under stringent conditions-for example, the inhabitants of all the Western world when exposed to tuberculosis? The mutation theory is not complete as it stands. The fact that races do not evolve against diseases to which they are not exposed demands the corollary that every useless mutation (e.g. capacity to resist measles) is also so harmful that nature eliminates the individual possessing it. Otherwise it is impossible to account for the fact-which we shall be in a position to perceive more clearly later-that races evolve only in useful directions. ${ }^{1}$

327. "It was said even at the time of Drelincourt that no less than two hundred and sixty-two groundless theories of sex had been suggested; and it may be added that since that time there has been no falling off of interest in the sex question if the number of new theories proposed is a criterion." The latest theory, that which I venture to formulate, is sure, therefore, to meet with scepticism. Nevertheless, the evidence supporting it when gathered together appears strong. Whether I am right or wrong, the correct interpretation in this case, as in other instances, probably lies obvious on the surface, provided we do not allow

${ }^{1}$ See chapter xiii. and especially $\$ 435$. 
ourselves to be blinded by that veil of familiarity which is so apt to obscure the significance of common facts. To me it seems likely that the failure to discover the function of sex is due largely to the circumstance that the attention of biologists has been concentrated, on the one hand, on progressive evolution, and on the other hand, on striking abnormalities. Retrogressive evolution, which is every wit as important, every wit as adaptive as progressive evolution, has received scant attention; and biologists, with hardly a recent exception, have attributed it to the same agency (direct selection) as produces progression. The fact that it occurs in the total absence of direct selection has not received due weight. Presumably, sex has some function. The two theories most in favour with biologists at preseut are, on the one hand, the hypothesis that it renders species variable and so provides materials for Natural Selection, and on the other, that it mixes parental qualities as marbles are mixed. We have already dealt with the Mendelian hypothesis. No one not a Mendelian doubts the swamping effect of sex on progressive variations. The function of sex, therefore, cannot be the provision of progressive variations as materials for the work of Natural Selection. Sex itself must have been evolved by the prolonged selection of pre-existing progressive variations. ${ }^{1}$ Moreover, it has been proved that parthenogenetic species display such variations in plenty. Indeed, a principal part of the work of Natural Selection seems to be to confine variations within useful bounds.

328. Before we can be in position to understand the effect and the function of conjugation, we must study normal intra-varietal breeding. We are quite incapable of observing its effects in plants and lower animals. Even in human beings, with which we are so familiar, our task is rendered very difficult by the constant presence of spontaneous variations arising independently of sex. Modifications, due to the action of the environment on the germ-cell as well as on the soma, also complicate matters. However, we have no reason to suppose that amongst human beings, members of a natural 'wild' species, the effects of conjugation differ greatly, if at all, when the breeding is inter-racial from what it is when it is intra-racial. Many human varieties differ so widely that when they cross we are easily able to note the effect of con-

1 Possibly it originated in attempted cannibalism amongst unicellular organisms, an attempt which resulted in beneficial fusion. Thence, perhaps, during the evolution which adapted species for conjugation, arose gradually those multitudinous sexual differentiations which we find in multicellular animals and plants. 
jugation. In every instance the result is a blend which persists in subsequent generations, not a Mendelian mixture. Here, very clearly, the effect of blending is retrogression towards the parental and varietal mean. If many races interbred it would imply retrogression, within the limits of their differences, towards the specific mean, consequently towards the ancestral type. Thus, if races which have undergone evolution against different diseases cross, the resisting powers of those races which have undergone progression are diminished in the descendants. ${ }^{1}$

329. We have used the term blending as including all degrees of prepotency up to the exclusive inheritance of the character of the one parent and the permanent loss of that of the other. Old established characters tend always to be prepotent over newer characters, as is proved by the fact that when parents differ the progressive variations of one generation tend not to be reproduced by the next. It is also proved by another set of facts, namely, that retrogression of even old-established parts is a constant accompaniment of cessation of selection. We have no reason to doubt that, apart from the effects of blending, offspring tend to vary from their parents retrogressively as well as progressively. Evidently these retrogressions which imply reversions to an old type, tend to be prepotent over the type which has become the normal of the race; hence, in part at least, retrogression in the absence of selection.

330. The little evidence we possess concerning parthenogenetic species points in the same direction. Their useless characters (e.g. receptacula seminis in Cypris reptans) are very persistent. Parthenogenetic types are exceptionally prolific of progressive varieties, a fact which clearly indicates comparative lack of regression and, therefore, of retrogression.

33I. The fact that under conditions of parthenogenesis (complete or partial) "thousands of forms may be cultivated side by side ... and exhibit slight but undoubted differentiating features, and reproduce themselves truly by seed " 2 is very significant. It affords evidence (I) that progressive variations are then plentiful, and therefore that bi-parental reproduction is not the cause of them; (2) that such variations are then especially persistent, and therefore that conjugation tends to plane them away, and (3) that fluctuations are unstable, not because they are due to "varying conditions of food supply, temperature, density, moisture, light," etc., but because they blend with one another in bi-parental reproduction. Otherwise,

1 See $\S 464$.

2 See $\$ 244$ (footnote). 
since persistent variations are so many in parthenogenesis, we must suppose that mutations are a thousandfold more numerous under that condition than when reproduction is bi-parental, which is, to say the least, unlikely.

332. Speaking generally, among animals that reproduce biparentally, varieties are few when the powers of locomotion are great-that is when mating between individuals derived from widely separated districts is common. Thus, avian species have few varieties except when spread over a very wide area or an area that is interrupted by natural barriers, and often, unlike parthenogenetic types, only a single variety inhabits a whole region. When there are effective barriers, varietal differentiation is usually noticeable. When the powers of locomotion are less, the number of varieties is greater. Thus, as Gulick noted, every valley in Samoa has its local species of land-shells, the differentiation between the varieties, species, and genera being proportionate to the distances which separate them. ${ }^{1}$ All this indicates strongly that the effect of sex is to blend characters. If the theory of unit segregation and gametic purity were true, and, as a consequence, that of blended inheritance untrue, species which reproduce sexually should possess at least as many varieties as those the reproduction of which is parthenogenetic, and those which range widely as many as those with poorer powers of locomotion. In that case there would be, not only no approach to uniformity through conjugation, but such a shuffing of characters that almost every individual would form a variety by itself.

333. The fact that, apart from the sexual traits, all the individuals of the same variety differ, with rare exceptions, only to the extent of fluctuations, is presumptive evidence that the function of sex is to blend parental characters. In that case the average experience of the whole race, rather than the, perhaps, exceptional experience of single individuals, becomes the determining factor in evolution. The advantage of this is obvious;; for most individuals are more likely to have an average than an exceptional experience. Moreover, as I say, blending when combined with Natural Selection is a selecting agency. Selection eliminates harmful progressive and retrogressive variations, and, on rare occasions, old-established characters that have become harmful. Blending, with its swamping effects, its general tendency to produce retrogression, eliminates useless characters and variations-characters and variations which are merely useless to the individual, but which, if accumulated, would become

${ }^{1}$ See Darwinism, by Alfred Russel Wallace, p. 43. 
harmful to the race. Selection may be compared to a sculptor who designs and rough hews a statue; sex to a colleague, more skilful mechanically, who chisels out the finer lines.

334. We never see racial progression except when selection, natural or artificial, is at work. The evidence is, that given sufficient time, retrogression invariably occurs when there is cessation of selection, its speed being proportionate to the recency of the antecedent progression. Now, when a character (e.g. limb) has been long established, constant interbreeding has so blended together all the strains of a race, that mating individuals differ comparatively little. At any rate, apart from mutations, which are comparatively rare and tend to be eliminated, individuals, exhibiting every gradation between the extremes of 'normal' variations, occur-the most numerous type and therefore the most influential as regards inheritance being those which approximate most closely to the specific mean. In such cases, therefore, the retrogression which follows the cessation of selection depends wholly on the prepotency of new retrogressive variations, and is a very gradual process. But when the character (e.g. any of the special points of prize breeds) has been newly established, mating individuals differ more, for the blending will have been less thorough, and retrogression, therefore, is more rapid. It is possible, indeed I think it is probable, that retrogression follows mere cessation of selection even in parthenogenetic species. But the prolonged persistence of useless structures in such rapidly reproducing types as Cypris reptans, as well as the fact that parthenogenetic species tend to break into a multitude of progressive 'varieties,' indicates that retrogression on cessation of selection is a slower process than when blending is present.

335. The higher animals are the most complex products of evolution. The multitude and differentiation of their structures is very great, and the fact that they are active moving machines renders necessary a very delicate co-adaptation of all their parts. A tree may differ, always does differ, greatly in shape from other members of the species; but, if a higher animal diverges much from his type in shape, he is a monster and cannot persist. But this very complexity of structure and closeness of adjustment increases the area of variability and the chance that variations will throw the whole machine out of gear. It is significant that the reproduction of all these animals is bi-parental. The blending of useful characters, combined with the automatic planing away of redundancies, relieves Natural Selection of half its task of adapting them to the environ- 
ment. When considering Natural Selection, we are apt to think of the progressive variations of the individual as passing unchanged to his descendants. As a fact, apart from his variations, the descendant is, so to speak, a composite photograph of ancestors from which useless characters are, more or less, excluded. It is largely because conjugation has planed away redundancies and blended into a congruous whole much that enabled the survivors in past generations to persist till they had offspring that the individual is so closely adapted to the average environment of his race.

336. Self-fertilization is not uncommon in plants, and it occurs also amongst vegetative marine animals. Doubtless it has arisen as a substitute for cross-fertilization, owing to the uncertain occurrence of the latter amongst the individuals of stationary and scattered types. The germ-cells of an individual differ amongst themselves, but not, on the average, to the same extent as germcells derived from different individuals. In self-fertilization, therefore, the influence of conjugation, though still potent, is less than in cross-fertilization. Presumably, however, it is sufficient for the needs of the comparatively simple types among which it is found. Parthenogenesis is comparatively rare, and occurs as a rule among simple forms, the multiplication of which is very rapid, and which thus provide very abundant materials for Natural Selection. The theory that the function of sex is to bring about the retrogression of useless characters by the blending of parental traits, therefore, accords well, not only with the facts of bi-parental reproduction, but also with those of self-fertilization and parthenogenesis.

337. The problem of sex occupies at present a disproportionate share of biolngical attention. The study of it, under the name of Mendelism, is very fashionable. Nevertheless, from a practical point of view, indeed from every point of view, it is of lesser importance than many other biological problems. A complex and difficult question, it cannot be solved except by examining it from all points of view, by utilizing all the evidence available, and testing our thinking carefully. It cannot be too often or too forcibly reiterated that the only extensive body of evidence in our possession relating to normal intra-varietal breeding and the crossing of natural varieties is that drawn from human races. That evidence indicates decisively that offspring blend parental characters. To restrict our data to the scanty and imperfect material obtainable by crossing domestic varieties for a few generations, and even then by observing only the inheritance of glaring parental differences, is to court 
failure. It would be as reasonable to isolate a party of English people, rear them for three or four generations under conditions as foreign as they could possibly be made, and then, while excluding all other evidence as non-experimental and therefore fit only for essayists, attempt from the data thus obtained to write an account of the mode of development of English society. I think a historian who adopted such a method would not be deemed wise, even if he were deemed a historian; but the illustration does not greatly exaggerate the method and claims of some experimental workers who esteem themselves austerely scientific. "Experiments on crossing can give nothing but laws of crossing; it may be possible that some of these laws may be applicable to the breeding of pure races, but this cannot be decided without definite trial." 1 A full appreciation of the importance of experimental work need not blind us to the value of evidence differently obtained, nor to the fact that there are problems of heredity other than the effect of conjugation. In truth, heredity is a subject much wider, much more important practically, much more fascinating intellectually than is implied in a good deal of recent work.

${ }^{1}$ Mr Udney Yule. 


\title{
CHAPTER $\mathrm{X}$
}

\author{
SUMMARY
}

Adaptation-Stimuli to growth-The confusion as to what is innate and what is acquired-Variations-Spontaneous variability and insusceptibility of the germ-plasm-Recapitulation-The function of conjugation-BlendingAlternative recapitulation-Fluctuations and mutations-The difficulties presented by the different sciences-Methods of inquiry-The method of comparing races-Materials supplied by human beings-The greater problems of Heredity.

338.

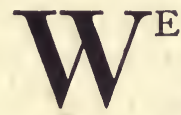

see then that the great outstanding feature of living beings is that, in part and whole, they are adaptations. For example, in man, the being we know best, very few characters exist the utility of which in past or present has not already been ascertained. Doubtless a proportion of his characters-for instance susceptibility to the charm of alcoholmay be mere by-products of evolution, accidental accompaniments of useful characters; but, speaking comparatively, they are rare. His nervous, circulatory, muscular, glandular, digestive, and other systems, the mass of his characters, his specific and varietal traits as distinguished from his personal variations, are nearly all demonstrably useful. We shall see that what is true of his body is true also of his mind. The variations of the individual are, of course, not all useful, but the spontaneous variability to which they are due and which supplies the materials for selection is an immensely useful trait which has been evolved through Natural Selection into an adaptation. Presumably adaptation is not less perfect in plants and lower animals than in man. Apparently they have been just as much subjected to selection. In all probability, therefore, if we are less able to trace the utility of all their characters, it is only because here our knowledge is less complete. It must be borne in mind, however, that nature can do no more than choose from the variations or combinations of variations that happen to be presented to her for selection. If, therefore, two sections of a species separated, but continued to exist under conditions which (were it possible) were identical, they would still be sure to differentiate; for neither the variations presented to nature for selection nor the accidents of survival would be quite 
the same. If, then, two varieties (e.g. of man) arise in different localities, it does not follow necessarily that in each locality they have diverged progressively in the most useful direction conceivable, but only that each has diverged in a useful direction.

339. Except actual injuries, as distinguished from vital reactions to injury, all the characters of living beings result from an interaction between the hereditary tendencies or potentialities of the individual and stimuli which awaken them to activity. In essence, all evolution consists in the evolution of these hereditary tendencies-that is, in the evolution of the germ-plasm.

340. There are many such stimuli to development. Amongst the principal are nutriment, use, and injury. In order to avoid unnecessary complications in the argument, I have ignored all stimuli save the three named, or rather I have roughly grouped under the head of nutriment all stimuli save use and injury. Here I have merely followed established biological custom, which distinguishes characters as inborn and acquired, and separates the latter into those resulting, on the one hand, from use, and on the other, from injury. Nutriment supplies, not only a stimulus to development, but the materials for all growth. All the 'normal' structures of many species appear to develop wholly under the influence of this stimulus, and many of the structures of all species so develop. In every case the beginnings of all growth are attributable to it, for, of necessity, it is the first stimulus to which each organism and structure responds. The capacity to develop under the influence of use or injury is present only in structures where it is useful, at a time when it is useful, and only to an extent which is useful. The power of responding by growth to injury (regeneration, healing) has been closely studied by biologists. That of responding to the stimulus of use has been more neglected. The utmost confusion prevails, for example, as to which characters in each species develop under the influence of nutrition, and which (if any) under that of use. When, however, we study mind we shall see that this question is the most interesting and practically important of all the problems of biology. Probably the great Lamarckian controversy, which still lingers, would have ended as soon as it began, if biologists had carefully defined their terms and noted in what species, in what characters, and to what extent the power of responding to the stimulus of use was developed : in other words, if they had studied this power as what it really is, an adaptation, a product of evolution, not a chance or necessary property of living protoplasm. 
341. Modern biologists are accustomed to divide the characters of multicellular living beings into those which are inborn or innate and those which are acquired, and to declare that only the former can be inherited by offspring. They are mistaken. No character, in any real sense, is more innate than any other. The child inherits nothing from his parents. These terms are convenient, inasmuch as they enable us to avoid circumlocution, but they are inaccurate. The true distinction between characters results from the stimuli under which they arise. The so-called acquirements arise under the stimulus of use or injury; the so-called inborn characters under other stimuli, especially that of nutriment. The true meaning of the statement, that acquirements are not transmissible, is that the characters which arise under the stimulus of use or injury in the parent are not reproduced in the child under the stimulus of nutriment. This sudden transference of characters from one category in the parent to another in the child certainly never occurs. In the first category they are products of evolution ; if they were reproduced in the second they would be products of miracle.

342. Offspring resemble their parents, but with minor differences. These differences are due either to differences in the play of stimuli on individuals, or to germinal differences. The latter are termed variations, and on them all evolution is founded. Massive evidence indicates that immensely the greater number of variations are spontaneous. This, in turn, implies that the germ-plasm is highly insusceptible to the direct action of the environment. A close examination of the evidence renders it clear that living beings could not have persisted on earth unless their variations had been spontaneous and their germ-plasm resistant.

343. Variations are either progressive or retrogressive. Since the general resemblance between parents and offspring is due to the fact that the latter in their own development closely recapitulate the development of the former, a progressive variation implies a prolongation of the parental development, a complete recapitulation with an additional step. On the other hand a retrogressive variation implies an incomplete recapitulation, an abbreviation. Since offspring recapitulate (with variations) the parental development, they necessarily recapitulate (with interpolated additions and subtractions due to innumerable interpolated progressive and retrogressive variations that occurred in the ancestry) the evolution of the species. It follows that retrogression implies a reversion to 
the ancestral type. It further follows that (as regards each character) ancestors are represented by the individual, not en masse, but in orderly succession. The so-called contributions of ancestors are nothing other than reversions, that is to say, failures to recapitulate the life-history beyond the points reached by the ancestors, or else the reappearance of hitherto dormant characters. The truth of recapitulation, though admitted by the majority of biologists, is universally ignored when theories of heredity are formulated. Biologists found their belief in recapitulation solely on very uncertain and fragmentary embryological evidence. They have not realized that, given facts which are admitted to be true, any method of development other than by a recapitulation of the life-history is unthinkable. This failure, together with the failure to distinguish the true nature of, the true distinction between, innate and acquired characters, has been in the past a principal obstacle to a scientific systematization of the facts of heredity, a main source of error, confusion, wild speculation, and endless controversy.

344. Evolution (i.e. adaptation) depends as much on retrogression as on progression. Direct Natural Selection is concerned almost exclusively with progression. Only seldom is it a cause of retrogression, which, with rare exceptions, occurs only on cessation of selection, and is due to the general tendency to reversion-i.e. the general tendency to abbreviate rather than to prolong the lifehistory. In the last analysis, however, this tendency is itself due to the Natural Selection of strains that possess it in the right degree. Retrogression planes away all redundancies, all useless variations and structures that have lost their utility.

345. The function of conjugation is to blend parental characters. The average experience of the race is thus substituted for the special experience of the individual. Moreover, in each blend retrogressive (i.e. the more ancient) characters tend to be prepotent over progressive (i.e. less ancient) characters. The effect of this blending, therefore, is to bring about the more or less rapid retrogression of useless characters - a retrogression which is only checked or reversed by selection. Sex, therefore, is as much a selecting agency as Natural Selection. Since every gradation occurs between equal blending of parental characters and the exclusive inheritance of the character of one parent only, it is convenient to class even exclusive inheritance under the head of blended inheritance. There is no such thing as alternative inheritance; there is only alternative patency and latency. When 
this occurs the child inherits the characters of both parents but develops those of only one. Blending then occurs between the patent traits of one parent and the latent traits of the other. Therefore, speaking practically, ${ }^{1}$ all inheritance is blended in biparental reproduction. Unit segregation, gametic purity, and independent inheritance of characters (in the Mendelian sense) are all myths that have been founded on experiment but have not been tested by it or in any other way. They are mere guesses. The evidence from which they have been inferred is very restricted and fragmentary, and, especially when considered in connection with other larger bodies of fact, really points to quite other conclusions.

346. In sexually dimorphic species every individual possesses one set of non-sexual characters, all of which are patent, and two sets of sexual characters of which only one set is patent. But if parents differ greatly in any non-sexual character, the reproduction of it tends to follow the sexual mode. This rarely happens in the normal intra-varietal breeding of natural varieties, for under such conditions individuals who differ greatly from the norm seldom survive and have offspring, but it is quite a common occurrence under artificial selection, which is distinguished from natural selection by the choosing of mutations rather than of fluctuations, the crowding together of newly formed varieties in the same locality, and their constant intercrossing through design or accident. All the evidence indicates that in nature varieties arise solely or almost solely under conditions of geographical separation. Artificial Selection and artificial varieties therefore differ profoundly from Natural Selection and natural varieties. In artificial varieties are found dormant traits which are practically unknown in natural varieties. Judging from the analogy of patent characters, it is an error to suppose that dormant traits persist for ever. Having no utility, they are not maintained by Natural Selection, and therefore tend to disappear in time. Thus when the horse is crossed with the Burchell zebra the reproduction of the long dormant ancestral stripes is very faint as compared to the vivid reproductions of ancestral coloration which may appear in offspring when two dull-plumaged breeds of poultry or two white varieties of garden flowers are crossed.

347. The progress of science depends first on the collection of verified facts, and second on their systematic arrangement. The facts must be verifiable or we cannot know them to be true. They 
must be systematized, or they are of no greater scientific value than the amorphous mass of data with which casual experience stores our minds. The methods by which facts are collected and arranged differ according to circumstances.

348. In some sciences, for example systematic botany, zoology, and anatomy, the facts are nearly all patent and, once ascertained, fall so naturally and easily into the right categories that the difficulty of systematization, of discovering the relationships between the facts, is reduced to a minimum. In others, the experimental sciences, for example physics and chemistry, very few facts are patent to our senses: the main difficulty lies in ascertaining them. Therefore special methods of investigation have been devised. In yet other sciences, for example heredity, though many facts are patent, others are obscured, and the relationships of nearly all are hard to ascertain. Consequently we have difficulties both in discovering information and in arranging it. The patent facts can be simply observed. The obscured facts must be rendered patent by means that clear away the obscuring conditions. The difficulties in arranging the facts must be met by testing our thinking with especial care. In this way only is it possible to achieve an approach to exactness.

349. Very remarkable notions as to what constitutes science are prevalent amongst sections of biologists. Thus medical men, who are biologists in the sense that they are students of a living being, frequently denounce deduction. Apparently they are unaware that the correctness of inductive thinking can be tested efficiently only by means of it, and that mathematics, the purest and most accurate of sciences, the model for the rest, is almost purely deductive. Again, systematic zoologists and botanists sometimes denounce 'speculation.' That is they denounce all thinking which is not very easy, all attempts to ascertain relations that are not very obvious. Yet, again, some experimental observers, appealing to physics and chemistry, sciences that are both accurate and experimental, often insist that biology must be made accurate by being made experimental. But physics and chemistry are not accurate because they are experimental, but because the data on which they are founded are capable of being as precisely measured as it is possible to measure anything, and because the students of them test their thinking with great care. In biology, we must, of course, when necessary, use experiment as well as every other means of ascertaining truth. But that is one thing. Quite a different thing is what has actually happened in 
practice-the limitation of thought to data provided by experiment, and the abandonment, from lack of material, of all attempts to ascertain the correctness of hypotheses. The experimental method can do no more than fill some lacunæ in our already wide knowledge of living, beings. In the past men have sometimes filled these gaps with imaginary facts and have speculated wildly. But such lapses from scientific caution afford no excuse for treating the gaps when filled by experiment with authentic facts as the whole of knowledge and then speculating still more wildly.

350. Splendid examples of scientific method, of combined induction and deduction, are the hypotheses of evolution and Natural Selection. The actuality of evolution was suspected even by the Greeks, but modern conviction, founded as it is on authenticated facts and carefully tested thinking, is more surely based. By linking together particular facts we infer (I) that it is possible to create new varieties of animals and plants by careful artificial selection, (2) that varieties, species, orders, and genera tend to shade into one another, (3) that species inhabiting the same or adjacent areas tend to have features more alike than those more widely separated, (4) that the remains of animals and plants preserved in geological strata indicate that related species, which were widely separated in time, were as a rule more unlike than those that were more nearly contemporary, and (5) that the structures of embryos are often more suggestive of lower types than of their own adult progenitors. Linking together all these inductions, as in reaching them we linked together particular facts, we are able to infer the hypothesis of evolution. In this way, not only is a deeper knowledge and a larger synthesis achieved, but we are able to perceive a new and very valuable aspect of truth which induction could never have established unaided by deduction, and which suggests many fresh inductions and deductions which, again, not only reveal still newer truths, but tend to test the aspect of reality, which was reached in part by deduction.

35I. Darwin accepted the theory of evolution and linked it with the inductions-which had previously been more or less familiar to all men-(I) that offspring tend to vary from their parents, (2) that some of them are better adapted to achieve survival than others, (3) that they tend to outnumber their parents, (4) that, nevertheless, the number of individuals in a species does not, as a rule, increase, and (5) that species are adaptational forms. Noting that all these inductions were in harmony and testing them rigorously, he linked them together in the hypothesis 
of Natural Selection. His reasoning was largely deductive, for, apart from the tests to which he subjected his thinking, his attention was not attracted by human disease and, consequently, he never observed Natural Selection actually at work. He was unable, therefore, to reach his wider synthesis through an actual observation of particular facts or test it by an appeal to them, but his work was fertile in suggestions-hence, for example, Poulton's attempt to demonstrate Natural Selection amongst chrysalises, and Weldon's attempt to prove its occurrence amongst crabs. Hence also many biometric inquiries.

352. Starting from the point at which Darwin stopped, accepting his theory of evolution by Natural Selection, and combining with it the hypotheses which we reach by induction, that species tend to retrogress on cessation of selection, and that offspring recapitulate the main features of the parental development, we are able to infer (I) that retrogressive variations tend to predominate over progressive variations, (2) that, with rare exceptions, variations are spontaneous, and that spontaneous variations tend to occur all round the specific mean, (3) that, therefore, the germ-plasm is highly insusceptible to change through the direct action of the environment, (4) that, apart from his own progressive variations, the development of the individual is an abbreviated and inaccurate recapitulation of the evolution of the race, and (5) that, therefore, every retrogressive variation is, in effect, a reversion. Here we formulate laws or generalizations of heredity which are probably as nearly fundamental as it is possible to reach in the present state of our knowledge. As in the case of Natural Selection, the process of reasoning, since we endeavour to ascertain whether our laws are in harmony with one another and with reality, is largely deductive. But, granting the premises from which we started, and none of them are seriously disputed, I believe we must not think at all, or we must think in terms of miracle, or we must accept the laws as true. And, very obviously, if they are true, if they are real laws, we have made a step towards rendering biology (or at least the study of heredity and evolution) a deductive science-towards establishing it on the basis of a few generalizations, to which a vast mass of data, already discovered or yet to be discovered, may be linked by chains of causation, that is to say, towards attaining that goal which is the aim of all science. ${ }^{1}$

1 " It will be seen hereafter that there are weighty scientific reasons for giving to every science as much of the character of a Deductive Science as possible ; for endeavouring to construct the science from the fewest and the simplest possible 
353. The method of inquiry on which Darwin principally relied, and which enabled him to affect so great a revolution in modern thought, was to compare varieties and races living under one set of conditions with those living under another. Having noted the

inductions, and to make these, by any combinations, however complicated, suffice for proving even such truths, relating to complex cases, as could be proved, if we chose, by inductions from specific experience. Every branch of natural philosophy was originally experimental; each generalization rested on a special induction, and was derived from its own distinct set of observations and experiments. From being sciences of pure experiment, as the phrase is, or, to speak more correctly, sciences in which the reasonings mostly consist of no more than one step, and are expressed by single syllogisms, all these sciences have become to some extent, and some of them in nearly the whole of their extent, sciences of pure reasoning; whereby multitudes of truths, already known by induction from as many different sets of experiments, have come to be exhibited as deductions or corollaries from inductive propositions of a simpler and more universal character. Thus mechanics, hydrostatics, optics, acoustics, thermology, have successively been rendered mathematical; and astronomy was brought by Newton within the laws of general mechanics. Why it is that the substitution of this circuitous mode of proceeding for a process apparently much easier and more natural, is held, and justly, to be the greatest triumph of the investigation of nature, we are not, in this stage of our inquiry, prepared to examine. But it is necessary to remark, that although, by this progressive transformation, all sciences tend to become more and more Deductive, they are not, therefore, the less Inductive; every step in the Deduction is still an Induction. The opposition is not between the terms Deductive and Inductive, but between Deductive and Experimental. A science is experimental in proportion as every new case, which presents any peculiar features, stands in need of a new set of observations and experiments -a fresh induction. It is deductive in proportion as it can draw conclusions, respecting cases of a new kind, by processes which bring those cases under old inductions" (J. S. Mill, Logic, Bk. II. chap. iv. §5). "The discoveries which change the method of a science from experimental to deductive mostly consist in establishing, either by deduction or by direct experiment, that the varieties of a particular phenomenon uniformly accompany the varieties of some other phenomenon better known. Thus the science of sound, which previously stood in the lowest rank of merely experimental science, became deductive when it was proved by experiment that every variety of sound was consequent on, and therefore a mark of, a distinct and definable variety of oscillatory motion among the particles of the transmitting medium. When this was ascertained it followed that every relation of succession or co-existence which obtained between phenomena of the more known class, obtained also between the phenomena which correspond to them in the other class. Every sound being the mark of a particular oscillatory motion, became a mark of everything which, by the laws of dynamics, was known to be inferable from that motion; and everything which by those same laws was a mark of any oscillatory motion among the particles of an elastic medium, became a mark of the corresponding sound. And thus many truths, not before suspected, concerning sound, become deducible from the known laws of the propagation of motion through an elastic medium; while facts already empirically known respecting sound become an indication of corresponding properties of vibrating bodies, previously undiscovered " (op. cit., II. iv. 7). "We have thus already come within sight of a conclusion which the progress of the inquiry will, I think, bring before us with the clearest evidence, namely, that in the 
differences between races and between conditions, he sought to account for the former by the latter. ${ }^{1}$ His very learning and the skill with which he used it contributed, through exhaustion of materials, to render the task of his successors difficult. The actuality of evolution was proved and the method of evolution demonstrated, but many problems of heredity remained. A great deal of splendid work was done subsequently, but it was felt that our knowledge of wild plants and animals was very vague, and an impression spread that laboratory methods alonewere capable of supplying information sufficiently exact.

354. But, clearly, if we are able to trace precisely the changes a race undergoes as a reaction to the conditions under which it dwells, if we are able to establish clearly the connections between causes and effects, we observe nothing less than an actual, a very perfect experiment. Such an experiment, since it is conducted by

sciences which deal with phenomena in which artificial experiments are impossible (as in the case of astronomy), or in which they have a very limited range (as in mental philosophy, social science, and even in physiology), induction from direct experience is practised at a disadvantage in most cases equivalent to impracticability; from which it follows that the methods of those sciences, in order to accomplish anything worthy of attainment, must be to a great extent, if not principally, deductive. This is already known to be the case with the first of the sciences we have mentioned, astronomy; that it is not generally recognized as true of the others is probably one of the reasons why they are not in a more advanced state" (op. cit., III. vii. 3 ).

1 "If what is called observation is at so great a disadvantage, compared with artificial experimentation, in one department of the direct exploration of phenomena, there is another branch in which the advantage is all on the side of the former.

" Inductive inquiry, having for its object to ascertain what causes are connected with what effects, we may begin this search at either end of the road which leads from one point to the other; we may either inquire into the effects of a given cause or into the causes of a given effect. The fact that light blackens chloride of silver might have been discovered either by experiments on light, trying what effect it would produce on various substances, or by observing that portions of the chloride had repeatedly become black, and inquiring into the circumstances. The effect of the urali poison might have become known either by administering it to animals, or by examining how it happened that the wounds that the Indians of Guiana inflict with their arrows prove so uniformly mortal. Now it is manifest from the mere statement of the examples, without any theoretical discussion, that artificial experimentation is applicable only to the former of these modes of investigation. We can take a cause and try what it will produce ; but we cannot take an effect and try what it will be produced by. We can only watch till we see it produced, or are enabled to produce it by accident.

"This would be of little importance, if it always depended on our choice from which of the two ends of the sequence we would undertake our inquiries. But we seldom have any option. As we can only travel from the known to the unknown, we are obliged to commence at whichever end we arc best acquainted with." (Op. cit., III. vii. 4.) 
nature under natural conditions, spreads over a great number of generations, includes the largest possible number of families and individuals, and results eventually in definite and unmistakable changes of considerable magnitude, is superior to any inquiry that can be devised in the laboratory, where only a very few generations can be studied, and where, since our power of observing and measuring the lesser variations is very limited, it is impossible to observe gradual but cumulative change. Provided the conditions of accurate observation are present, I think no thoughtful biologist would maintain that the method of comparing races is scientifically unsound. He might insist, however, that such conditions are never met. Undoubtedly they are difficult or impossible to find when we study natural species of plants and lower animals, and the study of domestic varieties is misleading. But one vast field of research has been left practically untilled by students of hereditythe human species. Here, if only because we are very familiar with the subjects of observation and, therefore, able to note small differences, our knowledge is more definite and precise than in the case of any other species. In certain particulars at least we are able to watch, under conditions insuring great accuracy, the tremendous and crucial experiments made by nature. ${ }^{1}$ We may see the differentiation of races occurring in such a way as enables us to trace the connexion between cause and effect. In a real sense we may extend our observations over hundreds of generations and thousands of years, for, in many instances, by comparing contemporary races, we are able to ascertain exactly the ancestral type of a race that has changed under the influence of a precisely known agent.

355. Occasionally I have expressed myself very positively in the preceding pages, and this will be displeasing to some readers. But the evidence on which my conclusions are founded is given in detail, and certitude is not necessarily dogmatic nor unscientific. I have tried, as best I am able, to test my thinking. I can only ask my readers, while bearing the evidence in mind, to think out the greater problems of heredity for themselves-to ask themselves whether evolution is ever anything but adaptation, whether it is possible for any class of characters to be in any true sense more innate or inheritable than any other, whether it is conceivable that the development of the individual can occur otherwise than by recapitulation, and therefore, whether retrogression can ever be other than reversion, whether the main mass of retrogression is 
due directly to Natural Selection, and, if not, whether it is not possible to formulate a rational theory to account for its occurrence, whether under the conditions in which life exists variations can be other than spontaneous, whether the power of growing under the stimulus of use is not just as much an adaptation as the power of repairing damaged parts under the stimulus of injury, whether the effect of conjugation is to mix parental characters as marbles are mixed or to blend them as colours are 'blended, and what in the one case or the other is the function, the utility, of the mixing, and above all to ask themselves whether adaptation is due wholly to the direct action of Natural Selection or in large part also to retrogression occurring in the complete absence of selection.

356. These are the greater problems of heredity. It is very possible that the solutions I have ventured to suggest are erroneous, but I am very sure that evidence exists which should enable us, if only we think clearly, closely, and comprehensively enough, to solve them, and that very much of this evidence may be obtained from a study of human beings. To that study we will now proceed. 


\section{CHAPTER XI}

\section{HUMAN DISEASES}

Neglect of the human species by modern students of heredity-The meaning of racial change-Progression and retrogression-The present evolution of manMicrobic disease-Air, water, earth, and insect-borne diseases-Toxins-Species that produce abundant extra-cellular toxins, and species that produce little or none-Phagocytes-The duration of disease and its cause-Acquired immunity.

357.

TORMERLY man, considered as a whole, as a bundle $\gamma$ of useful and related characters, occupied a good deal of scientific attention. Darwin traced his descent. Huxley sought to fix his place in nature. Spencer, Romanes, and Lewes dealt with his mental evolution-mainly from the standpoint of Lamarckism. But of late years the turn given by fashion to biological work has been unfavourable to an adequate study of him. He cannot be dealt with experimentally, at any rate not by inter-varietal crossings arranged by the worker. The results of crossings arranged by nature have not to my knowledge attracted much attention except in so far as is indicated by the surprising statement that human skin-colour is the only feature which crossed varieties blend perfectly. The alternative inheritance of various human abnormalities has been noted. Biometricians have devoted rather more attention to man than Mendelians and mutationists. They have endeavoured, on the one hand, to ascertain the degree of correlation between certain of his parts, and on the other to discover the extent to which some of his variations tend to be inherited. Only a very small portion of the total field has been covered, however, and some biometric work is vitiated by a failure to distinguish adequately between 'innate' and 'acquired' characters. ${ }^{1}$ The importance of drawing this distinction will become manifest as we proceed.

358. While studying heredity in connection with human beings we shall have to depend largely on patent facts-quite indisputable facts, however-collected by simple observation, and largely also on the special method of comparing races. The data left 
unused hitherto are extremely rich and suggestive, and will furnish, not only materials from which may be drawn some conclusions, which to me seem indisputable, but also the means of testing the thinking by which they are reached. We shall be embarrassed, however, by the mass of error which has collected around the subject. In all ages the thoughts of men have been concentrated on themselves, their parents, children, and fellows, and traditions have arisen which, though far from the truth, are yet-owing to a human mental characteristic of the highest general utility ${ }^{2}$-very hard to break down.

359. The reader must bear in mind certain conclusions which we have already reached. Like other living beings, man is manifestly a bundle of adaptations. Indeed, since he is better known, he is more manifestly a bundle of adaptations than any other type. Beyond doubt some human characters have no utility. Of these a few may be by-products of evolution correlated to more useful traits, the only one of the kind of any importance known to me being susceptibility to the charm of alcohol and other narcotics. Others, such as the colour of blood and bone, are chance qualities of useful characters-chance in the sense that in all probability they have not arisen through selection. These, however, do not enter into the account; of necessity blood and bone must have some colour. ${ }^{2}$ Yet others have lost their utility, and have become or are becoming vestigial. Speaking generally, in only a few instances has the past or present utility of a character not been ascertained. There can be no doubt, therefore, that the immense majority of human characters, whether in the trunk, limbs, or head, are adaptations. The growth of modern physiology implies merely an increased power of interpreting human traits in terms of their utilities.

360. We also reached the conclusion that all adaptation has resulted from the combined action of progression and retrogression, the former being a product of selection, the latter usually following cessation of selection. Given progression as an accompaniment of selection, and retrogression as a correlate of cessation of selection, close adaptation is obviously inevitable. We concluded, further, that this theory of evolution has for its necessary corollary a theory of heredity which supposes (I) that the great mass of variations are spontaneous, and, therefore, (2) that the germ-plasm, so far as its hereditary tendencies are concerned, is highly insusceptible to the direct action of the environment; (3) that ${ }^{1}$ See $\$ 663$.

See \$ 649 . 
retrogressive variations tend to predominate over progressive variations; and (4) that these three fundamental characters of living beings are themselves adaptations which have resulted from Natural Selection.

36r. Nearly all biologists at the present day accept the theory of Natural Selection; but some accept it with considerable reservations, and none, as far as I am aware, accept it in the form I have suggested. For example, the elimination of useless characters (variations and old-established parts) is still generally attributed to reversed selection. Sometimes progression is thought to result from an inherent growth-force that is only kept in check by Natural Selection, which therefore moulds the race by planing away superfluities. In other words, progression is thought to result, not from selection, but from a natural tendency for progressive variations to predominate over retrogressive variations. According to this theory, therefore, all species would progress were it not for Natural Selection. There is massive evidence, however, that this view, which appears to be especially popular amongst the supporters of the mutation theory, is mistaken. All parts, for example vestigial parts and the special features of prize domestic breeds (e.g. race-horses), which have no survival value, tend to disappear on cessation of selection, and we have only to observe Natural Selection in actual operation to perceive plainly that its special rôle is the causation of progression. ${ }^{1}$

362. My excuse for the positive attitude I assume must be that I offer, in the chapters that now follow, easily verified and, I think, very conclusive evidence of Natural Selection in actual operation; whereas the opinions I controvert are founded on a consideration of lower animals and plants, amongst which,

${ }^{1}$ As a rule the idea that one or the other kind of variations tends to predominate is implied rather than expressly stated in the theories of biologists. The problem has not often been especially formulated. I think most students of evolution believe, more or less vaguely, that progressive and retrogressive variations about balance one another. This view, however, leaves unexplained the retrogression of parts that have no selection value. Weismann's theory of Panmixia implies that retrogressive variations predominate, and he has attempted to account for the fact by his hypothesis of Germinal Selection. The view I have ventured to set before the reader differs from his merely in that $I$ attribute the predominance to a tendency evolved by Natural Selection, and do not speculate on the mechanism of processes in the germ-plasm concerning which I have no data. Mutationists and Mendelians ignore retrogression. At any rate, the subject is not to my knowledge mentioned in their works. Indeed the fact that retrogression constantly occurs on cessation of selection is not only quite ignored, but also is by implication controverted by many of them. See $\S 285$. 
admittedly, when wild, the operation of Natural Selection cannot be observed, and amongst which, when domesticated, it is largely in abeyance. Very many, if not all, biologists believe, also, that variations commonly result from the direct action of the environment on the germ-plasm, and found this opinion mainly on experimental evidence-that is, on evidence obtained from plants and animals which have been placed under conditions abnormal to the race and to the individual. But, as already noted, such experiments prove merely that it is sometimes possible to devise conditions in which the germ-plasm, like other living structures, is injured and yet not killed. They do not demonstrate that the variations of a species in its normal environment commonly arise in this way. It is impossible to prove a universal negative, and therefore, if only for that reason, we cannot demonstrate that variations are not so caused. Indeed, I do not maintain anything of the kind. I insist merely, on grounds which I believe are quite unassailable, that the conditions of life are such that it could not persist on earth unless the vast majority of variations were spontaneous-unless the germ-plasm were highly insusceptible to the direct action of the environment. We have already dealt with some of the evidence. But it will be well worth while to consider it more at length, especially as we shall thereby gain an insight into man's recent evolution, and be brought in contact with some practical problems of great importance.

363. We reached one other conclusion which bears on our present task, namely, that the predominance of retrogressive variations is less marked in the case of old-established than of more recently evolved characters. This, also, is an adaptation. The circumstance that continued selection tends to render characters more stable carries the advantage that ultimately a minimum of selection suffices to maintain a useful trait. The mortality resulting from selection is thus diminished, and the selection (and therefore the progression) of other useful characters is rendered possible without the death-rate exceeding the birth-rate.

364. Before discussing human heredity, it is necessary to define carefully the meanings we attach to such terms as 'evolution,' 'racial change,' and the like. They have both been used, especially by popular writers, to indicate two quite separate sets of phenomena, which, if we wish to avoid endless confusion, must be clearly distinguished. On the one hand are germinal or truly innate changes; on the other are changes due to a differential play of stimuli. Thus the colour difference between Scandinavians 
and negroes has a germinal origin, whereas that between Englishmen at home and in India is merely acquired. So, also, the mental difference between a naturally intelligent race and a naturally dull one is not the same as that between two races, one of which has been rendered more intelligent than the other by a better system of mental training. By such terms as evolution, racial change, progression, and retrogression we shall always in the present work imply germinal change. By so doing we shall follow an established biological custom, which, as a rule, is infringed only when the writer mistakes acquirements for germinal changes. It is often very difficult to disentangle the two, and the attempt to do so will form a main part of our future task.

365. Accepting for the moment, then, the hypothesis that racial progression is invariably due to selection, whereas retrogression results, as a rule, from cessation of selection, it is evident that, if we seek to ascertain the direction in which a human race is undergoing germinal change, we must study the causes of death. If we find that inferiority in any character results in a considerable mortality, or in a diminution of the average number of offspring, we may assume that in all probability the trait in question is undergoing progression; for here we have evidence that the race is not yet well adapted to the environment. On the other hand, total loss of utility, implying as it does cessation of selection, implies also a tendency to retrogression.

366. During the last few thousands of years man has altered his environment in one very important way and in that one way only. $\mathrm{He}$ has become increasingly civilized. Civilization implies, on the one hand, protection from most of the dangers which beset wild animals, and, therefore, cessation or diminished stringency of selection along those lines of evolution which raised man in the animal scale and ultimately made him human. On the other hand, it implies a fuller command over the resources of nature, which in turn implies a more abundant and regular supply of food, and that again implies a more crowded and settled population. The problem before us is whether this vast alteration of environment has resulted in appreciable germinal change-in appreciable progression or retrogression. Civilized man differs in many striking ways from the modern savage, and probably, therefore, from his remote ancestors. Our task is to ascertain how much of this difference is innate and how much due merely to a differential play of stimuli. We shall study first man's physical characters, reserving mind for a later section of this book. 
367. Popularly, and even by most scientific men, the human race is supposed to be undergoing progression in all sorts of directions. Others suppose that cessation of selection is resulting in general retrogression. But clearly, as in other species, the great majority of human physical characters are merely stationary. They are selected, but only to an extent which maintains an efficiency previously established. They fit the race sufficiently well to the environment, and only marked defect usually results in a failure to rear offspring. Judging from ancient remains and from a comparison with modern savages, civilized man differs little, if at all, from his remote ancestors in hands, feet, blood, bones, heart, lungs; and the like. Doubtless, owing to accidents of survival, to sexual selection, or to migration which brings different sets of selecting agencies into play, all races have undergone some structural changes; but these are comparatively unimportant. In obvious physical features, the features by which biologists are accustomed to measure physical change, humanity as a whole has undergone little innate alteration during the few thousands of years of civilisation. We are said to be more bulky than our ancestors of six or seven centuries ago, but there is no evidence that during the interval big individuals have been specially selected for survival. Probably, therefore, the increase in size is due merely to environmental changes, such as improved nutrition and treatment of the young, which permits better individual development. An analogous case is that the modern girl of the higher classes is taller on the average than her grandmother, and the modern field labourer than the factory hand. Some modern savages are taller on the average than the members of any civilized race, and some skeletons of the Stone Age are those of large men with well developed crania.

368. Of retrogression, also, there is little positive evidence. Thus, when suitably trained, civilized men appear to be, on the whole, just as keen of sight and hearing and as capable of enduring fatigue as savages. The Boers and Australian whites are cases in point. It is only when we compare man to the nearest lower animals that we seem to obtain positive evidence. Some of his senses, his hearing and sense of smell, for example, appear weaker. His jaws and teeth certainly, and probably his digestive apparatus, have undergone retrogression. $\mathrm{He}$ is less able to masticate and assimilate the raw coarse food on which his ancestors subsisted. But, even here, it is probable that much is attributable to the direct action of the environment on the developing 
structures. The senses of hearing and touch and possibly of smell become very acute in the blind, and civilized children are supplied with such soft food that their jaws are not stimulated to the utmost possible development. Their teeth, which owe nothing to the stimulus of use, and therefore attain their full size, are crowded irregularly together in the small jaws, and while retaining fragments of fermentable food in their interstices, are not cleansed by the tough fibrous substances which form a prominent part of the diet of savages. ${ }^{1}$ They are therefore particularly exposed to decay. African negroes have magnificent teeth; their relatives in America suffer much from caries; yet only a few generations separates the American black from a savage ancestry.

369. It follows that though man has not now so strenuous nor so active a struggle for mere existence as formerly, though exceptional strength, activity, endurance, beauty, or cleverness do not at the present day imply as a rule more than an average number of offspring; though men perish so very rarely and in such a haphazard manner from wild beasts and enemies and from privation that there is no real selection, though modern; weapons of war do not discriminate between the fit and the unfit in battle, yet since man's ancient characters are firmly established and since conspicuous defect still acts, on the average, as a bar to offspring, he has undergone little retrogression. Of progression on lines of ancient characters there is no valid evidence.

370. Nevertheless, since men still perish in great numbers before contributing their full quota of offspring to the race, some sort of selection is occurring. Ourfacilities for ascertaining its precise nature are particularly good. Indeed, this is the only instance in nature in which the operations of Natural Selection may be observed, not merely guessed at. In every civilized country tables of mortality are compiled from which we may learn exactly the causes which eliminate the inhabitants of civilized states, the number of deaths due to each cause, and the ages of those who perish. In the vast majority of instances civilized men die of disease. ${ }^{2}$ Disease

${ }^{1} \mathrm{~J}$. Sims Wallace, Decay in Teeth (London, Churchill).

2 In England and Wales 520,03I people died during the year 1905. Of these 19,437 perished of violence, mainly the result of accident in flood or field or in mines. Among the violent deaths, however, were 133 from homicide, I 7 from execution, and 3 from battle; 2 I persons died from starvation, 2638 committed suicide, but probably many of these suffered from physical or mental diseases; 813 are said to have died of plague. The remaining half million perished almost exclusively from disease, including from tuberculosis 55,759 , pneumonia 44,367 , bronchitis 38,9 I 5 , cancer 30,22 I, diarrhœe 20,534, measles I I,076, whooping-cough 8709, influenza 6953, diphtheria 5459, scarlet fever 3834, cirrhosis of liver 4008 , 
then is the only stringently selective agent amongst civilized men. The types it weeds out are those that are weak against disease, the survivors are those that are resistant to disease: it follows that the only racial progression, certainly the only considerable racial progression, that civilized human races undergo is one against disease.

371. This conclusion is likely to be controverted, and by none more strenuously than by students of evolution, most of whom have devoted attention exclusively to such peculiarities of shape, colour, size, and the like, as mark obvious differences between species and varieties, and who have not taken into account the actual causes which eliminate human beings under conditions of civilization. But, either heredity is of the same nature in man as in other species, or it is not. If it is not, how shall we account for the miracle of adaptation, and for the constant retrogression of useless parts? Man, while supplying indubitable evidence of progression when under selection, affords no clear instance of real progression in the lack of it. If retrogression constantly accompanies cessation or diminishing stringency of selection, it follows that only selection, which is sufficiently stringent, can be a cause of progression. Amongst civilized men, then, what are the causes of stringent selection other than disease? What types of men are being eliminated? In what direction precisely is progression occurring? I have known men who have attempted to answer these questions, but I have never known one who succeeded. At best they have merely guessed vaguely at lines of elimination the existence of which they were unable to demonstrate, or indicated lines of progression which they could not show were other than acquirements due to the better development (through improved conditions) of the descendants as compared with the ancestors.

372. The reader has only to refer to his own experience of life to perceive that not a single character save power of resisting disease is stringently selected. Except in extreme cases such traits as, for example, shortness of stature, muscular weakness, or lack of beauty or intellect, are not serious bars to offspring. But great susceptibility to disease is an absolute bar, for the individual perishes in early life. Relative susceptibility is a lesser obstacle, but individuals characterized by it are seriously handicapped, and $22 \mathrm{II}$ of alcoholism. Of the total number of deaths 280,302 were of people under 45 years of age, and 239,729 of older people; 18,87I infants died because prematurely born, 2343 of teething, and 12,933 of convulsions. Doubtless in the last three categories, disease, parental (through causing injury in utero) or filial, was a potent factor. 
and frequently perish without leaving offspring. It is conceivable, of course, that insusceptibility to this or that disease may be correlated to some other and apparently unrelated character such as height or colour or peculiarity of disposition, which therefore would undergo concurrent evolution as the race grew resistant to the disease. But, speaking comparatively, correlated characters of this sort are very rare, and the study of disease has revealed none, though the opportunities for observation are excellent; for we are able to compare races which are very susceptible to this or that disease with races which are highly resistant. Very certainly the comparison of such races reveals no such correlations.

373. Some biometric investigations, it is true, seem to indicate lines of progression and retrogression other than that related to disease; but the data on which they are founded are very scanty as compared with the evidence that can be gleaned from tables of mortality; they cover only a few generations, and make no very serious attempt to distinguish real variations from acquirements. In the case of disease we are able not only to obtain precise numerical information on a large scale as to the causes of death, but it is in our power to test such theories of evolution as we may found on that information in a very decisive way, by comparing races which have long and severely suffered from any disease with races that have been newly introduced to it. By this means we may discover, for example, whether the selective elimination which we suspect to be a cause of progression is a true cause, and whether the progression, if any, has correlated to it the growth of some other trait.

374. Of deaths due to disease by far the greater number are caused by microbic maladies, or by the abuse of such narcotics as alcohol and opium. Probably, with the exception of violent death, which is non-selective, and cancer, which occurs mainly during advanced life, and therefore exercises little or no selection, there are no other very important causes of elimination. Thus of the 76,844 deaths which occurred in England and Wales during the year 1905 from diseases of the heart and blood-vessels, it is probable that the great majority had a microbic or alcoholic origin, especially in the case of people who died before the end of the reproductive period. Doubtless, also, the deaths of very many of the $18,87 \mathrm{I}$ infants who perished because prematurely born, or who died of 'convulsions' (I 2,533), or of teething (2343), were due to the same causes acting directly on the children or through the parents.

375. Half a century ago the nature of microbic diseases was 
unknown. Thus when I was a boy in India, troops were marched across the wind to new camps in the endeavour to avoid cholera. At the present day even school children have some idea of the agencies which cause them. Sanitary authorities work with a knowledge of cause and effect, which is daily growing more exact, and hardly a year passes but some disease hitherto mysterious is traced to a microbic origin. As a consequence, both the prevention and treatment of disease are becoming more rational. Men hope with good reason to utterly abolish such terrible maladies as malaria, the treatment of which was formerly limited to attempts to cure, and which were supposed to be as irremovable a part of the natural features of a country as its mountains and rivers.

376. Human zymotic, infectious, or microbic diseases-tuberculosis, scrofula, lupus, influenza, measles, common cold, bronchitis, ${ }^{1}$ chicken-pox, smallpox, syphilis (great pox), and other venereal diseases, whooping-cough, diphtheria, pneumonia, rabies (hydrophobia), tetanus (lock-jaw), enteric fever (typhoid), scarlet fever (scarlatina), typhus, leprosy, septicæmia, peritonitis, malaria, dysentery, epidemic diarrhœa, cholera, yellow fever, plague, sleeping-sickness, erysipelas, rheumatic fever, puerperal fever, abscess, meningitis, cerebro-spinal (spotted) fever, mumps, opthalmia, phlebitis, tonsilitis (quinsy), beriberi, dengue, blackwater fever, Malta fever, and a host of others-are caused by the invasion of the body of man, and the multiplication within his blood or tissues of various species of microbes, minute unicellular plants or animals, which find their nutriment in him. Very many of these parasitic disease-producing (pathogenic) organisms have been seen under the microscope. In many cases the causal connexion of a species with the disease to which it gives rise has been demonstrated by an experimental inoculation of non-infected animals, and at the present day the doubts of a medical man as to the nature of the disease he is treating are often solved by the bacteriologist with his test-tube and microscope.

377. Some zymotic diseases are comparatively rare. The microbes may be uncommon, as those of spotted fever ; or they may not easily find entrance into the living body, as those of lock-jaw, or they do not readily establish themselves in the healthy tissues of the normal individual, as those of septicæmia. Others are so prevalent in some countries, and gain entrance to the body so readily that no man escapes infection unless he is "by nature"

${ }^{1}$ See The Sixty-eighth Report of the Registrar General of Births, Deaths and Marriages, p. cxiii. 
immune, or death, unless he possesses a high degree of resisting power-for example, the microbes of measles, common cold, whooping-cough, and tuberculosis in England, malaria in West Africa, and dysentery in India.

378. The species of microbes that cause disease in man, numerous as they are, form but a fractional part of the total number of parasitic species which afflict animals and plants, and these again are few as compared to the multitudes of unicellular organisms which find their nutriment in non-living matter. Some of the latter, the saprophytes, exist on the dead bodies of animals and plants, which but for them would cumber the earth unchanged. Each species fits its own particular niche in nature. All are capable of extremely rapid multiplication, a circumstance which not only secures the persistence of the species, but provides material for selection, and, therefore, facilities for quick adaptation to changing environments.

379. Of the microbes which cause human disease, some, for example those of typhoid, cholera, dysentery, and diarrhœa, enter the body in water or other nutritive substances. Since infected water is a very common vehicle, they are usually termed water-borne. The microbes of other species are commonly inhaled. The types which use this latter method of entrance are usually very minute ; they float in the air like fine dust, and therefore are termed airborne. Such are the microbes of influenza, smallpox, common cold, and many other maladies. Few of these types, owing doubtless in part to their small size, have been seen. While, seemingly, the microbes of all air-borne species are very minute, small size is of course not their only means of adaptation to their method of infection. For example, it appears likely that their mode of existence in the body is such that they are exhaled directly into the atmosphere by the breath of the sufferer, ${ }^{1}$ whereas the microbes of the water-borne maladies pass from him mainly in solid or liquid excreta. The air-borne microbes, owing to their mode of conveyance and their enormously rapid rate of multiplica-

1 There is evidence that the microbes of such diseases as scarlet fever may be carried into the atmosphere, or may infect the clothes through dried particles from the surface, especially in the later stages of the disease. In the latter case, the microbes are beyond the reach of the phagocytes or the enzymes (see $\$ \S 385-6$ ). This is probably the explanation of the well-known fact that a person recovered from scarlet fever is often infective to his fellows for a period which is long compared to that which is the rule with common cold or influenza. In its earlier stages it is practically certain that scarlet fever infects through the breath. Certainly it is very infective before there is any desquamation of the skin. 
tion, are exceedingly infective. Consequently, the immediate neighbourhood of a person attacked by them is dangerous to susceptible people. On the other hand, a person suffering from a water-borne malady may be approached with safety, the danger lying in the source, the nutriment (water or food) whence the disease was acquired, or in the solids or liquids he may excrete. Some species of microbes which enter by the breath, for example, those of tuberculosis, are comparatively large and heavy. They fall to the ground, whence, the sputum in which they are contained, having dried, they are swept up with particles of dust by currents of air. They are, therefore, termed earth-borne. As compared to the air-borne types they are in lesser multitudes in the air surrounding a sufferer whose neighbourhood, therefore, especially in well-ventilated spaces, is not so charged with infection. The microbes of other earth-borne diseases, for example those of tetanus, gain entrance through abrasions in the skin.

380. Yet other species, for example those of malaria and sleeping-sickness, are insect-borne; that is, they are conveyed from an infected to a susceptible person by blood-sucking insects. Lastly, some species, for example those which cause the venereal diseases, pass by direct contact from one person to another. Rabies, which is one of these 'contagious' maladies, is hardly ever, if ever, communicated by one human being to another, but is acquired through the bite of an infected individual belonging to some lower type of mammal.

38I. The terms contagious, earth, air, water, and insect-borne are convenient as indicating common modes of infection, but they do not necessarily imply that each of the various diseases infects in only one way. For instance, scarlet fever is usually air-borne and tuberculosis earth-borne; but it is probable that both are sometimes conveyed by milk. During the war in South Africa, enteric fever seems to have been spread by dust storms, or by flies which had been feeding on excreta. In England also flies are common vehicles for infantile diarrhœa during the summer months.

382. The bodies of the higher animals consist mainly of nutritive substances, and would be happy hunting-grounds for microbic types if they possessed no means of defence. Though the skin and food and air passages of the higher animals swarm with unicellular organisms, these are absent from the tissues of a healthy individual. The fact that the microbes of disease are often able to break down the defence and enter the tissues, whereas the 
harmless types are unable to do so, points to the fact that the former have special means of offence. When studying heredity in relation to disease, it is necessary to know something of these means of offence and defence, otherwise we are liable to fall into the error, which vitiates so much speculation on the subject, of mistaking somatic modifications for germinal changes.

383. Just as the different species of microbes have different means of entering the body, so, when once the entrance is affected, they tend to select different parts or tissues of the body for their habitation. Some, as those of malaria and probably of all 'general' diseases such as influenza, smallpox, measles, and scarlet fever, inhabit the blood or lymph. They multiply with great rapidity, and penetrate to every region. On the other hand, tuberculosis especially affects the lungs and lymphatic glands, more rarely the membranes of the brain, the joints, and the skin. The microbes of diphtheria are usually limited to the mucous membrane of the upper air passages, those of pneumonia multiply in the lungs, those of cholera, enteric fever, dysentery, and epidemic diarrhœa in the bowels. The microbes of tetanus are limited to the small area surrounding the wound by which they have gained entrance.

384. Though the area of body invaded by the microbes of a disease may be very small, yet in many cases the sufferer becomes extremely ill. Thus in tetanus he is thrown into convulsions, which resemble those produced by strychnine, and which indicate that his spinal column is in some way poisoned. We may search the cord in vain for bacilli. Again, it is possible to grow several species of pathogenic microbes in broth or other nutrient fluid outside the body. If this medium is filtered so that it is entirely free from living organisms, and then injected into the body, the symptoms of the disease may follow. It is evident, therefore, that at least some species of microbes elaborate poisons, or toxins as they are technically termed, which they set free in the medium surrounding them. In tetanus and diphtheria the toxins enter the blood stream at the small area affected, and so produce the symptoms of poisoning. Toxins, like pepsin and trypsin, which are digestive secretions from the cells of the alimentary tract of the higher animals, are evidently ferments; that is, substances which in some unknown way bring about chemical changes in certain other substances when the latter are exposed to their action.

385. The cells of the multicellular organisms are specialized 
for the performance of various functions that adapt the individual to his environment. The function of certain classes of cells is secretion. Thus the cells of the alimentary tract secrete the digestive ferments which prepare the food for assimilation, while such glands as the thyroid and the super-renal capsules manufacture 'internal' secretions which pass into the blood, and are essential to the well-being of the cell-community. Very important members of the cell-community are certain colourless cells, the white blood corpuscles or leucocytes, which float in the blood stream or wander in the tissue spaces. In a sense not altogether metaphorical, these cells are the sanitary inspectors, the police, the scavengers' of the community. They may be seen to approach intrusive microbes, enclose them within their own substance, and destroy them, apparently by digestion, or else perish themselves, apparently by poisoning. On account of this function of ingesting microbes they have been termed phagocytes.

386. The fluid part of the blood as distinguished from the corpuscles is termed serum. Several species of microbes, for example those of diphtheria, flourish in serum which has been withdrawn from the body and freed from blood-cells. It is, therefore, for them a nutritive, not a poisonous substance. But if microbes are introduced into the body enclosed in a capillary glass tube, the ends of which are plugged by a substance that permits the free diffusion of fluids, but prevents the escape of the micro-organisms or the entrance of the phagocytes, the latter collect about the tube in numbers, especially at the open ends where they form clusters. Presumably, as sanitary officials, they are attracted by the secretions (toxins) of the microbes. Presently the micro-organisms perish and disintegrate, apparently in much the same way as when actually enclosed in the substance of the phagocytes. Similarly during recovery from diphtheria the bacilli may be found perishing on the surface of the throat, even when not in contact with phagocytes. It appears, then, that the phagocytes, and possibly other cells of the body, secrete substances, as harmless to themselves as pepsin to the stomach cells, which act as countertoxins and poison the microbes, and that they are stimulated to this act by the presence of the microbes and their toxins. Presumably these counter-toxins are identical with or similar to the digestive ferments that destroy bacilli which are actually ingested by the phagocytes.

387. The toxins of the various species of microbes are not identical, as is proved by the fact that the symptoms of the 
different diseases are unlike; for example, the symptoms of the poisoning occasioned by diphtheria are different in type from those which occur in tetanus. Judging again by the symptoms, the virulence of the toxins of some diseases is much greater than that of others. Possibly there is not in nature a more deadly poison than the tetanus toxin. The microbes, few in number and located in a single small area, can produce only an exceedingly minute quantity, yet the sufferer is thrown into violent convulsions, and is often killed. On the other hand, even when large areas of the body are affected by leprosy, the person afflicted feels comparatively well. In measles, smallpox, influenza, and the other acute diseases, the infected person falls suddenly and violently ill. Evidently he is suffering from intense poisoning; but he may be infected for months by tuberculosis before he is even aware of illness ; and, even when he is dying, his symptoms are traceable to nutritive changes, and to the irritation and destruction of tissues caused by the microbes themselves, or to toxins and waste products produced by the microbes of normally saprophytic species, which have established themselves in the perishing areas of tissue, rather than to toxins secreted into the surrounding medium by the bacilli tuberculosis. Therefore, we may reasonably infer that, whereas some species of microbes produce virulent toxins which pass into the surrounding medium, the toxins of others are weak or are retained within the microbes.

388. This hypothesis is confirmed by another set of facts In acute diseases, such as diphtheria and tetanus, though the phagocytes crowd towards the infected area till the red and inflamed tissue surrounding it is full of them, yet, killed or paralysed by the concentrated toxins, they do not ingest the microbes, at any rate at first. If the sufferer dies they are unable to the end to cope with the invaders; but in cases of recovery they gradually gain the upper hand, and in the later stages of disease the disintegrating microbes may be seen within them. On the other hand, in such diseases as tuberculosis and leprosy, the phagocytes ingest the microbes from the first. The struggle is then, in a way, physical, not one conducted at long range like that fought out with the microbes that produce virulent toxins. Victory depends, so to speak, on personal prowess. If the microbes are victorious, they spread and multiply, and the sufferer eventually dies; if the phagocytes are victorious, the microbes are exterminated and the sufferer recovers.

389. Microbic diseases differ greatly in the suddenness of their 
onset, and in their duration. As a rule, the more sudden and violent the onset of a disease the shorter is its duration. Thus influenza, common cold, pneumonia, diphtheria, smallpox, and many others, develop and end quickly. After an illness of a few days the sufferer dies or recovers - at least he recovers from the actual disease, though injuries (sequelæ) inflicted by it may persist, for example deafness from scarlet fever. Before his illness he is free from microbes, as is proved by the fact that he does not infect his fellows; during it he swarms with them, for he is then very liable to convey infection ; after recovery, since he is no longer infective, he is free from them again. Something has banished the microbes. His body has undergone a profound and remarkable change. From being a soil in which the parasites were able to flourish and multiply, it has become impossible as an abiding-place to them. He has acquired immunity. He has developed, not under the stimulus of nutriment, but under that of experience (use). This kind of immunity, which follows recovery from disease, is obviously quite distinct from that power of resisting infection which develops under the stimulus of nutriment, and is termed inborn immunity, and is often seen in Englishmen, for example in relation to tuberculosis. In the case of some diseases, smallpox, measles and others, acquired immunity is usually permanent, but in the case of others, for example common cold, influenza, and diphtheria, it is generally temporary.

390. In strong contrast to the acute diseases are such chronic maladies as tuberculosis and leprosy. Here the onset of illness is marked by no sudden symptoms of deep poisoning; the complaint pursues a prolonged indefinite course; recovery, if it occurs, is as gradual as the onset, and is commonly accompanied by exacerbations and remissions of disease. The microbes are banished, not because that particular bodily change which is termed 'acquired immunity' supervenes, but because an improvement in general health, due to better nutrition, or surroundings, or some such cause, strengthens the individual, and with him his phagocytes, and enables them to destroy their enemies. Often, in spite of apparent recovery, the microbes may linger in the system for years; and if the health be lowered, the disease, uninfluenced by the past experience of the individual, may be started afresh by surviving microbes, or by a secondary infection from without, and so ultimately prove fatal. Recovery from such chronic maladies of indefinite length, therefore, is precarious; it affords no protection, no increase of resisting power, no 'acquired immunity.' Unlike a man who has recovered from smallpox, one who has experienced tuberculosis is always in 
peril-in deadly peril, if exposed to reinfection under unfortunate conditions. The fact that he has experienced the disease is evidence that he is susceptible to it, not that he has acquired increased resisting power.

39I. Between such diseases as smallpox which run a short, sharp, definite course during which acute poisoning occurs, and such maladies as tuberculosis which are unmarked by acute poisoning, and run a course of quite uncertain but always relatively prolonged duration, lie such complaints as enteric fever and syphilis. The onset, course, and recovery from enteric fever, unlike that from smallpox, is comparatively slow, but not so slow as in the case of tuberculosis ; nevertheless, there are very distinct symptoms of poisoning, and the duration of the disease is pretty definite. In syphilis, the onset of the disease is even more delayed, the symptoms of systemic poisoning as indicated by fever and a feeling of illness are, as a rule, hardly observable, ${ }^{\mathbf{1}}$ and the microbes may persist in the system for two or more years. Nevertheless, the disease ultimately confers an immunity which is usually permanent.

392. Malaria occupies a curious position. Its toxins are abundant and virulent, and the reaction to them is sharp and rapid. The individual develops high fever and other symptoms of poisoning; but presently the symptoms abate; apparently the microbes cease to produce toxins; the individual appears to acquire immunity. But this phase is of very short duration. After an interval, which may be measured by hours or days, the signs of poisoning recur. During the period of intermission the microbes seem in a resting stage, when, like the tubercle bacilli, they are highly resistant to the phagocytes. In individuals belonging to races which have had much experience of malaria a more permanent immunity slowly arises. Thus all, or nearly all, negro children on the West coast of Africa suffer long from malaria, and many perish of it, but the adults are more or less free of it. At any rate, they are much more resistant. Blacks from the American Islands, many of whom enter the country as soldiers in West India regiments, suffer severely, and it may be fatally at first, a fact which has led Koch and others to the highly erroneous conclusion that no race is more resistant than any other to malaria. But West Indian blacks, comparatively few of whom perish, suffer much less than the white officers. If they survive for some months

1 In syphilis the development of the rash is sometimes marked, as in measles, by a rise of temperature indicative of poisoning. Indeed, the evidence of the presence of toxins in syphilis is clear. See \$\$ 4I6-4I8. 
they acquire immunity; whereas the whites continue to sufferif anything more and more severely as they become weakened. The latter cannot acquire permanent immunity. They cannot rear families, and a white settlement in African swamps or forests would soon become as extinct as the British colony in Darien. The following from Miss Mary Kingsley is very much to the point :-

393. "Yet remember, before you elect to cast your lot with the West Coasters, that 85 per cent. of them die of fever, or return home with their health permanently wrecked. Also remember that there is no getting acclimatized to the Coast. There are, it is true, a few men out there, who, although they have been resident in West Africa for years, have never had fever, but you 'can count them on the fingers of one hand. There is another class who have been out twelve months at a time, and have not had a touch of fever; these you want the fingers of your two hands to count, but no more. By far the largest class is the third, which is made up of those who have had a slight dose of fever once a fortnight, and some day, apparently for no extra reason, get a heavy dose, and die of it. A very considerable class is the fourth-those who die within a month or a fortnight of going ashore.

"The fate of a man depends solely on his power of resisting the so-called malaria, not in his system becoming immuned to it. The first class of men I have cited have some unknown element in their constitutions that renders them immune. With the second class the power of resistance is great, and can be renewed from time to time by a spell home in a European climate. In the third class the state is that of cumulative poisoning; in the fourth of acute poisoning." 1

394. Now since acquired immunity arises most rapidly in acute diseases in which virulent toxins are most abundantly and quickly produced, since it arises slowly when toxins are less abundant or more gradually developed, and since it never arises when the evidence indicates that poisoning by toxins plays a minor part or no part at all in the disease, it is clear that acquired immunity is due directly to the presence of the toxins, not to the presence of the microbes. This hypothesis is confirmed by the fact just mentioned, that in acute diseases the phagocytes are able to approach and destroy the microbes as soon as the symptoms of acute poisoning are mitigated, whereas in the more chronic maladies, though from the first undeterred by toxins, they are

1 Travels in West Africa, pp. 526-7. Macmillan \& Co. 
unable to overcome the microbes except after a prolonged struggle from which no acquired immunity results.

395. It is a noticeable and very important fact that immunity acquired against any acute disease does not confer immunity against any other. Thus experience of smallpox or scarlatina affords no protection against measles and whooping-cough. The toxins differ, and therefore immunity to each disease must be acquired separately.

396. Obviously, the various species of microbes are adapted by different means to the environment. In the search for nutriment they enter the body in different ways. Some species defend themselves from the phagocytes by means of toxins which they secrete into the surrounding medium; others have evolved great powers of resistance which doubtless depend in part at least on toxins retained within themselves. In no case is the death of the host they inhabit of advantage to the microbes. They are adapted for the temperature, nutriment, and other conditions met in the living body. The dead body becomes the prey of the bacteria of putrefaction. The death of the individual, the cell-community attacked by them, is comparable to the destruction of a hive by the bee-keeper in an attempt to secure the honey. Continued multiplication of the microbes within the system, however, insures the death of the host, if only by interference with the functions of life. The preservation of their species, therefore, demands that the microbes shall pass through an unending succession of living individuals. In acute diseases this is secured by rapid multiplication and migration to fresh and susceptible persons during the short interval of safety afforded by the toxins before acquired immunity or death supervenes. In the more chronic maladies there is less need for rapid multiplication and migration. The host is not poisoned. His continued existence is an advantage as affording a continuous supply of nutriment and prolonged opportunities for infecting other individuals. However quickly the microbes may multiply, they never increase at the tremendously rapid rate which characterizes the organisms of the acute diseases. Thus, in a few days or weeks, measles, influenza, or smallpox may spread over a vast area of country, and infect millions of people. The progress of tuberculosis and leprosy is always much slower. 


\section{CHAPTER XII}

\section{ACQUIRED IMMUNITY}

Intra- and extra-cellular toxins-Susceptibility to disease-Methods of altering virulence-Parasites and saprophytes-Smallpox-Rabies-Anthrax-Diptheria -Active and passive immunity-Antitoxin-Syphilis-Snake venom, and vegetable poisons-Yeast-The nature of acquired immunity-Pasteur's theoryChauveau's theory - The theory of neutralization-The theory of habituationEhrlich's side-chain theory-Inborn and acquired immunity.

397.

7 HE nature of acquired immunity and the means by which it is acquired have long been subjects of debate. Many hypotheses have been formulated. Since the mass of facts is enormous, and the difficulty of tracking their relations very great, the subject is an exceedingly difficult and complex one. Probably a workable idea of it will be most easily conveyed to the reader if we begin by setting down an array of facts, all of which I believe have been verified; or, at any rate, few, if any, of which are disputed.

398. (a) Acquired immunity is evidently a reaction against toxins. The toxins of the acute and sub-acute diseases, against which alone immunity is acquired, are set free in the medium surrounding the microbes much as snake venom is set free in the blood-stream of the victim. Other toxins, intra-cellular toxins, are more or less retained within the microbes. Thus, if the bacilli of tuberculosis, the extra-cellular poisons of which are non-existent or very feeble, be triturated in a mortar and treated with water, a poison, tuberculin, may be obtained, which, when injected, produces symptoms markedly different from those which occur in the actual disease. (b) Some species of microbes can be cultivated in bloodserum outside the body-not only in the serum of susceptible individuals, but also in the serum of those who have acquired immunity. (c) Immunity is influenced by individual peculiarity, race, and age. Thus many Englishmen are under ordinary conditions immune to tuberculosis; "white rats, adult dogs, many kinds of birds, and frogs are naturally immune to the bacillus of anthrax, which is very fatal to cattle, common rats, field-mice, 
and man. Algerian sheep resist the organism, whereas other breeds of sheep readily succumb. Dogs are practically immune to tuberculosis, while guinea-pigs are killed by the most minute dose of the bacillus. Rats and mice are not susceptible to diphtheria. In none of these cases, however, is the immunity absolute. By altering the circumstances of the animal it is generally possible to render it susceptible to the disease. Thus, by keeping frogs at a raised temperature, it is possible to infect them with anthrax; and by overtiring animals by excessive work they may be made susceptible to infection with organisms to which they otherwise possess almost complete immunity." 1

399. (d) As a general rule parasites flourish best in the species of animal which they normally inhabit, doubtless because they have evolved adaptation to that particular habitat; for this reason human diseases more readily afflict allied species (mammals in general and monkeys in particular) than types less akin. (e) Microbic species, if removed from their normal habitat (e.g. from one species of animal to another, or from the living body to nonliving media), are apt to alter their degree of virulence. The longer they inhabit a species of animal the more virulent, as a rule, do they grow for that species till a degree of virulence, sufficient to enable the microbes to persist, is reached. Thus the microbes of rabies taken from dogs and passed through a series of rabbits have their virulence immensely exalted for rabbits, though it is said to be lessened for human beings. ${ }^{2}$ When transferred to non-living media microbes tend to lose their virulence and become saprophytes. The only rational explanation appears to be that in the former case selection of the microbes that are most virulent to the new type of host, and therefore best able to defend themselves from his phagocytes, causes progression; whereas in the latter cessation of selection leads to the loss of the now useless virulence. On the other hand, normally saprophytic organisms may be rendered virulent by placing them in the living body, first in situations where they are least exposed to the phagocytes, and then in situations where they are more exposed. Some organisms, normally saprophytic (e.g. the tetanus bacilli and those which cause putrefaction) are intensely poisonous. Apparently their toxins have been evolved, not through a struggle with phagocytes, but probably with other lowly organisms.

400. ( $f$ ) It has been conclusively proved experimentally that if smallpox be passed through a series of calves it becomes cowpox;

${ }^{1}$ Bosanquet, Serums, Vaccines and Toxines, p. I 3.

${ }^{2}$ Op. cit., p. 153 . 
in the horse it becomes horse-pox; transferred back to man it is vaccinia. By then the nature of the microbes has been profoundly altered. Owing probably to a loss of virulence (i.e. offensive and defensive power), they are unable to spread over the body, but are restricted to the spot of inoculation, where they and their toxins are most concentrated. The toxins, however, permeate the body, and induce a general change, from which results recovery from vaccinia and immunity to smallpox. The absence of the microbes from the blood-serum, and therefore from the lungs, prevents their diffusion by the breath. Probably it is for this reason that vaccinia, unlike smallpox, is not air-borne. Consequently, it does not infect people in the vicinity. It has become like rabies and syphilis, a disease which is communicated only by direct contact and under particular circumstances. ${ }^{1}$ It does not, during its passage through a series of human hosts, again evolve the ancient characteristics of smallpox because, on the one hand, the failure of its microbes to pass naturally from a sufferer to his fellows results in the extermination of all that are not artificially removed, so that there is no survival of the fittest as regards the power to migrate, while, on the other hand, man artificially enables the more and the less fit to migrate alike. Human races which have had no ancestral experience of smallpox, which have not been weeded out by it, have comparatively low powers of resistance. A party of Esquimaux, who visited Berlin and were vaccinated there, developed a general disease resembling or identical with smallpox, and perished of it. ${ }^{2}$ The microbes, therefore, were able to spread over the entire body. Doubtless if vaccinia were passed through a series of such people, and then through more resistant types, it would again become smallpox-just as harmless saprophytic organisms become virulent parasites if passed, under favourable circumstances, through a series of living hosts.

40I. (g) Rabies in dogs is a very deadly disease. If passed through a series of rabbits, it becomes still more virulent, at any rate, for rabbits. If passed through a series of monkeys it becomes milder. Pasteur secured complete immunity from virulent rabies by inoculation first with the mildest type (from monkeys), then with more virulent types, and lastly, with the most virulent type (from rabbits). Subsequently he discovered an even better method, that

1 Not all 'general' diseases, malaria for example, are air-borne. To be airborne it is necessary not only that the microbes shall find their way to the air passages, but also that they shall be adapted for transmission through the air.

${ }^{2}$ The Scottish Medical and Surgical Journal, April I900, p. 330. 
now in use. Rabies develops slowly in an infected person. The microbes inhabit especially the great nervous centres, the brain and spinal cord. Pasteur dried a series of cords from infected rabbits, made emulsions from them, and injected the emulsions into individuals who had acquired the disease, but had not as yet developed the symptoms. He began with a cord which had been artificially dried for fourteen days. Next he used in succession fresher cords, till he injected material from one which was absolutely fresh, and which would have infallibly communicated the disease had the previous treatment not been undergone. As a result the individual, so far from acquiring the disease, developed immunity.

402. (h) Pasteur subjected the bacilli of anthrax, for a longer or shorter time, to an abnormal degree of heat. He found they became 'attenuated' (i.e. lost their virulence) in proportion. If, now, sheep were inoculated, first with bacilli of little virulence, and then in succession with those of greater virulence, they could eventually be rendered immune to those of the greatest virulence. Attempts to pass directly from weak to strong bacilli led to the death of the infected sheep. (i) A few microbes are not, as a rule, sufficient to infect even a very susceptible individual. They are destroyed by the phagocytes, and tend merely to induce an increase of resisting power. Rabbits are susceptible to anthrax, and young animals more susceptible than older individuals. It has been found that not less than 16,000 bacilli must be injected into a young rabbit to induce the disease.

403. ( $j$ ) If diphtheria bacilli be cultivated in broth, outside the body, they render the broth intensely poisonous with their toxins. If the broth be injected, at first in small but afterwards in larger doses, into a horse, the animal acquires immunity, and remains in good health during the rest of the treatment. But immediately after a large dose his blood-serum is poisonous to other animals which have not been similarly treated. If, however, the serum be withdrawn from him after a fit period, it is not only harmless, but helps another animal (e.g. man), who is suffering from the actual disease, to recover; or, if not suffering, it renders him, for a time at least, insusceptible. This is the celebrated antitoxin treatment which has greatly reduced the mortality from diphtheria, and is yearly being extended to other diseases, the medical treatment of which was formerly of little value. (k) Roux found it possible to obtain at different times two lots of serum of equal value from a prepared horse, though no intermediate injection of toxin had 
been made. ( $l$ ) An injection of a right quantity of antitoxin confers a 'passive' immunity which is less enduring than the 'active' immunity which results from actual experience of the disease. Some authorities suppose that the former is due merely to the presence of neutralizing substances introduced with the injection of antitoxin, or afterwards produced under its influence by the individual, and that it persists only so long as these remain within the system; whereas active immunity is thought to be a vital reaction to the microbes themselves. But probably the truth is, that active and passive immunity are merely degrees of the same thing, and that in any disease the duration of immunity depends more on the severity of the illness, and on the consequent completeness of the reaction, than on the presence or absence of the microbes. A kind of passive immunity may be induced by the repeated injection of small quantities of microbes. Workmen in sewers acquire a passive immunity to enteric fever, doubtless through constant experience of small doses of bacilli. Dwellers in malarious countries, or in places where yellow fever is prevalent, live immune till after a sojourn in a more healthy climate. One vaccination mark confers an immunity which is more passive than that conferred by two, that conferred by two is less enduring than that conferred by more, and still less lasting than that acquired through experience of actual smallpox. Judged by its duration, immunity against such diseases as diphtheria, influenza, and common cold is always passive.

404. (m) Just as immunity acquired against any disease does not confer immunity against any other, just as vaccinia confers immunity only against smallpox, so the antitoxin of any one disease confers no resisting power against any other. (n) Within limits, a dose of toxin large enough to be poisonous may be rendered harmless by previous intermixture with the corresponding antitoxin. This 'neutralization' is somewhat less marked if the two be simultaneously injected into separate parts of the body, and still less marked if the antitoxin be injected some time after the toxin-which, in effect, is what is done when disease is treated by means of appropriate serum. A mixture of toxin and antitoxin, for example that of tetanus, in a proportion just harmless to a rather resistant animal of a susceptible species, for example a mouse, is poisonous to a more susceptible animal of the same species, or to an animal of a more susceptible species, for example a guinea pig. An animal which has acquired immunity to a disease (i.e. which has acquired the power of resisting infection) 
does not necessarily retain its power of tolerating large doses of toxin when these are injected.

405. (0) Syphilis is a disease of such long duration that it may cover several pregnancies in a woman. As a rule, the infants that are born during her illness suffer less and less as the disease progresses. Thus, speaking generally, if she have rapidly recurring pregnancies, at first she miscarries early in the pregnancy, then a dead but fully developed child is born, then a child which appears healthy, but soon wastes and dies, then one who survives but exhibits signs of disease, and lastly one which shows no signs of it. Even if both parents be diseased the successive children tend to suffer less and less. If a diseased woman bears children to successive men, then, whether the male parents be diseased or not, her successive children tend to suffer less and less as time goes on till they do not suffer at all. But, if a diseased man has infected children by a succession of women who were not previously diseased, the last child tends to suffer as severely as the first. Evidently, then, while a woman may mitigate the disease for her offspring, a man cannot. Though a woman suffering from the disease tends to confer immunity on her surviving children, yet a woman recovered from it confers none on children that are not infected-at least, judging from the analogy of other diseases, so we must suppose, for a mother who has suffered from and acquired immunity against measles, scarlatina, smallpox, or any other malady, confers no immunity on her offspring. A woman who has recovered from syphilis has never been known to bear a diseased child to an infected father. But a woman who has not, and never has had the disease, may bear an infected child to such a mate. The bearing of the infected child confers immunity on her, for she never contracts syphilis when suckling it, though healthy wet nurses may. A diseased woman may bear a healthy child which, presumably, is immune, since it does not acquire the disease when suckled by her. The proof that it is not infected is afforded by the fact that it does not convey the disease to a healthy wet nurse.

406. ( $p$ ) Snake poison (a toxin) may be swallowed in doses a thousand times larger than would suffice to cause death if injected under the skin. Immunity instead of death results. The Bushmen of South Africa are declared on good authority to seek and obtain safety from snake-bite by swallowing poison glands. Snake venom artificially attenuated is now successfully used as an antitoxin for the cure of snake poisoning. (q) Nicotine, opium, strychnine, and other vegetable poisons, many of which are used as medicines, are, 
in effect, toxins-that is, they are defensive poisons which have been evolved as means of protection against animal enemies, insects or herbivora. Usually such toxins, besides being poisonous, have an unpleasant taste. As is well known, use enables the individual to tolerate immensely increased doses of some vegetable poisons, such as nicotine and opium; in other words, a high degree of immunity may be acquired. The vegetable toxins abrin, ricin, and crotin are probably of a proteid nature, resembling the toxins of disease in having a very complex chemical composition. In the case of each of them it is possible to manufacture an antitoxin protective against the corresponding toxin. ( $r$ ) If the yeast fungus be placed in a solution of sugar it consumes the latter and excretes alcohol. Alcohol is not a toxin, a defensive weapon, but a waste product, comparable to the urine of man not to the venom of snakes. When it attains a certain strength (about 14 per cent.) in the solution the fungus perishes-just as the cells of a man's body perish if his excreta be retained as in kidney or liver disease.

407. Here we have a confused mass of data, culled from many sources. Our task is to ascertain the relations of the facts and so link them together, so systematize them, as to discover, if possible, how immunity is acquired. Pasteur supposed that each species of microbe finds in the body of its host a special pabulum, the exhaustion of which results in the starvation of the microbes, and therefore in immunity-permanent if the pabulum be not renewed, temporarily if it be renewed. But Nature has evolved both man and microbes as bundles of adaptations. It would be strange if man possessed a series of pabula which are not necessary to his well-being-for he lives very well after recovery-but which render him liable to disease. Chauveau supposed that the microbes like yeast, are killed by the concentration of their own excreta, and that immunity is permanent or temporary accordingly as these waste products are permanently bottled up within the body, or eliminated. But waste products are usually less inimical to the organism producing them than to most organisms to which they are strange. Thus yeast fungi are able to live in a solution in which the percentage of alcohol is as high as thirteen or fourteen ; but no man could live if alcohol formed anything like that proportion of his total body weight. Both Pasteur's and Chauveau's hypotheses are decisively negatived by the fact that microbes may flourish in sera taken from immune individuals.

408. Highly popular is, or was, the hypothesis that microbes or their hosts produce substances (the antitoxins) which chemically 
neutralize and render harmless the toxins-much as an acid neutralizes a base-and so enable the phagocytes to overcome their enemies. The supporters of this hypothesis point to the suggestive facts that, within limits, toxins are rendered harmless to an animal if the corresponding antitoxins are first injected in sufficient quantities, and that, if mixed with the antitoxins before injection, they are neutralized more thoroughly, and therefore are less poisonous than when injected simultaneously but separately, and, further, that when mixed outside the host a definite amount of antitoxin will render inert a definite amount of toxin. But a number of additional facts indicate that the neutralization theory is untenable. If a fatal dose of toxin be rendered harmless by a minimum quantity of antitoxin, and then another lethal dose of toxin be added to the mixture, the latter should become lethal once more. But it does not, it merely causes a very slight illness. It needs the addition of quite a number of lethal doses to make the mixture deadly. Again a mixture of toxin and antitoxin may be made which is harmless for one animal (e.g. mouse) but poisonous to another (e.g. guinea pig), a fact which proves that the reaction that results in immunity is, not merely a chemical, but a vital one. Yet again, if a mixture of diphtheria toxin and antitoxin which is harmless to a mouse be made, it is rendered poisonous by heating it to the boiling point of water. Similarly if the mixture be placed in a porcelain filter the toxin passes through, but the antitoxin remains, a fact which is decisive that the interaction between the two substances is not of the nature of a chemical combination.

409. Both toxins and antitoxins are substances of highly complex chemical composition ; each toxin is 'neutralized' only by the corresponding antitoxin; the power of producing its particular toxin has been evolved by every species of microbe; but antitoxins as shown by the acquirement of immunity may be produced by races which have had no previous experience of the corresponding diseases; antitoxins have been found in the blood of animals a few minutes after the injection of the corresponding toxins. Therefore the hypothesis of the chemical neutralization of toxins by antitoxins elaborated by the individual attacked involves the assumption that the animal body is a species of magic bottle which instantly produces, under conditions never perhaps experienced by the race, elaborate substances exactly suited to the needs of the moment. It is, in fact, a hypothesis of immunization by miracle. Equally founded on an appeal to the supernatural is the hypothesis, which is or was popular, that the microbes after producing toxins which 
defend their lives are good enough to produce antitoxins which compass their own destruction.

410. The theory of neutralization, so simple at first sight but so incredible when examined closely, is one that would naturally occur to students of chemistry which to some extent all medical men, and more especially all bacteriologists are by training. A theory hardly less obvious and certainly more probable, is more likely to find favour with students of evolution which no medical men are as yet, at least by formal training. Men get 'used' to opium and nicotine just as they get used to exertion or heat or anything else. Opium and nicotine are toxins in a real sense. No one has suggested that the power of tolerating increased doses of them is due to the production of neutralizing substances, any more than they have suggested that the power of tolerating heat or fatigue is due to the formation of substances which chemically neutralize heat or fatigue. In each case the growth of toleration, like the growth of the limbs after birth, is plainly one of that large class of acquired characters by means of which the higher animals achieve part of their development and by which they are made so adaptive. There does not seem to be any very valid reason why we should place acquired immunity to disease in another category and ascribe to it a miraculous origin. Certainly, antitoxins seem to neutralize toxins, but it is possible to find an explanation which savours less of the supernatural.

4II. The spinal cord of a rabbit in the later stages of virulent rabies contains microbes and their toxins, but, presumably, little or no antitoxin, for the animal, if not killed, invariably perishes. But, if we kill the rabbit and dry the cord, antitoxins soon begin to appear. Evidently, moreover, the quantity, or quality, or both, of this substance alters in a peculiar way from day to day; for, if we inject an emulsion from a cord of a freshly killed rabbit into an infected man, we do not cure his disease. Nor do we cure it if we make the emulsion from a cord which has been dried a fortnight. To achieve the desired result we must follow a certain routine; we must begin with a cord that has been thoroughly desiccated and then proceed by fresher and fresher cords till at last we inject one that is absolutely fresh and virulent. Here the theory of neutralization clearly breaks down. The cells of the cord are dead and dried and cannot produce a neutralizing substance; the microbes are dead or dying and are wildly unlikely to produce it ; most decisive of all, the immunizing agent is not found in any particular cord, but only in a succession of cords when these are used 
in a certain order. Again, if we pass smallpox microbes through a succession of calves, we attenuate the disease. Presumably, we so weaken the toxins that the microbes are unable to spread through the body. Vaccinia, with its weakened toxins confers immunity against smallpox, for, if a man has recovered from the former, his phagocytes are able to destroy the microbes of the latter when brought in contact with them; hence his acquired immunity. In the same way it is possible to immunize against rabies by passing the microbes through a series of animals (monkeys) which 'attenuate' it. Again by subjecting anthrax microbes to an abnormal degree of heat we are able to attenuate them. By inoculating first with much attenuated microbes, and then in succession with less attenuated microbes, we are able to bring about immunity against the most virulent form of the malady. Since in these cases, we introduce directly, not antitoxins but attenuated microbes, then if the theory of chemical neutralization be correct we must make the incredible hypotheses that the passage of rabies through monkeys, of smallpox through calves, and the subjection of anthrax to heat so alters the microbes that they produce substances which exactly neutralize the toxins which their ancestors produced.

4I2. It is very significant that microbes, attenuated to the right degree, will confer immunity on animals of a species somewhat resistant to the disease, but cause death with symptoms of the virulent disease in animals of a species that is less resistant. A parallel fact is that, while smallpox, measles and many other diseases destroy the less resistant, they confer immunity on the more resistant individuals of the same species. Obviously, therefore, the toxins of attenuated disease still remain toxins-but weakened toxins. Consequently we are driven to the conclusion that in rabies, smallpox, and anthrax experience of a mild variety of a disease helps to the acquirement of resisting power against the more virulent variety — not by introducing substances which chemically neutralize the toxins but by supplying weakened toxins, reactions against which act as stepping stones towards reaction against strong toxins. ${ }^{1}$ In other words, when the individual has

1 The alteration in the toxin of rabies in drying cords may arise in one of two ways: (I) the virulent toxin may be directly altered by the drying process, or (2) the microbes may be so altered that they produce attenuated toxins. Some authorities have surmized that as drying proceeds the microbes perish, first at the surface of the cord, and then progressively towards the centre, and, therefore, that the efficacy of Pasteur's treatment depends on the injection of small but progressively increasing quantities of microbes. But the microbes are already 
acquired the power of resisting weak toxins, he is in a position of advantage from which he can react to the stronger toxins and so acquire immunity. In these instances, then, acquired immunity is nothing other than a 'use-acquirement,' resulting from progressive habituation. The antitoxins educate the phagocytes till they are able to tolerate the strongest toxins and destroy the microbes producing them.

4I3. That habituation plays a great part in the acquirement of immunity is evident from the fact that, if we begin with small but increasing doses, we may eventually inject very large doses of diphtheria toxin into a horse, without making him ill, though his serum becomes poisonous to other animals, which, of course, it would not be were his immunity due to chemical neutralization. So also, by beginning with small doses a certain degree of immunity against morphia, nicotine, and arsenic may be achieved. It is evident, therefore, that immunity may be secured with comparative ease in two ways, by giving weakened toxins, or by giving small but increasing doses of unaltered toxins. The chemical composition of morphia and nicotine is comparatively simple. These substances, therefore, cannot be altered without destroying them as poisons. Arsenic is a still more simple substance. But the composition of the microbic toxins, that of such proteid vegetable poisons as abrin and ricin, and that of such animal poisons as snake venom is very complex. Therefore they may be altered in such ways as to leave them still poisonous but weakened in various degrees. If food be swallowed, it is digested by the pepsin and the other ferments secreted by the alimentary tract and passes into the system in a somewhat altered condition. Similarly if snake poison be swallowed, it is digested and somewhat altered so that it enters the blood in an attenuated state and educates the system against virulent venom injected under the skin. ${ }^{1}$ If the poison gland be swallowed, it is also digested. Now microbes, during recovery from disease or if enclosed in a capillary tube, perish though not in contact with phagocytes.

present in the infected person in numbers sufficient to cause disease, that is, they are present in such numbers that the phagocytes cannot overcome them; and their numbers continually increase. According to this hypothesis, then, if we wish to cure a disease due to an increasing number of microbes, we must add to the multitude from extraneous sources.

1 Digestion in gastric juice of spinal cords infected with rabies, has also been practised, especially in Italy as a means of attenuating the virus of that disease. If a series of injections of this digested material be made into sheep an antitoxic serum may be obtained. 
Presumably the same digestive substance which destroys them when enclosed within the phagocytes is secreted into the surrounding medium and destroys them there-just as pepsin, which is harmless to the stomach cells which produce it, digests food at a distance from them. Moreover, just as pepsin digests both snake venom and the gland which secretes the venom, so, presumably, the ferments secreted by the phagocytes digest both the microbes and their toxins. Of the existencel of these digestive ferments there can be no doubt. Thus, if we add blood from a person recovering from enteric fever to a medium in which typhoid bacilli are contained, the behaviour of the latter indicates injury.

414. If, then, this theory be correct, and I am not aware of anything known to us which contradicts it, antitoxic serum from a horse contains substances-ferments and enzymes-which are engaged in digesting the toxins and so attenuating them; for which reason a fatal dose of toxin is rendered harmless when mixed before injection with its equivalent of antitoxin while the effect of even larger doses is mitigated. But, what is even more important, it contains more or less attenuated toxins which form a scale up which the cells of the individual into whom it is injected react until he achieves the power of tolerating the strongest toxins.

4I5. Given suitably attenuated toxins and time for reaction, immunity against acute disease may almost always be secured. Thus, if an individual be infected with rabies, which develops slowly, there is usually ample time if treatment be begun early. In diphtheria, which develops quickly, days and hours may be of vital importance; but since we are able to pour in an ample supply of antitoxin, our treatment may be very successful. The vaccine treatment of smallpox, on the other hand, is preventive not curative. It is useless to vaccinate after the disease has manifested itself, for before the weaker toxin is elaborated, the sufferer is on the high road to recovery, or is past help. ${ }^{1}$ Presumably the normal process of acquiring immunity is in essence similar to that which occurs when antitoxins are artificially introduced. The severity of the disease depends in part on the resisting power of the individual attacked, and in part on the virulence of

1 It is remarkable that subcutaneous injections of considerable quantities of vaccine lymph does not appear to have been tried in smallpox. Theoretically such treatment should be excellent. It is probable, however, that the majority of deaths amongst sufferers from smallpox are caused not by that disease, but by subsequent septic infection and the absorption of the toxins of putrefaction. Even so, however, if the injection of vaccine lymph caused the vesicles to abort, the effect would be curative. 
the microbes. If the phagocytes are resistant they are able to produce enzymes which attenuate the toxins, thus providing antitoxins, and recovery follows. If their resisting power is low, they are paralysed or killed, enzymes are not produced, a scale of attenuated toxins is not provided, and the individual does not react, but perishes. The tobacco smoker does not acquire increased resisting power against any other toxin, for example, he is not rendered more resistant to opium. Similarly, since the toxins of every disease differ from those of every other, immunity (i.e. habituation) acquired against any one disease confers no increase of resisting power against any other.

4I6. Syphilis, which has been curiously neglected by students of acquired immunity but which is often quoted by medical supporters of the Lamarckian doctrine, affords very instructive and illustrative data. The disease is caused by a microbe, the spirochete pallidum. It has been said that offspring may be infected by either parent, but certainly infection is much more common through the mother. If it be true that healthy mothers sometimes bear infected children, then, in such cases, the microbes must have been travelling with the fertilizing spermatozoon, or in some other way which I cannot imagine have entered the ovum without invading the maternal tissues. The microbe of Texas fever, a disease of cattle, is known to travel with the germ-cells of the tick with which the cattle are infested. The child in utero, is, rightly speaking, merely a parasite on the mother. It adheres to her by the placenta, through which it receives nutriment, and in which the thin-walled maternal and fotal vessels lie in close contact so that fluids with dissolved solids and gases pass from one to the other, whereas solids such as blood corpuscles and microbes do not. In some diseases of which syphilis affords by far the greater number of examples, injury to the placental vessels may occur and result in the passage of solids, for example microbes. Apparently the toxins of syphilis are but slowly elaborated and are not very virulent. Consequently the disease is of gradual development and of long duration. It would appear that the microbes, while they are yet few in number, are at first confined to the place of entry, where they are protected by their concentrated toxins. Later when they have multiplied and the resistance of the phagocytes has been, for the time, overcome by the increased abundance of toxins, they spread over the whole system. The mother, then, gradually acquires immunity. Her phagocytes become tolerant of the toxins and produce enzymes which attenuate them. A 
scale is thus provided up which she reacts. Her fotus receiving enzymes and attenuated toxins from her tends to acquire immunity at the same time.

4I7. But young individuals are usually less tolerant of disease, less capable of acquiring immunity, more liable to be poisoned even by attenuated toxins than older individuals. ${ }^{1}$ Consequently in the earlier stages of the mother's illness the fotus tends to perish. In the later stages, protected by the mother's enzymes, and little injured by the much attenuated toxins, the child may be born with every appearance of health; but after birth, when both the enzymes and the attenuated toxins from the mother have disappeared, the microbes may reassert themselves, and the child may perish, or waste and sicken for a time till its own immunity is thoroughly established. If the placental vessels are uninjured, so that only the mother or the child is infected, the non-infected individual tends to acquire immunity more easily and quickly than when microbes with their concentrated toxins are present. It undergoes, in fact, a serum treatment strictly analogous to that which has proved so successful in diphtheria-or rather strictly analogous to that by which we secure immunity to a person in imminent danger of infection by diphtheria. Consequently a noninfected child, born to an infected mother, tends to acquire immunity and survive, though it is possible that its immunity, being ' passive,' may not be permanent. Doubtless if a mother, who has recovered, becomes pregnant of healthy children, she confers no immunity on them, for no toxins or antitoxins are present to cause reaction. So also a mother who has recovered from measles or smallpox confers no immunity on her children subsequently conceived. But doubtless, also, if she becomes pregnant of an infected child, she confers immunity ; for toxins elaborated in the child pass to her, and returning as antitoxins induce a reaction similar to that which arises in the children she bears in the later stages of her own disease. At any rate, as far as I am aware, no instance is

${ }^{1}$ Doubtless this is because they represent a stage in the life-history at which powers of making resistance had not been evolved, or so much evolved. Young embryos represent a stage when the very power of making use-acquirements did not exist. Probably, therefore, the survival even of fœtuses is determined less by their own resisting powers than by those of their mothers. It must be noted, however, that since variations occur in all stages of development, and since children as well as adults are selected by most diseases, they are usually more resistant than their remote ancestors to diseases of which the race has had experience-as is proved, for example, by the fact that English children are more resistant to tuberculosis than American Indians, who therein resemble the ancestors of the English (see § 442). 
known of a mother who had suffered from syphilis bearing infected children to a diseased father, though probably the union of such mothers with such fathers is not very rare. Obviously, a father can never confer immunity, for immunity is a reaction made by phagocytes and perhaps some other cells functionally concerned, not by the germ-cell, the function of which is reproduction.

4I8. It is clear, then, that in syphilis there is no transmission of acquirements in the Lamarckian sense-that is, the child does not develop under the stimulus of nutrition characters which the parent developed under the stimuli of injury and use. The child acquires the disease and, if it survives, immunity, as the parent did, through infection with a microbe which has previously lived in another person. Therefore the expression 'hereditary syphilis' is erroneous. It would be as reasonable to speak of a bullet which passed through the mother and lodged in the child as a hereditary bullet.

4I9. An explanation of immunity at present very popular amongst bacteriologists, is Ehrlich's 'side-chain' hypothesis, a chemico-physiological attempt to interpret the facts. This hypothesis resembles Darwin's theory of pangenesis, Weismann's theory of germinal selection, Mendel's theory of segregation, and many others, in that it is not founded on actual evidence. It seeks to account for facts believed to be true by assuming conditions not yet ascertained to exist. Ehrlich supposes that the cells of the body possess side-chains composed of molecules, receptors as they are termed, the primary function of which is to attach to the cells various kinds of food molecules, fats, carbohydrates, oxygen and the like. The receptors, therefore, are of different kinds. In addition to having an affinity for this or that kind of foodmolecule, this or that kind of receptor may have an accidental affinity for this or that kind of toxin-an affinity which is a positive and fatal disadvantage, for the cell is thereby rendered susceptible to poison. If much toxin be present, many receptors combine with its molecules and the cell perishes. If little is present, some of the side-chains alone perish and are shed, combined with the now inert toxin, and undergo subsequent disintegration in the blood serum. This may be termed the chemical part of the theory. The physiological part is the supposition that the loss of side-chains stimulates the cells to excessive production of them. The appropriate receptors are thus set free in the blood which consequently becomes antitoxic. Immunity is thus acquired. It is not stated whether this excessive 
production and shedding of receptors occurs normally in the presence of over much nutriment. Presumably, it does not, since the free receptors would render the food incapable of being taken up by the cells and thus over much nutriment would eventually be equivalent to too little, and starvation would result. In any case, the power of acquiring immunity is, according to Ehrlich's theory, an accidental accompaniment of the power of assimilating nutriment.

420. On the other hand, inborn immunity-the kind which prevents an animal ever acquiring a disease-is thought to depend (I) on a lack of receptors which have an affinity for the toxins of that particular disease, or (2) on the normal presence of free receptors in the blood-that is, on receptors which for some reason are free in the serum, though disease has not stimulated their super-production. Such non-bacterial poisons as morphia, nicotine, arsenic and the like-which are not of very complex composition, are supposed to affect not the side-chains especially, but the whole cell, which is thus poisoned or else acquires resisting power against them in ways that differ from that in which it acquires immunity against the proteid poisons.

421. Obviously, the physiological part of Ehrlich's theory attributes immunity to use-acquirement. It attempts to explain up to a certain point how toleration of toxins arises; that is, Ehrlich supposes that toxins stimulate cells to the production of receptors. But the central mystery of how it happens that the stimulation has the effect alleged, remains unsolved. Instead of direct digestion by enzymes, neutralization and subsequent disintegration (which must be due to some form of digestion), are imagined. But, since it is admitted that toleration of poisons of simple composition, such as morphine and nicotine, is not due to an increased production of receptors but merely to increased toleration by the cell itself, the necessity of postulating the existence of the receptors is not very evident. No account is taken of the evolution of the power of attack in bacteria nor of resisting power in the species attacked. In fact the whole indubitable truth of evolution is ignored. Disease is treated, not as part of the normal environment as it really is, but as an accident which is resisted by accident. Immunity, inborn and acquired, is regarded, not as a product of protective evolution, but as a fortunate 'fluke.'

422. Some varieties of animals (e.g. white rats) are normally immune to certain diseases to which allied varieties or species 
that use similar food are liable. Some individuals of a species (e.g. man) are immune to disease (e.g. scarlatina), to which other individuals are liable. Many species of animals normally immune to this or that disease, are liable to infection, if the health be lowered as by abnormal heat or cold. Antitoxins ('receptors') may be obtained from the dead or dying cord of a rabbit which was suffering from rabies and would infallibly have perished if it had not been killed; that is, though receptors are not produced in the living animal in sufficient abundance to neutralize the toxins, and though they cannot, of course, be produced by the dead cells of the drying cord, yet in the latter they are thought to be in abundance. Snake venom, in doses lethal a thousand times over if injected under the skin, confers immunity if swallowed. Whence the receptors in the latter case? All these facts are inconsistent with the side-chain theory, which with many other bacteriological hypotheses would, I think, never have been formulated had their authors taken the whole of the facts into account-had they paused to consider that living nature is a product of evolution, not of miracle.

423. If the reader bears in mind the facts of adaptation, if he remembers that almost every important character is an adaptation, I think that he must conclude that acquired immunity is not an isolated phenomenon nor a 'fluke,' but essentially a use-acquirement entirely comparable to other use-acquirements in the sense that the power of acquiring it has been evolved. I think, also, he will find the strongest reasons for believing that the process by which this habituation is achieved, is, in the case of virulent complex toxins at least, one of reaction from weak to stronger toxins, the weak toxins, the so-called anti-toxins, being nothing other than stronger toxins that have been more or less digested, and in this way chemically altered by enzymes secreted by the cells. ${ }^{1}$

1 All the evidence points to the fact that acquired immunity is especially a reaction to the extra-cellular toxins (exotoxines)-those that are secreted by the microbes into the surrounding medium, not retained within themselves. Thus, while an experience of a few days confers immunity to such diseases as smallpox and measles in which symptoms of toxin poison are much in evidence, life-long experience confers no increase of resisting power against tuberculosis or leprosyeven when the sufferer is but slightly infected and his natural powers of resistance are but little lowered. Effective antitoxic treatment, therefore, would seem impossible in such cases. Very scanty success, or no success has attended all attempts to deal thus with maladies which, being experienced, do not naturally give rise to acquired immunity in resistant individuals. The celebrated tuberculin treatment of phthisis is a case in point. Tuberculin is prepared from dried tubercle bacilli and contains therefore an intra-cellular toxin (endotoxine). It seems to 
424. The outline I have attempted to give of the process of digestion is, of course, even if correct in all its facts and inferences a mere sketch. Each species of bacteria may secrete not only one but two or more toxins complementary to one another. Thus there is ample evidence of the existence in acute diseases of toxins which are retained within the microbes, in addition to those which are passed into the surrounding medium. Probably the organisms of the chronic diseases (e.g. tuberculosis) are protected more especially by the former, those of the acute diseases (e.g. small-pox) by the latter, whereas those of the sub-acute diseases (e.g. syphilis) depend in a more equal degree on both. It is significant that an extract obtained from dead tubercle bacilli is very poisonous, while one similarly obtained from diphtheria bacilli is not. Again, there is some evidence that one kind of enzyme acts directly against the microbes and another against their toxins. The cells, therefore, may secrete, not one kind of enzyme, but perhaps several. The same enzyme may not protect against every disease. The process of digestion may not be continuous, but may proceed by well marked steps in which various cell products, globulins, lysins, alexines, opsonins, and the like successively take part. As far as I am able to judge, however, no matter how complicated the process, it is somewhat of the nature I have attempted to describe.

425. Inborn immunity on the other hand must in each case be

have some curative properties, which depend apparently on an increase of inflammation round the foci of infection which leads to necrosis and sloughing of the diseased tissues; but there is no evidence that it conduces to that toleration, that acquired immunity, to the attainment of which the antitoxic treatment of acute disease is so great a help. The antitoxic treatment of such troubles as alcoholism and morphomania has also been tried-with declared success by the originators of the treatment, with ill-success by all who followed them. Doubtless serum treatment of a tendency to fatigue by means of an antitoxin obtained from very tired animals will one day be attempted, and will meet with the same unsatisfactory sort of success. Some of the hypotheses formulated and acclaimed by bacteriologists are of a kind to fully justify the endeavour. The speed with which immunity is acquired bears an evident relation to the abundance of the extra-cellular toxins and the rapidity with which they are produced by the microbes. All the cells of the body are affected by them and all those which are functionally concerned in the acquirement of immunity tend to react. In the case of the intra-cellular toxins only those cells which ingest the microbes are affected. Possibly this is the reason why immunity is acquired in the one case and not in the other. The duration of immunity, which is usually life-long in some diseases and of short duration in others, depends on factors which are quite unrecognized. It is sometimes said that one attack of certain diseases (e.g. diphtheria) in which the period of immunity is short, predisposes to subsequent attacks; but the truth of this surmise is very doubtful. An attack of such a malady indicates that the individual is susceptible; subsequent attacks probably indicate no more than a recrudescence of this susceptibility. 
due to one of two very different causes; a very high resisting power on the part of the individual attacked, or to a low attacking power on the part of the microbes. The high resisting power is due to stringency of selection affecting the species possessing it ; the low attacking power is due to lack of selection amongst the microbes. Thus human races that have been much afflicted by tuberculosis are, as compared to other human races, especially resistant. Dogs on the other hand are almost quite immune. Since we have no reason to suppose that these animals have ever suffered from the disease, we are driven to the conclusion that the environment they offer is unsuitable to the tubercle bacilli. It is one to which their evolution has not adapted them. It must be noted, however, that an evolution, which fits a race for a particular environment, does not necessarily unfit it for existence in another, and, within limits, a different one. Thus many European plants and animals, including man himself, flourish exceedingly in New Zealand and elsewhere. So also the bacillus tuberculosis, which, so far as we know, has never afflicted wild guinea pigs, is very deadly to them in captivity. In rare cases inborn immunity appears to be a correlate of other and apparently quite unrelated characters. Thus, unlike coloured varieties, white rats are immune to anthrax.

426. The main purpose of the present chapter is to establish a clear distinction between inborn and acquired immunitybetween the kind of immunity which arises under the stimulus of nutrition and that kind which develops through individual experience of disease. To achieve this object we have been forced to discuss the rationale of acquired immunity. The two kinds are constantly confused. Thus in medical discussions, one has only to maintain that parental diseases and acquired immunity are not 'hereditary,' and some one is sure to retort that in both instances the contrary is constantly observed in syphilis. Many doctors are still convinced that 'common sense' demands a belief in the Lamarckian doctrine. Certainly nearly all doctors are convinced on grounds of common sense that ill-health tends to so affect the germ-plasm that, in consequence, offspring of unhealthy people are commonly born degenerate. In other words they believe that parental disease is a frequent cause of variations in offspring. We have already discussed this problem somewhat in detail, but it is of great practical as well as theoretical importance and will repay further study. The ground is now cleared of possible misconceptions and we may proceed to discuss it in comfort. 


\section{CHAPTER XIII}

\section{THE PRESENT EVOLUTION OF MAN}

The conditions under which infection occurs-Inborn immunity develops in the individual under the stimulus of nutriment-Acquired immunity under that of use-Theories of the causation of variations and the facts of disease-The decisive nature of the evidence furnished by disease-Malaria-TuberculosisThe air-borne diseases.

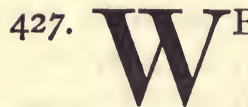

saw in the eleventh chapter that, with the exception of alcohol, opium, and perhaps one or two other narcotics, microbic disease is the only agency which is stringently selective amongst civilized men. Speaking generally, in England, for example, the people who survive and leave offspring are not those who escape contact with the microbes of such prevalent diseases as measles and whooping-cough, but those who are resistant to them. Selection implies, of course, variations in resisting power, and no one will deny that men differ naturally in this particular as in all others. Again, no one will deny that resisting power against one disease does not necessarily imply resisting power against any other. The selection is, in fact, 'specific.' Each disease chooses its own special victims, and is the cause of an evolution only against itself. On the other hand, no one who has studied diseases doubts that, owing to fluctuations in vitality and environment, individuals tend to alter from time to time in their powers of resisting it. The man who is very resistant, may become less resistant through the operation of some cause which lowers his vitality, while he who is very susceptible may be rendered more or less so through changes in health and surroundings. Nevertheless, the truth holds that the naturally more resistant tend always, under any condition, to survive in greater numbers than the more susceptible. Lastly, selection is not equally stringent in all environments, nor at all times in the same environments. It has been ascertained, experimentally, that the dosage of microbes plays a large part in the causation of infection, and, therefore, in the causation of acquired immunity or death. The microbes of disease are much more numerous in some 
situations than in others. We, in England, contract almost all our diseases in dwelling-houses or in places of popular assembly, such as schools and churches. In the slums of cities where overcrowding is greatest, the separation between the sick and the healthy most imperfect, the dilution of the dosage with fresh incoming air most incomplete, and the general nutritive and sanitary conditions worse, selection is more stringent than in better houses and in rural districts. Such very common diseases as measles, chicken-pox, and whooping-cough are usually always present (endemic) in towns, whereas in the country and even more in remote islands, such as the Hebrides, they tend to be more distinctly epidemic. That is, in towns the exposure of susceptible people to large doses is very frequent, while in the country it is more exceptional.

428. We saw also that all microbic diseases may be placed in one or other of two categories. On the one hand are those diseases against which immunity cannot be acquired, and, on the other, those against which it may be acquired-acquired immunity being an entirely specific reaction which follows individual experience of disease, and which in every case is of avail only against the particular disease which has been experienced. It is quite distinct from 'innate' immunity, which (though it is also specific) does not follow but precedes experience of the microbes, and which arises therefore like eyes, ears, hair, and teeth, as a nutritional character. Tuberculosis and leprosy are typical examples of diseases against which immunity cannot be acquired. No doubt, in a sense, immunity may be acquired against some of them; that is, owing to improved health and vitality, an infected person may recover and live immune; but this is not what is meant by the technical expression 'acquired immunity.' On the other hand, a person may be 'innately' immune against such typically acute diseases as measles and influenza. That is, he resists infection though exposed to doses which would give rise to disease in the ordinary individual who has not acquired immunity. But in the case of such diseases, in the vast majority of instances, people differ, not so much because they resist infection, for nearly all contract each disease if sufficiently exposed to infection, as because they recover more or less easily. ${ }^{1}$

${ }^{1}$ Inborn immunity, though rare in measles and so rare in smallpox that formerly when the disease was prevalent an unmarked skin was in itself regarded as an exceptional beauty, is common enough in some acute diseases. Thus, though constantly exposed to infection, I have never contracted diphtheria nor scarlatina. Probably, in the case of such diseases, the two forms of immunity are evolved concurrently. 
It follows, if there is any truth in the theory of evolution through Natural Selection, that a race much afflicted by a chronic disease-for example, tuberculosis-should evolve inborn immunity against it; whereas, if afflicted by an acute disease-for example, measles-it should tend to develop an inborn power of acquiring immunity. Both inborn immunity and the power of acquiring immunity arise in the individual through the experience his race has had of disease. They are nutritional characters. But actual acquired immunity arises in the individual through his own personal experience of disease. So also a man's hand and his power of acquiring callosities on it are due to an evolution in his race which enables him to develop those characters under the influence of nutrition; but the actual callosities are due to his own experience of rough work.

429. Again, we have seen that there are several theories of evolution, each with its corresponding theory of heredity. First, there is the theory of an internal adaptive growth-force, which supposes that variations tend, on the whole, to be adaptive. This hypothesis, which is founded purely on an appeal to miracle, need not be considered further. Science ought not to appeal to the supernatural until, at least, it has been demonstrated that natural interpretations are impossible. Second, there is the Lamarckian doctrine, which supposes that characters acquired by parents under the influence of use or injury, tend to be reproduced by offspring under the stimulus of nutriment. Third, there is that very popular theory of heredity which supposes that variations commonly arise through the direct action of the environment on the germ-plasm. This hypothesis of the causation of variations is usually associated with the doctrine of Natural Selection, but is clearly incompatible with it. Examined closely, we find that it implies a theory of racial change, but not adaptive change. It implies, in fact, that species drift helplessly under the influence of the environment in directions which may or not be adaptive, but which can very rarely be adaptive. Lastly, there is the theory that the mass of variations are spontaneous, and that they occur all round the specific mean, and, therefore, that the germ-plasm (in so far as its hereditary tendencies are concerned) is highly insusceptible to the direct action of the environment. This theory, and this alone, is compatible with the doctrine of Natural Selection. If only we think closely and clearly, we find also that it is the only theory which fits in with the facts of adaptation.

430. Now, which theory of heredity is borne out by human 
experience of diseases? If the Lamarckian doctrine be true, all races exposed to such chronic maladies as tuberculosis should steadily deteriorate; for by such maladies the individual is never strengthened in any known way, but, on the contrary, always injured. His resisting power is lowered. Therefore, no matter how slight and partial, how fitful and faint the inheritance of parental injury, yet the effects accumulating during many generations should at last cause the race to trend towards extinction. Is it, then, the fact that races afflicted by tuberculosis, or any other agency which causes chronic ill-health, are steadily deteriorating, steadily becoming less and less capable of existence? On the other hand, acute maladies confer a great measure of resisting power on individuals who have experienced them and survived. If this be inherited, no matter how faintly and fitfully, the race should in time develop absolute inborn immunity, absolute incapacity to acquire disease. Is it, then, the fact, that races which have long been afflicted by such acute maladies as measles, chicken-pox, whooping-cough, and the like, have become quite immune to infection? Evidently the Lamarckian doctrine implies racial effects due to disease which are very remarkable. Each chronic disease must weaken the race till it perishes, each acute disease must strengthen it till it is quite immune to that disease.

43I. The hypothesis that variations are caused by the direct action of the environment, though often confused with the Lamarckian doctrine, is quite distinct from it. ${ }^{1}$ It supposes, not that the characters which arise in the parent through use or injury are reproduced by the child under the influence of nutrition, but merely that nutritive substances, waste products, toxins, and the like, circulating in the blood, or otherwise acting on the germcells, tend to alter the hereditary tendencies in the germ-plasm in some definite way-in such a way that the individual who springs from the germ-cell is injured or benefited, weakened or strengthened. At first sight this hypothesis is much more probable than the Lamarckian theory. But it is only reasonable to suppose that if the germ-plasm is altered at all by the direct action of the environment, it is injured and devitalized, not benefited by exposure to the toxins of acute diseases or by the general interference with nutrition which accompanies the chronic maladies. At any rate, while it has often been held that parental ill-health is a cause of filial degeneration, no one has suggested that syphilis,

${ }^{1}$ See $\S 98$. 
tuberculosis, malaria, measles, or any other disease or ill-condition suffered by parents increases the vitality of their offspring. If, then, this theory is correct, all races afflicted by any sort of disease should steadily drift towards extinction.

432. Lastly, if the theory that the vast majority of variations are spontaneous, be true, and, therefore, if the doctrine of Natural Selection be correct, then every prevalent and lethal disease should be the cause of protective evolution against itself. Diseases which are not prevalent or not lethal should cause no racial change. Prevalent and lethal chronic diseases, since the survivors from them are people who resist infection, or who, if they recover from infection, do so only because their vitality is improved, not because they undergo that peculiar reaction known as acquired immunity, should cause an evolution of inborn immunity-an evolution of an inborn power of resisting infection. Prevalent and lethal acute diseases, since the survivors from them do not, as a rule, resist infection, but merely when infected tend to recover within a definite time, should cause an evolution of the power of acquiring immunity under the experience of disease. In other words, the evolution should be such that, in the case of chronic maladies, immunity should develop in the individual under the stimulus of nutrition, whereas in the case of acute maladies it should develop in him under the stimulus of use. Thus, if the theory of spontaneous variations and of Natural Selection be true, the individuals of a race that has been long and severely afflicted by tuberculosis should, as a rule, display a greater innate power of resisting infection, or, if infected, of withstanding the malady, than individuals of races that have been less or not at all afflicted. A race afflicted by measles should display no greater power of resisting infection than any other race, but a much greater faculty of recovering by the acquirement of immunity than races that have had less experience of the disease. On the other hand, races that have been afflicted by such non-lethal diseases as chicken-pox ${ }^{1}$ should possess a power of resistance no greater than that displayed by races that have had no previous experience. Moreover, in all cases the resisting power, whether inborn immunity or the inborn power of acquiring immunity, should be specific. That is, the effect of each disease on human races should be an increased

${ }^{1}$ In 1905 " chicken-pox is said to have claimed ninety-three victims, but it is at least probable that some of the latter were unrecognized cases of the graver malady" (smallpox).- Sixty-eighth Annual Report of the Registrar-General of Births, Deaths, and Marriages in England and Wales, p. Ixxix. 
power of resisting it and nothing more. The race should not gain in beauty or muscular strength or size or anything of the sort. ${ }^{1}$ Of course, it is possible that this or that character may in the future be found to be correlated to susceptibility to, or to power of resisting this or that disease, but, to the best of my knowledge, nothing of the kind has yet been discovered. ${ }^{2}$

433. Very plainly, disease furnishes ideal material for investigation of that most important problem of heredity, the causation of variations. The acquirements made under the influence of injury (e.g. in such chronic maladies as tuberculosis), or of use (e.g. in such acute complaints as measles), are very striking and peculiar, and in the case of many diseases and many races have been made by every generation for unnumbered generations. The germ-cells are often literally bathed in toxins, it may be for prolonged periods, as in syphilis and malaria. Their nutrition is interfered with for even longer periods in such complaints as tuberculosis and leprosy. Our opportunities for observation are particularly good, for every one of us has had personal experience of illness, and is acquainted with numerous cases of it. Any amount of precise statistical evidence, furnished by Departments of Public Health, concerning the different effects of disease on different races, is available. Every instance of the birth of a child to a parent who is or has been diseased constitutes an experiment which is as stringent as any that can be consciously devised, but which possesses in addition the advantage that it is made under conditions that are normal to the species. Like many other experiments, this experiment has the disadvantage that the effect produced on the offspring, if any,"may be so small as not to be observable, or it may be so confused with spontaneous variations as not to be detectable; but in the case of every disease we are able to compare many races which have suffered much and long

${ }^{1}$ It seems hardly necessary to insist on this, but various critics have thought it necessary to declare that there is no evidence that much experience of disease produces the 'finest race.'

2 The dark colour of negroes has been thought to be correlated to their high powers of resisting malaria. But many black races, for example some Polynesians, are highly susceptible, and some white races are relatively resistant. Thus malaria, which destroyed an invading British army as a fighting force, left the inhabitants of the island of Walcheren able to pursue their ordinary avocations. Biometric attempts have been made to discover correlations between physical and mental traits and disease by comparing individuals of the same race, but if we compare individuals of different races, in whom contrasts in resisting power, colour, and the like are so much more marked as to be easily observable, we find no such correlations. 
with many others which have suffered less or not at all. If disease produces any germinal change, then, no matter how small and imperceptible the difference between one generation and the next, or how confused with spontaneous variations, the constant accentuation of the alteration during hundreds, perhaps thousands, of generations, must make it at last manifest and unmistakable. The multitude of races and their diseases is so great that we are able to make endless comparisons and so reduce the chance of error to a minimum. The materials are so familiar that every man may verify them for himself; whereby that grave source of error and doubt, the 'personal equation' of the observer, is eliminated.

434. Manifestly, we are dealing now with a problem of the first importance, the question of the causation of variations, which, both from a theoretical and a practical standpoint, is the central problem of heredity. All theories of racial adaptation and degeneration, of the influence of environment, of likenesses and differences between parents and offspring, of plant and animal breeding, of far-reaching social effort depend on it. Disease affords material for solving the problem more conclusively than anything else to be met with in nature. The reader must, therefore, consider carefully what theory of the causation of variations fits in with the known changes undergone by races that have been in contact with disease. In other words, he must make a rigorous deductive inference of consequences, and then carefully compare these consequences to reality.

435. The facts are decisive. Nearly all human races have been exposed to disease for thousands of years, and in no instance is there to be found an iota of evidence that any race has, as a consequence, become degenerate, or that any race has transmuted acquired into inborn immunity. On the contrary, not only is every race resistant to every prevalent and lethal disease precisely in proportion to its past experience of it, but the resisting power is such that it can have been evolved only through the Natural Selection of the spontaneous variations of a germ-plasm that was insusceptible to the direct action of the environment, and did not transmute acquirements into innate characters. Without exception, those races which have been exposed to diseases (e.g.tuberculosis), against which immunity cannot be acquired by the individual, have tended to become, not enfeebled, but innately capable of resisting infection; whereas, races which have been exposed to diseases against which immunity may be acquired (e.g. measles), have evolved nothing other than an increased 
capacity of making the acquirement, of recovering from infection. On the other hand, races that have been exposed to non-lethal diseases (e.g. chicken-pox), are neither more nor less resistant, neither more nor less degenerate than races that have had no previous experience of them. Apparently they are in no way changed. The sufferings of races have not altered them at all. Only the elimination of the unfittest has altered them.

436. Clearly both the Lamarckian doctrine of the transmission of acquirements and the doctrine that variations are normally caused by the direct action of the environment on the germ-plasm are erroneous. The theory that the mass of variations are spontaneous is just as clearly correct. Only in that case can Natural Selection, the actuality of which as regards disease is obvious to every observer, have produced the particular racial changes that have resulted. We constantly speak of the acclimatization of an individual or a race. As applied to individuals, the term is a synonym for use-acquirement, for protective development, for a becoming accustomed or habituated to the new conditions-a habitation which may be 'active' (i.e. due to recovery from actual disease), or 'passive' (i.e. due to experience of microbes and toxins in doses too small to cause disease). As applied to races, it is a synonym for protective evolution, which, in the case of diseases against which immunity cannot be acquired, implies the increase of a power of resisting infection, and in the case of diseases against which immunity can be acquired, implies the increase of a power of recovering from infection and resisting it subsequently. When we talk of the deadly climate of West Africa and the healthy climate of England, we think only of ourselves. Not less deadly to the African negro, to whom the West Coast is healthy, is the climate of London with its prevalent tuberculosis. To the Polynesian or Red Indian, whose race has had even less experience of this disease, a residence in any one of our great cities is usually equivalent to a sentence of death.

437. All educated men know more or less clearly that every race that has long dwelt in any environment is especially well adapted to the conditions of that environment, including its diseases. Medical men share this knowledge. But most doctors believe also that races tend to deteriorate when exposed to injurious conditions. The two beliefs are utterly incompatible, and afford a good example of the confusion of thought that arises when the duty of making a rigorous deductive inference of consequences is neglected. The latter belief (that variations are commonly 
caused by the direct action of the environment) is based in part, apparently, on a tradition ultimately derived from popular notions and first acquired by us all in the nursery, and in part on experiments and statistics which as a rule have no bearing on the point at issue. The problem, as we have already noted, is not whether it is possible to devise conditions in which the germ-plasm is injured beyond recovery, but not destroyed, but whether under conditions more or less normal to the race variations are commonly caused by the direct influence of the environment. ${ }^{1}$ Disease, which at one time or another affects practically every individual in England and Africa, has for thousands of years been almost as normal an occurrence as birth itself.

438. The experiments on which medical men rely are such as are afforded by Clayton's beans, ${ }^{2}$ transplanted Alpine plants, Féré's alcoholized eggs, ${ }^{3}$ and the like. The experiments they ignore are those of nature which every day's professional work brings under their notice, but over which hangs the veil of familiarity. The statistics they rely on are such as alienists collect. ${ }^{4}$

1 See $\S 160$.

${ }^{2}$ See $\S$ I 34, I 56.

${ }^{3}$ I think it would puzzle anyone who tries to think clearly to explain how Féré's perpetually quoted experiments bear on the problem of the causation of variations. Féré exposed eggs to the vapour of alcohol and got some remarkably miserable chickens. This indicates that alcohol is bad for developing chickens. It does not indicate that the ill-effects are germinal and therefore 'innate' and ' transmissible' to descendants-any more than a plucked chicken indicates that the effects of plucking are 'inheritable.' Féré should have bred from his dedebauched chickens. The attitude of some medical men, who are interested in philanthropy, especially temperance reform, and who have been accustomed to insist on the 'degeneracy' which results from insanitation and drink, is instructive from the point of view of scientific education (see $\S$ I 16 , footnote). The statement that ill-conditions cause protective evolution, not racial deterioration, is often declared by them to be too 'philosophical ' or 'academic' or 'logical.' 'Honest facts ' are demanded. It is hard to say what is meant exactly-possibly nothing more than that the masses of fact on which the statement is founded are large and complex, and the thinking necessary before the relations can be ascertained somewhat difficult. The fact that diseases cause evolution, not deterioration, if not honest, is at least indisputable. I imagine by honest is meant experimental. The statement that "we know very little about heredity" has no doubt very often an element of truth; but the version that "very little is known about heredity" is distinctly inaccurate. Sometimes it is said that " the proposition that germ-cells are inviolable is sheer nonsense. They are just as liable to injury as other cells, and we have daily experience that somatic cells may be injured." But no one has maintained that germ-cells are inviolable. It has only been maintained that all kinds of cells, including the germ-cells of all species, have been so dealt with by Natural Selection that they hold to their hereditary tendencies almost as firmly as they hold to life.

4 See $\S$ I 3 I, I 35 . 
The statistics unused are those published by Departments of Public Health, which, especially in the conquests and colonies of Europeans, show clearly the real effects which have flowed from racial experience of disease.

439. It is hardly necessary to quote evidence that races are resistant to diseases in proportion to their past experience of them. ${ }^{1}$ The evolution of negroes against tuberculosis, however, is so very illuminating and typical that it is worth while to devote a paragraph to it. There are no accounts of negro conquests outside the limits of Africa, but from very ancient times a constant stream, of slaves has passed to Southern Europe and Asia, where they have been employed mainly in domestic service, and in more recent times to America, where their principal occupation has been agriculture. The invasion of Asia has continued to our own day; but, notwithstanding the great antiquity and volume of the stream, one may search from Spain to the Malay Peninsula, and, except in recent importations, find no trace of a negro ancestry. Yet slaves like cattle are valuable property, more cheaply bred than imported. In Eastern countries they have often been kindly treated, and many have achieved wealth and power. Yet they all perished in a few generations, the elimination of the unfit being so stringent as to cause extinction, not evolution. A permanent. colony of native Africans in the midst of an ancient tuberculosis-infested civilization is impossible. The fate of negro migration into America has been different. The race had undergone some evolution against tuberculosis in Africa, and therefore was more resistant than the vanishing aborigines. In its new home, employed in agriculture in a hot climate where white men and tubercle bacilli, also recent importations, were yet few in number, it was placed under the best conditions possible. Gradually, as the stringency of selection waxed, it evolved increasing resisting power. To-day the descendants of the migrants have undergone such an extent of protective evolution that they are able to dwell even in North American and European cities, though it is still said of them that "every other adult negro dies of consumption."

1 The reader who requires evidence will find it in convincing quantities in my work, The Principles of Hevedity, chapter xi. 


\section{CHAPTER XIV}

\section{EPIDEMIC AND ENDEMIC DISEASE}

The conditions of parasitic life-Probably every disease originated from a single centre-No new diseases are known-Disease in ancient and modern times -Contagious diseases-Insect-borne diseases-Air-borne diseases-Water-borne diseases-Earth-borne diseases-The antiquity of diseases-Pestilence-The invasion of the Western Hemisphere by disease-Diseases as empire-builders.

440. T TE may safely assume that the micro-organisms of disease evolved from saprophytic forms of life, the only alternative being the impossible hypothesis that these highly specialized types arose by spontaneous generation after the evolution of the higher animals and plants. Precisely how the passage from a saprophytic to a parasitic mode of life occurred is a matter of conjecture. We know only that there is perpetual war in nature, and that the bodies of the higher animal types contain nutritious material of which the lower types must always have tended to avail themselves. From the beginning, therefore, there must have been some sort of a struggle. The evolution of the parasite had two concurrent phases. First, the saprophytic species must have become capable of persisting for a longer or shorter period in living bodies in spite of their defences. Second, it must have evolved means of passing from infected to healthy but susceptible individuals. Some saprophytic species are very virulent, for example those of putrefaction. Apparently the toxins they manufacture are either digestive ferments or means of offence or defence against other lowly organisms. Under favourable circumstances they readily adopt a parasitic mode of life, causing disease; but, since they are unable to pass easily from one individual to another, they cannot maintain themselves as parasites. Thus, also, the microbes of vaccinia are unable to persist unless artificially propagated. The main difficulty to be overcome by an evolving microbic species, then, is not the development of virulence, but the evolution of faculties for passing from one individual to another. Possibly each species of pathogenic organism first established itself as a regular parasite during times when hardship, famine, 
or other causes had lowered the resisting power of a community of its present hosts.

44I. The microbes of the different diseases differ so sharply in the effects they produce, in their methods of protecting themselves when in the body, and in their modes of passage from one individual to another, that it seems more probable that each pathogenic species was derived direct from a separate saprophytic type than that any two species were derived from a common parasitic stock-an ancient disease ancestral to two modern diseases. Very possibly the air-borne species arose from saprophytic types that were originally air-borne, the water-borne species from water-borne saprophytes, and the contagious types from saprophytes that normally and harmlessly inhabited cavities of the body, and so on. Again, since the constant tendency in nature is towards differentiation, not approximation, in type, since the microbes of disease are highly specialized, and since the passage from saprophytic to parasitic ways of life is very difficult, whereas, when once the parasitic habit is established, the passage from one host to another, and so from one country to another, is comparatively easy, it seems probable that no disease had multiple origins in different parts of the world, but that every disease spread from a single centre. ${ }^{1}$ However, as to all this we have no direct evidence on which to base speculation.

${ }^{1}$ At least we must consider it improbable that diseases had multiple origins if we accept the selectionist theory of evolution. Doubtless, however, supporters of the mutation theory, who insist that the same mutation tends constantly to occur in the same species, would hold a contrary opinion. But probably recurrent mutations are in most, if not all cases, reversions (see footnote, \$ 297). None of them have been known to be adaptive, and a mutation which adapted a saprophytic species in all its structures to a parasitic mode of life would be a very wonderful thing. It would involve, not only the sudden development of great virulence and all that that implies, including a capacity to resist the enzymes of the host, but, at the same time, a power and a tendency to pass from the infected person to others in the neighbourhood. Moreover, this amazing conglomeration of correlated mutations would have to occur, not in one or a few microbes but simultaneously in thousands; for if only a few microbes invade the body they perish. Disease results from the invasion of very many (see $\S 402$ ). Thus the microbes of vaccinia are unable to persist naturally because as a variety they lack means of passing from one host to another. It follows, if evolution is by mutation, then only the simultaneous mutation of thousands of vaccinia or other microbes could render them independent of artificial propagation. On the other hand, if evolution proceeds by the selection of fluctuations, a passage through a few weakly (e.g. non-resistant or starving) persons might create the necessary virulence (see $\S 400$ ). It must be borne in mind that it has been experimentally demonstrated that microbes gain in virulence only slowly after the passage of many generations, and unless greatly injured, as by heat, lose their virulence as slowly. 
442. Somewhat better than mere guessing is the assumption that mankind, under the influence of constant persecution, has gradually grown more resistant, not only to particular diseases, but to microbic infection in general. Owing to the increase of population, and to the crowded, insanitary, wretched conditions under which many communities live, the facilities for the evolution of parasitic types are now greater than ever they were. Yet, though we have the whole world under survey, we never hear of a new disease. The so-called new diseases are merely maladies newly observed in their ancient habitats, or complaints which have invaded fresh territories. Presumably, therefore, the alteration of saprophytes into parasites (i.e. the evolution of a new disease) is now a more difficult and unlikely occurrence than it was anciently. This conjecture (that mankind has evolved against microbic infection in general as well as against particular diseases) is confirmed by the fact that children, who recapitulate the life-history, are, as a rule, less resistant to microbic disease than adults, even when the disease is one of which the race has had little or no experience. For example, English children succumb much more readily than older persons of their own race to malaria and dysentery. It must be remembered, however, that since variations occur during all stages of development, the race evolves in the stage of development reached by the child as well as in the adult stage. On this account African and Indian children succumb much less readily to malaria and dysentery than English children, though more readily than adults of their own race. ${ }^{1}$

443. On the other hand, we have no certain evidence that any disease has ever completely died out. The chances are all against such occurrences in the past. When once established as parasites, the microbes, owing to the continual growth of human populations, found a constantly augmented food-supply as well as increased opportunities of reaching fresh fields of conquest. Sanitary science is still in its infancy, and as yet has accomplished comparatively little. Under skilled direction it has done something towards diminishing earth-, water-, and insect-borne diseases. Tuberculosis, enteric and yellow fever, and malaria have diminished somewhat in a few parts of the world. Leprosy has disappeared from some countries. But air-borne diseases, which are propagated through a medium the flow of which is too swift and the volume too vast for control, are as common as ever. Almost every child acquires measles, whooping-cough, chicken-pox, mumps, common cold, 
bronchitis, and, in its modified form, smallpox. All are more or less exposed to scarlet fever, diphtheria, pneumonia, and influenza. 444. We have historical evidence of the antiquity of many diseases. Leprosy is mentioned in the Book of Exodus as prevalent in Egypt. By other accounts it was prevalent in India, China, and Japan about the same time. Contagious diseases (e.g. syphilis) were well known to the Greek and Roman writers. ${ }^{1}$ We have few accounts of them from the Middle Ages, when physicians regarded their treatment as degrading to professional dignity; but at the Renaissance the 'great pox' was a terrible scourge. "A pox on you" was a common Elizabethan malediction. "Consumption of the lungs may be traced with certainty in the writings of every period as far back as the earliest attempts of the ancient world to deal with medicine according to a method." 2 Granting that diseases had not multiple origins, their wide prevalence in the ancient world, when communities dwelt in far greater isolation than at present, argues extreme antiquity.

445. It seems probable that both man and his diseases arose in the Eastern Hemisphere, where "behind dim empires ghosts of dimmer empires loom." Still more ancient than the oldest empires are the traces of the early agriculturists, who in turn were preceded by the hunters and cave-men of the long Stone Ages. Amongst savages of the lowest types the problem of the foodsupply is always acute. Population, as till lately amongst the natives of Australia, tends always to exceed the available supply and is limited by recurrent famine. Man is naturally a gregarious animal, delighting in large communities, but the difficulty of procuring sufficient food forces a race of hunters, not only to scatter, but to assert rights of ownership over large areas of country, intrusion into which by strangers is strongly resented. Hence tribal jealousy, perpetual warfare, and frequently an isolation so profound and stringent that the inhabitants of near valleys may be of distinct types and speak a language unintelligible to their neighbours. Civilization implies a dense and settled community, which in turn implies a more abundant and secure supply of food, and that, again, implies comparative peace, combined with regular communications over great areas of land and even of water, whereby the deficiencies of one part are supplied from the super-

${ }^{1}$ Hirsch's Handbook of Geographical and Historical Pathology, vol. ii. p. 2 (New Sydenham Society). The notion is general that the venereal diseases were imported from America, but this would appear to be an error.

op. cit., vol. iii. p. 170 . 
abundance of others. Our many and vast English cities are possible only because our peace is more profound and our means of communication more perfect than ever before in the history of man. On our tables may be found fruit and spices from the tropics, wheat from Canada, Australia, and Russia, mutton from New Zealand, beef from the Argentine, rice from India and China, bacon from the United States, and fish from half the world. To-day we may eat cheese or drink wine that has been stored for years. So widely do we travel that there is on earth scarcely a distinct language but some Englishman is able to express himself in it.

446. The pressure of population, therefore, is usually greatest amongst the lowest types of mankind, and this results, not in intercommunication, but in isolation; in wars the object of which is extermination not subjugation; in a search for food, not for slaves, whom a stone or pointed stick would arm as well as their masters. Doubtless it was during this time of great pressure and desperate struggle that man, spreading slowly from a common centre, populated the Eastern and ultimately the Western Hemisphere.

447. The era of the early hunters, who subsisted solely on wild animals and vegetables, was followed by that of the primitive shepherds and husbandmen, who made provision, not only for the immediate present by hunting, but for the future by labour. The supply of food became more abundant and secure. Property in land tended to become personal instead of tribal. Men began to gather for mutual protection into communities, which differed from one another in their stored productions. Barter, and therefore intercommunication, become more general and widespread. Since the labour of a man now produced more food than sufficed for his own existence, warfare underwent gradual change. The conquerors sought slaves and tributaries rather than mere elbowroom. They subjugated rather than exterminated their enemies. Population increased immensely, and with it the microbes of disease, which, spreading;rom their centre of origin, ranged to and fro with travellers and raiders.

448. The microbes of contagious diseases pass directly from one individual to another. The living body furnishes their entire environment, and, therefore, they are able to exist in all climates and under all conditions. The long duration in the individual of syphilis, the chief of them, makes it especially capable of infecting and maintaining its hold on new communities. But the method by which 
contagious maladies pass from one individual to another renders their diffusion through a population comparatively slow. They are endemic from the first. They rarely cause great epidemics; but, once established, are never again absent from their new habitat.

449. The microbes of all other diseases alternate between the human body and the world outside, in which, on the way to fresh 'hosts,' they pass a longer or a shorter time. They are dependent, therefore, to a greater or lesser extent on this outer environment. The insect-borne maladies are found only in localities where the insect host is able to maintain existence. Malaria was once common in England, but the conditions became unfavourable to the mosquito, and the disease disappeared. Though many infected people from the tropics land on our shores, it cannot reestablish itself. In historic times it is said to have invaded Greece and Italy, and by some medical writers is supposed to have contributed to the downfall of the classic empires. In more modern times it invaded Mauritius. The fact that before the historical era malaria had spread to most parts of the globe where its intermediate host, the Anopheles mosquito, is capable of persisting, is evidence of its vast antiquity. Of all the host of maladies which now infest the Western Hemisphere it is apparently the only one of any importance which was not introduced by Columbus and his successors. Possibly it is the most ancient of all human diseases. The path by which it journeyed to America is quite unknown. Under present conditions of climate it can hardly have travelled by the Arctic circle. Before the era of European discovery man had crossed the Pacific, all the large islands of which are thickly populated. But, except near Asia, malaria is unknown in them. The difficulties of crossing the practically uubroken expanse of the Atlantic are obvious. The antiquity of the disease is evidently so great that the surmise that it may have reached America during a warm inter-glacial period is perhaps not altogether extravagant.

450. Plague, sleeping sickness, and yellow fever are other very important insect-borne maladies. Plague is a disease of rats, the intermediate host being a species of flea which is parasitic on these animals, but which will on occasion attack human beings. During an epidemic the rats tend to be exterminated and the disease to die out. It is endemic in India, but several great pandemics in which it spread widely are recorded in history. It was known in Europe before the Christian era. ${ }^{1}$ In the sixth century after 1 Hirsch, vol. i. p. 494. 
Christ it "spread over the whole Roman Empire of the East and West and even far beyond the limits of the empire; ... and wrought the most frightful devastation wherever it appeared; . . . it depopulated towns, turned the country into a desert, and made the habitations of men to become the haunts of wild beasts." 1 Another great pandemic, "known everywhere under the name of the Black Death as one of the great events in the world's history," "reached over the whole of the known world" and destroyed a third or a quarter of the inhabitants of Europe. The Great Plague of London furnishes an additional example.

$45 \mathrm{r}$. Yellow fever, like malaria, has the mosquito for its intermediate host. Its original habitat appears to have been the West Coast of Africa, but it is now endemic in the tropical parts of Eastern America. Till very recently sleeping sickness appears to have been confined to the West Coast of Africa. It was often carried by slaves to Brazil and the West Indies, where, however, in the absence of its intermediate host the tsetse fly (Glossina palpalis), it did not spread. The infected slaves were supposed to be suffering from nostalgia or home-sickness. ${ }^{2}$ Owing to the improvements of communications consequent on European conquest, it has spread very widely and has now almost reached the East Coast near Zanzibar, where it is likely to prove a scourge as terrible as it is already in Uganda. Doubtless the tsetse fly preceded the disease in Central Africa, but did no great harm till infected human beings arrived. The insect-borne diseases are the only complaints which, on the whole, afflict sparse populations more than they do denser communities of men.

452. Air-borne maladies are immensely infective but of short duration in the individual. After a brief illness the sufferer dies or acquires an immunity which may or may not be permanent. Consequently, in small and isolated communities, they can occur only in epidemic form, at any rate when the immunity they confer is at all lasting. Owing to the large number of susceptible persons, such a disease, when it reaches a new country, spreads like fire. If it has long been absent, it strikes down the whole population. So many are infected, so abundant do the microbes become, that no one escapes the chance of infection. As a consequence, the food-supply of the microbes is swiftly exhausted, and the malady disappears till re-introduced from foreign sources. On the other

${ }^{1}$ Hirsch, vol. i. pp. $495-6$.

${ }^{2}$ The Kingdom of Man, by Sir E. Ray Lankester, p. I 59 (London: Constable \& Co., 1907). 
hand, when a community is large, and especially when it enjoys constant intercourse with other considerable centres of population, air-borne disease prevails in endemic form, mild exacerbations of which are termed epidemics. Nearly every one suffers during youth. The non-resistant perish, the resistant acquire immunity. New births supply materials sufficient to secure the persistence of the disease, and provide for the so-called epidemics; but the microbes never become so abundant as when they invade a new country and infect the whole population. Therefore children may not be infected until several years after birth.

453. Disease, when endemic, is far less terrible than when epidemic. In the former case the sick are tended, the treatment is understood, and the business of the community is not impeded. In the latter, so great may be the number of adult sufferers that the sick receive scant attention, and so considerable the dislocation of business that famine may follow. Disease, when endemic, does the work of selection much more 'cleanly' and thoroughly than when epidemic. Practically every non-resistant person is eliminated, but few people perish from neglect or starvation. Epidemic disease, on the other hand, causes the death of numbers of people whose powers of resistance are considerable, while it spares during its intervals of absence many others whose resisting powers are low. It selects in a much less discriminating fashion. ${ }^{1}$

454. Formerly smallpox was endemic in Europe in the same way as measles is to-day. At present vaccinia is endemic. The disappearance of smallpox coincided with the prevalence of vaccinia. A considerable portion of the community, including some men distinguished in science or of great intellectual activity, insist that the two events had no causal connection, and therefore that vaccinia is not a preventive of smallpox. But, if they are right, it is difficult to conceive what can have banished the latter. It cannot have been sanitation, for smallpox is an air-borne

1 We noted that sanitary science has absolutely no control over air-borne disease. In England it is customary to close the schools during an epidemic of measles, chicken-pox, mumps, or whooping-cough. It is a question whether " the game is worth the candle," at any rate as a preventive measure. Speaking practically, every susceptible child-and innately unsusceptible children are rare-is sure to acquire the disease sooner or later. The danger from chickenpox and mumps is negligible. Measles and whooping-cough are more dangerous to infants than to older people, but not appreciably more dangerous to school children than to adults. If these diseases are inevitable, it is perhaps better that they should be done with early in life, when time is less valuable and the inconvenience not so great as later. 
disease, and no other disease of this kind has been so affected. It cannot have been isolation, for, if vaccinia is no protection, almost the whole community is susceptible, and doctors and nurses should contract and spread the disease. Isolation is possible only when susceptible individuals are comparatively rare. It has been denied, even, that there is such a thing as acquired immunity against any disease. But the fact that the microbes of acute disease, which at first swarm in an infected person, disappear within a definite time, so that the sufferer no longer conveys infection after recovery, is decisive proof. The fact that a vaccinated person cannot be made to contract vaccinia again soon after recovery is proof that it confers immunity against itself. The fact that he can be successfully vaccinated after an interval of years is proof that the immunity is not perpetual. The fact that smallpox, if experimentally transferred to the calf, becomes cow-pox, and if transferred back to man becomes vaccinia, is proof that the latter is a modified form of smallpox. The fact that recently vaccinated persons are able, with almost complete safety, to nurse people ill of smallpox, whereas unvaccinated persons are almost sure to contract the disease, is proof that experience of the attenuated malady affords protection from the more virulent type. The fact that in England most people who contract smallpox have been vaccinated, but not recently, is proof that the protection afforded by vaccinia is only transient-so transient, owing to the mildness of the disease, as to be midway between 'acute' and 'passive' immunity. Probably the agitation against vaccination would never have arisen in this country had the power of conferring permanent immunity from smallpox not been claimed for vaccinia by the medical men of a past generation. Reaction followed the discovery that most sufferers from smallpox had undergone vaccination. The public learns very slowly, but forgets no faster.

455. Water-borne diseases are almost as infective as the airborne type. They prevail as epidemics more rarely in isolated communities, but as such are not uncommon in densely populated centres. The microbes are very dependent on the environment outside the body of the host, therefore they are less able to travel than air-borne species, and seasonable changes may for a time purify the water-supply, even where they are most prevalent.

456. Earth-borne diseases, of which the only one that greatly affects human beings is tuberculosis, are always endemic. Direct sunlight is fatal to the microbes of tuberculosis. In effect, it can- 
not be contracted in the open air. Its microbes are found especially in such dark, ill-ventilated, and crowded dwellings as are built by the civilized inhabitants of cold and temperate regions. More especially it affects the larger towns and cities, and yet more the slums of these. Beyond all other complaints it is the disease of civilization. As in the case of syphilis, its long duration confers considerable powers of journeying to distant lands.

457. For all we know or can surmise to the contrary, distinctively human contagious and insect-borne maladies may have prevailed during the early Stone Age. Air-borne maladies can hardly have come into being till large continental tracts had been relatively densely peopled by hunters and nomads. The advent of water-and earth-borne diseases must have occurred later. Their existence implies large and settled populations. In the one case the watersupply, and in the other stationary dwellings must have been constantly used and infected. Probably, of all animals, human beings suffer most, or at least from the greatest number of microbic diseases. More than any other animal, man now dwells in large and settled communities, between which there is constant intercourse. Many insect-borne diseases of the lower animals, however, are known to be endemic in certain regions, as for example the Texas fever of cattle, which is carried by ticks, and the fly-disease in South Africa, which is borne by the tsetse fly. Races long afflicted by them tend to evolve resisting power, whilst, as in the case of man, immigrants suffer severely. Air-borne diseases, such as are perhaps the rabbit plague of Canada and rinderpest which lately more than decimated the herbivora of Africa, are the causes of the great murrains or pestilences which tend to appear whenever animal populations become dense. The distemper of dogs appears to have become endemic amongst the large and settled canine population supported by man, but amongst wild animals air-borne diseases appear always to be epidemic. Earth-borne diseases occur especially amongst browsing animals, which acquire them through herbage that has been tainted by infected individuals. Probably the disease which destroys hive-bees is earth-borne. Since recovery within definite time from microbic diseases implies immunity, and since the vast destruction of life greatly diminishes the available supply of nutriment for the microbes, epidemics amongst lower animals are usually very transient. Probably the starting-point of every great pandemic of earth-borne disease is some area where the animal hosts, having undergone evolution, are 
numerous but resistant, and where, therefore, the disease is endemic. Rinderpest is said always to start from Southern or Central Asia. ${ }^{1}$

458. In histories and poems dealing with recent times, and in the works of modern philosophers or people who attempt to found new religious sects, we rarely meet any mention of endemic diseases. These are accepted as part of the normal environment, and claim allusion only when some prominent person dies of a 'decline' (consumption) or the like. But should a great pestilence occur, it is eagerly noted, described, and made the basis of endless dissertations and exhortations. Yet among modern civilised people endemic disease claims by far the greater number of victims. So also ancient records, which were written not for us but for the contemporaries of the writers, tell as little of the endemic diseases prevalent at the time as they do of the everyday life of the people, but much of such great and striking disasters as epidemics and wars. We may be sure, however, that some endemic diseases, for example leprosy, the contagious maladies, and malaria, did exist very anciently.

459. A notable contrast between ancient and modern times is furnished by the fact that pestilence (i.e. epidemic disease) was

1 The microbic or other diseases of plants and lower animals, considered from the standpoint of the student of evolution, constitute a vast subject of great intellectual fascination and practical importance, which, it is to be hoped, some worker with more time and knowledge than I am able to claim will investigate. Like me, he will find his materials scattered through books of travel, histories, and technical works, but will have to surmount even greater difficulties than I have encountered in my very imperfect and fragmentary study of human disease. Many profitable industries have been ruined or are being checked in various parts of the world by plant or animal disease. Thus Phylloxera vastatrix (a. species of Aphides) caused great damage to the vines of France, and coffee-growing had to be abandoned in Ceylon. Pig and poultry farming on a large scale are unprofitable, because as surely as many animals are gathered in one place they are swept by epidemic disease. Wild animals are delicate in captivity, mainly because they perish of microbic diseases against which domesticated animals have undergone some evolution. The difficulties of the farmer illustrate very vividly the dangers which beset the human race in its earliest struggles towards settlement and civilization. Human selective breeding is at present outside the range of practical politics. Selective plant and animal breeding is practised all the world over ; but I am not aware that plants and animals, especially animals, have ever been bred to any considerable extent with the view of increasing their powers of resisting disease. Some horses and cattle, for example, are able to acquire immunity to the fly disease of Africa. Artificial selection achieves results much more rapidly than Natural Selection. It should not be difficult to establish a resistant race by continued breeding from animals that had shown themselves capable of recovery from infection. Since plants are almost incapable of making useacquirements, and since their diseases are usually caused by muticellular organisms, only ' inborn' immunity could be evolved amongst them. 
formerly much more common and severe. The Great Plague of I 666 was the last very destructive epidemic in England, but it was not nearly so destructive as the Black Death, which occurred some centuries previously. Both these pestilences had spread from regions in the East, where they were endemic, where the inhabitants were so resistant that the victims were comparatively few, and where they caused no more comment in histories, poems, and exhortations than malaria in Africa or tuberculosis in England. As has always been customary, preachers in Europe attributed them to the anger of the Deity, and called the peoples to repentance for sins committed against this or that religion, and to a more strenuous support of this or that sect. Similarly, while no great epidemic of our domestic diseases is recorded in history, they have occasioned some terrible pestilences amongst peoples to whom they were previously unknown, to whom we conveyed them, and by whom they were regarded as evidence of divine or diabolical anger.

460. The inference is irresistible that historians are mistaken when they attribute ancient epidemics to diseases which, owing to war, bad sanitation, or similar causes, came suddenly into being, but have since died out of the world. In each case in which the accounts are sufficiently ample and clear, every ancient pest may be identified with an air, water, or insect-borne malady now, as then, endemic in some part of the world, whence it has spread periodically whenever the conditions, such as a hot, dry summer and much intercourse between neighbouring nations, have become favourable. Bad sanitation tends, of course, to render a strange water- or insectborne disease epidemic when once it has arrived, but, other things equal, it tends also to perpetuate it afterwards in the endemic form. Yet, though sanitation did not noticeably improve, the Black Death, the Great Plague, and other exotic diseases retreated to their normal habitats in India or farther east. Both the former were evidently the same bubonic plague which is now ravaging India. They "spent their force," they disappeared from Europe because they were epidemic-because the disease became so prevalent that it exhausted its nutrient supply of susceptible rats or human beings so effectually that it could not persist. Very modern sanitation has rendered its return to Europe difficult. Rats are less numerous. Probably all our earth- and air-borne diseases, which are much better able to travel than insect and water-borne complaints and therefore sooner become permanently established, were endemic amongst our ancestors before the dawn of established history. But from remote periods, from myth and 
legend, we seem to gleam dim accounts of air-borne pestilences. Thus the disaster which befell the Assyrian host before Jerusalem and that which afflicted the Greeks before Troy had that appalling suddenness which characterizes an air-borne epidemic occurring in a population entirely susceptible.

461. To sum up, human microbic diseases seem to have originated in the Eastern Hemisphere, which has long been thickly populated, each disease from a single centre, whence it spreadfacts which appear to be confirmed by the apparently total lack of all microbic diseases, except malaria, in the various parts of the West before the invasion by Europeans and their subsequent establishment there. Earth-borne and contagious diseases must have been endemic from their origins, as also must have been some insect-borne maladies. Doubtless water-borne diseases early became endemic wherever the conditions, including a dense and settled human population, were favourable. Air-borne disease was probably epidemic at first, passing with great rapidity and fearful results from one region to another, but gradually became endemic in all thickly populated localities that were in frequent communication with surrounding areas. There can be no doubt that all our familiar maladies were rife in Europe at the time when Columbus set out on his fateful voyage, and initiated the greatest tragedy, the most tremendous event in human history.

462. On the one side of the Atlantic were peoples who for thousands or tens of thousands of years had been slowly evolving resisting power against a multitude of maladies-peoples whose increase had been very slow, largely because of the numbers of the unfit that had perished from disease, maladies that had gradually passed from the epidemic form in which the selection of their victims was haphazard and imperfect, to the endemic form, when selection was more thorough and clean. These Eastern peoples now dwelt under conditions that would have been fatal to their remote ancestors. Their civilization, with its dense communities and constant communication between distant parts, was absolutely conditioned by their power to resist many diseases. Their houses, especially in their great cities, were dark, ill-ventilated, and crowded both with men and microbes. Their clothes, which their climate necessitated and custom and religion ordained even in warmer regions, were vehicles almost ideally adapted for the conveyance of disease. On the other side of the Atlantic were peoples who had undergone no evolution against any zymotic malady except malaria, and against the latter only in the wooded tropical parts. 
To them came Eastern civilization, not by slow degrees, as it had dawned on the inhabitants of the Old World, but suddenly, and, in its consequences, fearfully. The aborigines could not in a few generations achieve an evolution which the Europeans had accomplished only during the lapse of thousands of years and at a cost of millions of lives.

463. At once the very ancient conditions of the Old World were reproduced. Air- and water-borne diseases began to sweep in great waves of pestilence over the whole vast regions of the West. The entire population was susceptible; and, therefore, almost every individual was stricken down. Each disease took its toll of victims, and then, its nutritive supply exhausted, passed, but only to return after intervals of years in the same epidemic form. Towns and cities of the European type, foci of endemic disease, arose on the seaboard, extended into the interior, and provided the starting-point of fresh epidemics. Measles, cholera, and especially smallpox, penetrated into remote prairies and forests, and piled the earth with the dead. Since the invasion by Europeans was rapid, and the aborigines had achieved no antecedent evolution, the epidemics were more frequent than anciently in the Eastern Hemisphere, and more deadly in their effects. Whole tribes and nations were exterminated. ${ }^{1}$

464. The part played by tuberculosis, though less striking and obvious, and therefore less noticed by historians, was even greater. Prevailing mainly in the North and South, in the neighbourhood of European settlements and missionary establishments, it spread with their extension into the interior and swept the land quite bare of its native inhabitants. It completed the destruction, begun by air- and water-borne diseases, of the natives even of the tropical West Indian Islands and parts of the adjacent mainland, especially in the neighbourhood of mines, in which aborigines were forced to labour and where the malady prevailed to a terrible extent. But in the mainland of tropical America, where the great forests were defended by malaria which prevented white settlement, and by heat which necessitated the building of airy dwellings, it did comparatively little harm. Moreover, the whites intermarried with the aborigines, and the mixed race which resulted, inheriting in half measure powers of resisting both malaria and tuberculosis, seems, as in Mexico, destined to survive. In North America and in the open pampas about the river Plate, the work of extermination was more thorough. Many unions occurred between the whites and ${ }^{1}$ Hirsch, vol. i. p. 136. 
the aborigines. But except on the distant frontiers the native women perished or were sterile through ill-health, and even the half-caste children could not survive under the conditions that were created. ${ }^{1}$ The natives melted away, and now are found almost solely in remote and thinly populated wilds, where a nomadic life affords some protection. The white colonization of Australasia is having similar results. In Polynesia, as soon as the trader brings his clothes and the missionary insists on his converts wearing them and attending crowded churches and schools, the work of extermination begins.

465. In Asia and Africa, where Europeans have made many conquests and native wars have been as many and as bloody as in the West, every European settlement has a flourishing native suburb. But throughout America and Australasia no considerable European town has a native quarter. This one fact brings into startling prominence the difference between the inhabitants of the East and West. The latter were able to found settled communities and even cities before the advent of tuberculosis. Since its arrival even nomadic life is hardly possible to them.

466. Writing of the Spanish occupation of the West Indies, the historian Froude declares, "The Carib races whom the Spaniards found in Cuba and St Domingo had withered before them as if struck by a blight. Many died under the lash of the Spanish overseers. Many, perhaps the most, from the mysterious causes which have made the presence of civilization so fatal to the Red Indians, the Australians, and Maoris. It is with man as it is with animals. The races that consent to be domesticated, prosper and multiply; those that cannot live without freedom, pine like caged eagles, or disappear like the buffaloes of the. prairies. Anyway, the natives perished out of the islands of the Caribbean Sea with a rapidity

1 The fact that half-castes abound in tropical America but are comparatively rare in the North has an interesting bearing on the Mendelian hypothesis. It has been surmised that the inheritance of susceptibility and resisting power to disease is alternative. But if that were true, and if the independent inheritance of characters be also a fact, half-castes should abound in the North as much as in the South, and be as capable of dwelling in tubercle-infested cities as Europeans. A proportion, while displaying a Mendelian mixture of Indian and European characteristics, should inherit fully the powers of resisting consumption possessed by their white ancestors. Obviously, since they persist in the tropics, where selection by tuberculosis is less stringent, but perish in the temperate regions, where it is more severe, the 'inheritance' of resisting power is blended, not alternative; wherein it accords with all other human racial traits except eye colour. 
which startled the conquerors. The famous Bishop Las Casas pitied and tried to save the remnant that was left. The Spanish settlers required labourers for the plantations. On the continent of Africa were another race, savage in their natural state, which would domesticate like sheep and oxen, and learnt and improved in the white man's company."

467. These sentences are typical of much that has been written concerning the decay of the New World races. Almost all writers unite in speaking of it as mysterious, and yet the facts are patent to all observers on the spot. There is no more mystery connected with their decay than with the extinction of the dodo and the bison. It cannot be doubted that the races of the New World have suffered or are suffering extinction in consequence of the introduction amongst them of Old World microbic diseases, and because of one other cause, also an importation, of which more hereafter. ${ }^{1}$

468. Like the imported African slaves that suffered from sleeping sickness, and perhaps also Swiss mountaineers who are said to die of nostalgia, but probably fall victims to microbic disease, they perish of physical, not mental decline. Their incapacity to achieve European civilization is mainly corporal, not psychical. That much is quite beyond dispute and may be verified by reference to the reports of all the Departments of Public Health in America, Australasia, and Polynesia. These show quite decisively that the proportionate mortality of the natives is very high, that they perish on the average at an exceptionally early age, and that the causes of their deaths are our imported diseases. Their families are abnormally small; but we need not, as so many writers have done, seek a mystical explanation. Presumably, in the New World as in the Old, amongst natives as amongst Europeans, ill-health is one of the causes of sterility.

469. Anciently the inhabitants of conquered territories were put to the sword, and thus were never again a menace to the invaders. In this manner the Jews established themselves in Palestine, and, apparently, the Saxons in England. Zengis Khan is said to have destroyed eighteen millions of people in China alone. Red Indians and Maoris sought to exterminate their enemies, as do African and Polynesian savages at the present day. Civilized peoples fight to conquer, not to destroy. Rome extended her sway over many lands, the inhabitants of which prospered and multiplied under her rule. Similarly, the Normans in England and

${ }^{1}$ See chapters xv. and xvi. 
the English in Wales merely administered the conquered territory. England and Russia govern empires vaster than the Roman, and, so far from making any conscious effort to root out the subject races, endeavour to render them peaceful and prosperous.

470. But if history teaches anything with plainness, it is the lesson that conquest, to be permanent, must be accompanied by the extermination of the natives; otherwise, in the fullness of time, the conquered expel or absorb the conquerors. The Greeks and Romans were expelled from their acquisitions. The Normans were absorbed. The Moors have left scarcely a trace in Spain. The Irish continually threaten the expulsion of the English, to whom the Welsh are united on equal terms. In parts of South America pure Spaniards have nearly disappeared. It is folly to suppose the European possessions in Asia and tropical Africa have any element of permanency. The local endemic diseases fight for the natives, who yearly grow more numerous, intelligent, and insistent in their demands for self-government. Presently the Europeans, like the Romans, will be expelled. South Africa is not effectually defended by diseases strange to Europeans; but they have imported none which the natives are unable to resist. Therefore at the Cape and in the north of the Continent, Europeans are threatened with absorption; or if, as is very unlikely, the two races remain for ever distinct, with subjugation. History affords no example of a race maintaining perpetual supremacy over a more numerous subject people.

47I. But in the Western Hemisphere conquest has usually been followed by extermination. Disease has wrought more widely and not less fatally than the sword. The races of nearly half the world are becoming extinct, and are being replaced by races from the other half, and in this great cataclysm our own people has played a predominant part. The Spaniards and the Portuguese, powerful maritime nations in the fifteenth and sixteenth centuries, had the first start in the race and chose the seemingly richer tropics. But, checked by malaria, European emigration soon dwindled. Possibly there are now fewer individuals of pure European descent in tropical America than there were three hundred years ago. But the Britons, who colonized North America and at a later date Australasia, multiplied enormously, and the stream of new colonists, so far from dwindling, ultimately gathered volume, till now by far the larger part of our race resides abroad. Owing solely to the vast void created by disease, a people 
who, four centuries ago, numbered no more than the present inhabitants of Greater London, has increased nearly fifty-fold. The greatest world-force to-day, it seems certain to maintain its position, for the sword as a means of founding permanent empires went out of date with the coming of civilization, and the regions that disease can lay waste are all occupied, not by mere conquerors, but by colonists rooted in the soil and resistant to every disease that is likely to afflict them. No other race in the world has such room for growth and expansion as the Anglo-Saxon. 


\section{CHAPTER XV}

\section{ALCOHOL}

The essential constituents of alcoholic beverages-The reasons why men drink-The types of men that drink for thirst, taste, and the cerebral effectsThe question as to whether alcohol is beneficial or harmless-The mental effects produced by alcohol - Normally no man drinks except for the pleasure that drinking affords-Inheritance and acquirement in relation to alcohol-The hypothesis that men drink in proportion as they do, or do not, exercise self-controlThe causes that determine the extent of indulgence-The inheritance of susceptibility to the charm of alcohol-Individuals differ as regards susceptibilitySelection in relation to alcohol.

472. T $T E$ see, then, that disease is the only factor, at any rate the only clearly recognized factor, of selective elimination sufficiently stringent amongst civilized men to be a cause of progressive evolution. The racial changes resulting from it have rendered many peoples of the Old World so resistant to the endemic diseases of their own countries that they are now able to dwell in large and settled communities and so achieve civilization. On the other hand, civilization, with its dense populations and constant intercourse with distant peoples, has conveyed to and rendered prevalent in every country the diseases to which the environment is suitable. The races to which civilization with its endemic diseases came so slowly that the birth-rate tended to exceed the death-rate, are adapting themselves to the new conditions. The rest have become extinct or are drifting towards extinction.

473. But parasitic micro-organisms are not the only causes of selective disease amongst civilized men. Certain narcotics, of which the principal are alcohol and opium, are causes of disease, disablement, and death, as stringently selective and as widespread as any parasitic species. Unfortunately, we now enter a region of discussion which has ever been a playground of passion and prejudice, born, on the one hand, of self-interest, and, on the other, of a fanaticism as unreasoning as any that has been displayed in religious controversy. Here the disputants have belonged mainly to classes peculiarly unfitted for the calm discussion of what are 
really abstruse problems in heredity and psychology. No statement has been too obviously absurd for acceptance or too obviously true for rejection by the one side or the other. Nevertheless, in dealing with alcohol we possess one great advantage : most people are able to appeal not only to the observations of others, but to their own sensations. If the reader has anything like an average knowledge of life, he will possess ample materials drawn from his personal experience on which to found a judgment. In science independent thought, founded if possible on first-hand knowledge, is not only a right but a duty. Applying the broad principles of heredity already laid down, I shall set forth conclusions which conflict with popular beliefs, but which, I think, the reader will find not altogether unreasonable.

474. The essential constituents of every alcoholic beverage in common use are alcohol, certain flavouring agents, and water. These three constituents attract three separate classes of drinkers; or, at any rate, they supply separate and unlike motives for drinking, one or other of which predominates in every drinker. Water is an essential part of our bodies. When the quantity within us becomes deficient, we crave to renew it, and this instinctive craving we term thirst. Many men drink alcohol only when they are thirsty. The thing primarily desired by them is water. Alcohol is added, as lime-juice might be, merely as a flavouring agent, as something which renders the necessary draught of water more pleasing. Drinkers of this sort usually prefer the lighter beverages, such as beer and wines, which contain a considerable proportion of water. Other men drink alcohol mainly to gratify the sense of taste-to produce pleasant sensations by stimulating certain nerveendings in the mouth. The average connoisseur is a typical example. He drinks for precisely the same reason as a child consumes sweets, and he always chooses the beverages most palatable to him. The third class of drinkers comprises people who desire to experience the various sensations which result from alcohol circulating in the blood and acting directly on the central nervous system, the brain-sensations of well-being, of exhilaration, of confidence, of general happiness, of comfort, of cheerfulness, of restfulness, of somnolence, and so forth, which in different drinkers mark different stages of intoxication. A drinker of this sort, especially if his desires are very strong and he is unable to procure anything more palatable, may drink thirst-creating and nauseous compounds such as methylated spirits.

475. Of course the three primary constituents of alcoholic 
beverages, the three sets of sensations to which they give rise, may all attract a given drinker at one and the same time; or a man may drink at first to satisfy his thirst or his palate, and later for the sake of the feelings which accompany intoxication; or in the beginning of his career as a drinker he may be influenced mainly by one or other of the former motives, but later in life mainly by the last. Nevertheless, the fact remains that the three main constituents are distinct, the desires they gratify are utterly distinct, and that, while some drinkers are influenced solely by one of these desires, all drinkers are influenced at any given moment more by one of them than by the others. The heated cyclist or cricketer, who gulps a quart of light beer and is satisfied, evidently seeks sensations which are not the same as those desired by the connoisseur toying with his choice wine, smelling, sipping, rolling it in his mouth so as to get all the enjoyment possible from its taste and aroma; nor the same as those sought by the man who desires a 'stimulant' or 'night-cap,' or who with deeper longing craves intoxication, and drinks perhaps with a shudder some strong and it may be distasteful spirit which increases his thirst.

476. Thirst and taste are not, by themselves, causes of excessive indulgence. Both are satisfied by a very moderate degree of indulgence; and the experienced drinker, who knows what sensations to expect from various amounts of the beverage he is imbibing, soon learns the quantity which will afford him the greatest gratification. Both thirst and taste are incentives to instinctive actions. The former impels to the drinking of dilute beverages, and ceases as soon as sufficient water is imbibed; while the palate is cloyed by a few glasses even of the choicest wine, just as it would be by the best of sweets. Excessive drinkers are drawn solely from the class that seeks the sensations produced by alcohol when acting directly on the brain. Of course, however, not every drinker who belongs to this latter class takes alcohol in excess. Many, if not most, in England at least, take no more than they would if impelled by thirst or taste.

477. It is a moot point whether alcohol in small quantitiesthe quantities that are drunk by the 'moderate' people we meet in society, who are able to go about the occupations of life apparently unaffected mentally-is injurious. Many people, including a minority of medical men, declare that the smallest amounts are harmful, and support their contention by an appeal to the statistics of insurance companies, some of which place in different classes total abstainers and moderate drinkers, and report that the 
former live, on the average, distinctly the longer. But all inebriates are not paragons of candour. None who seek to insure their lives will admit that they are other than moderate. Were they so honest as to admit excessive drinking they would not court inevitable rejection by presenting themselves for medical examination. ${ }^{1}$ There can be no doubt that many people who have insured as moderate drinkers really indulge to excess. Probably most medical examiners have experienced the humiliation of having recommended to insurance companies individuals who declared they were temperate and showed no signs that they were otherwise, but who were subsequently discovered to be much the reverse. If we accept the dictum that the smallest quantity of alcohol is injurious, we must believe, contrary to the evidence of our senses, that whole nations like the Italians suffer chronic ill-health. Some men are much more resistant to alcohol, physically and mentally, than others. I have known many excessive drinkers who have survived till old age. Others perish early in life. Doubtless the truth is that, while large quantities of alcohol are injurious to all men, they are less injurious to some men than to others; and that, while small quantities, such as are drunk by Italian peasants, are injurious to some men, they'are little, if at all, injurious to most men.

478. Probably no substance that is eaten or drunk produces quite the same sensations in every one. One man prefers beef, another mutton, a third pork; one prefers apples, another oranges ; one tea, another coffee; one delights in tobacco, another derives no pleasure from it. If their sensations were exactly alike, their preferences would be the same. Identical quantities of alcohol make some men cheerful, others maudlin, others morose, others amiable, yet others pugnacious; some are stimulated to greater activity, others are lulled to sleep. However, it is evident that whatever his sensations, the habitual and excessive drinker finds them pleasant, or he would not, in spite of heavy penalties, seek to renew them periodically.

479. In one particular all excessive drinkers agree": they pay for the pleasant sensations experienced when alcohol is circulating in the blood by feelings, following its elimination from the body, which are the reverse of pleasant-sensations of abnormal thirst, headache, nausea, nervousness, wakefulness, irritability, terror,

1 One Canadian company which does business in this country demands from its 'proposers' a declaration of the number of times they have been drunk during the past five years, and the date of the last occasion. If nothing else is elicited, at least a maximum of lying is. 
delirium, and the like, which indicate temporary or permanent impairment of health. In some cases, especially with habitual drinkers, these disagreeable sensations are temporarily alleviated by further indulgence in alcohol, the drinker taking "a hair of the dog that bit him." Doubtless the fact that drinking often. affords temporary relief from the after effects of previous indulgence conduces to continued indulgence. Some writers seem to hold a belief that it is the sole cause of all excessive drinking, at any rate of all excessive drinking that is of importance. Obviously, however, they are mistaken. A week's incarceration in prison, which may remove every unpleasant sensation, will seldom cure a habitual drinker. Sailors, who start on a long voyage in miserable case but return perfectly cured, are as prone as any class to deep indulgence. Years ago I lived in New Zealand with bushmen and "gum diggers." Alcohol was not obtainable in the neighbourhood. Some of my companions, men in splendid health and vigour, dreamed, as of heavenly delights, of the week or fortnight of furious drinking in which they were wont to dissipate the earnings of months. Clearly, then, the craving for drink is not always due to a longing to counteract the ill-effects of previous indulgence, but to a recollection of its delights. The relief which continued drinking affords is a contributory cause, but never, of course, the primary cause. ${ }^{1}$

480. The craving for alcohol is a resultant of two factors. The first is an inborn (nutritional) capacity to enjoy or develop a power of enjoying the sensations that alcohol awakens. The second is a recollection of the sensations aroused by previous acts of drinking. No one could possibly crave for indulgence in alcohol unless he were so constituted as to enjoy the effect-any more than he could crave for a draught of sea-water. On the other hand, no one is born with a knowledge of the sensations aroused by drinking, therefore none can crave for them without a recollection of previous experience. It is true that a man who has heard of the delights of drinking may desire alcohol in the sense that a boy desires tobacco, or an Englishman some tropical fruit; but this desire is quite distinct from that which besets the experienced toper. Both these desires, again, are distinct in type from hunger, or thirst, or the

1 This discussion, with much else in the present chapter, may appear childish to the reader-an attempt to prove the obvious. But it must be borne in mind that we are now dealing with a subject about which an incredible amount of nonsense has been written and accepted. It is necessary, therefore, to demonstrate even the very obvious. 
sexual craving. The latter are instincts, 'inborn ' characters, which develop inevitably as the body develops, and which, though they may be awakened to activity by fitting stimulus (the presence of food, water, or a particular mate), are not then created by it in the sense meant. In other words, they are not, like the drinker's longing or the boy's desire for tobacco, necessarily dependent on memory.

48I. Mental differences in relation to other narcotics are just as marked as in relation to alcohol. For example, some natives of China are satisfied with a very moderate indulgence in opium; in others the craving is so strong that, in effect, they commit suicide to gratify it. The insensibility which follows the inhalation of chloroform is a form of intoxication. Most people who have experienced it only once or twice describe the sensations of 'going off' and 'coming to' as horrible. But often pleasure attends wider experience. Some people find pleasure even from the beginning. Once a woman, who was inhaling the drug for the first time, said to me, "You are sending me into heaven." I knew a doctor's servant who was in the habit of stealing her master's chloroform and rendering herself deeply insensible.

482. The individuals of every species vary from one another in every particular. Probably there is not on earth an individual who is exactly like another in any character. We have seen that men manifestly vary in the sensations aroused in them by alcohol -in the amount of pleasure which drinking confers, and consequently in the degree in which they are tempted to repeat the remembered experience. Some men who have taken alcohol all their lives experience, apparently, not much more pleasure from the act than they derive from the eating of an apple. Their temptation to deep indulgence is of the slightest. From first to last they are attracted more by thirst or taste than by the cerebral effects. But beyond question the longing awakened in other men is very intense. A woman inebriate, in desperate straits for drink, once procured alcohol by a trick-the amputation of her own hand. Pathological specimens in hospital museums, gruesome preparations of cancers, diseased intestines, and the like, have been spoilt because people who had access to them drained the methylated spirits in which they were preserved.

483. Until very recently the vitally important fact that the degrees in which men are tempted by alcohol differ greatly was hardly recognized. ${ }^{1}$ Even now very many well-meaning people,

${ }^{1}$ As far as I am aware it was not recognized before 1896, when I published The Present Evolution of Man. 
who think it is in conflict with their schemes of temperance reform, endeavour to push it as much as possible into the background. At any rate it was and is quite ignored in all discussions on intemperance. Men are supposed to be temperate or the reverse solely in proportion as they exercise, or do not exercise self-control; or, what is very much the same thing, in proportion as they have or have not been well trained. ${ }^{1}$ But since there are men who, very plainly, are intensely tempted by alcohol, this hypothesis carries the corollary that all men who drink at all crave furiously for the sensations awakened by deep indulgence, and differ among themselves only because some of them exercise strenuous restraint. But let the reader turn to the best of all guides for him, his own experience of life. He is sure to have known many inebriates, men whose desires for alcohol were as those of a shipwrecked sailor for water. Some of them he has seen to struggle desperately if unavailingly against an overwhelming desire. As much as any man they seek to exercise self-restraint. Next let him examine

1 "It is often alleged, it is still more often assumed that the difference between the sober man and the drunkard is that the one possesses and the other lacks sufficient self-control to enable him to overcome his urgent and masterful desire for drink. The repetition from mouth to mouth, and from book to book, of this obviously false doctrine is one of the most striking instances of the ovine imitativeness of the human intellect, and of the ingrained habit of yielding unquestioning assent to authority. There are countless millions of sober men and women in the world, all of whom are ready to utter the parrot cry that they are sober because of their superior self-control, because they have the strength to resist temptation; and this they say in perfect good faith, when, if they would only think for one moment and interrogate their own consciousness in their own experience, they could not fail to know, "with irresistible conviction, that in fact they are not tempted to drink at all. Drink has no temptation for them. It offers them no allurement. It yields them no delight. It satisfies no craving. The taste of it finds them as indifferent as it leaves them. They are drink-proof, not because of any superior virtue, not because of any superiority of self-control, but because drink holds out to them no temptation And, not being tempted, they do not fall. They are no more meritorious for not getting drunk than a cat is meritorious for not wetting its feet, or a child is meritorious for not falling to the ground. Many such persons could not get drunk if they tried. The sensations produced by the ingestion of alcohol are to them so unpleasant that they are compelled to leave off long before they have taken enough to make them drunk. If, then, the difference between the drunkard and the sober person is not a difference in self-control, what is it ? . . The facts are that, on the one hand, when alcohol is applied in solution in the blood to the brain tissue of one person, there arises in that person pleasurable feeling. When applied in solution in the blood to the brain tissue of another person, there occurs in that person no such pleasurable feeling. . The feeling is neutral, or is unpleasurable, or is displeasurable." (Mercier, The Presidential Address on the Physical Basis of Mind, delivered at the sixty-seventh annual meeting of the Medico-Psychological Association, July 23 rd and 24th, I908.) 
his own emotions. Is he so miserable as to be afflicted by this intense and tormenting desire? Is he saved from that which will wreck home and happiness and life only by an effort as tremendous as and more sustained than that which keeps the sailor from draining his last drops of water? Or is it a fact that his desires for deep indulgence are very faint-as faint as the everyday desire of a landsman for water, so faint or non-existent that he is hardly, if at all, conscious of them? What of the people he knows best, his own relatives and intimate friends? Are the majority of them deeply tempted? Has he any valid reason for supposing that the lady whom he meets at dinner, and who sips her glass of wine in such seeming contentment, is earnestly desirous of draining the decanter? It is sufficient merely to ask the question to demonstrate its absurdity.

484. For the sake of comparison, it may be worth while to describe my own feelings at the present moment. If a glass of beer were before me, I should feel no inclination for it, though I am capable of enjoying beer when thirsty. If a glass of some sweet wine were here, I should enjoy perhaps half a glass, just as I should enjoy a morsel of chocolate. When I was younger and my delight in sweets greater, I should have liked more. A glass of whisky would awaken only repulsion. When sleepless or tired or when with friends I have often found it pleasant. I find, however, that even a small amount affects me disagreeably some hours later, and therefore as a rule I take none. I am not conscious at any time of having exercised any self-control worth mentioning. I dare say many people will doubt the truth of this introspection -especially abstainers who have had no experience, and people to whom even a glass of methylated spirits is a temptation. But I think a great many others will find a parallel in their own experience.

485. Plainly, then, as in the case of tobacco, a lifelong experience of alcohol endows some men with only weak and easily satisfied desires, whereas a much shorter experience awakens in others a keen longing which is satisfied with nothing short of deep indulgence, or is resisted only at the cost of acute suffering. Plainly again, not only does alcohol awaken deeper desires in some men than in others, but it awakens them more quickly. Selfcontrol, therefore, though a factor of great importance, is not the only or even the main factor in the causation of sobriety. It is argued sometimes that men are temperate or the reverse because those of the former class have, from the beginning and always, 
exercised self-restraint, and so have never acquired through experience the desires which increased indulgence creates in the latter. But we all know men and women whose whole lives are abandoned to an unrestrained search for pleasure, and who are nevertheless temperate in their drinking. Is it believable that these people constantly resist urgent temptation which they continually feed with inadequate indulgence? Abstainers form a class by themselves. They are not exposed in the same way to temptation. But probably the reader is a moderate drinker. Let him inquire of his own sensations.

486. Just as a limited experience enables the smoker to ascertain the amount of tobacco and the periods and methods of indulgence which are most agreeable to one of his type, so a certain experience endows the drinker with a similar knowledge. As we see, some men, influenced mainly by thirst or taste, are satisfied with a glass at meals. Others are satisfied with nothing short of complete insensibility. Some, desiring only the drowsiness which alcohol creates in them, take a "night-cap"; others must be sipping the whole day. In the latter case the drinker may not, and usually does not become intoxicated in the ordinary acceptance of the word; but a mental condition which borders on intoxication is so pleasant to him that he seeks to maintain it constantly during his waking hours, and in this way may imbibe a quantity of alcohol which may exceed the average amount taken by one who drinks when opportunity offers to the point of coma.

487. We must, however, always bear in mind that the drinking habits of men are not solely determined by the pleasure conferred by alcohol. Many men are unable to afford or are otherwise prevented from obtaining as much as they desire. Some are so trained that they regard all drinking with moral abhorrence, and from birth to death never indulge in it. Others, seeing the misery of their loved ones, or having the fear of physical, material, or social ruin in sight, become abstainers later in life. All normally constituted men are greatly influenced by fashion, that is, by the tone of the society in which they live. It is probable that our great-grandfathers encouraged one another to drink more, on the average, than they really craved. It is certain that under the present fashion many men voluntarily drink less than they would enjoy. But when all this is granted, it still remains true that a given experience of drink awakens stronger desires in some men than in others, that under any given conditions men on the average drink in proportion to their desires, that the men most tempted 
most often fall, and that the excessive drinker, whether actual inebriate or continuous soaker, is invariably one to whom deep indulgence is a strong temptation; whereas the moderate drinker is almost as invariably one who is so constituted that his temptation is much weaker. All deep drinkers suffer from the immediate or remote effects of poisoning, and know well to what their sufferings are due. It is almost inconceivable that any man would incur the inevitable penalties were he not greatly tempted. We never hear of men deliberately making themselves poor, contemptible, miserable, and ill with something, for example sea-water, which has no intoxicating effect. On the other hand, a moderate drinker who has had an average experience of alcohol and has in him the makings of a drunkard, is not common. The human will is seldom strong enough to resist the steady pull of vehement temptation when the latter is constantly fed by partial indulgence.

488. Some drinkers, it is true, are so constituted that alcohol affords them pleasure mainly as a relief from unhappiness. They are one variety, but on the whole a somewhat rare variety of excessive drinker. If the reader will again consult his own experience of life, I think he will find that nearly all the inebriates with whom he is well acquainted, slipped, after a longer or shorter experience, according to the ease with which strong desires were awakened in them, into bad habits under quite ordinary conditions of life. Again, bad companionship is said to lead to intemperance, and doubtless it often does. But usually the zest for such society owes its origin to alcohol, which can furnish it only to those who are susceptible to its charm. "Birds of a feather flock together." A man who craves for drink would hardly choose the society of abstainers or moderate men. The kind of pleasures that appeal to a more or less inebriated man are not those which appeal to respectable women, clergymen, scientific men, or athletes, as such.

489. Now, since susceptibility to the charm of alcohol is an 'innate' character which develops with the growth of the individual under stimulus of nutrition, it tends as such to be 'inherited,' in the same sense as, for example, the shape of his head. In this connection it matters not whether the individual does or does not drink. Awake or dormant, his susceptibility is still present. On that account drunkenness tends to run in families. The child is "by nature" similar to the parent, and under similar conditions tends to acquire the same trait. Often, however, it happens that a sober or a drunken parent is succeeded by a child 
who is the reverse. The child has two parents, and may inherit mainly or exclusively from the one or the other. Or the conditions of life under which parent and child develop may be different. Thus a man, impressed by the "awful example" of his father, may become an abstainer. But his son, falling amongst evil companions, and seeing at first more of the delights than the woes of drinking, may exhibit the character of the grandparent. The facts remain, however, that, though many people who are very susceptible to the charm of alcohol do not fall victims to it, yet, whether it be indulged or not, the susceptibility exists, is greater in some people than in others, tends to be inherited in its various degrees, and people on the average tend to fall victims to it in proportion to its strength in them. The clearest proof that degrees of susceptibility to the charm of alcohol tend to be reproduced by offspring is found when we examine races. In the midst of abundance of alcohol, generations of Spaniards or Italians lead temperate lives. Under similar conditions Englishmen are more drunken, Russians yet more drunken, while Red Indians furiously drink themselves to death. Races are merely aggregates of more or less nearly related individuals. Therefore, as in the case of disease, the individuals of each race must tend to transmit their degrees of susceptibility to offspring and descendants.

490. There can be no doubt, then, that some people are so constituted as to be more tempted by drink than others, that they tend to transmit this characteristic to offspring, and that, though a proportion of those who have it are, in England at least, abstainers, or in far rarer cases moderate drinkers, yet all really excessive drinkers are drawn from their ranks. Again, there can be no doubt that excessive drinking is injurious, and that a great number of people, all of a particular type, indulge in it. The question arises, then, whether it is so injurious and to so large a number of people, as to act, like a microbic disease, as a stringent agent of selection. In that case it must so shorten or in other ways so influence the lives of a certain type of people, that the total number of offspring and descendants they contribute to the race is much less than would have otherwise been reared. According to the RegistrarGeneral, 22I I people died of alcoholism in 1905, the rate per million of the population being only 65 . In the same year the deaths from tuberculosis were 55,759 , the rate per million being 1632. Judged, then, by the returns, excessive drinking is by no means a stringent agent of elimination. The number of deaths recorded must bear a very small proportion to the total number of 
excessive drinkers. Must we conclude, then, that alcoholism only very rarely destroys its victims?

49I. The statistics of the Registrar-General are compiled almost exclusively from medical certificates of death. When I turn over the counterfoils of my old books of death certificates, I find, here and there but very seldom, the word 'alcoholism.' The term implies that the person whose end is recorded drank himself quickly, violently, and relentlessly to death. He or she died, not from the remote effects of alcohol, but from poison actually circulating in the blood at the time of death, or during the few days or weeks preceding. De mortuis nil nisi bonum; but I could not truthfully enter anything else. Sometimes such a one, after a more or less prolonged career of drink and poverty, owed his actual decease to a windfall of money. More common is 'cirrhosis of the liver,' an expression which is usually, but, as Dr H. B. Donkin has shown, not always a synonym for chronic or soaker's alcoholism. The Registrar-General's returns indicate that 4008 persons died of this complaint in 1905 , a number which works out at a rate of I 7 per million living. This number, even when added to that due to acute alcoholism, is too small to indicate stringent selection.

492. But, looking through my counterfoils, I see recorded the names of many people some of whom as I knew, and more of whom as I suspected, had been injured by excessive drinking before they died of accident or contracted phthisis, pneumonia, bronchitis, apoplexy, or the more gradual forms of paralysis, erysipelas, syphilis, kidney, heart, or arterial disease, or this or that other complaint which was the immediate cause of death. The circumstances surrounding their ends make me very sure that in a large proportion of cases they would not have contracted the fatal disease, or would have recovered from it had they not previously weakened their powers of resistance by intemperance. Some years ago "The Harveian Society of London instituted an investigation and found that in London, of 10,000 persons dying over twentyfour years of age, the result was as follows :-

A. Deaths in nowise due to alcohol. . . . . 8598

B. Deaths accelerated or partly caused by its abuse . 1005

C. Deaths wholly due to it . . . . 397

These 1402 deaths constituted almost exactly I4 per cent. of the total deaths. If this proportion still continues... the total deaths in the United Kingdom for 1889 , altogether or partly caused by alcohol were 94,416 , of which 26,736 would be directly due to alcohol and 67,680 accelerated or partly caused by it. 
In that inquiry, ${ }^{1}$ conducted over totally different grounds, the deaths of intemperate males of over twenty-five were 30 per cent. of the whole, while 25 per cent. were careless drinkers, sometimes taking excess. This being the proportion among adult males, we find no difficulty in believing that about one death in seven is partly or wholly caused by alcohol, omitting for the moment those caused indirectly." 2

493. Very possibly the above estimate exaggerates the mortality due to alcohol, but that it is very considerable is shown by the statistics of insurance companies. Thus the United Kingdom Temperance and General Provident Institution, the policy-holders of which are separated as sheep from goats into abstainers and non-abstainers, found the mortality amongst the former 7 I.49 per cent. of the total expected, whereas amongst the latter it was 96.66 per cent. In the Sceptre Life Office the percentage was 56.86 and 77.6r. On the average the members of the Friendly Society of Rechabites, who are abstainers, live longer and have less sickness than Oddfellows and Foresters. The mortality amongst grocers has increased appreciably since they were permitted to sell wine and spirits. English and Russian soldiers, who consume considerable quantities of alcohol, perish more readily of wounds than Afridis and Turks, who consume little or none. Heavy drinkers tend to fall into poverty, the slums of our cities being full of them. Their children, who, if they survive, tend to inherit their characteristics, perish in greater numbers than the offspring of more temperate people. Women drinkers are often immoral and sterile.

494. Evidently the elimination due to excessive drinking is much greater than that indicated by the returns of the RegistrarGeneral, so much greater that there is every probability that alcohol is an agent of stringent selection. We are able to test this conclusion; but, first, it is necessary to note that stringency of alcoholic selection varies with time and place. It is greatest when and where drink is most abundant and accessible, and the prevailing social tone most favourable to its consumption. Thus alcohol is more accessible to Italians than to Englishmen and $a$ fortiori to Red Indians; to coal-porters more than to coal-miners. The social tones prevailing during the Victorian era and the Commonwealth were less favourable to its consumption than those which prevailed during the period of the Georges and of the Restora-

${ }^{1}$ Collective Investigation Committee of the British Medical Association.

2 Alcohol and Public Health, by J. J. Ridge, M.D. London: H. K. Lewis. 
tion. It is less favourable amongst clergymen than amongst publicans. All this is very obvious and need not have been mentioned were it not that a confused notion seems prevalent amongst writers on the subject that selection is not selection if it varies in intensity. Selection is selection, no matter what its intensity. It may not be stringent enough to cause progression, but selection it is nevertheless. Again, it must be noted that the stringency of selection is not to be estimated by the magnitude of the mortality due to the selecting agent, but by the greater or lesser completeness with which it weeds out the unfit. When the unfit are very numerous, a large mortality may indicate a lesser degree of stringency than a lower mortality when the unfit are less numerous. For example, in Italy, where people are 'naturally' disinclined to excessive indulgence, and where alcohol is very abundant and accessible, the deaths of quite a few excessive drinkers may indicate a greater stringency of selection than the elimination of a much larger number in England.

495. The people that alcohol eliminates are those who are so constituted mentally that they are tempted to take it in excess. Therefore "the tendency of evolution is to produce a race . . capable of sitting down in the presence of floods of alcoholic liquor ... without the desire to get drunk." 1 Is there any evidence of such an evolution? If the reader will call to mind all the races of the world of which he has a knowledge, he will find, as in the case of a prevalent and lethal disease, that every race is resistant (i.e. temperate) in the presence of alcohol in proportion to the length and severity of its past experience of the poison. There is no exception to this rule. Moreover, whenever we possess a sufficiently detailed and prolonged history of a race which is now temperate, we find that it was anciently drunken. To this rule also there is no exception. Of course, however, when we seek to compare the past with the present of a race, we must not commit the absurdity of comparing one year or decade with the next preceding or succeeding. Man is a slow-breeding animal in whose race germinal changes occur only very slowly, and the extent of his drinking is influenced, not only by the depth of his desire, but by many other considerations, such as the accessibility of alcohol and the tone of the society in which he is reared. Therefore we must compare past eras or centuries with much later times.

${ }^{1}$ Sir E. Ray Lankester, Fortnightly Review, Sept. 1896, p. 41 3. 


\section{CHAPTER XVI}

\section{THE EVOLUTION AGAINST NARCOTICS}

Alcohol in ancient and modern times-Among the lake-dwellers-In Egypt -In Greece-In Italy-Among Jews-In West Africa-In Great BritainIn Germany-In China-In the Western Hemisphere-Opium-The poisonous effects of narcotics-The origin of the susceptibility to the charm of narcoticsQbjections to the theory of narcotic evolution-Education-Strength of beverages -Civilization-The memory of past racial disaster-Convivial and industrial drinking-The alleged effect of alcohol on the germ-plasm.

496. T TRONG solutions of alcohol are products of the knowledge and skill which civilization brings. The lowest savages, for example the Tierra del Fuegians and Australians, are unacquainted with means of manufacturing intoxicating liquor. Settled tribes almost always possess intoxicants in some form, alcoholic or other; but, unless they are well advanced in civilization, the beverages they manufacture are weak and far from abundant. Thus it is recorded that the natives of Guiana were obliged to drink their cassava intoxicant (which, however, was not alcohol) for three days before a state of drunkenness satisfactory to them was achieved. Moreover, especially in cold and temperate climates, the alcohol made by savages is usually derived from the fermentation of articles of food-milk, honey, corn, and the like-which are rarely abundant with them. Owing, then, to the insufficiency of the supply, due partly to difficulties of manufacture and partly to lack of materials, excessive drinking amongst most savages is comparatively rare and spasmodic. Consequently the elimination of the unfit is far from stringent. But, as in the case of such a disease as tuberculosis, with growing civilization comes an increasing and more regular exposure to the evil-in other words an increasing and more effective stringency of selection. It follows that a race that has evolved its own civilization or very slowly absorbed that of another, tends to become resistant to alcohol and survive, whereas a race which is suddenly introduced to civilization with its strong solutions of alcohol, tends to drink to extinction.

497. Probably in all the world alcohol has been most abundant 
and uninterruptedly accessible in the vine-producing countries of the South of Europe and the forest region of West Africa. There is evidence that it was manufactured from wild fruits such as the raspberry and mulberry by the lake-dwellers of Switzerland and the South of France during the Stone Age. We do not know when the vine was first cultivated for alcohol, but the fact that at the dawn of myth and fable grape-wine was in use all round the Mediterranean, argues an immense antiquity. Probably palm-wine has been used in West Africa and elsewhere for almost as long. Both the vine and the palm enable men to manufacture abundance of alcohol without trenching on their food-supplies. Just as we are able to gather little from history about the endemic diseases of antiquity, so we are able to learn little about its endemic drunkenness. Usually the earliest references relate to the intoxication of some prominent person on some memorable occasion, and then the reference is made, not because the person was intoxicated, but because the occasion was memorable. Thus Noah is represented as drunk when Ham discovered his nakedness, as was Lot when he became the ancestor of the Moabites and the children of Ammon. So also the suitors of Penelope were inflamed with wine when Ulysses slew them, and we are told that Alexander died of a surfeit of drink. Clearer evidence is to be gleaned from laws and injunctions against excessive drinking, such as are common in the Old Testament, ${ }^{1}$ and of which many

1 For example, "Who hath woe? Who hath sorrow ? Who hath contentions ? Who hath babblings? Who hath wounds without cause ? Who hath redness of eyes? They that tarry long at the wine. They that go to seek mixed wine. Look not upon the cup when it is red, when it giveth colour to the cup, when it moveth itself aright. At last, it biteth like a serpent, it stingeth like an adder" (Prov. xxiii. 29-32). "Woe unto him that giveth his neighbour drink, that puttest the bottle to him, and makest him drunken also." " Blessed art thou, $O$ land, when thy king is the son of nobles, and thy princes eat in due season, for strength, and not for drunkenness" (Eccles. x. 17). "Woe unto them that rise up early in the morning that they may follow strong drink, that continue until night till wine inflame them " (Isa. v. I I). "Woe to the crown of pride to the drunkards of Ephraim ... that are overcome with wine" (Isa. xxviii. I). "But they also have error through wine, and through strong drink are out of the way; the priest and the prophet have erred through strong drink, and they are swallowed up of wine, and they are out of the way through strong drink: they err in vain, they stumble in judgment. For all tables are full of vomit and filthiness, so that there is no place clean " (Isa. xxviii. 7-8). Jews have still a feast, that of Purim, when intemperance is not only permissible to them, but is absolutely encouraged. Modern Jews show no disposition to avail themselves of the permission; but anciently it was different. "We need only mention the Purim, or deliverance of the Jews from the rage of Haman, which, until the reign of Theodosius, was celebrated with insolent triumph and riotous intemperance" (Gibbon, The Decline and Fall of the Roman Empire, vol. ii. p. 85, ed. Grant Richards, 1903). 
are addressed to races which are now so temperate as not to need exhortation. The oldest known Egyptian papyrus, which comes to us from an antiquity of 7000 years, contains such an injunction, which is repeated in many later writings. "The moralists reprove these excesses, and cannot find words strong enough to express the danger of them. Wine first loosens the tongue, even wresting from him dangerous words, and afterwards it prostrates him, so that he is no longer capable of defending his own interests. Do not, therefore, forget thyself in the breweries; be afraid that words may come back to thee that thou hast uttered, without knowing that thou hast spoken. When at last thou fallest, thy limbs failing thee, no one will help thee, thy boon companions will leave thee, saying, 'Beware of him, he is a drunkard.' Then when thou art wanted for business thou art found prone on the earth like a little child." 1 "Young men especially should avoid this shameful vice, for beer destroys their souls. He that abandons himself to drink is like an oar broken from its fastening, which no longer obeys on either side; he is like a chapel without its god, like a house without bread, in which the wall is wavering and the beam shaking. The people he meets in the street turn away from him, for he throws mud and hoots after them till the police interfere and carry him away to regain his senses in prison." 2

498. The fact that the Spartans made drunken their helots that they might serve as "awful examples" to the aristocratic youth is clear evidence of the danger and probably the prevalence of drunkenness amongst the latter. Lycurgus amputated the legs of drunkards and destroyed the vines. Solon condemned an Archon to death for being drunk. The Senate of Areopagus punished men for too prolonged drinking. Pittacus of Mytelene inflicted double punishments for offences committed in drink. It is related that thirty-six competitors perished in one of Alexander's drinking matches. The festivals in honour of Dionysus "became more and more dissolute both in Greece and Rome until they degenerated into saturnalia of the most disgraceful character." 3 Drunkenness is a constant theme of Homer and Aristophanes.

499. Apparently alcohol was not abundant in Italy when Rome was founded, for "Numa, the successor of Romulus . . . directed, from the great scarcity of wine that prevailed, that no man should besprinkle the funeral pile with it, and when the

${ }^{1}$ The Maxims of Ani, XVIII. Dynasty, about I $530-1330$ B.C.

${ }^{2}$ Maspero, Life in Ancient Egypt, translated by A. Morton, p. 3 I.

${ }^{3}$ Samuelson, History of Drink, pp. 75-9. 
sacrifices to the gods were permitted in wine, it was declared, with a view to encourage the plantation of vineyards, that all wine so offered should be the produce of such vine plants as had been cut and pruned." 1 The elder Pliny, Petronius, and other historians of later Roman times, mention great excesses. The sobriety of the modern inhabitants of the vine countries, including Jews, who until lately dwelt mainly in the warmer parts of Europe, is well known. For example, until lately, the Italian peasants drank at their taverns, for a very trifling sum, by the hour, not by the quantity. A publican who adopted such a custom among Englishmen would be ruined, amongst Red Indians he would be murdered. An English physician resident in Italy writes-

500. "I have met time after time Italians who confess without shame that they have never drunk anything but wine; they are never drunken. Drunkenness upon Italian wine I have seen, but only amongst my fellow-countrymen and women. Among my servants I find that water as a drink is considered bad for the stomach, and is feared just as water in a bath is feared, as a peril and a danger. ... On inquiry from old residents in the country I learn that, however abundant the vintage, the contadini preserve always their temperate habits, drinking their fill, but never becoming drunken. Drunkenness in the British sense is so very rare as to be a matter of great interest and discussion when it occurs ; . . . even thirty years ago, when the export trade was practically nil, and there was a great excess of production over consumption, there was no drunkenness. In those days, I am told, wine was given to horses, and whole barrels would be poured out in the road to make way for the new vintage, when the price was only a few coppers per flask ; . . . it is given to infants, children drink it regularly, and babies are bathed in it, but drunkenness in the English sense does not exist." 2

50I. The sobriety of modern Jews has been the subject of numerous homilies. In London, for example, a drunken Jew-or Italian organ-grinder-is practically unknown. Hebrew sobriety is often attributed by the members of that community to "the fact, recognized by many non-Jews, that the absence of drunkenness amongst Jews is, in the main, due to their training, from their earliest childhood, in the principles of their religion, which, amongst other things, inculcates temperance and moderation in all things." The Jews have the reputation of being as much addicted to

1 Morewood, History of Inebriating Liquors, p. I I.

${ }^{2} \mathrm{Dr}$ H. Laing-Gordon, British Journal of Inebriety, Jan. 1904. 
pleasure as other races, and Jewish abstainers are almost as rare as Jewish drunkards. If, then, Jews are temperate only through self-restraint, they must all be under the influence of that dire craving which the toper feels, but must very bravely resist it. I do not know if it is possible for anyone to believe this. Moreover, the claim of superior moral training carries a corollary. The Hebrew moral code inculcates a good deal besides sobriety-the Ten Commandments, for example. It will hardly be maintained that Jews are as conspicuously superior to the English, for instance in morals in general, as they are in sobriety. If, then, Jews are so teachable and so well taught as to be sober mainly through religious training, must we conclude that their religious training is, speaking comparatively, conspicuously lax and faulty with regard to morals in general? This inference flows logically from the Jewish claim, but no Jew will care to press it. Notwithstanding the fulminations in the Old Testament, some Jews maintain that their race has always been temperate. But, whether we trace their descent from Noah or the savages of the Stone Age, we arrive at an ancestry actually or potentially drunken. What caused the change of type? The only real alternative to miracle is Natural Selection.

502. Palm wine is cheap and plentiful in West Africa, but it is customary at missionary meetings to represent the natives as debauched by the European liquor traffic. However, evidence to the contrary is not lacking. "My business is to state facts, not to reconcile those facts with the representations or fancies of any other person... The phrases that 'liquor is a great scourge to the natives of this country,' that it 'commits great ravages' and 'possesses extraordinary power over the people,' are not true.... The last phrase is certainly applicable to Great Britain, but not to West Africa.... The whole subject of the attitude of philanthropists towards liquor in this country is of intense interest to the philosopher. . . Therefore understand that in my humble opinion the future of the people of this country is not in any danger from liquor. One would like to be able to say as much for Great Britain, and of her nearest neighbour, to which nations, in the natural and logical course of circumstances, temperance advocates should be sent from this country." 1

1 "Extract from a speech on the Liquor Question delivered by Sir W. MacGregor, K.C.M.G., in Legislative Council, on the I6th Oct. I90I, and republished for general information."-Southern Nigeria Government Gazette, Sept. 9th, 1908, p. 1285 . 
503. "The Honourable Mr Sapara Williams : 'I rise to support the remarks which have been made by His Excellency the Governor relative to the extracts which he has read from the speech delivered by Bishop Tugwell before the Native Races and the Liquor Traffic Committee, and published in the Diocesan Magazine of the Western Equatorial Africa for July 1908. I have read the speech as published, and standing here as an Unofficial Member of Council, I am bound to say that it is the duty of this Board to take special notice of the same and to denounce the utterances therein contained. Every one knows that I am an abstainer and one who is entirely opposed to the evils of intemperance; but that fact does not impose on me the obligation to shut my eyes to facts in order to endorse these false statements of the Bishop.'" 1

504. "I do not say that every missionary who makes untrue statements on this subject is an original liar; he is usually only following his leaders and repeating their observations without going into the evidence around him. ... I have no hesitation in saying that in the whole of West Africa, in one week, there is not one quarter the amount of drunkenness you can see any Saturday in a couple of hours in the Vauxhall Road, and you will not find in a whole year's investigation on the Coast one-seventh part of the evil, degradation, and premature decay you can see any afternoon you choose to take a walk in the more densely populated parts of our own towns." 2

505. "One of my native colleagues also gave a temperance address. I shall not soon forget the earnestness with which he exhorted his hearers to fight the gigantic demon strong drink. 'For,' said he, 'unless we put forth our best efforts in this direction, there is a danger of becoming as drunken as the people of England.' This was a rather startling way of putting the case, but it was decidedly refreshing, as I remember there were so many in England who appear to be under the impression that whole territories are being depopulated by the importation of spirits. The statement expressed by my colleague was forceful, but I unhesitatingly endorse it. . . A A friend of mine, a most ardent temperance reformer, was appointed District Commissioner of Cape Coast a few months prior to my first residence. At the end of his first term of service he took the opportunity to write to a temperance paper in this country to say that the friends of temperance would

1 op. cit., p. I 283.

2 Miss Kingsley, Travels in West Africa, pp. 492-5 
be glad to know that the ravages of the drink traffic were not so serious at the Gold Coast as was generally supposed. Out of the hundreds of cases tried at his court during the twelve months, not one was traceable to the abuse of strong drink." 1

506. "But what are the facts of the case? The author has for many years been favourably situated for ascertaining the condition of affairs in Africa. He has conversed with men of culture who have resided for many years on the coast at various places of trade, and the consensus of opinion as well as the facts that have been narrated to him point to a widely different conclusion. The exportation of strong drink from England to the West Coast of Africa is enormous. It chiefly consists in rum; and by far the larger portion of this is forwarded into the interior, and is drunk out of sight amongst the savage tribes who are rarely visited by Europeans."2

507. The ancient Germans were exceedingly intemperate. According to Tacitus, "intemperance proves as effectual in subduing them as the force of arms." "They gloried in passing whole days and nights at the table; and the blood of friends and relations stained their numerous and drunken assemblies. . . . Strong beer, a liquor extracted with very little art from wheat or barley, and corrupted (as it is strongly expressed by Tacitus) into a certain semblance of wine, was sufficient for the gross purposes of German debauchery. But those who had tasted the rich wines of Italy, and afterwards of Gaul, sighed for that more delicious species of intoxication. They attempted, not, however (as has since been executed with so much success), to naturalize, the vine on the banks of the Rhine and Danube; ... The intemperate thirst of strong liquors often urged the barbarians to invade the provinces on which art or nature had bestowed those much envied presents. The Tuscan who betrayed his country to the Celtic nations attracted them into Italy by the prospect of the rich fruits and delicious wines, the productions of a happier climate. And in the same manner the German auxiliaries, invited into France during the civil wars of the sixteenth century, were allured by the promise of plenteous quarters in the provinces of Champange and Burgundy. Drunkenness, the most illiberal but not the most dangerous of our vices, was sometimes capable, in a less civilized state of mankind, of occasioning a battle, a war, or a revolution." 3

1 Rev. Dennis Kemp, Nine Years at the Gold Coast.

'Samuelson, History of Drink, p. 9.

${ }^{3}$ Gibbon, Decline and Fall of the Roman Empire, vol. i. pp. 255-6. 
508. "After the establishment of Christianity the monks, who needed wine for sacramental purposes, introduced the cultivation of the grape to Gaul and Germany." 1 Drunkenness increased enormously, both amongst men and women. ${ }^{2}$ German intemperance became " a byword amongst the nations, as the edict of Karl IV. declared." ${ }^{3}$ The papal official at the court of Frederic III. wrote to his master, "Nilhic est aliud vivere quem bibere," 4 "Living here is nought but drinking." So plentiful was the wine that a contemporary rhyme declares-

\section{"In fifteen hundred and thirty-nine}

The casks were valued at more than the wine."

People of all classes drank the whole day long like modern soakers. "Amongst the vices," said a preacher in Germany in the ninth century, "feasting and drunkenness especially reign, since not only the rude and vulgar people, but the nobles and powerful of the land are given up to them. Both sexes and all ages have made intemperance into a custom ... and so greatly has the plague spread, that it has infected some of our own order in the priesthood, so that not only do they not correct the drunkards, but become drunkards themselves." 5 Charlemagne and his successors vainly attempted, by means of numerous edicts and laws, to control the prevailing intemperance. At the present day modern Germans and Frenchmen of the vine regions are as temperate as modern Greeks, Italians, and Portuguese.

509. Great Britain has always been intemperate, and has sought as long, as often, and as vainly as Germany to cure the evil by laws. Though the vine was never cultivated to any considerable extent, yet alcohol gradually became cheaper and more accessible as the wealth and civilization of the country advanced. British intemperance reached its culmination, therefore, at a later period than that of Germany. However, since the eighteenth century, when men got "drunk for a penny and dead drunk for two pence," and had clean straw in which to sleep away the effects for nothing, it has steadily diminished. At the present day, though not so temperate on the average as the inhabitants of the vine countries, most Englishmen are sober, notwithstanding the fact that they are not abstainers.

510. At the present time the Chinese are very temperate in the use of alcohol. The teachings of Confucius and his disciple

1 Samuelson, History of Drink, p. I04.

3 Op. cit., p. II2.

4 Op. cit., p. II2.

20
2 Op.cit., p. Iog.

s Op. cit., p. II 4 . 
Mencius contain so little about alcohol that it is probable that there was little excessive drinking, even in fifth century B.C. "Whilst the princes and people are warned against voluptuousness and extravagance, we seldom find drunkenness referred to as a dangerous and prevalent vice." 1 But in the "Announcement about Drunkenness," an imperial edict believed to have been promulgated about III6 B.C., it is stated that "our people have been greatly disorganized and lost their virtue, which can be traced to their indulgence in spirits." "Yea, the ruin of states great and small " is invariably traced to the same cause, the use of spirits. ${ }^{2}$ Prince Fung, to whom the Announcement is addressed, says, "The people of Yin had followed the example of the sovereign, and the vice of drunkenness with its attendant immoralities extensively characterized the highest and lowest classes of society. . . . The disastrous consequences of drunkenness are strikingly set forth; he is called to roll back the flood of its desolation from his officers and people." 3

5II. The furious drinking of those savages, and those only, who have had little or no racial experience of alcohol, Red Indians, Esquimaux, Tierra del Fuegians, Australasians, and others, is too well known to need description. They tend to drink themselves to death at a single bout. ${ }^{4}$ Often, as in the case of the ancient Germans, their intoxication is accompanied by homicidal mania, a cerebral effect that tends to procure the speedy elimination of the drinker, and in its extreme forms is rarely found amongst peoples that have long used alcohol.

5I2. The facts concerning opium are very similar. It has been used in India for hundreds of years. So rarely do the natives of that country take it in excess that none of the scientific witnesses who appeared before the late Royal Commission on Opium had ever seen an instance. It was introduced into China about two centuries ago with disastrous consequences, but already many Chinese take it in moderation. In Burma, Polynesia, and Australia, where it was introduced within the memory of living man, the aborigines take it in such excess and perish of it in such numbers that their white rulers are obliged to forbid its use to them, while permitting it to natives of India, Chinese, and other aliens-just as in Australasia and Canada they are obliged to forbid the use of alcohol to the aborigines, while permitting it to the whites.

513. Narcotics, like most diseases, have two sets of poisonous

1 Samuelson, History of Drink, p. 19.

2 Op. cit., p. $20 . \quad 3$ Op. cit., p. 20.

4 See Alison's History of Europe, vol. i. p. 2 I, seventh edition. 
effects, the immediate and the remote. The former are those which are experienced at the time of indulgence or within a short period after, and are due mainly to the direct action of the drug on the nervous system. Probably the earliest effect is always a greater or lesser degree of paralysis, which besets at first certain 'inhibitory centres' of the brain, and, therefore, may present in the beginning an appearance of stimulation. Thus, after a small dose of alcohol the heart tends to beat faster, owing to paralysis of the centres which inhibit and control its movements, and there may also be mental changes involving some loss of self-control. A deeper degree of paralysis, involving other nervous centres, constitutes obvious intoxication. This initial paralysis, with its associated pleasurable sensations, is exchanged, some hours later, when the poison is eliminated from the body, for the well-known after-effects-the temporary illness which follows a bout of excessive indulgence. The remote effects are mainly gradual degenerative changes in many tissues, including the brain. As a rule they do not occur until after chronic excessive indulgence of some months, or, more commonly, years. In England few deaths occur from the immediately poisonous effects of alcohol, but amongst Red Indians and others, whose tendency to very deep indulgence is greater, they are more common.

514. Alcohol is an excretion, a waste-product of the yeast fungus, comparable as such to the urine or carbonic acid excreted by man. Opium and nicotine, on the other hand, are real toxins elaborated by the poppy and tobacco plants. They serve, like all other vegetable poisons and like the toxins of microbic diseases, as a means of defence against enemies. We have seen that an acquired immunity to a disease is a capacity, developed under the stimulus of use, to tolerate a toxin which was previously more poisonous. We have seen, also, that many individuals of human races that have had no previous experience of measles and other acute lethal diseases, are able to acquire immunity against them, though individuals of races that have had long experience are able to acquire it more easily. It follows that, in addition to having evolved special powers of acquiring immunity against particular diseases, man has evolved a general power of becoming able to tolerate toxins, which again is a part of the general power of making use-acquirements. Doubtless, it is on this account that experience makes the individual so tolerant of opium and tobacco that he is able to consume, without immediate poisoning, immensely increased doses.

515. But no amount of experience enables a man to increase 
his power of resisting tuberculosis nor greatly increases his power of tolerating alcohol. That is, he cannot acquire immunity to the former nor learn to take, without intoxication, doses of the latter, a waste product (the chemical composition of which is much less complex than that of the toxins) ${ }^{1}$ a hundred-or a thousand-fold larger than those which were immediately poisonous to him at the beginning of his experience. Therefore, in the case of acute diseases and opium and nicotine, man has a means of protection which is absent in the case of alcohol and chronic complaints such as tuberculosis. Consequently a race that is for the first time introduced to an acute disease or to nicotine or opium does not start "from the scratch." It is advantageously situated. It has undergone a previous evolution which, by enabling it to acquire immunity, places it in a position of advantage. In the case of tuberculosis and alcohol it has to undergo an evolution which, in each instance, must be begun almost at the beginning. Under severe modern conditions, the races of the New World are able to survive acute diseases and undergo evolution, but tuberculosis exterminates them. Therefore, evolution against tuberculosis is much the more difficult and tedious. Similarly, evolution against alcohol appears to be more difficult than that against opium. An evolution of a few hundreds of years has rendered the natives of India 'immune' to opium, but an experience of thousands of years has rendered the British only in a measure immune to alcohol in the sense that Italians are immune to it.

516. Tobacco may be compared to chicken-pox. Nearly all sufferers quickly and easily acquire immunity against this disease, and all tobacco-smokers are moderate in the sense that they do not desire to reproduce the immediately poisonous effects which they felt as beginners. So few people perish from the one or the other that no evolution results. Therefore races that have long been afflicted by chicken-pox suffer as severely as races that are newly exposed to it, while races that have long used tobacco indulge in it as deeply as those who have had no previous experience.

517. Opium may be compared to malaria. Malaria lies midway between tuberculosis and chicken-pox. It is more acute than

1 The power of acquiring a toleration does not, however, depend entirely on the chemical complexity of the toxin. Thus, while it is said to be possible to increase the toleration for arsenic, an absolutely simple substance, no appreciable increase of toleration follows the use of many vegetable toxins, for example digitalis and strychnine. See § 4I 3. 
the former and more chronic than the latter. But since native West Africans suffer more from it as children than as adults, and since adult negro immigrants from non-malarious countries suffer more at first than the native adults and more than they themselves do later, it is evident that individuals of a race that has long been exposed to it are capable of acquiring a considerable degree of immunity. Apparently, average Englishmen gain no such increase of resisting power. ${ }^{1}$ Nevertheless, judging from the direction taken by the evolution, it is probable that members even of a race that has had no previous experience have some power of acquiring resisting power-a power which, though it does not afford much protection to a majority of individuals, yet has selection value. Similarly, opium lies midway between alcohol and tobacco. The individuals of all races are capable of increasing their power of resisting opium; that is they are able to acquire the power of taking greatly increased doses without immediate poisoning, without intoxication. But individuals of races that have long experienced it tend to take it in less excess, tend more to be satisfied with non-intoxicating doses than races to which the drug is new. Experience of opium, therefore, tends to place a race in the same position of advantage that it occupies from the first with respect to tobacco.

518. It should be noted that the term 'immunity,' when applied to microbic diseases and to narcotics, has not identical meanings. Immunity to disease implies a physical power of resisting infection. Immunity to a narcotic is a mental phenomenon implying such an insusceptibility to its charm that the individual is not tempted to excessive indulgence. Nevertheless, the analogy between diseases and narcotics is very close.

519. We see, then, that alcohol and opium act as agents of selection in the same way precisely as lethal and prevalent diseases. They eliminate particular types of individuals. Individuals differ in their susceptibility. The more susceptible are eliminated and as a consequence the race undergoes protective evolution. When thinking of narcotics, the reader must bear in mind evolution in general. All progressive evolution depends on selection, all stringent selective elimination results in evolution. When the whole of the facts are taken into account it is not believable that alcohol and opium, which are so enormously destructive of life and so plainly selective, can be an exception to the rule. It has been objected that, since alcohol awakens unlike sensations in 
different men, thereby causing them to drink for diverse reasons, it does not eliminate a particular type. The objection is merely verbal. Whatever the shades of difference, all men drink because the act awakens pleasurable sensations-because it gives actual pleasure or because it soothes discomfort or suffering. The type which survives is one in which acute pleasure is not awakened nor suffering soothed by deep indulgence. It would be as reasonable to argue that carnivora are not causes of evolution in antelopes because some of the latter are eliminated on account of dull hearing, others for defective sight, others for insufficient speed, and so forth. Nor, as we have seen, is the argument valid that selection is not selection because it varies in intensity with time and place, and is never absolutely thorough. Some people ask how the susceptibility to the charm of narcotics can have arisen in the human race, and appear to think that they have indicated a valid objection. Probably it is a by-product correlated to mental evolution in general-a by-product which was harmless while mind was evolving, and only became injurious when men discovered that certain vegetable poisons were capable of producing delightful sensations. But the question as to how it arose is one thing. The question as to whether it, being existent, is a cause of selective elimination is quite another thing. It certainly exists.

520. The theory that alcohol and opium, like lethal and prevalent diseases, are causes of protective evolution, is so opposed to popular notions that it is apt to be received with initial incredulity. I can only beg the reader to test the chain of fact and reasoning link by link. I believe that he will conclude ultimately that, if evolution has occurred in any instance, then it has occurred in this instance also. Is it undeniable that men differ in the sensations awakened in them by alcohol (and opium); that some are so constituted that after a given experience they are much more tempted to excessive indulgence than others, for the reason that their sensations are more pleasurable; that, as a general rule, men yield to temptation in proportion as they are strongly tempted; that the average moderate drinker shows no indication that he is strongly tempted to deep indulgence; that the average excessive drinker shows every indication that he is so tempted, for otherwise no one would do actions so painful and ruinous, both in the immediate and remote future; that he tends to transmit his degree of susceptibility to descendants; that drink tends to shorten the lives of excessive drinkers and otherwise lower the number of their possible descendants; that the amount of this elimination is large; that 
therefore alcohol is an agent of stringent selection; that every race on earth that has had little or no experience of alcohol tends to be excessively intemperate when afforded the opportunity; that therefore this excessive susceptibility is the primitive condition of humanity ; that every race that has had previous experience is temperate in the presence of abundant supplies of alcohol in proportion to the length and severity of its experience; that therefore there is no escape from the conclusion that such races have undergone protective evolution? The subject requires careful and unprejudiced thought. Hitherto men have been content to accept without question the simple belief that men are sober or intemperate in proportion as they do or do not exercise self-control-a belief which involves the corollary that, since drunkards are vehemently tempted, therefore all moderate men are as much tempted but oppose even more vehement resistance. The reader should test this hypothesis by examining his own psychology and that of his intimates.

52 r. Formerly it was universally believed that certain races (e.g. Italian and Spanish) were 'naturally' more temperate than others (e.g. English and Red Indian). But since the theory of alcoholic evolution was formulated, the surprising discovery has been made that the races hitherto supposed to be the most temperate are really the most drunken of all. Thus it is stated that the per capita consumption in the South of Europe is greater than in the North, and much greater than among Red Indians or Australian blacks, and the 'obvious' inference is drawn that South Europeans are much the most intemperate of all. According to this theory, if a party of one hundred men consumed two bottles of spirits, each man taking his share, while one member of a party of similar strength drank a whole bottle by himself, there would be twice as much intemperance in the former party as in the latter. In the South of Europe practically every man, woman, and child takes alcohol; but excessive drinkers are rare. In the North multitudes of men, more women, and nearly all children take no alcohol at all; multitudes take it in moderation; but a considerable minority are excessive drinkers. The Western aborigines are rarely able to procure alcohol; but, when they have the opportunity, they all of them almost invariably take it in excess.

522. Racial differences with respect to alcohol are admitted by most people, but are attributed to all sorts of causes other than evolution. Climate: Since South Europeans are sober, a warm temperate climate has been thought to be conducive to moderation. But only those races inhabiting warm temperate climates which 
have undergone prolonged alcoholic selection are moderate. All the rest, for example Andamanese, Red Indians, Polynesians, and Australasians, are very drunken. Jews and South Europeans are temperate in all the climates to which they migrate. West Africans are temperate in the torrid zone.

523. Education, and general mental training. What is there in the education of Jews and South Europeans that should render them more capable of exercising self-control than North Europeans? The upper classes in England and elsewhere amongst civilized races are supposed to be better trained mentally than the lower. Certainly they have more information derived from reading and are also less inclined to excess. But the upper classes of the present day are in general derived from those of former times, and therefore are precisely the classes which have had the greatest command of alcohol, and have been most weeded out by it. Recruits from lower to higher classes are almost always temperate people; contrariwise intemperance has lowered many families from a higher to a lower stratum. There has been, in this particular instance, a process of Social as well as Natural Selection. It must be remembered that though education may create a moral abhorrence, it cannot alter sensations-neither those created by alcohol, nor tobacco, nor salt, nor sugar, nor anything else. The sensations created by excessive indulgence in a majority of people in the upper classes are apparently different from those experienced by many in the lower, in that, on the average, they are so much less pleasurable that they awaken desires which are very much less keen. Poverty and its accompaniments, hardship, want, overcrowding, dirt, insanitation, and lack of intellectual pleasures are supposed to conduce to the intemperance of our poorer classes. Doubtless they do, but to a very limited extent. Under similar or worse conditions, for instance in the East End of London, Jews and Italians are extremely temperate. In their own countries the lower classes of South Europeans are as temperate as the upper. ${ }^{1}$ The fact that South Europeans (e.g. Italians and Spaniards) living in cities or engaged in manufactories, tend to be more intemperate than their rural compatriots, has been supposed to demonstrate the influence of the environment (i.e. mental training) as against that of heredity. But the influence of the environment has been denied by no one. It is maintained

$:$ It is interesting to note that while the intemperance of the English lower classes is often attributed to their poverty, the comparative sobriety of the poorer Chinese has been assigned to the same cause (see History of Drink, p. 32). 
merely that the degree to which any individual indulges depends on the interaction of two factors, one of which (susceptibility) is innate, and the other of which is environmental. Proof that the environment is influential is not proof that innate susceptibility has no influence. ${ }^{1}$ It is known that when men are gathered together in large numbers in cities or manufactories they tend, owing to the relative lack of restraint, to be more intemperate in every way than when living in the country. The question, therefore, is not whether the individuals of the same race are more intemperate in one environment than in another, but whether, when individuals or races are placed under conditions that are practically identical, the one individual or race tends to be more intemperate than the other. Compare, for example, Italian peasants with English peasants, and Italian ice-vendors and organ-grinders in London with English street musicians and costers.

524. Strength of beverages. Races are supposed to be drunken or temperate according to the strength of the beverages they consume. No doubt a man who desires to get drunk will, as a rule, if he have the opportunity, choose a strong solution of alcohol, whereas one who is prompted mainly by thirst or taste will select a more dilute and palatable drink. But it is quite as possible to get drunk on a weak as a strong solution, and it is done very often. More Englishmen are intemperate on beer than on spirits, and on the average their alcohol is twice or thrice as dilute as the wine of South Europeans. ${ }^{2}$ Those savages who are able to manufacture only very dilute solutions-or, indeed, no alcohol at allare the most drunken people on earth when opportunity offers.

525. Civilization is supposed to conduce to moderation. This is certainly true, but not for the reason implied. North Europeans are more civilized than South Europeans and West African savages, but more drunken. Civilization has always implied abundance of alcohol. Therefore all races capable of living under civilized conditions have undergone alcoholic evolution. The incapacity of Red Indians to resist alcohol is as much a bar to their survival under modern conditions as their inability to withstand tuberculosis.

526. The memory of past disasters. Races, like the Italians, which have suffered much - two or three thousands of years agoare supposed to bear in mind so keen a recollection of their miseries that they are now temperate in consequence. Whereas races like the English who have suffered less-but who are now

1 See $\S 693$.

'See The British Medical Journal, I5th Dec. I900, p. I733. 
suffering, and for two or three thousands of years have been suffering, are supposed to be proportionately less aware of the dreadful results of intemperance, and on that account to be more drunken. The existence of a temperance propaganda and of prohibitory laws amongst North Europeans and their colonists, and the absence of them amongst South Europeans, is a fit commentary on this shocking nonsense.

527. Convivial and industrial drinking are terms which have been applied to indulgence occurring during leisure and work time respectively. Other things equal, men drink, smoke, play cricket, or indulge themselves in any other way in proportion to their desires. One of the "other things" is opportunity for indulgence, which varies with time, place, and occupation. Thus I have two occupations, medicine and authorship. I smoke a great deal when I am writing, but not at all when I am attending to sick people. So also a judge or preacher smokes less during the performance of his duties than the average gardener. A coal-miner is forbidden under the severest penalties to smoke or drink during his working hours. He is even searched before descending into the pit. A coal-porter on the other hand, is restrained from immoderate indulgence in alcohol only by expense and the fear of the police. It does not require a very high degree of intelligence to conclude that, other things equal (e.g. a desire for alcohol), the average porter will consume more during his working hours than the average judge or miner. Again, some occupations demand delicate manipulative skill, others nothing more than violent muscular effort. The imbibition of a quantity of alcohol which interferes little, if at all, with the latter, spells inefficiency in the case of the former. For example, a watchmaker or engraver will become incapable on smaller quantities of alcohol than a dock-labourer or market-porter. As a consequence, others things equal, skilled workers tend-if only by the dropping out of the incapables-to be more temperate than rough labourers.

528. In two kinds of occupation, then, is excessive drinking during working hours comparatively rare-those in which drink is more or less inaccessible to the workman, and those in which it quickly renders him inefficient. Speaking practically, all 'industrial' drinkers are 'convivial' drinkers also. So that in effect the former imbibe all day long, whereas the pure convivial drinker, who is also a worker, imbibes only in the evening. Doubtless it often happens that the man who has been half drunk previously becomes wholly drunk when his work is finished and he 
is comparatively free from restraint. Consequently, as might be expected, industrial soakers consume more, and therefore suffer more, than merely convivial drinkers. In point of fact, occupations in which industrial drinking is easily possible show higher proportions of illness and death from excessive indulgence than occupations in which it is difficult or impossible.

529. In every occupation, even the coarsest, excessive drinking, owing to the expense and loss of efficiency, is a bar, and is universally known to be a bar, to success. If, then, a man, in spite of pains and penalties-loss of health and efficiency, liability to dismissal from employment, and the like-drinks to excess during his working hours, it is reasonable to conclude that he is greatly tempted by alcohol. Indeed, we may fairly conclude that on the average such a one is more susceptible to its charm, more tempted by it, than his fellow who abstains from prudential motives and drinks only in the evenings when he is at leisure. That, at any rate, I think, is the conclusion which ninety-nine people of normal intelligence out of a hundred would reach. But a belief that men drink alcohol for enjoyment and that individuals vary in the degree and kind of enjoyment conferred by it leads inevitably to a theory of alcoholic selection, a conclusion greatly disliked by many people, especially temperance enthusiasts. Recently, therefore, a new interpretation of the facts has been formulated, ${ }^{1}$ a consideration of which will at any rate serve to demonstrate the dreadful kind of thinking which finds favour with many earnest reformers. We are told, in effect, that it is absurd to suppose that men differ in the degree in which they are tempted by alcohol.

530. "It has led some less responsible students of the question to assume that the view conveniently expressed in the formula, that inebriety is a symptom of disease offers a complete and valid explanation of all the facts of alcoholism. Thus it has been asserted that intemperance is always a manifestation of a definite brain condition which creates a specific craving for alcohol. And some enthusiasts have even gone further, and, assimilating this hypothetical drink-crave to a peculiarity of anatomical structure, have regarded the potentiality of being a drunkard as a simple inborn trait, which, we are gravely assured, being clearly unfavourable to its possessor, must secure his early elimination in the struggle for existence, and so lead through natural selection to the evolution of a race immune from drink!

${ }^{1}$ See, for example, Alcoholism, by W. C. Sullivan, M.D. London: James Nisbet \& Co. 
53I. "Extravagances of this sort, apart from such direct error as they may engender, are likely to have a mischievous influence, in that they divert attention from the real biological aspects of the question, and make an unnatural divorce between the organic and social causes of alcoholism, which, as we have already pointed out, can only be understood in their mutual relations.

532. "For the proper apprehension of the question we must, therefore, at the outset get rid of this figment of an inebriate diathesis and replace it by the rational view, that the explanation of inebriety is to be sought, not in any specific tendencies of an abnormal brain, but in the reaction of the normal organism to the ordinary physiological effects of alcohol." 1

533. Now very obviously the foregoing passage contains numerous very grave, if unconscious, misrepresentations of the facts and reasoning which led to the belief that there is such a thing as selection by alcohol and that it has resulted in protective evolution. Doubtless the alcoholic diathesis, the susceptibility, has often been regarded as a symptom of a diseased mind. Indeed, in England chronic drunkenness is in many cases associated with mental defect, ${ }^{2}$ possibly because feeble-minded persons have small powers of restraint. $^{3}$ But no reasonably well-informed student of heredity has ever held, as is implied in the passage, that mental defect is a normal accompaniment of the alcoholic diathesis. Were the latter a symptom of a diseased mind we should have to consider all primitive humanity and half the races now inhabiting the world, including the whole of the aborigines of the Western Hemisphere, as mentally diseased. Every one knows men and women of great intellectual power who drink to excess, and it is not believable that North Europeans are on the average more defective mentally than South Europeans. Doubtless, the diathesis, through the indulgence it tends to provoke, is a frequent antecedent of disease, but by itself it is no more a disease than temerity is a wound.

534. Again, no students of heredity and evolution, not even "less responsible students," have assimilated this hypothetical drink-crave to a peculiarity of anatomical structure, unless by that is meant the belief, common to all psychologists, that mental facts are correlated to cerebral facts. If the "potentiality

1 Op. cit., pp. 58-9.

${ }^{2}$ See Branthwaite, British Journal of Inebriety, Jan. 1908.

3 See $\$ 699$. 
of the drunkard" is not in the last analysis "a simple inborn trait," then, in the name of clear thinking, what is it ? Even if we suppose it to be an acquirement, we must in the last analysis, as in the case of other acquirements, postulate an inborn foundation for it. Whether the inborn foundation is simple or compound is irrelevant. The word 'simple' has not to my knowledge been used by any adherent of the theory. Possibly we are all potential drunkards in the sense that, under very exceptional circumstances, we are all capable of acquiring a liking for intoxication; but it is beyond doubt that some of us are so constituted that we become drunkards more readily than others. This liability to acquire a liking for intoxication is the diathesis, the inborn trait, the susceptibility. If the writer from whom we quote means to imply nothing more than that the diathesis is not the only factor in the causation of inebriety, then his language implies also that some one has stated that it is. But, in point of fact, no one has been so excessively foolish. The influence of education, fashion, selfrestraint, accessibility of alcohol, and the like have all been fully admitted. For example, it has been admitted that abstainers, who will not drink, and Fuegians who cannot procure drink, may have the diathesis strongly developed and yet not be drunken. It has been held only that the craving for drink is the resultant not of one factor, but of two, inborn susceptibility and previous experience, and that the greater the susceptibility the more easily does the experience awaken the craving. Yet again, no one has so much as hinted that alcoholic evolution would lead to a race 'immune to drink' in the sense implied. Evolution is never perfect. It has been maintained merely that evolution tends so to decrease the susceptibility of a race exposed to selection that it becomes increasingly temperate, even in the presence of increasing supplies of alcohol.

535. During the Middle Ages the belief was prevalent that alcohol-presumably in moderation-was beneficial to health and strength. Rightly or wrongly-wrongly, I think, except in certain cases of depressed health-this belief has survived to the present day, particularly amongst medical men and nursing mothers. I suppose, however, that no human being who has an average experience of life, and is not a real lunatic, believes that alcohol taken in excess is beneficial. By excess I mean here an amount which obviously impairs health and efficiency. Certainly no sane excessive drinker, however ignorant, no man who has experienced the resulting temporary paralysis, indigestion, loss of 
appetite, headache, thirst, 'hot coppers,' nervousness, delirium tremens, and the rest, is under any such delusion.

536. It is true, of course, that, if a soaker of the lower classes be questioned, he may lie, and, like sots of a higher social standing, may excuse himself by declaring that he takes alcohol for his health's sake, or his work's sake, or his stomach's sake; but the last to believe him will be his own mates, who perceive in him the consequences of heavy drinking. To ascertain his real sentiments it is only necessary to ask his opinion of some other soaker-of one who drinks to the same excess as himself. Indeed, when sick, sorry, and repentant, he will admit his fault, and swear an amendment, which, as a rule, temptation subsequently annuls. He knows, of course, that "a hair of the dog that bit him" will temporarily mitigate the unpleasant feelings aroused by previous drinking; but he knows also that the only permanent cure for his miseries is abstinence or moderation. In many cases, lack of money will afford experience of the advantages of sobriety. That his virtuous assumption of drinking for his work's sake is a pure fiction is proved by the fact that, when he has lost his job and is idle, he drinks, if he can, as much as or more than ever. Moreover, for thousands of years laws against excessive drinking have been common, and denunciation of it an everyday affair. Almost every pulpit, schoolroom, and reputable newspaper conducts a campaign against it. The temperance question is, and has long been a burning one with the public. Our workmen have a colloquialism, "drinking himself to death," which is commonly applied to chronic soakers. Slums, workhouses, prisons, and asylums are full of the victims of alcoholism. Whenever possible, employers insist on sobriety. In every workshop and factory the men who abstain, at any rate during working-hours, are known to achieve better results and greater prosperity than their fuddled comrades.

537. In spite of all this-by way of proving that there is no such thing as alcoholic selection-we are now assured that the British workman who imbibes to excess, drinks, not because he enjoys drinking, but merely as an aid to labour. "This is the fundamental factor." 1 It is supposed that he is poisoned day by day and ultimately perishes, not because he is tempted by pleasure, but on the altar of duty; whence it follows, that the people eliminated by alcohol are not those who are especially tempted by it. The fact that every race is temperate precisely in proportion to the length and severity of its past experience of drink is quietly

${ }^{1}$ Alcoholism, by W. C. Sullivan, M.D., pp. II5; see also pp. 6I, II4, II6. 
ignored. It is admitted that the "worker who does his labour with the help of alcohol is sure to have recourse to the drug for his ideals of pleasure," 1 and that "exceptions to this rule are rare." 2 In other words, while it is admitted that after five or six o'clock the worker drinks for the sake of enjoyment, it is assumed that before that time he drinks from altruistic motives. With the setting of the sun his sensations are supposed to undergo a radical change. We are told, therefore, that, to wean workmen from excessive drinking, nothing more is necessary than to convince them that the habit is injurious to health and work. Thereupon industrial drinking, the only harmful form of indulgence, will cease. ${ }^{3}$ 538. The unconscious humour of this surprising hypothesis is

1 op. cit., p. 62.

2 Op. cit., p. 87.

3 The hypothesis under discussion is interesting as a type of, and is really not much nore absurd than a great deal that has been written on the problem of, alcoholism since the theory of alcoholic evolution was formulated. For example, if we seek to demonstrate the existence of alcoholic selection and its corollary evolution, plenty of people will at once inform an astonished public that universal intoxication is advocated as a cure for national drunkenness. If we protest that we advocate no such thing and that we would object to it as strongly as to universal disease, the reply is that it follows logically from our opinions. It is quite useless to indicate to intelligences of this order that their logical conclusions are not necessarily ours, especially when we have strongly and categorically repudiated them; that unpleasant truths are not the less true for being unpleasant, and that the only rational way of refuting opinions they dislike is not to make mis-statements but to break the actual chain of alleged facts and the inferences drawn from them. It may be thought that I am unfair in saying that the drunken British workman is described as sacrificing himself on the altar of duty. But clearly, if drinking makes a man feel better, if it creates in him a " sense of wellbeing," if his dislike of labour is lessened thereby, he is susceptible to its charm. The hypothesis that men who vary in all known characters do not vary in this one particular of receiving pleasure from alcoholic indulgence is too grotesque for serious discussion. It has been trumped up by people unfamiliar with the facts of biology to meet the exigencies of the moment. Therefore, if it be said that men drink because of the sense of well-being which the act creates in them, then, since it is admitted that alcohol is a considerable cause of ill-health, incapacity, and death, that poison must tend to eliminate especially people who are most susceptible to its charm. It follows that the hypothesis under discussion, which is supposed by its supporters to controvert the theory of alcoholic selection, can do so only when it premises that men at work drink alcohol, not because they like it, not because it creates a sense of well-being, but from some other cause. The cause alleged is a superstition, said to be derived from the Middle Ages and still prevalent amongst workmen, that an amount of drinking, which anyone not a lunatic would know to be injurious, is an aid to labour. Apart from its amazing inferences, the volume from which I have quoted is an excellent work. It is well and clearly written, contains a mass of useful statistics, and proves-if proof be needed-quite conclusively that, other things equal, men drink most when their opportunities are greatest, and that the man who drinks to excess all day long suffers, as a rule, more than the man who drinks only in the evening. 
greatly enhanced when we read the facts and inferences on which it is founded. "The petition of the Middlesex magistrates, for example, in support of the Gin Act of 1736 especially singles out journeymen and apprentices as the classes particularly addicted to this sort of excess" (i.e. industrial drinking). Surely it is sufficiently clear that the action of the magistrates indicated a knowledge that excessive drinking in work time is injurious, and that the drinking of the journeymen and apprentices was excessive. The notion that industrial drinking is a comparatively modern institution is absolutely erroneous. In many parts of Europe, for thousands of years, water has only exceptionally been used as a beverage. Even as regards our own country, it may be laid down as a general rule that, before the introduction of tea and coffee, men, whenever able, used alcohol at all times of the day, both when working and playing. Beer and cider were common industrial drinks with our ancestors, especially with agricultural labourers, who were the great majority of the nation, and to whom it was customary to make a regular allowance. The condition of affairs now prevalent in Russia, as depicted in the following extract, formerly obtained in England.

539. "Feodor asked me if I would like to go to the haymaking next day. ... 'Is Feodor at home?' I asked. Then a man appeared from a neighbouring cottage and said, 'Feodor is in the inn-drunk.' 'Is he going to the haymaking ?' I asked. 'Of course he is going.' 'Is he very drunk ?' I asked. 'No, not very ; I will tell him you are here.' Then a third person appeared, a young peasant in his Sunday clothes, and asked where I was going. I said I was going to make hay. 'Do you know how to?' he asked. I said I didn't. 'I see,' he said, 'you are just going to amuse yourself. I advise you not to go. They will be drunk, and there might be unpleasantness.' . . Then the haymaking began. The first step that was taken was for vodka bottles to be produced, and for every one to drink vodka out of a cup. Then was a great deal of shouting and an immense amount of abuse. 'It doesn't mean anything,' said Feodor. 'We curse each other and make it up afterwards.' Then they drew lots for the particular strip they should mow; each man carried his scythe high on his shoulder. ('Don't come too near,' said Feodor; 'when the men have taken drink they are careless with the scythes.')" 2

540. The following are descriptions of devoted workmen who

${ }^{1} Q$ p. cit., p. II.

2 Maurice Baring, A Year in Russia, pp. 29I-3. London: Methuen \& Co., 1907. 
are supposed to believe that really tremendous drinking beneficially affects their working powers : "We may take as a type for our description the originally healthy workman who is engaged in a trade that encourages, or at least allows, the habit of regular drinking throughout the working day, and who probably goes in also for an occasional convivial bout at the week-end or on special festivals. After a period, longer or shorter according to the intensity of his excesses, such a drinker will begin to show signs of more or less persistent disorder, usually in the digestive and nervous functions; he will be aware of a diminished and more capricious appetite; he will suffer from pain and oppression referred to the region of the heart; he will often have nausea or vomiting on awaking in the morning; his sleep will be disturbed by disagreeable dreams, by attacks of muscular cramp, by electric starts in the limbs; more or less tremor in the hands and feet, and in the tongue and muscles of the face, will be evident early in the day.

54I. "As the intoxication continues, and by its own effects supplies the motive for increasing excess, all these symptoms become aggravated ; the morning sickness is regular, and is often accompanied by the vomiting of blood; the tremor is constant; nightmares and insomnia divide the hours of rest, and foreshadow characters which will appear later in the full development of delirium tremens." 1

542. "As can readily be imagined from the amount of the docker's average wages, the non-alcoholic part of his dietary is apt, under any circumstances, to be deficient, and the money which he spends on liquor leads necessarily to a further lowering of his ordinary food allowance. Not only does his dinner money very frequently go on beer before the meal time arrives, but even when he brings his dinner with him from home he will often sell it for a few pints to some more temperate comrade. This condition of under-feeding contributes, of course, to the more rapid development of the lesions of chronic alcoholism; and it is usual for industrial drinkers at this work to suffer from gastric catarrh and severe nervous disorders before they reach the age of forty." 2

543. "The industrial drinking habits which we have described in the ordinary docker class reach a further degree of development in some special varieties of waterside labour. We may take as an instance the Thames Street fruit porters. Amongst these porters the method of work and payment is this: The overseer takes on a

1 op. cit., pp. 49-50.

op. cit., p. 81. 
limited number of men, each man paying a nominal deposit for his knot ; the porter then starts work, receiving at the warehouse door for each box he takes in a brass ticket marked with the amount of his fee, Id., $2 \mathrm{~d}$. or $3 \mathrm{~d}$., according to the size of the box. These tickets he can get changed for money later in the day by the clerk, or he can bring them at any time to a public-house which enjoys the special privilege of cashing them, the porter taking a certain proportion of their value in drink. The second alternative is, of course, nearly always adopted, and, as a consequence, a large number of these men do their work almost exclusively on beer. As soon as its effect is sufficiently visible to suggest risk of accident, the man has to give up his knot for the day, and is replaced by a fresh hand. These conditions produce the maximum development of industrial alcoholism." 1

544. The belief that parental drinking commonly tends to alter the germ-plasm, and so results in filial degeneration, was universal until recently, and is still very prevalent amongst medical men. As in the case of diseases it is founded mainly on 'common sense,' and on statistics which indicate that the progenitors of physical and mental 'degenerates' have often been intemperate. Every reason which is valid against microbic disease as a cause of degeneracy is valid against alcohol. It is not to be denied, of course, that parental drinking may sometimes (when the germplasm has varied unfavourably in such a way that it has lost its power of resistance) be a cause of filial degeneracy, but in view of the fact that races which are now temperate are not degenerate, but were originally drunken, it is impossible that it can be a common cause. It must be remembered that in the case of many races alcohol like malaria affects practically every individual. We have all known people, derived from drunken stocks, who were physically and mentally fit and capable-that is, people whose germ-plasm was resistant to the direct action of alcohol. It follows that, even if alcohol does tend commonly to alter the germ-plasm, especially on its first introduction to a race, the constant elimination of susceptible strains and survival of resistant types would infallibly tend to establish a high degree of insusceptibility.

545. To sum up-the questions the reader must decide when studying alcoholism from the point of view of heredity and evolution are (I) What, fundamentally, are the motives which induce men to become intemperate? Do they drink to excess because they enjoy the sensations thus awakened, or from some other motive,

1 Op. cit., pp, 83-4. 
such as a sense of duty? (2) Do men differ in their 'by nature' susceptibility to the charm of alcohol? (3) Is alcohol a lethal agent ; and, if so, is it selective? (4) Bearing in mind the universal truth that races are temperate when exposed to alcohol in proportion to their past experience of it, but show no other change that can be traced to it, is alcohol a cause of protective evolution or of degeneration? The very obvious truths that men of the same race drink, as a general rule, more when their opportunities for drinking are greater, and that the amounts they drink depend to some extent ou their previous moral training, are not in dispute.

546. Two problems of heredity are of outstanding importance. The first is the problem of the causation of variations. The second is the problem of the proportion which the 'innate' characters of any species bears to its 'acquirements.' In the section of this work just concluded we have considered, by the light of the evidence furnished by microbic diseases and narcotics, the problem of the causation of variations, and have concluded that, when we take all the evidence into account, it is inconceivable that the great mass of variations can be other than spontaneous. In the next section we shall consider the second problem. For this the study of mind affords peculiar facilities. 


\section{CHAPTER XVII}

\section{IDEALISM AND COMMON SENSE ${ }^{1}$}

Mental adaptation-Objections to the hypothesis that minds are products of evolution-Memory-Sense-impressions-What a sheet of paper meansWe are directly aware of nothing but a stream of feelings-The mental differences between the higher and the lower animals-How we construct external objectsThe differences between feelings ordinarily recognized as such, and those which are thought of as properties of external objects-Idealism and common senseCoherent thought and common sense-Except at rare intervals all men think in terms of common sense-There are no thorough-going idealists-The two views are quite incompatible-Invariable succession and necessary succession.

547.
'characters,' which, in turn, are compounded of
smaller characters. With the possible exception of a few traits (by-products of evolution) correlated to more useful traits, the larger and older characters-hands, limbs, hair, nails, and the like-are almost all adaptations; or, if vestigial remains, have been adaptations. The smaller and newer characters, the

1 Many of the words used in the following discussion (chapters xvii., xviii. and xix.) have gathered special meanings during the course of controversy. By matter I mean something which is not mind, but which exists external to mind, and has properties that excite sensations in it, and is thus mediately known to it. By feeling I mean not merely sensation, but any state of consciousness. By mind I mean the stream of feelings. By idealism I mean the philosophy which supposes that " the sensations, which, in common parlance, we are said to receive from objects, are not only all we can possibly know of the objects, but are all that we have any ground for believing to exist," and which declares "we have no evidence of anything which, not being itself a sensation, is a substratum or hidden cause of sensations," and that "such a substratum is a purely mental creation to which we have no reason to think that there is any corresponding reality exterior to our minds " (J. S. Mill, Examination of Sir William Hamilton's Philosophy, 3rd ed.). By common sense I mean merely the notion, held consciously or unconsciously by every one during ordinary thought, that our minds tell us of a universe of real or material things external to the minds. I do not use the word as implying immediate or intuitive cognition of real things. I have thought the discussion necessary, partly because I wish, as far as possible, to establish the nature of the relation between mind and body, and partly (since it is sometimes said that science explains nothing, and that there is no such thing as necessary truth) because I wish to define what appears to me to be the scope of science, and the significance of certain terms that I use, such as cause, invariable succession, necessary succession, necessary truth, explain, and understand. 
variations, are not necessarily adaptations, but they are the materials out of which nature manufactures adaptations. The constant occurrence of them is an adaptation. Similarly, the minds of animals are compounded of characters-sense-impressions, emotions, memory, and the like. Doubtless, individuals and races differ, on the average, as much in mind as in body; yet, here again, the older and the more important, the specific or varietal characters, the 'faculties,' are all or almost all adaptations. We can think of no animal that affords evidence of possessing mind, but we find that it is as certainly adapted mentally as physically to its environment. For example, human sense-impressions, emotions, instincts, and memory all fulfil this function. Therefore, when studying mind as when studying body, we must ever keep this great truth of adaptation before us. The facts are such, that if our hypotheses, our interpretations of the facts, do not accord with it, they are certainly erroneous. ${ }^{1}$

548. We saw that, when the whole of the facts are taken into account, only two hypotheses of the causation of physical adaptation are conceivable, or at any rate have been conceived, with any degree of clearness-miracle, and natural selection. This is not less true of mind. We are bound in science not to appeal to the supernatural till it has been demonstrated that natural explanations are inadmissible. Nevertheless, some writers, even amongst those who believe that the human body has evolved under the action of Natural Selection, have been inclined to attribute to mind a supernatural origin. On the one hand, they have insisted that mind is so unique that it can have been evolved out of nothing else in nature; on the other, they have asked how it is possible that such human 'faculties' as the mathematical, the musical, and the devotional, which, seemingly, have never contributed to the preservation of life, can have been brought into being by the action of Natural Selection. Mind is certainly unique; nevertheless, as we shall see, this difficulty is not absolutely insurmountable. At any rate, it is not a unique difficulty. It is not a greater difficulty than many others which science first ignores and then proceeds as if they had been surmounted, leaving the consideration of them to metaphysics, which seeks to be more thorough. ${ }^{2}$ The study of memory, intellectually the most important of all the faculties, has been constantly neglected, and we shall find that the evolution of this one character, easily explainable by the theory of Natural Selection, furnishes the key to all such appa-

\footnotetext{
${ }^{1}$ See $\$ 616$ and 648 .
}

2 See $\$ 590$. 
rently unsolvable riddles as that of the existence of the devotional and mathematical faculties.

549. The moment we begin to discuss mind in any but a very superficial way, we encounter the problem of the connexion between mind and body. Unless I start by dealing with this problem, and making quite clear my own beliefs and the reasons why I hold them, some of my readers will presently raise hands of horror, and declare that I am not scientific, but metaphysical. Probably some of them will do so in any case. For my own part, I propose, in this and the two succeeding chapters, to be much horrified at the metaphysical attitudes of other people. In the end I think I shall be in a position to insist that, if I am metaphysical, I am so only to an extent that every one must be metaphysical.

550. I am writing on a sheet of paper. For the moment I am thinking of it, in the 'common-sense' way usual with me and other people, as a real substantial thing, of the existence of which I am aware through my senses. My sight and touch tell me that it oblong, thin, and smooth. My sight tells me that it is white, and marked in parallel blue lines. Sight, touch, and hearing assure me that it is crisp. Smell informs me that it has a faint, pleasant odour. Taste would inform me that it has a flavour which is not pleasant. Muscular sense declares that it has weight. Thus, in ordinary colloquialism, the sheet has 'caused' certain sensations in me, by means of which I perceive it, by means of which I gain a perception of it. Memory supplements this information; I link up the sensations I have just received with other information which I received in the past, and with thoughts concerning it. I recognize this thing which now lies before me as what I have been accustomed to term a sheet of paper. That is, I form a conception of it as a sheet of paper.

55 I. But to go deeper, what is the real meaning of the statement that my senses and thoughts have given me information? Obviously I can be aware of nothing, including my own body and even my mind, except through sensations, recollections, and thoughts, through my feelings. They are the only conceivable means of communication between the universe and that inner essential me which I am accustomed to regard as possessor of mind and body, and the observer of my universe. ${ }^{1}$ Now, still using the language

1 This is not to say that there is any such inner essential " 1 ." There may be. I do not know. I am conscious only of a changing stream of feelings amongst which are feelings by means of which I contemplate (recollect or foreshadow) other feelings which are past or which may arise. I say only that in my everyday thinking I am accustomed, more or less unconsciously, to postulate this being. 
of common sense, of everyday life, consider the sense of sight. We are told that I see the paper because light, which is some sort of vibration in the ether that surrounds us, passes from a luminous object (e.g. the sun) to the paper, where some of it is absorbed, and whence the remainder is reflected to the retina of my eye, where it sets up chemical changes. These chemical changes set up other chemical or molecular changes in my optic nerve. These, yet again, set up other similar changes in my brain. Thereupon there dawns within that dark box, my skull, a feeling which I call a sight of the paper. In an analogous way my other senses convey their items of information. Now, plainly, feeling, which has neither extension, nor colour, nor weight, nor any other of the properties which I am accustomed to ascribe to material things, cannot in the remotest degree resemble the chemical changes in my brain, optic nerve, or retina, or the vibrations of the ether that lies between the retina and the paper. Nor can it, an immaterial thing, resemble the material thing, the paper. It can, at best, be only a sign and a symbol to me of the real thing, the paper.

552. I find that some of my feelings are so unlike that I can institute no comparison between them. Thus the 'crisp' sensation of touch which I get when I indent the paper is quite unlike the crisp look of it, or the crisp crackle I hear. I use the same word in each instance, but the several sensations it describes are not at all similar. I may say, indeed, that all these sensations are faint or vivid, but then I indicate no essential likeness except the degree in which they impress me. If feelings may be incomparable, how much more incomparable must be feelings and material things? Besides, how can light, undulations in the ether, passing between the sheet of paper and my eye, convey anything at all like a real sheet to my retina? How can the series of changes in my optic nerve convey anything of the sort to the brain? How can the series of changes in the latter create or accompany a feeling which is remotely like the paper?

553. I write the word 'paper,' and it stands to me for a sign and a symbol of the sound, the spoken word 'paper,' which it is incomparably unlike. Similarly, the spoken word is only a symbol of the seen, felt, heard, tasted, smelt, or weighed thing, 'paper.' So also, in a sense even more thorough, my several feelings of paper are only signs and symbols of the 'real' thing, which, as I suppose, awakens them. That real thing is altogether outside the circle of my consciousness, within which are nothing but feelings. By no 
manner of means can I gather anything more than a symbolic knowledge of it.

554. Indeed, to go yet deeper, it is a question whether I can gather even a symbolic knowledge. I have surmised that my feelings have been awakened by a real object, but it is impossible to test that hypothesis. The symbolism of my dreams, if symbolism it be, is, I am accustomed to believe, very misleading. How can I know whether the symbolism of my waking hours is of a more truthful kind? My waking feelings seem to link together better than my dream feelings, but the fact that they link better together does not prove that they are symbols of anything. At any rate this is certain, that what I term the 'properties' of the material object, extension, weight, colour, and the like, are really feelings in me. Strip the material object of these properties, of these my private feelings, and what remains of it? The remainder, if remainder there be, the 'matter in itself,' the noumenon, is quite inconceivable. For me it has no thinkable existence. I close my eyes, and have a recollection of the sheet. That recollection is not more a mental and less a material thing than the perception I had when my eyes were open.

555. It is evident that the only existence of which we are really aware is a stream of feelings. That stream of feelings is the whole of consciousness. For us the universe is a universe of appearances, of phenomena, which we construct out of our own feelings. Time and space are only modes of thought, the former being concerned mainly with appearances that occur in succession, the latter with appearances which co-exist. We speak of mind and matter, but from first to last we know of nothing but mind. We are conscious of nothing else. Matter is a metaphysical abstraction, an unknown and unknowable something which has been surmised by common sense, but only surmised, to be in some way the cause of phenomena. But our minds are so constructed that we are impelled to attribute material existence to many appearances. Thus this sheet of paper appears to me a thoroughly material object. If I tear, or burn, or crumple it, I seem to be wrecking a material thing with material hands. It is only when I pursue a very unusual line of thought that I am able to realize that I am dealing throughout with groups of feelings which experience tells me tend to follow one another in certain sequences. The sight of the paper, the intention to tear it, the sight, and sound, and tactile sensations of tearing, and the feeling of an intention accomplished are all equally mental phenomena.

556. We shall see later that it is probable that animals low in 
the scale of life, for example most insects, intuitively regard the objects revealed to them by their senses as real existences. On the other hand, it is likely that the human mind begins as a chaos of feelings, some of which, by a process of gradual and unconscious inference, are ultimately referred to external bodies as properties or qualities of them. In other words, the insect has an intuitive belief in the existence of the universe which his mind constructs, while the human being slowly builds up a similar belief. ${ }^{1}$ The latter then divides his feelings into classes, one of which is regarded as a collection of mental happenings, and the other as a collection of external objects. Thus the pain caused by a cut from a knife is regarded as a feeling, whereas the colour, extension and weight 'of the knife' are supposed to be properties of a material object. Ultimately the human being finds it extremely difficult to think and speak of his ' physical ' universe as other than a collection of external objects. Both thought and language become moulded on that belief. Thus I, while insisting that material objects are unknown to us, have just used words which imply an unquestioning faith that insects, human beings, and knives are realities external to me. I have also used the words 'we' and 'us,' and have written this book on the assumption that other people will read. But other people, other minds, are just as much unknown to me, just as much outside the circle of my consciousness as sheets of paper. Indeed, other minds are, if anything, more outside the circle of my consciousness than material bodies; for I can only arrive at the notion of these other minds by observing that bodies move in such a way (talk, gesticulate, etc.) that they seem to be, like my own body, under mental control.

557. And now I wish to fix the reader's attention on a matter which is of very great importance, but which is never accorded due weight. He must bear with me if I use such words as 'reader,' 'nurse,' 'wall,' and the like, and so constantly express myself in common-sense terms. Language affords no other way of indicating my meaning. If I had always been an idealist, if from the beginning I had ever regarded my feelings merely as feelings, if I had never draped them about supposed external objects as properties of the latter, if I had never put a materialist construction on them, I should now be living in a chaos of unmeaning feelings. We do not know to what extent a newly born infant has an intuitive belief in an

1 Of course, I do not mean that the human infant at first regards all his feelings merely as feelings and afterwards changes his opinion. Like the insect he does not think about it. I mean merely that he learns to do that which the insect does intuitively. 
external world, but there are good reasons for believing that his intuitions cover but a very small portion, if any portion, of his total mental field. He is emphatically a creature wholearns. When I was born, a flood of new, and strange, and doubtless incomprehensible feelings beat upon me. Probably, for example, the walls of my room were not conceived, as they were later when my education was well advanced, as material things having extension and other properties; they were only patches of light and shade, and were not even thought of as such. The nurse consisted only in similar patches. Her movements resulted in changing patches which were not distinguished from the changes caused by the purposeless movements of my own eyes. Probably the sounds she made as well as the feelings that came from my skin and other organs were not differentiated, were not understood, in any way.

558. But gradually my wonderful faculty of memory did its work. By means of it I stored impressions in my mind, and learned with ever-increasing power to link them together. ${ }^{1}$ And this linking was performed, not merely by recording likenesses and differences, coexistences and sequences, but also by processes of inference which were none the less real because at first rudimentary, and of only gradual growth in scope and accuracy. In this way groups of feelings gained coherence, and linked themselves to other groups. Thus, at length, I gathered the notion that certain patches of light and shade proceeded from a permanent material wall were properties of the wall. Thereupon all the feelings successively excited by the wall began to fall into their places, like pieces in a well-designed mosaic. Though the patches changed when my position was changed, or when there was more or less light, or when the direction of the light was changed, or when the nurse moved about, yet I began to connect all such changes not with changes in the wall, but with changes in my position, and so forth. Another vast group of feelings linked themselves together in the notion of a material nurse, and cohered notwithstanding even greater changes in her. An even vaster group were gathered in the notion of a body, consisting of head, trunk, and limbs, which I came to regard as peculiarly mine, which was I, and from which I derived some feelings (e.g. ' physical' pain and pleasure) that were unlike those excited by all other bodies.

559. Here then is the point I wish to emphasize-the truth that $I$ have been able to classify by feelings, and become a rational being, only because from early infancy $I$ have referred them to ${ }^{1}$ See $\S 666$. 
external objects. In other words unless groups of my feelings had draped themselves about a supposed material wall, and others about a supposed material nurse, and so on, they would not have cohered at all. It was the hypothesis, which gradually and inevitably, owing to the constitution of my mind, I formed of the existence of external objects that gave them meaning and order, and, therefore, coherence. Otherwise my memory could no more have held them together than it now can the shifting colours of a kaleidoscope. Though all the evidence indicates that our memories are particularly retentive when we are young, ${ }^{1}$ yet I can remember nothing of what happened to me during early infancy. It is hard to explain this fact except by the hypothesis that my world was then for me a very complex kind of kaleidoscope that furnished not only sensations of sight, but multitudes of other sensations as well, which, because they were then incoherent, could not be remembered. It is, therefore, the coherence of my sensations about supposed external objects that has rescued me from chaos.

560. To illustrate by an example how my ' material' world was created: a little while ago I linked together a group of entirely dissimilar feelings (visual, tactile, etc.), by conceiving them as properties of a sheet of paper. This group I then linked with other groups, other sheets of paper, the recollection of which had dwelt more or less vaguely in memory, and by thinking about which I had reached the general conception of a sheet of paper as distinguished from perceptions and recollection of particular sheets of paper. The economy of thought achieved by means of such general conceptions is very great; thus I do not now need to remember thousands of individual sheets of paper, but only a conceptual one which is my notion of that kind of object. Now, again, as I sit thinking, I link up the group of feelings which represents to me the sheet of paper with another group which represents the table on which it is lying. The compound group thus constituted, forms part of a more highly compounded group which represents the room in which I am. That, yet again, is linked with memories of the room, with memories of the house and its inmates, the street, the town, the surrounding country, the whole of my world. So the universe as conceived by me was constructed. Very early a division was made between feelings which were recognized as feelings (e.g. emotions and recollections) and groups of feelings which were supposed to be material objects in the external world. Only long after manhood was reached did I begin to suspect that I 
have never been really aware of anything but my private feelings.

56I. Between the feelings which I ordinarily recognize as mere feelings (recollections, imaginings, and the like), and those which I refer to external objects as properties of them or as caused directly by them (i.e. my sense-impressions), there exists a vast difference. Thus, what I suppose to be the sight of a dog, or the pain caused by his bite, is immensely more vivid than my thoughts about such an event. Moreover, sense-impressions follow one another in an infinitely more coherent and orderly way than do thoughts. Indeed, so orderly is the procession of the former that we feel intense surprise when the usual order is apparently broken, and we immediately cast about to restore it, or, in other words, to discover a cause for the break, which we firmly believe to be only apparent. Thus, if I had the feeling of a dog biting me when no dog was in sight, I should feel unbounded astonishment, and should immediately form all sorts of hypotheses to account for the event.

562. Doubtless, it is this peculiar vividness, and above all coherence of sense-impressions, the way they link themselves in related groups, which cannot be altered at will but may be verified by repeated observation even after a length of time, that has given rise to my notion of external objects (included amongst which is my own body); whereas the lack of a similar coherence and vividness amongst other feelings (e.g. thoughts) has led to the unhesitating recognition of them as mere mental phenomena. Thus, all the impressions which I gather from the objects in this room cohere together so strongly that as they are collectively to-day, so, very nearly, they were yesterday, and will be to-morrow. They are invariably so similar, so closely repeated in the same groups, that they have driven me to think they represent the same external objects-the same table, the same chair, the same walls, and roof and floor. If I wish to see again what once I saw on the way from New Zealand I can do so; for, if the journey be repeated, a long train of similar sense-impressions will follow one another in similar array. Forgotten scenes, appealing to awakened memory, will insist as strongly as anything I can still recall on a reality independent of my mind. Even when I read a description in a book, I am able to verify it by examining the place or object described. But in my thoughts I can pass, and habitually do pass, in a moment from the room to India or Honolulu, or Mars, and in two consecutive instants can be concerned in a dog-fight round the corner, or a Saxon coronation. It is no wonder, then, that I 
have been led to refer thoughts to a mental world, and sense-impressions to external objects as properties of them, or as caused directly by them. Indeed the coherence and vividness of my sense-impressions is my only ground for inferring the existence of a reality which is beyond them, and which, as I suppose, they symbolizefor I do not think they can do more-to my mind. So coherent and capable of being tested in diverse ways is the story told by sense-impressions, that nothing is more certainly true than the existence of the reality they insist on-except that which is very much more certain, the existence of the sense-impressions that tell the story.

563. Evidently, as I say, my feelings have gained coherence and meaning, and I have achieved such knowledge and skill in thinking as I possess only because I have thought in the commonsense way to which I have been driven, as it seems, by the very constitution of my mind. Without that previous common-sense thinking, my thoughts would now be infinitely more chaotic than any that occur in my dreams. For, at least, in my dreams my feelings do not constitute a meaningless procession. All of them are grouped or linked together by being thought of as properties of, or as caused directly or indirectly by, external objects. Even now, when for an hour or more I have forced myself to think in idealist terms, and have decided that I know nothing of what lies beyond my feelings, yet, so strong is my tendency to think in a common-sense way, that my wife and child have only to come into the room, and the whole philosophic tangle is instantly dissipated. I return forthwith to common sense. They become warm, living material realities. Realities, too, become the room and all within it, the house, the street, the whole universe.

564. Now, if my feelings have gained coherence only because I have draped groups of them about external objects, which $I$ do not know to be other than illusions, of what value is the coherence? If the objects are illusions, the coherence is delusive. How am I to know that my thinking is in any way better than the imaginings of a madman (phenomenal to me), who, like me, depends on his feelings, and is unlike me only in that he perceives some groups or links together some groups differently? It would be absurd to argue that my mind is normal and his abnormal. For the thoroughgoing.idealist there are no such things as mental normality and abnormality. He knows no more of other minds than of material bodies. Indeed, the assumption that there are other minds, normal or abnormal, is tantamount to a complete abandonment of the whole idealist position. For if there are 
other minds that have perceptions similar to those which occur in my mind, there is no reason to doubt that there are bodies, that there is a something, a reality, external to those minds, which causes the similarity of feeling. That something is what common sense, which postulates other minds, designates as "matter." It follows, that, though the coherence which common sense has bestowed on my feelings is (regarded as nothing more than coherence) very real, and though it lingers when I think as an idealist, yet, beyond the satisfaction it affords me, it has no value to me (as an idealist); for, as far as I know, it is founded on illusion. Idealism, then, leads to a cul-de-sac, from which there is no escape; and the thoroughgoing and consistent idealist has no right to proceed beyond a wondering and bewildered contemplation of his own feelings.

565. But there are no thorough-going and consistent idealists. There never have been any. All idealists have thought more or less coherently, and have failed to note that, if common sense is based on illusion, coherent thinking is based on it also. Very certainly, notwithstanding ardent effort, $I$ am no consistent idealist. I find that I constantly act under an intense common-sense conviction that I am a material item in a material world, which contains many similar items. As it is with me, so I suppose it is with these other items, these other men. Now and then, though seldom, a rare individual may think for a little space in futile idealistic terms - terms which, as we see, have become possible to him only because common sense has previously trained his thinking powers. But ever his mind tends to swing back to the common sense that has become engrained in his nature. Therefore he makes provision for his family, and performs other altruistic actions, engages in politics, distinguishes between immaterial minds and material brains, is pleased or vexed with his friends, speaks to other people, and writes books for them to read-even, it may be, books which demonstrate more or less logically and conclusively that neither they nor the books have any known existence. As Clifford, who declared, "I do believe that you are conscious in the same way as I am; and once that is conceded, the whole idealist theory falls to pieces," ${ }^{1}$ but who, nevertheless, wrote and lectured at great length as an idealist, said very properly, "A true idealism does not want to be stated, and conversely, an idealism that requires to be stated must have something wrong about it." 2

1 Lectures and Essays, Letter to Sir F. Pollock, p. 33.

2 Lectures and Essays, Philosophy of the Pure Sciences, p. 209. 
Formerly, these inconsistent idealists, while tacitly admitting the existence of other people, denied that of matter, for which "Berkeley placed God; Kant and after him Schopenhauer placed Will; and Clifford placed Mind-Stuff." 1 Now, to crown all, Professor Karl Pearson, pinning his faith to Hume, and declaring that he knows nothing beyond the veil of his sense-impressions, is highly contemptuous of the opinions of these metaphysiciansand so joins their company. Even Hume wrote for other minds. ${ }^{2}$

1 Pearson, Grammar of Science, ed. 1900, p. 68, footnote.

2 In this question of other minds lies the crux of the whole problem. When I declare that the only existence known to me is the stream of feelings which constitutes my own mind, and that this stream, especially when I divest it of the coherence conferred by common sense, does not furnish evidence of the existence of anything outside of it, I am on safe ground. But when I suppose that there are other minds, I make a guess which I cannot test, and which is therefore of the same nature as that made by the materialist. Attempts have been made by idealists, who deny or doubt the existence of material objects, to demonstrate the existence of the other minds to which they appeal. For example, J. S. Mill (Examination of Sir William Hamilton's Philosophy, 3rd ed.), declares, "I do not believe that the real externality to us of anything, except other minds, is capable of proof" (p. 239). He distinguishes between actual sensations and Permanent Possibilities of Sensations. The latter are those groups of sensations which we drape, or under fitting conditions might drape, about supposed external objects; and which, so grouped, may recur again and again. Calcutta is such a Permanent Possibility. "I do believe," says Mill, " that Calcutta exists, though I do not perceive it, and that it would still exist if every percipient inhabitant were suddenly to leave the place, or be struck dead. But when I analyse the belief, all I find in it is, that were these events to take place, the Permanent Possibility of Sensation which I call Calcutta would still remain; that if I were suddenly transported to the banks of the Hoogly, I should still have the sensations, which, if now present, would lead me to affirm that Calcutta exists here and now. We may infer, therefore, that both philosophers and the world at large, when they think of matter, conceive it really as a Permanent Possibility of Sensation. But the majority of philosophers fancy that it is something more ; and the world at large, though they have really, as I conceive, nothing in their minds but a Permanent Possibility of Sensation, would, if asked the question, undoubtedly agree with the philosophers" (p. 235). In spite, then, of the circumstance that these Permanent Possibilities may exist before the percipient mind, and survive after it, Mill rejects, as unprovable, the notion that they are caused by matter-by that which is not part of the mind, but external to it, and which possesses properties that excite in the mind sensations which symbolize those properties., Nevertheless, because a certain Permanent Possibility (the recurring group of sensations which he calls his body) is constantly associated with his mind, he endows this part of his mind with all his mind ; at any rate, because other of his Permanent Possibilities (which he calls the bodies of other people) resemble his body, he supposes that these Possibilities have minds of their own. In other words, he supposes that Possibilities which are not external to his mind have themselves minds which are external to it. From groups of his sensations he thinks he can legitimately infer the existence of other minds; but, though he believes that other minds can have identical sensations only when placed under identical conditions (e.g. at Calcutta), he thinks he is not in a position to infer the existence of an external 
566. These two views, entirely incompatible but often intermixed, the idealist and the common sense, carry with them consequences that are immensely important to the thinker. Since the idealist supposes that he is aware of nothing but the phenomena happening in his own mind, he is unable to account for their appearances, their coexistences, and their sequences. ${ }^{1}$ Even after being trained by common sense to think coherently, he can do no more than observe and record them. Thus he observes that in the compound phenomenon ' $\mathrm{dog}$ ' there tends to coexist the subsidiary phenomena canine trunk, head, limbs, and tail ; and that, when the phenomenon dog has the appearance of biting the phenomenon idealist, there tends to succeed the phenomenon pain in the phenomenon leg. But he has not the remotest idea why certain phenomena tend to coexist with the phenomenon canine body, nor why the appearance of biting should be followed by the feeling of pain. $\mathrm{He}$ is, or should be, bound by the terms of his philosophy not to pass outside the circle of his own feelings, and therefore cannot, or at any rate should not, explain the dog and the feeling of pain by the theory of evolution through Natural Selection. For, of course, the notion of Natural Selection (which is supposed to have occupied millions of years) occurring amidst the phenomena, the mere phenomena, of his mind is exquisitely absurd. It is true that most modern idealists, like most cultured people whose culture is not entirely in the air, are adherents of the theory of Natural Selection; but when they are so they are thinking in common-sense terms, and an absurdity is none the less because an inconsistency is added. It follows that all notions of causation, in the meaning the word ordinarily bears in the language, are as inconceivable to the consistent idealist as are material bodies. For him the word implies nothing more than mere invariable' succession-not a succession which is invariable because it results from the unvarying action on each other of material bodies which have unvarying properties. Accordingly he declares, now with some approach to consistency, that the mission of science something which causes the common sensations. To me, on the other hand, it seems that we have no title to believe in other minds unless we first believe in real bodies external to our own minds. That, moreover, appears to be the order in which the human mind acquires these beliefs. To this day, as when giving chloroform, I draw my inferences about other minds by the behaviour of what I conceive to be the material bodies associated with them.

1 "Another inference, apparently more paradoxical still, needs to be made, though, as far as I am aware, Dr Hodgson is the only writer who has explicitly drawn it. That inference is that feelings, not causing nerve-actions, cannot even cause each other " (James, Principles of Psychology, vol. i. p. I33). 
is merely to describe phenomena and record their coexistences and sequences.

567. On the other hand, to the common-sense thinker, who supposes that both minds and material bodies exist and that the former can be aware of the latter, causation implies more than mere invariable succession. It implies necessary succession. ${ }^{1}$ For, believing that material bodies have properties or qualities which bring them into certain relations with one another, he believes also that, given these properties and relations, certain consequences must follow. Thus, though he perceives that day and night invariably succeed one another, yet he repudiates the idea that they alternately cause each other. He knows of no property in the one which can cause the other. On the contrary, he supposes that day and night succeed because the round, opaque, material earth rotates and so presents successive parts of itself to a luminous body the sun. Given these conditions, the non-occurrence of day and night would be inconceivable to him. Given the absence of the opacity of the earth, or of its rotation, or of the luminosity of the sun, and he would be forced to believe that day or night would be eternal, though he has no experience of such a condition. A single chemical experiment will convince him that a certain substance exists because two or more elements have united; but no amount of experience will convince him that fine weather is the cause of the rain that succeeds it. In other words, there is for him a distinction between post hoc and propter hoc.

1 See $\$ 578$. 


\section{CHAPTER XVIII}

\section{NECESSARY TRUTH}

Idealism and experience-Common-sense and experience-Kinds of properties-The limits within which we can know and think-Truths reached through simple enumerations-Necessary truths-Mathematical axioms-Cause and effect -Causation is a common-sense notion-Science is common sense-Thoroughgoing idealism is unassailable, but leads to a cul-de-sac.

568.

LL knowledge, all thinking is founded ultimately on
experience that has been stored by the memory.
Experience begins with sense-impressions. The one thing that no one can doubt is the existence of his own feelings. There lies before me what common-sense regards as a sheet of paper. I may, after much thought, have doubts about the existence of that sheet, but I can have none about the existence of the sensations that seem to reveal it to me nor of my thoughts about them. If I think as a thorough-going idealist, I can note these feelings; if I go so far as to use the coherence of thought that common sense has established, I can also observe and remember their co-existences and sequences. But there the matter ends. The origin of them and of their co-existences and sequences remains an unfathomable mystery. On the other hand, if I make the assumption which common sense makes, if I suppose that the sheet has a real existence external to me and that my feelings are aroused by the properties or qualities of it, then the matter does not end there. A limitless field of thought opens up. If this object has a real existence and certain properties, then innumerable other objects have real existences and properties which are not merely my feelings but the exciting cause of them. Above all, just as the properties of the sheet of paper and those of my mind bring the two things into relation, so are all other things brought into relation by means of their properties. In this way the universe is linked together and becomes a unity.

569. If, then, the assumption that there is a universe external to my mind, and that it is revealed to me by my feelings, be given, it follows that, though knowledge begins with sense-impressions, 
which reveal the qualities of things, it does not end with them. They constitute but a minor part of it. The main portion of it is reached through the discovery of relations, a process which involves, not merely perception, but thought. Thus, through thought we link together dissimilar impressions (tactile, visual, etc.) in groups by recognizing their relations to one another, and so, by referring our impressions to the qualities of external objects, construct our notions of the latter. For example, we construct the notion of a sheet of paper by thinking that it has certain properties. Facility in linking together impressions (i.e. skill in thinking, skill in perceiving relations) is laboriously acquired during infancy; but later, when the facility has become so great that we accomplish the task without effort, we forget the initial difficulty and are apt to suppose, with the old school-men, that the thinking is performed through intuition. In this way, also, we learn the relations between the various objects thus constructedfor example between the ink, the pen, the paper, the table, the room, the house, the street, and so on. Here again the relations are not all perceived at a glance, but are thought out by a process that becomes more and more complicated, but more facile, as experience multiplies, knowledge increases, and skill grows.

570. I notice that, though the number of objects in nature appears to me to be limitless, the number of the different kinds of properties they possess is much smaller. Objects have properties in common, for which reason we are able to classify them. I observe that some properties, for instance extension in space and persistence in time, are possessed by all real things within my experience. Indeed I cannot conceive a real thing that does not possess them. Others, for instance definite and persistent shape, colour, and weight that I can feel, are very common. Others, such as certain colours, are rare. Yet others, such as a particular shape or place (e.g. the precise shape and place in the universe of my face at this moment), are possessed by one object only. Though the number of the different kinds of properties which I can perceive are limited, yet they are so numerous and may be possessed by objects in such varying degrees and combinations that an infinite variety of objects is thereby rendered possible. Thus a beetle has some properties (e.g. extension and weight) that are possessed by a house; but, because some of his properties (e.g. vitality) differ from those of the house in kind while others differ in degree, the two objects are very different and therefore distinguishable. 
571. The coherent thinking of common sense is founded on these likenesses and differences between the properties of things and on the fact that the properties of all things are conditioned by the properties of other preceding or co-existing things. Thus, owing to some of its properties, a certain object is classified by the postman as a letter, and, owing to others is distinguished from all similar objects as one belonging to a certain person in a certain country, town, street, and house. The postman, the letter, and the person to whom it is addressed, have relations to one another which are conditioned by their respective properties. Now when we speak of cause and effect, what we always mean is that the properties of a thing, or set of things, are, or have been, conditioned (altered or preserved) by the properties of another thing or set of things. Thus, some of the properties of the letter (e.g. its situation) are altered by the postman because he has certain properties (e.g. a desire to perform his duty), and because the person to whom it is addressed has certain properties (e.g. a certain name and situation). The words in the letter are preserved because the paper on which they are inscribed has the property of prolonged persistence.

572. If we follow any line of thought sufficiently far we are sure in the end to reach limits which mark, apparently, the beginnings of the unknowable and the unthinkable (e.g. the nature of things in themselves, and the terminations, if any, of space and time). Nature is parsimonious and adapts our minds, like our bodies, to practical uses. But within those limits experience stores our memories with data which enable us to reason. All, or nearly all, our reasoning consists in a tracing of cause and effect-in a tracing of how the objects we think about are, or have been, or will be, conditioned by their respective properties. At first, especially during infancy, we trace the more obvious relations. But we build continually on the data thus gathered till our reasoning, tracing effects from many combined causes or from causes that are far remote, grows increasingly complex and subtle.

573. The limits within which we can think seem to be fixed more by our perceptive than by our thinking powers. We can think only of things that have properties of the same kinds (extension, persistence, colour, etc.) that we have already perceived. Thus, though I can think of a dragon or a demon unlike anything that ever was on land or sea, yet I find I can confer on it only those kinds of properties, however strangely combined and exaggerated, that are already known to me. I am as little capable 
of conceiving new kinds of properties as a congenitally deaf man is of imagining sound or a congenitally blind man colour-which a blind man once imagined to be like the sound of a trumpet. Men are sometimes paralysed as regards sensation and movement, that is, they lose in some parts of their bodies the tactile and muscular senses. Conceivably they might be born and might exist entirely without them. Judging from the analogy of congenitally blind and deaf men, if I had never possessed any senses but those of smell, taste and hearing, I could have no idea of extension. So slowly did I develop mentally after birth that-apparently I gradually built up my idea of it. It was, therefore, not an intuition, an instinctive bit of knowledge, but an acquirement, an induction from experience. And, if my notion of space was not an intuition, it is hard to believe, so inconceivable does existence which is not spatial now seem to me, that I have any other intuitive ideas. Moreover, as we shall see later, all ideas are founded on memory, and, therefore, cannot be intuitive or instinctive. An instinct is a mere impulse to action. ${ }^{1}$

574. On the other hand, I have no doubt that, if I could see the colours that lie beyond the red and violet ends of the spectrum, I could think in terms of them. Probably, therefore, if a new power of perception, analogous to sight or hearing for instance, were suddenly conferred on me, my intellectual powers would suffice to enable me to use the materials provided by it for purposes of thought.

575. It is certain that our senses, even when at their best, are not sufficient to enable us to perceive completely all the properties of objects; thus we can see only within a certain range of the spectrum and smell odours and taste flavours of a certain intensity. It is practically certain that we cannot perceive at all some of the properties of objects; thus, apparently, a bat has some sense lacking to us which enables it to perceive some property unknown to us, for it is said to be able to avoid obstacles when flying in what appears to us absolute darkness. Again, we are unable to perceive that property in material bodies which acts across apparently empty space, and the effects of which we term gravitation. We are able to perceive only how it affects the relations of material bodies to one another. It is possible that all the properties of some real things are altogether outside the range of our perceptions, and, therefore, that some real things exist of whose existence we cannot be aware (at least directly)-whose properties are inconceivable to us, 
576. Our perceptive powers are mainly 'innate,' that is, the infant is born with senses that do not greatly develop afterwards under the stimulus of use. Since, then, these powers are 'inherited,' we can perceive only within that very limited range within which our ancestors perceived. But our thinking powers are mainly ' acquirements'; they develop under the stimulus of use (under increase of knowledge and practice in using it) to meet all sorts of contingencies; and, therefore, by perceiving different objects (not different kinds of properties) and by combining the materials supplied by our perceptions differently (by perceiving or imagining different relations) we can think not only in a different, but often in a more far-reaching way than our ancestors. Our sciences have grown in this way. It is not his senses, his perceptive powers, but his powers, depena'ent on memory, of growing mentally under the stimulus of use, that confers on man his intellectual pre-eminence over brutes. But of this more anon.1

577. I do not know how it happens that real objects have (for instance) extension in space and persistence in time. My senses supply no data, and, therefore, in this case also, I seem to have reached the limits of the thinkable. Here I cannot trace cause and effect; I can only note that all things known to me have these properties. But the fact that all the real objects I have met are possessed, in some degree, of extension and persistence, has given me the notion that they are present in all real things. That notion, however, may not be correct, for real objects lacking them may exist and I may be unaware of them because my senses are incapable of being influenced by their kinds of qualities. It is true that we can use a form of words and declare that a point is that which has no magnitude-no extension; but such a point, a thing without qualities that we can perceive, cannot reallybe thought of. $^{2}$ We do not conceive a new thing when we use that form of words; we merely try to strip a thing we know of the qualities by which we know it. I seem, then, to have reached the notion that all real bodies have extension and persistence by an induction from a 'simple enumeration'; that is, I have concluded that what has always held good within my experience always would hold good were my experience universal. In the same way I have reached the notion that all bodies in nature are susceptible of movement (of having their spatial relations to one another altered), and the notion that matter is impenetrable (that no two bodies in nature can occupy the same part of space at the same instant of

${ }^{1}$ See $\$ 624$, et seq.

${ }^{2}$ See J. S. Mill, Logic, Bk. II. chap. v., § I. 
time). Thus also I have reached other general notions. It seems then that I have gathered many notions of what seem to me uniformities in nature ('laws' of nature) by this process of simple enumeration. Such a process is a form of reasoning, but it is the most rudimentary form. "This is the kind of induction which is natural to the mind when unaccustomed to scientific efforts"1 "True knowledge is knowledge by causes. For that knowledge that proceeds by simple enumeration is a puerile thing, and concludes uncertainly, and is exposed to danger from any contradictory instance, and for the most part pronounces from fewer instances than it ought, and of these only from such as are at hand." 2 . "Popular notions are usually founded on induction by simple enumeration; in science it carries us but a little way, we are forced to begin with it ; we must often rely on it provisionally in the absence of means of more searching investigation. But for the accurate study of nature we require a surer and more potent instrument." 3 It is a remarkable fact, however, that in much recent work (e.g. in the statistical work of idealist thinkers) it is maintained, or at least implied, that the method of simple enumeration is the only means by which correct (and, therefore, scientific) results can be ensured.

578. But, after I have learned by such enumeration that real things have properties that vary in kind and degree and that certain uniformities exist in nature, I have data that enable me to think in a new way. I can now say to myself, "If it is true that real bodies exist and have properties which bring them into relation with one another in definite ways, and if it is true that certain uniformities run through nature, then given certain conditions, even combinations of conditions of which I have no previous experience, certain results must follow, and can be thought of by me even when I have had, and can have had no previous experience of them. When I think thus, I think in terms of cause and effect, and have passed from the notion of invariable to that of necessary succession. The latter is not identical with the former; it is a superstructure reared on it.

579. Thus, after I have acquired through experience definite ideas of plane surfaces and of lines which are straight, perpendicular, and parallel, I am able to pass the bounds of direct experience and declare that straight lines, on the same plane surface and perpendicular to another straight line, will never meet,

op. cit., Bk. III., chap. iii., § 2.

${ }^{2}$ Bacon, Novum Organon, i. 105.

3 J. S. Mill, Logic, Bk. III., chap. iii., \$2. 
no matter how far they be produced. Given these real things (surfaces and lines), those qualities (plane and straight) and that relation (perpendicular), then, that other relation (parallelism) necessarily follows and from that also the necessary truth that parallel straight lines will never meet-for the notion of straightness, combined with that of parallelism, implies that lines having that quality and that relation will always preserve the same distance, and, therefore, will never approach, and, therefore, never meet. ${ }^{1}$ It matters not that I have never seen surfaces that are absolutely plane nor lines that are quite straight, perpendicular and parellel, but only surfaces and lines that nearly approach perfection in these particulars. ${ }^{2}$ 'Absolutely' and 'nearly' are relative terms indicating, not different kinds of qualities, but only different degrees in qualities. Therefore, when I have experience of things that are nearly straight, perpendicular and parallel, I can think in terms of things that are absolutely so.

580. Again, I can through direct experience, through actual measurement, reach the notion that the three angles of a certain triangle, which is bounded by straight lines on a plane surface, are equal to two right angles. Moreover, by repeating these observations on other triangles I can, through simple enumeration, reach the notion that what is true of one such triangle is probably true of all similar triangles. But a notion so reached will appear to me merely an expectation, not a necessary truth. It will only seem to be the latter, if I deduce it, as Euclid does, from the previously known and admitted properties and relations of lines, angles and triangles.

$58 \mathrm{I}$. In mathematics we are able to discover many necessary truths, for here the conditions are, speaking comparatively, so little complicated that we are able to perceive and conceive things and their properties and relations with greater completeness and certitude than elsewhere, and, therefore, can think more exactly and confidently. Nevertheless, in everyday life we are continually endeavouring to deduce such truths. That is, we are continually endeavouring to deduce from the previously known and admitted properties and relations of things, effects that, given those pro-

${ }^{1}$ I am informed by Professor H. H. Turner that much or all of this is controverted by the "New Euclidians." But the example even if faulty will serve to illustrate my meaning. It may be noted also that in mathematics we do not deal with sequences of events. We suppose, however, that, given certain facts, certain other facts must necessarily be true also, and we proceed to discover the latter. The thinking, therefore, is of the same nature as when we deal with sequences of events-with cause and effect.

S See Mill, Logic, II. v. 147. 
perties and relations, must follow. Thus, given that my cook has certain properties (e.g. the will and the ability), given the existence of food in the house, given a multitude of other things, it follows necessarily that my housemaid will presently summon me to breakfast. Here, I do not know all the conditions as perfectly as in geometry, but, if I did and could think as adequately about a matter so complicated, I could be just as sure in the one case as in the other. The important point is that in this case also I have reached the expectation that I shall be summoned to breakfast otherwise than by an induction from a simple enumeration of instances of former like events. I could have ignored the qualities of the cook and the other things concerned and reached the expectation by remembering that I have been so summoned for a long series of mornings. But, as a fact, I did not do so. It is when we think in terms of cause and effect that the need for testing our thinking by making a deductive appeal to reality arises. Such a testing is necessary even in the mathematics in which the conditions are relatively simple and clearly defined, and the chances of error are correspondingly slight. Thus, when we multiply one number by another (e.g. 123 by 345 ), we are able to make sure that the product, the conclusion to which we have led by our reasoning, is correct only by some such test as is furnished by dividing it by one of the factors and then ascertaining whether the quotient agrees with the other factor. The more difficult the problem, the more complex the conditions or the more prolonged the chain of reasoning, the more necessary it is to apply tests ; as in biology, where the conditions are often exceedingly complex and so obscure that it is often difficult to separate essentials from non-essentials, real causes and effects from accidental accompaniments.

582. In science, as in everyday life, we endeavour to deduce effects from causes, or causes from effects, and so link up our knowledge in uniformities, which, though based ultimately on results obtained by simple enumeration, are other, and additional, and as superior to them as a temple to its foundations. All the sciences in which we have succeeded best in this endeavour are the most 'scientific,' the most deductive, the most completely knit into compacted wholes. ${ }^{1}$ Mathematics and physics are examples. Zoology and botany formerly rested on a simple enumeration of likenesses, differences, co-existences, and sequences. Doubtless they had practical uses and satisfied scientific workers of that 
class which is content with such a catalogue as a grocer makes of his stock. But consider the improvement effected by Darwin, when, deducing necessary truths from given data, he linked together plants and animals by chains of causation. Knowledge was then supplemented by understanding. Something more than a mere memory of perceptions came into play. That something was Reason.

583. What, then, do we mean by the expression 'necessary truth'? How is it to be distinguished from 'invariable' occurrence? A necessary truth is not one reached by intuition. Apparently no such truths are known to us. Nor is it one reached by induction from simple enumeration; for that merely endows us with a sense of probability, of expectancy. It is always one which we have reached through what seems to us valid reasoning from an apparently complete consideration of all the relevant properties and relations of real things. It is not, of course, more certainly true than the existence of those real things and of their properties and relations ; but, given those existences, those properties, and those relations, it is certainly true provided we have reasoned correctly and taken all that is essential into consideration. It is an effect that we have inferred from its cause, or a cause that we have inferred from its effect. It is a conclusion drawn from premises antecedently formulated or implied, and true if the premises are true.

584. If the reader will try to think of any notion the truth of which appears necessary to him, he will always find that it bears this character of an inference from premises which have previously been granted. For example, though we are told that every axiom of geometry is a 'self-evident' and 'fundamental' truth ; " that is, its truth should not be deducible from any other truth more simple than itself"; yet, as a fact, we do, in every instance, derive geometrical axioms from truths which we regard as more fundamental, simple, and self-evident than themselves-from the qualities and relations of the things we are considering. Some reasoning is always done before we admit the truth of an axiom. Thus the axiom that "Things which are equal to the same thing are equal to one another," is but another way of saying that "If things are equal to the same thing they are equal to one another, for the reason that they will then have identical properties and relations." The reasoning may be so swift and easy that only more thinking reveals its existence, so swift and easy that an attempted exposition of it appears like stating the same thing in another and a more involved way; the inference may be so obvious that only 
careful consideration reveals that it is an inference; but the given premises, the reasoning, and the inference are always there. An axiom may seem self-evident to us who have unconsciously drawn the inference a thousand times a day for years; but it is not selfevident to an infant who must not only acquire a knowledge of the data on which it is founded, but also skill in thinking sufficient to enable it to perceive their relations and how they affect one another. ${ }^{1}$

585. To take other examples; we do not regard a line as necessarily straight unless we have reasons for doing so, unless we draw an inference, unless we think it traverses the shortest distance between two points and apprehend what that means. Unconsciously we reason out that a line that does not traverse the shortest distance cannot be straight. A straight line is not necessarily perpendicular to another unless it makes equal angles with it. Straight lines are not necessarily parallel unless they make equal angles with a third straight line on the same plane. Straight lines on the same plane will necessarily meet if produced far enough unless they are parallel. Evolution through Natural Selection may be true; but it is not necessarily true unless offspring resemble their parents on the whole but differ from them in details, unless they outnumber them, unless only the fittest survive on the average, unless the fittest belong to types, and so on. In brief, for us a necessary truth is always an effect or cause which we have been able to trace so perfectly to its cause or effect that no doubt of the nature and reality of the connexion remains in our mind. It is a truth which we think we not only

1 Dr Whewell and others have held that "it is not experience which proves the axiom; but that its truth is perceived a priori by the constitution of the mind itself, from the first moment when the meaning of the proposition is apprehended, and without any necessity of verifying it by repeated trials, as is requisite in the case of truths really ascertained by observation" (Mill, Logic, v. 4). This view is opposed by Mill who thinks that we learn the truth of axioms wholly through experience. The two opinions do not appear to be absolutely contradictory. Whewell admits that we must have experience-that the meaning of propositions and all that that implies in the way of experience, must be apprehended. Mill admits as necessary truths those "necessarily following from hypothesis." I think I learned originally through experience that two halves make a whole ; but now that I have learned " the meaning of the proposition" I hold that belief on other grounds as a necessary truth. In this way, at first mere experience led me to believe in the mathematical axioms and most of the other necessary truths that I now hold as such ; but they did not become necessary truths to me till I had reached them in another way. The view I suggest differs from those of Whewell and Mill in that axioms, as such, seem to me products of reasoning, not of intuition or of mere repeated experience. 
know, but which we think we can account for in terms of other admitted truth. Moreover, it is always a truth which has been or which can be reached otherwise than by simple enumeration and which, therefore, can be tested in one or more ways, for example by simple enumeration. Thus, after declaring that, given certain conditions (e.g. universal gravitation, the rotating earth, and the moon), a certain result must follow (e.g. the tides), we can test our supposed necessary truth by appealing to instances which occur in reality. Therein it differs from a truth which as yet has been and can be founded only on simple enumeration (e.g. the statement that all real bodies have extension); for such a truth, which has not been deduced from any other, can be tested, if that deserves to be called testing, only by continuing the enumeration.

586. We see then that a necessary truth is one which we have reached by tracing the relation of cause and effect. We observe that certain bodies in nature have certain properties or qualities, for example extension and gravity, by means of which they are brought into relation with one another and so affect one another. A law of nature is a uniformity that we have observed in nature. Usually we mean by the term a uniformity of sequence; that is, a uniformity in the way in which bodies affect one another. Uniformities of sequences imply uniformities of qualities and relations, and, therefore, of consequences. Doubtless, it is this notion of necessary consequences that has given rise to the term 'law.' 1 Thus, if it be true that material bodies attract one another, it must be true also that they tend to approach one another. A necessary truth is merely a particular instance of a general uniformity, or else it is a consequence correctly deduced from such a uniformity. When we formulate such a truth, we declare, in effect, "If the general law is true, then, since the bodies we are considering have qualities and relations that bring them within the range of that law, this particular instance or consequence of it must be true also." In practice the term necessary truth is used, as a rule, in cases in which the inference is not very obvious-in cases in which consequences rather than instances are dealt with, in which more than one quality and relation and therefore more than one uniformity is concerned, and in which, therefore, the conclusion is reached by

1 "A law, then, in the strict scientific sense is a fully established statement of universal and necessary connexion . . . more loosely, the term is frequently used to denote mere empirical generalizations. . . These, however, express no necessity and, consequently, are not laws in the strict sense . . . 'empirical law' is, correctly speaking, almost a contradiction in terms." (Welton, Manual of Logic, vol. ii. p. 200.) 
reasoning that is more or less complex or prolonged. The declaration that a conclusion is a necessary truth amounts to a claim that we have reasoned correctly from premises (law or laws) that are admittedly true. No matter how simple or complex the case, when we declare that we have 'explained' or 'understood' an occurrence, we mean merely that we have interpreted it in terms of one or more uniformities of which we are aware. Moreover, this tracing of instances and consequences to general uniformities, and therefore to the qualities and relations of objects, is all that we mean by 'tracing a chain of causation.' Thus, knowing the common uses of pokers and that iron can be raised to a high temperature by fire, we explain the hotness of a poker, we describe the cause of the hotness, by saying that the poker has been in the fire. On the other hand, knowing the qualities of ice, we should, if we found a hot piece of it, be at a loss for an explanation. Knowing that intact brains are essential to the existence of human beings, we suppose we have the explanation of the death of a man when we discover a bullet imbedded in his brain. We explain the rise of the tide and the fall of a stone by the same law. We explain the rise and fall of water in a harbour by the tides of the ocean, the tides by the influence of the moon, the influence of the moon by the law of gravitation. We cannot as yet explain the law of gravitation by reference to a wider uniformity; it is for us an ultimate law; but between it and the rise and fall of water in a harbour lies a chain of explanation, of causation, and of necessary truth. When Huxley declared that, "Fact I know : and law I know: but what is this Necessity, save an empty shadow of my own mind's throwing?" 1 he referred to these ultimate uniformities which as yet we cannot explain, and which perhaps we shall never be able to explain by reference to more general laws. He referred to necessary truth when he said " even thoughtful men usually receive with surprise the suggestion, that the form of the crest of every wave that breaks, wind-driven, on the sea-shore, and the direction of every particle of foam that flies before the gale, are the exact effects of definite causes; and, as such, must be capable of being determined, deductively, from the laws of motion and the properties of air and water." 2 "The very postulate of knowledge compels us to think every variation and every detail, even the smallest, as so determined by conditions that, under the circumstances, it could not possibly be other than it is.

1 Essays, vol. i. p. I6r.

When the conditions of every detail

2 Hume, p. 122. 
of a phenomenon are so fully and exactly known that not only a phenomenon of this general character, but just this very phenomenon, with exactly these details, and each in exactly this amount, must follow from those conditions, and from those only, then that phenomenon is fully explained." 1 But when we think of necessary truth, we must do so in terms of that reaiity to which our minds seem to testify. If we question the reality, we can declare, "Fact I doubt : and law I doubt : and, therefore, necessity I doubt." But, given the fact and law, then necessity is beyond all doubt.

587. When tracing cause and effect, we do not refer explicitly to all the qualities of the objects we are considering. Every object has many qualities. Thus iron has persistence in time, extension in space, weight, colour, frangibility, ductility, hardness, and a multitude of others. It could not become hot, it could not even be an object for us, unless it had at least many of them, for example, persistence and extension, yet we do not think or say that the poker becomes hot because it has persistence and extension. Many pokers are not hot; even ice which cannot become hot has persistence and extension. We refer especially to the quality in iron, its temperature, which is changed by the fire, and the quality in the fire which is concerned in the change. The last is termed the cause, the change in the first is termed the effect.

588. More than one quality may be concerned in a cause; thus both the momentum and the hardness of a bullet are concerned in the effect produced when it strikes another body. More than one quality may be concerned in an effect; thus the position, shape, and temperature of a piece of iron may be changed when it is struck. Nevertheless, no matter how many of the qualities of an object are concerned when it acts on or is acted on by another object, in practice we think only of those which are especially concerned; or indeed, only of those of with which we are especially concerned. Of course, no cause or effect stands isolated in the external world; we merely isolate it in thought. In reality it is connected not with a chain, but with a circle of cause and effect. Thus a bullet, on which certain properties were conferred during manufacture, flies because it is impelled by the explosion of powder, ignited by a spark in a gun, which was fired because a man, who was present through a concatenation of circumstances that reaches back through eternity, pulled a trigger because he was made angry by certain events, and so on. Yet we say only that the one man was ${ }^{1}$ Welton, Manual of Logic, vol. ii. p. I88. 
shot because he angered the other man. A knowledge of the whole circle of causation would imply perfected science, a thorough knowledge and understanding of the whole universe during all past time. In a sense, at any instant, we can point to the termination of effect, for at any instant the effect has not proceeded beyond that instant; but the circle of causation had its beginnings only in the beginnings, if any, of time and space. But this totality, both when we consider the qualities of objects, and the circle of causation, is utterly unwieldy. Therefore, as in practice we select for purposes of thought certain qualities of objects, so also when tracing causation we select certain parts of the circle. We suppose a chain, and, even then, think only of those links that appear to serve our purpose best.

589. In this way, by supposing that objects have reality outside our minds, and by supposing that they have qualities by means of which they act and react on one another in a uniform way, we gather the notion of a universe which, so far from being chaotic, is orderly to its minutest details; and in reference to which, as far as we are able to perceive the order and trace cause and effect, we are able to use such words as ‘ 'understand,' explain,' 'why,' 'reason,' and the like with intelligible meanings. If we did not, and more especially if we had not, supposed a material universe, our thoughts, as we have seen, would be without coherence. Or rather, we would have no thoughts, but only feelings. Very emphatically, then, the entire notion of causation belongs wholly to common sense. It has, or should have, no place in idealist thinking.

590. All science, like all ordinary thinking, is founded on common sense. "Every science assumes certain data uncritically, and declines to challenge the elements between which its own ' laws' obtain and from which its deductions are carried on.... Of course these data are themselves discussable, but the discussion of them (as of other elements) is called metaphysics." 1 That is, every science thinks in terms of what are supposed to be real existences and omits to discuss the question whether they are, or are not, real. "All physical science starts from certain postulates. One of them is the objective existence of a real world." 2 It is true that we are told that " a scientific law is related to the perceptions and conceptions formed by the perceptive and reasoning faculties in man; it is meaningless except in association with these; it is the

1 James, Principles of Psychology, Preface, v-vi.

${ }^{2}$ Huxley, Essays, vol. iv., p. 60. 
résumé or brief expression of the relations and sequences of certain of these perceptions and conceptions, and exists only when formulated by man. ${ }^{1}$... The law of gravitation is not so much the discovery by Newton of a rule guiding the motion of the planets as his invention of a method of briefly describing the sequences of sense-impressions, which we term planetary motion. . . . We are thus to understand by a 'law of nature,' a résumé in mental shorthand, which replaces for us a lengthy description of the sequences among our sense impressions." 2 According to the law of gravitation, all bodies in nature attract each other proportionately as their masses and inversely as the squares of their distances. Now in what intelligible sense can the sense-impression, the earth, be said to attract the sense-impression, the sun? What are their masses, and what the squares of their distances? To all appearances the planets, the sun, and the other stars are of no great magnitudes, at no great distances, and float lighter than feathers through the air. Even if they be conceived as nothing more than sense-impressions between which, after a previous training by common sense, Newton was able to discover relations which are quite unlike their apparent relations, how do we know that Newton existed as a thinking entity? As a fact, the law of gravitation has been accepted by astronomers and physicists as describing a relationship between material bodies external to the human mind -bodies and a relationship between them which the human mind is thought to be aware of at present, but which existed long before the evolution of any such mind. Obviously also, since the word 'attraction' is used, the relationship indicated, as in the case of many other laws that have been formulated, is one of causation. It is not a mere invariable succession such as that of night and day, the notion of which is reached, not through a consideration of the qualities of the related objects, but through simple enumeration. I say 'formulated,' for the laws that are reached through simple enumeration (e.g. the law that all material bodies have extension), are, as a rule, discovered by every one and therefore are not worth formulating by anyone.

591. "In the scientific sense, a law is a statement of a necessary connexion; it is not something imposed upon reality from without, but is the outcome of the nature of reality itself. The perception of natural laws is due, no doubt, to the synthetic activity of mind, but it is possible only because the connexion actually exists in reality. It is because the world is a systematic unity that we are

${ }^{1}$ Pearson, Grammar of Science, ed. 1900, p. 82. op. cit., p. 86-7. 
compelled to think it as such. A law, then, in the strict scientific sense, is a fully established statement of universal and necessary connexion." 1 As a simple matter of fact, no chemist, astronomer, zoologist, botanist, or medical man, for example, ever does, under his ordinary conditions of thought, conceive elements, worlds, animals, plants, or human beings, as groups of his private feelings. They are to him concrete realities existing external to himself, and a principal part of his labours is to gather information from and impart it to other concrete people. It is just this fact that science, in more senses than one, is "organized common sense" that imparts to it its intellectual richness and splendour, that rescues it from the intellectual leanness, meanness and barrenness of the grocer's catalogue.

592. Nevertheless, though idealism is possible only to the man who has learned to think in terms of common sense, though it is utterly unable to advance beyond the fact that his feelings exist, though the idealist philosopher can do nothing but wonder impotently, its position seems quite unassailable. That is, the position of through-going idealism seems quite unassailable. That kind of idealism which uses the whole paraphernalia of common sense, which appeals to the phenomena that common sense has compounded and so rendered coherent and to other minds, which is idealist only during occasional asides when it proclaims itself idealist, that kind is assailable at every joint. It is only a tour de force by people who in their appeals to other people assume the very thing they profess to deny. To sum up: my own attitudeI must be egotistical when I speak in terms of idealism-is this; I admit I cannot pass beyond the circle of my feelings, which may lie to me, or which, if they tell the truth, can tell it only in a symbolic way. I do not positively know that there is a universe external to my consciousness in which exist material objects and minds like my own, which know or can know the things that I know. But, having made that admission, I return wholly, or almost wholly, to common sense, which has rescued, and still rescues, me from mental impotence. I assume that the universe exists as a reality, and that my feelings, and those of other people, symbolize it much as written words symbolize spoken words. I suppose that the real things of which the universe is constituted have properties which bring them into relation with one another, and that thence arise relations of cause and effect, and ultimately my notions of necessary truth. I can test the evidence furnished

${ }^{1}$ Welton, Manual of Logic, vol. ii. p. 200. 
by common sense in so many ways, and if the tests are conducted with sufficient care, I so invariably find the evidence consistent with itself, that it seems to me highly probable that my assumptions are correct. As to the actual nature of the reality which I think my mind symbolizes, I make no guess. I use the word ' matter,' but only as a convenient name for a something of which I have no immediate knowledge. Since I suppose that my mind symbolizes reality, I suppose also that the uniformities which I detect in nature are due to a uniformity of causation which depends on the qualities and relations of material objects, not on a mere invariable and utterly unaccountable succession of mental happenings, which is all that the idealist has a right to postulate. Therefore, though I do not know the whole circle of causation, but only a little part of it, yet, within the narrow limits of my knowledge, the universe appears to be comprehensible, and I am able to say that I understand and can explain. I am, then, in one respect, an idealist, and in another a materialist. I am an idealist in that I know I know my feelings, but nothing else. I am a materialist in that I believe my feelings symbolize reality. My attitude differs from the common sense of everyday life and of science (which accepts appearances for what they seem to be) in this matter of symbolism. I suppose I am like a man who is deaf, and knows that he is deaf, but who can read. I am not like a deaf man, who, being able to read, supposes that he can hear, or, at the other, the idealist, extreme, like one who, being able to read, supposes he can neither hear nor read.

593. Granting the existence of material objects, then evidently there are two distinct ways in which we may classify facts, and so create science. On the one hand, we may arrange our data accordingly as the objects we have under consideration have, or have not, properties, or, as they are termed in biology, 'characters,' in common. Thus, since a man possesses certain characters in common with other living beings, we classify him as an animal, a vertebrate, a mammal, and so on. In this way systematic zoology, botany, and anatomy have been developed. Here the mental faculties we employ are principally observation, recollection, comparison, and the like. On the other hand, we may arrange our facts in chains of causation. This is the method adopted in mathematics, physics, to a great extent in astronomy, and in biology when we discuss problems of heredity and evolution. Here, regarding the properties of objects as causes or effects, we proceed principally by formulating hypotheses, which 
we test and establish (or disprove) by making a deductive appeal to reality. In other words we reason. The kind of thinking employed in the one case differs sharply from that employed in the other. For example, if a systematic botanist wishes to demonstrate the truth of a disputed statement, his main task, as a rule, is to prove the correctness of the data from which he starts his thinking. In other words, he has to demonstrate that he has observed, recollected, and compared correctly; for, while his facts are often multitudinous and complex, the thinking founded on them is usually relatively simple, and therefore unlikely to be wrong if the facts are correct. The mathematician, on the contrary, usually founds thinking that is relatively difficult on facts that are comparatively simple. His main task is to prove the correctness of his reasoning. We shall see later that skill in thinking, whether of the kind employed by the systematist or that which is used by the mathematician, is an 'acquirement.' It develops under the stimulus of use. Moreover, skill in the one kind of thinking does not necessarily imply skill in the other kind. No one disputes that we have created true science when we have arranged our facts 'systematically.' Nor has any one ventured to deny that mathematics and physics, in which causal relations are traced, are products of real scientific thought. Occasionally, however, a biologist, while neither denying the facts nor controverting the thinking founded on them, denounces 'speculation' and 'theorizing.' 1 It is unscientific, of course, to found speculations on imaginary facts or formulate guesses which are incapable of proof or disproof; and, certainly, hypotheses of this kind are not uncommon in biology. But, judging from the language sometimes used, it is not merely the faulty use of a right method of work that is condemned, but the method itself. Manifestly, however, if it be possible to link facts in chains of causation, it is proper to do so. Knowledge is then supplemented by more knowledge, and by understanding besides, and the structure of science is all the more complete because we have added the woof to the warp of it. Since verified facts are abundant in biology, and since as living beings we are familiar with the conditions of life, it should not be beyond the powers of the human intellect, which has discovered the calculus and the law of gravitation, to indicate in general terms the uniformities of sequence under which the structures of plants and animals have arisen. At any rate the attempt to do so is not unscientific. ${ }^{2}$ 


\section{CHAPTER XIX}

\section{THE RELATION OF MIND TO BODY}

Huxley and Clifford-Their inconsistencies-Mind and body are causally related-Professor William James-Theories of the relation of Mind to BodyProbably mind is the work done by the brain-The bodies of human progenitors and descendants are more closely alike than their minds.

594.

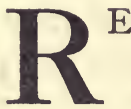

ETURNING now altogether to the thinking of everyday life and of science, common sense tells us that we have minds and bodies, and that these two sharply distinct things influence one another-have causal relations with one another. This causal relation has been denied, however, and sometimes on very high authority.

"The consciousness of brutes would appear to be related to the mechanism of their body simply as a collateral product of its working, and to be as completely without any power of modifying that working as the steam-whistle that accompanies the work of a locomotive engine is without influence on its machinery. Their volition, if they have any, is an emotion indicative of physical changes, not a cause of such changes. ... Much ingenious argument has at various times been bestowed on the question. How is it possible to imagine that volition, which is a state of consciousness, and, as such, has not the slightest community of nature with matter in motion, can act upon the moving matter of which the body is composed, as it is assumed to do in voluntary acts? But if, as is here suggested, the voluntary acts of brutes-or, in other words, the acts which they desire to perform-are as mechanical as the rest of their actions, and are simply accompanied by the state of consciousness called volition, the inquiry, so far as they are concerned, becomes superfluous. Their volitions do not enter into the chain of causation of their actions at all. . . The soul stands related to the body as the bell of a clock to the works, and consciousness answers to the sound the bell gives out when it is struck. ... Thus far I have strictly confined myself to the automatism of brutes. . . . It is quite true that, to the best of my judgment, the argumentation which applies to brutes holds equally 
good for man; and, therefore, that all states of consciousness in us, as in them, are immediately caused by molecular changes of the brain-substance. It seems to me that in men, as in brutes, there is no proof that any state of consciousness is the cause of change in the motion of the matter of the organism.

"If these positions are well based, it follows that our mental conditions are simply the symbols in consciousness of the changes which take place automatically in the organism; and that, to take an extreme illustration, the feeling we call volition is not the cause of a voluntary act, but a symbol of that state of the brain which is the immediate cause of the act. We are conscious automata." 1

595. "All the evidence that we have goes to show that the physical world gets along entirely by itself, according to practically universal rules. .. . The train of physical facts between the stimulus sent to the eye, or to any one of our senses, and the exertion which follows it, and the train of physical facts which goes on in the brain, even when there is no stimulus and no exertion-these are perfectly physical trains, and every step is fully accounted for by mechanical conditions. . . . The two things are on utterly different platforms-the physical facts go along by themselves, and the mental facts go along by themselves. There is a parallelism between them, but no interference of one with the other. Again, if anybody says that the will influences matter, the statement is not untrue, but it is nonsense. Such an assertion belongs to the crude materialism of the savage. The only thing which influences matter is the position of surrounding matter, or the motion of surrounding matter. ... The assertion that another man's volition, a feeling in his consciousness which I cannot perceive, is part of the train of physical facts which I can perceive - this is neither true nor untrue, but nonsense ; it is a combination of words whose corresponding ideas will not go together. ${ }^{2}$... Sometimes one series is known better, and sometimes another; so that in telling a story we speak sometimes of mental and sometimes of material facts. A feeling of chill made a man run; strictly speaking, the nervous disturbance which coexisted with that feeling of chill made him run, if we want to talk about material facts; or the feeling of chill produced the form of sub-

${ }^{1}$ Huxley, Animal Automatism, quoted in part by James, The Principles of Psychology, vol. i. p. I 3 I.

${ }^{2}$ W. K. Clifford, Lectures and Essays, Body and Mind, pp. 262-3, quoted by James. 
consciousness which coexists with the motion of the legs, if we want to talk about mental facts. ... When, therefore, we ask, 'What is the physical link between the ingoing message from chilled skin and the outgoing message which moves the leg ?' and the answer is 'A man's will,' we have as much right to be amused as if we had asked our friend with the picture what pigment he had used in painting the cannon in the foreground, and received the answer, 'wrought iron.' It will be found excellent practice in the mental operations required by this doctrine to imagine a train, the fore part of which is an engine and three carriages linked with iron couplings, and the hind part three other carriages linked with iron couplings; the bond between the two parts being made up out of the sentiments of amity subsisting between the stoker and the guard." 1

596. In the passages quoted both authors, professed idealists however, draw a common-sense distinction between brain and mind on the ground that the former is a material object, whereas the latter is not. Huxley thinks it impossible that mind can affect matter; but, regarding mind as a 'product' of the working of the brain, believes that matter can affect mind. It is hard, however, to conceive how one thing can directly affect another without being affected back again. If light reaches an object, it is reflected or absorbed; if the sun attracts the earth, the earth attracts the sun; if I touch a stone, the stone touches me; if my arm makes a movement, the arm suffers change. All relations are mutual. If it be true that brain can affect mind, then, judging by all analogy, it should be true also that mind can affect brain.

597. Clifford is even less consistent. He supposes that mind and matter do not affect one another; but his mind was evidently aware of, was affected by, guards, stokers, engines, carriages, and pictures, which he describes as material existences. He derides the 'crude materialism of the savage,' but the materialism of common sense, which he here adopts, is every whit as crude. $\mathrm{He}$ declares that "all the evidence that we have goes to show that . . the physical facts go along by themselves, and the mental facts go along by themselves. There is a parallelism between them, but there is no interference of one with the other." But to imagine this unrelated but exactly coincident working is to imagine the greatest of miracles. Moreover, the hypothesis involves the corollary that mind is useless, and that the unfeeling, unthinking brain is by itself responsible for all the more wonderful

1 Op. cit., Right and Wrong, pp. 328-9, quoted by James. 
activities of the higher animals, including man. To the student of evolution, who realizes the extreme parsimony of nature, and the universality of adaptation, such a notion, Huxley notwithstanding, should be unbelievable. ${ }^{1}$

598. Common sense tells me that there is at this moment a cup of coffee on my table, which I see and smell, because I have material eyes and a material nose, but which I would neither see nor smell if I had lost the eyes and nose. I become conscious of a desire to drink, and, apparently because of that desire, my hand, a thing as material as my brain, goes out and seizes the cup. I drink with a material body, and feel a satisfaction which is mental. My entire life is filled with such convincing experiences, and so, as far as I am able to judge, is the life of every one else. At any rate, the evidence is such that every human being over the age of a few weeks, and I think every conscious animal that can think at all, is convinced, in effect, that mind and body influence one another-that, for example, he can move his body at will, and that when his body is injured he suffers pain as a consequence. The whole of our private and public conduct, the entire structure of the family and of society, is founded on this belief that mental and bodily actions are not links in separate chains of events, but links in the same chain. Thus we bestow material gifts, seek to please those we love, and imprison and even hang men who have consciously broken the law-whose minds, as we suppose, have influenced their bodies. But we do not punish or even blame a man when he unconsciously develops a cancer which offends sight and smell, or an infectious disease which starts a destructive epidemic. Continually, therefore, we blame the mind for the doings of the body, and punish the body for the doings of the mind.

599. In fact, given the existence of the body, then, as far as the evidence goes, there is nothing in the whole range of experience so certain apparently as that mind and body affect one another. It is not the lack of evidence that is the obstacle to belief, but the difficulty of conceiving how a material thing can affect an immaterial thing, and vice versa. But matter itself is inconceivable. Having swallowed the camel, we have no right to put on an air of delicate discrimination and strain at the gnat. When evidence is massive and apparently conclusive-as it is in this case, if anywhere-the probabilities are that the apparent inconceivability of a thing is due, not to its non-existence, but to our mental limitations-to our ignorance, lack of the right perceptive powers, and

1 See James, Principles of Psychology, vol. i. pp. I38-I44. 
the like. We have no senses by which we can perceive minds; therefore we are unable to conceive how they affect or are affected by brains; but that does not confer on us the right to declare dogmatically that there is no relation save a parallelism. Other existences, though equally inconceivable, are readily believed in by all of us, including Huxley and Clifford, merely because sufficient evidence demonstrates their existence. Thus both authors accepted the theory of gravitation as a true statement of fact; that is, they supposed that the earth attracts bodies to its surface by means of an immaterial something, which, acting across millions of miles of space, affects even the sun and the stars. "One cannot thus blow hot and cold. One must be impartially naif or impartially critical." 1

600. For the idealist, material bodies (e.g. brains) do not exist. $H i$ insurmountable difficulty is to account for the coexistences and sequences of phenomena. ${ }^{2}$ It is, for example, no easier for him to imagine that iron (a mere appearance) can couple railway carriages (also mere appearances) than to imagine that feelings of amity can perform the task. To adult people, thinking in common-sense terms, the idea that immaterial minds can influence material brains seems, on the face of it, very absurd-as absurd as that sentiments of amity can couple railway carriages. On the other hand, the notion that the chains of physical and mental facts are distinct and separate seems reasonable-as reasonable as that feelings cannot connect railway carriages. But why does the one seem absurd and the other reasonable? Only, I think, because experience has impressed on us, till we accept its teaching as a natural law, that, while sentiments do not directly influence such things as railway carriages, physical things do. An infant would feel no sense of absurdity in the one case or of reasonableness in the other; nor should we had our experience been different. We consider things reasonable or absurd accordingly as they do, or do not accord with previous experience. Our experience is that sentiments do not influence such things as railway carriages, with which they have no direct connexion. Therefore, though the evidence that brains and sentiments are directly connected, and that they influence one another is immensely massive, we, or at least some of us, reasoning from analogy, tend to suppose that neither connexion nor influence exist. It would be quite as reasonable to suppose that there is no such thing as gravitation or magnetism.

1 James, Principles of Psychology, vol. i. p. I37.

2 See $\$ 566$ (footnote 2 ). 
60I. Like Huxley and Clifford, many modern writers insist on " these two notions, of the absolute separateness of mind and matter, and of the invariable concomitance of a mental change with a bodily change." 1 "But this 'concomitance' in the midst of 'absolute separateness' is an utterly irrational idea. It is, to my mind, quite inconceivable that consciousness should have nothing to do with a business that it so faithfully attends." 2 ... "To comprehend completely the consequences of the dogma so confidentlyenunciated, one should unflinchingly apply it to the most complicated examples. The movements of our tongues and pens, the flashings of our eyes in conversation, are of course events of a material order, and as such their antecedents must be exclusively material. If we knew thoroughly the nervous system of Shakespeare, and as thoroughly all his environing conditions, we should be able to show why at a certain period of his life his hand came to trace on certain sheets of paper those crabbed little black marks which we for shortness' sake call the manuscript of Hamlet. We should understand the rationale of every erasure and alteration therein, and we should understand all this without in the slightest degree acknowledging the existence of the thoughts in Shakespeare's mind. The words and sentences would be taken, not as signs of anything beyond themselves, but as little outward facts, pure and simple. In like manner we might exhaustively write the biography of those two hundred pounds, more or less, of warmish albuminoid matter called Martin Luther, without ever implying that it felt.

"But, on the other hand, nothing in all this could prevent us from giving an equally complete account of either Luther's or Shakespeare's spiritual history, an account in which every gleam of thought and emotion should find its place. The mind-history would run along the side of the body-history of each man, and each point in the one would correspond to, but not react upon, a point in the other. So the melody floats from the harp-string, but neither checks nor quickens its vibrations; so the shadow runs alongside the pedestrian, but in no way influences his steps." 3

"My conclusion is that to urge the automaton theory upon us, as it is now urged, on purely a priori and quasi-metaphysical grounds, is an unwarrantable impertinence in the present state of psychology." 4

1 Chas. Mercier, The Nervous System and the Mind, p. II.

2 James, Principles of Psychology, vol. i. p. I 36.

3 James, Principles of Psychology, vol. i. pp. 1 32-3.

4 op. cit., vol. i. p. I 38. 
602. Assuming, then, that brain and mind influence one another, what is the connexion between the two? It has been said very crudely, but I think with some element of truth, that the brain secretes thought as the liver secretes bile. It has been said equally crudely that the mind, like heat and electricity, is a form of motion. It has been said that mind and body are different aspects of the same thing, a statement which is merely verbal in the sense that it conveys no intelligible idea. It has been said that mind and body are distinct but temporarily related things, and that mind bears the same relation to body as the musician to his instrument; in which case the actions of the body are comparable to the music. It has been thought that mind is the music that proceeds from the instrument, the body, and that the environment is the musician, the thing that stimulates the body, the brain, to produce the music. This is the common-sense, and, I think, the correct, notion. But it is a notion which is capable of expansion.

603. To me, bearing in mind the theory of evolution, it seems probable that thinking and feeling are forms of doing, and that the brain is the doer; or, in other words, that mind is one of the products of brain activity, one of the functions of the brain, one of the things that it does. I say one of the things, for not only does the brain, as I suppose, think and feel, but it receives and despatches stimuli. I do not know how brain produces mind any more than I know how one material body attracts another across space. But, as far as I am able to judge, the evidence indicates that mind arises only when there is an expenditure of energy in the brain;-when there is no brain there is, apparently, no mind; when a portion of the brain is destroyed or injured, and ceases to function, or functions imperfectly, a corresponding portion of the mind is eliminated or is imperfect; accordingly as the brains of the higher animals are complex and ample so are their minds; mental exhaustion bears a relation to physical (brain) exhaustion; and so on. I assume, therefore, as the most probable conjecture, that mind is somehow a consequence of the work of the brain. When energy is expended in the muscles of the arm, molecular movements occur, from which finally result the muscular contractions, by means of which the arm is moved and the hand does its varied work. So, apparently, when energy is expended under conditions which exist in certain forms of nervous tissue (e.g. brains), mind results, and the brain does its varied work. But when I think of the chain of events that end in the movements of the hand, I think from first to last of nothing but matter in motion. If I had a 
sufficiently powerful microscope, I should be able to perceive the whole chain, and, within narrow limits, to think of it in terms of causation. Even now my perceptive powers are such that they supply me with materials which enable me to imagine it. But no microscope, however powerful, could enable me to perceive how the molecular movements in the brain result in mind. Even if I could see the movements of the molecules of another man's brain, even if I learned that certain movements in certain regions of his brain always coincided with certain of his feelings and thoughts, I should yet be unable to perceive the link between the two trains of happenings, the mental and the physical. And, since my perceptual powers, however aided, are such that I could not perceive the link, therefore I cannot think in terms of it. ${ }^{1}$ Nevertheless, this is not the main difficulty. It is merely part of the larger difficulty raised by the idealist-the difficulty of conceiving how, even if material bodies exist, our minds, which are so profoundly different from material objects, which do not occupy space, and have none of the properties by means of which material objects act and react on one another, can be affected by such objects, can become aware of them. But, if we accept the commonsense view, and suppose that material bodies both exist and affect our minds, then, though we cannot conceive how the minds are thus affected, it seems to me that we have no alternative but to suppose that our minds are produced by our brains. As far as we know, our minds are nothing other than streams of feelings, states of consciousness. Ex hypothesi, some of these feelings symbolize material objects. When we become aware of an object, that object causes-in the full sense of the word causes-that feeling to arise. But its action on the mind is not direct. It first produces a change in a sense-organ. This change produces in turn a change in a nerve, and that again a change in the brain. Thereupon we become aware of the object. It follows, that if material objects exist, and if our minds are arvare of them, then our brains producein the full meaning of the word produce-the feelings that symbolize them. And, if it be admitted that they produce these items in the stream of feelings, we have no grounds for denying that they produce any of the items, even the highest flights of thought.

604. When we know the conditions exactly, we are able to measure work in terms of the energy expended; for example, we are able to say that under certain conditions a certain expenditure of energy will raise a certain mass a certain distance. We are 
unable to measure feeling in this way-to say that a certain feeling arises in relation to a certain part of the brain when there is a certain expenditure of energy in the latter. Consequentlymerely because we cannot measure feeling in this way-it has been argued, in effect, that there is no connexion between the two. A vast amount of nonsense has been written on this basis. For example, we are told that the thoughts of a Shakespeare and those of an imbecile bear no relation to the amount of energy respectively expended by them. Here the excellence of the thinking, as excellence is estimated by us, is taken as a criterion, and it is assumed that the greater the excellence the greater should be the expenditure of energy. The assumption is obviously absurd-as absurd as if it were argued that, because good and bad railway engines give different results, therefore the expenditure of coal has nothing to do with their movements. Moreover, this hypothesis, that sensation and thought and the concurrent expenditure of energy, bear no proportion to one another, is contrary to the universally accepted belief that every mental change, every feeling, is correlated with a brain change; for it is not denied by any one that cerebral changes, like other physical events, result from, and are proportionate to, the expenditure. It is certain that feeling is unknown to us except in connexion with the work of nervous tissue. But, since the conditions under which the expenditure occurs are but little known, we cannot estimate the amount of it in terms of feeling; and, apparently, because our perceptive powers are limited, we are unable to conceive how the working of the brain gives origin to mind.

605. Of course, as just indicated, mind may be considered as comparable, not to the music that proceeds from the instrument, but to the player who plays on it. But this hypothesis of an independent agent seems, at least, unnecessary. We shall see immediately that there is convincing evidence that mind is a product of evolution. As a function of nervous tissue, we can conceive of it as coming gradually into being under the influence of natural selection. Or rather, we can conceive the brain as evolving and becoming capable of doing more and more varied work, just as we can conceive the hand as so evolving. But, if we think of mind as an independent player who operates on the brain, and so directs the activities of the body of which the brain is a part, how shall we account for the existence of this player? Is there a definite amount of mind in the universe as there is of substance and energy, and is he somehow evolved out of this fund? Is a 
certain amount of mind somehow captured and attached to each brain, human and brute? Or is he and others of his kind miraculously created out of nothing, when brains, including those of lower animals, come into being and begin to function? In any case the player, if he exists, lies, if anything, even farther outside the range of observation than even 'matter in itself.' We have no means of knowing anything about him. All we know quite certainly is that there is mind, and the mind tells us that it is associated with a brain, and with expenditure of energy. To us mind is a function of the brain.

606. The physical characters of animals develop in response to stimuli, in directions and within limits that have been closely fixed by evolution. On that account the body of every normal man is very like the body of every other normal individual of the same variety. Thus the lungs and brain of one person are very like the lungs and brain of any other. We attribute these likenesses to the fact of a common descent. But, in spite of this common descent, the mind of every man so develops as to be immensely different from the mind of every other man, past or present. For example, while my father acquired a knowledge of military science, my mind has grown in such a way that I know-more or less imperfectlythe art of medicine. My neighbour, the sailor, likes and dislikes one lot of people; I quite another lot. He is familiar with one set of scenes ; I with quite other scenes. If we suddenly exchanged bodies we should scarcely be conscious of the change till the mirror or the behaviour of other people revealed it; if we exchanged minds we should be as lunatics in one another's environments. Had I been captured as an infant by savages, and reared by them, I should now resemble my educators mentally much more nearly than my progenitors. For example, I should know only the things that they know-the scenes, the people, the aspirations. Obviously then intellectual characters cannot have been evolved in the same sense as physical characters. Thus, a knowledge of medicine can never have been evolved in my case in the same sense that brains have been evolved. How then does it happen that I possess a knowledge of medicine? It seems to me, as the only probable explanation, that mind is not a thing distinct from the brain, but only the work of the brain-just as manipulation is the work of the hand. Nature has not directly evolved immaterial minds any more than she has evolved manipulations. She has merely evolved brains and hands capable of doing work useful to the individual. The kind of brain she has evolved in the human 
species is particularly capable, within limits that vary with individuals, of learning to do infinitely varied and complex work, with a constantly increasing degree of facility and skill. For that reason my brain has done, not what the brains of my predecessors could not do, but what they did not do (e.g. the work which results in the knowledge of medicine). I believe, then, if we consider these two facts together, the facts that human brains are much alike, but human minds very different; and if, moreover, we think in terms of common sense and accept the theory of evolution, then we are forced to conclude as the only course open to us, that mind is the work, or rather part of the work, of the brain. 


\section{CHAPTER XX}

\section{REFLEX ACTION, INSTINCT, AND REASON}

Mind is associated with nervous tissue-It is adaptive-It is related to movement-The evolution of mind-Reflex and voluntary actions-Intelligence and reason-Memory-Traditional knowledge-The mental characteristics of antsThe instincts of man - Sucking - Crying - Weariness-Hunger-Thirst-Imitativeness-Sexual and parental love-The mutation theory-The importance of mental acquirements.

607.

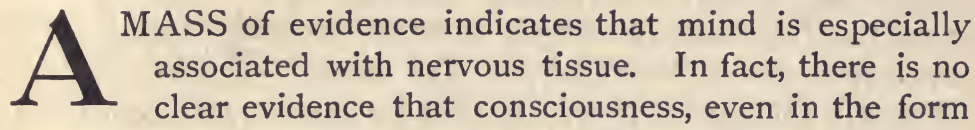
of the most rudimentary feeling, is anywhere present except in conjunction with those masses of nerve cells which are termed ganglia, the largest of which is the brain. Mind appears to be rudimentary, or even non-existent in animals in which ganglia are relatively little developed or absent. It is found in greater amplitude in the higher animals in which the ganglia are better developed. In the highest animals it appears associated exclusively with the brain. Moreover, mind seems invariably associated, not only with ganglia, but also with sense organs-eyes, ears, tactile, and taste organs, and the like-which are mostly situated at, or near, the surface of the body, and which receive from the external world and transmit to the ganglia messages (stimuli) derived from light, sound, contact, and the like-messages which in some way awaken an activity which is correlated to consciousness.

608. Mind is useful to the animal that possesses it only in so far as it controls his actions by initiating, guiding, or inhibiting them. If the animal is of a relatively simple type, living in a comparatively simple environment, his equipment of nervous tissue and mind is correspondingly simple. If, on the other hand, he is fitted for more complex surroundings, and if, therefore, his activities must be adapted to meet a number of more or less remote contingencies, to achieve more or less far off aims, his brain and mind display a corresponding complexity. Compare, for example, a sea-anemone, whose consciously directed movements, 
if any such occur in him, are adapted only to meet the needs of the moment, with a man, who, in early youth, may form a plan (e.g. the achievement of wealth), and pursue it undeviatingly in ways remote from the end during all the varied scenes of a long life.

609. Since mind is clearly adaptive, and since it increases in amplitude and complexity from the lower to the higher animals, it is apparently as much a product of evolution as the function of any physical organ, for example the hand. Certainly, we have as much reason to suppose it is such a product-to suppose that the functions of the brain have undergone evolution in the same sense, in the same way, and by the same means as the functions of the hand. It is, in fact, a 'character' (a reaction to stimulus) of the individual, which, as far as we are able to surmise, was, like other useful characters, absent in remote ancestors, had its origin in variations, and, passing through simple to more complex types, underwent evolution (as a function) by the action of Natural Selection. There is evidence that we are able by careful selection to increase or decrease in any desired direction the intelligence of our domesticated animals. In parasites and other animals which have forsaken an active existence, mind appears to have undergone retrogression through cessation of selection. Again, some animals, which lead an active existence early in the individual life, but which lapse into an inactive adult stage, seem to lose their minds just as they lose their larval organs. In brief, mind owes its existence to its utility.

6ro. Various authors have supposed that, "the beginnings of mental life date back from the beginnings of physical life. The question of the origin of mental development thus resolves itself into the question of the origin of life." 1 If they are right we are

1 Wundt, Principles of Physiological Psychology, Eng. Trans., vol. i. p. $3 \mathrm{I}$. Here Wundt is very unconvincing. We are told that "the beginnings of a differentiation of mental function can, however, be found even in the protozoa. .. The only sense that is plainly functioning is the sense of touch . . . the cilia with which these infusorians They function as organs of touch, and sometimes appear sensitive to light as well " (pp. 33-4). But reaction to stimulus does not necessarily imply sensation. The environment of infusorians is so simple, their reactions to it so few, that sensation superimposed on the reactions made by them would appear to be a useless epi-phenomenon. No sensations, as far as we are aware, accompany much more complex reactions in our own bodies, for example the normal movements of our intestines. "In the compound organisms we observe a more radical differentiation of mental function and its bodily substrate. The cellmass of the yolk, originally homogeneous, divides first of all into a peripheral and a central layer of different structural character, while the cleavage cavity gradually 
guilty of great cruelty when we boil our vegetables alive. Other writers, influenced by the uniqueness of mind, attribute some sort of mentality even to lifeless objects. Judged, however, from the standpoint of evolution, there does not appear to be any warrant for these fanciful speculations. As we shall see, the function of mind is adaptation. It is difficult to believe that a thing so clearly useful, so evidently bearing the marks of evolution, can have existed prior to its usefulness. If there was any unique

widens out to form the future body cavity. At this stage sensation and movement appear to reside exclusively in the outer cell-layer, the ectoderm, while the nutritive functions are discharged by the inner layer or entoderm." Here, then, according to Wundt is a phenomenon hitherto non-existent in naturei.e., living cells devoid of sensation which nevertheless perform perfectly well their complex function of elaborating nutriment for themselves and other cells. Loss of sensation is attributed to evolution. "At a higher level of evolution a third layer of cells, the mesoderm, forms between the two. This discrimination of organs is accompanied by differentiation of the elementary constituents of the tissues. When the separation of ectoderm and entoderm is first accomplished the cells of the former discharge the combined functions of sensation and movement. The initial step towards the separation of these two cardinal functions is apparently taken in the hydridæ and medusæ, where the ectoderm cells send out contractile precesses into the interior of the body. The sensory and motor functions are still united in a single cell, but are distributed over different portions of it. In the next stage the properties of sensation and contractibility pass to special and spatially separated cells" (p. 36). At this stage, then, the stage reached by the higher animals, Wundt supposes that sensation has departed from the contractile cells. Nevertheless they contract on receipt of stimulus precisely as they did before its departure. But the only evidence of sensation they ever afforded was contraction. That evidence still remains and is as inconclusive in the one instance as in the other. If contact alone caused an infusorian to contract efficiently, the sense of touch would be as useless to it as to a muscle cell. Before we attribute sensation to such low animals we should at least feel sure that this wonderful product of evolution is so useful to them as to be an important factor in securing survival ; that is we should feel sure that touch alone without the sense of touch is not sufficient for their needs. The functions of life, concentrated in a single cell in the case of the unicellular organism, are distributed amongst skin, nerve, muscle, and other cells of higher types. But this separation of function does not involve the corollary that no new functions (e.g. sensation) have been evolved. Touch is a discriminating agency; but discrimination does not necessarily imply a mental element. Thus, apparently unaided by sensation, gland cells discriminate between the substances conveyed to them by the blood. It follows that an infusorian may well display tactual discrimination and yet feel no sensation. Besides infusorians, many cells have cilia. In multicellular organisms " all the cilia of adjoining cells do not move at once, but in regular succession, the movement travelling from one cell to the other, but how this coordination is brought about we do not know. At least, it is independent of the nervous system, as ciliary movement goes on in isolated cells (i.e. cells that have been scraped away) and in men it has been observed two days after death." (Landois and Stirling's Physiology, vol. ii., p. 6r3). Here the cell, which is admittedly devoid of sensation and is cut off from nerve stimuli, behaves exactly like an infusorian which has been stimulated by touch. 
thing in nature out of which it was evolved, a thing outside the range of our perceptive powers, then that thing was not mind in any real sense any more than nitrogen and other chemical elements are muscle.

6II. Granting that mind is a product of evolution, that it originated in connection with movement, and that it occurs only in connection with nervous tissue, to me it seems probable that the order of evolution was as follows. First, through Natural Selection, cells, the function of which was contraction and without which movement would have been impossible, appeared in multicellular organisms. Many unicellular organisms are contractile, and some have contractile flagella. Doubtless the contractility of the cells of the higher types was derived from them. Next, as animals became larger and more complex, as other cells underwent differentiation, as the masses of the cells which were contractile increased in size, number, and complexity, a nervous system was evolved, which consisted of lines of communication (nerve fibrils) and one or more central ganglia. The ganglia received stimuli through the 'afferent' fibrils from the surface, and translated them into other stimuli which passed outwards through the 'efferent' fibrils and awakened the activities of various other cells, especially the contractile cells. The function, therefore, of nervous cells was to co-ordinate the reactions of the organism and so adapt it to the environment. Next there were evolved on the surface, at the ends of the nerve fibrils, organs which delicately discriminated between different classes of stimuli-light, heat, contact with foreign bodies, and the like. Nerve currents, passing from these surface organs, were transmuted in the ganglia into appropriate stimuli for the several masses of contractile and other cells. As yet there was no consciousness.

6I 2. Next, the surface organs which received stimuli from the environment evolved into sense organs, where originated nerve currents that gave origin in the ganglia to sensations-delicately discriminating stimuli which indirectly (through the efferent nerve currents to which they gave rise) exploded the right stores of energy latent in this or that mass of contractile cells. Throughout the process was one of increasing adaptation to the environment. Thus mind dawned. It will be noted that here I attempt nothing more than a description of the conditions under which, as I suppose, mind appeared. I indicate when, not how, it appeared. I do not know how nervous tissue does this work. I only suppose that it was rendered possible to nervous tissue through the selection 
of favourable variations. Lastly, through the continued action of Natural Selection, sensations took on those peculiar tones which we designate as pleasure and pain. Thereupon desire and will came into being. The animal was now no longer an automaton in the ordinary sense of the word. It felt, and in a real sense desired; and some of its activities were under the control of its desires, or rather of the will which the desires awakened. The whole process, I suppose, involved a very gradual co-ordinated change in which other cells besides nerve cells participated. There was no mutation, no sudden appearance of nervous tissue or of mind; for such a sudden development could have been useful only if it had involved simultaneous changes in several classes of cells-changes so numerous, complex, and adaptive, that their simultaneous occurrence would have been altogether miraculous.

6I3. All the movements of living beings, which result from their vital activities but are not under nervous control, may be termed ' protoplasmic.' The movements of plants and unicellular animals and ciliary movements are examples. Movements made under nervous control are either reflex or voluntary. All movements under nervous control, but with which no feeling is associated, are reflex. There are many such in our bodies, for example the normal movements of our heart and intestines. But some movements, which are rightly termed reflex, have a great deal of feeling associated with them. Sneezing and the convulsions resulting from tickling are examples. Here, not only does feeling accompany the action, but it initiates it. Neither sneezing nor the convulsions of tickling occur in the absence of feeling; for example, in people under the influence of chloroform. If I cough because I choose, the action is voluntary; if I cough because I must-because an irritation in the larynx compels me to coughthe action is involuntary. But often I can control the reflex, and delay, and in some cases even prevent it. A reflex action, therefore, is not necessarily one which is not associated with sensation, nor one which is not initiated by sensation, nor one which is not controllable by the will. It is one which is under nervous control but which is not directly INITIATED by the will. This definition is unlike those commonly accepted, but I think it can be justified.

6I4. The same action may be voluntary at one time and reflex at another. If the reader will think of all the reflexes he can call to mind, he will find that their distinguishing peculiarity is that they cannot be set agoing by the direct impulse of the will. Thus no matter how strong his willing he cannot really sneeze when he 
wishes. It is true he may set himself sneezing by voluntarily introducing an irritant into his nose; but the irritant, not the will, is the direct cause of the act. So also he is able to make himself sick by tickling the back of his throat, but he cannot vomit by a pure act of will. Swallowing is another example, and a very good one. The act of swallowing seems at first strictly voluntary. Really it is purely reflex. We are able to swallow only when we have something to swallow; that is, only when the mechanical stimulus which starts the reflex is present at the back of the throat. The reader can easily test this by swallowing his saliva and then trying to repeat the act. He will succeed only after he has collected more saliva-supplied more mechanical stimulus.

6I5. The reflexes are all 'inborn and transmissible,' that is they all develop in the individual under the stimulus of nutriment, and reappear in all normal offspring. With rare exceptions, they are extremely useful to him, indeed essential to his survival. For example, no child lives who does not inherit the cardiac and intestinal reflexes which were possessed by his ancestors. The exceptions are one or two reflexes which seem to be mere byproducts of evolution-traits correlated to more useful traits. The convulsions of tickling are examples. When feeling is associated with the adaptive reflexes it always contributes to their usefulness. In some cases (e.g. the feeling caused by food in the larynx, which in turn causes swallowing) feeling is the discriminating spark which awakens the reflex. In other cases, by promise of pleasure or relief from pain, it awakens desire, which, as in the case of hunger, awakens the will to perform those voluntary actions which necessarily precede and supply the stimulus for the reflex act. In some instances, as in the case of the reflexes of the bladder and lower bowel, the will acts usefully, not because, as in the case of swallowing, it initiates the performance of voluntary actions which in turn supply stimuli for reflexes, but because for a longer or shorter time it controls or inhibits the reflexes-because it is able to delay their performance till a more convenient time. Such reflexes are always initiated by a feeling which tends to awaken, not only the reflex, but also the will that inhibits it. The duration and amount of the control which the will is capable of exercising is always proportionate to its usefulness. Thus it is short and slight in the case of sneezing-as a rule no longer than gives time to swallow food already in the mouth. It is a little longer in the case of breathing. In the case of coughing it is short in proportion to the severity of the irritation. On the other hand the control is 
more persistent and more perfect in the case of bladder and bowel. But always the reflex impulse tends to grow stronger, till at last it overpowers the will. No adaptive reflex which is not associated with feeling (usually pleasure or pain) is under the control of the will. Thus we cannot voluntarily control the beatings of our hearts nor the movements of the middle portions of our alimentary canals. All adaptive reflexes which are normally associated with feeling are directly or indirectly under the control of will. Thus, as we see, the bladder reflex which promises relief from pain is directly controlled by the will, whereas the swallowing reflex which promises pleasure is indirectly controlled by it, for the will provides those voluntary actions, the provision of food, the chewing and removing of it to the pharynx, which in turn awaken the reflex impulse to swallow.

6i6. We see then how clearly the reflexes are adaptive, how plainly they bear the marks of evolution. On the other hand, we see how complex they are both physically and mentally; and yet how perfectly they link up with the rest of the physical and mental activities of the individual. Every complex animal has these wonderful reflexes. Except through actual miracle such manifold co-ordinated activities could not have arisen by mutation. Unless, therefore, we attribute them directly to a supernatural origin, we must assume that they arose gradually by the continual selection of fluctuating variations-by a process which gradually and concurrently altered the whole organism. Here also we have an example, emphasised as we shall see by the whole study of mind, of facts which are familiar to everyone, which nevertheless since their relations are complex and difficult to trace, cannot be classified except after careful thought. 'Obvious inferences,' such as are all that are needed in anatomy and that kind of botany and zoology which takes no account of the sequences of events in the evolution of the race, here lead, and have repeatedly led, only to confusion and error.

6I7. Voluntary acts are either instinctive or (in the lack of a better term) rational. As far as I am aware, no one but myself regards instinctive actions as voluntary. Usually they are classed as a kind of reflex. However, I feel confident I am right. A voluntary act is one which is prompted by the will. If we think carefully we find that the will, in turn, is always prompted by desire; and that desire again is always prompted by present or prospective pleasure or pain. The desire may be so faint that we can hardly detect it ; but unless it is present we do not, we cannot, 
act voluntarily. It may be so strong as to be irresistible, in which case it enlists a corresponding strength of will. Desire cannot be awakened except by present or prospective pleasure or painnot necessarily 'physical ' pleasure or pain, but pleasure or pain of some sort-for example a whimsical pleasure in demonstrating that we are able to disregard 'physical' pain, in which case the desire to achieve the pleasure transcends the desire to avoid the pain.

618. An instinct may be defined as an 'innate and inherited' mental impulse or inclination to do a certain definite act, the instinctive act, on receipt of a certain definite stimulus. Therefore it is a mental character which is developed in the individual under the stimulus of nutriment, but which is awakened to activity by experience (the appropriate stimulus). For example, the sexual instinct is an 'innate' character that is aroused by the presence of an individual of the opposite sex. I think psychologists believe instinctive actions to be types of reflex action only because, when studying the question, they have limited their attention mainly to the instincts of the lower animals. We have then, very often, no means of knowing whether any given action is, or is not, prompted by desire and directed by the will. But when we consider our own instincts we are on safer ground. Our knowledge is not then derived by inference from the actions of another animal, but from actual experience of the mental phenomena. We know our own instincts therefore with an intimacy with which we cannot know those of the lower animals, amongst which all our own instincts have their counterparts. Consequently it is safer to reason from our instincts to those of lower animals than to adopt the reverse process. Since we are derived from lower types, presumably, our instincts are of the same nature. They may differ in degree, they can hardly differ in kind.

6I9. Consider, then, our own instincts, for example the instinct to sleep periodically, to rest when tired, to sport when rested, to eat and drink when hungry and thirsty, the instincts of imitativeness and curiosity, and the deferred instincts of sexual and parental love. In the case of each we feel strongly, if sometimes vaguely, the prompting desire to seek pleasure or to avoid pain which is the actual and direct stimulus of the will to do, which, in turn initiates the instinctive act, and which therefore sharply differentiates it from the reflexes which are never directly prompted by the desire to do an action. Thus, sleepiness prompts us, strongly inclines us, voluntarily to assume attitudes of repose, as 
also does weariness. Clearly it is the presence of the desire that renders the act voluntary. Every instinctive impulse is nothing other than a desire, even when the object of desire is not easily recognised. Were the desire not present we should not act. Hunger inclines us to procure and chew our food; both actions are distinctly voluntary. Imitativeness, curiosity, sexual, and parental love, also prompt us to acts which are entirely voluntary. It is true that sometimes, when the prompting desire is very strong, we are colloquially said to act 'against our wills.' As a fact, however, except in the case of the reflexes, we never do act against them, though we often act against our judgments. Wise and good actions are certainly all voluntary; but, equally certainly, not all voluntary actions are either wise or good. When the starving sailor kills and eats his comrade he may abhor and struggle against his desire. Nevertheless from the act of killing to the act of chewing his actions are voluntary. He performs them simply because his desire, his will to eat, is stronger than his will to abstain.

620. It must be borne in mind that, especially in the case of beings like men whose desires are complex, two or more impulses inciting to different actions may be in operation at the same time. When one is distinctly stronger than the other, we yield to it at once; when they are equally balanced, we have a feeling of irresolution. Suppose a man falls in love with his friend's wife, the feeling would be an instinct, and would impel to instinctive acts. Opposed to it might be a desire to do right. He will certainly yield to the stronger desire, and, in each case, his action will be voluntary. It is very probable that before taking one course or the other he will seek to reinforce his desire to do right by picturing all the consequences of doing wrong. But if he acts wrongly he will not be acting against all his desires, but only against one of them; not against his whole will, but only against the weaker incitement of it. No one is held blameworthy for his reflex actions. It is felt that he does not initiate them by his will, and that even when he is able to some extent voluntarily to control them, he must sooner or later yield. But for every kind of instinctive act he may be held blameworthy. Therefore it is recognised that they are initiated by his will. On that account they involve questions of right and wrong, meriting praise or blame.

621. Apart from his reflexes, what man ever acted except under the influence of desire? The anchorite abstains from 
pleasure only because he desires and anticipates greater pleasure in heaven. The patriot dies because his desire for his country's good overmasters his desire for life. The tigress that has captured prey goes hungry because her instinctive desire for food is opposed by a yet stronger desire to feed her cubs. If I deliberately burn myself, it is only because my desire to do so is stronger than my desire to avoid pain. In every case the action, whether instinctive or 'rational,' is prompted by desire, and, if prompted by desire (which awakens the will) is plainly voluntary, plainly different from those distinctly non-voluntary actions which we term reflex. Compare, for example, the actions to which hunger prompts with the peristaltic action of the stomach when hunger is satisfied. The former are instinctive, the latter are reflex. Wherein lies the distinction? Clearly in the circumstance that the instinctive actions are prompted by desire and initiated by the will, whereas the reflex actions are neither prompted by the one nor initiated by the other.

622. The term voluntary is sometimes limited to deliberate acts. Thus, if I avoid a danger by taking thought, I am quite rightly thought to act voluntarily. But, if I impulsively start away from it, my action is held by psychologists to be involuntary or even ' reflex.' But we shall see later that speed and absence of deliberation imply, not necessarily lack of will, but only a quick and smooth working of it, one principal condition of which is absence of opposing desires. When we are hungry and have only one dish, we set to work without hesitation. If half-a-dozen dishes are placed before us, we may deliberate before setting to. But our action in the one case is just as voluntary as in the other. Will is one thing, deliberation is quite another thing. All deliberate actions are certainly voluntary; but, quite certainly, not all voluntary actions are deliberate. When we are beset by two or more nearly evenly balanced desires, we hesitate, reflect, deliberate. But these mental processes are quite distinct from will. They may incline the scale in one direction or the other and so end the paralysis of will which results from balanced desires. But obviously they are no part of the will, nor even a necessary accompaniment of it.

623. Voluntary action then may be defined as action which is prompted by desire. An instinct, we see, is an 'innate' and 'inherited' inclination, an impulse, to act in a certain way under certain conditions. At a fixed stage in his development the caterpillar builds himself a cocoon. His dwelling is a wonderful 
structure, but from our human point of view, the most wonderful thing is that he does not learn to build it. He may never have seen a cocoon before, and he constructs only one in his life. Yet his work is perfect, or at least excellent, and it is as good at its beginnings as at its endings. Evidently he owes nothing to experience beyond the stimulus to act, but is impelled and guided throughout by that 'innate and inherited' faculty which we term instinct. Since his cocoon-building instinct does not appear at the beginning of conscious life, it is termed a deferred instinct. $\mathrm{He}$ has many such, for instance the instincts of flight and mating, which do not appear until after he has undergone his metamorphosis into a butterfly.

624. On the other hand, man, unlike the caterpillar, cannot build his house unless he first learns to build it. He depends, not on instinct, but on stored experience. The faculty by which experience is stored in the mind is termed memory. The faculty by which we use stored experience is termed intelligence. When the contents of memory are very vast, and the processes of thought by which they are utilised comparatively difficult and complex, intelligence is termed reason. INTELLIGENCE and REASON depend, therefore, on MEMORY, on ability to learn, on capacity to profit by stored experience. Memory is not the whole of intelligence, but it is the basis of it. Without memory, there could be sensation and emotion, but no thought; for the materials for thought would be lacking. Thinking depends on such processes as association, comparison, discrimination, inferring, and the like, which in turn depend on memory.

625. In a sense, the caterpillar has discrimination; thus, he can distinguish his food or his mate from other objects; but he has not the kind of discrimination which is so much used by man. His discrimination does not depend on a comparison of present things with things formerly known or imagined, but merely on differences of feeling awakened by different objects. He does that which he feels is pleasant, not that which he has learnt is pleasant. If a man had no memory he could compare no two objects or ideas. He could have no ideas. He could perceive men and women who might awaken emotions and impulses in him, but he would not know them for men and women, nor as big or small, dark or fair, near or far, nor would he know the ends of the actions to which he was impelled. Such thoughts, depending as they do on association and comparison, could not arise in his mind, which, at the end of a long life, since he had 
learned nothing, would still be as blank as that of a newly born infant.

626. We always measure the intelligence of an animal by its power of profiting by experience. Thus, a cat is more intelligent than a rabbit, because it can learn more; a dog, for the same reason, is still more intelligent. An animal with a purely instinctive mind, with 'innate and inherited' impulses to act in this or that way but no memory, can have no conception of its past, and therefore no idea of its future. It lives wholly in the immediate present, feeling but not thinking. It acts entirely on simple inclination, not on reflection. Like the caterpillar building his cocoon, it makes provision for the future, not with any idea of providing, but simply because stimulus from the environment causes it to react in a certain way, gives it an impulse to a certain course of action the performance of which bestows pleasure of the kind that a child derives from playing or eating, and with the ultimate result of which it is no more consciously concerned than a playing child. If a caterpillar sheltered in a hole with the idea, founded on experience, of avoiding danger, his action would be intelligent. If, appealing to a memory, in which a great number of complex experiences were stored, he took thought and designed himself a shelter in which provision was made for all sorts of remembered or imagined dangers, his action would be rational. But if, making no appeal to the past nor taking any thought for the future, he builds only because impelled by an 'innate' inclination, then, no matter how elaborate the edifice he rears, his action is instinctive.

627. In proportion as animals are low in the scale of life, they appear incapable of learning. Thus, memory seems rudimentary or absent in most insects. But often they are wonderfully equipped by instinct. To take, again, the example of the caterpillar: on emerging from the egg, driven by an innate inclination which is the instinct, the animal moves from place to place. It co-ordinates its muscles without practice perfectly at the first attempt. In other words, its movements are instinctive in a double sense; they are prompted by instinct and they are co-ordinated instinctively. The animal does not learn to do them. In the human being, on the other hand, even when the prompting is instinctive, the power of co-ordinating the movements for the performance of the instinctive act is for the most part slowly and laboriously acquired through experience. For example, maternal love is an instinct; but facility in all the movements to which the instinct prompts the mother is 'acquired.' 
628. Prompted by another 'inherited' 'inborn' impulse, the caterpillar seeks its proper food. Yet another instinct prompts it to inhabit such situations as afford concealment from its enemies. Next, at a certain stage of its growth, an instinct prompts it to provide shelter for its helpless pupal stage by spinning a cocoon or by some other device which has been followed invariably by its ancestors. As a butterfly, it is under the influence of an entirely different set of instincts. Inhabiting an altogether different environment, it moves instinctively by means of other organs, adopts new devices to escape its enemies, seeks and feeds in a different way on different food, pursues the female and mates with her. She, impelled by an instinct unfelt by the male, deposits her eggs in one of the few spots in the vast and complex world in which she moves in which her offspring will have a chance of surviving.

629. The caterpillar has sense organs and a nervous system; we have every reason to believe that it feels. But there is little or no evidence that it remembers or thinks. Memory would be little use to it; therefore parsimonious nature bestows little or none. Cast adrift in a hostile world, it must come into existence ready armed for the battle of life. The necessity of following, by its own unaided efforts, the right line of action is as pressing at the beginning of life as subsequently. It has no time to learn, and during the rapid and strange changes in its career little opportunity of acquiring knowledge which would beneficially affect its future. Many of its most important actions, such as cocoon building and mating, are done only once. Since memory and its corollaries, comparison, discrimination, idealism, imagination, reflection, intelligence, reason, and all that they imply are most developed in the higher animals and are imperceptible lower in the scale, since they gradually increase in number, range, and utility, they are clearly later and higher products of evolution than instinct. Memory, itself, the faculty by means of which we learn, arises under the stimulus of nutriment. Therefore it is 'inborn.' But all that is stored in the memory, all the mental growth which arises through its use and the use of its contents is 'acquired.' Therefore intelligence and reason are 'acquirements.' A man who has no memory, whose mind is blank of all that memory supplies, and who, consequently, cannot compare, discriminate, or perform any of the operations which the possession of a stored memory would enable him to accomplish, is not intelligent. ${ }^{1}$ The only mental operations possible to him are sensations and instinc- 
tive emotions. But, though intelligence is altogether an acquirement, I think no one will deny that we speak correctly when we say that it has undergone evolution in the higher animals. Like many instincts and physical characters, it arises late in the development of the individual (for it arose late in the evolution of the species), but it is none the less as essential a part of his mental growth as any trait which arises under the stimulus of nutriment. In fact, though students of the subject have never realised that intelligence is an acquirement, yet it has been universally recognised that the mental evolution of the higher animals has consisted mainly in the evolution of intelligence and reason-that is, as we see when we think closely, of the evolution of memory and of the mental growth which the possession of memory renders possible.

630. Family life, which is practised by all the higher animals, is not only a product of memory but is also that which has rendered possible the evolution of a voluminous memory. The offspring are re-cognized by the mother, and in the case of some animals by the father who then recognises his mate also. This recognition implies some degree of memory and consequently intelligence. The young, instead of being cast adrift at birth to fend for themselves, are watched and protected, and, by the highest animals, taught. Time and opportunity are thus afforded for learning about the world, and, more particularly, of acquiring the traditions, the stored experiences of the race. Animals which have no family life, for example most fish, amphibians (e.g. frogs), and reptiles, may possess a memory, but it is always very rudimentary. They may learn a little from experience, but only a very little; their intelligence is very low, their thoughts must be few and simple. The recognition of offspring by parents is usually, but erroneously assumed to be instinctive. It is certainly acquired through experience after the birth. But once the child is known, the more intelligent the animal the more perfect is the subsequent recognition. The hen will adopt strange eggs and chickens, and even ducklings and pea chicks. The ewe, the cow, and the human mother may have strange offspring foisted on them immediately after parturition, but they are not easily deluded after they have performed the first offices of tenderness.

631. With the opportunity which parental protection affords to the young to profit by experience comes the ability to profit by it, and with the latter a proportionate retrogression of now useless instincts. Intelligence is substituted for unthinking impulse. All the instincts are not lost, but in the higher animals we find no such 
elaborate impulses as in the lower. Such a being as the caterpillar is able to fend for itself from the first, but just in proportion as animals are intelligent they are helpless at the beginnings of consciousness, and correspondingly capable later. A young pig can run as soon as it is born, but the acquirements of the 'learned' pig are small compared to those of a dog which is more helpless at birth but so teachable, so capable of learning, that it becomes the companion of man. All our domesticated animals, except such small and harmless types as silk-worms, are teachable, and for that reason we are able to tame them. A tiger or a leopard cannot easily be domesticated because instinct forms too large and memory too small a proportion of its total mentality. Domestication, in the sense meant, implies intelligence, and therefore memory.

632. Of living beings man is by far the most helpless at birth. He cannot even seek the breast. In him instinct is at its minimum and memory at its maximum. For him, more than for any other animal, is prolonged and elaborate tuition necessary. But, so vast is his memory and so great his power of utilising experience stored in it, of growing mentally under the stimulus of use, that in later life he is beyond comparison the most capable of the inhabitants of the earth. Compare what even a dull man knows, including the words of a language with their inflections and articulations, with what is acquired by the cleverest dog or monkey and the immensity of the difference is at once apparent. We may take a frog and rear him solitary from the egg in an aquarium. If, when he is adult, we remove him to a pond, he will take his place with his fellows at once. He has little if anything to learn. Instinctively he 'knows' his food and how to seek it, his enemies and how to avoid them, his mate and how to deal with her. But how forlorn and helpless would be a man reared from infancy out of sight and sound of his kind and then turned into a world where his experienced fellows struggled for existence.

633. Traditional knowledge-that is knowledge imparted by one generation to the next-is common enough amongst the higher animals and forms no inconsiderable part of their mental equipment. Thus we may see the hen, impelled by an instinct, teaching her chickens, as she herself was taught, to seek food, and the cat teaching her kittens how to ambush mice. The young animals, on the other hand, discover an instinctive interest and pleasure in copying their elders. While insects and others of the lower type learn nothing from the presence of man, birds and mammals learn much. When inhabiting desert islands they have 
none of that fear of him which in our country they learn from dire experience. We have a saying, "as wild as a hawk"; but Darwin relates how he almost pushed a hawk from its perch with the muzzle of his gun in the Galapagos islands. Sea-birds round our coasts, where they are molested, are exceedingly shy; at London Bridge they feed from the hand. Formerly Arctic seals, impelled by fear of polar bears, inhabited the outer margin of the ice floes ; now they have retreated from the more dangerous neighbourhood of man to the landward edge. Antarctic seals, harried by the great carnivora of the ocean, are watchful in the water; on land or the surface of the ice, where, till lately, they met no danger, they may be slaughtered like sheep in the shambles. They are capable of profiting by experience, but they are slow to learn, and can acquire but little. Judged by our human standard they are very stupid. The means of escape adopted by Arctic seals compared with the means of capturing them, the ships, guns, and the rest, afford a measure of the intellectual difference.

634. When animals are social and so have the opportunity of learning, not only from their parents but also from other members of the species, the power of making mental acquirements and consequently of handing on the traditions of the race is often correspondingly great. It reaches a remarkable degree of development even amongst insects, some species of which live in great communities. Young ants are carefully tended and are said to receive instruction from their elders. A capacity to profit by experience implies, of course, memory. There is, however, more decisive evidence. Some species of ants have the habit of capturing the pupæ of other species and of so training the young individuals that develop from them that they peform duties which were quite unknown to their ancestors. Therefore it is clear that the slaves profit by experience. Consequently their actions are intelligent not instinctive.

635. So important are the duties of the slaves, so much do they learn, so long has the habit of slave-making been followed by their masters, that in some species the latter have evolved into mere fighting machines, incapable even of feeding themselves. Many important physical and mental characters have undergone retrogression in them-not through lack of use, for doubtless the animals used while they possessed them, nor even through lack of utility, for powers of self-feeding must have always been useful, but solely through lack of sufficient survival value. That is to say, the parts, however much used and useful, underwent retrogression because the acquirements of the slaves supplied a 
substitute so efficient that individuals (or rather the communities) that possessed them in higher degrees did not survive in greater numbers than those that possessed them in lower degrees. Since ants possess such high powers of making mental acquirements, it is only reasonable to suppose that their instincts, like those of man and like many of the mental and physical parts of the slave-holders, have undergone retrogression. Whence it follows that many of their so-called instincts-for example the habit of warring in disciplined hosts, of keeping pets, of preserving aphides for the sake of their sweet secretions, of clearing areas of ground, growing food plants on them, and storing the seed, of nipping the rootlets of seed to prevent germination, and so forth-are quite possibly, not instincts but items of traditional knowledge and habits entirely comparable to that traditional knowledge, to those traditional mental attitudes, those ways of thinking and acting, which play so large a part in human lives.

636. The instincts of man, though comparatively few and simple and though overshadowed by the enormous mass of his acquired mental characters, by his intelligence, are yet essential to his existence. Some of his actions are wholly instinctive, for not only are the inclinations to perform them innate, but also the ability to do so. The act of sucking in a young infant is an example. This action is often termed a reflex. But it is entirely voluntary in the adult. No man ever sucks unless he chooses to suck. If, then, it is reflex in the infant, it must altogether change its character later which no other reflex is known to do. Thus, though we may learn to breathe and cough, yet we continue to do both because we must. Sucking, on the other hand, is always voluntary in the sense that it is invariably prompted by an emotion, a desire, and that it is initiated by the will. Doubtless the new born infant does not consciously desire milk (of which lit has no previous experience) when it sucks, any more than a child desires bodily development when it plays. But when the emotion is awakened by the mother's nipple the act itself is delightful and is instinctively performed as long as the delight continues-that is while the milk flows and the hunger is unsatisfied. Were there no prompting emotion, no delight, the act would not be performed. Crying, an act prompted by discomfort or pain, is also entirely instinctive at first. The function of the cry is an appeal for aid; but here again the infant has no notion of its real meaning. It cries only because it is impelled by a certain emotion, the instinct, to a certain act, the instinctive act. Yet again when tired or 
sleepy, we instinctively, without learning, place ourselves in an attitude of rest. Doubtless we learn in time what attitudes are most comfortable, but no learning is needed to secure cessation of movement and relaxation of all our voluntary muscles and the consequent assumption of a restful position.

637. Hunger and thirst are terms which are applied both to the instinctive desires to take food and water and to the antecedent sensations which awaken them. Thus, when we say we are hungry we may mean either the feeling of discomfort in the region of the stomach, or the wish for food, or both. The recognition of food as such by the sense of taste is also instinctive. Normally we eat and drink only nutritive substances, those which contain food or water, with pleasure. Probably, however, this instinct is more perfect in the lower animals than in man in whom it seems to have undergone some retrogression. The infant, protected as it is by its mother's intelligent care, tends to swallow any small object that comes to hand; the caterpillar and the puppy, which is aided by its sense of smell, are rarely in fault. In all animals the amount of food desired is limited by the needs of the individual, though even in this case the instinct appears more perfect in the lower animals than in man. Instinctive also are those voluntary movements of tongue and jaws which occur when food or water is placed in the mouth. Unlike the caterpillar, however, man must learn all those actions by means of which he procures food.

638. The impulse to play is an instinct, but it prompts to no action for which our muscles are instinctively co-ordinated. Its sole function is to impel us to make physical and mental acquirements. Under its influence we use our bodies and minds which develop in response to the stimulus thus supplied till we are fitted to the ancestral environment. We have already dealt at length with physical use-acquirements. ${ }^{1}$ The mental acquirements resulting from play are strictly homologous and not less important. They enable us to co-ordinate our muscles for purposeful action and so perform our voluntary actions as swiftly and surely as reflexes. The vague movements of the infant become purposeful if awkward; his awkward movements become easy and sure; and these easy movements at length become 'automatic.' Only those animals sport which make physical and mental acquirements, and they sport only when play is useful, and in a way that is useful. The caterpillar does not sport; he has no physical and

1 See chapter $\mathrm{i}$. 
mental 'acquirements' to make; his actions are all entirely business-like. It is true that gnats and other insects indulge at certain seasons in dances which appear to us sportive; but there is every reason to believe that they are impelled, not by the playing, but by the sexual instinct. On the other hand, young ants are said to be sportive. The young of the higher animals are playful in proportion to the extent to which their bodies and minds are capable of development under the stimulus of use. In each instance the sport is exactly adapted to fit the individual for the future business of life. The little girl 'naturally' turns to her doll, which she nurses as she will her baby. The play of the boy as naturally involves contests which foreshadow the grimmer struggles of adult life. As he grows older the character of his sport changes. More and more it becomes an affair of wit and dexterity, an appeal to wider experience, and a means of adding to it. When the girl's play with her doll and the sports of the little boy have served their purpose and ceased to be useful, they cease also to excite interest. Compare the ambush and pounce of the kitten, the ardent chase and overthrowing of the puppy, and the climbing proclivities of the monkey and the kid. When full physical and mental development is attained, the desire for sport wanes. It lingers longest in the most intelligent animals, those whose memories are the most capacious, who are most capable of profiting from experience. Little remains of the instinct in the adult cat or horse; the dog retains it longer; while man, who is capable of learning even in old age, retains it in some measure to the end. Hence, for example, his pleasure in billiards and cards.

639. Imitativeness and curiosity are instincts which impel the young individual to store his mind with useful traits. Like sportiveness, they are developed in every species in proportion to the power of making mental acquirements, and they persist in the individual only as long as they are useful. Imitativeness impels him to copy his progenitors, and so make for himself those acquirements which enabled them to exist and rear offspring in their world. The kitten, the puppy, and the monkey may be seen observing with interest and subsequently imitating their elders. The most imitative of all living beings is a child, who from the time he copies his elders in walking, speaking, and manipulative dexterity, to the time when he acquires his ultimate political and religious notions, is constantly under the influence of this instinct. A large part of a little child's sport consists in pretending to be such and such a person, or to do such and such a 
thing. All acting is imitating, and much of a child's play is acting. Fashion is nothing other than a manifestation of imitativeness; the infectious fury of mobs, and the steadfast purpose of men in armies, is little more. Human society, which could not exist unless the units composing it were much alike, is founded on it. It is the principal means by which the traditions of the race are transmitted to remote generations.

640. The function of curiosity is obvious. Like imitativeness, it impels to the acquirement of useful mental characters. Almost as strong in the adult as in the child, it persists in men throughout life. It is alike conspicuous in the aged gossip and in the man of science.

641. Sexual love is an instinct which prompts to actions which, like swimming, are instinctive in the lower animals, but which man acquires the ability to perform. At any rate, the ability to perform all the actions which lead up to the sexual act are acquired by him. The instinct is better developed, as a rule, in men, who play an active part, than in women, who play a passive part. No man could have offspring unless he had the instinct; but women, who in past times have often been the helpless slaves of their masters, may have offspring in the absence of sexual inclination.

642. Parental love is an instinct which impels to the care of offspring; but none of the actions prompted by it are instinctively performed by human beings. The lower animals are innately capable of tending their young; but the human mother must not only learn during her own infancy to co-ordinate her muscles for the tending of a child, but she must learn from other mothers how to do it. However, some innate maternal impulses, which prompt to actions that need no special learning, survive. Thus almost every human mother delights in teaching her child to walk and speak, characters which are essential to its well-being. ${ }^{1}$ Very beautiful and illuminating is the pleasurable participation of even dull and care-worn women in the play of their babies and very young children. Parental love is more highly developed in women than in men. In " the state of nature" in which the race evolved,

1 Apart from the parental instinct, the pleasure we feel in imparting information seems as instinctive as the pleasure felt in receiving it. Every normally constituted individual delights to awaken interest in this way. To-day, for example, we bought my little boy, for use in school, a pair of drawing compasses, a protractor, and a set square. It is many years since I handled these instruments, but I remembered some of their uses, and I am sure the child was not more eager and pleased in learning than I was in teaching. The utility of both the imparting and the receiving instincts are very obvious. 
a child might survive its father's, but not its mother's neglect. The mother who lacked parental instinct left few offspring behind her. Complementary to the mother's instinct to tend her offspring is the latter's delight in and acceptance of her care. For example, the cat's instinct to carry her kitten in a particular way (by the skin of the neck) is associated with the passive attitude of the kitten-in all except the tail, which is tucked out of harm's way-when it is so carried.

643. To sum up, the caterpillar, and all animals at a similar stage of evolution, are mentally a mere bundle of sensations and instincts. The latter, which lie latent till they are awakened in due season by the former, develop under the influence of nutriment, and are nothing other than emotions or desires which impel to the performance of certain definite actions, the instinctive actions, the capacity to perform which is also innate. On the other hand, man has; not only sensations and instincts, but a memory. $\mathrm{He}$ develops mentally, not only under the stimulus of nutriment, but also under that of experience (use). His power of feeling sensations and instinctive impulses is innate. His memory, his power of making mental acquirements under the stimulus of experience, is also innate. But all that is stored in his memory, all that comes to it through the sensations and emotions he feels and the conscious and unconscious inferences he draws from them is acquired. Mentally, therefore, he is a bundle of (I) sensations ; (2) instincts, which in him are, in most cases, merely incitements to make acquirements; (3) capacities for making acquirements (i.e. memory); and (4) actual acquirements. Since the caterpillar feels, he has a mind. Man not only feels, but thinks. He has not only sensations and instincts, but, in addition, an intellect. The evolution of mind is beautifully recapitulated by the stages of the mother's care for her offspring. Up to the time of birth, the care is entirely reflex. After birth, it is instinctive in the higher animals. In still higher animals, intelligence steps in, and both aids, and to some extent replaces, instinct. Thus it is intelligence, not instinct, that impels us to send our children to school.

644. It is not possible, of course, to indicate precisely the stage of evolution (i.e. the kind of animal) in which sensations first appeared as sparks to explode reflex action, and in which, therefore, mind dawned. Nor is it possible to indicate when sensations first took on tones of pleasure and pain, when desire and will awoke, and instinct and voluntary action appeared. Nor again can we indicate when memory began, and brought in its train as it evolved 
thought and intellect, intelligence and reason. But the fact remains that all these faculties are products of evolution, means by which individuals and races are adapted to their environments. They all arose from small beginnings, and increased gradually with the needs of the species as its relations with the environment grew more and more complex. Sensation followed reflex action, instinct followed sensation, memory followed instinct, and intelligence memory.

645. The mental traits-sensations, desires, instincts, memory, intelligence-fit in exquisitely with the physical traits-skin, bone, blood-vessel, nerves, reflexes, and all the rest. In mind and body the living animal is an immensely complex and wonderfully adjusted machine-a machine vastly more complex and perfectly adjusted than anything that has ever been invented by man as the work of his hands and brain. A single important adaptive change in this machine cannot occur separately. It must involve a thousand or ten thousand readjustments, a thousand correspondingly important changes in mind and body. Thinking of all this, we realize the full force of the statement that the organism is a bundle of adaptations and co-adaptions. We realize also the full force of the main objection to the mutation theory. It is possible to accept that theory if we think only of such things as the colours and shapes of leaves, or other changes which do not necessarily ruin co-adaptation. But when we take the whole of the facts into consideration, when we think of the complex way in which the mental and physical adjustments of the higher animals interlock and how fine the adjustment is, we are driven to the conclusion that evolution can have been founded only on the continual selection and blending of multitudes of small variations, by means of which all co-adapted parts underwent change together, and the organism was moulded as a whole. In other words, we are forced to believe that nature selected for survival, not individuals who varied widely from the rest of the species in this or that particular, but individuals who varied comparatively little as regards the size of their variations, but much as regards the number of their variations, and who, therefore, were better adapted on the whole to the gradually changing environment than their fellows that perished; and who blended their variations, so that it was not so much the successful appeal of the individual that counted in the racial struggle for persistence, but the experience of the entire species or variety. Indeed, notwithstanding the wealth of material furnished by fluctuations, so vast is the complexity of the higher 
organisms, and so delicate the adjustment of their parts and faculties, that many thinkers have been driven, in spite of the fact that there is apparently no alternative, to doubt whether Natural Selection is adequate for the task. Our faith is restored only when we contemplate the special and beautiful devices which Natural Selection has evolved to help its work of adaptation and coadaptation - on the one hand, bi-parental reproduction and the tendency of retrogressive variations to preponderate over progressive variations, both of which automatically plane away all redundancies; and, on the other, the power of developing under the stimulus of use which brings about co-adaptation in the characters of the individual through the influence which related parts and faculties exercise over one another's growth.

646. Again we have seen how much neglected has been the study of 'acquirements' ; it has even been supposed that evolution is founded entirely on 'inborn' traits. But consider what man would be without his mental acquirements. The congenital idiot and the normal man start life equal, except in one particular. The idiot has little or no memory, no power of learning, no power of growing mentally under the stimulus of experience, whereas the normal man has that power. This, at birth, is the sole difference between the two. ${ }^{1}$ But an empty memory, a mere possibility of learning, is nothing. If the normal man had not filled his memory, he also would be an idiot. Now is, or is not, the intelligence of the normal man, which has arisen only because he, personally, has made acquirements, a product of evolution? Is not this mental growth, which consists entirely in acquirements, and which separates him mentally from such animals as the idiot and the caterpillar, as much a part of his normal growth as his head or his instincts? If it is, then surely, the study of acquirements, including the entire field of the intellect, is at least as well worth the attention of the student of nature as the study of any other class of character.

647. It. may be argued that 'the task of the biologist is complete when he has traced the growth of memory, which is all that is inborn, and, therefore, all that has undergone evolution.' But to this there are two sufficient answers. First, no serious attempt has ever been made to trace the evolution of memory. Though it is the foundation of all intelligence, it has, of all mental characters, been the most neglected by biologists in general and psychologists in particular. Indeed to this day there are some

1 See $\$ \$ 762$, et seq. 
who suppose that memory (like extension in space) is a property of all living protoplasm, and who attribute it, therefore, even to the protozoa and to plants. Others, declaring that without memory there can be no feeling, place the beginnings of the faculty at the beginnings of conscious life. Second, if we regard the power of growing mentally under the stimulus of experience as the essential thing, and the intelligence and reason which result from the exercise of this power as accidents, even then, if we are consistent, we must regard all the characters of living beings, including memory, as accidents ; for, in exactly the same sense, all 'inborn' characters arise merely because there has been evolved a power of developing under stimulus -in this case that of nutriment. Here, then, is the point on which I wish to fix the reader's attention. As he peruses the remaining chapters of this book, he must constantly bear in mind, not only that he is studying 'acquirements,' but that acquirements are products of evolution, parts of normal growth, characters without which the individual, who belongs to a species which has evolved the power of making them, cannot achieve complete maturity. If we study mind from this point of view, we shall not only observe it in a light that is new, but also in one which will, I think, enable us to penetrate more deeply into its mysteries than has been possible hitherto.

648. The reader perceives that $I$ am what is termed an ' extreme' Darwinian or Selectionist. Indeed I am a very extreme Darwinian. For me the great outstanding truth concerning living beings is the wonderful closeness with which, notwithstanding the vast multitude and diversity of species and characters, they are all adjusted by all their structures, faculties, and chemical reactions (for the individual is a complex chemical factory) to their environments - an adjustment which includes an equally close adjustment of their parts and faculties to one another, as well as such fundamental characteristics as the degree of variability of each character, and the normal limitation of variability to a portion of the germ-tract. Experience tells me that just in proportion as I increase my knowledge of any species, I am forced to believe that parsimonious nature has bestowed on it little or nothing but adaptations. Of necessity I know my own species best; and I, a medical man, can think of hardly a human structure or faculty to which I am unable to assign a useful function. I know, and as a human being can know, comparatively little about species that are far remote from my own, such as beetles, butterflies and plants; and of the uses of their structures and faculties 
I am correspondingly ignorant. But, when I see in such a species a structure, the function, the utility, of which I am too ignorant to perceive, I do not leap to the conclusion that it has no function, or no useful function. I remember my own species, and, bearing in mind that all species apparently fit their environments as well as my own, I seek, if possible, to learn the function and usefulness of the structure. Even when I fail, which is very often, I yet remain convinced that almost certainly both the function and the usefulness exist.

649. From the time that Darwin taught that plants and animals had undergone evolution for their own benefit, not for that of men, this opinion has been, until very recently, the accepted scientific view. But now a new school of thought, or if not of thought at least of opinion, has arisen, which (as may be judged from the following passages, examples of many that might be quoted) tells us that more correct and less dogmatic is the opinion that, in many instances at least, the structures of remote species have not been evolved because of their usefulness, but, on the contrary, have come into being and have persisted in spite of a complete uselessness. "The claim of the opponents of the theory that Darwinism has become a dogma contains more truth than the nominal followers of this school find pleasant to hear; but let us not, therefore, too hastily conclude that Darwin's theory is without value in relation to one side of the problem of adaptation; for, while we can profitably reject, as I believe, much of the theory of natural selection, and more especially the idea that adaptations have arisen because of their usefulness, yet the fact that living things must be adapted more or less well to their environments in order to remain in existence may, after all, account for the widespread occurrence of adaptation in animals and plants." 1 "To imagine that a particular organ is useful to its possessor, and to account for its origin because of the imagined benefit conferred, is the general procedure of the followers of this school." 2 The writer is mistaken. The 'claim' that evolution is other than adaptation appears, not so much unpalatable as absurd to the followers of Darwin; the reasoning by which it is supported excites, not chagrin, but amazement. The following is a case in point. "As an example of a change in the organism that is of no use to it may be cited the case of the turning white of the hair in old age in man and in several other mammals. The absorption of bone at the angle of the chin in man is another

1 T. H. Morgan, Evolution and Adaptation, p. ix.

Op. cit., p. 453. 
case of a change of no immediate use to the individual. We also find in many other changes that accompany old age, processes going on that are of no use to the organism, and which may, in the end, be the cause of its death. Such changes, for instance, as the loss of the vigour of the muscles, and of the nervous system, the weakening of the heart, and partial failure of many of the organs to carry out their functions." 1

650. "Though there is no question of absolute perfection in nature, it appears that, under given conditions, adaptation is and was sufficiently perfect to make it very difficult to put one's finger on any defect. When we think we can do so, it generally turns out that the defect is in the mind of the critic rather than in the organism criticized." 2 Occasionally we are told that the palæontological evidence lends support to the mutation theory in that an examination of the records stored in the strata of the earth indicates, as a rule, not gradual changes of type, but sudden changes. The nature of this evidence may be judged by any one who makes a careful examination of the cut surfaces of ditches and canals. If by means of such an examination he is able to achieve a knowledge of the fauna and flora now inhabiting our planet sufficiently minute and detailed to enable him to indicate the mode of origin of our new varieties, then, allowing for the destructive effects of immensely longer time, he will have some title to declare that strata deposited in past ages furnish evidence of value concerning not only changes of type, but of the mode of change. In that case, however, since the changes found in geological strata are very vast, and since the connecting links, if any, are absent, he must decide in favour, not of the hypothesis that animals and plants have arisen by mutation, but in favour of the hypothesis that they have arisen by special creation; for not even the most ardent mutationist will maintain, for example, that marsupials suddenly gave origin to placental mammals.

1 Op. cit., p. 25 ; see also $\S 359$ of the present work.

- Dr D. H. Scott, Presidential Address, Linnean Society, May 24th, 1909. Nature, July 22nd, I909, p. I I 5 ; see also $\$ 86$. 


\section{CHAPTER XXI}

\section{THE EVOLUTION OF MEMORY}

The evolution of mind-Definition of memory-Distinction between memory and its contents-Memory stores most actively in the young-Memory does not grow with use-A vast memory is the distinguishing peculiarity of the human being-The mental distinctions between ancient and modern, savage and civilized men-Distinction between man and the lower animals-Conscious and unconscious memory-Distinction between reflex and automatic action-The stereotyping of mental acquirements-As we sport with our bodies, so we sport with our thoughts-There is no impassable mental gulf between man and the lower animals - The neglect of memory by writers on evolution-Darwin-SpencerRomanes-Lloyd Morgan-Baldwin-The Mendelians-The Biometricians.

651.

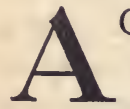

CCORDING to the theory of mental evolution formulated in the preceding chapter, mind is not derived from the non-mental. Just as life was quite a new thing in the world when it first appeared, just as nervous tissue however gradual its evolution was new, just as manipulation was new, so was that particular function of nervous tissue which we term mind. No doubt living cells and the chemical elements of which they are compounded preceded nervous tissue, but that particular combination of a certain class of living cells and fibrils to which we apply the term had no previous existence. So also its function -one of its functions-mind had no previous existence, nor even an antecedent which remotely resembled it. If this hypothesis be correct, mind in its earliest beginnings was associated with a series of variations of nervous tissue which proved favourable. It appeared at first in the form of rudimentary sensations which served as sparks to explode reflex actions-dim and faint perceptions of light, heat, sound, touch, and the like, which were more delicate and discriminating than the stimuli that had hitherto awakened action. The individual now received stimuli from a wider and more complex environment; and his actions, which became correspondingly more numerous and varied, were adapted to meet more and more distant contingencies, and achieve ends more and more remote. Later to mere sensations were gradually added tones of pleasure and pain, with which came desire and its corollary the will, and with all a further increase of complexity of action and 
remoteness of aim. Compare, for example, the simplicity of the internal sensationless reflexes of a butterfly, and the immediate ends to which they are directed, with the complexity and remoteness of aim of the actions to which he is aroused by feelings of hunger or sexual desire, or by the sight of danger. Lastly when, owing to the evolution of many reflexes and instincts, the nervous system had grown highly complex, memory arose, though not of course as a necessary consequence of the complexity. This also was new and unlike anything that had formerly existed in nature. Memory, the name we apply to the power of making mental acquirements, of growing mentally in response to the stimulus of experience, is strictly the counterpart, the homologue, of the power of growing physically in response to the stimulus of use. Indeed the physical power may be termed the physical memory, while the additions made to our bodies through it are the homologues of the mental growth, the stored experience, which results from the exercise of the mental power. In both cases, as we have seen, the powers are 'innate' or nutritional characters found only in the higher animals, and in their greatest developments only in the highest. Probably they originated about the same time, and perhaps in the same type of living beings.

652. Memory is the power of making mental acquirements. Though we speak of our recollections of past events as memories, yet, strictly speaking, these acquirements are only the contents of memory. The memory itself is purely innate, and is not increased by experience, though the volume of its contents is so increased. In other words, the power of learning does not increase from birth forwards. On the contrary, it diminishes. A young infant, for example, is more capable of learning than an older person. At birth, the mind of a child, except for sensations and a few instincts, is blank. Sights and sounds and other feelings convey no meanings to it. But soon, so great is its power of learning, the messages sent by the organs of sense are understood. In a few weeks it evolves order out of chaos, and comprehends to a wonderful degree the world around it. It learns to co-ordinate its muscles, and in a year or two is able to walk and speak a language, and do a vast deal besides. In these early years, the years of man's greatest mental activity, he makes his most valuable and fundamentally indispensable physical and mental acquirements. But, as he becomes more and more completely equipped for the battle of life, his powers of adding to the store slowly decline. In adult life the gains tend to be balanced by the losses. In old age the mental, 
like the physical losses, exceed the gains. Compare the perfection with which the young are able to acquire the manners of a society, and every accent, inflection, and intonation of one or more languages, with the imperfections displayed when the task is undertaken later, and the superiority of the youthful powers of learning at once becomes manifest. There are many things which we are better able to acquire later in life than during infancy; for example, mathematical knowledge; not, however, because our powers of learning are greater, but only because we are able to build on acquirements previously made. Similarly, while a child, whose power of growing physically is so great, cannot in a few weeks or months acquire the muscles of an athlete, a man, whose development is sufficiently advanced, but who is less capable of growth, may do so. It is sometimes said that an infant cannot make the physical and mental acquirements which are possible to a child, nor a child those which are possible to an adult, merely because the anatomical developments of brain and body are not yet sufficiently advanced. This statement is true, of course. But the hypothesis it is meant to support-that the developments which render possible various actions arise independently of, not in consequence of, previous use-is not true. The question is easily tested. Would a child who had not previously learned to walk or do simple arithmetical problems, develop body and mind sufficiently to enable it to play cricket and learn the calculus?

653. As we add to our mental stores our minds grow, but not our memories, not our powvers of learning. So, also, as we add to our store of physical acquirements our bodies grow, but not our power of growing physically. Systems for improving the memory, of which many have been invented, do not improve it in the least. They merely direct the attention more strongly to facts which are considered worthy of remembrance, and, by making them form mental associations with familiar objects or common happenings, seek to secure their recall to mind. ${ }^{1}$

654. Our minds are as busy storing experiences when we are merely 'passing the time' as when we consciously endeavour to memorize; but in the former case there is so much that is almost identical with other experiences that it cannot be recalled in detail. On that account we can recall but few of the common, the unremark-

${ }^{1}$ See James, Principles of Psychology, vol. i. pp. 659, et seq. "No amount of culture would seem capable of modifying a man's general retentiveness . . . all improvement of memory consists, then, in the improvement of one's habitual methods of recalling facts." 
able happenings of our daily lives. So, also, if we saw a thousand sheep jump in succession over a gate, we could recall, not the action of every sheep, but in the main only a general notion. The ultimate result is, that our minds are not burdened with a mass of unnecessary details, but store only what is likely to be useful. Our general impressions are concentrated, condensed recollections. Concentrated memories also, as we shall see presently, are all our acquired dexterities in thinking and acting. Moreover, apart from the concentration which occurs in the formation of general impressions, much that is seemingly lost and forgotten is none the less stored, and waits ready to be dragged into the conscious mind by some associated fact or idea. Thus when walking down the street we may see a face which is recorded but not recalled unless a second meeting awakens the memory.

655. Every animal species is fitted by its structures and their associated faculties to its particular place in nature. In some cases it holds its own largely through the great evolution of some one structure or group of structures in co-adaptation with which the whole mind and body is modified. Thus the bat is especially distinguished by the great development of its fingers and of the web between them, and the elephant by its trunk. The principal distinguishing physical peculiarity of man is the enormous relative size of that upper part of the vertebrate brain which is termed the cerebrum, and which we have every reason to believe constitutes his organ of memory and thought, and of all the mental processes and physical co-ordinations that depend on them. The cerebrum, especially the human cerebrum, is one of those organs which, after birth, develop under the influence of use, its growth being correlated to the growth of the mind under the stimulus of experience. In evidence of this is the fact, that if one hemisphere of the cerebrum be injured, as by disease, the other tends to hypertrophy under the stimulus of increased use.

656. Associated in a special way with man's great brain are his organs of speech and manipulation. These three structures, the brain, the vocal apparatus, and the hand, undoubtedly underwent concurrent evolution by the constant survival, during what must have been a period of intense competition, of those individuals who were 'naturally' (i.e. innately) the best capable of receiving and storing experience, of using it for the intelligent manipulation of objects, and of communicating it to their fellows and descendants through the medium of speech. Even the highest of the lower animals are able to learn from one another 
only by example, or through such very elementary verbal signs as calls, growls, or cries of alarm, which express no more than simple emotions. Therefore their traditional knowledge is as nothing compared to that of man, who, by means of articulated speech, indicates not only sense-impressions and emotions, but also complex items of knowledge and processes of thought which have been garnered, elaborated, and systematized during tens of thousands of years by millions of predecessors. Without speech or some such method of communicating abstruse information, his great brain and its special functions would be useless. Knowledge and powers of thought are of no avail, unless they can be translated into action, and for this the hands are necessary. To set free the forelimbs, which had hitherto been organs of locomotion, for their new function of manipulation, man gradually became a biped, and assumed the erect attitude-not, of course, by any conscious effort, but by the constant survival of the fittest, those best structurally adapted to walk erect.

657. Savage man differs from lower animals mainly in that he has, relatively to his size, a larger brain, a more capacious memory, and greater powers of using its contents and communicating them to others of his species. Modern man differs from ancient man because he is heir to a greater accumulation of traditionally transmitted experience. Civilized man differs from the savage chiefly in that he has invented and more or less perfected certain artificial aids to memory, thought, and speech -written symbols by means of which he is able to store in an available form information immensely more abstruse and voluminous than would otherwise be possible. His books are artificial memories and vehicles of communication of unlimited capacity and unerring accuracy. Moreover, by means of these symbols he is able, as in the mathematics, to perform feats of thinking quite beyond the powers of his unaided mind, just as by means of mechanical contrivances, he is able to perform physical feats beyond the unaided powers of his body. Obviously, the mental change which occurred when savage races achieved civilization did not necessarily imply germinal alteration. But, because each generation communicated its growing traditions to the next, it so exactly mimicked evolution that innumerable writers have been deceived. The belief is almost universal that the savage differs innately in mind from the civilized man. ${ }^{1} \mathrm{He}$

1 Dr Alfred Russel Wallace is a rare exception. See his very interesting article on Evolution and Character in the Fortnightly Review, Jan. I908. 
may differ, and very possibly does differ, to some extent, but the evidence on which an opinion may be founded is lacking.

658. To memory, then, is due the advance of the savage beyond the lower animal; to tradition, the child of memory, the advance of modern man beyond ancient man; to facts and thoughts stored in books the advance of civilized man beyond the savage. To written symbols also are due his vast potentialities for future advance. In the past of the earth the brute, the mammoth, the bear, the tiger, the horse, the ox, and the sheep, became ever more and more helpless in the presence of a knowledge and an ingenuity, the outcome of organized knowledge, which gathered direfully with the rolling years, and which, though accumulating for ages, were yet comparatively new things in this enormously old world.

659. The lower animals in proportion as they lack memoryin proportion as they are incapable of profiting from experience, but develop more or less exclusively under the stimulus of nutrition-move in a narrow, instinctive groove. Their mental traits are 'innate and inherited,' and, therefore, every individual follows nearly exactly in the footsteps of its predecessors. Since they cannot learn they cannot adapt themselves to circumstances. Removed from the ancestral environment, they perish. Cast in a rigid inexpansive mould, they resemble one another of the same species as much mentally as physically. It is different with man. $\mathrm{He}$ is pre-eminently the educable, the reflective, the adaptable animal - the animal who develops mentally under the stimulus of use. Since men can learn, and since the experiences of no two men are quite similar, they differ in knowledge, ideas, aspirations, modes of thought, and motives for action. Therefore none are very closely alike mentally. The child does not follow exactly in the footsteps of his parent. So great is human educability (i.e. adaptability) that though the mind of the savage differs immensely in all except instincts and powers of learning from that of the civilized man, yet if the child of the latter were trained from birth by the former he could not be other than a savage. Indeed the offspring of civilized parents captured by savages (e.g. American Indians) have actually developed into utter savages. On the other hand, some savages, for example the Maoris of New Zealand, have passed in a single generation from barbarism to civilization. The Maoris, as I judge from personal experience, are not innately inferior mentally to Europeans. They are unable to compete with the intrusive whites only because they are more susceptible to 
imported diseases. The average individual amongst us, and doubtless amongst the Maoris, may be trained to fill the rôle of a beggar or a king, a scientist or a monk, a thief or legislator. $\mathrm{He}$ is so intelligent, so adaptable that he is able to dwell in the tropics or in the Arctic, in the city or the wild, on land or on sea. Memory, knowledge, intelligence, reason, adaptability, are all links in a single chain of efficiency.

660. Memory is of two sorts, conscious and unconscious. The conscious memory contains experiences which can be re-collected, such as scenes we have beheld, or the words of a language. The unconscious memory contains impressions which cannot be recalled to mind, but which are none the less important. Indeed, many of its contents were never present in consciousness in the sense that a sight, an emotion, or an idea is present. Thus we learn to use our legs in walking, a process that involves a precise but unconscious adjustment of the actions of numerous muscles, the very existences of which are unknown except to the anatomist. In a sense, therefore, we know as little about walking as any insect. All our acquired dexterities, both in thinking and acting, belong to this category. They are mental acquirements stored by the unconscious memory. Thus we learn to reason; but, though we recall some of the times and lessons by which we learned to think well, and though results may make us conscious of a growing facility in reasoning, yet the facility itself, like dexterity in walking, is a thing that is outside our consciousness. So also, to some extent at least and especially in youth, we unconsciously imitate our fellows, adopting in great measure their mental tones and attitudes without knowing how or when we were influenced. Many experiences, too, once capable of being distinctly recalled, are added to that hidden store, and, though apparently lost, remain potent for good or evil. Our minds are like floating icebergs, of which the visible part is but a fraction of the whole, and which are moved by deep currents in a seemingly unaccountable way. ${ }^{1}$

1 I speak of the ' unconscious memory.' The expression is in common use, and is convenient. But, properly speaking, memory is the faculty for storing and recalling to consciousness feelings that have been there before. An unconscious memory, therefore, is a contradiction in terms. Probably what we call unconscious memory is nothing other than a growth in efficiency, or in size, or in complexity, or in all three, of brain; a growth which enables that organ to perform work which till then it was incapable of performing, or which it was incapable of performing as easily or as well. In physiological and psychological writings this cerebral change is usually spoken of as an "opening up of paths," in the brain. If by that is meant a building-up in the brain analogous to that building which occurs when an addition is made to our means of telephonic communication, the 
66r. We learn to do new things, acquire new knowledge, and think new thoughts with toil. But practice confers facility. In the end we know or perform with ease that which was acquired with difficulty. We cannot unlearn, however, as we have learned by an act of will. The facility (the brain change) lingers, and, as a consequence, since we tend to lose our powers of making new acquirements, our action and thoughts, our mental tones and attitudes, our whole outlook on life, becomes more or less ' automatic' or stereotyped. ${ }^{1}$ In other words, our acquirements come at last to resemble reflexes and instincts. Not only do they take the place of those reflexes and instincts which retrogressed when memory was evolved, but they are infinitely more numerous. So like are they to reflexes and instincts that they are often so misnamed. Thus psychologists usually place automatic actions in the reflex category. As a fact, reflexes and automatic actions are as poles apart; but of this more presently. ${ }^{2}$

662. Again we often apply the term 'instinctive' to emotions and 'physical' dexterities which have been acquired. For example, deceived by the quickness and readiness of the act, we speak of such actions as that of a boy when he dodges a blow as instinctive. But the boy has learned to dodge, and would learn to dodge still better and quicker if he went into training for the prize ring. When untaught by experience, he can no more dodge a blow than he can solve a problem in quadratics. His action is intrinsically different from that of a house-fly, which learns nothing from experience, and dodges as well at the first attempt as at the last. It is the mode of origin, not the quickness, readiness, or facility in performance which constitutes the difference between reflex and

expression may pass, though it is apt to mislead. But, if by it be meant that 'channels' are somehow made in the brain analogous to those which water wears on or under the ground, the expression is, probably, not only misleading, but wrong in every sense. There is nothing to justify it except an impossible analogy. It is admitted that mental happenings are correlated to cerebral happenings. If mental growth occurs, for example increased thinking capacity, we have every reason to believe that this growth is correlated with actual cerebral growth analogous to muscular growth-though possibly this growth may often imply little or no increase in the size of the brain, but only an adjustment which increases its working power.

${ }^{1}$ It would be well for the avoidance of subsequent confusion if the reader carefully noted the meaning here given to the word ' automatic.' An automatic action is one which we have learned to perform, but which, through practice, has become so easy of performance that, like a reflex action, it is done, apparently, without mental effort. It is sharply distinguished from a reflex action in that it is a product of individual experience.

${ }^{2}$ See $\$ 676$ et seq. 
instinctive actions on the one hand, and, on the other, actions which are often termed automatic, or intelligent, or rational, but which as a class are best distinguished as actions the performance of which depends on stored experience, on memory. So alike are our acquired emotions and dexterities to their 'innate' counterparts that a being from another planet, who for the first time saw a man walking, cycling, or reading, could not distinguish the nature of these acquirements from such instinctive actions as the running, flying, or building, of an insect. The patriotic emotion of a Spartan or a Japanese differs from that of a bee or an ant merely in its mode of origin. Were the religious fervour of a Salvationist or a dervish, or the hatred of religion which characterises the atheist, innate, it would be an instinct. But these emotions, like many of our 'physical' dexterities, are obvious acquirements. This, then, is the main distinction between man and the low animal; - the emotions and (apart from the reflexes) the dexterities of the man are mainly HABITUAL or 'acquired;' those of the low animal are wholly or almost wholly instinctive or 'inborn.' The former develop under the stimulus of use; the latter under that of nutriment.

663. A principal function of our faculty of making mental acquirements, of our conscious and unconscious memories, is to supply us with those automatic and stereotyped ways of thinking and acting which are our substitutes for numerous reflexes and instincts. Our conscious memories supply us in part with our stereotyped mental attitudes-desires, beliefs, aspirations, habitual ways of thinking, and so forth. Our unconscious memories, not only aid in building up our mental attitudes, but give us our stereotyped ways of acting-those automatic ways of acting (e.g. walking, reading, cycling, etc.), we have just considered. At the same time our physical 'memories' (our powers of making physical acquirements) enable us to develop, in perfect co-adaptation with our mental acquirements, our bodily parts, our brains, limbs, lungs, heart, and the like. As we grow older our imitation reflexes and instincts increase in number and importance; they form a larger and larger portion of our total reaction to the environment. They equip us for the battle of life with a thousand faculties for acting readily, quickly, and easily. In the aggregate they are immensely superior to real instincts and reflexes for they render the individual adaptable; they enable him to grow into fitness to the particular environment in which he is reared; and, even if he changes it, to grow to fit the new one. Whether he be artist or sailor, beggar 
or king, he develops just such dexterities in thought and action as are necessary to him, but is burdened with no more. Our human memory, our power of growing mentally in response to the stimulus of use and experience is a device by means of which nature has surmounted the difficulty of evolving an untold number of reflexes and instincts. Each real reflex and instinct demands for its evolution and maintenance a separate process of selection. For example, the reflexes of the heart and the bowels, and the instincts of hunger and sexual love are entirely separate things not evolved by the same process of selection and not equally well developed in every individual. If the individual is defective in any important reflex or instinct, he leaves no offspring. Therefore nature must maintain all. When they are very numerous, individuals defective in one or another are so many that the death-rate tends to exceed the birth-rate. Therefore there is a limit to their number. In other words there is a limit to the complexity of the individual in innate characters, and therefore a limit to the number of innate reactions he is able to make to the environment. On the other hand in memory nature evolves and maintains only one thing. But that one thing, as I say, is capable of supplying any number of emotions and automatic reactions which answer precisely the same purpose as innate reactions.

664. It is a principal business of our lives to acquire these dexterities in performance (e.g. walking), these imitation reflexes (e.g. quick withdrawal from a cause of pain) and these imitation instincts (e.g. patriotism). But, though a great advantage is gained when the acquirements are made, one almost as great is lost. We then think and act more quickly in familiar situations. When the adult stage is reached we are by virtue of them as fit or more fit than an insect to face the battle of life. But each important acquirement is written, as it were, in indelible ink on the sheet of the mind. This matters comparatively little in the case of those automatic actions which take the place of reflexes. They are almost always useful, as for example, the automatic co-ordinations of our muscles by means of which we walk, speak, cycle, swim, knit, or dodge blows. But our bad as well as our good acquirements are imitation instincts. Once acquired, they tend perpetually to pull us down. Moreover, as we grow older, we tend to lose our splendid human capacity for learning. Old habits, good or bad, persist ; new habits cannot easily be acquired.

665. In addition to the acquired dexterities and mental attitudes which depend on records unconsciously retained, our 
memories supply us with a vast fund of facts, ideas, emotions, and the like, which may be more or less vividly recalled to mind and which, therefore, afford the materials for such mental processes as association, comparison, and discrimination - in brief with the materials of all that is comprised under the term thought. Byappealing to our conscious memories we are able to note sequences of events in the past and deduce necessary truths and so to foresee to a very useful extent the sequences of events in the future, and in this way make provision for coming events and dangers. By virtue of the intellectual powers thus conferred we are rational beings in the strict sense of the word. Unlike the purely instinctive animal which is impelled merely by emotion, we guide our more complex and less habitual actions by thought, by intelligence and reason. But though the whole domain of the intellect pertains to the conscious memory, yet we are able to labour usefully in it only by virtue of powers conferred by the unconscious recorder of our experiences. We learn to think, to reason just as certainly as we learn 'physical dexterities,' but, as in the case of the latter, the springs of our 'mental' dexterities cannot be recalled to mind in the sense that an event or a fact may be recalled. That is, though we may remember that we are skilful in thinking, and though we may call to mind the ways in which we acquired the skill and the results we obtain by the exercise of it, yet the skill itself depends on concentrated experiences which cannot be recalled to mind any more than those on which skill in walking depends. The skill is useful, but the power of picturing it in mind would be of no practical utility; and therefore nature does not bestow it.

666. An instinct impels us to sport with our limbs and so supply them with the stimulus necessary for growth and our minds with the practice which confers dexterity in using the limbs. This growth of 'physical ' dexterity is, as we see, really a mental growth. So also, an even more imperious instinct impels us to sport with our thoughts. From birth forwards we think perpetually and cannot help doing so. Fresh experiences recall antecedent experiences with which they are compared, and, these again call up yet other experiences. In our idlest moments we are busy thinking. In this way we learn dexterity in thinking, and this acquired dexterity is an essential part of intelligence and reason. Obviously, therefore, reason is not, as all psychologists imply, an ' innate' character like memory. It is learned just as surely as reading and walking are learned. No child is born capable of reasoning, and much of our school-room education is a 
formal endeavour to develop the faculty by means of special exercises.

667. The range of thought possessed by any species is always proportionate to the greatness of the conscious memory, which records not only items presented by the senses, but also those acquired through the imagination and intelligence. On this account the dog has a greater range than the cat, and man than the dog. Man's unique intellectual powers depend wholly on his vast and capacious memory by means of which he acquires both the materials (facts and ideas) and the methods (association, discrimination, and the like) of thought-materials and methods which render him intelligent, rational, 'self-conscious,' capable of 'conceptual thought' and of 'apprehending universals,' and abstract ideas such as those of right and wrong, and of performing feats of thinking as in mathematics, philosophy, and science, or of imagination as in music, poetry, and fiction, or of ingenuity as in engineering and warfare, or of becoming devout or patriotic, and so forth. The fact that he alone, of all animals, is capable of these mental operations has caused many writers to declare that an impassable gulf separates him from the rest of living nature. But plainly there is no impassable gulf. None of the traits mentioned are 'inborn' or special products of evolution or creation. The infant possesses none and can possess none until his memory is stored with the materials of thought and he has learned through imitation and practice fit methods of thinking. He differs from lower animals only in that his memory is so large that it alone is capable of being stored with these traits amongst many others. That which has been created by evolution is his huge cerebrum, the organ of memory, the thing which does the work which is memory, reason, and all the rest which results from acquirement. Both cerebrum and memory are found in lower animals, and there is no greater difficulty in conceiving man's mental evolution through the continued selection of mentally superior individuals (that is, of individuals who are mentally superior because they have a superior quality of brain) than of conceiving the evolution of the elephant's trunk, the bat's wing, or any other structure that especially distinguishes this or that animal.

668. Not only may memory vary as a whole, but it may vary in particular directions in different individuals. Thus, while one man may possess exceptional powers of acquiring the facts and methods of mathematics and of learning to use his knowledge for purposes of fresh discovery, another man may be similarly dis- 
tinguished with respect to music, literature, engineering, or warfare. But there is nothing more wonderful in this than in the fact that some part of a structure (e.g. a nose) may be relatively more developed in one man than in another. Men often differ very greatly in mental capacity, in innate power of making this or that acquirement, and doubtless these mental differences are associated with innate cerebral peculiarities, and tend, therefore, to be transmitted to offspring; but it must be borne in mind that mental differences need not imply correspondingly great cerebral differences. When we attempt to estimate the latter by means of the former, we examine them, as it were, through a magnifying glass. The amount of nervous tissue in the brains of ants is very small and the differences apparently are not very great, yet the species differ, vastly in their mental characters. Small cerebral variations, therefore, may imply great psychological differences.

669. None of the objections by which it was sought to controvert the doctrine of man's mental evolution from loweritypes could have been maintained had the faculties (mathematical, devotional, musical, and the like) concerning which controversy raged been traced to their true source, memory. The evolution of memory can be traced with the same degree of certainty as that of brain or any other physical structure; but I think I am right in saying that hitherto no one has attempted to trace it. Its importance has not been realised; and only very scanty space is devoted to it in standard works on evolution. The root of the mischief has lain in the initial failure to note the real nature of the difference between 'inborn' and 'acquired' characters, and to compare the parts they have played in evolution and development. Acquirements, as I say, have been regarded as transient unimportant traits, mere accidents, appearing in one generation and disappearing in the next. Some attention, indeed, has been given to the faculty of growth under the stimulus of injury (regeneration), but wonderfully little has been attracted by the more important faculty of growth under the stimulus of use and experience. ${ }^{1}$

670. The older students of psychological evolution supposed that memory was an invariable accompaniment of consciousness and therefore had no notion that it was a late and high product of evolution. Darwin, as far as I have been able to ascertain, devotes only one short paragraph to it, in which he merely relates three incidents which tend to show that memory is present in baboons, dogs and cats. ${ }^{2}$ He discusses, however, at greater length,

2 See $\$ \$ 20$, et seq.

2 Descent of Man, p. II2. 
imagination, imitativeness, and reason, all of which depend on memory but which he treats as independent faculties. Spencer regarded instinct as compound reflex action and the precursor of intelligence, which he thought resulted from still further compounding. On the other hand, Lewes, thinking, like Spencer, in terms of the Lamarckian doctrine but arriving at an opposite conclusion, believed that the intelligence of ancestors becomes the instincts of descendants. That is, he believed that mental characters which developed in the ancestors under the stimulus of experience were 'inherited' by descendants-i.e. were transmuted into characters that developed under the stimulus of nutriment. He failed to note the fact, fatal to his hypothesis, that the human being, the latest product of evolution, is of all animals the most equipped by acquirements and the least by instinct. Richet insisted that "without memory no conscious sensation, without memory no consciousness." Romanes declared: "The most fundamental principle of mental operation is that of memory, for this is the conditio sine qua non of all mental life." 1 "The power of learning by individual experience is therefore the criterion of mind." $\mathrm{He}$ adds, however, "But it is not an absolute or infallible criterion ... it serves to fix the upper limit of non-mental action more precisely than it does the lower limit of mental; for it is probable that the power of feeling is prior to that of consciously learning." 2

$67 \mathrm{r}$. When a particle of food goes the wrong way I cough. That particular reflex is quickly abolished by chloroform or even a local anæsthetic. Therefore it is initiated by sensation, by mind. But a newly born infant coughs just as well as a man. Here it has nothing to learn. The act is quite involuntary and has no connection with memory. But it is highly useful. Therefore

${ }^{1}$ Mental Evolution in Animals, p. 35, Romanes proceeds: "But memory on its obverse side, or the side of physiology, can only mean that a nervous discharge, having once taken place along a certain route, leaves behind it a molecular change, more or less permanent, such that when another discharge afterwards proceeds along the same route, it finds, as it were, the footprints of its predecessor. And this, as we have seen, is no more than we find to be the case with ganglionic action in general." This notion of nervous discharges making paths which render more easy the passage of subsequent similar discharges is found in almost all psychological works, and furnishes an easy but as we have already noted a fundamentally deceptive illustration. In low animals, in which only reflexes and instincts occur, nothing is added to the mental and nervous equipment by experience. In higher animals, in which memory is present, the stimulus of experience, so far from opening a path, adds a growth. Hence the increase in volume and complexity both of the child's mind and of his brain.

Mental Evolution in Animals, p. 60. 
mind without memory is useful. Romanes himself, by means of a somewhat cruel experiment, afforded clear evidence of the lack of memory in low animals and the usefulness of sensation in its absence. "I am more surprised with my failure in this respect with the higher Crustacea; for although I have tried similar experiments with them I have never been able to teach them the simplest things. Thus, for instance, I have taken a hermit crab and put it into a tank filled with water, and when he had protruded his head from the shell of the whelk in which he was residing, I gently moved towards him a pair of open scissors, and gave him plenty of time to see the glistening object. Then, slowly including the tip of one of his tentacles between the open blades, I suddenly cut off the tip. Of course the animal immediately drew back into the shell, and remained there for a considerable time. "When he again came out I repeated the operation as before, and so on for a great number of times, till all the tentacles had been progressively cut away little by little. Yet the animal never learnt to associate the appearance of the scissors with the effect which always followed it, and so never drew in till the snip had been given. Nevertheless, that memory does occur among the higher Crustacea is proved by an observation quoted in Animal Intelligence (p. 233), concerning a lobster mounting guard upon a heap of shingle beneath which it had previously had hidden some food." 1

672. That memory is absent or very rudimentary in hermit crabs is proved by the experiment. That the action of the lobster indicates memory is not so certain. ${ }^{2}$ Though named in the margin, memory had no place in Romanes' famous diagram which precedes his works on Mental Evolution and which was "intended to represent in one view the whole course of mental evolution," 3 and concerning which he wrote, "I feel confident that the general structure of our knowledge concerning the evolution of mind is now sufficiently coherent to render it highly improbable that this diagrammatic representation of it will, in the future, be altered in any of its main features by any advances that science may be destined to make." 4 In his list of the products of intellectual development memory is placed before the primary (i.e. the earliest) instincts. ${ }^{5}$

673. Recent writers make also as little mention of memory as a factor in mental evolution as Darwin. The mental 'plasticity'

1 Op. cit., pp. 122-3.

2 See § 689.

${ }^{3}$ Mental Evolution in Animals, p. 63.

'See Romanes' diagram.

4 Op. cit., pp. 63-4. 
of Baldwin, Osborn, and Lloyd Morgan ${ }^{1}$ is of course memory, but the fact is nowhere recognized. Nor, indeed, is the importance of the so-called plasticity appreciated. "Professor Morgan here develops (Chap. XIV., Habit and Instinct) a suggestion which has been put forth by Professor H. F. Osborn, and independently reached by the present writer, as Morgan points out, namely, that by learning intelligently and imitatively to do the things which are essential, certain animals are screened from the operation of Natural Selection, and so hand on their capacities to future generations, while the race accumulates further congenital variations in the same directions (what Morgan calls 'Coincident variations'). Thus evolution takes the direction marked out in the first instance by the individual's learning." 2 In other words, it is supposed by Baldwin, Morgan, and Osborn that useful acquirements may enable a race or a line of individuals to survive until the descendants have varied in such a way that the useful traits appear in them, not under the stimulus of experience, but under that of nutriment. ${ }^{3}$ In this way, as according to Lewes' theory though not as he supposed by the transmission of acquirements, that which was intelligent in ancestors becomes instinctive in descendants. For example, if learning to swim or to speak a language enabled ancestors to survive, it is supposed the descendants may vary so as to swim or speak instinctively. That is, unthinking impulse is supposed to be substituted in descendants for learning, thought, intelligence, and reason in ancestors. This theory, of course, affords an instance of the prevalent biological obsession that nutritional characters are more important, more innate, more a possession of the species than characters which develop under the stimulus of use and experience-the latter being regarded as mere accidents limited to the individual and not like nutritional characters, part of the heritage of the race. The whole course of the evolution of the higher animals demonstrates the erroneousness of this opinion. So far from the 'innate ' replacing the 'acquired,' the contrary is the invariable rule. For example, in man the highest and the latest product of evolution, that which is 'inborn' is at its minimum, while that which is 'acquired' is at its maximum. It is not realized by the writers mentioned that to the animals that have the power of making acquirements, these characters are

${ }^{1}$ See, for example, Development and Evolution, by James Mark Baldwin (The Macmillan Company), and Animal Behaviour, by C. Lloyd Morgan, p. I 72 (Arnold). Neither of these authors mention memory. At any rate I can find no mention, and the word does not occur in their indices.

Development and Evolution, pp. 390-I.

3 See § II 4 . 
infinitely more useful than similar 'innate' characters could possibly be. They render the individual adaptable. I do not think I overstate the case when I insist that, until this truth be grasped, all biological speculation concerning the evolution of the higher animals must be, in great measure, futile.

674. Very recent biological work has been mainly Mendelian or biometric. Memory has been discussed by neither the one school nor the other. Mendelian experiments could, at most, tell us nothing more than whether the reproduction of memory is or is not alternative, and it would not be easy to devise experiments which would elucidate even that much. They would not indicate the nature of memory. The problem is one for the thinker, not the experimental observer. Biometricians, as we shall see, have worked on the assumption that various characters which seem clearly part of the contents of memory are 'innate' or instinctive. Indeed, it is probable that, more than any other section of biologists, they have failed to realize the importance of the acquired factor in the human mind. 1

675. Speaking generally, very recent writers who deal with mental evolution suppose that first reflex action, then instinct, and lastly intelligence and reason were evolved; other faculties such as imagination, discrimination, and the power of drawing inferences, being variously placed in the general scale. There seems good reason to believe, however, that the true order of the evolution was first reflex action, next instinct, and lastly memory. Imagination, intelligence, reason, and the rest are merely operations which animals perform because their memories have undergone sufficient evolution. Without memory there could be no such faculties; for they are concerned solely with the contents of memory, associating, comparing, discriminating, adding to them. In a real sense they are themselves part of the contents of memory; for infants learn to think just as truly as they learn to walk or learn the facts about which they think. The range and quality of the thinking are entirely conditioned, on the one hand, by knowledge, and, on the other, by the power of learning to think ; that is, from first to last by memory.

1 See $\$ 706$ et seq. 


\section{CHAPTER XXII}

\section{NATURE AND NURTURE}

Acquired emotional impulses are copies of instincts, and automatic actions of reflexes-Distinction between reflexes and automatic actions-The way in which our dexterities arise-The fovea centralis of attention and will-Automatic actions differ from reflex actions in that they are controlled by the attention and the will-Dreams-The distinction between recollection and bearing in mindThe difference between physical and mental acquirements-Nature and nurtureCapacity and mental acquirements-Ability and efficiency-The factors of efficiency-Instincts and acquirements-Imbeciles-Racial mental characteristics -The influence of religion-Opinions founded on biometry.

676. NPEAKING generally, the impulses to action (emotions) which we gradually acquire and store in our memories, for example, patriotism and religious enthusiasm, are all close copies of instincts. They resemble instincts in all except their mode of origins. Like them they prompt, with various degrees of urgency, to voluntary actions. The resemblance is made all the closer by the fact that, just as a great deal of our acquired physical growth is a prolongation, a mere continuation, of growth that was previously made under the stimulus of nutriment, so some of our acquired impulses to action (e.g. love for some one woman, a curiosity concerning some particular science), are prolongations or reinforcements of instincts. In other words, many of our permanent impulses to action are partly instinctive and partly acquired; and the acquired part of the emotion so closely resembles the inborn portion that we cannot distinguish where the one begins and the other ends. The resemblance is made yet closer by the fact that the performance of only a few of the actions to which human instincts (e.g. the sexual and the parental) prompt are instinctive in the sense that they can be performed without previous learning. Thus the human mother not only acquires the special learning which enables her to tend her child, but during her own infancy acquired the necessary power of co-ordinating the muscles of her limbs. The question, then, arises whether imitation reflexes (automatic actions) are as close copies of real reflexes as acquired emotions are of instincts. We learn to do habitual 
actions, for example, walking, speaking, writing, reading, sewing, cycling, swimming, and the like, with toil and difficulty. At first the performance is awkward, and the action on which we are engaged requires our 'undivided' attention. Gradually, however, performance grows more facile till at length we are able during it to do some other less habitual action at the same time. The latter, then, in turn requires our 'concentrated' attention, until, perhaps, it also becomes habitual. When an action has become so easy of performance, so habitual, that we are able to do it 'unconsciously,' it is said to be automatic. It is then so close a copy of reflex action that psychologists usually include it in that category.

677. A true reflex, as we have seen, is not initiated by the will, and is, besides, an action which the individual is born with the power of performing. Therefore, it resembles an instinctive action in that the power of perfoming it is innate, but differs from the latter in that it is involuntary; whereas it differs from an automatic action in that the power of performing it is innate, not acquired. The problem before us is whether automatic actions resemble true reflexes in being quite involuntary. The question is important, for, as I say, psychologists usually include automatic actions amongst the reflexes, just as they often include acquired emotions amongst the instincts; with the result that the full importance of the distinction between the inborn and the acquired, and, therefore, the part played by acquirement (i.e. the contents of the memory) in the building up of the normal human mind is lost to sight. We have here no mere question of nomenclature, nor even merely a problem of pure science. Immense practical issues hang on the conclusions we reach. For example, if we were able to attain a thorough knowledge and agreement as to how the human mind developed-what it is possible and what impossible to create or eliminate by means of careful mental training-we should also, in all probability, be in equal agreement as to the best methods of educating our children. Even if a thorough knowledge and agreement be unattainable by us, we shall, nevertheless, be nearer both if we found our conclusions on careful investigation, instead of careless assumption.

678. A few facts may be first noted. (I) Every automatic action (e.g. knitting, cycling) was clearly voluntary when first we began to learn it. (2) As a rule, any action which has become automatic is still clearly voluntary when we begin to perform it; thus, we usually begin to walk through an impulse which is plainly 
due to an exercise of will, though afterwards, when we are thinking of other things, the performance becomes automatic. (3) Every clearly voluntary action is now in part automatic, for we have learned a general automatic power of co-ordinating our muscles for every kind of action. (4) Every automatic action becomes voluntary directly our attention is concentrated on it; thus, the automatic action of a woman who, when she is knitting, thinks of other things, becomes clearly voluntary when she concentrates her attention on her work.

679. At the back of the eye is a small spot, the fovea centralis, which is the region of the most acute vision. When we wish to see an object distinctly, we turn our eyes so that the retinal image of it falls on the fovea centralis. That constitutes the action of fixing our eyes on the object. The images of the other objects within the area of sight are cast on the peripheral regions of the retina surrounding the fovea, and are seen much less vividlythose nearest the fovea being most, and those farthest least distinctly seen. Normally, when we are attending to the things we see, the major part of our attention is concentrated on the object on which the sight is fixed. But we are not entirely oblivious of its setting, for any unexpected occurrence within the area of sight immediately attracts our concentrated sight and attention, which could not happen were we quite unconscious of it. This diffusion of some of our sight and attention while most of them are concentrated on particular objects is extremely useful; for thereby the concentrated faculties are continually guided to fresh objects of interest or importance. Thus, having sufficiently admired one flower on a bush, our sight and attention does not wander vaguely round in search of other objects of admiration, but swiftly passes to flowers which previously were marked on the peripheral portion of the retina. The recollection of the first flower is then stored in the memory, and to it, as our concentrated sight and attention wander about, we are able to add memories of other flowers. Consequently, we do not live solely in the immediate present. We are able to compare the various flowers on the bush with one another and with flowers seen perhaps long ago. Thus our minds are built up.

680. It seems, then, as regards the objects of sight, we are able to attend to more than one thing at a time. At any moment, our attention is more or less concentrated on one thing, but some of it is diffused, with varying but much lesser degrees of concentration, on other things. The object on which our sight is for the time being 
fixed is, as a rule, of all the objects within sight, the most interesting to us during that time. For that reason we 'look at' it. For that reason it 'claims our attention.' Sometimes, indeed, but then only by a conscious effort, we do not concentrate our attention on the object of sight on which our eyes are fixed, but on some other object in the peripheral area of vision. Thus, in a drawing room, though we may be gazing full at the face of a friend, we may really be watching through the 'corner of our eye' the pretty girl beside us, who is talking to some one else. More commonly, however, when, as is often the case, we are 'not attending' to the object that falls on the fovea centralis of the retina, our attention is concentrated, not on any object of sight, but on something else. Thus, while we are almost entirely oblivious of our friend or the pretty girl, we may be concentrating our attention on some interesting gossip that is being whispered behind us. Our concentrated attention, the fovea centralis of our attention, wanders, like the fovea centralis of the eye, from one sensation (auditory, tactile, etc.) to another; or it may ignore sensations altogether, and, as when we are in a brown study, be concerned only with our thoughts. Our concentrated attention is what is colloquially termed 'active,' while the diffused attention is termed ' mechanical.' Thus, when we are thinking of the pretty girl or the gossip, we are actively attending to one or the other and mechanically to our friend. But the latter attention, however mechanical, however diffused, is still attention. We are not quite oblivious of the friend. We still converse mechanically.

681. The degree of concentration of attention varies greatly. It may be so concentrated that we are almost unconscious of the rest of our surroundings. It may be so diffused that it is hard to say which object is claiming the most of our attention. But there always is some concentration and some diffusion. Now, we invariably remember best those things on which we have concentrated our attention most. Thus, as a rule, we can recall only the seen things on which we have fixed our sight, and even then only when we have paid attention to them at the time. All the rest we have seen, or otherwise felt, is vague in recollection, if it be recollected at all, in proportion to the degree of the diffusion of the attention. Thus, I have been invited to see an interesting town, by a friend who was even more interesting, and have walked miles with him, and have come away with hardly a recollection of the place, but full of vivid remembrances of what my friend said. If he had been less interesting I should have remembered more of 
the town. Doubtless, in that case, my concentrated attention would have fluctuated back and forth from him to it. Since, however, only my diffused attention was given to the town, I remember little of it. Only enough attention was given to enable me to walk about and avoid obstacles.

682. The will is associated with the attention. We never will to do anything unless our attention is attracted to it. We are able to do several actions at once. Thus a few minutes ago I was standing, smoking, pouring water into a cup, stirring its contents, and trying to think of a good example of several actions done simultaneously. My attention was concentrated on the last-mentioned act, the others being more or less automatic. Were they, or were they not under the constant control of my will? Suddenly it occurred to me that my action at the time afforded the example I required. Thereupon, as I noticed immediately, I ceased to smoke, to incline the kettle, and to stir the cup. I do not know how it is possible to explain these cessations, except by supposing that the new thought occupied so much of my attention, and therefore of my will, that the latter ceased to direct the smoking, inclining, and stirring, though the still more automatic actions of standing and holding the kettle continued. Had my attention been very strongly concentrated it is possible that even they might have ceased. I remember, as a student in Edinburgh, watching with mischievous expectation a party of tourists in whose near neighbourhood a signal cannon, unsuspected by them, was about to be discharged. At the sound of the explosion almost all action ceased for an instant in the crowd. It stood as if paralysed. Many of its members let fall sticks and, parasols, and many seemed ' ready to drop' - to cease the automatic action of standing. Similarly, anything that interests us very greatly tends to cause cessation of automatic action unless some of our attention is directed to it by conscious effort. On the other hand, during ordinary preoccupation, we are able to hold within the peripheral parts of our attention things we desire to bear in mind. This happens even during sleep, for often we are able to wake, if we desire it, at an earlier hour than usual. It seems, then, that even the most automatic of our actions is maintained only because some part of our attention is directed to it, and some part of our will engaged in its performance.

683. I think, therefore, there can be no doubt that automatic actions differ from reflexes, not only in that the ability to perform them is acquired, but also because they are always initiated, controlled, and guided throughout by the will. Therefore they 
are not such close copies of reflex actions as certain other actions are of instinctive doings. While we are learning to perform them they engage our attention and will strongly. But as they become habitual and are performed with increasing ease, we are able to distract more and more of the attention and the will to less habitual actions; but never the whole attention and will. The will does not fall into abeyance as we become dexterous, it merely works very smoothly and easily so that little mental effort is required. As already noted, it is one of the main businesses of our lives to acquire the power of performing frequently recurring actions automatically, and so set free the concentrated part of the attention and the will-the fovea centralis of our attention and will-for other objects.

684. It is said sometimes that lower animals differ so much from us in brain and mind that we can form no conception of their mental states. Doubtless this is true in a great measure. The senses and, therefore, the outlook on the world as well as the instincts of many animals differ greatly from ours. Moreover, no other animal possesses such an enormous memory as man; none, therefore, learns so much about the world or is so preoccupied with the past and the future. On the other hand, since most of our instincts and those of lower animals are derived from progenitors that were common ancestors, our innate emotional impulses cannot be very different; and, though our powers of thought are transcendently great, yet all that is conferred by our unconscious memories, our 'physical' dexterities and mental attitudes, including many of our prepossessions and prejudices, tend to become close copies of instincts and instinctive activities. It is possible to imagine a state of mind in which experiences are not recognised as old acquaintances-a state of mind in which nothing appears familiar or unfamiliar; in which everything is taken as a matter of course and nothing causes surprise ; in which there is no idea of the past or of the future but only a consciousness of the immediate present; in which, while most sights, sounds, tastes, tactile sensations, and the like leave us unmoved, some of these concentrate our attention and will and incite more or less vehemently to definite actions the aims and ends of which are unthought of. Such a state of mind should be very like that of purely instinctive animals. Things are familiar or unfamiliar only because memory differentiates between the two, and surprising things are surprising only because they run counter to our previous experiences. ${ }^{1}$ 
Doubtless, an African elephant or a dragon from the pit would excite no greater interest or surprise than a rabbit in a new born baby or in a beetle of any age.

685. Probably our dream world resembles in great measure the real world of a purely instinctive animal. In proportion to the depth of our slumber, our memory, or some part of it, seems in abeyance. We may " know sleeping thoughts at the moment they arise, and not retain them the next moment." 1 As a medical man I am frequently rung up at night, and, having formed the habit of taking note, I observe that, invariably, I awaken out of a dream, which I remember if I mentally record it without delay, but which otherwise I forget almost immediately, especially if I am roused out of very deep slumber. In lighter slumber, when I hover on the borders of waking, memory also is more awake. It is then I have my 'vivid' dreams, those I remember best. I think, therefore, that my mind as a whole never sleeps. In deepest sleep I dream and weave the sensations that come to me from my body into the fabric of my dreams. Of all my mind my memory alone rests, more or less profoundly, when I sleep; and, when I awake, it is my memory that awakes.

686. The main differences between our sleeping and waking mental experiences arise from the fact that we are unable to store, to profit from, the former. They are not retained in the memory to anything like the same extent as the latter. Moreover, when dreaming we refer to experiences recognized as past much less than when awake. We live mainly in the immediate present, accepting experiences, as they arise, without to any extent consciously associating them with what has gone before. It is thirty years since I studied for the Royal Engineers and more than twenty since I sought to become a medical student. Nevertheless, occasionally I am still rendered miserable when asleep by the thought that I may be 'ploughed' in Latin. All that has happened between youth and middle age is quite forgotten. It is true that the objects we see in our dreams have usually been stored in our memories whence they are evoked; but they do not come to us surrounded, as it were, by a halo of recollections. They are not usually greeted as remembered things. We feel that they are good or bad, delightful or horrifying, just because they seem by nature to be so, not because of the recollections with which they are invested-as if the physical characters in the brain with which they are correlated had developed through the stimulus of ' Isaac Walton. 
nutrition, not through that of use and experience-as if they had developed as Adam's navel is said by the theologians to have developed. Just so might an insect see his hitherto unimagined mate or enemy with delight or fear.

687. I dreamed lately that I sat in a railway carriage suffering from headache, which a commercial traveller who was next to me tried to cure. I felt extremely grateful. At the same time, as quite an independent person who knew nevertheless he was the same person as the sufferer, I sat opposite, and marked the proceedings of the traveller with some professional contempt and disapproval. The personalities of sufferer and observer alternated rapidly, but I accepted without question the fact that I was both. Because in my waking hours I appeal to past experience and therefore am able to reason from it, I know now that the situation was an impossible one; but I did not appeal to it then. In another dream I looked at the top of my own head as I lay asleep and noted with regret but no surprise that I was extremely bald. In my dreams I have flirted with Queen Elizabeth and in the same night have been in heaven and hell and have not been astonished that the one bestowed no more delights than the other. Dreams, being thoughts, are as 'swift as thought': between two intervals of waking-five minutes by the clock-I have dreamed volumes. Yet of all the immense mass of our dream experiences how little we remember! Though, as I say, I appear always to dream when asleep I recollect nothing that occurs during deep sleep unless I am suddenly awakened and at once make the record. Even of the visions of light sleep I commonly remember little. It is significant, that though recollection slumbers, yet all the instinctive emotions may be felt during sleep. On all counts, therefore, it seems evident that the faculty that slumbers more or less deeply during sleep is memory, and more especially the part of memory which is concerned in recording (not recalling) experiences. In other words though we have plenty of experiences during sleep our minds do not then grow under their stimuli. We become like instinctive animals; we neither record facts nor grow dexterous in using them. Since reason deals with the contents of memory and is itself one of the contents, reason also sleeps with a soundness which is in proportion to the soundness with which the memory slumbers. ${ }^{1}$

1 See Sir Arthur Mitchell's most interesting work Dreaming, Laughing, and Blushing, pp. 5-9. It is sometimes said that intoxication is marked by a pro. gressive loss of faculties beginning with the highest, the last evolved, and ending 
688. Do purely instinctive animals sleep? If they do, what is the faculty that slumbers? When they rest, as a fly in darkness, and hardly any stimulus awakens their senses, are they unconscious in a truer sense than we are during sleep? Or do they also see visions? Our visions are supplied by a more or less paralysed memory; what supplies their visions, if they have any? Most of the experiences stored in our memories are acquired through the sense of sight. Is the fact that all animals with well developed memories close their eyes during sleep connected with the fact that their memories need rest? It is true that the eyes of such animals are especially vulnerable, but that peculiarity may be the effect not the cause of the fact that eyes are covered by eyelids. Apparently the eyes of insects see well enough; and yet they are not protected.

689. Many insects, for example solitary wasps, are not protected during the beginnings of conscious life by their parents. Therefore, of necessity, they are fully or almost fully equipped for the battle of life by instinct. Nevertheless they are able to return burdened with loads of food to the cells in which they have deposited their eggs. By what means do they find the road-by instinct or memory? The word memory is used in two senses, one of which implies recollection, and the other bearing in mind. We recollect when we recall an experience which was formerly in consciousness but has passed out of it; we bear in mind when we keep an experience in consciousness. Thus I recollect a blow if, after the memory of it has passed out of consciousness, a sight of my opponent recalls the experience. I bear in mind if I continue to dwell on the experience after having been struck. Probably the kind of memory which is absent in purely instinctive animals is the power of storing experiences so that they may be recalled. Therefore it is possible that the wasp may instinctively bear in mind, during all the vicissitudes of her hunt for food, the situation of her nest; in which case, she is, like higher animals, able to attend to more than one thing at a time. On the other hand, if she recalls the situation of her nest, of which she was previously unmindful, she possesses a true memory; but in that case, since she is very incapable of learning other things ${ }^{1}$ she has a memory with the lower, the more anciently evolved. The interpretation of this appears to be that intoxication, like sleep, is a kind of paralysis by which the memory in particular is affected. The highest faculties and the most skilful actions are those which depend on the greatest amount of stored experience. The ' brute ' muscular strength and the innate mental and nervous characters (instincts and reflexes) are relatively little affected.

${ }^{1}$ See Lubbock, Ants, Bees, and Wasps. 
which is capable of storing only a particular and a very limited set of facts. The latter hypothesis seems, at least, improbable.

69o. From birth to adult age our physical development is due mainly to the stimulus of use. After the attainment of manhood almost all growth which is not pathological or a mere storing of spare nutriment as fat, is due to that cause. The growth thus made during youth is enormous, but it occurs only on certain predestined lines and within well-defined limits. By limiting the amount of use to which this or that structure is put we may limit its growth; by putting the structure to a more than normal amount of use we may increase its size somewhat beyond the 'normal.' But there our power ends. The hand is put to a greater variety of uses than any other organ in the body. Yet, even if we begin with the infant, we can only make it large and coarse, or small and fine. It still remains a hand which closely resembles in size and shape altogether normal hands. It is very different in the case of mind. Consider the millions of 'physical' dexterities we are capable of learning. These are all really mental. For example, our hands do this or that thing dexterously because our minds have learned to direct them readily and rightly. The 'normal' dexterity of the penman differs widely from that of the woodcutter, the sailor's from that of the surgeon. Yet any of them may be acquired by the average young individual. But physical dexterities are as nothing compared to the rest of our mental acquirements ; they are as nothing compared even to the mental acquirements that are most closely related to them. Thus, the architect's manual skill with ruler and pencil is but a small part of his total mental acquirements as architect, and that again is but a small part of his total mental equipment as a man of the world, his other physical dexterities, his acquired mental attitudes, his knowledge of men and things. The average man is capable of becoming an average architect; or, instead, he may acquire skill in one or more of a thousand other occupations.

691. Physically most of us could fill the places of most other men; that is our physical structures are capable of doing the work that their physical structures do. Mentally not one of us is capable of filling the place of any other. I, for example, though as large and strong in body, could not mentally fill the place of a workman with whom I am acquainted. I have not the same skill and mental attitudes which have fitted him into a certain niche, the same prejudices, the same ethics, the same knowledge of his world based on past experience. My religious and political convictions 
are different. I do not love the same old people, the same wife, the same children, the same fireside, the same friends. Yet I might well have fitted his niche had I been reared to it.

692. It is evident, therefore, that while human bodies can grow in only one way, human minds may grow in any one of a thousand ways. As already noted, if the child of refined and educated English parents were reared from birth by African cannibals, then in body, when grown, he would resemble his progenitors more than his trainers. Does anyone believe that the same would be true of his mind? All Anglo-Indians know the disastrous effects of too much association with natives on the plastic minds of white children. They become unfitted for the environment of the average Englishman. Even when an adult young Englishman migrates to the United States or the colonies and returns after twenty years, we perceive marked mental differences between him and his stay-at-home contemporaries. Indeed, he seems to us to have become quite similar to the people with whom he has dwelt, though, doubtless, Americans or colonists, whose observation is sharpened by familiarity can detect differences. The English child we imagined as reared by African savages would certainly display no hint of the language and general knowledge of his parents, no tincture of their moral, social, religious, and political ideals and aspirations. He would ruthlessly murder and enjoyingly eat the stranger. He would harry the stranger's property and annex the stranger's wives by the wool of their heads whenever practical. He would treat his own wives as beasts of burden, and perhaps thrash them as a matter of routine. His rsthetic ideals would be satisfied by a little paint, some beads, and plenty of grease; his moral ideas by a homicidal devotion to the tribal chief. His god would be the tribal fetish, to whom he would offer human sacrifices. He would go naked and unashamed. The common-sense of mankind has universally recognised this radical difference between man's mind and body. We allow our children to train their own bodies, being satisfied that they will develop physically well enough under the influence of sufficient food and the exercise to which the instinct of play impels them; but to the training of their minds we devote the most anxious care. We mould them, and we know we mould them. No one fears that his child will be made short or dark by association with short or dark companions; but everyone dreads that his child may become silly or bad if his associates are silly or bad. Clearly, then, the mind of the individual is 'shaped' by 
his immediate surroundings in a much greater degree than his body.

693. Sometimes it is asked if nature or nurture plays the more important part in the mental development of the human being. As a fact this question is nonsensical. It is as if we asked whether the locomotive or the steam played the greater part in transporting the train. The truth is that nature has rendered man transcendently responsive to the nurture of use and experience. The question should be "To what extent has nature rendered man responsive to the stimulus of nutriment and to what extent to that of use and experience?" Nature has rendered all living beings equally responsive to nurture. The caterpillar is responsive to the nurture of nutriment. Man differs from the caterpillar in that nature has rendered him responsive in addition to the nurture of use and experience. He differs from other animals in that he is immensely the most responsive of all to this kind of nurture. It is the one peculiarity which strongly differentiates him. Mental experience is, after all, only a form of use. We use our minds when we store experiences; or rather, if mind is a function of brain, we use our brains. When, for example, we acquire a new manual dexterity, our brains acquire a new function; through use they learn to do a new thing. Doubtless the acquirement is accompanied by actual growth of brain, which however, especially in adult life when gains are being balanced by losses, need not imply an increase in bulk, but only a relative increase in certain of the brain constituents such as nerve cells or connections between nerve cells. In youth, when man is making the great mass of his mental acquirements including the more important of his 'physical' and mental dexterities (e.g. walking and reasoning), there is rapid growth in the bulk of the brain, which is most rapid just when the acquirements are being most rapidly made. In old age, though the individual may still be making mental acquirements, he is usually losing more than he acquires. Then, while the total mass of his brain does not decrease, the nervous elements tend to be replaced by fibrous tissue.

694. If we wish to avoid hopeless confusion it is necessary to distinguish sharply between two entirely different things; between, on the one hand, capacity to make mental acquirements, and, on the other, the mental acquirements themselves, between the memory and the things that are stored in the memory. The capacity is an 'innate,' a nutritional character; the acquirements arise under the stimulus of experience. Thus a man may have 
exceptional mathematical ability by means of which he achieves considerable mathematical learning if afforded the opportunity. The ability is 'inborn' and tends to be 'inherited' by offspring ; the acquirements are not. That is, the child, if he lives and grows at all, is sure to have food and therefore to develop, unless he has varied, the parental capacity; but he is not sure to have the parental training, the nurture of the same use. Therefore, even if he 'inherits' the parental ability, the child of a great mathematician may, or may not, achieve similar distinction. But such a one is more likely than the child of an ignoramus, not only to inherit ability, but to receive the right training. Without the training the ability is naught; just as without coal the engine is naught. Nutriment, therefore, bestows on the child the parental aptitudes; association with the parent tends to bestow the parental acquirements. Consequently children tend to resemble their parents for a double reason; first, because they tend to have much the same capacities, and second, because they make much the same acquirements. We must bear in mind, however, that the parent is not the only influence in the child's environment. Therefore, not only may the child vary from the parent in capacity, but also he may differ in acquirements.

695. Mathematical efficiency depends on mathematical acquirements made by virtue of mathematical capacity and in response to mathematical experience. The efficiency is impossible without the acquirements, and the acquirements without both the capacity and the experience. The same is true of all other intellectual characters. But the part played in the creation of efficiency by capacity on the one hand, and by experience on the other-by nutriment on the one hand and use on the other-varies with different characters and in different species. The caterpillar apparently owes nothing to acquirement; he has no substratum of capacity wherewith to store experience and therefore cannot utilize it. The acquirements of the cat, for example the increased efficiency in hunting which practice bestows, are, as a rule, nothing more than mere extensions of pre-existing instincts comparable to those extensions of its physical structures which are bestowed by use. Some of man's acquirements are also mere extensions of instincts. Thus, both parental and sexual love tend, within limits, to be increased by association with the objects of affection. Speech is an extension of the infant's tendency to utter useful but inarticulate cries. In this case, however, the extension is immeasurably greater than the thing extended. But unlike extensions 
of instinctive actions and unlike his physical acquirements, most of man's intellectual characters cannot, except through very strained interpretations, be regarded as extensions of previously existing innate characters. Thus many of his manual dexterities, his efficiency in reading, his devotion to a religion, or an ethical code, resemble nothing instinctive in him. They are wholly ' acquirements.'

696. We must distinguish not less sharply between instinct and acquirement, than between capacity and acquirement. A glance at the literature of the subject demonstrates that numbers of human characters are accepted as innate on inadequate evidence. Modesty is supposed to be innate. But what kind of modesty? that of the Mohammedan women, or that of the nun, or that of the savage? Savage women have no modesty in the European sense, but I have known mission natives exhibit an extraordinary, and, as it seemed to me an Englishman, an absurd degree of prudishness. In Tonga, for untold centuries, the natives went about naked and unashamed, and, judged by English standards, sexual intercourse amongst them was almost promiscuous. A few years later mere flirtation was regarded as a horrible crime and was legally punishable. It is impossible to doubt that English women trained by savages would show no trace of the traditional modesty of their race. Modesty is not an instinct, but an acquirement dependent on memory. The only instinct with which it is connected is that of imitation. Morality also is supposed to be an instinct. But what kind of morality? Like modesty, morals vary with time and place. Real instincts are universal in the species, and in measurable time unchanging. We often read of the special instincts of savages, for example their instinct for tracking game. But no savage, having all the while no notion of the aim or end of his actions, has an innate impulse to follow certain marks on the ground till he catches a kangaroo or a deer. Manifestly his so-called instincts are acquirements. He excels the civilized man for the same reason that the native Frenchman excels the foreign resident in the perfection of his French-because, he has learned at the most receptive age.

697. Even characters which have a large instinctive element are extended, modified or even in some cases in a sense suppressed in the human being by acquirement. Appreciation of sexual beauty is an instinct, but the kind of beauty admired depends greatly on acquirement. As a rule we admire women who are decorated in the latest fashion. At the present day we can 
perceive no element of beauty in the chignon or crinoline of the, as it seems to us, terribly inartistic Victorian era; but our fathers were ravished by the appearance of the women who wore them. Various savages admire mutilations which are horrifying to us. Indeed almost all races practise some form of mutilation and find it beautiful; thus we crop and shave the magnificent human mane which, judging from its great evolution, must have been a principal instrument of fascination amongst our remote ancestors. I, personally, am nauseated by the sight of long hair on a male human head. Doubtless sexual jealousy is in a measure instinctive; yet while the husband is jealous of the lover, the latter is seldom jealous of the former; were the emotion purely instinctive they should be equally jealous. The jealousy of an Australian black is as nothing compared to that of the Mohammedan. Parental affection is an instinct, yet many people have cheerfully practised infanticide; and the average parents of various races are good or bad according to the prevailing fashion. Perhaps the most convincing evidence that parental actions are mainly founded on acquirement is afforded by the fact that civilized parents whenever possible tend to delegate them to the hands of servants. The actions to which they are prompted by purely instinctive impulses, for example thirst, hunger, weariness, and sexual love are never delegated.

698. To sum up: the distinguishing peculiarity of the human being as compared to other animals is that his characters arise much less under the stimulus of nutriment and much more under that of use. Most of his nutritional characters are mere foundations on which 'acquirements' are reared. Biologists commonly suppose that innate characters are much more important than acquirements. But if, were it possible, we deprived an adult human being of his acquirements nothing would be left but the likeness of a new born infant which possessed the sexual instinct, and a few adult physical traits such as hair, teeth, external ears, and organs of generation. Manifestly the physical and mental maturity of the normal human being depends mainly on acquirements, the making of which is just as essential a part of adult development as the growth of heart and limbs is of fotal development. This truth, though opposed to scientific tradition and on that account rarely recognized, is really a matter of common knowledge and is so plainly true that I think it has only to be formally stated to be recognized as true. It may be that there is more of what is innate and less of what is acquired in this or that human character than I have been led to 
believe. But the fact that human acquirements, especially mental acquirements, are of immensely greater magnitude and importance than is commonly supposed cannot, I believe, be doubted.

699. A little thought renders it evident that the essential defect of the feeble-minded person, the idiot, and the imbecile, is lack of memory. ${ }^{1} \mathrm{He}$ is unable to profit like the normal individual from experience. This defect of memory may be, and usually is, general, so that the individual is able to learn very little. Or the defect may be limited to some particular; for example the individual may be unable to acquire the code of morals prevalent in the community in which he exists-an acquirement which on the average and in the long run would be of great advantage to him. The truth that the higher intellectual faculties are less developed in the feeble-minded than lower faculties is due entirely to the fact that the former can be acquired only by people whose receptive powers are considerable. In effect and in fact the feeble-minded person is an instance of reversion to a pre-human mental state. Judged by the human standard every dog and monkey is an imbecile. But the reversion of the imbecile is not complete; for, while he has lost part of his power of profiting by experience, he has regained no part of the lost power of being guided by instinct. Therefore he is correspondingly helpless as compared to a lower animal. On the other hand, the instincts (e.g. the sexual) which normal human beings still possess often appear unduly prominent in him; but only because he cannot learn to control them.

700. But though human beings develop mainly under the stimulus of use and experience, doubtless they differ 'by nature' amongst themselves just as much as animals that develop solely under the stimulus of nutriment. In the last analysis all variations are nothing other than variations in powers of responding to stimuli-nutriment, use, and injury-and we have no reason to suppose that variations in the capacities for responding to the stimulus of use are smaller or fewer than variations in the capacity for responding to the stimulus of nutriment or injury. Doubtless, therefore, men vary not only in general mental capacity (i.e. power of making mental acquirements) but also in particular departments of capacity. At the one extreme is the absolute idiot who has no power of storing and utilizing experiences, and, at the other, the genius who has exceptionally great powers. It has been observed that some imbeciles have considerable capacity in some particular department. Thus, they may have great powers of remembering 
this or that class of facts, but no power of learning to utilize them. So also, while it is possible that some geniuses may be men endowed with exceptional all-round capacity, they are usually distinguished from the average type by exceptional capacity in some particular department of mental activity. It is probable, for example, that Shakespeare had more poetic capacity (i.e. power of responding to poetic experiences, of recording and learning to utilize such experiences) and less artistic capacity than Michael Angelo, who presumably had less mathematical capacity than Newton, who in turn had less military capacity than Napoleon, who again was inferior in philosophic capacity to Darwin.

701. But, admitting all this, it must still be borne in mind that (I) the stimulus of nutriment is capable of developing the bodies and minds of men in only one way; that is, we cannot by changing the kind of nutriment which the human being is capable of assimilating, produce different kinds of bodies and minds; under all kinds of nutriment a man will still have much the same sort of limbs, lungs, sensations, instincts, and memory; (2) changes in the kind of stimulus of use cannot produce very great changes in the bodies of men; as in the case of nutriment, they may develop this or that organ more or less according to the amount of use supplied, but that is all ; limbs and lungs and other structures will still be of the same kind no matter what the stimulus; (3) but differences in the stimulus of experience supplied to the mind are capable of causing tremendous differences in mental development. For example, while the bodily parts of a yokel (e.g. his brain) differ little in appearance from that of a cultured man, the mental difference is obviously very great. It follows, that in comparing men or races we are on safer ground when we suppose that their bodily differences are innate (i.e. germinal) than when we suppose that their mental differences are innate. Doubtless, as I say, differences in mental capacity are just as great as innate physical differences ; but they are apt to be overshadowed and concealed by vaster acquired differences.

702. Perhaps the most convincing evidence that human mental and moral characters arise mainly under the stimulus of experience is afforded by the history of races and nationalities. Like individuals, races differ in their mental characteristics. Thus the English have one set of characteristics, (knowledge, ideals, and so forth), the Japanese a second, the Russians a third, and West Coast Africans a fourth. Most historians and men of science 
adopt the easy view that the mass of these differences are innate in the same sense that physical differences are innate.

703. Man is a very slow breeding animal. Innate changes of importance cannot occur in his race at all swiftly-in the course of a few centuries-except under excessively stringent selection; and, owing to the strong tendency to retrogression thereby created, such changes can only be maintained afterwards by selection almost as stringent and continued over a very long period. But, in the absence of all recognisable selection, some races have changed their mental characteristics with great rapidity. Thus the Greeks and Romans, separating quickly from the surrounding barbarians, displayed, for a few centuries, extraordinary intellectual qualities, and then sank, quite as suddenly, into abysmal degradation. At the Renaissance and the Reformation, after more than a thousand years of stagnation, various European nations began to display qualities comparable to those of the great Pagans. Their material and intellectual progress has continued to the present day; but even now, when we are able to watch contemporaries, we are unable to detect any form of stringent selection save that by disease. The Japanese furnish a very striking modern instance. Another similar instance is afforded by the fact that, in a single generation, the ferocious cannibals of New Zealand have become progressive and law-abiding. Nothing apparently prevents them taking an equal place with the whites as citizens of a progressive and civilised state except their physical inability to withstand imported disease.

704. Every race that has changed its religion has immediately undergone a great moral and intellectual change. The inhabitants of Syria and North Africa are examples. At first they were Pagans and shared to some extent the Greek and Roman characteristics; next they became typical Christians of the Dark Ages; lastly they became typical Mohammedans,-fierce, turbulent, fanatical, stagnant. Races of diverse origin, which follow a given religion, resemble one another more closely in mental characteristics than sections of the same race which follow diverse religions. Thus all Mohammedan nationalities have mental peculiarities which separate them sharply from Christians on the one hand and Pagans on the other. Mohammedan Greeks mentally resemble their co-religionists more nearly than their compatriots. The Teutonic Catholics of Ireland are quite indistinguishable from the Celtic Catholics, but they differ sharply from Protestant Teutons. Catholic negroes are typical Catholics of the more ignorant type ; 
the negro Baptists and Methodists of the United States are in many respects typical Baptists and Methodists resembling those of Wales. Jews who abide by their religion retain their characteristics; those who abandon it merge indistinguishably into the surrounding population. Indeed all the world over peoples of the same colour are divided mentally much more sharply by religious differences than by anything else.

705. To me it appears, if only we think carefully enough and take all the facts into account, that it is impossible to come to conclusions other than the foregoing. They amount to nothing more than is implied in the statement that man is mentally an extremely adaptable, malleable, educable, intelligent, and intellectual animal; and, therefore, that he is much more a creature of habit than of instinct. Educability is nothing other than a power of growing mentally under the stimulus of experience; intellectuality is nothing else than a having so grown.

706. Some authorities, however, founding themselves on statistical inquiries, are of a contrary opinion. At least they claim to have proved that man is less educable, less a creature of acquirement, than I have supposed. Thus Professor Karl Pearson ${ }^{1}$ issued schedules to school-teachers and requested them to supply information as precise as possible concerning certain mental and physical characters of (I) pairs of brothers, (2) of sisters, and (3) of brother and sister. Some of the physical characters were capable of being measured. Others were estimated, as, of course, were all mental characters. The physical characters included health, athleticism, length, breadth, and height of head, colour, smoothness, waviness, or curliness of hair, and colour of eyes ; the mental characters included ability in various studies, noisiness or quietness, self-consciousness, self-assertion, shyness, conscientiousness, popularity, and temper. Pearson chose children rather than adults owing to the difficulty of obtaining correct information concerning the latter. "In the first place it seemed to me absolutely impossible to get a quantitative measure of the resemblances in moral and mental characters between parent and offspring. You must not compare the moral character of a child with those of its adult parents. You can only estimate the resemblance between the child and what its parents were as children. Here the grand-parent is the only available source of information; but not only does age

1 "On the Inheritance of the Mental and Moral characters in Man, and its Comparison with the Inheritance of the Physical characters." The Huxley Lecture for 1903. The Anthropological Institute of Great Britain and Ireland. 
affect the clearness of memory and judgment, the partiality of the relative is a factor which can hardly be corrected and allowed for." 1 He argued that resemblances between children of the same family is as clear a proof of inheritance as resemblance between parent and child-in which, doubtless, he is right.

707. It was found that, on the average, the children of the same family resembled one another as closely mentally as physically. Pearson admits that certain physical characters such as size of head and body are influenced by 'nurture, food, and exercise,' but since brothers and sisters resemble one another in these traits in the same degree as in such physical characters as the cephalic index, and the colour of hair and eye, which are not affected by 'home influence,' he concludes that the environment is not a disturbing factor when calculations are made respecting the degree of resemblance due to inheritance. A similar line of reasoning is applied to mental characters. In effect his conclusion amounts to this-that if, as regards any set of characters, brothers and sisters resemble one another on the average in the same degree, then all the resemblances must be due to the same cause. For example, if the resemblances are equal as regards geniality, probity, eye-colour, hair-colour, and the cephalic index, then geniality and probity must be inherited in the same sense as the physical characters mentioned. "The sameness surely involves something additional. It involves a like heritage from parents. The degree of resemblance between children and parents for the physical characters in man may be applied to the degree of resemblance between children and parents for psychical characters. We inherit our parents' tempers, our parents' conscientiousness, shyness and ability, even as we inherit their stature, fore-arm and span." 2

708. But eye-colour cannot be influenced by environment, whereas there is at least some reason to believe that geniality and probity may be so influenced, and the environment of different families differs greatly. Pearson meets this difficulty by the statement that "We are too apt to overlook the possibility that the home standard is itself a product of parental stock, and that the relative gain from education depends to a surprising degree on the raw material presented to the educator. We are agreed that good homes and good schools are essential to national prosperity. But does not the good home depend on the percentage of innately wise parents, and the good school depend quite as much on the children's capacity, as on its staff and equipment?" 3 According
3 P. 180.
2 Loc. cit., p. 204.
${ }^{3}$ Loc. cit., pp. 179, 180. 
to Pearson, then, there must be, on the average, a tremendous germinal difference between Englishmen of the upper classes and those of the lower, between Englishmen of the country and those of the town, between Englishmen of to-day and those of a century or two ago, between Englishmen and foreigners, between Greeks and Romans of the period of national greatness and their immediate predecessors and successors, between Greek Mohammedans and Greek Christians, and so forth; and an English child reared by African cannibals should develop the moral and intellectual as well as the physical characteristics of his progenitors not of his educators.

709. Moreover, "If the conclusion we have reached to-night be substantially a true one, and for my part I cannot for a moment doubt that it is so, then what is its lesson for us as a community? Why simply that geniality and probity and ability may be fostered indeed by home environment and by provision of good schools and well equipped institutes for research, but that their origin like health and muscle, is deeper down than these things. They are bred, not created. That good stock breeds good stock is a common-place of every farmer; that the strong man and woman have healthy children is widely recognized too. But we have left the moral and intellectual faculties as qualities for which we can provide amply by home environment and sound education."1... "Looking round dispassionately from the calm atmosphere of anthropology, I fear there really does exist a lack of leaders of the highest intelligence, in science, in the arts, in trade, even in politics. I do seem to see a want of intelligence in the British merchant, in the British professional man and in the British workman. But I do not think the remedy lies solely in adopting foreign methds of instruction or in the spread of technical education. I believe we have a paucity, just now, of the better intelligences to guide us and of the moderate intelligences to be successfully guided. The only account we can give of this on the basis of the results we have reached to-night is that we are ceasing as a nation to breed intelligence as we did fifty or a hundred years ago. The mentally better stock in the nation is not reproducing itself at the same rate as it did of old; the less able, and the less energetic are more fertile than the better stocks. No scheme of wider and more thorough education will bring up in the scale of intelligence hereditary weakness to the level of hereditary strength. The only remedy, if one be possible at all, is to alter the relative fertility of the good and bad stocks in the community. Let us have a census 
of the effective size of families among the intellectual classes now and a comparison with the effective size of families in the like classes in the first half of the last century. You will, I feel certain, find, as in the case of recent like censuses in America, that the intellectual classes are now scarcely reproducing their own numbers, and are very far from keeping pace with the total growth of the nation. Compare in another such census the fertility of the more intelligent working man with that of the uneducated hand labourer. You will, I again feel certain, find that grave changes have taken place in relative fertility during the last forty years. We stand, I venture to think, at the commencement of an epoch which will be marked by a great dearth of ability. If the views I have put before you to-night be even approximately correct, the remedy lies beyond the reach of revised educational systems; we have failed to realize that the psychical characters, which are, in the modern struggle of nations, the backbone of state, are not manufactured by home, school and college; they are bred in the bone; and for the last forty years the intellectual classes of the nation, enervated by wealth or love of pleasure, or following an erroneous standard of life, have ceased to give us in due proportion the men we want to carry on the ever-growing work of our empire, to battle in the fore-rank of the ever intensified struggle of nations.

7 10. "Do not let me close with so gloomy a note. I do not merely state our lack. I have striven by a study of the inheritance of mental and moral characters in man to see how it arises, and to know the real source of an evil is halfway to find a remedy. That remedy lies first in getting the intellectual section of our nation to realize that intelligence can be aided and can be trained, but no training or education can create it. You must breed it, that is the broad result for statecraft which flows from the equality in inheritance of the psychical and physical characters in man." 1

7 II. Pearson adopts the ordinary view that the body and mind of the individual are compounded of 'innate ' and 'acquired ' characters, that only the former tend to be 'inherited,' and, therefore, that evolution (or any sort of intrinsic racial change) depends entirely on them, acquirements being mere somatic 'modifications.' On the other hand, the view upheld by me has been that no kinds of characters are more innate or inheritable than any other kind, that the terms 'innate' and 'acquired' are misnomers and the causes of endless confusion, misunderstanding, and misinterpretation,

1 Loc. cit., pp. 206-7. 
that the so-called innate characters are simply characters which have developed under one kind of stimulus (that of nutriment) whereas the so-called acquirements are equally important characters which have developed under other kinds of stimuli (those of injury and use), that all evolution (or any sort of intrinsic racial change) consists in a germinal alteration which implies increased or decreased capacity to develop in a definite way under the influence of this or that stimulus. According to this view the superior innateness and inheritability of nutritional characters are only in seeming. They develop more certainly than acquirements merely because the stimulus of nutriment is always present in every individual who develops at all, whereas the stimulus under which this or that acquirement arises may be absent. Moreover, I have supposed, not only that the evolution of the higher animals has consisted mainly in a continually increasing power of developing under the stimulus of use, but also that it has consisted in a concurrently decreasing power of developing under the stimulus of nutriment-the former power possessing great advantages over, and therefore displacing the latter.

712. Of all this evolution, which to me appears to be so manifest, Pearson seems unaware. As results of our differing opinions, the moral and intellectual characters are to Pearson instincts; whereas I cannot conceive how such characters can be instincts. An instinct, as I understand it, is merely an emotional impulse, the prompting of which the individual is sure to follow unless it is opposed by other instincts or acquirements. It is utterly unintelligent, utterly non-moral. It has nothing to do with thought except as a subject of thought, or with a sense of right and wrong except in so far as this sense approves or disapproves of its promptings. Moral and intellectual characters, as proved by unlimited evidence and from their very nature, are acquirements. Taking all the facts into account, I cannot imagine a purely instinctive animal, a beetle or a caterpillar, as moral or intellectual, or a human being as such except by virtue of the characters supplied by his memory. In effect, the moral and intellectual beings that Pearson imagines, since they are not moral or intellectual through acquirement, are imbeciles. ${ }^{1}$

713. The differences between Pearson and me are so great that it is useless to discuss details in this place. Directly or indirectly I have already done so, supporting my own opinion in almost every chapter of the present volume by a mass of evidence which is enormous, and which, however new (or newly applied), 
cannot, I think, be controverted. ${ }^{1}$ As far as I am able to judge, I have drawn no illegitimate inferences. Pearson's reasoning, in the very cases which he instances, appears to me demonstrably wrong. His main inference is that, since offspring resemble one another in the same degree in, for example, probity and eye-colour, therefore these characters are both 'innate.' But, if his reasoning is valid as regards the mental and physical characters named by him, it should be valid also as regards other characters not named. Now English school children resemble one another absolutely in that they all possess heads, lungs, livers, and so forth. Here the degree of resemblance is ' unity.' They reproduce these characters with much greater certainty than eye and hair-colour and variations in forearm and span. They also resemble one another absolutely in that, when they have reached a certain age, they can all read, write, and speak the English language, and in that they have English notions of modesty with respect to clothes. Here, again, the degree of resemblance is roughly 'unity.' At any rate they reproduce these characters with much greater certainty than they do eye-colour and the like. Moreover, in thecase of the boys, there is a mental tendency, almost as universal as their sex, to wear trousers; in the case of the girls, to wear frocks. The children would be infinitely reluctant to appear at school without these garments. Must we suppose, then, that this sameness involves a like heritage, a similarity of the germ-plasm, and that English children 'inherit' these characters, which are universally regarded as acquirements and would probably be admitted as such by Pearson, with a degree of certainty which is almost as great as that with which they inherit heads, livers, and sexual organs? I do not mean in the least to be offensive in the examples I have drawn. I have selected them merely in order to indicate as strongly and vividly as possible the reasons that have led me to think that Pearson's thinking, founded as it is solely on biometric data, though much more massive and conclusive evidence is available, and quite untested as it is by any appeal to reality, is totally mistaken and illegitimate. Biometry is capable of demonstrating the degrees in which given characters tend on the average to be reproduced by offspring under given conditions. But, if we wish to ascertain the category to which any of these characters belongs -whether it is 'inborn' or 'acquired '-we must draw an inference which biometry, a mode of observing not of thinking, cannot help us to draw ; and which, therefore, must be tested as carefully as when our facts have been otherwise gathered.

1 See also chapter xxv. 
714. When we compare one modern species with another, the more intelligent type invariably has, proportionately to the size of its body, a larger brain. Thus the dog has a larger brain than a rabbit, and a man than a dog. Presumably, therefore, intelligence and size of brain are correlated in some degree at least. Doubtless the correlation is not absolute; for brains vary in their constituent elements, for instance, in the relative amounts of grey and white matter. "The leading feature in the development and separation of man from amongst other animals is undoubtedly the relatively enormous size of the brain in man and the corresponding increase in its activity and capacity. It is a very striking fact that it was not in the ancestors of man alone that this increase in the size of the brain took place at the same period, viz. the Miocene. The great mammals, such as the titanotherium, which represented the rhinoceros in early Tertiary times, had a brain which was in proportion to the bulk of the body, not more than one-eighth of the volume of the brain of the modern rhinoceros. Other great mammals of the earlier Tertiary period were in the same case; and the ancestors of the horse, which are better known than those of any other modern animal, certainly had much smaller brains in proportion to the size of their bodies than has their descendant."1

7I5. Biometricians, however, founding their opinions on statistical data, have denied that the law that intelligence is correlated to size of brain obtains in the case of human races and individuals. According to them "there is no marked correlation between skull capacity and intellectual power." What, however, is the real meaning of their facts, for there can be little doubt that the facts are correct? The mind manifestly grows under the stimulus of experience. Mental facts are correlated to cerebral facts. Therefore, we have every reason to believe that the brain grows under the stimulus of use. We know, in fact, that if one hemisphere of the brain atrophies from any cause, such as disease, the opposite hemisphere tends to hypertrophy; and we cannot account for this increase except by supposing that it results from increase of function. After birth, then, the growth of the mind and brain is conditioned, on the one hand, by the inborn capacity to grow under the stimuli of experience and use, and on the other, by the amount of stimulus. Individuals may differ both with respect to the capacity and the amount of stimulus their minds and brains receive. It follows, within limits that vary with the individual,

1 The Kingdom of Man, by Sir Ray Lankester, p. 22. 
that the growth of the brain after birth is in some degree proportionate to the extent to which it is used. But the degree of intelligence achieved does not depend solely on capacity and amount of experience. It depends also on the kind of experience. An individual may acquire a false and foolish conception of his total environment with as much toil as a true and wise one. Mediæval monks had on the average larger brains than their contemporaries, and the Chinese as a race have larger brains than most races, but it does not follow necessarily that they were, or are, more intelligent. The real problem, therefore, is not whether there is a correlation between skull capacity and intellectual power, but whether the capacity to develop a large brain is correlated to a capacity to become exceptionally intelligent in one or more directions.

716. The right conclusion to which the biometric facts, combined with the rest that we know, lead us, appears to me, not that the men who can develop large brains have on the average no greater power of becoming intelligent than men who can only develop small brains, but that the former may be worse trained and therefore less intelligent. In other words, the biometrical facts tend to demonstrate that right mental training is of as much importance as capacity. 


\section{CHAPTER XXIII}

\section{PHYSICAL DETERIORATION AND MICROBIC DISEASE}

Summary-The factors in development-Methods of improving development -Human selective breeding-The effects of diet-Of exercise-Of mental training - Physical deterioration-The opinions of biometricians-Of medical men-The right method of improving the physique of urban populations-Microbic diseasesExternal and internal sanitation-Contagious, water-borne, insect-borne, earthborne, and air-borne diseases.

717.

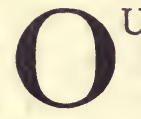

UR study of heredity from the purely scientific standpoint is ended. We have reached certain conclusions, certain broad principles, 'laws,' or, 'brief, simple, and comprehensive classification of facts,' from which necessary consequences may be deduced. ${ }^{1}$ No characters are really hereditary; or, if the term be admitted, all characters are equally hereditary. Characters differ, not because some are inborn and inheritable while others are acquired and not inheritable, but because they arise under different classes of stimuli. Evolution is only another name for adaptation, and in the last analysis all adaptation results from the Natural Selection of favourable variations. A variation is due to an alteration in the germ-plasm. Variations are either 'spontaneous' or due to the direct action of the environment on the germ-plasm. The latter class of variations are relatively rare, and can very seldom, if ever, be other than unfavourable. Therefore Natural Selection builds solely, or almost solely, on spontaneous variations, and a main part of its work is, on the one hand, to provide for the occurrence of spontaneous variations and regulate their magnitude, and, on the other, to render the germ-plasm insusceptible to the direct action of the environment. It follows that the variability of species and characters is not a fixed quantity; on the contrary, each species and each of its characters tends to be variable, on the average, in a degree best suited to secure adaptation. In other words, the tendency to vary, displayed by all living beings, is itself an adaptation which is subject to variations, by means of which Natural Selection regulates the amount of variability and provides for the occurrence of variations all round 
the specific mean. It follows also that the germ-plasm of every species is less susceptible to the direct action of influences to which it has long been subjected than to influences of which it has had little or no previous experience. All, or nearly all, the variations which furnish material for Natural Selection are fluctuations; that is, they are small plus or minus variations of characters previously existing, and, even so, not of the whole character but only of parts or qualities of it. By their accumulation and blending during ages, old characters are lost and new characters created; or, more commonly, old characters are so modified as to be in effect new creations. All development is an abbreviated and inaccurate recapitulation of the life-history of the species. Retrogression plays as important a part in evolution as progression. Variations are either progressive or retrogressive. Natural Selection has so dealt with species that retrogressive variations tend to predominate somewhat over progressive variations because they tend to be more numerous, or larger, or prepotent in the blend, or on all these accounts. As a consequence all characters tend to retrogress on cessation of selection. Species fit their environments so closely, and their physical and mental parts and qualities interlock so exquisitely, that in nature mutations can rarely be adaptive. Owing to their size, and to the alternative mode in which mutations tend to be reproduced, they have an appearance, but only an appearance, of great stability. When reproduction is bi-parental and the species is sexually dimorphic, all individuals have three sets of characters, a non-sexual set of characters all of which are patent, and two sets of sexual characters of which one set is patent and the other latent. The function of sex is to blend characters, and the ultimate effect of blending, combined with the prepotency of retrogressive variations, is to cause the retrogression of useless variations and characters and to make evolution depend, not on the persistence of the variations of individuals, but on the persistence of the blended variations of the race as a whole. All biparental inheritance is blended, the apparent non-blending of the sexual and Mendelian characters being due to the fact that the patent set from the one parent blends with the latent set from the other. Natural Selection differs from Artificial Selection in that the former is founded mainly on fluctuations, whereas the latter is founded mainly on mutations. Mendelian reproduction is an anomaly of sexual reproduction whereby non-sexual characters are reproduced and blended in the same mode as sexual characters, one of each allelomorphic pair being patent and the other latent. 
The present progressive evolution of man, at any rate of civilized man, is chiefly, if not exclusively, against disease which is, apparently, the only selective agency acting on him sufficiently stringent to do more than merely maintain characters previously evolved. Human beings differ from lower animals mainly in that they owe their development, especially their mental development, to a much greater extent to the stimulus of use and experience.

718. The evidence on which these conclusions are based is very massive and appears to me conclusive. But the reader is now in a position to judge for himself. Some evidence remains for consideration, but it can be dealt with conveniently as we apply our 'laws' to the practical problems of human life. The following are the factors in all development: (I) capacity for growth in directions more or less fixed in every species, but so differing with different species that the latter differ in their characteristics; (2) stimulus which awakens the capacity; and (3) nutriment which supplies the material for all growth (as well as the stimulus for much of it). Capacity for growing physically and mentally, for responding in such and such a way, in such and such a degree, to such and such a stimulus, depends on the antecedent evolution of the race and the variations of the individual. A variation, indeed, is nothing other than an alteration of capacity to grow, an alteration which is founded on an alteration of the germ-plasm. Capacity arises in the race through slow processes of selection; and can be altered in it only through selection or cessation of selection. It is that with which every individual begins life, and is wholly an 'inborn' or nutritional character which cannot be increased in the individual by use though it may be diminished or lost in him through injury. ${ }^{1}$ It must be borne in mind, however, that the effect produced by such alteration of stimulus is limited by the capacity of the individual ; thus, no matter how a given man be fed or trained, he cannot achieve more than a certain degree of height, strength, or capacity.

719. If, then, we desire to improve a human race, two ways of attaining our aim are conceivable. (I) We may follow the plan of Nature and of plant and animal breeders and alter by selection the racial CAPACITY for growth in this or that direction; or (2), by altering the conditions under which the individuals of the race

1 If anything may be rightly described as innate it is capacity. In a sense all capacities for growth lie latent in the fertilized ovum, and, with variations, are passed on to the descendent germ-cells. Thus mathematical knowledge is a pure ' acquirement ' in every sense of the term; but that which enables it to arise under the stimulus of experience has its roots in the ovum. 
develop, we may alter the kind and amount of STIMULUS they receive. Thus, if we wish to increase the size and strength of Englishmen, we must breed from individuals who grow big and strong under present conditions, or we must try so to improve the surroundings that, with the same average capacity for growth, individuals will grow bigger and stronger than they do at present.

720. Human selective breeding presents obvious difficulties. It is possible that in a future not very remote some control will be exercised by law or an enlightened public opinion over the multiplication of particularly undesirable types, for example, imbeciles and people very susceptible to tuberculosis or the charm of alcohol. But selection with a view, not merely to prevent marked deterioration, but to raise the general standard of the race is at present an impracticable dream. Moreover, even were such selection practical it would have to be undertaken with the greatest caution. Mistaken notions as to what characters are desirable in human beings might entail disastrous and not easily remedied consequences. That mistakes in human breeding might easily be made is evident from the fact that many races, including our own, practise mutilations as a means of improving beauty, and that the majority of the people of all races greatly admire and strive to perpetuate a very real kind of stupidity. ${ }^{1}$ On the other hand, it is comparatively easy both to alter the conditions under which development occurs, and, even in the very next generation, to remedy altogether any mistakes that might be made. Clearly, therefore, it is better to exhaust the possibilities which may be achieved by such means as improved food, housing, and physical and mental training, before we attempt to tread the difficult and dangerous path of selective breeding.

72I. It is important, therefore, to ascertain as precisely as possible what characters in human beings it is practicable to improve by altering the environment, to what extent they may be thus improved, and by what means the improvement may be brought about. Only so shall we reach that bed-rock of fact and clear understanding without which the discussion of such great problems as physical and mental deterioration, alcoholism, public health, social and moral reform, education, and the like, is vague and unprofitable. I am not aware that any very thorough-going endeavour to analyse the characters of living beings, especially human beings-any endeavour to separate the 'inborn' from the 'acquired' - has been attempted hitherto. I say especially human

See $\$ \S 805$ et seq. 
beings because, owing to more intimate knowledge, we are better able to analyse our own characters than those of other types. As far as I am able to judge, the apparently fundamental divergencies of opinion which separate various classes of scientific workers (biometricians, Mendelians, and those who endeavour to take other classes of facts into account), social reformers, educationalists, and the like, are due mainly to this neglect. Every section appears to the others to be building on a basis of unproved assumption. A common platform is lacking. In attempting the task of analysis it is only too probable that like most other pioneers I shall often be in error. Nevertheless, however wrong I may be in the conclusions I reach, yet at least the reader will be placed in possession of a good deal of material evidence which he will be able to link up with that already set forth in this volume, and apply to the problem as to whether it is possible to obtain improvement in this or that character (e.g. intelligence) by altering the conditions under which individuals develop, or whether improvement is possible only through selective breeding. It should be borne in mind constantly that selection alters only capacity for development, whereas improved surroundings alter only the stimuli which awaken capacity. Apart from selection, then, improved human development may be achieved by altering the supply of nutriment, by removing causes of injury, and by altering the training which the body and mind receive.

722. All human diets contain certain necessary constituents, water, proteids, carbohydrates, various salts, and the like. Almost all diets, whether vegetable, animal, or mixed, contain in excess one or more of these constituents, and a deficiency of others. If in any diet the quantity of a necessary constituent is deficient, the instinct of the individual prompts him to eat till the deficiency is supplied, the excess of the other constituents being wasted, or within limits which vary with the individual, stored in the tissues. ${ }^{1}$ For this reason a mixed or varied diet is usually the most economical, though the variations of the individual and the kind of life he leads must be taken into account. We tend instinctively to grow tired of a diet which contains this or that constituent in excess, and to turn with avidity to one which has less of it, but more of a constituent in which the first was deficient. Under

1 This power of storing excess of nutriment in the tissues, especially as fat against a period of scarcity is, speaking relatively, of little use to the average civilized man whose supply of food is regular and secure; but it must have been very useful to his remote ancestors, as it now is to many lower animals-for example, bears during hybernation or insects when undergoing metamorphosis. 
special conditions, as when doing hard muscular work in cold climates, we long for the particular constituents, for example, fat and sugar, which are then especially needed. Excess of this or that constituent may cause injury (e.g. gout) in susceptible individuals ; individuals may vary unfavourably so as to desire excess; and often individuals develop abnormal and injurious tastes, as for alcohol or opium. We shall consider these abnormal desires separately; but meanwhile it may be stated in general terms that instinct impels the individual to consume about the right quantity of nutriment, and to combine the right constituents in about the right proportions.

723. Such nutriment as the normal individual receives, penetrates to all parts of his body and nourishes all his tissues. It is possible to control the quantity taken and so produce the general effects of extreme starvation, or over-feeding, or anything between ; but beyond this, our power is very limited. For example, we cannot by special forms of diet increase the size of the muscles as compared to the glands, or suppress one mental trait (e.g. the sporting instinct) while developing another (e.g. mathematical talent). ${ }^{1}$ Apparently when nutriment is in excess no tissue uses an excessive quantity of food, though some structures eliminate the excess, and others store it for the future needs of the whole body. When the supply is defective, some tissues suffer less than others. Thus, inactive structures which need little nutriment, such as bones, or very important structures (e.g. the heart), seem then to develop better than more active or less vitally important parts such as the voluntary muscles. Again, wholly 'inborn' parts, those for which nutriment supplies not only the material, but the stimulus for growth, appear to suffer less than structures which grow under the stimulus of use. Thus the hair, teeth, eyes, external ears, instincts, memory, and the like, of a half-starved child, seem to develop better than its limbs or its mental acquirements, and the structures of a fœtus, all or nearly all of which are wholly 'inborn,' appear to have the advantage in their competition for nutriment with the physical acquirements of an ill-nourished mother.

724. Except in the case of certain unfortunate sections of the community, the British are a well nourished race. Both the quantity and the quality of their nutritive supply are sufficiently

1 Though this is true of human beings it is not true of all animals. Bees, for example, develop into workers or queens accordingly as they are fed on one kind of food or another. 
good, and where they are defective, the effects and the remedy are obvious. It follows, since human diet, consisting as it does of certain essential constituents, cannot be greatly varied, since nutriment penetrates to all parts of the individual, since the supply of nutriment is usually sufficient, and since instinct prompts to the consumption of about the right kind and quantity of nutriment, that we cannot hope to improve materially such a people as the English by altering its nutritive supply. Especially we cannot hope to improve its inborn traits-its capacities for development, and those characters which arise under the stimulus of nutriment. If, therefore, we wish to alter them, the only conceivable method is selection. Probably, however, no one would wish to use a method so difficult to manage rightly, so destructive of liberty, so fraught with suffering to the innocent as selection, merely to change the shapes and sizes of such physical structures as ears, eyes, lungs, and the like, and no one who understands the functions of the instincts would lightly wish to tamper with these exquisite mental adaptations. It would be excellent, of course, if we exalted the potential physical and mental powers of Englishmen by increasing their innate capacity to develop under the stimulus of use and experience; but it is so much easier, so much more within the range of practical politics, to alter stimuli by changing the conditions of life than to alter capacities by selection that, before attempting the latter, we should, as I have indicated in general terms, be very sure that the conditions under which the British dwell are such as ensure the utmost development and the best kind of development possible. Only after we have ascertained beyond reasonable doubt that nothing more is achievable by altering the conditions can selection be desirable.

725. The different parts of the human body develop under the stimulus of use on lines almost as rigidly fixed as those on which they develop under the stimulus of nutriment. Indeed, the physical growth made under the former stimulus is usually a mere extension of that made under the latter. For example, a human limb develops after birth on lines very similar to those on which it developed before. When the direction or character of the growth is changed it is usually by injury, not by use. But while nutriment penetrates to all parts of the body and is thus, except as to the quantity consumed, largely beyond control, we are able to regulate in great measure the extent to which the different parts of our bodies are used. Thus we may lead a hard laborious life as that of a navvy or hunter, or a sedentary one as that of a clerk 
or a tailor; we may use our legs much or little as compared to our arms ; during childhood we may have opportunities of indulging in many active games, or we may be cramped in a factory or the slums of a great city. Though the diet of the navvy may be less appetizing than that of wealthier people, yet it is usually sufficient in all essentials to secure full physical development. Not so the work of the clerk or the tailor. It follows, that when individuals, especially individuals belonging to different sections of the community, differ in those characters which develop under the stimulus of use, the difference is less certainly founded on germinal differences than when they differ in the characters which arise under the stimulus of nutriment, for example, eye-colour.

726. Probably, on the average, some sections of the community, for instance the men of the leisured classes who take plenty of exercise, develop under the stimulus of use nearly as well as their capacities permit. It is quite certain, I think, that other sections, for example, clerks and slum-dwellers, would, under other conditions, develop better than they actually do. In the case of ' useacquirements,' unlike 'inborn ' traits, it is evident, then, that there is scope for considerable physical improvement in certain classes without any resort to selection. But, here again, the means of securing improvements are so obvious as to be non-controversial. Therefore they need not be discussed at length. The debated point is the extent to which slum-dwellers, for instance, owe their physical inferiority, on the one hand, to the conditions under which the individuals are reared, and, on the other, to germinal peculiarities which, as is alleged, constitute them an instrinsically degraded race. Speaking generally, biometricians assume that the inferiority is germinal and, therefore, beyond cure except by selection. Medical men believe it is partly acquired, partly due to the inheritance of parental acquirements, and partly a consequence of the direct action of the environment on the germ-plasm of unfortunate or vicious parents. Believing that good surroundings cause intrinsic racial improvement and bad conditions degeneration, they suppose that only a prolonged revival of good conditions will remove the degeneracy. To me it seems overwhelmingly probable that slum-dwellers, factory hands, and the like, are physically inferior, not because they are as a class incapable of developing as well as the best sections of the community, but mainly, if not solely, because their surroundings are such that they have not had the chance of developing as well as they might. I think we have only to improve their surroundings sufficiently and 
the deterioration will vanish in the very next generation. Judging, however, by the evidence given before various Royal Commissions by multitudes of medical men and others, I have been very much alone in this opinion until recently. We shall deal more at length with the question immediately.

727. If the physical use-acquirements are mere extensions of growth made under the stimulus of nutriment, the same is more rarely true of mental acquirements. Sensations, instincts, and memory (all 'innate' traits) differ sharply from the contents of the memory (all 'acquired' characters.) It is true that a few instinctive emotional impulses (e.g. the parental) are extended by acquirement. It is true also that we acquire through experience emotional impulses to action and 'physical' dexterities which are close imitations of true instincts and instinctive movements. The latter emotions and dexterities, however, are not usually extensions of previously existing characters. For example, nothing like an inborn love of a particular religion or of dexterity in handling a billiard cue is inborn in the individual. These and their like are entirely new creations due to experience and developed in the individual in lieu of any one or more of a thousand alternative emotions and dexterities equally possible of attainment.

728. It follows that, in the case of mind more than in the case of the body, there is scope for improvement without resort to selection. The human being is capable of developing mentally so greatly, in so many directions, and under the influence of so many varieties of experience, that probably no man, no matter how careful his training, nor what ideals-moral, social, religious, intellectual, practical, and so forth-his educators had in mind, ever received a perfect mental up-bringing, ever developed mentally in as perfect adaptation to his total environment as was possible to him. In the absence of perfect knowledge and wisdom, the choice of his guardians has always fallen short of perfection. A man cannot be taught all knowledge, and many useful or admirable mental habits and attitudes are incompatible with others. Nevertheless, as fitting the individual for his environment, the mental training of most men somewhat approaches the ideal. And this is more especially the case in savage and therefore comparatively simple states of society. Sport, imitativeness, curiosity, and the instinct to play with the contents of the memory, lay the foundations, and the child's educators, consciously and unconsciously, strive to reproduce in him the traits which enabled them to survive in the common environment. Other traits, even if ideally desirable, 
would not, speaking generally, be useful. Thus, under a dominant church, clear, far-reaching, fearless love of truth conduced more often to death by torture than to survival during the dark ages of Europe. Even now they are apt to handicap the individual in many walks of life; though under our more complex and enlighted conditions a greater diversity of mental type is both possible and beneficial to him.

729. From the point of view of the social reformer the important fact is that man's intellectual powers, including his capacity to learn even in old age and to communciate his acquired knowledge, thoughts, and mental attitudes to his fellows, confer on him great powers of altering the mental and material environment and of adapting himself, and more especially the next generation, to the alteration. Improved mental development may thus be made, if not positively beneficial to the individual in his struggle for existence, at least not positively injurious. For example, in the course of one or more generations a savage environment may be exchanged for one more civilized; or an atmosphere of prejudice and superstition may be made to yield to one relatively more enlightened. Modern Japan, in which ideas are now openly prevalent the expression of which only a few years ago would have entailed death, is a case in point. Though the mind of this race has altered in some very important particulars, though it has some intellectual characters different from those which it lately possessed, yet we have no reason to suspect any germinal alteration such as would probably be indicated by a marked change in physical growth.

730. To sum up: racial capacities for physical and mental development cannot be altered except by selection, or lack of selection, or in rarer instances (as in the case of European breeds of dogs in India) through injury to the germ-plasm. Speaking practically, physical and mental characters which develop under the stimulus of nutriment cannot be improved in the mass of the population except through selection. Physical characters which develop under the stimulus of use may be considerably improved without selection in many sections of the community; but each improvement, however beneficial, can consist only in mere extensions of growth previously made under the stimulus of nutriment. On the other hand, it is probable that the mental characters which develop under the stimulus of experience may be immensely improved in many directions in all sections of the community by careful training and without resort to selection. 
In considering any practical problem, therefore, we must first of all determine what we propose to improve-whether germinal potentialities, or characters which developed under the stimulus of nutrition, or of use, or of injury-and then consider in what way they may best be improved-whether by selection or by altering the stimulus, and if the latter, how the stimulus may best be altered. These, then, are the general considerations we must bear in mind.

731. Physical deterioration in slum-dwellers, factory hands, and sedentary workers generally, may be due to one or more of several conceivable causes. It may be 'innate' or germinal, in which case it must depend on antecedent selection, or on lack of it, or on injury to the germ-plasm, such as is said to occur in European dogs in India; or it may be due, not to inferior capacities for development, but merely to inferior growth resulting from lack of sufficient food, or exercise, or from injury (e.g. by disease) to the growing bodies, the soma, of the young. As already indicated, speaking generally, biometricians and medical men believe that the inferiority is, in great 'measure, innate. But they do not trace the alleged 'degeneracy' to the same source.

732. Biometricians suppose that in the past a process of social sifting has separated the innately superior from the innately inferior families of the population, ${ }^{1}$ the former rising to the upper and middle classes, the latter becoming the dregs that settle in the slums and other habitations of the wretched. But, if this be true of the town, it should not be less true of the country. Presumably the sifting process has occurred everywhere, and in former centuries as well as at the present time. Our towns and factories have only during the last few generations absorbed a considerable proportion of the population. If, then, the biometric hypothesis be correct, agricultural labourers should be almost as physically inferior to members of the upper and middle classes as slumdwellers and factory hands. But, to say the least, of this there is no evidence. Doubtless some of the inhabitants of the slums, as of the country, are people who have sunk through the germinal physical inferiority (i.e. incapacity to develop as well as their

1 May I remind the reader that the words 'innate' and 'acquired' may be used in two senses, one of which is correct and the other incorrect. None of the characters of the same individual are more innate or acquired than any other. If any of his characters are innate, then all are innate. If any are acquired, then all are acquired. But different individuals are innately alike if their germ-plasms were alike; they are innately different, if their germ-plasms were different. They are alike, or unlike, by acquirement if stimuli from the environment have so moulded them that they have grown alike, or unlike. 
fellows under similar conditions of life) of themselves and their progenitors. But selection is keenest where the struggle for life and comfort is hardest-in the lowest stratum of society. Amongst the very poor in cities, the offspring of the physically and mentally weak are often of necessity more exposed to neglect and disease than the children of their stronger fellows. Moreover they suffer more from a lack of good and sufficient food. Consequently in slums, far more than in the country, there is still a real, though limited, elimination of the 'naturally' weak in bone and muscle such as occurs under savage conditions, an elimination which tends to counterbalance any social sifting. The sifting, if real, is further off-set by the fact that the town, offering better wages and superior opportunities for enjoyment and openings for ambition, attracts the best, the most robust and enterprising of the peasant stock. But the main objection to the biometric hypothesis lies in the patent fact that in the modern civilized world the rise and fall of men in the social scale is due much more often to mental than to physical peculiarities, and most of all to good or bad fortune occurring during development. In the slums are, doubtless, some people who have fallen because they are innately somewhat feebleminded; that is, people whose mental capacity to profit by experience is so inferior to that of the average of their race that they have sunk in consequence. A far larger number have sunk through a mental susceptibility to the charm of alcohol which, on the average, weighs alike on them and on their descendants. But probably the largest number have descended, not through any germinal inferiority, but through sheer misfortune, including the great misfortune of a bad mental training. Owing to the growth of machinery and population there is not enough work in the land for all the hands that are capable of doing it. Therefore even some of the capable must fail. Once a family has fallen into the slums, it is exceedingly difficult for it to emerge again-partly owing to the lack of opportunity which extreme poverty entails, and partly because the physical and mental traits acquired in the slum are such as tend to unfit the individual for successful life in any other environment. The statistics hitherto compiled by biometricians demonstrate the precise average degree of physical superiority or inferiority present in one section of the population as compared to another. But-if the thing must be done statistically-before we can decide whether the difference is innate or acquired, a further inquiry is needed in which the children derived from one section are compared to those derived from another after all the 
children have been reared under identical conditions. This inquiry might be made, though with difficulty; for in the slums are any number of people immediately derived from robust English and Irish peasant stocks, and these, if biometricians are right, should be physically superior to the rest of native slum population.

733. Medical men trace physical deterioration to the direct effect on the germ-plasm of such agencies as insufficient food, bad hygiene, disease, and alcoholism. They have discovered that apparently no British families of a purely urban descent of four generations are in existence, and believe, therefore, that the germplasm is poisoned so rapidly under urban conditions that the town renews itself from the country in every two or three generations. But the very magnitude of the migration into the town, combined with constant intermarriages between the urban and rural populations, renders practically impossible a purely urban descent of four generations. The absurdity of attributing the rarity of families of pure and ancient urban descent to germinal deterioration is seen very clearly when we consider the case of the Jews. These people, who intermarry amongst themselves, are often desperately poor, and are not specially clean and sanitary in their habits and dwellings. Indeed numbers of them inhabit the worst slums. Derived by an almost pure descent from the inhabitants of those terrible slums, the ghettos of the middle ages, and rarely inhabiting rural parts, they are the most extremely urban type in the country, and are, nevertheless, under town conditions, the most hardy and prolific type of all. Medical writers attribute the relative immunity of the Jews to the superior care bestowed by Jewish women on offspring. But while Jewish mothers in slums are, on the average, noticeably more healthy ${ }^{1}$ and temperate, ${ }^{2}$ and, therefore, better able to rear and suckle children than English women in the same surroundings, neither they nor their children are appreciably cleaner, nor are their dwellings better than those of the natives. The fact appears to be, then, that Jews are especially healthy and temperate in towns, merely because they are derived from a race which has been fitted by prolonged selection to urban conditions. At any rate, if all the facts, including the recent evolution of civilized races, are taken into account, I can think of no other explanation; and none other has been suggested except that the Deity takes particular care of His chosen people, or that the religious training of the Jews results in better maternal acquirements than Christian training, and so forth. The Chinese appear 
to be quite as resistant and prolific as the Jews, and it will hardly be maintained that they are a specially favoured or well-trained race.

734. It follows that urban conditions, except perhaps in rare and isolated instances, do not cause deterioration of the germ-plasm, but merely of the individual exposed to them. As far as the evidence indicates, the child of peasant parents, if reared in the slums, suffers quite as much as the offspring of a town-bred family. Indeed the survival of the town family indicates some degree of fitness to the environment. It must be borne in mind that the physical dangers of urban life arise mainly from microbic disease, which, though less concentrated, is by no means absent, even in the most sparsely populated localities in Britain. The great cities of the present day have grown from the hamlets and villages of the past, and their slow increase has enabled the race to adapt itself to the altered conditions. Even now our villages are training the race for existence in cities.

735. The conclusion we reach, then, is that the physical deterioration which accompanies urban life may be removed in a single generation by improving the conditions of life under which the poor dwell-by providing better food, housing, sanitation, and greater opportunities for active games to the young. Much, indeed, has been done already. The task before us is difficult, but not nearly so difficult as is supposed by biometricians and medical men. We need not resort to selection, unless we wish to evolve rapidly a race capable of vigorous life in slums. It would be an easier and quicker proceeding to abolish the slums. We need not wait till the imaginary innate deterioration caused by generations of degradation is remedied by generations spent in better surroundings. Our main difficulties arise from the selfishness of the governing classes, the intemperance of the poor, and the ignorance of both. If all classes understood the causes to which physical deterioration is due, desired its removal, and were willing to work and spend with that object in view; if we saw to it that even the children of drunkards had proper food, care, housing, and exercise, physical deterioration would cease to characterize sections of the people, though we should still see it, as amongst the upper classes at present, in individuals who were congenitally incapable of attaining average physical development.

736. Microbic disease, the principal source of physical injury and death amongst civilized peoples, affords several practical problems of great interest and importance. In combating it, two 
methods of procedure are conceivable: (I) we may attempt, by what may be termed external sanitation, by rendering the environment external to the human body unfavourable to the microbes, to banish the organisms that cause disease, or (2) we may strive to render human beings more resistant, and so practise what may be termed internal sanitation. If we adopt the latter course we may $(A)$ raise the innate resisting power of the race by altering the germ-plasm by artificial selection, or $(B)$ that of individuals by conferring artificial immunity such as that which is acquired to smallpox through vaccination. Artificial selection, even when pressed to a point to which Natural Selection is never pressed, would merely increase the resisting power of the race. ${ }^{1}$ That is, it would mitigate the severity of disease, but would not banish it. On the other hand, acquired immunity, if universally acquired, would banish disease ; for the micro-organisms, deprived of their nutritive supply, would become extinct. It must not be forgotten, however, (a) that immunity to one disease does not confer immunity to any other, and $(b)$ that while it is possible to acquire immunity to some diseases (e.g. diphtheria and smallpox), it is not possible to acquire it to others (e.g. tuberculosis and leprosy). Therefore, while we may hope to banish utterly the microbes of such a disease as smallpox, either by external sanitation or by internal sanitation, we can hope to banish the microbes of such diseases as tuberculosis only by external sanitation. It is true that by improving the surroundings we are able to increase the power to resist tuberculosis (and its consequences) in people who have lived under weakening conditions (e.g. in slums); yet, since many people living under the best conditions take the disease, it is impossible, merely by making people healthier and stronger, to prevent frequent infection and therefore propagation of the microbes.

737. Our power of banishing the microbes of the various diseases by external sanitation is largely conditioned by the mode in which they pass from one human being to another. The contagious diseases, ophthalmia, various skin complaints, the venereal maladies, and the like, are, for obvious reasons, particularly

${ }^{1}$ Selection by any ill-condition merely increases the racial power of resisting that ill-condition. It cannot procure absolute immunity for the reason that, when the severity of selection is relaxed, retrogression tends to follow. Absolute racial immunity to any ill-condition results only through selection by agencies other than that condition. Thus the higher animals are not immune to the assaults of spiders because they have undergone thorough selection by spiders. They are immune because they were otherwise selected. 
controllable. Were the public sufficiently well-informed, intelligent, and careful, they should disappear within a very few years. Even as it is, with the exception of the venereal diseases, cleanly people rarely suffer from them. The venereal diseases are prevalent mainly because our systems of religion and morality are still so primitive that effective sanitation, like personal cleanliness in the early ages of Christianity, is regarded as morally reprehensible. Wilful poisoning with arsenic is a criminal offence. Wilful poisoning with syphilis, even of an innocent wife or an ignorant boy, is not a legal crime.

738. The water-borne, the 'filth' diseases, enteric fever, cholera, dysentery, epidemic diarrhœa, and other maladies which affect particularly the alimentary canal, are also very controllable by external sanitation. Except under special conditions they do not spread rapidly, and their mode of existence outside the human body is such that they are extremely open to attack. Careful sanitation has almost eliminated them from many areas where they were formerly prevalent. There is no apparent reason why, with increasing knowledge and efficiency of preventive measures, they should not be banished altogether. As in the case of the contagious diseases, artificial selection is unnecessary, and artificial immunity only desirable in localities where sources of infection outside the human body are yet too numerous and widespread to be controlled by the system of sanitation practised or practicable.

739. In all probability insect-borne maladies, also, will be banished in no very distant future by external sanitation. The destruction of the intermediate hosts supplies a ready means of attack. Already Europeans, owing to the conditions under which they dwell, are rarely infected by plague in India, even when it is raging amongst the natives. Malaria has been greatly diminished in many parts of the world through the destruction of mosquitoes. The same means have been effective against yellow fever in Havana. Comparatively little as yet has been achieved against sleepingsickness, but the disease has not long been studied, and the life history of the intermediate host, the tsetse fly, is not fully known. If it be true that the malady is conveyed only by one species of insect which is confined to the shady banks of streams, it may prove possible to banish it more easily and effectively than has been found possible in the case of any other insect-borne disease. In the case of insect-borne diseases, then, selection is clearly out of place.

740. As far as we are able to judge at present, air-borne diseases will never be banished by external sanitation. They 
pass from host to host through a medium, the air, which is essential to human existence, and which, blowing where it listeth, cannot be controlled nor efficiently disinfected. Speaking practically, these maladies are independent of climatic or other local conditions except density of population. Since they spread with a rapidity which is proportionate to the number of susceptible individuals, and since immunity to them is conferred by illness and recovery, the more thoroughly they are banished from a community, the greater grows the number of susceptible persons, and therefore the liability to pestilence-to disease in its fearful epidemic form. In England, for example, only the endemic prevalence of air-borne disease, and the consequent acquired immunity of the major portion of the population, preserves us from disaster. If air-borne diseases were restricted to a single islet for a generation, the rest of the globe would stand in danger of depopulation. When they broke out from the island habitat they would sweep, as in ancient times, over the whole world-not merely in one great and terrible pandemic, but in many recurrent and devastating epidemics, each with its attendant famine, till in the process of ages the epidemics, growing more frequent, but less disastrous, at last settled again into endemic disease. Therefore, even were it possible to banish measles, and whooping-cough, for example, from our midst by external sanitation, it would be disastrous to do so while they yet lingered in any quarter of the globe, and it would always be hard to ascertain with any degree of certainty that some such spot did not exist. In civilized countries unavailing attempts are constantly made to restrict the spread of measles and whooping-cough, as by closing schools during such slight epidemics as occur amongst us, but only with the result of aggravating future epidemics. Isolation is quite impracticable except when the great majority of the members of the community have already been infected and thereby have acquired immunity. ${ }^{1}$

741. Clearly, then, in the case of air-borne diseases we have nothing to hope from external sanitation. From artificial selection, also, little or nothing could be gained. Already Natural Selection is very stringent; only people resistant to measles, whoopingcough, influenza, and probably such complaints as bronchitis, pneumonia, and rheumatic fever, survive. Very prolonged and effective artificial selection could only render air-borne maladies somewhat milder to the mass of the population. There remains internal sanitation - the production of acquired immunity by means 
of vaccination ; that is, by means of an artificially attenuated form of each disease. By such measures smallpox, the most dreaded of all air-borne maladies, has been almost banished from various parts of the civilized world, and would have quite disappeared from the globe had the whole human species for the term of a generation been vaccinated at intervals of a few years. Thereafter, the virulent microbes, having become extinct, vaccination itself would have become unnecessary. This conclusion may appear extravagant; but, if we take all the facts into account, it is difficult to escape from it. In Germany, for example, smallpox seems never to appear except as an importation from abroad. What is possible in one country is possible in others.

742. Whether internal sanitation can be made as effective a weapon against air-borne disease in general as it is against smallpox in particular can only be known in the future. As yet we have made no more than a beginning. It may be useful only against such diseases as usually confer permanent immunity, and perhaps it will ultimately prove impossible to attenuate many air-borne diseases in the way smallpox has been modified-that is, to bring about such an attenuation in the nature of the microbes, that, unable to exist in the blood-stream, they are confined to a single locality in the body, and so are incapable of reaching the air passages and infecting the atmosphere. Antitoxins, since they induce only a 'passive' or temporary immunity, cannot serve the same purpose. Moreover, in order to manufacture antitoxins, it is necessary to keep alive the virulent microbes.

743. Immunity to leprosy and tuberculosis, the principal earthborne diseases, cannot be acquired by the individual. Therefore, internal sanitation supplies no weapon against them. The infectivity of leprosy, however, is very low. Owing apparently to the isolation of sufferers, and the increase of personal cleanliness, it has been banished from most civilized communities. There can be little doubt that ultimately it will disappear altogether. On the other hand, tuberculosis, principally a disease of the air passages, is, relatively, highly infectious. ${ }^{1}$ External sanitation-improve-

1 I have termed tuberculosis an earth-borne disease, because its bacilli rest when outside the body mainly on the floor, walls, and furniture of dwellings. But since they are inhaled with floating dust it is also in a real sense an air-borne malady. To me the evidence that it enters the body in the vast majority of cases through the lungs appears overwhelming. But many medical men, notably Behring, favour the hypothesis that the alimentary canal is the main portal of infection, and that the milk of tuberculous cows is the principal vehicle. It is supposed that bacilli, imbibed during infancy, "remain latent until they find 
ments as regards light, ventilation and air-space-has somewhat reduced its prevalence, and may continue to do so for a time. But the power of resisting tuberculosis is a direct product of evolution. A fall in the mortality due to it implies lessened stringency of selection, which in turn, if carried far enough, implies retrogression. Therefore to procure a permanent fall in the death-rate, it is not

their opportunity [whatever that may mean] for development, and that individual predisposition is of little account." But how in that case is it possible to explain the fact that consumption tends to run in families even when individuals are separated by continents and generations? It can hardly be that the members of families of low resisting power are naturally prone to use tuberculous milk. Besides, the very people who suffer most from tuberculosis when exposed to it in infected dwellings are precisely those who have had no opportunities of being infected during infancy; who have never drunk any milk but their mother's milk; who, indeed, in many instances, have never seen a cow, let alone an infected cow ; for example, Esquimaux, Tierra del Fuegians, Polynesians, and the like. Deaths from the various forms of tuberculosis occur in about the same proportion in Japan as in Europe; but, according to Kitasoto, tuberculosis is unknown amongst the Japanese cattle, infants are never fed on other than human milk, and cow's milk is so little used that the daily per capita consumption by the human population averages no more than three-quarters of a teaspoon. In fact, cow's milk is not a general article of diet in Japan. Additional evidence that tuberculosis is an air-borne disease is afforded by the fact that a sufferer with phthisical lungs is apparently a much greater danger to his fellows than one who suffers from tuberculosis of the skin (lupus). It is possible that some human beings, especially infants, in whom the alimentary canal is the principal seat of disease, have contracted the malady from tuberculous milk; but, since tubercle is always found in the bronchial glands of infected children, it is not at all probable that the majority of sufferers are thus infected. More especially, it is improbable that pulmonary tuberculosis occurring in adults is commonly due to bacilli imbibed in milk during or after infancy. The Royal Commissioners "Appointed to Inquire into the Relations of Human and Animal Tuberculosis," after conducting a long series of experiments, maintain that human and bovine tuberculosis are similar in kind. Since tuberculosis has never been observed in wild animals, it is practically certain that the bovine disease is derived from human sources. Doubtless, therefore, they are right, but their labour was unnecessary. " The fact that the bacillus of bovine tuberculosis can readily by feeding as well as by subcutaneous injection give rise to generalized tuberculosis in the anthropoid ape, so nearly allied to man, and indeed seems, so far as our few experiments go, to produce this result more readily than in the bovine body itself, has an importance so obvious that it need not be dwelt on " (Second Interim Report, p. I4). But the susceptibility of anthropoid apes only proves, as might have been expected, that their bodies furnish an environment favourable to bacilli which have undergone evolution in the allied human species. But apes, unlike Englishmen, have undergone no evolution against tuberculosis. They are in the position of Polynesians. It does not follow that Englishmen can be as readily infected as apes. Judging from analogy, we have every reason to believe it probable (I) that bovine tuberculosis is derived from the human disease; (2) that the microbes of it are becoming adapted to the new environment; (3) that they are becoming less adapted to the old environment; and (4) that consequently they are less virulent to human beings than the microbes derived from the human disease. 
enough to improve the sanitation; to balance the increasing retrogression continuous improvement is necessary. Doubtless, if the poor Jews of the slums, who as a class are probably the most resistant in the community, were removed to better surroundings, they would have lower death-rates from tuberculosis than before; but only for a few generations. Gradually the rate would rise until the mortality balanced the retrogression. Even among our wealthier classes consumption claims its toll of victims, and it is difficult to believe that the environment in cold and crowded England can ever be made so unfavourable to the bacilli as it already is in the warm, sun-bathed, and sparsely populated Polynesian Islands where the native races are undergoing extermination. Isolation, such as that practised in sanatoria, is, owing to the vast multitude of sufferers, impracticable as a general preventive measure. Speaking practically sanatoria effect cures only amongst highly resistant people who have been weakened in worse surroundings A great number of those who improve in them return to perish in their own homes. Consumption is never detected in its earliest stages. The victim does not suspect his disease until it is well advanced. Long before it has been recognized, the bacilli have been disseminated by means of his coughnot only in large masses of sputum, but in a fine spray. It is conceivable of course that we shall one day discover a medicine which will act as effectively in tuberculosis as mercury in syphilis and quinine in malaria; but, since tuberculosis has so long been studied, this is, at least, improbable. It would seem, then, that the only hope of permanently reducing the mortality from tuberculosis lies in selection-probably, in the first instance at least, not a selection enforced by legal penalties, but one due to the presence of an enlightened public opinion which will regard as morally reprehensible the fertile marriages of phthisical types. 


\section{CHAPTER XXIV}

\section{INTEMPERANCE AND INSANITY}

Immunity to alcohol-Evolution and retrogression-Attempts to suppress drinking in Mohammedan countries-In modern civilized states-In the United States, Canada and Australasia-Failure of prohibition-Insanity-Two distinct kinds-Lunacy-Feeble-mindedness-Definitions of idiotcy, imbecility and feeble-mindedness-The Royal Commission on the Care and Control of the Feebleminded.

\section{4.

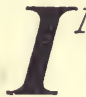

NTEMPERANCE. Alcohol is capable of bestowing keen pleasure on many, and probably some pleasure on all people. But in England and many other countries the amount of pleasure conferred by it is greatly outbalanced by the suffering. In these countries, if no one drank, the gain would be great, though not so great as if all men drank, as most men smoke or as the Jews drink, in moderation. Like the microbic poisons, it is a cause of disease through its action on the tissues of the body. There is, however, this immensely important difference between the microbic poisons and alcohol; people 'take' the former in spite of their desires, the latter because of their desires. They suffer from a microbic disease, because they have a certain weakness of body; from intemperance, because they have a certain peculiarity of mind. It follows that the term 'immunity,' when used in reference to microbic disease and alcohol, has unlike meanings. Used in reference to disease, it implies that the individual is physically incapable of being infected under the normal conditions of life. But, though a given quantity of alcohol is less poisonous to some men than to others, no man is physically incapable of swallowing poisonous doses; and many men, even the most resistant physically, take it in such doses. Consequently, Nature, taking as always the most direct course, evolves races which are mentally, not physically, immune. Immunity to alcohol implies, therefore, an incapacity to be tempted by the narcotic in harmful quantities. A kind of immunity to alcohol may be created by fostering a moral abhorrence of intemperance; but this voluntary avoidance of temptation bears no likeness to what is termed immunity to disease-neither to the inborn immunity 
which prevents infection, nor the acquired immunity which results from infection and recovery. It resembles more closely that external sanitation by means of which we endeavour to prevent the infection of susceptible people.

745. Susceptibility to the charm of alcohol, like that to infection by a disease, is an 'innate' character. It may be extended or decreased by acquirement, by circumstances affecting the mental state of the individual (e.g. misery or happiness), just as susceptibility to microbic infection may be altered by circumstances affecting his bodily state. But it may be, and often is, present in people whose circumstances are otherwise of the happiest. As we have seen, it is much more pronounced in some individuals and races than in others. On the other hand, the actual craving for alcohol is purely an acquirement, a mental growth, which occurs only in susceptible persons who have had previous experience of the poison. Within limits, which vary with the individual, the craving tends to increase with added experience. Herein the disease of alcoholism differs sharply from such a malady as measles, experience of which gives origin to acquired immunity. ${ }^{1}$ It follows that internal sanitation, the procurement of immunity through experience of the poison, is impossible in the case of alcohol. Therefore, if we wish to benefit the race, our choice of action is limited to the banishment of alcohol or the elimination by selection of the susceptible individual.

746. Alcohol, a waste product of the yeast fungus, is derived from sugar. Speaking practically, both sugar and yeast are present in every part of the habitable globe. Their area of distribution is much more nearly universal than that of any disease. Obviously, it is impossible to eliminate either the one or the other. Alcoholic fermentation occurs constantly in nature, but human art is necessary to produce the narcotic in intoxicating quantities. The art was discovered by savages at some period enormously remote in human history, and has been preserved and improved ever since. At the present day, in addition to its consumption as a beverage, alcohol is useful as a fuel, a solvent, a preservative, and the like, in many of our industries. Obviously again, it is practically impossible to suppress the manufacture of intoxicating solutions. The question then arises whether it is possible by legal means to

1 It will be noticed that I compare actual alcoholism, not the susceptibility to it, to a disease. The term disease is often applied to the susceptibility. It would be as reasonable to describe a person who had never been infected by measles as suffering from that malady. Questions of nomenclature are not trivial when a wrong use of words indicates confusion of thought in the speaker, and is a cause of confusion in his audience. 
suppress its use as a beverage, or at any rate its immoderate use. Probably every one will agree that purely moderate drinking cannot be enforced by law in a race many members of which are capable of enjoying immoderate drinking. Human nature is unable to resist the strong, steady pull of a constantly nourished temptation.

747. There remains, then, the total suppression of alcohol as a beverage. Suppose for a moment that this were possible, and that a law enforcing it were passed. Then a race like the British, which had undergone some evolution, would tend to retrogress towards that primitive state in which the average racial susceptibility to the charm of alcohol was much greater than it is at present. Therefore, if at any future period this law were repealed or passed into abeyance, the last state of the race would be worse than the first; for the race began its evolution when alcohol was hard to procure, and, in any case, could be obtained only in very dilute solutions. The longer the law was effectually enforced the worse would be the ultimate disaster. Our literature, both ancient and modern, is full of references to the delights of drinking, and the instinct of curiosity is strong in man. Any one who could read and possessed fruit, sugar, or starch, could manufacture alcohol for himself in secret. Many races, the South Europeans, for example, derive great pleasure and little suffering from alcohol. They could hardly be persuaded to pass a self-denying law which forbade its use, and which benefited, not themselves, but only distant races. The world grows increasingly cosmopolitan every day. Merchants and travellers conveying foreign products and habits pass more and more from every quarter of the globe to every other. As surely as the sun shines, travellers from lands where the use of alcohol has been suppressed, would learn its use abroad, and seek, in the end successfully, to reintroduce it to their own countries. Judging, then, from the biological standpoint, external sanitation against alcohol cannot be perpetually successful. Temporary success would merely expose the race to dangers similar to those which would menace it were a prevalent, lethal, and highly infectious disease banished from the country but not from the world. Since alcohol cannot be banished from civilized communities, great insusceptibility to its charm, implying evolution and therefore selection, is essential to the existence of a civilized race.

748. However, it may be argued that "Our business as practical men is with the present, and not with a remote and problematical future. In the past, men have rarely sought any- 
thing more than immediate ends; yet modern civilization has arisen. Posterity may safely be left to redress its own evils. Doubtless it will discover means of doing so at present unknown to us." Is it possible, then, in the immediate future, if nothing more, to suppress or considerably reduce excessive drinking by legal or moral methods? An immense body of evidence bearing on this problem is available. All history indicates that as soon as any race gains possession of considerable quantities of alcohol, that is as soon as it reaches the earlier stages of civilization, it begins attempts at temperance reform. Moderation was preached at least 7000 years ago in Egypt. Prohibition was decreed more than a thousand years before the Christian era in China. The ancient Rechabites, the Gnostics, the followers of Montanism, and the Manicheans were professed abstainers. It is probable that every century in the history of every European and Asiatic community, which had not already achieved moderation through selection, has been marked by repeated temperance edicts, some of them of savage severity. The final result is that the use of alcohol is now less controlled and more nearly universal than at any former period of the world's history.

749. Under very primitive conditions the human being enjoys almost as much freedom as a wild animal. In fact he is a wild animal. As society organizes itself, and the individual is brought into closer and more constant contact with masses of his fellows, his freedom is restricted till a point is reached when the nation is regarded as the property of some person who is believed to have, or at least claims and enforces, a divine right to deal with his subjects as he pleases. Later, with advancing organization, there is a return.towards the primitive condition of individual freedom. In modern England, for example, the individual is in many respects as free as the utter savage. The general tendency of such legal restrictions as bind him is merely to prevent him curtailing the liberties of his fellows, or breaking such engagements as he has voluntarily contracted.

750. Some laws which are possible at one stage of civilization are impossible at another. If incompatible with the spirit, the general tendency of the times, they quickly pass into abeyance. Thus during the Dark Ages of Europe edicts for the maintenance of uniformity in religion were often terribly effective. They would be absurd at the present day. A year or two ago a man was forbidden to marry his deceased wife's sister; it is probable that in the future he will be permitted to marry his grandmother. Laws 
prohibiting drinking are just such interferences with individual liberty as cannot be enforced in modern times. It will be observed that I do not maintain that such laws are inherently wrong. I insist merely that they cannot be enforced. It will be observed also that I speak of prohibition, of attempts to prevent all drinking, not of regulation, of attempts to place the consumption of alcohol under such conditions that moderation is encouraged, so that, as at an ordinary dinner table, the drinker does not interfere with the rights and comforts of his fellows.

75I. Under semi-civilized conditions prohibitive laws have occasionally met with temporary success. In some Mohammedan countries they have had prolonged success. Nevertheless, intemperance has been common among many, and especially among the more enlightened Moslem communities. Others have substituted opium for alcohol. Religious fanaticism has tended to keep some Mohammedan communities both sober and barbarous; but it cannot now long resist the influences which are so swiftly changing the face of the modern world. Presently Nature will pass the races of the near East through the fire which has hardened their neighbours against the stringent conditions of civilized life. Today, the comparatively drunken Englishman and Russian are at a disadvantage in the struggle for life and wealth as compared to the temperate Jew. To-morrow the Mohammedans will face a yet heavier handicap. This forecast may be disputed, but we have the facts. Has a highly civilized community ever existed which had not already passed through the fire?

752. Prohibitory laws, when enacted, in highly civilized states, have almost invariably failed, and as a rule worse than failed. I say 'almost,' because in some very sparsely settled colonies where temperance sentiment has been very strong, where each inhabitant is known to all his neighbours for many miles round, and where the channels of supply and communication with the outer world are few and well known, prohibitory legislation appears to have achieved a temporary, I had almost said a momentary, success. I say, 'worse than failed,' because in places where the population is dense, where men are acquainted with few even of their near neighbours, and where many channels of supply and communication exist and more may easily be organized, attempts at repressive legislation are always followed by increased intemperance, and by even greater evils.

753. No modern civilized government has attempted the gigantic task of abolishing the use of alcohol throughout its 
dominions. But various areas, possessing local self-government, in the English-speaking settlements, have sought to enforce abstinence on their working-classes by prohibiting, not drinking or the importation of alcohol, but only the manufacture and sale of it. This modified form of prohibition has been adopted at various times by seventeen entire States of the American Union. "It is now retained only by three, and in those it cannot be looked upon as a success. Parts of prohibitory States have always been in open rebellion against the law; drinking has never been impossible; the sale of liquor has always been profitable, and seldom disreputable in the eyes of the public. Violation of the law has been open and avowed. Federal law requires the payment of a special annual tax by retail liquor dealers; and most States, including those under prohibition, provide that the payment of the tax shall be prima facie evidence of a sale of liquor having been made, thus utilizing the Federal officials in the detection of illegal traffic. During the fiscal year ending June 30th, 1906, the number of retailers in malt liquors were, in the State of Kansas, 4019; in Maine, 599; and in North Dakota, 1582-these being the three prohibition States.

754. "In urban districts, at any rate, it has not been found possible to find any sound ethical basis for the law, or to persuade the majority to regard its violation as immoral. Without the backing of public opinion, no enforcement of prohibition has been obtained except at the price of raising animosities between rival factions of such intensity as seriously to disturb the community. Juries have violated their oaths; judges have hesitated to impose statutory penalties; blackmail and corruption have been directly instigated, and the law in general has been brought into contempt. Persistent disregard of the liquor laws is supposed to encourage disobedience to other enactments, and an example is cited in Kansas, where the fact that an anti-gambling law is almost a dead letter has been attributed to the lax enforcement of prohibition in the cities of the State.

755. "It should be mentioned that prohibition prevents a community from passing any laws for reclaiming or protecting its drunkards. Where in theory there is no drinking, in theory there can be no intoxication; but from the practical point of view such arguments cannot be justified." 1

${ }^{1}$ Report on the Liquor Traffic Legislation of the United States, prepared by Mr R. C. Lindsay, Second Secretary to His Majesty's Embassy at Washington, and issued by the Foreign Office in April 1907. 
756. The difficulty of enforcing prohibition under the ordinary conditions of civilized life is shown by the fact that only very thinly populated states of the American Union have continued the attempt. In I890, North Dakota had four persons to the square mile, Kansas seventeen, and Maine twenty. None of these States had a town of 50,000 inhabitants. Speaking practically, in no town, not even in what we in England should term a village, was there more than a pretence of prohibition. At that date the States that have abandoned prohibition had on the average a population four or five times denser than the population of those that have retained prohibition. Nineteen per cent. of the inhabitants of the former dwelt in towns of more than 50,000 inhabitants. The difference has since increased. Westmoreland, the most sparsely populated English county, had, in round numbers, in 1891 , a population of eighty persons to the square mile, Lancashire and Surrey had 2000, and Middlesex I I, 500.1

757. Prohibition applied to more limited areas than entire States is termed local option. Owing to the greater ease with which alcohol may be obtained from neighbouring areas it is correspondingly easy to enforce as regards the manufacture and sale of drink, but is even less effective as regards the consumption of drink. It has been enacted in many States of the American Union, in Canada, and in Australasia. Like 'total prohibition,' it is possible that it has achieved partial and temporary success in some very wild and sparsely inhabited districts such as parts of Dakota. It is certain that it is a complete failure in every town and densely inhabited rural district. ${ }^{2}$

758. Mere failure would leave matters no worse than before; but here failure is accompanied by many evils. For open drinking under the eyes of the public and police, whose business it is to prevent excessive drinking, is substituted secret and uncontrolled debauchery. The actual amount of intemperance is increased; men make sure of drinking their fill while they have the opportunity; they drink in surroundings where the greatest possible amount of harm results, and are given every facility for excessive indulgence. Drug stores, normally conducted by a very respectable class of men, become drink-shops which sell in addition immense quantities of such narcotics as morphia,

1 For evidence concerning the statements in this paragraph see The Temperance Problem, by Messrs Rowntree \& Sherwell, pp. I 15-249.

op. cit., pp. $250-369$. 
laudanum and cocaine. ${ }^{1}$ The police turn aside from an impossible task. Successful evasion of one important law tends to produce contempt for all law. Widespread demoralization follows. Crying evils exist unredressed; politics become merely a struggle between prohibitionists and their opponents. Politicians, seeking place, support one side or the other regardless of their private opinions and practices. These statements are very sweeping, but they are supported by any amount of evidence. ${ }^{2}$

759. Other things equal, large towns tend to be more intemperate than smaller towns, and floating populations than fixed populations. In 1898 , the latest year for which statistics are available, the inhabitants of New York numbered 3,500,000, of Chicago $1,850,000$, of Boston 582,463, and of Portland, the capital of Maine and the classic prohibition city, 4I,508. Eighty per cent. of the population of Portland were native-born. The convictions for drunkenness per 1000 inhabitants were in New York 13, in Chicago 23, in Boston 45, in Portland 42. New York, Chicago and Boston permit the sale of drink ; but Boston is a 'safety-valve' in the midst of a prohibition area from which came 44 per cent. of the inebriates convicted in its courts. Nor was drunkenness confined to it. Cambridge, one of its suburbs, is the largest prohibition city in the States; since it adopted 'no license' it has doubled its own convictions per thousand of population. When prohibition was first introduced into Portland, the convictions for drunkenness averaged I6 per 1000. ${ }^{3}$ General Neal Dow, to whom more than to any other one man its establishment was due, stated in his evidence

1 " On August 31st, I899-a week prior to our visit-another ' raid' was made on a few of the saloons (including one bottling factory), and more than a thousand gallons of beer, wine and spirits, were seized, of which nearly one-half ( $475 \frac{1}{2}$ gallons) was seized at one establishment-a so-called 'drug-store' with a large bar in the rear of the shop. This raid, however, like its predecessors, had to be made over the heads of the city and county officials" (The Temperance Problem, p. 195). "In the regular drug-stores, and in 160 of the 172 general stores in the State of Vermont (then under prohibition), they sell every month 3,300,000 doses of opium, besides what they dispense in patent medicines, and besides what the doctors dispense ( 90 per. cent of the doctors dispense their own medicines), which gives one and a half doses of opium to every man and woman in the State of Vermont above the age of twenty-one years every day in the year. (By dose I mean I grain of opium, $\frac{1}{8}$ of a grain of morphia, $\frac{1}{2}$ ounce of paragoric, and 20 drops of laudanum). And the amount consumed would average a dose to every man, woman, and child in the State of Vermont every day in the year" (Dr Ashbel P. Grinnell).

2 Readers desiring fuller evidence than I have space for will find it set forth in, amongst other works, The Temperance Problem, and the Reports of the American Committee of Fifty to Investigate the Liquor Problem.

${ }^{3}$ The Temperance Problem, p. 701. 
before the Royal Canadian Commission, "When I was Mayor (I85I) every man who indicated he was drunk was arrested, but now they do not do that unless the man is noisy and disturbing the peace." Another witness, a "Past Most Worthy Patriarch of the Sons of Temperance," on being told that evidence had been given that men drunk but not disorderly were not arrested, and that in the preceding year "men shook their fists in the face of the Police in Portland, and dared them to arrest them," said, "I have no doubt of it." "And that would naturally reduce the number of arrests very largely in the city of Portland?" "Yes." 1 The following sums up the case very fairly : "Every word of. Mr Morris" (Rev. Phillip H.) arraignments of the prohibitory law is true. $\mathrm{He}$ might have made it much stronger and still kept within the borders of truth .... Prohibition has remained upon the Maine statute books for half a century, for the single reason that it has never been enforced. One year of genuine enforcement, and it would be abolished as soon as the legislature could be got together ... 'Make the law respected where it is a law,' said Mr Moore. There is the solution. Make the law respected, and everybody except the theorists who now sustain it will demand its repeal." 2

760. The evidence concerning the failure of prohibition and local option in other parts of the United States, in Canada, and in Australasia is similar in kind and quite as conclusive. Of Canada, the Report (1895) of the Royal Commission says, " in short the law as an aggressive weapon has been abandoned." In Australia one 'town,' the Moonta mines township, is said to be under 'prohibition.' Another, Mildura, has closed its public houses, but alcohol is sold in clubs. "There is no haughty exclusiveness about these clubs. The members appear to be anxious to extend the right hand of good fellowship to all comers, so that no man need go athirst." 3 New Zealand has a law of local option. At the election of I905 ' no license' was adopted by six out of sixty-five districts. It has been in operation longest in the Clutha district, and the King country, the inhabitants of which are aborigines. Its success may be gauged by the following: "The Maori chiefs in the King country, New Zealand, have asked the Government to substitute a limited licensing system for the prohibition which is in force at

${ }^{1}$ Quoted by Rowntree \& Sherwell, op. cit., p.16o.

2 Biddeford (Maine) Daily Record, April 24th, I899, quoted by Rowntree \& Sherwell, op. cit., p. 198.

${ }^{3}$ The Report to the Victorian Board of Public Health, by Dr Robertson, quoted by Edwin H. Pratt. The Licensed Trade, p. I4I (London, Murray). 
present, and under which liquor of bad quality is being sold everywhere. Mr Seddon, the Premier, approves of the proposal. He told a deputation that the chiefs and the police were unanimous in stating that prohibition had spread the evil it had been intended to exclude. Sly grog-selling is rampant and could not be stopped. The same thing was going on in the Clutha district in Otago, where there were no Maoris, and where prohibition was enforced by popular vote." 1

76r. A law forbidding the use of alcohol is as much an anachronism as one enforcing a belief in the Thirty-nine Articles, or the Darwinian Doctrine of Descent. Prohibition merely deprives the authorities of much of their power of controlling intemperance and tends to make them partners, pensioners, or blackmailers of law breakers. The whole habit of modern thought and behaviour, the whole fabric of civilized society is against its effective administration. But that which cannot be altogether prevented may to some extent be regulated and diminished. Up to a certain point it is possible to control the drink traffic; attempts to pass that point usually end in worse than failure. Drinking there is sure to be, but the more openly it is conducted the less imperfect are the facilities for control. If, were it possible, all public houses and clubs had glass walls, intemperance would be reduced to a minimum. Very little drunkenness occurs in the cafés of the French Boulevards. If, then, we wish to benefit the race in the immediate future our endeavour should be, not to prevent drinking, for that is impossible, but to bring all places where excessive drinking is likely to occur more thoroughly under the hand of the public authorities. That is the only method of external sanitation with respect to alcohol which, in civilised communities, has been at all successful in the past or is likely to be successful in the future.

762. Insanity. Two very distinct kinds of insanity are observable:- $(A)$ The individual may possess, and usually does possess in average degree, every mental faculty found in the normal human being except one-memory. Thus he may have all the human instincts, imitativeness, curiosity, sexual inclination, and the rest; but he is more or less incapable of storing experienceincapable of filling his conscious memory with data which may be recollected, and his unconscious memory with 'acquired" dexterities and habits. If his lack of memory is very great, he is an idiot, unable perhaps to learn to walk or speak. A lesser degree of incapacity to learn constitutes imbecility. A still

1 The Morning Post, Oct. 29th, 1900. 
lesser degree, feeble-mindedness. There is, however, no sharp line of demarcation: imbecility and idiocy are merely graver phises of feeble-mindedness. The defect of memory is usually general : but in some cases it is more or less limited to particular departments of mental acquirement. Thus there are 'moral imbeciles' who, while able to make other mentai acquirements, are unable to acquire, in the form of imitation instincts, the code of morals prevalent in the community in which they are reared. The low tone of morality, especially sexual morality, so common amongst the feeble-minded, however, is more often due to general incapacity: the individual possesses normal instincts which, like other animals, he tends to obey, but which he does not learn to control like the average human being. To lack of memory, also, is attributable his deficiency in the 'higher faculties,' for example intelligence and reason. Probably, in comparison to the rest of our powers, we are all feeble-minded to some extent in one or more particulars. Thus, while possessing fair capacity in most departments of mental acquirement, we may be relatively incapable of accumulating musical, lingual, or mathematical efficiency. The connection between weak-mindedness and incapacity to learn is clearly seen when we consider that if we lacked, as some people do, such an instinct as the sexual or the parental, the function of which is not to impel us to make acquirements useful to ourselves, we should not necessarily seem feeble-minded. But lack of imitativeness or curiosity would almost necessarily lead to a condition of mental dullness closely resembling imbecility.

763. (B) The individual may be a 'lunatic,' quite normally capable of recollecting experiences and learning dexterities and habits. But the universe his mind constructs for him differs markedly from that created by the minds of normal people. $\mathrm{He}$ feels and thinks abnormally. His experiences impress his mind in an unusual way, and he draws unusual inferences from them. He has hallucinations and delusions. What to the normal person is a shadow on the wall, may seem to him the devil. Common sounds may have an awful significance,

"As the Lord were walking near,

Whispering terrible things and dear."

The sight of two people, perhaps strangers, in conversation may create a conviction that they are plotting great good or harm to him. The ordinary behaviour of his fellows may give rise to the notion 
that they are persecuting him, or on the other hand, to a belief that they admit his claim to be monarch or deity.

764. Obviously, then, the difference between the two kinds of insanity is very great. Idiocy is a condition in which there is little or no capacity to learn; lunacy a condition in which there is capacity to learn but in which there is much zorong learning. Both kinds of insanity can be imitated in greater or lesser degree by people who are quite capable of normal development. Thus training may so close a man's mind as to render him more or less unable to profit by experience, or it may endow him with strange and what seem to non-believers absurd delusions, as in the case of the adherents of many religions. Feeble-mindedness may arise from injury to the brain at any period of life, and it is not an unusual accompaniment of extreme old age; but it is seen typically most commonly in people who from birth forwards are more or less incapable of making mental acquirements. On the other hand, lunacy, at any rate clearly marked lunacy, almost invariably manifests itself during or after adolescence.

765. Probably most alienists will accept my description of lunacy as correct. There is nothing new in it except perhaps the stress laid on memory in the statement that this form of mental defect is due to wrong learning. But the description of feeblemindedness is new. If it be correct, then, the importance of the part played by memory in human mental development has been as insufficiently realized by alienists as by students of normal psychology. However, when we study their descriptions of the condition, we find that simple incapacity to learn is always implied, though, owing to non-recognition of the fundamental truth, other mental states which, on consideration, would be admitted as not especially those of imbecility, are sometimes included. Consider, for example, the following description supplied by the Royal College of Physicians to the Royal Commission on the Care and Control of the Feeble-minded.

766. "'Idiots,' i.e. persons so deeply defective in mind from birth or from an early age that they are unable to guard themselves from common physical dangers, such as, in the case of young children, would prevent their parents leaving them alone.

"'Imbeciles,' i.e. persons who are capable of guarding themselves against common physical dangers, but who are incapable of earning their own living by reason of mental defect from birth or from an early age.

" 'Feeble-minded,' i.e. persons who may be capable of earning a 
living under favourable circumstances, but are incapable from mental defect existing from birth or from an early age: $(a)$ of competing on equal terms with their normal fellows; or $(b)$ of managing themselves and their affairs with ordinary prudence.

"'Moral' imbeciles, i.e. persons who from an early age display some mental defect coupled with strong vicious or criminal propensities on whom punishment has little or no deterrent effect." 1

767. Or consider "The very good definitions submitted to us by Sir James Crichton-Browne, of the 'imbecile' and the 'feebleminded" " which "do not turn on the "capacity of earning a living, but have in some measure to define the child by anticipation, by standards applicable to a later time of life than early childhood, such as prudence, independence, and self control,' or 'taking care of himself in the common affairs of life.'" These definitions are : - "An imbecile is a person who, by reason of arrested development or disease of the brain dating from birth or early years, has the use of his observing and reasoning faculties so restricted as to incapacitate him for education in the ordinary sense or for taking care of himself in the common affairs of life, and who in appearance and manner generally evinces his mental short-comings.

"A feeble-minded person is one who, by reason of arrested development or disease of the brain dating from birth or from some age short of maturity, has his reasoning faculties partially weakened, so that he is slow or unsteady in his mental operations, and falls short of ordinary standards of prudence, independence, and self-control.

"The moral imbecile is a person who by reason of arrested development or disease of the brain dating from birth or early years displays at an early age vicious or criminal propensities which are of an incorrigible or unusual nature, and are generally associated with some slight limitation of intellect." 2

768. Both these descriptions imply incapacity to learn. Those furnished by the Royal College of Physicians, however, merely describe the social and material consequences of the defect rather than the defect itself-consequences which might conceivably arise from lunacy, or mere "backwardness" due to illness, or exceptionally bad training. Sir James Crichton-Browne goes deeper, for while he also describes the effects, he attributes them to

${ }^{3}$ Report of the Royal Commission on the Care and Control of the Feeble-minded, vol. viii. p. 188 .

${ }^{2}$ Op. cit., vol. viii. p. 189 . 
a weakening of the observing and reasoning faculties due to disease of the brain or the arrested development (of a faculty not mentioned, and due to a cause not named). There is, however, no evidence that the observing faculties of imbeciles are defective; their senses are usually as acute as the normal, and, apparently, they are as much guided by them as other animals. But they cannot store their observations. Their reasoning faculties are defective only because reason itself is an 'acquirement'-only because they cannot gather sufficient materials for complex thought nor learn to use skilfully even such materials as they do gather. The defect is, as I say, essentially an incapacity to profit by experience, an initial lack or subsequent failure of memory, which prevents the imbecile learning and retaining all that the normal person learns and retains from infancy forwards. I include failure of memory because there does not appear any good reason for the arbitrary limitation of the term feeble-minded to " arrested development or disease of the brain dating from birth or from some age short of maturity." Take away, as by injury, from the normal adult all that he has learned and at once he becomes an imbecile. Failure of memory in the aged, whereby stored experience is lost, produces precisely the same effects as congenital defect. The individual becomes equally 'incapacitated for education or of taking care of himself,' and, as a fact, is taken care of by his friends or the State in just the same way as a congenital imbecile.

769. A better description occurs in a Statement on Heredity in Relation to Feeble-mindedness, written by Sir E. Ray Lankester at the request of the Commission. "There is every reason to regard this condition as a case of atavism-a relapse to a primitive animal condition of cerebral activity-memory, and with it selfcontrol (depending very closely as it does on memory), is defective. Some savage races (e.g. Australian blacks) are-as compared with more highly developed races-normally in a condition of feeblemindedness. ${ }^{1}$

"Such throwing back in brain character among the more advanced races of man is certainly transmitted to offspring, and there seems no properly based argument for the view that feeblemindedness is not always congenital. In most cases it can be shown (90 per cent. of carefully examined cases) to be due to

${ }^{1}$ I think it would be hard to prove this statement concerning Australian natives. A school of native children once obtained the first place in an Australian colony, and most people (e.g. Sir John Cockburn, who told me personally) who have personal knowledge of the blacks rate their natural intelligence very high, and suppose that training, not incapacity, has made them savages of a low type. 
hereditary taint, although, as in other cases of reversion, it may from time to time appear as a 'sport,' that is to say, without any history of parental taint." 1

770. In other words imbecility is both a reversion and a mutation. "A little thought renders it evident that the feebleminded person is always one with a defective memory.... In effect and in fact the feeble-minded person is an instance of reversion to a pre-human mental state. But the reversion is not complete; for while he loses some part of his power of profiting by experience, he regains no part of the lost power of being guided by instinct. Therefore he is correspondingly helpless as compared with a lower animal. The instincts (e.g. sexual) which normal human beings still possess often appear unduly prominent in him, but only because he cannot learn to control them." 2

77I. But if imbecility is a mutation, the reproduction should tend to be Mendelian. It should be patent or latent in descendants. It "is a sharp contrast to normality. Here, then, apparently we have an explanation of the well-known fact that while the children of a defective may all be normal, yet amongst the descendants may appear individuals who are defective."3 The Royal Commission states in its Report, "In this connection the probable nature of the swamping is of some importance. Speaking generally, the trend of the evidence indicates that the offspring of feeble-minded persons are either apparently normal or distinctly feeble-minded. In the same family occur both types, the same person may have both normal and feeble-minded offspring and descendants; the defect may skip one or more generations and appear later. It would appear, therefore, that the swamping may be due not so much to a gradual obliteration of the defect by a blending with normality, as to its becoming latent or overlaid by the latter. It would seem also that the probability of its re-appearance amongst descendants is in some degree proportionate to the frequency with which it has occurred amongst ancestors." 4 It seems, then, that the inheritance of imbecility is comparable to that of such a trait as eye-colour. We saw that black eye-colour is strongly dominant over one of a lighter shade, but that after two or more infusions of the latter (that is, after the lighter shade is derived in a patent or latent

1 Op. cit., Appendices, vol. v. p. 247.

$2 O p$. cit., Note on the Hereditary Transmission of Mental Deficiency, by G. Archdall Reid, Appendices, vol.v. p. 247.

${ }^{3}$ Note on the Hereditary Transmission of Mental Deficiency, by G Archdall Reid, vol. v. p. 248.

Op. cit., vol. viii. p. 184 . 
condition through both parents) it tends to be reproduced by descendants. So also normality appears strongly dominant over imbecility; but, when the latter is derived through both parents, normality, like black eye-colour, becomes less dominant and the alternative condition tends to be reproduced-to become patent.

772. In every civilized country, during the last half century, the number of persons registered as insane has increased out of all proportion to the increase of the general population. For example, in Ireland, "while one person in every 657 of the population was registered as insane in the year $185 \mathrm{I}$, the proportion had risen to one in 178 in I90I." 1 There is now one officially known lunatic to 301.32 individuals of the general population as against one to 335 nine years ago, and one to 536 in $1859 . " 2$ "From Ist of January I 858 to Ist January I 908 the total number of lunatics officially known to the Board . . . has increased from 5,824 to 16,288 , showing an increase of 10,464 . . . Since 1858 the number of lunatics under the protection of the Board has increased I 80 per cent." 3 Opinions differ as to the cause of the increase. Some of it, or most of it, is undoubtedly due to a more complete registration. ${ }^{4}$ But probably some of it is due to actual multiplication. ${ }^{5}$ We possess no data on which it is possible to found a

${ }^{1}$ Macpherson, Edinburgh Medical Journal, May, I903, p. 398.

${ }^{2}$ Fifty-fourth Report of the Commissioners in Lunacy, I90I, England and Wales.

${ }^{3}$ Op. cit., Scotland.

"See The Fiftieth Report of the General Board of Commissioners in Lunacy for Scotland, pp. 9 et seq.

5 " Every one knows that a large number of the mothers of illegitimate children are of weak intellect; that their issue are frequently of the same type; that a large number of the habitual inmates of workhouses are of the same low standard of mind ; that much of the petty crime of this country is committed by persons below the average in intellectual power.

" One of the Poor Law inspectors saw in a workhouse in Somerset, an imbecile woman with an illegitimate imbecile daughter, who had her own illegitimate daughter in her arms. Precisely the same picture has been seen in the County of Cornwall, and these are but pictures in small of a fact which is to be seen in very many of our workhouses.

"But the ranks of the insane as well as of the imbecile are recruited from the children of the feeble-minded. The fearful increase of late years of insanity in this country has necessarily created alarm, and I cannot but believe that one of the sources of this fact is to be found in the imbecility of the parents.

Sir James Crichton-Browne entirely agrees in this view. He has written to me to the effect that a terrible increase of insanity is going on, and that it is undoubtedly not merely due to increased diligence or improved diagnosis, but in some measure to the cause named, viz. propagation of the weak-minded, and ' I am confident,' he adds, that permanent provision for imbeciles of both sexes, but especially girls, however costly it might be in the first instance, would ultimately result in saving of the rates. In a word, imbecility, insanity, bastardy, 
sure conclusion. In any case, however, the number of the insane is so large that their existence is a source of vast misery and hardship to very many people and a considerable and constantly growing burden to the State.

773. The problem of the causation of insanity-of the causation of its first appearance in a line of descent - has been much debated. Both imbecility and lunacy are so often reproduced in families in which they have once appeared, that it is certain that there is present, in some instances at least, an 'innate and transmissible' weakness, a predisposition to one or other form of mental unsoundness. ${ }^{1}$ Since lunacy is due to wrong learning, since the individual is a lunatic because he receives wrong impressions from his surroundings, the direct influence of the environment must necessarily, in a sense, always play a part in its causation. But, since imbecility is due to no learning, or a lack of sufficient learning, since the individual is an idiot or an imbecile because he does not mentally acquire anything or enough from his surroundings, the environment need not play a part in the causation of his weakness, $^{2}$ for he may be defective through a spontaneous variation which occurred in him or in a progenitor, and which

and crime are now paid for by the ratepayer, and any method of diminishing these at reasonable cost must be to his benefit.

"In the ruder state of society which has passed away little heed was taken of these unfortunate children, and many of them, no doubt, died comparatively early in the struggle for existence. But we have learned to think more tenderly of the inferior members of our race, and we seek to protect them from the calamities and sufferings to which they are naturally exposed, and to preserve their lives to the utmost. But in so doing, and in so doing rightly, we incur, as it appears to me, another responsibility, namely, that of preventing, so far as we reasonably can, the perpetuation of a low type of humanity, for otherwise the beneficence of one generation becomes the burden and the injury of all succeeding ones. The past increase in the number of lunatics in the country, to which I have already alluded, demands our most serious consideration of every means which can be legitimately used to protect the race from physical and mental degradation, and I regard the segregation of imbeciles, first in childhood and in youth, and subsequently throughout life, as one of the means which is most open to us" (Sir James Fry, Evidence before the Royal Commission on the Care and Control of the Feeble-minded, vol. i. p. 312).

1 "Undoubtedly those who have great experience in the care and supervision of persons suffering from mental defect in these forms, such as 'idiots' or 'imbeciles' .. . state that in a very large proportion these persons are the offspring of mentally defective parents or are members of families in which other nearly related members are mentally defective. Op. cit., vol. viii. p. I8I.

2 The reader must bear in mind that by the direct influence of the environment we mean such influence as causes change in the individual or the germ-plasm by acting directly on them. By indirect action we mean selection or cessation of selection. 
renders him incapable of being so affected by the environment as to learn from it.

774. But in another sense the environment-through violence, disease, alcoholism, business worries, religious and sexual excitement, and the like-may act directly as a cause of insanity by injuring the brain of the sufferer, or by injuring the germ-plasm contained in his germ-cells. That is to say, it may cause immediäte insanity, or be a cause of a tendency to insanity in descendants. Cases of insanity, especially of lunacy which is a disease of adult life, plainly traceable to injury or disease sustained by the sufferer himself, are not uncommon. We need not dwell on such instances, the causation being obvious; the difficulty lies, not in perceiving the right means of prevention, but in applying them. Our interest centres in those more obscure cases in which the individual is born insane (i.e. more or less incapable of learning), or born predisposed to become insane (i.e. to become a lunatic.) Such cases are due either to injury sustained by the germ-plasm, or to spontaneous variation.

775. Probably injury to the brain occurring during intra-uterine life (especially from maternal disease or alcoholism) accounts for some cases of filial insanity. Probably, however, such injury is much more frequently a cause of death occurring before birth or shortly afterwards. It may be that the high infantile mortality of urban as compared to rural areas is due in a measure to this cause. ${ }^{1}$ Cerebral injuries, whether acquired before or after birth, are not transmissible. But germinal defects tend to be inherited. If the variations which result in insanity are usually spontaneous, it is beyond our power to diminish their frequency. We can only, by controlling the output of offspring by the 'innately' insane, prevent their perpetuation in descendants. If, on the other hand, they are usually caused (on the occasion of their first appearance in a line of descent) by the direct action of the environment on the germ-plasm, it may be within our power, not only to prevent their perpetuation, but also, by improving the environment, their initial occurrence.

776. But every argument which tells against the hypothesis that variations in general are due to the direct action of the environment tells equally against the hypothesis that those

I "It is not necessary to dwell upon some other causes of mental defect, such as disease or accident in early life, or even before or at birth, operating through injury to the brain. Cases of this kind are recognized by all ; and do not complicate the present question. They occur in comparatively few instances (op. cit., vol. viii. p. I85). 
particular variations that cause insanity are due to it. As we have noted, medical men have been accustomed to attribute all sorts of filial defects to all sorts of parental and ancestral illconditions. ${ }^{1}$ Such evidence has often been tendered to Royal Commissions and other State inquiries, and has guided or misguided them in their decisions. The Report of the Royal Commission on the Care and Control of the Feeble-minded, the most recent of such State inquiries, however, indicates a rapid and a very remarkable change of medical opinion. After discussing the evidence in detail and noting that the weight of evidence is entirely in favour both of the spontaneous origin and subsequent inheritability of feeble-mindedness, the Report continues :-

777. "The view that injurious conditions in the environment which affect the parent or the child in utero or after birth are of importance in the production of feeble-mindedness needs some further practical comment. The Memoranda quoted in the preceding paragraph $(549)^{2}$ contain strong arguments against any likelihood of such causes being in any way operative as causes. Many races have been exposed to one or other of all the ill-conditions which have been alleged as causes of filial deterioration. In every case the only apparent effect has been to render these races capable of dwelling comparatively unharmed under such conditions. It is not to be conceived that a race which deteriorates in every generation can emerge from the struggle not weakened, but strengthened. Moreover, almost conclusive disproof of this hypothesis is furnished by the facts submitted to us by the medical investigators. These observers show that feeble-mindedness is practically as common in rural as in urban districts, and probably no less prevalent amongst the well-to-do than amongst the poor. It is clear that if the contentions of these witnesses who place predominant stress on adverse environmental influences as a cause of feeble-mindedness were just, there would be an unquestionable prevalence of this affliction among the urban poor-the chief victims of poverty and disease." 3

1 Here is an example. "The Feeble-minded in Iveland. When a country with few industries except agriculture has its life blood drained by the emigration of the young and fit, and when those who remain are depressed by the discouraging results of trying to keep their heads above water by tilling the soil, it is small wonder that the commissioners charged with the task of deciding what steps should be taken for the better care and control of the insane and feeble-minded, should have found a grave state of things to exist." (Leading Article, British Medical Journal, Sept. I2th 1908.)

2 The allusion is to contributions by Sir E. Ray Lankester and the present writer.

${ }^{3}$ Op. cit., vol. viii. p. 184-5. 
778. Medical men have often declared that the insane tend to be infertile, and that, therefore, the type tends to die out. The Report states:- "With regard to the allegation of the small fertility of mentally deficient persons, the reports of some of our medical investigators and other witnesses, both medical and otherwise, tend to show that although, as might be expected for several reasons, there is great mortality among the children of these persons, there is also a very marked degree of fertility and survival to adult age, especially among those of the higher grades who are termed feeble-minded. Miss Dendy, Honorary Secretary of the Lancashire and Cheshire Association for the Permanent Care of the Feeble-minded, a very experienced witness, has stated elsewhere that from her personal knowledge she can show that the higher grades of feeble-minded persons (who are the most numerous and dangerous) tend to have very large families; and she can prove this from the detailed records of 1,000 cases." 1

779. In conclusion, the Commission sums up the general effect of the evidence as follows :-

"(I) That both on grounds of fact and theory there is the highest degree of probability that 'feeble-mindedness' is usually spontaneous in origin - that is not due to influences acting on the parent-and tends strongly to be inherited.

"(2) That, especially in view of the evidence concerning fertility, the prevention of mentally defective persons from becoming parents would tend largely to diminish the number of such persons in the population.

"(3) That the evidence for these conclusions strongly supports measures, which on other grounds are of pressing importance, for placing mentally defective persons, men and women, who are living at large and uncontrolled, in institutions where they will be employed and detained; and in this, and in other ways, kept under effective supervision so long as may be necessary."

780. "The question naturally rises whether it is desirable and practicable that any steps should be taken to place obstacles in the way of the marriage of persons ascertained to be mentally defective, over and above the restrictions on marriage and procreation which would ensue from the detention in institutions of mentally defective persons in whose case such procedure is deemed necessary; and if there be a disposition to answer the question in the affirmative, the problem must be faced, to which of the classes of the mentally defective should such additional restriction be applied.

${ }^{1}$ op. cit., vol. viii. p. 185 . 
781. "Such legislation would not be an absolute novelty in English speaking communities; but there is little or no reliable information as to the practicable results of the tentative efforts in this direction which have been made in New Jersey and elsewhere ; and it is clear that such legislation however carefully restricted would in effect operate less by imposing an easily enforceable legal prohibition than by guiding and directing the advisory functions of medical and other authorities and in other ways educating public opinion to the proper consideration of a very serious evil.

782. "We are of the opinion that it would be unwise to modify or supplement the existing law with respect to the marriage of 'persons of unsound mind ' [i.e. lunatics]. It is perhaps unnecessary to refer to other objections to any such proposal; it is clear that the limited and often temporary nature of this particular mental disability renders it impossible either to prohibit permanently the marriage of such persons or to set out in practically useful legislative proposals the conditions under which such marriages might take place.

783. "No such obstacle, however, presents itself in the case of those persons who exhibit the congenital and incurable forms of mental defect [i.e. imbecility], and we believe that a legislative prohibition affecting these classes would have useful direct and indirect effects." 1

1 Op. cit., vol. viii. p. 185 . 


\section{CHAPTER XXV}

\section{EDUCATION}

The most important practical problem of biology-Our power of influencing mental growth-Self-education-Formal education-The three aims of the school-master-Subjects taught, and methods of teaching them-The teaching of young children; of older children; of young adults-Religious teachingThe religious attitude is an acquirement-The antagonism between religion and science-Methods of teaching religion-Prejudice and superstition-Economic and social effects of methods of teaching religion-Inborn and acquired feeblemindedness and lunacy-The teaching of the classics-Scientific teaching-Science teaching-Some sciences are founded on few and some on many facts-The former are mainly interpretative, the latter mainly descriptive-Systematists-Darwin's effort to make biology interpretative-Description is the warp and interpretation the woof of science-The teaching of biology-The importance of the scienceConclusion.

784.

$\mathrm{F}$ all the practical problems on which the study of
heredity sheds a light, by far the most important is
that of the education of the young, if only for the reason that the solutions of all other problems, if soluble they be, depends on the intelligence of the race that takes them in hand. Here, since the mental growth which the normal human being makes under the stimulus of experience is so vast, since according to the stimulus supplied this growth may take any one or more of a thousand different directions, and since the kind and amount of stimulus is very greatly under the control of the guardians of the child, our power for good and evil is at its maximum. Training may endow the child with wide and useful knowledge, or with knowledge which, though great, is narrow and useless, or it may leave him very ignorant; it may bestow on him habits, tones and attitudes of mind which enable him to utilize such knowledge as he acquires to the utmost limit of his capacity, or which render the widest knowledge and the highest capacity unavailing. There is abundant evidence that a child of normal capacity may be trained to a degree of stupidity resembling innate feeble-mindedness, or to a degree of wrong-headedness resembling insanity, or, on the other hand, to a degree of intelligence which, relatively speaking, resembles genius. We may differ, indeed men do differ very 
widely, in ideals of mental training; we may not agree as to what kinds of knowledge and mental habits are the best; but no one will deny that a knowledge of what may be, and has been achieved by mental training, and of the means best adapted to achieve any desired result, would be a great advantage to the teacher. Every one will agree also, in theory at any rate, that wide and useful knowledge and high intelligence are desirable qualities, and that ignorance and stupidity are not. Unfortunately, useful knowledge and high intelligence are eulogistic terms which are applied by different men to very unlike things. For example, the terms would hardly have had the same meaning for Huxley and the curates whose 'cackling' he abhorred. In his opinion the curates were ignorant and unintelligent; in their view his knowledge was not right knowledge, and his intelligence damnable.

785. We have seen that memory is nothing other than a power of growing, of developing mentally under the stimulus of use, and that on this faculty depends all thought, all intelligence, all mental adaptability. Great intelligence must have for its basis a power, correspondingly great, of profiting by experience; or, in other words, a great memory. Man is intelligent because his memory is very capacious. It contains not only such things as words, scenes, events, and processes of reasoning, which can be recalled to consciousness, but also dexterities, habits, bents, tendencies, tones, and mental attitudes which cannot be consciously recalled or pictured in the mind, but which are every whit as important. We have seen also that, not only our 'physical' dexterities, such as walking and the delicately co-ordinated movement of the lips and tongue in speaking, but also such 'mental' dexterities as are involved in associating, comparing, discriminating, and reasoning depend on memory. Lastly, we have to see that, just as the instinct of sport impels the child to play with his limbs till he is able to use them dexterously, so a homologous instinct impels him to play with the contents of his conscious memory till he is able to use them skilfully. Probably this impulse to play with the contents of the conscious memory, to think, is the most imperious instinct in our nature. At any rate, it is the one which is the most ceaseless in its promptings. A man may resist hunger, or thirst, or love, or sleep, but not for a moment can he resist the impulse to thinkthe impulse to learn to use the contents of his conscious memory skilfully and thereby store his unconscious memory.

786. Prompted, then, by the instincts of play, curiosity, and imitativeness, the child undertakes the earlier part of his own 
education and performs its task supremely well. This phase of education, because directed by instinct, is full of pleasure and interest, and follows the same lines in the children of all races. With the aid of the hunting instinct it bestowed on primitive man almost all the acquirements that enabled him to maintain existence. But as soon as man became man, and more especially when he became civilized, labour, a new mode of activity to which he was compelled by the environment he himself had created but to which he was not prompted by instinct, and which, therefore, was not pleasurable, became necessary. Labour is seldom associated with pleasure, unless interest is awakened by some element of sport, curiosity, imitativeness, or some other instinct. At first, doubtless, all labour was associated with some instinctive promptings, but as civilization advanced, as life grew more complex and labour aimed at ends more and more remote, its instinctive promptings withdrew further and further into the background, and were replaced by motives derived from intelligence. Through labour men learned new dexterities. They fashioned tools, and learned to use them. They invented a mode of recording words and thoughts by written symbols and learned to become skilful in interpreting them. Ever, as the mental horizon widened, toil played a greater and greater part in education. At present our children spend many of their most receptive years at such labour; and when we speak of education we mean, as a rule, only the toilsome part of it.

787. This laborious, or schoolroom, training is formally designed to achieve two aims. An endeavour is made, on the one hand, to impart knowledge of a kind that cannot be gathered by the child during the course of his non-laborious education outside the schoolroom, and, on the other, to train the mind to deal skilfully with the ordered knowledge thus acquired, and in this way develop powers of thought (i.e. skill in thinking) more far-reaching and accurate than could be otherwise attained. A third aim to which the schoolmaster is supposed to devote attention while he is the immediate guardian of the child is the formation of 'character.' 'Character,' however, is not acquired with the same obvious toil as knowledge and skill in thinking. It arises, more or less unconsciously, as a reaction between the child's capacities and the total environment, of which the schoolroom is only a part, though it may be and often is, an important part.

788. The three aims of the schoolmaster are separable in theory, but not in practice. If facts be taught to the pupil, he tends to 
think about them; and, according to the way in which he thinks his character will be influenced subtly and profoundly. All arrays of facts do not afford equal facilities for acquiring skill in thinking. Some, for example, the lengths of rivers, the heights of mountains, the dates of history, and facts about the bones and muscles of animals, may be useful as items of knowledge, but by themselves they afford poor materials for the play of thought. Others, like the data given in mathematical exercises, may be useless as items of knowledge, but exceedingly well adapted as materials for mental gymnastics.

789. Facts are relatively useful or useless as items of knowledge and as materials for creating powers of thought, not only in proportion as they are true and afford exercise in hard thinking, but in proportion as they do or do not link up with the subsequent experiences of the learner. Thus, since the elementary truths of arithmetic are used almost every day in our lives, they are remembered and have a greater influence on the mental career of the average individual than the higher mathematics he may have learned; for the latter, though they require more strenuous thinking, are seldom used, and, therefore, being forgotten, tend to lose their influence. Again, facts as materials for thought derive their value for the pupil very largely from the way in which they are acquired; for on this depends the way in which he will think about them, and the way he thinks about them will in turn influence his whole habits of thought. Thus the facts of geometry are comparatively valueless if learned by rote, but excellent if the chain of reasoning by which they are demonstrated be understood and assimilated.

790. The teaching of bald collections of facts is comparatively easy. Any man, who has a book of reference and means of inflicting pain, may force his pupils to learn the dates of history, the outlines of zoology, or the words of a language. Unfortunately the ease and lack of intelligence with which this kind of teaching may be conducted, the little labour entailed on the teacher, and its effectiveness for purposes of examination or other displays of mere knowledge, constitute a strong temptation for its employment. It is very widely used, not only for the tuition of little children, but also for that of young men and women on the threshold of independent life. Indeed little children are usually better taught than older people. Their thinking processes are so simple, their instinctive curiosity and tendency to play mentally with all they learn so strong, that even the inferior teacher, taking 
the line of least resistance, finds himself joining in their play, asking and answering questions, suggesting analogies and other relations; in brief, giving lessons in careful thinking. Anything so good as the object lesson or the kindergarten system is rarely found in the tuition of older boys and girls. Moreover, in England, the majority of children attend the public elementary schools, the teachers in which have been trained for their functions, are under strict and competent supervision, and know how to make lessons interesting. It is quite common for poor children to prefer the school-term to the holidays.

791. A principal qualification of a good teacher lies in his power of interesting his pupils, a thing that can be done only by arousing their emotions, their instincts, or the imitation instincts which the teacher helps to create. Rewards and punishments may be made the means, but a much better way is to appeal to curiosity, imitativeness, and, above all, to that strongest of instincts which impels us to play with the contents of our memory, and so, by ascertaining the relations, particularly the causal relations, between the objects of thought, to unify the world our minds construct. If the teacher is dexterous, curiosity will cause his pupils to ascertain and store facts, imitativeness will cause them to copy his own processes of thought, and the instinct to play with the contents of memory will impel them to become skilful in thinking. The best of all teachers is one who does not merely state, nor even explain, the relations between facts, thereby doing little more than add new facts to the rest, but who so guides his pupils that they are led to do their own thinking and make their own discoveries. Both facts and thinking then acquire enhanced value. Not every subject affords full scope to his talents. But usually he has some power of choice, if only between the parts of a subject, and something may be done even with facts the relations of which are so simple and obvious that they are perceived without effort even by children. Unhappily, skill in teaching is rarely one of the qualifications by means of which the tutors who train the children of the governing classes secure their appointments. Doubtless many tutors, through private study or because they are of a thoughtful cast of mind, learn to teach well; but this ability is not, as in the case of teachers in public elementary schools systematically fostered by compulsory training.

792. Probably, as a body, the least skilful teachers are those who complete the training of young men of the professional classes. As a rule they are people who have achieved success in the 
practice of some lucrative profession, for example, medicine. They have knowledge, but they are seldom young, and, consequently, in many cases they are not very capable of learning such a new thing as how to teach. Indeed they rarely have any notion that there is anything to learn. Doubtless there are amongst them many 'natural' teachers whose processes of thought, through constant, if unconscious, self-training, combined perhaps with superior capacity to learn to think well, have become so clear and logical that they are able to present the objects of study interestingly and suggestively to their pupils. But probably every student of a profession hears lectures, attendance at which, though compulsory, is not only a waste of time but is also positively harmful to a developing and imitative mind. The method too often adopted is, not the Socratic plan of teaching the student, whenever possible, how to think in order that he may discover what to think, but that of the Catechism which asks only stereotyped answers to stereotyped questions. Among the pupils are future teachers, and in science, as in religion and other human affairs, evil as well as good traditions tend to be perpetuated.

793. Except as regards a supply of facts, some professional courses, for example the medical, hardly attempt to continue intellectual development. It is not reasonable to suppose, however, that the preparatory schools have already accomplished all that is possible to stimulate the reflective powers, or that mere accumulation of knowledge will do it. Men who have continued their formal education for some years after leaving school, usually possess much greater knowledge than their less fortunate fellows; but it is not equally obvious that their skill in thinking has developed a corresponding superiority. In brief, the teachers who direct the concluding stages of professional education, having been trained by an imperfect method, are usually unable to do other than train their pupils by it. They are compelled thereto, not only by the requirements of examinations which have been framed by men like themselves, but by their own attitudes of mind. When, as is not unusual, they exclaim that they are able to supply their pupils with knowledge but not with brains, they condemn their own methods. On the average they deal with normal human beings; and since 'brains' grow under the stimulus of use, it should be a main part of their business to develop them.

794. It is, of course, out of the question to select as teachers to young men about to enter a profession any but its most eminent practitioners. It is equally out of the question to expect 
men who have achieved distinction in any important branch of study, and who have, as is often the case, made considerable additions to knowledge, to acquire in middle or old age the sort of skill that is considered essential in good pupil teachers. Moreover, students who adopt a professional career must acquire the data on which practice is founded; and the array of facts which have to be committed to memory is often so vast that little time is left for thinking about them. Many of the subjects in the curricula are such that the best of tutors are forced to restrict themselves to teaching facts and habits of close observation. Nevertheless, a good deal might be done to awaken science teachers to a conviction that the function of higher education is to impart more than knowledge and habits of observation. Some of the facts and subjects on which students must pass examinations have no bearing on practice and might be exchanged for others which place a greater strain on the reflective faculties; or, at least, the teaching of them might be so modified that the reflective faculties are more exercised than at present.

795. If we train a man well in any department of 'physical' activity, such as penmanship or cycling, he tends to become particularly dexterous in it. But the influence of special training does not altogether end with the acquisition of special dexterity. Thus an individual who has learned to co-ordinate his muscles for walking has made a step towards learning to co-ordinate them for cycling. So, also, if we train an individual to think well in any sphere of 'mental' activity, for example, chess, mathematics, or physics, he will not only become especially skilful in it, but his acquirement will tend to colour his whole mental state by exerting a greater or lesser influence in other departments of thought-a greater influence in kindred departments, a lesser influence in departments that are psychologically more remote. By themselves facts are isolated things ; each fact must be acquired separately, and, unless linked by thoughts to others, held separately. But mental dexterities, habits, attitudes, link together inevitably; one furnishes a stepping-stone to another. Nevertheless, just as we cannot make a man a good cyclist by teaching him to walk, nor even by providing him with plenty of bicycles, so we cannot insure that he will be a good thinker in any department (e.g. the ordinary affairs of life) by training him in another department (e.g. mathematics), nor even by providing him with the materials of thought, the facts, in the chosen department. To insure the best results our teaching must be direct; that is, we must not only provide the right facts, 
but we must also train our pupils to think about them in the right way.

796. To sum up: skill in thinking, like other dexterities, depends partly on innate capacity to learn, partly on the amount of practice, and partly on the kind of practice. Innate capacity, though it varies with individuals, is a fixed quantity in each individual. But the amount and kind of practice is within our control. We deliberately train young children to think skilfully, and the younger they are the more direct is our teaching, the more closely adapted to the end in view and the needs of the subsequent career. But as they grow older and ripe for the attainment of higher grades of skill, we tend to slacken our efforts, or to seek our ends in a very indirect and ineffectual way. The human being tends to remember only impressive things, or those which are hammered into his mind by repeated experiences, and he tends to think only of that which interests him. Often, by way of mental gymnastics, in the vague hope that the youth will remember and think, we supply him with facts which, rightly used, might furnish excellent materials for thought, but which we do not impel him to use, and which he does not use of his own initiative, because by themselves they are uninteresting and of such a kind that his subsequent experiences do not keep them in mind. We put him on the tread-mill and expect him to become speedy in the race. ${ }^{1}$

1 I remember a professor of zoology telling me some years ago, that thougb he did his best to convince his pupils, principally medical students, that acquirements were not transmissible, his teachings were 'spoilt' by the physicians and surgeons through whose hands the young men subsequently passed. He quite recognized the importance of the matter, and alluded sorrowfully to the medical evidence given before various Royal Commissions by former members of his class. His lectures consisted in more or less detailed anatomical descriptions of certain types-starfish, sea-urchin, earthworm, leech, cockchafer, lobster, dogfish, cod, snake, rabbit, and so forth-animals which, by way of practical work, the pupils were required to dissect. His examinations were designed to discover the extent to which these teachings had been committed to memory. Once or twice during a session he mentioned the theory of evolution and expressed his agreement with it. Once or twice, also, he told his pupils that, though the matter was still sub judice, he was convinced that acquirements were not transmissible, and added an account of various experiments, such as the amputation of rats' tails. The anatomical studies of his pupils were so conducted that nothing was learned but facts, their co-existences and resemblances-that one species was related to another because such and such structures were possessed by both, that the lobster had certain organs and the rabbit certain others, that the dogfish had no bones but only cartilages in such and such numbers and shapes, but that the cod had bones of which there were just so many in his head, and that the precise shape and spatial relations of these bones were this and that-facts that had no bearing, or none at least that he made clear, on the problems of heredity which he recognized as so important; and, indeed, so little bearing on anything else in 
797. The extent to which thinking power may be influencd by the formal training received during early manhood cannot easily be observed in the individual. When trying to analyse the degree of intelligence displayed by him, we do not know what part to attribute to innate capacity, what to formal training, and what to various other influences, such as those exercised by his companions, all of which contribute to his intellectual development and status. If we meet a clever man and approve the system by which he was trained, we are apt to attribute his efficiency mainly to training; if we disapprove of the system, we tend to assign it to innate capacity. Owing to lack of systematic inquiry, this question of what is 'innate' and what is ' acquired ' in the human mind, though the most important of all practically, is yet the most neglected problem of psychology. The consequent uncertainty, combined with the difficulty of ascertaining the precise results of this or that system of mental training and then comparing them with the results obtained by other systems, has led to a vast diversity of opinion as to which system is the best, some authorities advocating one, some another, and some holding, apparently, that so much is innate that all systems produce nearly the same results in the end. ${ }^{1}$ It is possible, however, to overcome the difficulty in some measure.

798. We found that the solution of the problem of the causation

the medical curriculum or the affairs of everyday life that, when the examination on them was over, they were promptly forgotten by all except the few students who adopted zoology as a profession or as a hobby. The professor's convictions were countered by the convictions of other professors-of physiology, pathology, medicine, surgery, and the like. His experiments were met by accounts of other experiments (extraordinarily misinterpreted), by details about cats, almost as legendary as Bo-Peep's sheep, that had left their tails behind them in traps and doorways and had borne tailless offspring ever afterwards, by stories about human mothers who had seen moving sights and borne marked children, and about consumptives, alcoholics, syphilitics, and imbeciles whose children were similarly affected. Their reasoning was founded on credulity and confusion of thought, but the students had been so trained that they were unable to perceive its weakness. But, if the zoologist, instead of stating his convictions or appealing to evidence which his pupils could not verify, had carefully explained the distinction between variations and modifications, pointed out the difficulty of believing that the latter could affect the germ-plasm in such precise ways as to be reproduced in the child as 'innate' characters, and had then appealed to the facts of ordinary experience and of human evolution against disease, he would have supplied his audience with data and thoughts pregnant and unforgettable because they would have linked up with subsequent experiences, and with a mental training which would have enabled them to appraise at their true worth the puerilities of professors who argued, for example, that " acquirements must be transmissible, for every character must have had a beginning, and therefore must have been acquired by some one who handed it on to his descendants."

1 See $§ 708$. 
of variations is impossible as long as we observe only individuals, but that we are able to arrive at definite and almost certainly true conclusions when we compare races that have evolved under known but unlike conditions, and more particularly when we are able to compare the present of a race with its known past. The same method will serve in the endeavour to ascertain the effects of mental training in early manhood. We shall thus, if only we think carefully enough, be able to compare different systems of mental training and separate the effects produced by them from those produced by the other influences to which the individual is exposed. All systems profess to teach, on the one hand, knowledge, and, on the other, right and efficient ways of thinking. The amount and kind of knowledge imparted by any system is easily observable. Our object, then, is to ascertain the intellectual effects other than mere knowledge-the kinds and qualities of the thinking resulting from the principal methods of teaching. We shall see more clearly than ever that, while knowledge, as is very obvious, results from the things that are taught, the quality of the thinking depends in a degree that is rarely realized on the way in which they are taught.

799. The effects of religious teaching offer very favourable materials for study. All sorts of religions have been taught, in every variety of way, to races all over the world and in every stage of culture and civilization. Some religions have been held by the same races for many consecutive centuries. Other races have rapidly and repeatedly changed their religions. Some religions have been held by many diverse races, while in other cases sections of the same race have followed two or more diverse religions. In some countries the inhabitants are all of one religion, which is taught to every one in much the same way; in others, systems, strongly contrasted in the things they teach and in the way they teach, divide the inhabitants. In different times and places the same religion has been taught by different methods. As in the case of diseases, the range and diversity of the phenomena thus presented enable us, through processes of analysis and comparison, to reach conclusions otherwise unattainable.

800. At this stage I must beg the reader to bear in mind constantly the fact that we are discussing, not the truth or untruth of religious doctrines, but only the mental effects resulting from methods of teaching. If we strictly limit the discussion to this question, and if our reasoning be right, we may, however much we may disagree as to religious doctrine, be able to reach an agreement as regards method. At any rate, we shall have 
gathered materials which may help us to agree ultimately. Even if doctrines be regarded as Divine revelations, yet the ways in which priests, parents, and schoolmasters teach them are necessarily human devices, and therefore legitimate subjects of criticism. It is admitted that good teaching tends more than bad teaching to create intelligence; and a religion like any other subject may be well or ill taught. Again, since few people are able to think without bias of their own faith, and since it is probable that the reader is an average individual, may I beg him, if I happen to mention his religion, to try to think in terms of some other. Thus, if I make mention of the Christian sect to which he belongs, let him try to conceive some corresponding Mohammedan sect and suppose we are discussing that.

80 I. All religious knowledge is, of course, acquired, as are also the kinds of thinking and the attitudes of mind that result from religious teaching. It is because they are universally recognized as highly important acquirements that so much care is taken in teaching them. Since religion offers immense rewards and punishments, covers the whole field of morals, inculcates particular views concerning the universe, influences the individual in all his public and private relations, and tends, therefore, to be revived in his thoughts more often than any other subject that is formally taught, probably none other so profoundly affects his intellectual status. But children, though they may be coerced to religious thoughts and observances, are comparatively little affected by them. They are too completely under the sway of strong instincts which impel them to thinking which is, at least, non-religious; their minds are very ductile and therefore incapable of those passionate convictions and unalterable mental attitudes which so often characterize the adult, and which we term perverse and fanactical in people who differ from us; their stage of development is such that they are but little capable of appreciating the surpassing importance of ' religious truth,' or of being influenced by religious motives in the way that the adult is influenced. To them religion is but one of the many wonderful things that they learn from the adult - the occasion of a story or a lesson, of a game or of a labour. The fervour of the saint or the dervish, the stern conviction of the puritan, and even the hysteria of some sects is not for children. Only after adolescence is reached does religion exercise its typical influence.

802. Speaking generally, in proportion as the mental influence of a religion is wide, the intellectual status with which it is asso- 
ciated is low. This, of course, does not necessarily imply that the doctrines of all religions are untrue and degrading. If the religion is true, it may imply merely that many of the doctrines taught by it have, through the advance of science, been established on verifiable evidence, and, therefore, have passed from the category of things believed to be true into that of things proved to be true-into that category which it is the hope of all religious people that their articles of faith will be found at the awakening after death. They then become a part, not of 'religious' truth, but of that ordinary secular knowledge which, when systematized, is termed science. For their acceptance an "act of faith" is no longer needed. Thus, if all the doctrines of Mohammedanism were verified, they would be distributed amongst the various sciences, and, since no indisputably verified fact is ever regarded as a religious truth, the religion, as such, would cease to exist. It would be rejected, not by the irreligious, but only by the ignorant, who, as a rule, would be prejudiced adherents of some other faith. Probably, too, the emotional tone of true believers would undergo change. Verified truth is not regarded with that peculiar devotion to which the adherents of religions are trained. There is no need for it. Thus, in science heated controversies occur only about matters that are doubtful. No one was ever fanatical about a mathematical demonstration. It follows, that as verified truth accumulates, the area covered and emotionally coloured by religion tends to shrink. For example, our ancestors held as religious truth an immense body of belief concerning the visible universe, but nobody claims any part of our present verified knowledge as an item of faith, and, if any of our fellows now held that the earth is flat, we should think them foolish, not wicked. But "when we examine the general culture of the savage we find that it is entirely religious. The savage not only has religion, but apparently has nothing else." ${ }^{1}$ The heavens and the earth, every echo, rainbow, and lightning flash, every disease and accident, even the things he eats, and the rocks and trees he dwells under, are interpreted by him in religious terms. Compare the area covered by his religious convictions with the shrunken area covered by the modern Christianity of the more enlightened peoples. In one sense, then, science is always antagonistic to religion. Whatever is true it tends to render non-religious; whatever is false it tends to overthrow; and invariably it tends to alter the emotional tone,

1 The Rev. A. E. Crawley, "The Origin and Function of Religion," Sociological Papers, vol. iii. p. 245, Macmillan \& Co., 1907. 
the mental attitude. If the reader doubts this statement, it would be well if he tried to think of a truth which has remained religious after having been verified, or of a verified truth which he regards with emotional devotion.

803. Most religions have, or had, doctrines about the shape of the world. The ancient Christian belief that the world is flat has been abandoned, and, therefore, may furnish a text, the discussion of which, while permitting a measure of freedom, will arouse a minimum of prejudice. This 'fact,' that the earth is flat, may be taught in three distinct ways. First, it may be stated to the pupil, after the manner of much science teaching, as an obvious truth, which he may observe for himself by looking at the horizon all around him; so that, though he is supplied with material for thought and not forbidden to think, no further attempt is made to awaken thought or to influence its direction or quality. Or, to speak more accurately, while the pupil is supplied with facts which he usually learns to arrange according to their co-existences and resemblances, he is not taught to classify them according to their invariable or necessary sequences. Therefore, since his notions as to what constitutes evidence and what proof are not derived from his teacher, and since much of the thinking, indeed, nearly all the difficult thinking, of human beings is in terms of cause and effect, the main part of his intellectual development is neglected. Examples of this kind of instruction, which is little more than a storing of information, and which is regarded by modern educationalists as a necessary evil that should be reduced to a minimum, are seen when the lengths of rivers, the dates of history, or the facts of systematic zoology and botany are committed to memory. Facts concerning the shape of the world are so impressive and suggestive and tend so to link up with subsequent experiences that, unlike many 'scientific' facts, they are sure to be remembered and thought about; but the use that is made of them by a pupil taught according to this system is left more or less to chance. The mental attitude created is such that, though the pupil is left free to learn the actual truth from other people, he is not very likely to correct the initial error himself.

804. Second, not only may the pupil be told that the world is flat, but, as an essential part of the teaching, he may also be made to proceed to a rigorous deductive inference of consequences and a comparison of these consequences with reality. In other words, he is taught to classify his facts not only accord- 
ing to their co-existences and resemblances, but more especially, as in the ordinary teaching of mathematics and physics, he is trained how to arrange them according to their sequences. Acquiring deftnite ideas as to what constitutes evidence and what proof, he values his facts and hypotheses in proportion as they are capable of verification and of being linked with other facts and hypotheses. Thus, given the flatness of the world as an initial fact, he may be told that it follows necessarily that, if armed with a powerful telescope he stands in clear weather on the seashore, a ship will never sink below the horizon but will be visible from truck to waterline all the way to America, that a ship that sails continually to the West will never come back by the East, that the sun and stars must revolve round the earth, and that, since they are so many, since they rise and set in slightly different places every day of the year, and since their apparent positions in relation to one another are more or less fixed, it is likely that they must pass beyond the edge of the flat earth and not through holes in its surface; and so forth. The initial statement made to the pupil is untrue. He will start his reasoning from false premises. But, at least, he will reason and will treat his apparently established fact as material for thought and as the starting-point for the acquisition, examination, and systematization of more facts and thoughts. Moreover, facts and the thinking founded on them are distinct things. As was often the case in Pagan Greece, very good thinking may be founded on false premisses-thinking so good that presently the premisses are likely to be discerned as false, as not in accord with reality. But, whether the premisses be true or false, the main feature of this kind of teaching is that it tends to endow the pupil with an acquisitive, an open, a reflective habit of mind. Not only is he left free to learn the real truth about the shape of the world from others, but, because he will try to link up his belief with so many phenomena, he is apt to discover it for himself. Not only does he tend to think freely and carefully about his past experiences, but he remains alive also to fresh experiences, which, again, he tends to think about, and link up with, and use as a test for that which he has already stored-a mental attitude which tends continually to increase his store of material for thought and his skill in using it. He learns to remain a learner and become a thinker. $\mathrm{He}$ takes every possible means to avoid error. The universe his mind constructs tends continually towards that organized whole which is the goal of all good thinking and all good science. 
805 . We have seen that a vast capacity and tendency to profit by experience is the distinctive attribute of the human being, and particularly of the child. Through this arises what we call intelligence. In one sense the adult is more intelligent than the child; for, having had greater opportunities, he has profited more from experience; but in another and a very real sense, the child is the more intelligent; for, as a rule, he is more receptive, more capable of assimilating fresh experience to that already stored. As may be seen by comparing a Darwin with a Mohammedan ecclesiastic, or average men trained in modern Europe with those trained in mediaval Europe or in Thibet, the degree in which the adult preserves the youthful tendency to reach out the tentacles of the mind and knit together the materials thus gathered depends on the formation of a HABIT, which, in turn, depends, in great measure, on the kind of formal training he receives. The kind of teaching we are now discussing, though not especially employed for science subjects, is usually termed scientific by modern educationalists. It was practised in its highest perfection by the Pagan Greeks, and was, as it seems to me, the source of their greatness. We have only to read, for instance, the dialogues of Socrates, to perceive how strenuously they strove to create not only skill in thinking, but also that open, receptive mind which is its necessary accompaniment. At any rate, every nation on earth is, and has been, great in proportion as it has practised it. At the present day, owing to the freedom of thought permitted by modern civilization, we all tend to receive, in greater or lesser measure, this kind of training from our general surroundings ; but in our formal education it is used comparatively little except, as we have already noted, in teaching young children.

806. It has been used only very rarely for religious teaching, and can be used for it only when the religion is in no way opposed to known truth, or when the teachers have no dread that newly discovered truths-deceptions of the devil-will oppose it. If the religion is in any way false, if it covers and colours a wide area of the mental field, and if the contemporary science is rapidly advancing, the two are apt to come into sharp conflict; and then the scientific method is liable to be abhorred by some, at least, of the exponents of religion. On the other hand, when science has made but small advances, the current religion, even if false, may offer as good an interpretation of observed phenomena as can be conceived, and therefore may be very tolerant. This, to an exceptional degree, was the case in Pagan Greece. The excellence of Greek 
training in habits of thought was due, apparently, to an unusual combination of accidents. There existed an enthusiasm for mental development, the religion offered little or no opposition, the supply of verified and systematized knowledge was small; therefore the teachers could train, and did train their pupils in little besides methods of thought. In modern times the supply of facts is so enormous that the tendency is to teach nothing else-facts of language, history, geography, zoology, and the like.

807. Third, the 'fact' that the world is flat may be conveyed to the pupil in such a way that he holds it, as an article of faith, with a sentimental devotion that has for its correlate an active dislike or blank disregard of all evidence that tends to controvert it. Of course, all men do not hold their faiths in this way. Faith, even religious faith, may be associated with an open mind. But there is evidence all around us that men may be taught to hold it thus. This kind of thinking is essentially sectarian, and is very different from that which we have just considered. It, also, deals with the relation of cause and effect; but the criteria for evidence and proof are not the same. The learner is taught to appeal not to reality, but to authority, and to value his facts and hypotheses not in proportion as they are capable of verification and of being linked together by chains of reasoning, but in proiportion as the facts have been gathered in a particular field (e.g. Crabistian, Mohammedan, Hindoo, experimental) and the hypothesces have been formulated by particular persons. Under the binfluence of such teaching, not only is a given 'fact' accepteed by the pupil, but an attitude of mind is created which co-reliegionists (e.g. modern Mohammedans) describe as one of simple: or steadfast faith, but which opponents (e.g. modern Christ jians) term superstitious or fanatical. Both descriptions are correct. A superstition is a religious prejudice which is fanatical whenc the emotional bias is very strong. It is not necessarily an untrue, but only an unverified belief held in a certain obstinate, unreasoning way, which renders the mind more or lepss incapable of profiting from fresh experience. A faith that wo ill be maintained in spite of evidence that it is untrue, is both rsimple and steadfast, and superstitious and prejudiced. Obviously, then, as regards mental habits, it is not the truth or untruth of a doctrine that matters, but the attitude of mind. Doubtless, all of us hold many untrue beliefs. They are not prejudices if founded on ignorance or on mere bad judgment. On the other hand, a truth, which its pioneers discovered because they had open minds, 
may be held as a prejudice by followers whose minds are closed. Thus, if evidence were now produced which conclusively proved the flatness of the earth, it is very certain that many minds, even minds of scientific men, are so trained that they would cling to the notion that it is round with unalterable devotion. I think I have met people who held the theory of natural selection in this way. The kind of teaching which most commonly produces a faith, that turns from opposing evidence with abhorrence or contempt, is what is usually termed dogmatic. It is generally practised by sects which take a pride in being 'orthodox'-in holding opinions which are quite identical for all the members and every generation of the communion.

808. It is interesting to note how readily we are able to recognize as such superstitions and prejudices to which we have not been trained and which conflict with our own beliefs. Thus, every Mohammedan is able to perceive, not only how slight is the foundation of fact, or how great the misinterpretation of fact, on which Hindoos have reared the monstrous edifice of their faith, but also to perceive that nothing but intense prejudice prevents, if not a recognition of the truth (the doctrines of Mohammedanism), at least a recognition of falsehood (the doctrines of Hindooism). If we place irrefutable evidence before the ardent adult Mohammedan or Hindoo, and preach it for half a century, we shall not shake his faith in the least. Moreover, and this is the important point, his mental inertia, his habit of thought, extends beyond the articles of his faith. It determines his whole attitude. It is hard to interest him in new ideas or set him thinking on new lines. Thus, though he may be forced to perceive the advantages of a new gun or some other mechanical device, he will seldom invent or improve one. $\mathrm{He}$ is capable of patient labour on traditional lines, and even of prolonged and passionate outbursts of energy when under the influence of strong emotion; but he is quite incapable of that other sort of patient thought and sustained effort, that wide appeal to experience, that rigorous deductive inference of consequences, and that receptivity, which make individual men, or those collections of men we call nations, pre-eminently human, thinkers of new thoughts, learners and discoverers of new truth, and therefore progressive and successful over competitors who are worse equipped mentally. Very rarely does his race produce a man distinguished in any field of human thought and endeavour except religion. Examples of his great men are Peter the Hermit and 
the Mahdi. Great men in fields of labour other than religion are invariably thinkers of new thoughts; and if, by chance, such a one appears in an orthodox environment, he is apt to be treated with derision or dislike as insane or wicked, and so to perish unrecognized, ineffective, and inglorious. The children of the orthodox, on the other hand, are quick and receptive, for, given the opportunity, they are easily trained to recognize new truth. Plainly, then, the adult has been made incapable of learning. He has acquired a stupidity which is inborn in the lower animal and the congenital imbecile. Contemporary Mohammedanism and Hindooism afford examples of religions that cover and, since they are taught dogmatically, colour so large a portion of the intellectual field, that a habit of mind is created which is carried into every relation of life. Hence the stagnation of their adherents.

809. As compared to the lower animal and the imbecile, the normal human being is distinguished by his control over his instincts and passions, which prompt him as strongly and less imperiously. What is true of the normal man as compared to lower types is true of the more reflective as compared to the less reflective man. If only because the former is more far seeing, he tends, on the average, to be more 'reasonable,' more orderly and law-abiding. Therefore, open-minded and progressive communities not only tend to secure improved governments, but they are also more easy to govern. Among them individual freedom, mild laws, and a civil unarmed police tend to replace tyranny, savage laws, and a military government. Compare the social states and the governments of Morocco, Turkey, Persia, Thibet and China, with the domestic peace of many Christian communities, and of Japan since its type of mental training changed. Compare modern 'orthodox' Christian communities with those in which a greater play of the reflective faculties is permitted by the religious teaching. Note the comparative statistics of murder and other crimes of violence, the acts of brigandage and dacoity, the rebellions, civil wars, and conspiracies against the established government, and the acts of tyranny committed by the government. ${ }^{1}$ Note, also, the

1 All sorts of hypotheses have been formulated to account for the relative prevalence of crime. Here is a curious example from a particularly sane book (Recollections, by David Christie Murray, p. 247). "My sympathies are with the old exploded prize-ring. Rightly or wrongly I trace the growth of crimes of violence to the abolition of that glorious institution. I want to see it back again, with its rules of fair-play, and its contempt for pain, and its excellent tuition in temper and forbearance." Students of the question should study statistics. But they should not, as is so very common when it is desired to 
output of great men whose labours have rendered their nations illustrious. It will be found, no matter how fervently the priesthood inculcate a reverence for thrift, law and authority, that, of any two communities, the more orthodox is almost invariably the more poverty-stricken, the more turbulent, and the more savagely governed. All orthodox communities are flourishing in proportion to the number of heretics they include. Wherever the heretical (e.g. Protestants) and the orthodox (e.g. Greek Churchmen) mingle on equal terms, the former tend to be the more law-abiding and to occupy the more responsible and lucrative posts, whereas the latter tend to become hewers of wood and drawers of water. The great men produced by orthodox communities are relatively very few,

demonstrate, for example, the injurious effects of secular education, compare material derived from localities (e.g. new colonies) that are undergoing rapid change with data gathered from places where an old order standeth. Nor should the statistics of the country be compared without reservation with those of the town. Rural populations always tend to be the more law-abiding (see \$ 523). Here are some statistics of murder from an author (see Crime and its Causes, by the Rev. W. D. Morrison) who attributes crime to climate, but who has neglected to compare the relative amount of crime in Australasia and South America, both of which have warm climates:-

\begin{tabular}{|c|c|c|c|c|c|c|c|}
\hline \multirow{2}{*}{ Countries } & & \multirow[b]{2}{*}{$\begin{array}{l}\text { Population over } \\
\text { ten years of age. }\end{array}$} & \multirow[b]{2}{*}{ Years } & \multicolumn{2}{|c|}{ Tried. } & \multicolumn{2}{|c|}{ Convicted. } \\
\hline & & & & $\begin{array}{l}\text { Annual } \\
\text { average. }\end{array}$ & $\begin{array}{l}\text { Per } \\
\text { roo,000 in- } \\
\text { babitants. }\end{array}$ & $\begin{array}{l}\text { Annual } \\
\text { average. }\end{array}$ & $\begin{array}{l}\text { Per } \\
\text { roo,000 in- } \\
\text { habitants }\end{array}$ \\
\hline Italy . & - & $23,408,277$ & I 887 & 3,606 & 15.40 & 2,805 & II. 98 \\
\hline Austria . & . & $17,199,237$ & $1883-6$ & 689 & 4.01 & 499 & 2.90 \\
\hline France. & - & $31,044,370$ & $1832-6$ & 847 & 2.73 & 580 & 3.87 \\
\hline Belgium & - & $4,377,8 I 3$ & I $88 \mathrm{I}-5$ & I 32 & 3.02 & IOI & $2.3 \mathrm{I}$ \\
\hline England & - & $19,898,053$ & $1882-6$ & $3^{8} \mathrm{r}$ & 1.60 & I5I & 0.76 \\
\hline Ireland : & - & $3,854,588$ & $1832-6$ & 129 & $3-35$ & 54 & 1.40 \\
\hline Scotland & • & $2,841,94 I$ & I $882-6$ & 60 & 2.11 & $2 I$ & 0.74 \\
\hline Spain . & - & $13,300,889$ & $1883-6$ & 1,584 & II.9I & 1,085 & 8.16 \\
\hline Hungary & - & $10,82 \mathrm{I}, 558$ & $18 S 2-6$ & & & 625 & 5.78 \\
\hline Holland & - & $3,172,464$ & I $882-6$ & 35 & I.10 & 28 & 0.88 \\
\hline Germany & - & $35,278,742$ & I $88 z-6$ & 567 & $1.6 \mathrm{I}$ & 476 & 1.35 \\
\hline
\end{tabular}

The population of England and Wales is now in the neighbourhood of thirty-five millions. Probably the Anglicans number about fifteen millions, the Nonconformists about the same, while the Roman Church claims about a million and a half. The prison population, March 28, 1906, was 21,580. Of this total the Anglicans are returned as contributing 16,089 , the Nonconfarmists 1,094 , and the Catholics 4,397 (Parliamentary Return on Prisons, Religious Creed's of Prisomers, April 1906). Doubtless, the numbers assigned to the Anglicans are too high and those to the Nonconformists too low. Prisoners, like soldiers, tend to "follow the band." In Scotland, at the same date, Catholics, a small minority, contributed 97 I to a prison population of 2,812 . 
and, with scarcely an exception, rebels against clerical authority or actual defaulters from the faith. Such, for example, were Voltaire and the men of the French Revolution, Garibaldi, Mazzini, Cavour, de Lesseps, Renan, Victor Hugo, Jaurez the great President of Mexico, and Tolstoi. If the reader will consider the matter he will be able to recollect hardly a man, not a cleric, who was both orthodox and great. I can call to mind only Pasteur.

810. I feel sure that many people will dissent from the opinion that such great social and intellectual differences as exist, for example, between England and Morocco are due mainly to differences in the way religion is taught; or rather, since religious instruction tends everywhere to be of the same dogmatic type, to differences in the extent to which this method of teaching is influential in moulding the minds of young adults and thus determining their final mental attitudes. I can only ask the readerwhile bearing in mind ( $I$ ) that dogmatic teaching obviously tends to close the mind and to create an unchanging outlook on life, (2) that teaching of this kind is much more influential in such places as Morocco where nearly all the formal and much of the informal mental training is religious than in England where it preponderates much less, where the many divergent sects adopt a tolerant attitude towards one another, where much of the formal teaching of other subjects is deliberately designed to create a receptive tone of mind, and where indeed the whole surroundings are such that they tend to a far greater degree to encourage independence of thought, (3) that in proportion as dogmatic teaching has been influential the adherents of each of the religions of the world have tended to have much the same social and intellectual status, though in other respects (e.g. climate) the environment has often been very different, and (4) that in proportion as religious teaching has excluded other subjects, races have been stagnant and barbarous-to try to conceive interpretations unlike the one I have suggested. If the facts be granted, only two other suppositions are conceivable, or, at any rate, have been conceived with any degree of clearness.

8I I. First, there is the hypothesis that racial mental differences are mainly innate, and, therefore, that the social state of Morocco differs from that of England because Moors and Englishmen are "naturally" as much unlike mentally as physically. We have already discussed this hypothesis rather fully, but consider again what it implies. It implies that Englishmen and Moors are incapable of becoming alike mentally, and, therefore, that there is no 
hope that Moors will form a civilized community except after ages of selective elimination; in other words, it implies that they are now as a nation so incapable of learning as to be extremely feeble-minded in the technical sense. All orthodox Mohammedan communities, in proportion to their orthodoxy, in proportion as their faith is steadfast and fanatical, exhibit much the same social phenomena. Amongst them are descendants of the Pagan Greeks, who, also, as well as most Hindoos and Buddhists, must be feebleminded. How, in that case, shall we explain the fact that a few generations back, during the middle ages, the ancestors and coreligionists of these same Moors were, in Spain, far more enlightened, progressive, and orderly than our own contemporary ancestors? We derive the beginnings of modern science from them. During our own Dark Ages Europe was in a state of social and intellectual stagnation, perpetual foreign war, and domestic disorder identical with that of Morocco of to-day. If, then, the social state depends on innate racial characteristics, what caused the rapid and tremendous change that occurred at the Reformation in some of the countries of Western Europe? At that time the various peoples turned from the teachings of an infallible church to that of a book which, it is true, they believed infallible, but on which each man exercised his wits and which he interpreted in his own way. Hence the diverging heresies, the clashing sects, which in themselves were indications of, and conduced to, divergent and independent thought. Was the greatness of the Greeks and Romans at a time when the teaching tended to develop the reflective faculties due to race, and their decline when the teaching tended to crush independent thought due to race also? How did it happen that the tiny community of Athens, which in the former period produced more really great thinkers, poets, historians, philosophers, and the like, and more great men of action-who of necessity were men of great thought also-than all Europe during its Dark Ages, never again produced any? Did the Chinese achieve civilization because they were less feeble-minded than their contemporaries? Have they stagnated for centuries because they are more feeble-minded? Were the Japanese feeble-minded half a century ago? Are they no longer so? How is it that, except when the teaching is more liberal or less liberal than the ordinary, the adherents of each religion, who may be of many diverse races, are so much alike mentally, but so dissimilar from the adherents of other religions even when of the same race?

8 I 2. Whether, or not, connected as cause and effect, there can be 
no doubt that, in proportion as the prevailing religious teaching tends to create a habit of prejudiced thinking, races are stagnant and turbulent. Must we suppose, then, that inferior races tend ' naturally' to follow 'orthodox' religions? But, if that is so, then, once again, what was the origin of the astonishing germinal change which caused the Pagan Greeks and Romans to embrace orthodox Christianity and the progressive Protestant nations to abandon it ? No one, not a fanatic, denies the sincerity of the great majority of the ecclesiastics of every religion. How does it happen that ecclesiastics, especially those of the more 'orthodox' religions and sects, have ever as a body resisted new truth more strenuously than the laity? They are of the same race, but they have been much more carefully trained dogmatically. ${ }^{1}$ Most people place a high value on religious teaching and education generally, but of what value is any sort of teaching if 'nature' be so powerful as to be the main source of racial and social differences? Moreover, if the human being is so mentally blocked out by 'nature,' in what particular does he differ from the lower animal mentally? It cannot be by reason of superior educability.

813. Second, there is the hypothesis that the actual doctrines, the actual 'facts,' taught by religions are the source of the mental peculiarities of their adherents. Within limits, this is true, no doubt. If a child be taught, for example, that the world is flat, he will have, no matter how well trained, some difficulty, especially in adult life when his receptive powers are beginning to wane, in changing to a conviction that it is round. A man, who believes all the fiction taught by Fetishism, cannot well be other than a

1 The whole atmosphere of modern civilization, like that of Pagan Greece and Rome, is antagonistic to rigid orthodoxy. The various sects now mingle freely, almost every one can read, and to every one who is able to read is accessible a voluminous literature crowded with diverse opinions. Some freedom of thought is inevitable. Religion may flourish in this atmosphere, as witness the continual growth in England and America of ardent Nonconformist sects which, notwithstanding their fervour, permit considerable latitude of belief. But orthodoxy perishes, as witness the enormous secessions from the Roman church amongst the more enlightened peoples of the Continent, and the fact that, in spite of a stream of immigration which has been flowing for many years, the Catholic population of Great Britain and the United States does not increase proportionately. The doctrines of Catholicism are not demonstrably less true than those of Protestant sects. It seems evident, then, that the mental atmosphere created by the method of teaching is the cause of the decay of the former ; and the fact that the ecclesiastics of the more rigid churches persist in maintaining a mental environment which renders the prevalence of their own doctrines difficult, is a conspicuous instance of incapacity to profit from experience. The Greek church, also, is beginning to suffer. Christianity loses fewer adherents through Anglican teaching. Nevertheless, Nonconformity has:gained largely from this comparatively rigid sect. 
savage. Therefore religions, in so far as they are false, are bars to the discovery of truth. Nevertheless, false beliefs owe their stability mainly to the way in which they are taught to the pupil. For instance, it is possible to teach Mohammedanism and Buddhism, as in the case of the Spanish Moors and the Japanese, in such a way as to leave the mind open to fresh evidence. Moreover, it is not credible that the Pagan Greeks and Romans were intellectually greater than their Christian descendants of the Dark Ages merely because their religious beliefs were more true, or at any rate less false. Nor is it credible that the comparatively trifling doctrinal differences which separate Protestant races from their mediæval ancestors, and, in a lesser degree, from modern Catholics and Greek Churchmen, are the causes of their considerable social superiority.

814. As in the case of susceptibility to alcohol, racial intellectual peculiarities have been attributed to all sorts of causes. For example, climate has been alleged as a cause. But both stagnant and progressive races have existed in all the zones of the earth, and while climates change only in geological time, races may change their characteristics in a generation. Moreover, the effect produced by climate is either germinal, or one which is 'acquired' by every generation in turn. If germinal, it comes under the heading of innate; if acquired, under that of mental training. However, all thinking that has connected mental peculiarities with climate has been excessively vague. Because individuals, whose race has evolved in fitness to a cold or temperate climate, have found a warm climate enervating, the latter, in spite of natural selection, has been supposed to enervate the race that has evolved in it. On the other hand, hot climates and fiery suns have been thought to conduce to 'hot' and 'fiery' natures. Equally absurd is the notion that 'young' races tend to be vigorous and 'old' races decrepit, or that there is a law of nature that causes races to swing continually between states of high and low efficiency. Every human race is of the same age. Indeed, if it be true that living beings are genetically related, all species known to us are derived from an equal entiquity.

815. To sum up :-the method by which religion is taught brofoundly affects mental development. If it be such that the Jupil, as in the case of fanatical Mohammedans, is made to hold is beliefs as a mass of obstructive prejudices, then, no matter what the truth of the religion, it becomes a cause of social 
stagnation and intellectual inefficiency. The results of such teaching, observable as they are in every age and country, and in connexion with almost every religion, are so universal and manifest that the relation of cause and effect is certain. Precisely in proportion as the prevailing religion is used to render the mind incapable of estimating the value of conflicting evidence and of assimilating new truth, the individual is rendered unintelligent and the race stagnant and lawless. Such teaching, which every trained teacher would condemn as abominable if used for a secular subject, tends to produce a condition of feeble-mindedness which differs from the congenital kind only in that it has a different origin and is less profound inasmuch as it affects only the reflective faculties - the unconscious, not the conscious, memory; skill in thinking, not ability in storing facts. Now, since children are at that stage of mental development in which the individual acquires the main part of his knowledge and mental habits, it is impossible to close their minds permanently. Thus, while it is easy to convince them of the truth of any set of religious doctrines and prejudice them against any other set, it is almost as easy to obliterate the impression. Habits of thought become permanent only later when the youth develops into the man, when the instinctive open-mindedness and receptivity of the child wanes, and is replaced by a habit of being open-minded or the reverse. Consider how small, speaking relatively, would be the intellectual ill-effects of Mohammedanism, Hindooism, or any other religion which we believe to be false, if the method of instruction, even if faulty for children, were good when adults were taught.

816. I have discussed religious teaching only in order to demonstrate the truth that it is as necessary to bestow attention on the thinking faculties of young adults as on the increase of their knowledge. The years during which the youth changes into the man are, from the standpoint of mental development, the most important of all; for at that period the mental habits, like the physical parts, tend to settle into relative stability. To achieve the best results we must not only train little children to think well, but we must continue the process to the latest stage possible. As we use the child's knowledge for a foundation on which to build the larger knowledge of the adult, so we should use his mental skill as a stepping-stone towards the acquirement of greater powers of thinking. It is agreed on all hands that a. good educational system must teach knowledge and mental skill which will be useful in the widest sense to the pupil- 
useful not merely in this or that occupation, but in bestowing general intellectual power. Knowledge, and skill in using it, are useful only when they link up with the experiences of the subsequent career; that is, when they are actually useful in themselves, or when they serve as a ladder towards the acquirement of a more extended and directly useful knowledge and skill. It would be folly, for example, to teach even elementary mathematics to one who, were it possible, could never be helped by it in any way. Therefore, since environments vary, the same instruction is not always equally valuable. Thus an acquaintance with Latin and Greek is not as useful to-day as it was four centuries ago, when the former was a 'bread-and-butter' subject and the only path to knowledge, and the latter the sole means of reaching the higher planes of thought. At present three principal systems of mental training are advocated, the so-called classical, the so-called scientific, and the scientific.

817. The first is classical in name only. It consists mainly in a formal teaching of the words and the relations between the words of two foreign languages which are no longer spoken; a method of acquiring a language so unnatural, and therefore uninteresting and ineffective, that a decade of toil confers on the pupil less than was acquired without labour by a Greek or Roman infant of two. The Greek children and young adults studied no foreign language, and the Romans only Greek, and that only when their greatness was beginning to wane. It may be doubted whether the classical system of verbal signs contributed appreciably to the quality of the thoughts of which they were the means of expression. On the contrary, it is probable that good thinking needed, and therefore invented, good means of verbal expression. Classical literature can teach modern man nothing about the universe his mind constructs except some valuable human history. Its beauty is very great, and, considering the limited knowledge of the ancients, the thinking it reveals quite the most skilful known to us. But, judging from results, such instruction in Greek and Latin as is given to boys, and even to older pupils, does not very commonly awaken a very keen appreciation of the beauty or bestow great skill in thinking. Moreover, the thoughts have all been expressed in English by scholars with at least as much precision as is possible to the learner. Doubtless, since English is in part derived from Latin, a knowledge of the latter helps us somewhat to an understanding of the former; but the benefit is hardly so marked as to justify years of labour; and just as it was anciently possible to speak and write good Greek 
and Latin without a profound knowledge of the derivations of the words, so, in the case of English, it is still possible. While constructing Greek and Latin sentences or translating them into English, the student is forced to think strenuously. Doubtless, he derives benefit from this mental gymnastic; but the skill achieved is of a very special kind. It does not link up very-obviously with anything in the subsequent career of most students. That is to say, the teaching is extremely indirect. In any case classical teaching is as the poles apart from that which was practised when Greece and Rome furnished examples of mental training for all time. Modern classical scholars are often remarkably indifferent to science; but it is certain that the Pagans, activeminded and filled with intense curiosity, would have hailed our science with rapture, would have taught it to their young men, and, above all, would have taught them how to think about it in such a way as to turn it to the best intellectual and practical account.

8I 8. The expression 'scientific teaching' is used with two very different meanings. On the one hand it implies tuition so given that the pupil is stored with knowledge and skill that develop his intellectual powers to the utmost. This kind of teaching, which is scientific in the sense that means are consciously and effectively adapted to achieve clearly recognized ends, need not deal with the data of any of the sciences. But, since science is nothing other than verified and classified truth, since it deals with reality, since many of its problems are sufficiently abstruse to afford exercise in strenuous thinking, and since the pupil who learns to think well will be able to link up much of his scientific knowledge with the experiences of his subsequent career, it probably affords the best materials for the general culture of young adults. On the other hand, the expression is often used to imply nothing more than instruction in the data of one or more of the sciences of such a kind that little is required from the pupil save recollection and observation. This kind of tuition is sometimes termed 'science teaching,' a description which is valuable as marking a distinction of real importance.

819. We saw that some sciences, for example systematic zoology and botany, are based on facts that are patent to observation, whereas nearly all the facts on which others, for example chemistry, are founded are so obscured by the conditions in which they occur that special devices must be adopted before they can be observed. No one will maintain formally that this difference in the way in 
which facts are gathered ought to make a difference in the kind of thinking by means of which they are welded into sciences. There is, however, a difference between sciences which is more important in that to it is due a vast difference in the thinking by which they are created. Some sciences are founded on a multitude of facts, and some on a very few. The mathematical sciences are examples of the latter class. Thus, "All the inductions involved in all geometry are comprised in those simple ones, the formulæ of which are the Axioms, and a few of the so-called Definitions. The remainder of the science is made up of the processes employed for bringing unforeseen cases within these inductions; or (in syllogistic language) for proving the minors necessary to complete the syllogisms; the majors being the definitions and the axioms. In those definitions and axioms are laid down the whole of the marks, by an artful combination of which it has been found possible to discover and prove all that is proved in geometry. The marks being so few, and the inductions which furnish them being so obvious and familiar; the connecting of several of them together, which constitutes Deductions or Trains of Reasoning, forms the whole difficulty of the science, and, with a trifling exception, its whole bulk; and hence Geometry is a Deductive Science." 1 The definitions are precise descriptions. The axioms are laws. We have defined a law as a description of a uniformity in the sequence of phenomena, and we saw that it is usually possible to deduce necessary consequences from it about sequences of phenomena.

820. Physics in all its branches and throughout its whole vast extent is another science that has been developed from a few essential facts which have been welded into laws from which the rest of the science has been deduced. Relatively speaking, very little mere recollection is needed by the mathematician or the physicist. His 'knowledge,' however toilsomely acquired, consists mainly in a special skill in thinking about sequences.

$82 \mathrm{I}$. Astronomy is a science which is founded on an immense number of facts. Thus there are millions of stars, only a few of which the astronomer has named and attempts to recollect. If he merely classified his facts according to co-existences and resemblances, for example if he classified the heavenly bodies according to their apparent magnitudes, luminosities, and positions relative to one another, astronomy would be a very incomplete science, and the best astronomer would be a powerful photographic camera. 
But every astronomer is also a mathematician and physicist, and it is the application of mathematical and physical laws to astronomy that has given to this science all its intellectual splendour-that has converted the astrologer into the astronomer, that has linked together the facts of astronomy in chains of causation, that has made it in great measure a deductive science founded on laws.

822. Chemistry is based on an enormous number of facts, but, as far as I am aware, has only one law of considerable importance, Dalton's Atomic Theory, which is usually regarded by chemists as their most valuable possession.

823. Systematic zoology, botany, anatomy, and the other systematic sciences that relate to living beings are also founded on a great number of facts, probably on a greater number of recorded and described facts than any of the other sciences. But they have no laws. They are descriptive not interpretative; their facts are classified according to co-existences and resemblances, not in chains of causation. In effect, they consist of a multitude of definitions. Thus a systematic zoologist will tell us no more about a man than that he possesses certain characters, and that therefore he is an animal belonging to the sub-kingdom Vertebrata, class Mammalia, order Bimana, and so forth. The faculties required by the young learner in these sciences are mainly observation and recollection. No doubt, a great deal of thought-comparison, discrimination, association, and the likeis needed also; but, as compared to the amount required in physics and mathematics, it is very small in proportion to the number of facts. Moreover, it is not the same kind of thoughtnot deductive, not interpretative thought, not thought about the sequences of phenomena, not a linking together of facts in the relation of cause and effect, not a process of inferring the previously unknown as a necessary consequence from the previously known, not ratiocination.

824. If a student has exceptional powers of recollection and observation he may become an exceptionally good systematist. But no such powers by themselves will enable him to become a great mathematician or astronomer. The difference between the interpretative and descriptive sciences is vividly illustrated by the circumstance that I am able in a short time to ascertain all that I want to know, indeed all that is known to the average systematist, about any animal or plant, by turning to the books on my shelves; whereas no number of books will enable me to 
understand the reasoning of the mathematician or astronomer without a prolonged preliminary training in thinking. The mental attitudes of the followers of the two kinds of sciences are markedly different. Thus, I believe every student of the interpretative sciences would regard it as better science if the theory that the individual recapitulates the life-history of the race were first inferred as a necessary consequence from the familiar truth that offspring recapitulate with variations the development of their parents, and then proved by an appeal to the less familiar facts of embryology; whereas I think some biologists would regard such a proceeding as unscientific, and would prefer to begin at the other end and found an untested hypothesis on the evidence furnished by embryology alone. Biology is remarkable for the number of untested and often untestable hypotheses which its followers have formulated. Witness the numerous hypotheses concerning inheritance, sex, immunity, and cancer. On the other hand, if a physicist formulates a hypothesis he is almost sure to endeavour to furnish not only the evidence on which it is founded, but also that by which he thinks it is proved. Witness Kepler's rejection of nineteen of his own hypotheses, which he disproved by a deductive appeal to reality, before he arrived at the true statement of planetary motion, and Newton's suppression for fifteen years of a true theory of the moon's motion, because it did not accord with data which were subsequently found to be wrong. ${ }^{1}$

825. Obviously, since the mathematician has only a few data on which to found his thinking, his science must be deductive from its very beginnings. On the other hand, the biologist, like the astronomer, must begin by classifying his facts according to co-existences and resemblances, and only afterwards, when they have thus been rendered manageable, proceed to construct chains of causation. At any rate, every science, the facts of which are very numerous and which has passed beyond the descriptive stage, has grown in this way. ${ }^{2}$

826. Darwin attempted to make this second step. In his theory of Natural Selection he formulated that which, if true, is a real law. He described a uniformity in the sequence of events from which necessary consequences could be deduced. He strove to interpret facts about living beings, and so to link them together in chains of causation. Consciously or unconsciously he sought to do for biology what Newton had already done for physics and 
astronomy. ${ }^{1}$ If mathematics and physics are sciences, his work also was scientific, for he formulated a hypothesis which was capable of being tested. But this arranging of facts in sequence was a kind of science to which systematists, as such, were not accustomed, and which, therefore, some of them put aside austerely as that which was not science but 'speculation.' "Parenthetically we may notice that though scientific opinion in general became rapidly converted to the doctrine of pure selection, there was one remarkable exception. Systematists, for the most part, kept aloof. Every one was convinced that Natural Selection, operating in a continuously varying population, was a sufficient account of the origin of species, except the one class of scientific workers whose labours familiarized them with the phenomenon of specific difference. From that time the systematists became, as they still in great measure remain, a class apart. A separation has been effected between those who lead theoretical opinion and those who by taste or necessity have retained an acquaintance with the facts. ${ }^{2}$ The consequences of that separation have been many and grievous. To it are to be traced the extraordinary misapprehensions as to the fundamental phenomena of specific difference which are now prevalent." 3

1 "We claim for Darwin that he is the Newton of natural history, and that, just so surely as that the discovery and demonstration by Newton of the law of gravitation established order in place of chaos, and laid a sure foundation for all future study of the starry heavens, so surely has Darwin, by his discovery of the law of natural selection and his demonstration of the great principle of the preservation of useful variations in the struggle for life, not only thrown a flood of light on the process of development of the whole organic world, but also established a firm foundation for all future study of nature " (A. R. Wallace, Darwinism, p. 9). Nothing in the histories of scientific men is more remarkable than Dr Wallace's attitude. No one would suspect from his own writings that he was Darwin's co-discoverer of the theory of natural selection. He assigns the whole credit to the elder thinker. I imagine that posterity will be more just.

${ }^{2}$ Among those who 'lead theoretical opinion,' but who, to judge from Bateson's language, have not 'retained an acquaintance with the facts,' are Wallace, Hooker, Thiselton-Dyer, Lankester, Weismann, Osborn, and Poulton.

${ }^{3}$ Bateson, Mendel's Principles of Heredity, p. 3. Bateson continues, "If species had really arisen by the Natural Selection for impalpable differences, intermediate forms should abound, and the limits between species should be on the whole indefinite. As this conclusion follows necessarily from the premises, the selectionists believe and declare that it represents the facts of nature." Bateson is mistaken. Selectionists believe that evolution has resulted, not from the selection of impalpable, but from the selection of fluctuating differences. The distinction is important. Natural selection amongst human beings is the only instance of this process observable by us, and the reader is now in a position to judge whether, for instance, the difference between a man who perishes of consumption and one who survives in the same environment is 'impalpable.' If selection amongst men were such that short and weak individuals were eliminated, while tall and strong individuals survived, if men, for example, fought for their 
827. The question arises whether the attitude of those systematists who have 'kept aloof' from speculation can be justified. Here speculation means nothing other than an attempt to link together facts concerning animals and plants in chains of causation. Of course, speculation may be what even students of other sciences consider illegitimate; that is, it may be founded on data which have not been verified, or it may be conducted by thinking which is not tested. But no such considerations seem to have guided the systematists that 'kept aloof'; for, as their aloofness implies, they did not attempt to impugn either the facts or the thinking, and they preserved the aloofness even after the actual occurrence of Natural Selection and of evolution consequent on it was demonstrated amongst men, the only living beings with whose lives they were at all intimately acquainted. It is not alleged that their attitude was due to lack of time, or of interest, or of knowledge of the facts. Apparently they have been guided by the more or less clearly formulated notion that science is created, not by ascertaining chains of causation, but solely by classifying facts according to co-existences and resemblances. Whether they are right or wrong is, in the absence of any attempt at demonstration by them, a matter of opinion; but it is worthy of notice that that which is condemned by them is, in effect, not merely the science of Darwin, but also that of Bacon and Newton, not merely the theory of Natural Selection, but also mathematics in its entirety, physics almost in its entirety, and astronomy in the best part of its extent. It is worthy of notice also, that unless human beings linked together in chains of causation the facts they learn during the ordinary course of their lives they would not be rational beings, and could not maintain existence. The circumstance that they are able

mates, the differences selected would not be impalpable. It is true that, if we arranged all the members of a variety in a row in the order of their heights or strengths we should, as a rule, find the differences between each individual and his next neighbours very small. But nature would do nothing of the kind. On the contrary, she would simply select for survival, as a general rule, the especially tall and strong, and for elimination the especially short and weak. Forms intermediate between living types actually abound - forms that are intermediate between one kingdom and another, one class and another, one order and another, one species and another, or one variety and another. No selectionist believes that all the links have survived or are known. The complete chain could be reconstructed only if every generation were known to us. Mutationists do not doubt that the organic world has arisen by evolution. But their links are missing equally with those of the selectionists; for none will maintain that the vast gaps which exist between many living types and their nearest known relations, gaps which involve thousands of co-ordinated changes, can ever have been crossed by a single leap. 
usefully to employ this mode of thinking in ordinary life is, at least, presumptive evidence that it can be usefully employed in science where especial care is exercised.

828. My object, however, is not to assail the attitude of the systematists. "The isolation of the systematists is one of the most melancholy sequela of Darwinism." 1 They are doomed to extinction; for facts arranged in chains of causation are so very much more interesting to normal human beings than facts arranged in any other way, and normal people are so driven to think, are so trained to think, in chains of causation, by the ordinary conditions of their lives, that even the severest systematic training can hardly repress, and rarely quite represses, the tendency; the instinct, to think in that way. Pure systematists are now a small minority, even among biologists, and as a type are unknown, and have always been unknown, amongst the students of all other sciences. The great weight of scientific practice and opinion has ever been strongly against them. They are a peculiar product of biological training which still remains almost purely systematic; for, though Darwin greatly altered the course of biological thought, his influence has not yet affected the method by which students are instructed to nearly the same extent. Like monks and nuns, systematists afford a remarkable example of the importance of acquirement in human mental development, and of its power to nullify even an instinct.

829. My object is to draw attention to the circumstance that, while the majority of biologists, impelled by instinct and informal training, think about biological facts in terms of causation, yet, unlike mathematicians, physicists, and astronomers, they receive little or no formal education which especially fits them to deal with their data in that way. Here they are, in fact, untrained thinkers. I make this statement with no offensive intention. It may be verified in any centre of biological teaching. All biological curricula are designed to impart only "systematic" knowledge. If it is unscientific to think in terms of causation, it is never explained to learners why it is unscientific. The result is that numbers of students are left with what can only be very misty or wrong notions as to what constitutes science. The circumstance that biologists are divided by real sectarian differencesthat is, differences founded on an improper acceptance or rejection of evidence or modes of thinking ${ }^{2}-$ is, alone, sufficient proof that

1 Bateson, Darwin and Modern Science, p. 89 (footnote).

${ }^{2}$ See $\S 45$. 
their training is defective. Systematists tacitly repudiate science which is created by linking facts in chains of causation; most scientific men regard it as the most perfect form of science. Experimentalists seek to secure accuracy in thinking by, in effect, limiting the materials of thought to facts furnished by experiment; most men of science believe that one of the essential conditions of accuracy is the careful taking of all authentic and relevant facts into account. Medical men, whose professional thinking is entirely in terms of causation, frequently denounce deduction; most men of science suppose that the truth of hypotheses can be tested only by means of it. Even biometricians, who are mathematicians, rarely test their thinking deductively. In biology they follow the prevailing fashion, using mathematical formulæ, but not mathematical methods of securing accuracy in thinking. The science they create is purely empirical. Judging by their work, they appear to suppose that thinking which is founded on simple enumeration and which, therefore, is isolated and untested, is superior to thinking which is founded on a discovery of causes, and which, therefore, is linked with other truth and can be tested by it. Most scientific men believe the contrary.

830. Not only does the mathematician always endeavour to test his thinking, but he invariably prefers a deductive to an enumerative test. Thus, if he has multiplied one number by another, he has a greater sense of certainty that he has thought correctly when he has divided the product by one of the factors and found that the quotient agrees with the other than when he has repeated his multiplication a dozen times. In the former case he thinks that he has proved the correctness of his thinking; in the latter he supposes that he has merely raised an expectation that it is correct. Yet, since the conditions of thought are usually relatively simple in the mathematics, it is here, if ever, that testing by simple enumeration should be useful; for the simpler the conditions the less likely it is that any essential factor has been omitted or wrongly thought about, or that any non-essential factor has been included. On the other hand, the conditions are usually very complex in biology. We are constantly liable to confuse the essential with the non-essential. Here, if ever, simple enumeration should be dangerous and deduction comparatively safe and useful. Nevertheless, many biologists, though professing admiration for the mathematics as the type of all that is accurate, express or imply the utmost contempt for testing (i.e. deduction) when used in their own science. Thus, the fact that long lost ancestral traits 
sometimes appear in pure-bred individuals seems, according to the mathematical conception of science, to furnish conclusive proof that the Mendelian doctrine of segregation is erroneous. If, however, we mention this test to a member of the experimental school, we are sure to find that it carries no conviction. He will not attempt to demonstrate that the supposed test is no test. He will merely declare that the problems of heredity are not to be solved in the arm-chair by essayists, logicians, or dialecticians, but only by real men of science labouring in the laboratory or the breeding pen. If we insist that the "dialectician" is utilizing all the experimental facts and more besides, that it is needful that even "real" men of science shall test their thinking, that thinking can be carried on as well in an armchair as a breeding pen, and that the experiments on which he relies prove, not alternative inheritance, but only alternative reproduction, he will, by way of retort, adduce similar experiments on rabbits. If we raise the same objections to these, he will adduce like experiments on fowls. And so on, and so on. Ultimately we are left to wonder helplessly whether he is unaware of the true nature of science, or whether every one who has both thought and expressed himself clearly about it is unaware; whether biology, which, from the scientific standpoint of the mathematician and physicist, seems so pregnant with great possibilities, so laden with tremendous issues, is in truth so mean and narrow a thing, a mere guessing about the abnormalities of sexual reproduction, or at most no more than a study of the function of sex; whether a new and more accurate science is really being created by exclusive devotion to experiments and abnormalities, or whether we are spectators in a kind of confidence trick in which a display of experiment is used to obtain a fictitious appearance of accuracy.

$83 \mathrm{I}$. Whoever is right some one must be wrong; and a teaching which results in such a babble of opinions must be wrong also. A mathematician will seldom affirm contradictories, and any statement by him is usually accepted or rejected by his fellows on grounds of pure fact and reason. The same is true of physicists, chemists, and astronomers. But a biologist-as when he upholds both the theory of recapitulation and the Law of Ancestral Inheritance, both the theory of Natural Selection and the notion that variations are caused by the direct action of the environment, both the hypothesis that harmful conditions cause races to decline and the hypothesis that such conditions cause races to progress in the same characters at the same time-often 
displays a power of affirming contradictories which is not exceeded by, and is much less excusable than that displayed by the adherents of any of the creeds. The latter do not profess to be guided solely by verified truth and pure reason. Moreover, in many instances the statements of the biologist are judged on grounds of pure prejudice by many of his fellows.

832. Biologists often insist that 'the time has come' when the traditional education of young adults shall be replaced by a training in science. But of all modern sciences the most fundamentally important is the science of education; and the training accorded to young biological students is precisely that kind which is most condemned by modern educationalists who denounce, for example, traditional, classical, historical, and geographical teaching as consisting too much in a mere learning and comparison of words, dates, lengths of rivers, and the like. They insist that it is not enough to store the memory with facts ; intelligence must also be developed ; and by intelligence they always mean skill in thinking in terms of causation.

833. Systematic knowledge is necessary to biologists, but, to judge by what the students of other sciences consider essential, it is not all that is necessary. If it be right that scientific men shall have their powers of precise thinking about sequences developed to the utmost by formal training; if the most complete science is created not merely by classifying facts according to their co-existences and resmblances, but also by classifying them according to their causal relations; if the former method of classification is the warp and the latter the woof of perfected science; if facts should be arranged not only according to one relation, but according to every relation; - then there ought to be a considerable change in biological education, and learners should receive a formal training in the method of classification which Darwin has done so much to introduce as well as in the method of which Linnæus was so great an exponent.

834. It is not merely the education of biologists that is in question. All education is in question. And very much more than education ; for on its mental training depends almost absolutely, both in war and in peace, the weal and woe of every human race. Whoever has attempted to bring about any sort of reform-from that of a slaughter-house to that of a state, from that of a nursery to that of a religion-knows well that his chief difficulties lie, not in the ignorance of those he would influence, for facts however laboriously gathered are readily imparted, but rather in mental states engendered by an education which does little to create a 
habit of open-mindedness to new ideas. Only biologists are in a position to know the fundamental facts-to ascertain what is "inborn" and what "acquired" in the human mind, and to discover how any desired quality may best be evolved or developed, and any defect eliminated. When we think about what is good or bad in education, we think in terms of causation. Not until biologists have trained their students by direct methods to become skilful in the interpretation of evidence, and thus have raised a body of workers capable of becoming effective thinkers about education, not until their science teaching has become scientific in method, will they be in a postion to speak with the authority that is their right. And not until then will the mental training of the general public receive a reform of which there is urgent need, and the practical utility and intellectual splendour of biology be recognized by all men.

835. In this book, now finished, I have tried, however unsuccessfully, to follow the only method by which all thinkers who have expressed themselves on the subject have supposed it possible to trace sequences of cause and effect with any degree of certainty-the method of using some (as many as possible) of the available, authentic, and relevant facts for the foundations of hypotheses, and the remainder (which of necessity are usually by far the greater number) to test the correctness of the thinking -the method of fitting each inference to all the facts, no matter how numerous or how gathered, and so of never resting content until every devisable test had been satisfied. "An hypothesis is any supposition which we make (either.without evidence, or on evidence avowedly insufficient) in order to endeavour to deduce from it conclusions in accordance with facts which are known to be real; under the idea that if the conclusions to which the hypothesis leads are known truths, the hypothesis itself must be, or at least is likely to be, true." 1 The reader has perhaps noted that, while I have rarely, if ever, contradicted alleged facts, many of which in common with all other people I have been obliged to accept at second hand on what appeared good authority, I have often controverted inferences, none of which we are obliged to accept at second hand, which seemed to me, when tested, to conflict with well-known and undisputed truths. "An opinion which stands in need 'of much illustration can often receive it most effectually, and least tediously, in the form of a defence against objections. And on subjects concerning which speculative minds are still

$$
1 \text { J. S. Mill, Logic, iii. xiv. } 4 \text {. }
$$


divided, a writer does but half his duty by stating his doctrine, if he does not also examine, and to the best of his ability judge, those of other thinkers." 1 However completely I may have failed in all else, I am not without hope that I have demonstrated at least that much evidence, hitherto neglected, particularly that furnished by human diseases, is, if used in accordance with the methods employed in the other sciences, valuable to the student of heredity and evolution in his strivings to create a coherent body of thought and knowledge, the various parts of which shall interlock as smoothly and consistently as those of any other deductive science.

836. We have journeyed in a wide and in parts untravelled region of thought, and have ambitiously endeavoured to solve the greater problems of heredity. If in any instance we have succeeded, then in each such case a law of nature has been formulated which, as far as I am able to judge, is as nearly fundamental as can be discovered in the present state of our knowledge. Of these laws, if any of them are real laws, the most important from the scientific standpoint, because the most basic, is the generalization that the vast majority of variations are under the immediate control of natural selection, and therefore are spontaneous in the sense that they arise independently of the direct action of the environment, and that on these spontaneous variations only is evolution built; for they only are products of the normal growth and change of the germ-plasm, all others being results of injury to it. If this be a truth, the close and continuous adaptation of persisting species to their environments necessarily follows. On the other hand, the conclusion that the "acquirements" of the individual are as much a part of his inheritance as his "inborn" traits is, from the practical standpoint, the most important of all. If this be a truth, we have a valid explanation" of the principal line of evolution of the higher animals, and a potent means of influencing our own species for good and evil.

837. The matters we have dealt with have been complex and difficult, and I fear it is more than probable that the reader has detected numerous errors and inadequacies of fact and reasoning. For them I ask no sort of indulgence-more especially as I have often challenged him to indicate material misstatements and omissions, or, given the facts as true and comprehensive, to indicate how any conclusions other than those reached are conceivable. But I have the right to claim, either that I shall be

1 Op. cit., ii. vii. I. 
judged accordingly as I have, or have not, followed the established rules under which all science has been created hitherto, or that the reader shall plainly think out his reasons for rejecting them. Conceivably these established rules are wrong. But in that case. they should be shown to be wrong. To imply, as is so often done, that they are wrong merely by setting up private, unexplained, and apparently indefensible standards for evidence and proof is to destroy the distinction between scientific and sectarian thinkingis to render scientific discussion as futile and scientific dissension as unappeasable as the discussions and dissensions of religious factions.

838. "Principles of Evidence and Theories of Method are not to be constructed $\grave{a}$ priori. The laws of our rational faculty, like those of every other natural agency, are only learnt by seeing the agent at work. The earlier achievements of science were made without the conscious observance of any Scientific Method; and we should never have known by what process truth is to be ascertained if we had not previously ascertained many truths. But it was only the easier problems that could be thus resolved: natural sagacity, when it tried its strength against the more difficult ones, either failed altogether, or if it succeeded here and there in obtaining a solution, had no sure means of convincing others that its solution was correct. In scientific investigation, as in all other works of human skill, the way of obtaining the end is seen, as it were, instinctively by superior minds in some comparatively simple case, and is then, by judicious generalization, adapted to the variety of complex cases. We learn to do a thing in difficult circumstances by attending to the manner in which we have spontaneously done the same thing in easier ones.

839. "This truth is exemplified by the history of the various branches of knowledge which have successively, in the ascending order of their complication, assumed the character of sciences; and will doubtless receive fresh confirmation from those of which the final scientific constitution is yet to come, and which are still abandoned to the uncertainties of vague and popular discussion. Although several other sciences have emerged from this state at a comparatively recent date, none now remain in it except those which relate to man himself, the most complex and most difficult subject of study on which the human mind can be engaged. ${ }^{1}$. . .

${ }^{1}$ Mill was mistaken. He was thinking of the study of human mind and society in terms of causation. But already Darwin had published his view of the origin of species, and Wallace his famous companion essay. Stimulated by them, 
840. "If, on matters so much the most important with which the human intellect can occupy itself, a more general agreement is ever to exist among thinkers; if what has been pronounced ' the proper study of mankind' is not destined to remain the only subject which Philosophy cannot succeed in rescuing from Empiricism; the same process through which the laws of many simpler phenomena have by general acknowledgment been placed beyond dispute must be consciously and deliberately applied to those more difficult inquiries. If there are some subjects on which the results obtained have finally received the unanimous assent of all that have attended to the proof, and others on which mankind have not yet been equally successful; on which the most sagacious minds have occupied themselves from the earliest date, and have never succeeded in establishing any considerable body of truths, so as to be beyond denial or doubt; it is by generalizing the methods successfully followed in the former inquiries, and adapting them to the latter, that we may hope to remove this blot on the face of science." 1

84I. To sum up: whether we consider the histories of individuals, or peoples, or sciences, or religions, or peace, or war, or education, or commerce, or invention, or legislation, indeed of almost any field of human endeavour, one truth is seen to stand forth vividly and unmistakably the moment our attention is drawn to it-the truth that human progress and success depend, and have ever depended, chiefly on a power acquired through antecedent training, a habit, of thinking skilfully about causes and their effects. Thus only have men, by studying the present, been able with any degree of certainty, to realize the past and anticipate' the future. This kind of thinking is skilful only when it is carefully tested and comprehensive. Beginning with generalizations from experience, it proceeds, therefore, to establish these generalizations and extend the area covered by them by a thorough and relentless appeal to reality. Only those individuals have achieved enduring success that have thought in this way. Only those nations have been great and progressive that

many other men besides were at work to discover the causal relations that link together the different kinds of plants and animals that have appeared on earth. As we have seen, all Mill wrote was then, and is now, strictly applicable to the whole study of causation in relation to living beings-that is, to the whole study of heredity and evolution, and all that it implies. To this day, because of fundamental differences concerning evidence and method, biological thinkers, however right they may be, can, as a rule, convince only minorities of their fellows.

1 Op. cit., vi. i. 1 . 
have trained their youth to think in it. Only those religions that have not greatly obstructed this method of reaching truth have been associated with growing civilizations. Only those sciences have become interpretative in which it has been systematically recognized and followed. The races that have been barbarous, the religions that have caused stagnation and disorder, the systems of education that have been futile, the sciences that have been cockpits of factions and tumbling grounds of whimsies, the armies that have been defeated in war, the individuals that have laboured in vain-these have all owed their misfortunes to lack of thoroughness in thinking; that is, to an insufficient testing of suppositions, and, therefore, to an incomplete survey of the facts. 


\title{
APPENDIX
}

\section{AN ATTEMPT TO REPRESENT DIAGRAMMATICALLY SOME OF THE IDEAS CONNECTED WITH INHERITANCE}

BY

\author{
HERBERT HALL TURNER, D.Sc., LL.D., F.R.S. \\ Savilian Professor of-Astronomy, Oxford
}

\section{r.}

TNTRODUCTORY.-The perusal of Dr Archdall Reid's Principles of Heredity, and of the present work, suggest that there is a closer resemblance between biological and geometrical reasoning than might be supposed. Starting from certain axioms in geometry, a considerable superstructure is erected by following necessary laws of thought. In biology, there are no axioms of the same kind; but there are some fundamental truths which may to some extent take their place; as, for instance, that children resemble their parents. The precision of an axiom is absent : so is the self-evidence: but the universality of the truth and its universal acceptance, when once the limits of application are understood, are comparable with those of a geometrical axiom. Every one knows that the offspring of an elephant will be an elephant, of a dog a dog, of a butterfly a butterfly similarly marked. The resemblance between parent and child only extends to broad features and breaks down at details, and hence the necessity for specifying limits of application. But there is no difficulty about this unless we try to be too precise. We know with equal confidence that children differ from their parents; i.e. that no two beings are ever precisely alike; each of these two apparently contradictory propositions is undeniably true within its own limits of application : and though these limits cannot be precisely defined, they are well understood.

2. Now, Dr Archdall Reid has shown that we may build upon such truths as these in a manner not very different from that in which geometers build upon their fundamental truths. For instance, he deduces Recapitulation from the two principles just quoted, and little more; he insists that we need not have derived it from independent observation, but could have deduced it (as a necessity of thought) from other principles already known and more familiar.

3. To a mathematician such a view is fascinating, and his impulse is immediately to seek mathematical analogies or diagrammatic representations, which help him to picture the process of thought. Thus, he may think of the resemblance between the growth of a child and that of its parent as represented by two similar tracks-let us say the tracks of the front and hind wheel of a bicycle. They are nearly, but not quite, the same. If a third, fourth, fifth wheel followed in similar sequence, we 
should have a representation of ancestry by the successive tracks which would appeal specially to a mathematician, who is accustomed to curves and their properties. Given this tendency of one wheel, or say of one cyclist, to follow the preceding, it is suggested at once that the general path will remain much the same unless there is some persistent disturbing causesay the gradual wearing away of a bad patch on the road. Further, it is possible that each cyclist, though following the path of his predecessor, may ride just a little quicker and farther; so that what was once the whole extent of the ride becomes ultimately only an early stage of it passed over with great rapidity: and we thus arrive very simply at a conception nearly related to that of Recapitulation in biology.

4. Nothing is proved by this: but when Dr Archdall Reid challenges his readers to think of any possible alternative to Recapitulation, such analogies help the mathematician by presenting the corresponding difficulty of travelling from one point to another without passing through intermediate positions. One may "go round," but this is to lose time : and for living beings, natural selection makes it important to save timeto reach strength and maturity early. The cyclist we have pictured above can save time in two ways only: by going quicker or by shortening the route. If their predecessors had laid down a circuitous route, those following later may shorten it by "cutting across": but we must remember that time was important for the predecessors also, since they too were naturally selected: and hence they were forced into the most direct route they could find. The path is thus constantly tending to become straight, and to be ridden quicker.

5. This illustration will serve to show the kind of temptation which besets a reader, whose training has been mathematical, to think in his own terms; and to feel that ideas are clearer, to him at any rate, when expressed in those terms. It will thus explain the origin of the following essay in diagrammatic representation of biological conceptions. The crudity and imperfections of the attempt are too obvious to be covered by any apology: but they may stimulate some abler pen to a more successful endeavour.

6. Diagrammatic representation. - Let the size of an organ or feature

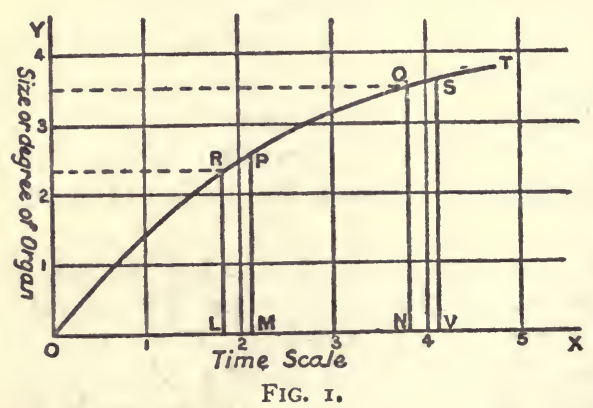
(length of arm, weight of brain, etc.) or its degree (clearness of vision measured in some selected manner) be represented in the familiar way by a diagram, in which one ordinate is the time scale measured along $\mathrm{OX}$ in any convenient units, I, 2, 3, 4, 5, etc., which may be years for a man, or days for an insect; the other axis, OY, being devoted to the size or degree of the organ. Then the growth of the organ will be represented by a path, ORPQS, of some kind, which has the follow- 
ing properties : (a) It starts from $\mathrm{O}$, since all organs must have a beginning. (b) It ends at some point, T, when the animal dies. (c) The ordinates, RL, PM, QN, SV, etc., tend generally to increase. There is nothing impossible in a decrease such as $P Q$ (Fig. 2), much less in a stationary period such as PR in Fig. 2 ; but the general rule in Nature is growth, and for the present the exceptions may be left out of consideration.

7. Resemblance to parents.-Now let the path for a certain organ in a parent be OQP, PD being the size at a time OD selected for observation or measurement. At a corresponding time in the life of one of the offspring let the corresponding organ be observed or measured, and found to be $\mathrm{pD}$. Then we know from experience that-children resemble their parents - (I.), and hence that $\mathrm{pD}$ will not be very different from $\mathrm{PD}$ on the average. But also we know that-children are not exactly like their parents, and may differ from them either way-(II.),

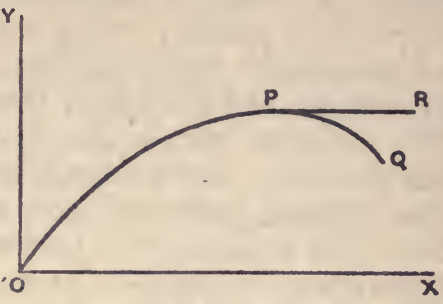

FIG. 2.

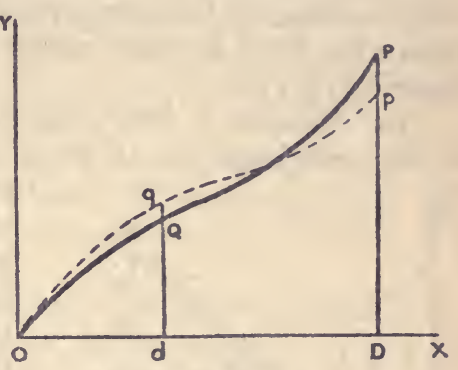

FIG. 3 . and hence $\mathrm{pD}$ will usually differ from PD; and the difference may be in either direction.

8. But the two elementary principles just stated tell us more than this. The resemblance and the difference are not applicable to a single moment only such as OD; they extend throughout life, and consequently if we observe the corresponding organs at any previous time Od, we shall find that $\mathrm{dq}$ is not very different from $\mathrm{dQ}$, though, as a rule, not exactly equal to it.

It is easily seen that the path Oqp for the child will thus lie near to

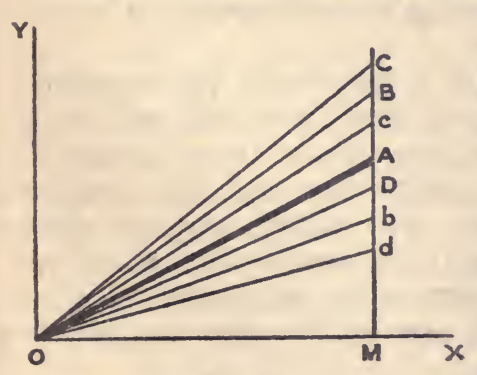

FIG. 4. that $\mathrm{OQP}$ for the parent throughout its whole length; or in other wordsthe development of the child is a recapitulation of the parental development-(III.).

9. The tendency to diverge. - Let us now suppose that the path $\mathrm{OA}$ is followed by a certain individual. (For convenience it and other paths are drawn straight, but this in no way affects the argument at present.) His children may, and generally will, follow paths near this, such as $\mathrm{OB}$ and $\mathrm{Ob}$, which may differ from $\mathrm{OA}$ in opposite directions. The children of $\mathrm{OB}$ will similarly, in 
general, follow either a path Oc, which is a return towards the ancestral path $\mathrm{OA}$, and may even go on the other side of it, or a path $\mathrm{OC}$, which is a further deviation from the ancestral. We are not yet in a position to say anything of the relative chances of these two possibilities, but experience of a wide kind tells us that both are possibilities; and similarly it is possible for the children $\mathrm{OD}, \mathrm{Od}$ of $\mathrm{Ob}$ either to return towards the ancestral type (as OD) or to diverge further from it as Od. Hence there is a continual tendency to produce descendants differing more and more from the original type in both directions, and if this tendency were unchecked we should get animals of all sorts of miscellaneous shapes. There are, however, three important checks to this tendency which may not be entirely independent, but which we will consider separately in the first instance; they are (a) the sexual, (b) the ancestral, $(c)$ the retrogressive. The first two we can deal with at once: the third is more conveniently deferred until we come to natural selection ( $\$ 55)$.

ro. Influence of Two Sexes.-Most living forms are the children not of one individual but of two. Let the path of one parent be OQ, and

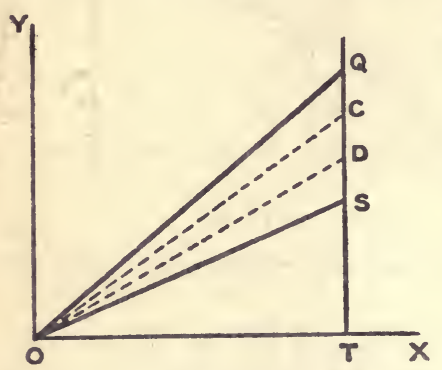

FIG. 5. of the other OS. What will be the path of the child? We know from wide experience that - as a rule children resemble both parents-(IV.), and thus the child's path will, as a rule, be intermediate between the paths $\mathrm{OQ}$ and $\mathrm{OS}$; not necessarily half-way between them, but somewhere intermediate. The child's path may be such as $\mathrm{OC}$ nearer to $\mathrm{OQ}$, or $\mathrm{OD}$ nearer to OS. There might, for instance, be a tendency for the child to resemble the father rather than the mother; and if $\mathrm{OQ}$

be the path of the father, the child's path would be like OC. But if for every pair of parents, $O Q O S$, in which $O Q$ represents the father there exists another pair in which $O Q$ represents the mother (V.), then for every child OC above the half-way path we shall have another child OD equally below the half-way path, and on the average the children will follow the half-way path.

I I. The condition italicized is roughly in accordance with experience. There are tall men with short wives and short men with tall wives. Whether there are more of the former than the latter we cannot say without statistical inquiry: but it is easily seen that the condition must be roughly fulfilled. For take any ten married couples: divide the men into tall and short-five of each : the women also into tall and short-five of each. Suppose three tall men marry tall women: then two tall men marry short women. There are thus left five short men married to three short and two tall women.

I 2. The argument is quite general. If we take $\mathrm{n}$ tall men and $\mathrm{n}$ short men: $\mathrm{n}$ tall women and $\mathrm{n}$ short women : and if $\mathrm{x}$ of the tall men have tall wives: then $\mathrm{n}-\mathrm{x}$ have short wives. And since $\mathrm{x}$ tall women are 
already disposed of, the remaining $\mathrm{n}-\mathrm{x}$ tall women must have short husbands, just as many as there are tall men with short wives. Hence the generality of the proposition is only limited by two assumptions tacitly made, viz. :-

(I) That there are equal numbers of tall and short. We can always secure this by arranging the individuals in series and counting to the half-way. But the separation will in general be rather arbitrary since many men (and women) will be closely of medium height, and there will be other striking features of distribution which are neglected in this rough assortment.

(2) We have assumed quite independent standards for men and women. This is an assumption already familiar in statistical work. Sir Francis Galton at the outset of his essay "Natural Inheritance" adopts the artifice of "never dealing with female measures as they are observed, but always employing the male equivalents in the place of them": and without this artifice he declares that he "hardly knows how we should have succeeded in making a fair start."

I3. With these qualifications the condition (V.) is seen to be in accordance with general experience: and hence it follows-that on the average the child's path will fall just midway between those of its parents-(VI.).

I4. But we must remember that this result is only the extension to the case of two parents of the law assumed for one parent-i.e. that a child resembles its parent. It is therefore liable to modifications due to any departure from this law such as we shall presently consider.

I 5. Sexual restriction of deviation from type.-Now suppose OP is the general type of a species, and some individual follows the path $\mathrm{OQ}$ differing from $\mathrm{OP}$. The path of the mate may be on either side of OP, but will on the average be along $O P$ : and hence the children will on the average follow the midway path OR, which diverges from $\mathrm{OP}$ only half as much as $\mathrm{OQ}$. Hence we see how the deviations of the parents are reduced in their offspring, and the tendency to diverge indefinitely, noticed in $\S 9$, is checked. We shall presently show that definite limits are assigned to the divergence, but

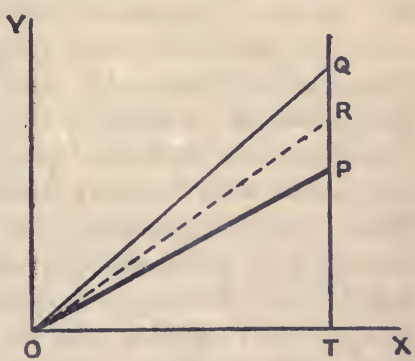

FIG. 6. first there is a special point calling for notice, which might modify the conclusion just reached.

I6. Assortative mating.-An assumption (italicized) was made in the last paragraph, namely, that the mate is chosen indifferently from the community at large: and that there is no systematic tendency, for instance, for the parents to resemble one another. This is in rough accordance with our experience : a tall man does not as a rule choose a tall wife; indeed there are often ludicrous exceptions, which strike the attention so much that we should say that the tendency was the contrary. 
It is doubtful, however, whether ordinary impressions are sufficient to decide with accuracy what is the general rule. We should probably be equally surprised to find on careful inquiry, that tall men chose tall wives in as large a percentage of cases as they chose short wives: though a small percentage would not be surprising. We should probably admit that the matter was essentially suitable for statistical inquiry, though we should not expect any very startling results. If "like on the whole chooses like," then the divergences of parents are not reduced by so much as one half : if "like chooses unlike," then the reduction is greater. Now each parent has many qualities, and the deviations are not all in the same direction. A tall man may have long arms and long legs, but he may be short in temper: and his choice of a mate may depend as much on his short temper as on his long arms. Hence, on the whole, though statistical inquiries may give interesting results in special cases, we should reasonably expect to find that the path of the child was that of the "midparent," and that the deviations of the parents from the type were halved in each generation.

I 7. Definite limits for divergence.-Now suppose that a snail were to start crawling away from a wall, and were to crawl one foot per hour. If it were undisturbed it would ultimately reach an indefinite distance from the wall. But suppose at the end of each hour some one put it back just half-way to the wall. Then the course of events is as follows :-

After I hour it has crawled to I 2 inches: put back to 6

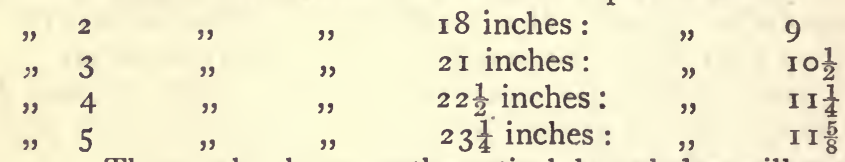

and so on. Those who have mathematical knowledge will see at once that the snail never gets two feet away from the wall, although it gets continually nearer and nearer to that distance: and indeed without mathematical knowledge the facts are fairly obvious : for so long as the snail does not reach the two foot mark he must be put back behind the one foot mark, and thus has more than a foot to go. This proves the first part of the proposition-that he never reaches the two foot mark. 'The second part (that he gets continually nearer) follows from the fact that his failure is halved each time. At the end of the first hour he fails by a whole foot: at the end of the second by six inches: at the end of the third by three inches: and so on. The defect is halved each hour.

I8. The application of this illustration is also tolerably clear. Children have a tendency to deviate from their parents, and if unchecked the deviations from the ancestral type might accumulate (like the distance of the snail from the wall) to any amount. But if the deviation is halved every generation, a certain limit cannot be surpassed, though it is con tinually approached. If the case were as simple as that of the snail this limit would be twice the deviation of any generation from the preceding(VII.): but the problem is really much more complicated. Nevertheless, we may reasonably anticipate a result of about the kind indicated. 
19. Limits of deviation.-The limits thus assigned will be of course on both sides of the ancestral type. They are not necessarily at equal distances from it, for (owing to natural selection or other causes) there may be more tendency to diverge on one side than on the other: and the limit will be proportionately greater. But it will be seen that we have arrived at an important conception of the path followed by a descendant. If $O P$ be the ancestral type, then the paths of the descendants are confined between certain limits, OQ, OR, on opposite sides of the ancestral type. These limits are not actually defined so sharply as we have drawn them; they are more like the edges of a shadow ; and an accurate mathematical expression for them would express a gradual shading off-a fall rapid at first and very gradual afterwards. But

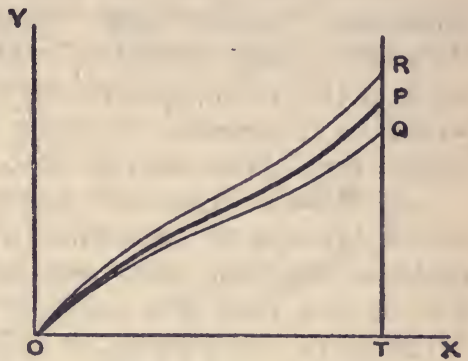

Fig. 7 . if for simplicity we think of actually well defined limits, we shall not be far from the truth, and the results arrived at will have corresponding exact propositions, differing from them only in detail.

20. Transmission of sexual characters. - The general law of transmission above considered has however a class of important exceptions, applying to cases where the differences between parents are not small, and are not continuously graded. The most conspicuous and important of these is presented by the sexual characteristics themselves. The child of male and female parents is not something midway between (with possible extremely rare exceptions), but either the one or the other. We are clearly in the presence of a totally different law of transmission.

2 I. We can illustrate the difference graphically by adopting separate planes for the representation of the two sets of characters. Our diagrams

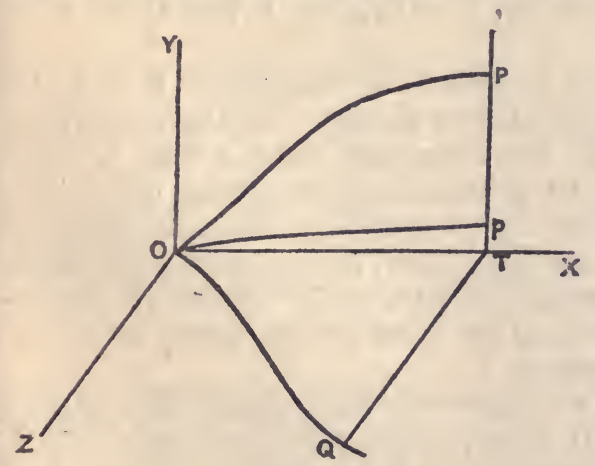

FIG. 8.

have hitherto been drawn in one plane, OXY : if we devote this plane to paths such as OP, representing the growth of a female organ, we shall not in general be able to represent the growth of the corresponding male organ on the same diagram, as the definitions would not apply. But we could take another plane, OXZ, in which OX still represented the time scale, and $\mathrm{OQ}$ the growth of the male organ. In

so far as the organ in one sex is rudimentary in the other, we could of course represent it by a line near $\mathrm{OX}$ in the other. Thus if OP represents the growth of breasts 
in a female, the corresponding growth for a male might be a path $\mathrm{Op}$, very close to $\mathrm{X}$, since the growth is slight. And if the organ of one sex were actually non-existent in the other, it could nevertheless be represented by the line OX itself, which represents zero growth throughout.

22. So that each sex would be represented by two sets of sexual characters, "patent" and "latent" as Dr Archdall Reid calls them. "Patent" female characters would be represented by paths such as OP far from OX, in the special female planes : and "latent" female characters would be represented by paths near OX (or coinciding with it) in the special male planes such as $\mathrm{XOZ}$.

23. Now we know that our principle (IV.) no longer applies in this case. From wide experience we know that-in sexual characters the children represent either one parent or the other: not both-(VIII.). Let us see how this principle can be represented diagrammatically. To avoid the complexities of solid geometry, let us make a cross section of Fig. 8 through the lines PTQ, as represented in Fig. 9. The

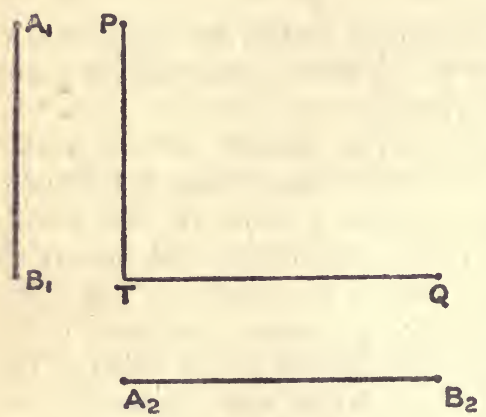

FIG. 9. point $\mathrm{T}$ represents rudimentary or zero characters, $P$ well developed female, and $\mathrm{Q}$ well developed male. Now the child has two sets of qualities : we may represent him by a pair of points, $\mathrm{AB}$, connected together, or rather kept separate by a rigid rod. (The notion of keeping separate is appropriate, because he has one set patent and the other latent : not both patent or both latent, i.e. $\mathrm{A}$ and $\mathrm{B}$ must not coincide.) Experience then tells us that there are two possible positions of $\mathrm{AB}$, as crudely represented in Fig. 9 : either $A$ falling on $\mathrm{P}$ and $\mathrm{B}$ on $\mathrm{T}$, or $\mathrm{A}$ on $\mathrm{T}$ and $\mathrm{B}$ on $\mathrm{Q}$.

24. This result would follow at once if we conceive of $P, T$, and $Q$ as centres of attraction for the ends, A,B, of the rod. And this conception would be quite in keeping with what has gone before: thus in Fig. 7 we might regard the type path, OP, as attractive, so that other paths would be attracted near it. If we took a cross section of the path as we did for those of Fig. 8, we should get a single point, P, representing the parent, attracting the single point, $R$, representing the child. When we come to sexual characters we must represent the child by two points as above: and we then get a new interpretation.

25. It is scarcely necessary to say that the diagrammatic analogy proves nothing whatever. It is merely a convenient summary of facts which we learn from wide experience. But it may help us to realize the connexion, with these fundamental and well known facts, of others which are not so well known.

26. For instance, it may help us to realize how Mendelian inheritance 
is analogous to sexual inheritance, as Dr Reid has argued. Mendelian inheritance deals with the combination of two characters, one of which is patent and the other latent or non-existent. It could be represented graphically by the same artifice used for sex. But properly to develop the consequences requires a more careful study of the phenomena than the present writer has been able to undertake.

27. Ancestral resemblance.-Let us now return to those qualities which are transmitted non-sexually. We have hitherto dealt simply with the resemblance of children to their immediate parents; but we know that inheritance is not from parents alone. From wide experience we know thata child has a tendency to resemble, not only its immediate parents, but its ancestors, even when the parents differ from them-(IX.).

Hence if $\mathrm{OP}$ be the ancestral type, and $O Q$ the path of a parent, the path of the child, Oq, will tend to lie between $\mathrm{OQ}$ and $\mathrm{OP}$ : but there is

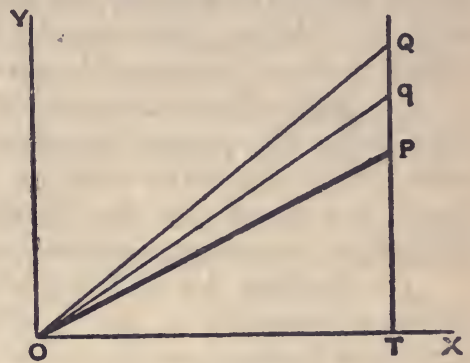

FIG. Io. no reason why it should be midway between $\mathrm{OQ}$ and $\mathrm{OP}$ : probably it lies nearer $O Q$, though we may not be able to deduce the numerical law from theoretical considerations. Sir Francis Galton found in 1889 that in certain cases $\mathrm{Pq}=2 \mathrm{Qq}$ : or $\mathrm{Pq}=\frac{2}{3} \mathrm{PQ}$ : i.e. the deviation of a child was $\frac{2}{3}$ of the deviation of a parent: and this may quite possibly be a general law deducible from theoretical considerations. On the ocher hand, the numerical factor may differ in every case. For the present, we shall merely use Sir Francis Galton's result for the purpose of illustration without attempting to explain it, or inquire into its generality.

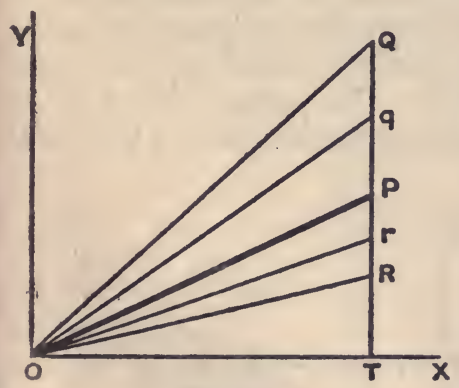

FIG. II.

28. Corrected statement of sexual effect. - Thus if one parent alone (OQ) were concerned, the child would tend to follow the path $\mathrm{Oq}$, where $\mathrm{Pq}=\frac{2}{3} \mathrm{PQ}$. If the other parent (OR) were alone concerned, the child's path would tend to be Or, where $\operatorname{Pr}=\frac{2}{3} \mathrm{PR}$. When both parents are concerned, the child's path will therefore tend to lie midway between $\mathrm{Oq}$ and Or, not midway between $\mathrm{OQ}$ and $\mathrm{OR}$ as previously stated. The deviation from OP will consequently be $\frac{2}{3}$ of that previously stated, which we found was, on the average, one half that of the parents. Hence the deviation will be reduced every generation, not in the ratio $\frac{1}{2}$ which is the sexual effect, but in the ratio $\frac{2}{3} \times \frac{1}{2}=\frac{1}{3}$, which is the combined sexual and ancestral effect. Returning to the analogy of the snail, if at the end of each hour one person sets him back to half his distance, and another person to $\frac{2}{3}$ of that half, he will always be set back to $\frac{1}{3}$ of his total 
distance : and it is then clear that his limit will be reduced from 24 inches to 18 inches. So long as he is short of the 18 inch mark he will be set back behind the 6 inch mark, and will thus have more than a foot to do to reach 18 inches. He will thus never get there, though he will continually approach it. The effect of ancestral inheritance is to reduce the limits of deviation from type which we arrived at in $\S 10$.

29. Stability of type.-We have thus arrived at a very definite notion of the stability of a type when no cause such as natural selection interferes with it. Mating alone would suffice to prescribe certain limits of deviation from the ancestral type which could not be exceeded.

30. These limits are narrow-a simple analogy suggests as average limit twice the average deviation of a single generation: and though a more suitable but more complex calculation might give a rather different result, the limits are not likely to be many times greater than the deviations from one generation to the next.

3I. Similarly the tendency of descendants to resemble their ancestors causes a reversion to the type in each generation, which narrows the limits still further.

32. We shall presently point out an important difference between

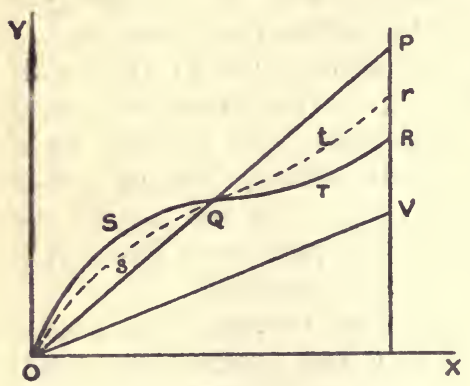

FIG. I2.

the action of these two agencies, the sexual and the ancestral; but at present we are concerned with their concurrence. Together they restrict the deviation within narrow limits and render the type stable.

33. Paring down of irregularities.It is perhaps worthy of note that the two agencies combine to destroy, not only deviations of the whole path $\mathrm{OV}$ from $O P$, but also irregular deviations such as OSQR, which may cross the type path. The portion, OSQ, which lies above OP, will tend to be replaced in the next generation by OsQ, still above but less above: at $Q$ there is no tendency to change, and in the portion, QTR, the influence is to raise the path towards OP. Thus the path of the child tends to straighten out irregularities in those of the parents. Indeed it is easily seen that irregularities of this kind have, owing to the sexual effect, far less chance of surviving than have deviations of the path as a whole, either above or below, for the paths of the parents may easily both deviate in one direction as a whole, but their

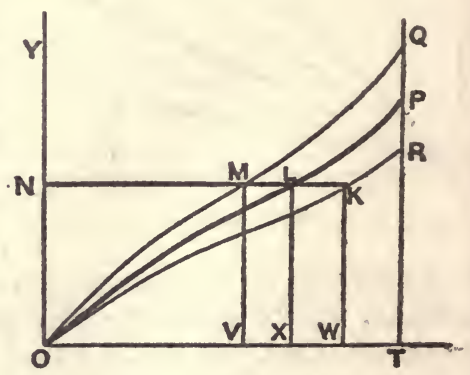

FIG. 13. irregularities would be unlikely to coincide, and would tend to annul one 
another. Hence if OP be the type-path, we may take as representative individual paths OKR and OMQ, lying near OP, but not as a rule crossing it-(X.).

34. Earlier development.-Now this has an important consequence. Instead of drawing, as hitherto, ordinates such as QPRT, parallel to the OY axis, draw one NMLK parallel to the OT axis. Then NM or OV represent the time at which the child, $\mathrm{OQ}$, has the organ under consideration of the same size (MV or LX) as the parent at the time OX. The child, $O Q$, reaches this stage earlier than the parent ; and since the same is true throughout the curve we see that this child passes through all the successive stages (in regard to the organ in question) earlier than its parent. Similarly the child, OR, develops more slowly than its parent, throughout. We may say generally that-individuals strong in any organ develop early; those weak develop late-(XI.).

35. Relation of organs.-But we have so far considered one organ alone, whereas the characteristics of any animal depend on the relation of its parts-small head and long neck in a giraffe: large head and short neck in a lion. What is true of an isolated organ may not be true of a combination, and we must consider how the relationship of one to another enters into the questions before us. In the first instance, take any two organs such as length of leg and weight of brain : and, dropping any question of time for the moment, let us make a diagram to represent their relationship, setting off the length of

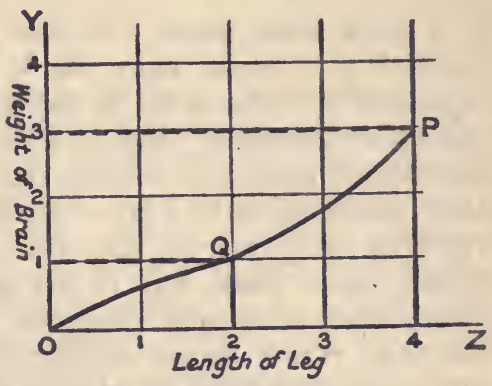

Fig. I4. leg along $\mathrm{OZ}$, and the weight of brain along $\mathrm{OY}$. Thus the point $\mathrm{P}$ represents the fact that when the length of leg is 4 feet, the weight of brain is 3 ounces or pounds, or any other suitable unit. Previously (at Q) the

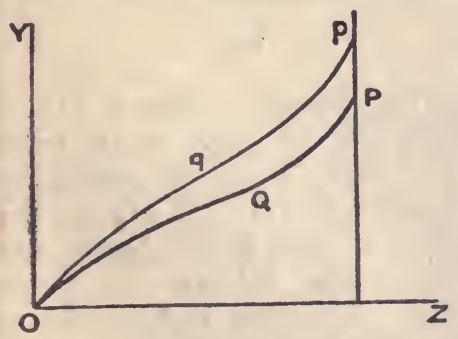

FIG. I5. length of leg was 2 feet, and the weight of brain was I oz. or lb. Since the dimensions are all zero at first, the path $\mathrm{PQ}$ will start from $\mathrm{O}$, just as that representing the relation between any organ (say Y) and the time.

36. Now it will easily be seen that most of the considerations already advanced in the case of the paths for the growth $Y$ of an organ, with the time $\mathrm{T}$, will apply equally well to the path for the growth of one organ

relatively to another. If $\mathrm{OQP}$ is the path for a parent, and Oqp that of a child, the facts that children roughly resemble parents, but that they may differ from them in either direction, and so on, still apply to these new paths. Hence we get the same rules about sexual and ancestral 
effect, and we are led in the same way to the limits of deviation from the type.

37. If $\mathrm{OP}$ be the type-path it may indeed be a more devious curve than those we have considered. But the descendants will have paths lying

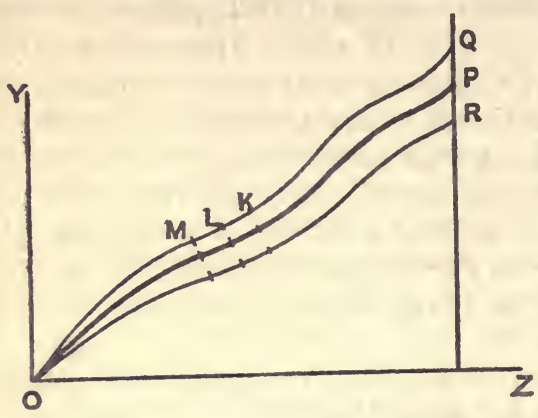

FIG. I6. within narrow limits, such as OQ and OR. And the same will be true of any pair of organs: so that the infinite complexities of growth and structure of the whole animal will all be reproduced within narrow limits in the developments of their descendants.

38. Earlier and later development.-But now let us reinsert the time element. Of the individuals which follow any given path, $\mathrm{OQ}$, reaching any stage, $\mathrm{L}$, on the average at a given time, some will have developed rather more rapidly and reached $\mathrm{K}$; others more slowly only and reached $\mathrm{M}$. We could represent the time scale in the same diagram by marking off various epochs on the curve. But we should always have to remember that some individuals, while still keeping near the type, arrived at any given stage early, and some arrived late. It is not difficult to see that the rule XII. specified in $\S 34$ applies, not to a single organ alone, but to the whole complexities of animal structure. We can thus return to our curves representing the variations of a single organ with the time, knowing that the results obtained can be extended in this way.

39. Order of the quantities. - It is perhaps worth while to consider for a moment the size of the quantities involved-what a mathematician calls their "order." Thus if PT represents

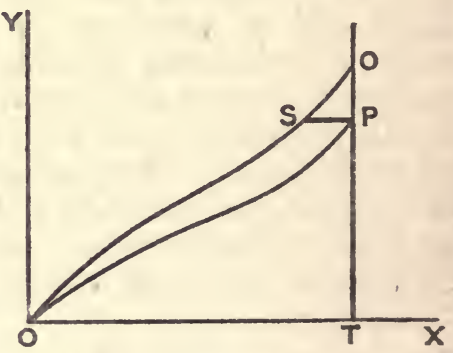

FIG. I7. the height of a parent, say 6 feet, then the average deviation, PQ, is

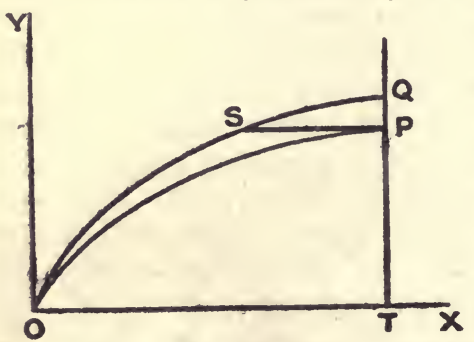

FiG. 18. something like an inch or two-say $I$ in 50 : and SP will be nearly the same fraction of OT. If OT be the time taken to grow to full height, say 25 years, then SP will be about 6 months. The child who is going to be taller than his parent by $\frac{1}{50}$ th part will, on this computation, reach a similar height about 6 months earlier. But usually growth is rapid at first and slow nearer maturity, so that the figure should be like Fig. 18 rather than Fig. I 7. It is easily seen that 
he child will under these conditions anticipate the parent by more still. This anticipation, or early development, is of importance as will be seen presently.

40. Natural selection.-We now come to the agency that modifies the above conclusions, natural selection, which works by the destruction of the defective. Let us represent the growth of the particular character which, by defect, renders the animal liable to destruction by paths between $O Q$ and $O R$, in the manner already familiar. Then if the animal does not reach a certain lower limit, $\mathrm{OD}$, in this character, it is liable to destruction-for simplicity let us say it is actually destroyed. But this liability does not commence at birth: animals are usually protected by their parents, sometimes for long

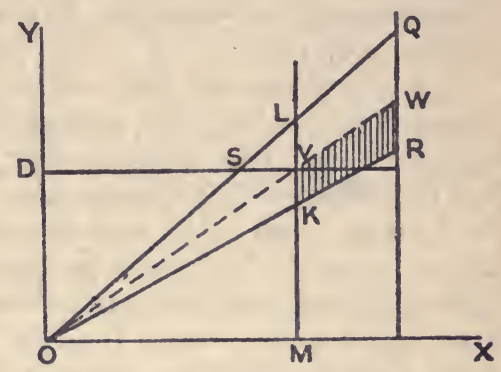

FIG. 19. periods, after birth. The period of protection may be represented in the time scale by OM, so that the animal is not liable to be destroyed until its path has reached the ordinate MV. If then all who have not reached the limit, OD, are destroyed, the survivors will be those following paths between OVW and OLQ. This is the main argument stated in its most elementary form : but in this form it is scarcely applicable to real life: we must generalize the details.

4I. Period of protection.-First let us consider the period of parental protection, which is often evanescent. If the argument were no longer applicable in such cases, we should have a serious difficulty. But parental protection is only one form of a more general protection in which chance plays a conspicuous part. Let us first consider a number of seeds scattered on the ground and liable to be eaten by birds. A seed may be snapped up directly it is scattered, or it may never be eaten at all : and though there is thus no definite protection, there will, according to the laws of probability, be an "expectation of life" of a seed-an average period for which it may expect to survive. And this period will not be determined by inherent qualities of the seed, but by external circumstances, such as the number of depredating birds, the nature of the soil, and the skill of the sower. There is something very similar in the case of young animals too feeble to depend on their own exertions for escape from destruction. In later life an animal may depend on its speed fo- escape, and the speediest will have advantages over those less speedy: but the speed of the very young may not suffice in any case to protect them: slow and speedy may be open to similar risks, like two seeds or two eggs in the same nest.

42. Hence the notion of the period of protection when generalized will be somewhat complex : it will include not only the period before birth (for viviparous animals), or the period in the egg and in the nest after hatching: but also an additional period before the powers are 
sufficiently developed to give one individual sensible advantages over another: the protection will in general be only partial, and the period will vary greatly in length for different individuals. It will be definite only in the sense in which an average is definite: and its essential characteristic is that it is not determined by differences between individuals, but by external causes : so that it is properly represented by a vertical ordinate, MKL in Fig. 19, which cuts all paths at an equal distance from OY.

43. The destructive limit. - Let us now consider the destructive limit, represented by a line, DV, cutting all the paths at an equal distance from the other axis. One criticism which may be made is that this representation is not always appropriate, since the quantity of any character required for escaping destruction may vary during the life-time of the individual. Thus if an animal depended for escape on length of leg, doubtless shorter legs would suffice in its youth to carry it into safety than when it became older and heavier : hence it might be said that the line, DV, ought to slope upwards. But we can make a horizontal line suit such cases by changing the character represented in the diagram. Instead of length of $l e g$, let us take actual speed: then the speed required in all cases would be the same (namely, that which outstrips the enemy), and hence the horizontal line would be appropriate. By choosing the right variable, we can make our diagram suit other cases.

44. Effect on next generation.-We may now consider the effect of this destruction on the next generation. The survivors represented by paths between OVW and OQ in Fig. I9, are stronger in the particular quality than those destroyed, and their children will tend to be like them. We shall have to take account, in this connexion, of sexual and ancestral influences; but let us omit these in the first instance, for separate consideration later. We thus simplify the problem to the case in which children merely tend to resemble their parents.

45. By a slight extension of the significance of the diagram we can use it to represent the members of one generation in all stages of growth. Thus those destroyed in a generation are represented by the figure VWRK, the survivors by LVWQ, and those who are still being protected by the triangle OKL. These last may in general be dismissed from consideration when we are discussing the next generation: for usually breeding does not commence until the period of protection is over, and the animal is thrown on its own resources. The parents of the next generation will thus be represented simply by the figure LVWQ, and their children will thus tend to follow paths between OW and OQ.

46. Thus the primary result of destroying the unfit is to improve the next generation. The type-path tends to rise, and will continue to rise as the destruction is repeated until the paths all clear the point V in Fig. I 9: that is to say the character represented will gradually improve. It will probably go on improving even after the point $\mathrm{V}$ is cleared: for $\mathrm{V}$ itself will tend to rise, since one animal is often the prey of another, and the struggle between them will tend to improve both.

47. The point $\mathrm{V}$ may also move to the left. For the children of the 
survivors run through the stages when they need protection more quickly than the generation before them. Now the protection itself is a product of natural selection, and will not be maintained without reason. Hence as each generation needs it for a shorter period (on the average) it may shorten automatically. Hence $\mathrm{O}$ moves to the left and upwards from a position such as $V_{1}$ to another such as $\mathrm{V}_{2}$; and the path representing the growth of the character which determines destruction or survival will move from $\mathrm{OP}_{1}$ to $\mathrm{OP}_{2}$ : that is, its gradient will become steeper, at any rate until it

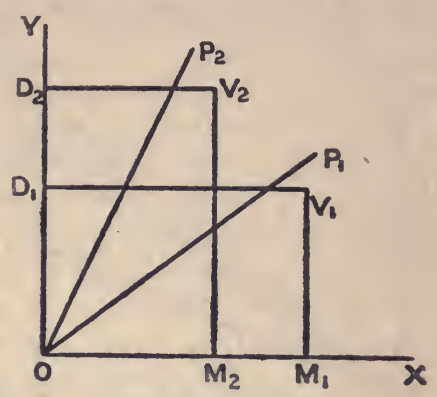

FIG. 20. has surmounted the point V. As to what happens after this we will inquire presently.

48. Now in $\S I_{5}$ it was pointed out that this means earlier development of the organ or character; and thus we see the effect of natural selection is to make organs on which it acts develop early.

49. Other organs. - Again there is nothing impossible in the association of an early development of organ $\mathrm{Y}$ with retarded development of organ $\mathrm{Z}$. But if $\mathrm{Y}$ and $\mathrm{Z}$ are both acted on by natural selection there will be a tendency for both to develop early; and the relative proportions of the animal, so far as these two organs are concerned, will tend to be preserved while at the same time its development is accelerated. But natural selection acts on an immense number of characters in a complex animal, and the same argument will extend to them all. Hence there is a continual tendency to preserve those individuals which run quickly through the copies set them by their parents, at any rate until they reach the destructive limit.

50. Modification of curve.-But now what happens after reaching this limit? We return to the case of a single organ for simplicity. Once the

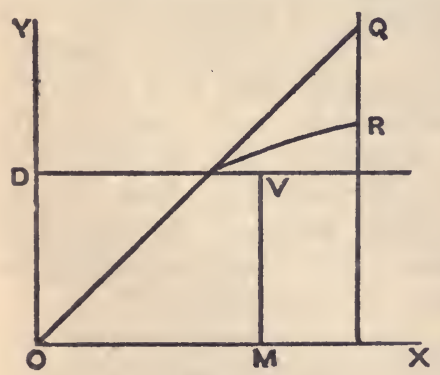

FIG. 2r. limit is attained, and the individual is secure of survival, he may, so to speak, please himself whether he goes further. Those who survive at $\mathrm{V}$ may go on to $\mathrm{Q}$ or only to $\mathrm{R}$, and their children will be equally numerous. Now we have hitherto dealt with straight paths and regarded such a path as OVR as unusual: but this was before the idea of natural selection was introduced; and we shall presently see reasons for modifying the results previously arrived at. We shall find in fact that influences tending to depress the portion VQ to VR; so that after a certain point the growth is slower than at first. This is a wellknown feature of the growth of all animals and plants : after a certain point they reach "full growth," and further development is comparatively slow. 
5 $\mathrm{I}$. Sexual effects combined with natural selection.-We now turn to the modifications introduced by mating. Let us suppose that the destruc-

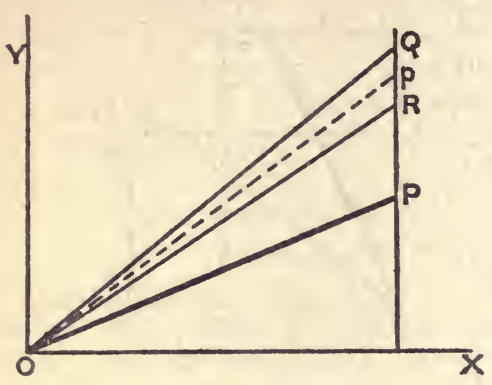

FIG. 22. tion of the unfit has produced a rise from the ancestral type $O P$ to Op. Then the reasoning of $\S$ I 5 shows how mating sets certain limits $\mathrm{OQ}, \mathrm{OR}$ for deviation from the type Op : but suggests no general tendency to return towards OP.

52. But there is a particular tendency of this kind arising from the sexual effect, in connexion with the paring down of irregularities. If $O P$ be the type, then we have remarked that such irregular paths as OrKR tend to disappear because in effect there are equally likely paths, OqKQ, with which they may be mated. But under natural selection the paths are not equally likely. The path OrKR climbs over the $\mathrm{V}$ and indicates survival: the paths OqKQ does not surmount $V$, and means destruction. Thus paths such as OrKR get the advantage and survive. This is the first cause for the depression of path noticed in $\S 50$.

53. Ancestral inheritance and

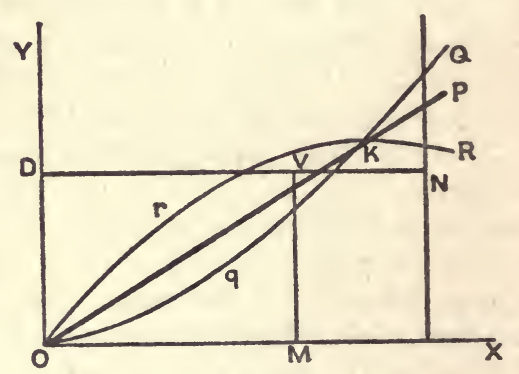

FIG. 23. natural selection.-Let us now consider ancestral inheritance. As before,

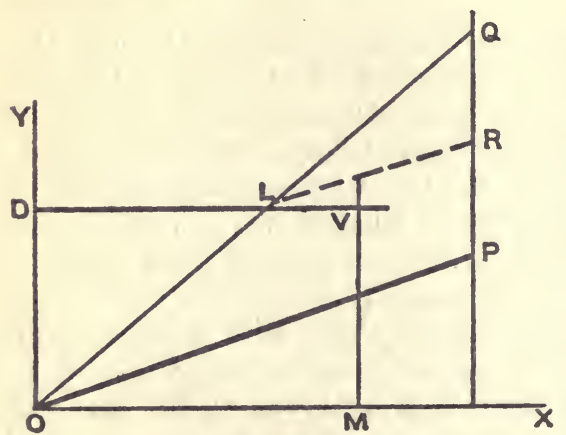

FIG. 24 . let $\mathrm{OP}$ be the ancestral type, DV the destructive limit, and suppose the type has risen, under the influence of natural selection, to OQ. Then by virtue of ancestral inheritance there is a continual tendency for every point of $\mathrm{OQ}$ to return to $\mathrm{OP}$; and this acts in the contrary direction to natural selection, and, at any rate, checks indefinite departure from the original type.

54. But is it sufficient to arrest it? In whatever form we think of ancestral influence, we cannot avoid contemplating it as becoming weaker in each succeeding generation. Suppose for a moment that for a particular generation the tendency of natural selection to raise the type were just counterbalanced by the tendency of ancestral influence to depress it. For the next generation the depressing influence would be weaker, and 
the type would rise : there seems no escape from the continual rising at any rate so far as the critical limit $D V$. But beyond this limit, natural selection ceases to work, and ancestral influences act unchecked. Hence LQ will tend to move nearer OP, i.e. in the direction LR. This is the second cause for the depression of the path noticed above.

55. Retrogressive tendency.-But as yet we have found nothing to arrest continual and indefinite departure from the original type up to the point $\mathrm{V}$. There is nothing which can permanently balance natural selection, so that the type may remain stable in the presence of its natural enemies. And yet our experience is that such stability is reached. If we admit the existence of such stability, then we may put the inference as follows : Let any generation consist of $\mathrm{A}$ individuals who are destroyed before breeding, and B individuals who survive and have offspring. If a condition of stability is reached the next generation will be the same$A$ unfit and B fit. But all these are by hypotheses the offspring of the $\mathrm{B}$ members only of the preceding generation. These $\mathrm{B}$ members do not, therefore, produce offspring $\mathrm{B}$, fit like themselves (on the average), but partly $B$ and partly $A$; the average of the offspring is lower than that of the parent : and this goes on continually. The parents are of a constant type $B$ : the children are of a constant inferior type between $B$ and $A$. If the difference remains really constant, ancestral resemblance cannot explain it, for this is all the time growing weaker. We seem to be thrown back on some innate tendency to retrogression, which may possibly be a universal tendency. We assumed at the outset that children might deviate from their parents in either direction: but the deviations may not be equal. We should have a vera causa of the kind we are looking for if the tendency to retrogression were the stronger. It is not necessary that it should be always stronger; it would suffice if it became stronger with growth.

56. Dr Archdall Reid has, however, no hesitation in stating the principle in a universal form. He wrote to me on July 16, 1909: "In all structures retrogressive variations tend to predominate, and do in the long run predominate over progressive variations. Therefore we get-

(a) Retrogression on relaxation of selection, and more especially on cessation of selection.

(b) Stability of type when selection balances the tendency to retrogression.

(c) Progression when the stringency of selection is sufficient to overcome the tendency to retrogression (reversion)."

He adds that-

"Retrogressive variations tend to be prepotent in a blend, i.e. the retrogressive parent is on the average better represented in the blend."

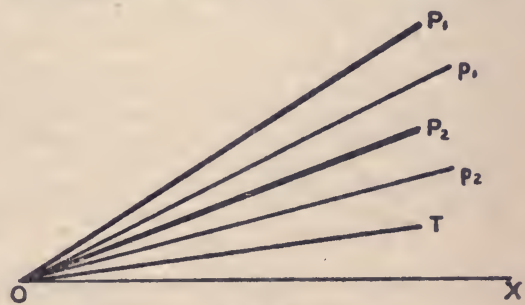

FiG. 25.

57. I am not clear whether this is really an additional effect.

Suppose $\mathrm{OP}_{1}$ and $\mathrm{OP}_{2}$ to represent the parents, $\mathrm{OP}_{2}$ being the retrogressive. Then according to the law of individual retrogression the child 
of $\mathrm{OP}_{1}$ would in any case regress, say to $\mathrm{Op}_{1}$ : and the child of $\mathrm{OP}_{2}$ to $\mathrm{Op}_{2}$. Hence without any additional effect due to blending the child of both parents would tend to be midway between $\mathrm{OP}_{1}$ and $\mathrm{OP}_{2}$, which is nearer to $\mathrm{OP}_{2}$ than to $\mathrm{OP}_{1}$ : so that the retrogressive parent would be better represented in the blend in any case. Is the prepotency intensified by the blending? It is clear that we are now on totally different ground, where we no longer depend on our every day experience, but require the guidance of specialists, who have observed the phenomena more closely.

58. This essay then comes to a natural termination. But if the reasoning is correct, we have been able to show that we can deduce from well known laws, combined with the principle of natural selection.

(I) The value of sex in restricting deviation from type.

(2) The tendency to run through early development more and more quickly, thus compressing the past history and leaving time for adding to it.

(3) The cessation of growth, or comparative slowness of growth, on reaching maturity.

(4) The probable existence of an inherent tendency to retrogression, other than the ancestral.

It follows then that such facts as (2) and (3), which we can independently verify from wide experience, are independent verifications of the wide working of natural selection. 


\section{GLOSSARY}

ACQUIRED, acquirement; terms intended by biologists to indicate alterations in the individual caused by the action of environment on his mind and body. Since, however, all characters arise as a reaction to the environment the terms are misleading. They really designate traits that arise under the stimulus of use and injury.

AllELOMORPH; an 'alternative' unit, believed by the followers of the Mendelian hypothesis to exist in the germ-plasm, which is supposed to be compounded of such units.

BIOMETRY ; the measurement and statistical treatment of biological phenomena.

BI-PARENTAL; see cell.

CELL; a living entity, usually microscopical, typically possessing a cell-wall, with living protoplasmic contents (the cytoplasm) in which is embedded a nucleus. Within the nucleus is a substance termed chromatin, which is differentiated from the rest of the nucleo-plasm. Cells multiply by self-division, the mother-cell constricting itself and dividing into two daughter-cells. Before division occurs the chromatin of the nucleus usually gathers itself into masses termed chromosomes, the number of which is definite for each species. On the nucleus depends, apparently, the nature of the cell, and therefore the nature of the individual that develops from the germ-cell. Consequently, it is probable that the nucleus contains all or most of the germ-plasm, the substance which is the 'bearer of heredity,' which carries the hereditary tendencies or developmental potentialities. The germ-plasm has been identified with some show of reason with the chromatin. Unicellular animals and plants consist each of a single cell, the daughter-cells of which separate and become distinct individuals. Each cell therefore performs all the functions of life. On the other hand, a multicellular organism is compounded of two or more cells. It takes origin in a single cell (which is usually a fertilized ovum). The daughter-cells in the successive generations do not separate. Consequently the individual may consist of billions of cells, all of which are differentiated to perform special functions-e.g. locomotion, secretion, reproduction of new individuals (cell-communities). The cells of the multicellular organism which are immediately concerned with the reproduction of new individuals are termed germ-cells. All the others (e.g. muscle, skin, and nerve cells) are termed somatic cells, and together constitute the soma, the main mass of the body of the individual. Since unicellular organisms are, in effect, germ-cells, they have, of course, no soma. The germ-cells of multicellular organisms are termed sperms (spermatozoa) and ova. In the case of plants they are termed pollen-grains and ovules respectively. Sperms are derived from the male, ova from the female reproductive organs. A fertilized ovum is formed by the union of a sperm with an ovum. Apparently this act of conjugation consists essentially in a union of nuclei which involves a more or less intimate union of the contained chromatin. In some species each individual is an hermaphrodite; that is, it possesses both male and female organs and produces both sperms and ova. In the great majority of species, including of course all those types the individuals of which are male or female and produce only sperms or only ova, the germ-cells of the individual conjugate only with germ-cells from another individual. The reproduction is then bi-parental. In some species, however, there is self-fertilization; that is the sperms and ova of the same hermaphrodite conjugate. Some species produce only ova, which, without being fertilized, develop into individuals. This mode of reproduction is termed parthenogenesis. Between the germ-cell, fertilized or not, whence a multicellular individual is derived and the germcells contained in his (or her) own body, lie generations of cells, the ancestors of the germcells, which, like the cells of the soma, do not conjugate with other cells. These are the generations of the germ-tract. When reproduction is by means of germ-cells it is termed sexual; when it is by means of slips, buds, suckers, and the like, it is a-sexual.

Cell-community; see cell.

CHARACTER ; any trait of an individual-e.g. his head, a hair, the colour of a hair.

Chromatin ; see cell.

Chromosome; see cell.

Conjugation; see cell.

Cytoplasm; see cell. 
DARWINIAN ; a term sometimes used to designate that school of evolutionists which denies the transmission of acquirements, and sometimes to designate the school which supposes that evolution is founded, not on mutations, but on fluctuations.

DAUGHTER-CELL; see cell.

DEDUCTION ; the chain of reasoning, the inverse process to induction, by means of which we pass from a consideration of general notions to a consideration of particular facts. It is most useful when employed to test the correctness of inductions and bring within their range facts not previously included.

DEVELOPMENT; the growth or unfolding of the individual from the germ.

EMBRYo; the individual in his earliest stages of growth after development from the ovum.

ENDEMIC disease; strictly speaking a local malady which has no tendency to spread. Usually, however, the term implies the continuous presence of a disease in a locality.

ENZYME; a ferment secreted by living cells.

EPIDEMIC; a term applied to a considerable outburst or increase of a disease.

Evolution; an adaptive change undergone by a race.

EXCRETION; a term applied both to the act of eliminating waste or used up material from the organism and to the waste material itself.

Fertilized ovum; see cell.

Fluctuation; an unstable variation.

FcETUS; the individual in that stage of development that intervenes between the embryonic condition and birth.

GAMETE; a germ-cell before conjugation.

GERM-CELL ; see cell.

GERM-PLASM; see cell.

GERM-TRACT; see cell.

GERMINAL ; pertaining to the germ-cell, or having origin in the germ-plasm.

HÆMOPHILIA; a disease or state in which the blood lacks coagulating power.

HEREDITY; the organic relation between progenitors and descendants.

HEREDITARY tendencies; see cell.

HERMAPHRODITE; see cell.

HYBRID; an offspring or descendant derived from the union of individuals belonging to distant species.

HYPOTHESIS; an inference not yet proved to be true.

INBORN or innate; a term employed to designate characters which are supposed to differ from acquirements in that they are especially rooted in the constitution of the individual. Really it designates traits that develop under stimuli other than use and injury.

INDIVIDUAL; see cell.

INDUCTION; the process of thought by means of which we found general notions on a consideration of particular facts. Thus, having observed that offspring recapitulate the parental development, we reach through induction the notion that they recapitulate the life-history of the race.

INHERITABLE; a term used to designate characters that arise under a stimulus other than use or injury. Really no characters are inheritable, or all are equally inheritable.

LAMARCKIAN doctrine; the doctrine that offspring tend to reproduce miraculously under the stimulus of nutriment traits which evolution fitted progenitors to acquire under the stimulus of use or injury.

LARVA; an insect in that stage of development which precedes the final stage.

LAW, natural ; an established theory; a description of a uniformity in the sequence of events the actual existence of which has been proved.

LIFE-HISTORY; the stages of the evolution of the race.

MATERNAL impression; a term having origin in the popular belief that maternal mental impressions are apt to produce analogous physical peculiarities in the child. 
MENDELISM ; a biological doctrine founded by Mendel.

MICROBES ; unicellular animals and plants which cause infectious disease in higher types.

MODIFICATION ; a term commonly used to designate a character which has resulted from use or injury; an acquirement.

MONGREL ; the offspring or descendant of parents that were derived from distant varieties.

MONSTER; an individual that diverges very greatly from the type of his race.

MOTHER-CELL; see cell.

MUlticellular; see cell.

Mutation; according to de Vries, a wide departure from the normal type, a 'sport'; according to Punnett and others, a stable variation.

Mutation hypothesis; the doctrine that evolution is founded on mutations.

NUCLEUS; see cell.

NUCLEO-PLASM; see cell.

OVUM; see cell.

Ovule; see cell.

PANDEMIC; a very wide-spread epidemic.

PARASITE; an organism that obtains its nutriment from the tissues of living beings.

PARThenogenesis; see cell.

PATHOGENIC; disease-producing.

PLACENTA; the organ which connects a mother with her unborn child.

Pollen-Grain ; see cell.

Progression; an increase, due to variation, of one or more of the qualities of a character -e.g. size, complexity, power.

Protoplasm; the physical basis of life.

RECAPITULATION; the recapitulation (always with variations) by the individual of the parental development and the racial life-history.

REGRESSION; the tendency displayed by the progenitors or descendants of exceptional individuals to be nearer the racial mean.

RETROGRESSION ; a decrease, due to variation, of one or more of the qualities of a character.

REVERSION; a return to the ancestral type.

SAPROPHYTE; an organism that obtains its nutriment from non-living matter.

SELECTION; is of several kinds; artificial, when man consciously chooses the progenitors of a race; natural, when unconscious nature exercises the choice; reversed, when the selection "affects not increase of an organ, but decrease of it"; germinal, when (as has been supposed) the selection occurs among the units of the germ-plasm.

SeLEctionist; an adherent of the doctrine that evolution is founded on the selection of fluctuations.

Self-FERTILization; see cell.

SeXuAL; see cell

SOMA ; see cell.

Somatic; pertaining to the soma.

SPERM or spermatozoa; see cell.

SPONTANEOUS; a term used to designate alterations in the germ-plasm caused, not by external, but by internal conditions.

TELEGONY; a term having origin in the popular belief that if a mother has offspring by two mates, the children of the second father will exhibit the influence of the first.

THEORY; an inference or hypothesis which has been proved to be true.

Toxin ; a poison, offensive or defensive in function, secreted by a living being. 
UMBILICAL cord; the structure which passes from the navel of the child to the placenta. UNICELLULAR; see cell.

UTERUS; the womb.

VARIABILITY; the tendency to vary.

VARIATION ; a filial departure, founded on a germinal change, from the parental type.

WASTE-PRODUCTS; the final products of tissue metabolism. They are excreted by living beings, and are comparable to the ash, carbonic acid, water, etc., which result from the combustion of coal.

ZYGoTE; the fertilized ovum. 


\section{INDEX}

A

ABILITY, biometric investigation of, 136-7, 428 et seq. ; nature of, 42 I-2

Abnormalities, 22

Aborigines, decay of, 279

Abrin, toleration of, 247

Abstinence from Alcohol, ancient, 459

Acclimatization, meaning of term, 263

Acquired, right use of term, 20

Acquirements, real nature of, I4 et seq. : importance of, II et seq., 245, 424, 485 ; superiority to inborn traits, 69 et seq., $40 \mathrm{O}$, 424 ; mental, 379, 4 or

Actions, protoplasmic, $37 \mathrm{I}$; reflex, $37 \mathrm{I}$; instinctive, 373; rational, 373 et seq .; voluntary, 373 et seq. ; deliberate, 376; automatic, 400

Adaptation, universality of, $21,56-7$, 106, $182,187,206,219,236,324-5,373,390-2$, 396 ; of microbes, 238 ; of the individual, 57 ; test of, 222 ; mental, 324, 388 et seq.

Adaptability of man, 38I, 428

Africa, West, malaria in, 234-5; alcohol in, 299, 302 et seq.

Alcohol, a waste product, 243; effect on health, 286-7, $3^{17}$ et seq.; immediate and remote effects, 306-7; craving for, 288 et seq., 457 ; variations in craving for, 289 et seq. ; influence of environment on consumption, 292-3; as agent of natural selection, 294 et seq. ; artificial selection for, $457-8$; evolution caused by, 297, 308 et seq., 458 ; compared to tuberculosis, 308 ; civilization, $3 \mathbf{I} 3$; climate, 3 II -2 ; occupation, 3I 4 et seq. ; strength of beverages, $3 \mathrm{I}_{3}$; ancient use of, 299 et seq. ; laws prohibiting use of, 300, 306, $45^{8}$ et seq.; average racial consumption of, 3 II ; convivial and industrial drinking, $3^{\mathrm{I}} 4$ et seq. ; susceptibility to charm of, $289,293-4$; susceptibility not a disease, 457 ; alleged cause of racial deterioration, 322 ; antiquity of drinking, 299 ; drinking of Jews, 299, 301-2 ; Greeks, 300 ; Italians, 301 ; West Africans, 302-3; Germans, 304-5; British, 305 ; Chinese, $305-6$; savages, 306 ; Mohammedans, 460

Alcoholism, and disease, 457 ; chronic, $3^{18}$; altruistic, 318 et seq.; impossibility of preventing, 457,465

Alison, on drinking of savages, 306

Allelomorphs, I55; compound, $16 \mathrm{r}$; nonexistence of, 188

Aiternation, of generations, 23 ; of progression and retrogression, 25 ; of sexual traits, I9o

Alternative reproduction, conditions of, $\mathbf{5} 78$

Anatomy, systematic, 33, 504

Ancestral Inheritance, Law of, 126 et seq.

- resemblances, 523
Andalusian Fowls, I55

Ani, maxims of, 300

Animals, lower, minds of, $380,398,415,425$; dreams of, $4 \mathrm{I} 8$; social, 382

Ant, mental operations of, 382-3; brains of 405

Anthrax, lethal dose of for rabbit, 86 ; attenuation of, 240

Antitoxin, preparation of, 240 ; mixed with toxin, 24I, 243-4

Aphides, latency of male characters, II3; variability of, $\mathrm{I}_{4} 8$; cause of disease in vines, 276

Aptitudes, mental, 422

Areopagus, laws against drinking, 300

Aristophanes, on drinking, 300

Arsenic, toleration of, 247

Astronomy, scientific method in, 503

Athens, great men in, 496

Attention, area of, $4 \mathrm{I2}^{-3}$; concentration and diffusion of, 4I3-4; association with the will, 4I4

Attractions, sexual, 145, 189

Australia, temperance legislation in, 462, 464

Automatic actions, 400 et seq. ; compared to reflexes, 4IO-I ; characteristics of, 4II-I2

Automaton hypothesis, 356 et se. 9

Axioms, mathematical, 346

B

BACON, Francis, on the power of words, 19-20; service to science, 50 ; on knowledge by causes, 343

Bacot, Mr A., on repertoire patterns, 60

Baldwin, Prof. Mark, on use-acquirements, 72 ; on plasticity, 408

Bantam, Sebright, 146, 165, 189

Baring, Mr Maurice, on drinking in Russia, 320

Bateson, Mr W., on dandelions and hawkweeds, 148 ; on segregation, 153,154 ; on central doctrine of Mendelism, I54; on imperfect dominance, I55; on compound allelomorphs, $16 \mathrm{r}$; on discontinuous origin of Mendelian characters, 162 ; on ferments, I64; on limitations experimentation, I72; on scientific method, $184-5$; on fertility and Mendelian inheritance, 190 ; on Mendelian inheritance of sexual characters, I9I ; on inheritance of stature, 193; on systematists, 506 ; on origin of species, 506

Bathmic hypothesis, 60

Beauty, appreciation of, 423-4

Bees, latency of male characters, $\mathrm{II}_{3}$; diseases of, 275

Berberis vulgaris, repertoire patterns of, 60

Berkeley, on Idealism, 334

Bible, exhortations against intemperance, 299 
Biologists, as essayists, I84-5; contrasted with students of other sciences, 509-10 ; education of, 5 ro et seq.

Biometry, 36, r33 et seq., 226, 26r, 409, 428 et seq., 443, 447, 509; wasted labours of, I36 et seq.

Black Death, the, 27I-2

Blending, abnormal, of sexual traits, I46 ; normal, r95; of Mendelian traits I60-1, 196; of crossed human races, I73-4; of fluctuations, 189; meaning of term, r94-5; the only exception to, I95; of mutations, I96 ; effects of, I97

Bosanquet, Dr W. C., on immunity, $23^{8}$; on virulence, 238

Botany, systematic, 33, 504; method of, 39,504

Brain, relation to mind, 362 et seq. ; evolution of, 396-7; relation of size to intelligence, 434-5

Branthwaite, Dr, on alcoholism and feeblemindedness, 3 I6

British, the, intemperance of, 305 ; alleged mental deterioration of, 430

British Medical Association, investigation on alcoholism, 295-6

Brown-Séquard, on transmission of acquirements, 73

Bumpus, on Natural Selection, 136

Bushmen of New Zealand, drinking habits of, 288

C

CANADA, temperance legislation in, 462

Canaries, varietal crossings of, 156

Cancer, 103

Capacity, for growth, 6 ; mental, 421 et seq. ; variations in, 425 ; definition of, $43^{8}$

Carnivora, immunity to septic poisoning, 89

Casas, las, Bishop, philanthropic efforts, 280

Castle, Prof. W. E., on dominance, $\mathrm{I} 57$; on gametic purity, I6I; on mutation hypothesis, r7o; on Mendelian inheritance of sexual traits, rgr

Caterpillar, development of, 120; instincts of, 376-7, 379; lack of memory, 379, 422 ; mental growth of, 42 I

Catholics, Roman, mental traits of, 427,495

Causation, chain of, $47-8,349$; circle of, $35^{\circ}$

Cause and effect, 343,350

Cells, in uni- and multicellular organisms, I ; multiplication of, $\mathbf{r}$; daughter-, $\mathbf{I}$; specialization of, I; contractility of, and mind, 368-70

Cell-community, $\mathbf{x}$

Cephalic index, 429

Cerebrum, association with memory, 396

Chapters in development, missing, 27

Characters, relation to germ-plasm, 8 ; inborn and acquired, I5-16; changes during evolution, Ir8; non-adaptive, 2I9, 39I-2

Chauveau, Prof., on immunity, 243

Chemistry, study of compared with that of biology, 3I, 34, 504

Chicken-pox, deaths due to, 260; compared to tobacco, 308

Child, mental peculiarities of, 394 ; education of, 480 ; effects of religious training on, 487
Chinese, drunkenness among, 306 ; brains of, 435

Chloroform, craving for, 289

Chromatin, as bearer of heredity, 3

Chromosomes, numbers of, I3I

Civilization, effects of, 222 et seq.; and disease, 269 et seq., 278 ; and alcohol, 298 ; and prohibition, 459 et seq.

Civilized man, distinction from savage, 397

Classical, teaching, $50 \mathrm{I}$

Classification of facts, 36 et seq.; of feelings, 330-I

Clayton, experiments on beans, 84, 97

Clifford, on science, 37 ; on idealism, 335 ; on relation of mind to body, 357

Climates, healthy and unhealthy, 263; and intemperance, 3 II

Cocaine, use as substitute for alcohol, 463

Cochin fowl, 155

Cockburn, Sir J., on intelligence of Australian Blacks, 469

Coherence of thought, 329 et seq., 340

Colour-blindness, inheritance of, 189

Columbus, voyage of, 278

Committee of Fifty, investigation by, $46_{3}$

Common sense, definition of, 324

Conception, mental, 326

Confidence trick in science, 5 ro

Conjugation, not a necessary antecedent of reproduction, 2, I42 ; effects of, I44, I88; function of, 202

Contributions, ancestral, I26 et seq.

Controversy, utility of, 45

Cope, Prof., on evolution, II

Correlation, 70-r, 182, 26r ; between size of brain and mental capacity, 434

Cowpox, 238

Crabs, changes of sex, II4; blind, r23; lack of memory in, 407

Crawley, Rev. A. G., on religion of savages, 488

Crichton-Browne, Sir J., on mental defects, 468

Crime, causation of, 494

Criteria, for facts and thinking, $32,505^{-6}, 5^{12}$

Crucial instances, 165

Cuba, imported disease, 280

Cuénot, Prof., theory of factors and determiners, I64, I66

Curiosity, instinct of, 385 ; in education, $478-9$ Cypris reptans, stability of, $20 \mathrm{I}$

D

DANDELIONS, parthenogenesis in, $\mathbf{r}_{4} 8$

Darbishire, Mr A. D., experiments on mice, I63

Darien, British colony in, 235

Dark Ages, diseases in, 269; mental training in, 497

Darwin, Charles, hypothesis of pangenesis, 5,66 ; belief in the transmission of acquirements, 6I ; on retrogression, II2; on latency, II3-4, I65, I77; belief in blended inheritance, 150 ; on reversion in pure-bred races, $165-6$; on artificial selection, 176 ; method of work, I85, 2I4-5; on effects of crossing, I98; use of deduction by, $212-3$, 346 ; his teaching, 212, 39r, 505; on memory, 405 ; the Newton of biology, 506 
Darwin, Mr Francis, on the transmission of aoquirements, 74

Daughter-cells, I

Davenport, Prof. C. B., on Mendelian traits of poultry, $\mathrm{I}_{55}, \mathrm{I}_{72}$; on blending of Mendelian traits, I60-I

Deaf-mutism, inheritance of, 189

Death certificates, 295

Deduction, nature of, 42-3; function of, 46 , $5^{1-2}$; medical condemnation of, $30,21 \mathrm{I}$, 509 ; in physics, $47,49,211,214-5,503$; in mathematics, 47, 2II, 344, 355, 503, 505, 509 ; in biology, 49-50, 345, 509; Darwin's use of, 212-3; when necessary, 509

Deductions, from fact of immunity, $257-8$

Dendy, Miss, on fertility of the feeble-minded, 475

Desire, emotion of, $373-4$

Deterioration, physical, 446 et seq.; cure of, 449

Determinants, 109

Determiners, 164

Development, method of, 23; of human beings, 26-6; periods of slow and rapid, I 20-I ; independent, I54, I60.

Devotion, to truth, $4 \mathrm{I}$; to beliefs, 488

Devotional faculty, 325

Dexterities, physical and mental, 4I9

Diets, human, 440

Digitalis, hybrids of, ${ }_{57}$

Dimorphism, sexual, 142, 144, 188-9

Diphtheria, antitoxic treatment of, 240,248 ; effects of sanitation on, 450

Discrimination, 377

Diseases, regarded as experiments, 39-40, $216,26 \mathrm{I}$; evidence furnished by, against mutation hypothesis, I99; in England, 224; selection by, 225; list of microbic, 227; frequency of, 227; air-borne, 228, 239, 272, 278, 45I-3; contagious, 229, 270, 450-I ; earth-borne, 229, 274-5, 453; insect-borne, 229, 27I-2, 45I ; water-borne, 228, 274, 45I ; general, 230, 239 ; cause of duration, $233-4$; spread of, 236 ; epidemic and endemic, $257,275,277,279,452$; as material for study of beredity, $26 \mathrm{I}$; resistance of human races to, 263 , of lower animals, 275-6; evolution of, 266 et seq. ; extinction of, 268 ; places of origin, 269 ; migration of, 270-I ; antiquity of, 269,275 ; conditions of prevalence, 275 ; invasion of Western Hemisphere, 278 et seq.; as empirebuilders, 28I et seq.

Disuse, as criterion of nature of growth, 7 ; alleged as cause of retrogression, I08

Dogs, deterioration in India, 84,98 ; distemper of, 275 ; crossing of large and small breeds, 198

Dominance, $\mathrm{I}_{53}$; imperfect, $\mathrm{I}_{55}$; of recessive characters, 155 ; influenced by sex, 156 ; by environment, 156 ; by race, 157 ; by idiosyncrasy, $\mathrm{x} 57$; a phenomenon of sexual reproduction, 192

Dominants, pure, impure, and extracted, $\mathbf{r}_{53}$, I 58

Donkin, Dr H. B., on cirrhosis of liver, 295

Dow, General Neal, on prohibition in Maine, 463-4

Dreams, $4^{16} 6$ et seq.

Drelincourt, on speculations about sex, 199

Drift racial, caused by environment, 92
Drinkers, the three classes of, 285,286

Dyer, Sir W. Thiselton, on latency in a natural species, 176 ; method of work, 185

\section{$\mathrm{E}$}

EARTH, shape of, 489 et seq.

Education, and alcohol, 312 ; importance of, 477 ; in early life, $478-9$; in school-room, $479-80$; of young adults, $48 \mathrm{r}$ et seq.

Educability, 398, 428

Efficiency, 422

Egypt, disease in, 269; intemperance in, 300 , 459

Ehrlich, hypothesis of immunity, $25 \mathrm{x}$ et seq.

Embryo, prototypes of, 27; adaptations of, II9

Emotions, acquired, 410

Enumeration, simple, 54, 342-3, 509

Epidemic and endemic diseases, 272, 452

Error, sources of, 36

Esquimaux, effects of vaccination on, 239

Essayists in biology $184-5$

Europe, use of alcohol in, 299

Evidence, when admissible, 35 ; neglect of, 59,136

Evolution, universal belief in, 4 ; recapitulation of, 24 ; theories of, $59-62,258$; of toxins, 88 et seq.; of human races, 180 ; against disease, 224-5; against alcohol, 297

Ewart, Prof. J. Cossar, on causation of variations, $8 \mathrm{I}-2,85$; on retrogression, $\mathrm{II}_{2}$; on horse-zebra hybrid, $x_{5} 6$

Exact methods of inquiry, 34 et seq. ; sciences, 36,211

Exclusive inheritance, $\mathrm{I}_{5} \mathrm{I}$

Exodus, book of, on leprosy, 269

Experience, stored in mind, 377

Experiment, object of, 33 ; two ways of using, 43 ; scope in biology, 45, 50, 5I, 510; failure of, 44-5, 8I-5, 87-9I, 98-9, I7 I- 3 , I 84 , 204-5, 22I, 264, 409; as cause of controversy, 45

Experimental, sciences, 36, 502 ; evidence in favour of Lamarckian doctrine, 73 ; school, 3O-I, 184

Explanation, nature of, 349

Extracted dominants and recessives, 158

Eye-colour, reproduction of, $145-6,149,174$, I78, 428 et seq.

Eyes, retrogression of, 123,223 ; protected and unprotected, $4 \mathrm{r} 8$

$\mathrm{F}$

FACTS, admissible, 32 ; patent and obscured, 33 ; scientific value of, 38,480 ; teaching of, 480

Factors, colour-, I64

Faith, 488

Family life, 380

Fashion, influence of, 204, 292, 386

Feeble-mindedness, 425, 465 et seq.

Female characters, reproduced by male, Ix 4

Féré, on alcoholized eggs, 264

Fertility, biometric investigation of, $137-8$, 430-1; evolution of, $137-8$; of the insane, 475

Fertilization, not essential condition of reproduction, 2, 142 
Filial regression, law of, I34

Fluctuations, 150,506 ; blending of, 176 ; as causes of racial change, $179,183-4$

Food-supply, 269; in manufacture of alcohol, 298

Fovea centralis of eye, $4^{\mathrm{I} 2}$; of attention, $4 \mathrm{x}_{3}$

Fowler, I., on knowledge by causes, 54

Fowls, Mendelian traits of, $1_{55}, 1_{56}, 1_{57}, 1_{60}$, $\mathrm{r} 65, \mathrm{r} 67, \mathrm{r} 72$

Frog, instincts of, $38 \mathrm{r}$

Froude, on disease in West Indies, 280

Fry, Sir J., on increase of insanity, 47I-2

Function, of cells, I; of sex, 197; definition of term, 188

\section{G}

Galton, Sir F., an Law of Ancestral Inheritance, 126 et seq. ; on kinds of inheritance, I5I

Gamete, I 53

Gametic purity, 157

Garden plants, variability of, 100

Gärtner, on latent characters, 176

Genetics, new science of, 188

Geniality, 429

Genius, 426

Geology, systematic, 33

Geometry, 343, 347, 480, 503

Germans, ancient drinking customs, 304-5

Germ-cells, 2; potential immortality of, 5 ; cause of numbers of, 96 ; alleged inviolability of, 264

Germ-plasm, nature of, 3 ; continuity of, 5 , I24, I34; quantitative and qualitative division of, $17-8$; necessity of thinking in terms of, $\mathrm{r}_{5}, 25$; causation of variations in, 80 et seq. ; resistance to direct action of environment, 92 et seq. ; as living entity, 98

Germinal selection, rog; changes, 221-2

Gibbon, on drinking customs of Ancient Germans, 304

Gilbey, Sir A., on race-horses, III

Gordon, Dr H. Laing, on drinking in Italy, 301

Gout, transmission of, 74

Gravitation, law of, $47,352,360$

Greece, drinking in, 300 ; teaching in, 490-I

Growth, factors of, $6,422,43^{8}$; of mind and body compared, 420,477

Growth-force, 60

Guinea-pigs, susceptibility to tuberculosis, 255

Gulick, Rev. J. T., on snails, 202

\section{$\mathrm{H}$}

HABITS, 40I et seq. ; 483, 515

Habituation to toxins, 247,253

Hæmophilia, alleged transmission of, 75

Hair-colour, 145,428

Hair-texture, I74-5

Halteridium, 8I

Hapsburgh lip, r8r

Hare, Dr F., on deduction, 30

Hartog, Prof. M., on transmission of acquirements, 74

Harveian Society, on alcoholism, 295

Hawkweeds, parthenogenesis in, 148

Heart's-ease, evolution of pansy from, II2
Henslow, Prof. G., on transmission of acquirements, 74

Hereditary tendencies, definition of, 3 ; alteration of, 79

Heredity, material for study of, 34 ; not an exact science, $35^{-6}$; experimental study of, 34 et seq., 184, 510 ; greater problems of, 216-7

Hermaphrodite, 143,146 ; human, 146

Herschell, on science, 37

Hewitt, on variations in ducks, 83 ; on reversion in bantams, 165

Hinny, 157

Hipparion, hoofs of, 123

Hippuris, 60

History, life-, 24

Historians, on disease, 276-7

Hodgson, Dr, on Idealism, 336

Hoffmann, experiments on plants, 83

Homer, on drinking, 300

Horse, retrogression in, 123; American trotting, 179 ; latent traits in, 196

Human beings, mutations of, 175 , 179; racial differentiation, I80-I ; racial crossings, I73 et seq., 200-I ; as subjects for study, 216, 220-I ; errors about racial changes of, 223 ; lack of instinctive movements, 378-9 ; distinguishing peculiarity, 424; adaptability, $38 \mathrm{r}, 428$; ways of improving, 438-9; artificial selection of, 439

Hume, on mind, 335

Hunger, instinct of, 384

Hunter, Dr W., on maternal impressions, 75

Hurst, Mr C. C., experiments on poultry, ${ }_{5} 6$; on rabbits, 163

Huxley, method of work, 185 ; on necessary truth, 349 ; on science, $35 \mathrm{I}$; on relation of mind to body, $356-7$; on curates, 478

Huxley lecture, 428

Hybridization, almost confined to artificial varieties, 176

\section{Hydroida, 27}

Hypotheses, 39-40 ; untested, 49-50 ; of chemists and physicists, 50 ; bathmic, 60 ; nature of, $5^{12}$

\section{I}

IDEALISM, definition of, 324 ; thorough going, 333 ; futility of, $334,336,338,360$

Idiot, mind of, $389,425,465$ et seq.

Imagination, the scientific, 52, 89

Imbecility, 53 ; as variation, 425 ; nature, 425 , 465 ; in slums, 447 ; causation, $472-3$; prevention, 475

Imitativeness, 385

Immunity, inborn and acquired, 233, 235, 253, 254,257 ; conditions of, 237,248 ; active and passive, 24I; Pasteur's hypothesis 243; Cheauveau's, 243; Behring's, 243 et seq.; Ehrlich's, $25 \mathrm{I}$ et seq.; in acute and chronic disease, 257; inference of consequences from, 258-9; evolution of, 225; meaning of term, 309; artificially developed or evolved, $450,453,455$

Impure dominants, 153

Inborn and acquired characters, true distinction between, I6-7; general failure to distinguish between, 439-40 
Individual, derivation from ovum, 5.6

Induction, nature of, 42

Inertia, mental, 493

Insanity, the two kinds, 465 ; increase of, $47 x$; causation of, $472-3$

Instinct, definition of, 374 ; human, 374-5, 383; imitated by acquirements, $400-\mathbf{x}$, $4 \mathrm{IO}$; extended by acquirements, 422 ; distinguished from acquirements, 423 ; modified by acquirements, $423-4$; for food, 440 ; to think, 403,478

Intelligence, and fertility, $13^{8}, 47^{8}$; defined, 377 i measure of, 378 ; distinguished from reason, 378 ; substituted for instinct, 380 et seq.; adaptiveness of, 398,478 ; Pearson on, 431 ; relation to size of brain, 434 ; of adult compared with that of child, $49 \mathrm{I}$

Isolation, physiological, $143,178,18 \mathrm{I}$

Italy, drinking in, 300-I

\section{J}

JAMES, Prof. W., on science, $35 x, 360$; on Idealism, 336 ; on separateness of mind and body, $36 \mathrm{I}$; on memory, 395

Japan, social and intellectual changes in, 445,497 ; tuberculosis in, 454

Jealousy, instinct of, 424

Jennings, Mr H. S., on paramœcium, 94

Jevons, on framing of hypotheses, $42 ;$ on the Newtonian method, 52

Jews, ancient intemperance of, 299 ; modern sobriety, 30I ; mental peculiarities of, 428 ; effects of urban life on, 448

\section{$\mathrm{K}$}

KANT, on mind, 335

Kemp, Rev. D., on drinking in West Africa, 3०3-4

Kepler, on planetary motion, 43,48

Kindergarten system, $48 \mathrm{x}$

Kingsley, Miss M., on malaria, 235; on drinking in West Africa, 303

Knowledge, sources of, $33^{8}$; traditional, $38 \mathrm{I}$; useful, 478

\section{L}

LABOUR, 479; laborious mental training, 479 Laboratory methods, when useful, 33,35 ; scope in physics and biology, 49

Lake dwellers, use of alcohol by, 299

Lamarckian hypothesis of evolution, II, $6 \mathrm{r}$, 62,76 ; controversy, $15-6$; strongest evidence against, 73 ; applied to disease, 259

Landois and Stirling, on ciliary action, 369

Lankester, Sir E. Ray, on retrogression, 22 ; method of work, 185; on alcohol, 297 ; on size of brain and intelligence, 434; on imbeciles, $469-70$

Latency, distinguished from retrogression, II 3 , II5 ; temporary, II3 ; permanent, II4 ; of sexual traits, I45, I59, I95; of Mendelian traits, 160 et seq.; of eye-colour, 174 ; Darwin on, 177 ; de Vries on, 177 ; occurs only in artificial varieties, $\mathbf{1 7 6}$
Latent characters, alternation of, $115-6$; common in artificial varieties, $16 \mathbf{r}$ et seq. ; absent in natural varieties, 275 et seq.

Law, natural, definition of, 47,348 ; of Ancestral Inheritance, 126 et seq.; of filial regression, 134; laws of heredity, 213

Laws, against excessive drinking, 300, 305, $306,45^{8}$ et seq., 465

Leprosy, antiquity of, 269

Lewes, idea of evolution, II ; on intelligence, 406

Life insurance and alcohol, 287, 296

Linnæus, scientific method, 5 II

Lobster, lack of memory, 407

Local option, 462,464

Lock, Mr R. H., on experiment, $3 \mathrm{I}$; mulattos, 174 ; on study of human species, 180 ; on claims of Mendelians, 188

Locomotion, power of, effects on number of varieties, 202

Logic, Port Royal, 42

Love, sexual and parental, $3^{86}$

Lubbock, on wasps, 418

Lunacy, 466 et seq., $47 \mathrm{I}$

Lycurgus, punishment of drinkers by, 300

\section{M}

MACAULAY, on scientific method, 42

M'Gregor, Sir W., on drinking in West Africa, 302

Mach, on scientific facts, 47

Macpherson, Dr J., on increase of insanity, $47 x$

Malaria, in pigeons, 8I; an insect-borne disease, 229; duration of, 234 ; racial resistance to, 235, 309; migrations of, 271 ; evolution against, 309

Male characters, alternation with female traits, 145 et seq. ; reproduced by female, II 3,165

Man, crossed races of, 173 et seq. ; neglect of, by biologists, 216,218 ; place of origin, 269 ; helplessness at birth, $38 \mathrm{I}$; differences between savage and civilized, ancient and modern, 397; distinction from lower animals, 404; mental differences between individuals, 426 et seq.

Manipulation, association with brain, 396

Maoris, mental traits of, 398 ; social state, 427 ; and alcohol, 464

Maspero, on intemperance in Ancient Egypt, 300

Maternal, impressions, 75,76 ; love, 378, 386

Mathematics, not an experimental science, 36; method of, 343 et seq., 503, 509, 517

Mathematical knowledge, factors of, 422

Matter, meaning of term, 324, 354; in motion, 362

Mauritius, invasion of malaria, 271

Medical men, and deduction, 30, 509; Royal Commission on, 74 ; belief in Lamarckianism, 75, 255, 443; belief in direct action of environment on germ-plasm, 8I, $84,264,448$; on Jewish mothers, 448 ; evidence before Royal Commissions, 474 ; study of heredity, 74

Medical statistics, $81,84,264$; certificates of death, 295 ; education, 482 


\section{Memories, concentrated, 396}

Memory, importance of, $325,330,33^{8}, 377$, 380 ; defined, $377,379,394,478$; evolution of, $380,394,405$; neglected study of, $389 ;$; superiority in youth, 394 ; systems for improving, 395 ; organ of, 396 ; conscious and unconscious, 399 ; range of, 404 ; variations of, 404,422 ; in crustacea, 407 ; in wasps, 418 ; abolished in dreams, 416 ; bearing in mind, 418 ; distinguished from " memories," 421 ; defective in the feeble-minded, 425,465 ; instinct to play with contents of, 403,478

Mendel, experiments on peas, $\mathbf{I}_{52}$, I $_{5} 8$

Mendelian hypothesis, chief points of, I54I $5^{8}$; exceptions to, I59; central doctrine of, I54; disproof of, I60 et seq. ; proportions, I53, I58, I67; exceptions to proportion, 158 ; inheritance, right interpretation of, $16_{3}, 165,167$; inheritance of mutations, I69; traits of poultry, I72; of peas, I 53 ; sexual and Mendelian traits compared, r89-9I; Mendelian inheritance a human creation, I92; deductive inference of consequences, I93

Mendelism, not tested by experiment, r84; concerned only with problems of sex, I88; and disease, 280; and science, 510

Mental traits, co-adaptation of, $388 \mathrm{et} \mathrm{seq.}$

- training, importance of, 435 ; of child, 40I, 478; of young adults, 48I et seq.

Mercier, Dr C., on transmission of acquirements, 74; on craving for alcohol, 290; on separateness of mind and body, $36 \mathrm{I}$

Method, of development, 22, 24; of science, 42, I 85, 345, 35I, 502 et seq.

Methods, exact, 34, 35, 184; experimental and biometric, 140

Metzger, experiments on maize, 83

Mice, crossed varieties of, 162,163

Microbes, variations of, 87 et seq. ; cause of disease, 227 ; evolution of, 88,266 ; our power of banishing, 450

Microbic diseases, effects of sanitation, 450

Middle Ages, physicians of, 269 ; social state of, 497

Migrations of negroes, 265 ; of human races, I8I, 270; of disease, 270 et seq.

Mill, John Stuart, on deductive method, 48, $2 \mathrm{I}_{3}-5,502,5 \mathrm{I} 4$; on the statistical method, 140 ; on matter, 335 ; on knowledge, 343 ; on the use of hypotheses, 512 ; on controversy, 512-3

Millais, Sir E., on breeding of Basset hounds, I26

Millardet, on false hybrids, I59

Mind, uniqueness of, 325 ; alleged supernatural origin of, 325 ; not comparable with matter, 327 et seq. ; growth of in infant, 329 et seq. ; relation to body, 362 ; the work of the brain, 362 ; relation to expenditure of energy, $363-4$; evolution of, 364 ; a product of evolution, 369 ; but not a direct product, 365 ; association with nervous tissue, 367 ; utility of, 367 ; beginnings of, $368-9,378,393$; a product of Natural Selection, 368; alleged universal presence of, 368 ; order of evolution, $370,387,393$, 409 ; of man, 374 et seq., 377, 426, 444 et seq.; bearing in mind, 418 ; development of, 4I9; growth compared with that of body, 420, 426; effects of training on, 444

Minds, of other people, 333 et seq.; closed and open, 492 et seg.

Miracle, 59, 65, 90, 325

Missionaries and disease, 280; and alcohol, 302 et seq.

Mitchell, Sir A., on dreams, 417

Modesty, 423

Modifications, I5, 2I ; distinguished from variations, 79

Mohammedans and alcohol, 460

Monks, cranial capacity of, 435

Morality, 423 ; effects on of temperance legislation, 462 ; of imbeciles, 466

Morgan, Prof. Lloyd, on use acquirements, 72 ; on plasticity, 408

Prof. T. H., on experimentation, 30-I ; on latency of Mendelian traits, I60; on evolution and adaptation, 391

Morocco, social state of, 497

Morrison, Rev. W. D., on crime, 495

Mosaic inheritance, $\mathbf{I} 55$

Mothers, Jewish, 448

Mulatto, skin colour, I74

Mule, I 57

Multicellular organisms, I

Murray, David Christie, on crime; 494

Mutilations, alleged transition of, 75

Mutation, meaning of term, I70

Mutations, 22, I5I ; alleged stability of, I50, r69-70, I7I, I79, r83; human, I75, I79, $\mathbf{I 8 I}$; in wild nature, I79; of 0 . Lamarckiana, I79; inutility of, I79; reproduced in the mode of sexual traits, 189 et seq.; blending of, I95-6 ; retrogression of, I7 I, I96

Mutation hypothesis, I50, I69; grounds for maintaining, 175-6; grounds for rejecting, I8r et seq., I99; not tested by experiment, I84; inference of consequences from, r94; and reflexes, 373 ; and mind, 388 ; and palæontology, 392

Mutationists and biometricians, $\mathbf{I}_{50}$

\section{$\mathrm{N}$}

NAMES, importance of, 18

Narcissus, from cross between jonquil and daffodil, ${ }_{5} 6$

Narcotics, immediate and remote effects, 306 Natural Selection, theory, contrasted with Lamarckian hypothesis, 66 et seq. ; objections to, 107, 185, 391

Natural varieties, blending of when crossed, I76; lack of latent traits, I76

Nature and Nurture, 421, 429

Naudin, on crossed artificial varieties, I77

Necessary truth, $47,346,349$

Negro, teeth of, 224 ; and malaria, 234 ; and alcohol, 302 et seq. ; migrations of, 265

Nervous tissue, association with mind, 367 ; evolution of, 370

Neutralization of toxins, hypothesis of, 243 et seq.

New Zealand, aborigines of, 427 ; temperance legislation in, 464

Newton, on orbit of moon, 43 ; scientific method, 52 ; on gravitation, 352 ; compared with Darwin, 505-6 
Nicotine, a toxin, 243, 307

Nucleus, of cell, the bearer of heredity, 3

Nutriment, as material for growth, 6 ; as stimulus, 7; and inborn traits, I4-r6; alleged cause of variations, 99

\section{O}

OBSCURED facts, 33

Offspring, relation to parent, 5

Old age, mind in, 421

Opium, a toxin, 243, 307 ; toleration of, 247 ; racial susceptibility to, 306-7; evolution against, 309 ; compared to malaria, 308 ; substituted for alcohol, 460,462

Organisms, unicellular and multicellular, I ; primitive, 9

Orthodoxy, in religion, mental influence of, 492 et seq.

Orton, Mr J. H., on hermaphroditism, II4

Osborn, Prof. H. F., on acquirements, 72 ; method of work, I85; on plasticity, 408

\section{$\mathrm{P}$}

PAGANS, ancient, social state and religion, 427 ; mental training of, 490,500

Palæeontology, 392

Palm wine, 299

Pandemics, 272, 275, 452

Pangenesis, hypothesis of, 5, 66

Pansy, evolution of, II 2

Parasites, evolution from saprophytes, 87

Parental affection, $3^{86}, 424$.

Parsimony, law of, $60,100,166$; of nature, 96, I38, I96, 202, 402; how caused, II8 et seq., 197 et seq.

Parthenogenesis, 2, I42; variations occurring in, 147 ; and Mendelian reproduction, 188 ; conditions of occurrence, 204

Parthenogenetic types, stability of, 201

Particulate inheritance, I $5 \mathbf{I}$

Pasteur, on rabies, 240 ; on anthrax, 240 ; on immunity, 243

Patency of sexual traits, I59; of Mendelian traits, 60 et seq.

Pearl, Dr, on paramœcium, 93

Pearson, Prof. Karl; on science, 37 ; on variability, 94, I49; on Filial Regression, r34; on metaphysicians, 335 ; on natural law, $35 \mathrm{I}$; inheritance of mental and moral traits, 428

Peas, Mendel's experiments on, I52 ; Mendelian characters of, I53; sweet, compound characters of, I6I

Perception, 326; limits of, 340

Pestilence, 276

Phagocytes, functions of, 23I-2, 247

Phylloxera, 276

Physics, study of, compared to biology, 30, $34,5 I, 2 I I, 505$; as exact science, 35, 48, 50,345 ; as deductive science, 214,503

Physiological isolation, I43, I78, I8I

Pictet, experiments on butterflies, 83

Pigeons, Ewart's experiments on, 81, 85 ; latent traits in pure breeds, 165

Pittacus, on drunkenness, 300

Plague, human, 271, 276; rabbit, 275
Plants, regeneration in, II; stability when propagated by cuttings, 94 ; retrogression in, 121; reversion in, 156

Play, function of, $384,403,478$

Pleasure in obeying instincts, 479

Pleasure and pain, evolution of, 37 I ; association with the will, 373

Pliny, on drunkenness in ancient Rome, зо

Poisoning, by microbes, 230; by alcohol, 306,456

Polar bodies, I3I

Polish fowls, I46, I89

Population, pressure of, 269

Poultry, Mendelian traits of, $155, \mathbf{I}_{56}, \mathrm{I}_{57}$, 160, 167, 172 ; blending of Mendelian traits, I6o

Predominance of retrogressive variations, Io9, III , I97, 220

Prejudice, defined, 492

Prejudices, biological, 32, 51 I ; religious, 492

Prepotency, meaning of term, I95

rimitive organisms, 9

Primrose, evening, 179

Probity, inheritance of, 429

Problems of heredity, the greater, 217 ; practical, 446

Progression, conditions of, III; of human races, 223

Progressive traits, 22

Prohibition in China, 306, 459; among Mohammedans, 460; in United States, 46I

Properties of objects, 339-34I

Protoplasmic movements, 37 I

Prototypes of embryo, 27, I I9 et seq.

Public health, statistics of, I36, 264, 28I

Punnett, Mr R. C., on stability of mutations, 169-170

Pure dominants, I53, I57 ; recessives from, $15^{8}$

Pure races, Mendelian traits in, I65

Purim, feast of, 299

Purity, gametic, I54

R

RABBITS, Ewart's experiments on, 82,85 ; effects of crossing, 162 ; lethal dose of anthrax, 86

Rabies, cure of, $239,245,246$

Races, human, differentiation of, 181 ; rapid mental change of, 427,497

Racial, change, definition, 221 ; inferiority, 497

Reality, deductive appeal to, 4 I

Reason, defined, 377; distinguished from instinct and intelligence, 378

Reasoning, nature of, 340

Recapitulation, of parental development, 2 I ; of the evolution of the race, 24 et seq., Ir6 et seq., $250,268,387$; occurs only amongst multicellular types, 23 ; inaccuracy of, 25, I 8 et seq. ; evidence furnished by embryo, 28 ; correct method of proving, 54, 505; utility of, 57; of ancestors, 125; Prof. Turner on, 517 et seq.

Recessives, I $_{53}$; dominants from, $\mathbf{I}_{58}^{8}$

Reduction of chromosomes, I3I

Reflexes, 37I ; imitated by automatic actions, 4 II ; distinguished from instincts, 374

Reformation, the Protestant, 427, 497 
Regeneration, ro

Regression, filial, 134

Reid, Archdall, on Mendelian hypotheses, I74, I80; on imbecility, 470 ; on mental deterioration, 474; on scientific method, 517

Rejuvenescence, I 47

Relations between facts, 38,46

Religion, mental effects of, 427 ; and science, 488

Religious teaching, mental effects of, 486 , 488

Renaissance, the, 427

Reproduction, alternative, I45, I49; blended, I49, I93, I95

Resemblance between parents and offspring, 21

Retrogression, explanations of, ro8; conditions of, Iro; the cause of total loss of traits, II5; identical with reversion, II6; utility of, II9; part played in evolution by, I21, 533 ; causation of, 122 ; of mutations, I7 I, I96; caused by blending, I97 et seq. ; of human races, 223 ; of instincts, $380,3^{84}$; in relation to alcohol, $45^{8}$

Reversion, the two kinds, II7; masking of, II7; in pure races, 165 ; of imbeciles, 425 , 470

Reversed, selection, I08; function of, 123

Revitilization by conjugation, hypotheses of, I47

Richet, on memory, 406

Ricin, acquired toleration of, 247

Ridge, Dr J., on alcohol, 296

Rinderpest, 275

Romans, rise and fall of, 427

Romanes, on evolution, II ; on transmission of acquirements, 62 ; on memory, $406-7$

Roux, experiments on serum, 240

Rowntree and Sherwell, on temperance legislation, 462 et seq.

Royal College of Physicians, description of the feeble-minded, 467

Royal Commission, medical evidence before, $74,444,484$; on tuberculosis, 454 ; Canadian, on intemperance, 464 ; on Care and Control of the Feeble-minded, 467 et seq.

Russia, drinking in, 320

S

SAMUELSON, on drinking in Greece, 300 ; in West Africa, 304; in Germany, 304-5; in China, 306

Sanders, Miss, on segregation, I $_{54}$

Sanitation, improvement of, 227, 273, 277 ; external and internal, 450

Saprophyte, evolution into parasite, 88, 266 ; retrogression from parasite, 88

Savages, food supply of, 269; and alcohol, 298, 306; English child trained by, 420, 430 ; instincts of, 423 ; religion of, 488

Scarlet fever, infectivity of, 228

Schools, epidemic disease in, 273; influence on children, $430,433,481$; biological, 31 , 508-9

School-master, aims of, 479

Schopenhauer, on mind, 335

Science, nature of, 36 et seq. ; warp and woof of, 355,511 ; antagonisms to religion, 488
Sciences, differences between, 33 et, seq., 502 et seq.; exact, 35 et seq.; the most scientific, 47 , 345 ; deductive, $47,2 \mathrm{I}_{3}$ et seq., 503 et seq.. 514 ; systematic, 354,502

Scientific distinguished from science teaching, 502

Scott, Dr D. H., on adaptation, 392

Sebright bantam, 146, 165, r89

Sectarian differences, definition of, 32 ; in biology, 32, 508-9

Segregation of allelomorphs, I 53 ; the central doctrine of Mendelism, I54; disproof of, 165, 173 et seq.

Selection, natural distinguished from artificial, 177 ; by violent death, 66 ; by slow decay, 67 ; when operative, 92 ; among germ-cells, 96 ; varying stringency of, 1 ro et seq., 256 , 296 ; experimental evidence, 73 ; by disease and alcohol, 98 ; by alcohol and opium, 309 ; in slums, 447 ; effects attainable by, 450 ; test of, 222 ; germinal, rog ; reversed, I08, 123; function of, 202, 529; human, 224

Selection theorist, $\mathbf{I}_{5} \mathbf{I}, 390$ et seq.; inference consequences from, I94-5

Selective breeding, human, 439

Self-control, 290, 425

-fertilization, 2, r94, 204

Sensations, 370 ; caused by alcohol, $285,28 \mathrm{~T}$ et seq.

Senses of civilized man, 223

Sense-impressions, 332 et seq., $33^{8}$

Serum, antitoxic, preparation of, 240 ; nature of, 248

Sex, an adaptation, I44; effects of, I88, I93; function of, 144, 148, I49, 193, I97, 199, $202,204,209,389$; diagramniatic representation of, 517 et seq.

Sexual traits, latency of, II3, I45 ; transference to opposite sex, 146 ; alternation of, 145, 159, 189; dominance of, 189; normal blending of, I95; abnormal blending of, 146 ; transmission of, 523

- dimorphism, 142

- beauty, appreciation of, 423

Sherwell and Rowntree, on temperance legislation, 462 et seq.

Sight, sense of, 327

Skill in thinking, 339, 342, 400, 40 r et seq., 485 et seq., 500 et seq., 504,5 I I

Skin colour, reproduction of, 174

Sleeping sickness, 229, 271

Slums, 39 ; prevalence of disease in, 257,275 ; alcoholism in, 296; physical deterioration. in, 446 et seq.

Small-pox, 273; change to cow-pox, 238

Smith, Mr Geoffrey, on Rhizocephala, I I4

Snails, differentiation of, I8I, 202

Snake venom, attenuation of, 242, 247

Sneezing, 37I

Solon, condemnation of intemperance, 300

Somatic cells, relations to germs, 3 , 5 ; resisting power of idio-plasm, 94 ; function of, 124

Space, knowledge of, how acquired, 330, 34I

Spanish plantations in West Indies, 280; empire in America, 282

Spartans, drinking customs, 300

Species, ever-sporting, 180

Specific mean, 66 
Speculation, 355, 506; legitimacy of, 507

Speech, 396, 422

Spencer, Herbert, theory of evolution, II ; on living body as crystal, $65 ;$ on evolution of higher animals, 72 ; on memory, 406

Sperms, union with ova, 2 ; equivalent with ova as bearers of heredity, 2 ; traits of, 143

Sports, or large variations, 22

Sporting instinct, $3^{84}$

Stability of type, 526

Stag, horns of, ro

Stagnation, mental, 427

Starvation, effects of on mother and foetus, 44I

Statistics, vital, 224

Sterility, on blending of alternative traits in, rgo

Stimulus for growth, $6,7,8,43^{8}$; nutriment as, 8 ; use as, $7, \mathbf{I I - I} 3$; injury as, $7,9-\mathbf{I I}$

Stone age, diseases in, 275

Stupidity, natural, 381, 425; artificial, 439, 477,494

Succession, invariable, 336 ; necessary, 337 . 343

Sucking in infants, $3^{83}$

Sugar, in manufacture of alcohol, 457

Sullivan, Dr W. C., 315 et seq., $318 \mathrm{et} \mathrm{seq.}$

Superficial thinking, 15,76

Superstition, defined, 492

Susceptibility of germ-plasm to environment, 66 et seq., 98 ; to charm of alcohol, 289 , 293, 457

Sutton \& Co., on culture of plants, II2

Symbolism, mental, 327

Syphilis, alleged inheritance of, 75,242 ; duration of, 234; recovery from, 249 ; antiquity of, 269 ; migrations of, 270

Systematists, rejection of Darwin's work by, 506

Systematic sciences, 39, 354, 504

$\mathbf{T}$

TEACHER, the skilful, $48 \mathrm{r}$

Teaching, for the professions, 482 et seq.; biological, 484, 510 et seq. ; religious, 486 et seq.; classical, 501 ; science, 502 ; scientific, 489,502

Teeth, human, 223

Telegony, 75-6

Tests, for hypotheses, 39, 345

Texas, fever of cattle, $82,27.5$

Think, instinct to, 403,478

Thinking, in terms of germ-plasm, I5, 25 ; means for securing accuracy, 42 et seq.; coherent, origin of, 330,340 ; skill in, 339, 342, 400, 401, 485 et seq., 500 et seq., 504, 5 I I

Thirst, $285,286,384$

Thought, limits of, 340

Tickling, 37r

Tobacco, racial effects of, 308

Toleration, of poisons, 245,247 ; of opinion, 497

Tonga, morals in, 423

Towns of Europeans in Asia and Western Hemisphere, 280

Toxins, evolution of, 88 et seq.; counter-, 231 ; intra- and extra-cellular, 232, 237, 253; produced by plants and higher animals, 242 ; toleration of, 245,247
Traditional knowledge, 38 r, 397

Training, mental, possibilities of, 477,483 ; in school-room, 479

Transmission or transmutation of acquirements, 19, 6r et seq., 25I, $25^{8}$

Truths, necessary, 344,346 et seq., 349

Tse-tze fly, 275

Tuberculin, 253

Tuberculosis, microbes of, 229; recovery from, 233 ; spread of, 274, 279; compared to alcohol, 308 ; effects of sanitation, 450 , 453 ; human and bovine, 453

Tugwell, Bishop, on drinking in West Africa, 303

Turner, Prof. H. H., on New Euclidians, 344 ; on resemblance and divergence from parents, 517 ; on the influence of sex, 520 et seq. ; on assortive mating, $52 \mathrm{I}$; on transmission of sexual traits, 523 ; on stability of type, 526 , on abbreviation of life-history, 527 ; on natural selection, 529 ; on retrogressive tendency, 533

Twins, identical, 17

\section{U}

UEBERWEG, on crucial instances, $r 65$

Understanding, nature of, 349

Unicellular organism, I

United States, temperance legislation in, $46 I$ et seq.

Units, ancestral, 124

Urban conditions, $44^{8}$

Use, as stimulus for growth, 7, II et seq., 442-3 ; mistaken idea about, II et seq. ; not stimulus for growth of all characters, 12, 89; hypertrophy of brain from, 396

Use-acquirements, utility of, 69 et seq. . $3^{89}$ : magnitude of, $7,7 \mathrm{I}$; limited to higher organisms, 12, 90 ; in disease, 247, 253

Utility, of old-established traits, II2

\section{V}

\section{VACCINIA, 239, 248, 273}

Variability, 98 et seq. ; an adaptation, roo et seq.; established by Natural Selection, ro2 ; biometric investigations, $13^{8}$; occurring apart from conjugation, 147 ; diagrammatic representation of, 518 , et seq.

Variations, from parents, 2I, 80, 196; progressive and retrogressive, 22, 220 ; continuous and discontinuous, 22, 150 ; nature of, 2I-22 ; interpolated, 26 ; difficulty of accounting for, $5^{8}$; distinguished from modifications, 22,78 ; spontaneous, 79 ; caused by direct action of environment, 79, 92,96 ; of unicellular organisms, 89 et seq. ; place of occurrence, I02-3; bud-, $\mathrm{IO}_{3}$; cause of, 105, 147-8, 259, 474; elimination of useless, II 8 ; in parthenogenesis, I47-8 ; definition of, $43^{8}$

Varieties, crossings of natural and artificial, 173 et seq. ; relative numbers of, 202

Veil of familiarity, I3

Vernon, Dr H. M., on correlated traits, 70 ; on experiments on beans, 84 ; on Law on Ancestral Inberitance, $\mathbf{1}_{28}, \mathbf{1}_{30}$; on Natural Selection, 136 ; on reversion in plants, 156 


\section{8}

Vestigial structures, 27, 108

Virulence, evolution of, 88

Voluntary action, 37 I et seq. ; defined, 376

Vries, Prof. H. de, on variations of dandelion and hawkweed, I48, 20I ; on mutation theory, I5I ; on Mendelian phenomena, I66; on latency, I77; on mutations of $O$. lamarckiana, 179 ; on correlated mutations, 182

\section{W}

WALCheren, British expedition to, 26I

Walker, Mr C. E., experiments on hens, II4; on chromosomes, I3I

Wallace, Alfred Russel, on transmission of acquirements, $6 \mathrm{I}$; method of work, 185 ; on snails, 202; on evolution of mind, 397 ; on Darwin, 506

Wallace, Mr J. Sims, on teeth, 224

Warren, Dr E., on variations in Daphnia magna and aphides, 148

Wasp, memory in, 418

Waterton, on male traits in hens, II3

Weismann, experiments on butterflies, 83 ; on continuity of germ-plasm, I24; on criminal selection, Io9; on revitilization, 147 ; method of work, 185

Weldon, Prof. W. F. R., on the Law of Ancestral Inheritance, I30

Welton, Prof. J., on universe as systematic unity, 37 ; on hypothesis and proof, 39-40; on scientific method, 42 ; on proof, 46 ; on natural law, $46,348,35^{2}$; on crucial instances, 164
West Indies, disease in, 280

Whewell, on scientific method, $4 \mathrm{I}$; on hypotheses, 48 ; on gravitation, 49 ; on mathematical axioms, 347

Whichura, on crossed natural varieties, $x_{77}$

Wicking, on latent traits in pigeons, II6

Will, the, 373,374 et seq.; association with attention, 4r4

Williams, The Hon. Mr Sapira, on drinking in West Africa, 302

Wine, sacramental, 304

Working hypotheses, 39

Wright, Dr Strethill, on Hydroidae, 27

Written symbols, 397

Wundt, on origin of mind, 368

\section{Y}

YeAST, production of alcohol by, 457

Yellow fever, 272

Youatt, on sheep, 70

Yule, Mr Udney, on Law of Ancestral Inheritance, 129-30; on experimental crossing, 205

\section{Z}

ZEBRA, crossed with horse; I56, 196

Zoology, systematic, 33 ; method by which it is created, 504

Zygote, 153

Zymotic disease, 227 


\section{A SELECTION OF BOOKS PUBLISHED BY METHUEN}

\section{AND COMPANY LIMITED}

\section{ESSEX STREET LONDON W.C.}

\section{CONTENTS}

\begin{tabular}{|c|c|c|c|c|c|}
\hline & & PAGE & & & PAGR \\
\hline General Literature & - & I & Little Library . & - & 20 \\
\hline Ancient Cities. & - & 15 & Little Quarto Shakespeare & & $2 I$ \\
\hline Antiquary's Books. & - & 15 & Miniature Library & - & 28 \\
\hline Arden Shakespeare & - & 15 & New Library of Medicine & . & 21 \\
\hline Classics of Art & - & 16 & New Library of Music & - & 22 \\
\hline "Complete" Series & - & 16 & Oxford Biographies & - & 22 \\
\hline Connoisseur's Library & . & 16 & Romantic History & - & 22 \\
\hline Handbooks of English & Church & & Handbooks of Theology & & 22 \\
\hline History . . . & . & 17 & Westminster Commentarie & & 23 \\
\hline $\begin{array}{l}\text { Illustrated Pocket Lib } \\
\text { Plain and Coloured }\end{array}$ & $\begin{array}{l}\text { prary of } \\
\text { Books }\end{array}$ & 17 & & & \\
\hline Leaders of Religion & 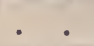 & 18 & & & \\
\hline Library of Devotion & . & 18 & Fiction & - & 23 \\
\hline Little Books on Art & . & 19 & Books for Boys and Girls & & 28 \\
\hline Little Galleries & - & 19 & Novels of Alexandre Dumas & & 29 \\
\hline Little Guides . & - & 19 & Methuen's Sixpenny Books & & 29 \\
\hline
\end{tabular}

APRIL I9IO 


\section{A SELECTION OF}

\section{MESSRS. METHUEN'S}

\section{PUBLICATIONS}

In this Catalogue the order is according to authors. An asterisk denotes that the book is in the press.

Colonial Editions are published of all Messrs. Methuen's Novels issued at a price above 2s. $6 d_{\text {., }}$ and similar editions are published of some works of General Literature. Colonial editions are only for circulation in the British Colonies and India.

All books marked net are not subject to discount, and cannot be bought at less than the published price. Books not marked net are subject to the discount which the bookseller allows.

Messrs. METhunN's books are kept in stock by all good booksellers. If there is any difficulty in seeing copies, Messrs. Methuen will be very glad to have early information, and specimen copies of any books will be sent on receipt of the published price plus postage for net books, and of the published price for ordinary books.

This Catalogue contains only a selection of the more important books published by Messrs. Methuen. A complete and illustrated catalogue of their publications may be obtained on application.

Addleshaw (Percy). SIR PHILIP SIDNEY. Illustrated. Second Edition. Demy 8vo. ros. 6d. net.

Adeney (W. F.), M.A. See Bennett (W.H.).

Ady (Cecilla M.). A HISTORY OF MILAN UNDER THE SFORZA. Illus trated. Demy $8 v 0$. ros. 6 d, net.

Aldis (Janet). THE QUEEN OF LETTER WRITERS, MARQUISE DR SEvigne, DAME dE Bourbilly, r626-96. Illustrated. Second Edition. Demy 820. 12s. 6 d. net.

Alexander (William), D. D., Archbishop of Armagh. THOUG H T A N COUNSELS OF MANY YEARS. Demy I6mo. 2s. 6d.

*Allen (M.). A HISTORY OF VERONA. Illustrated. Demy 8vo. 12s. 6d. net.

Amherst (Lady). A SKETCH OF EGYPIIAN HISTORY FROM THE EARIIEST TIMES TO THE PRESENT DAY. Illustrated. $A$ New and Cheaper Isswe. Demy 8vo. 7s. 6d. net.

Andrewes (Amy G.) THE STORY OF BAYARD. Edited by A. G. ANDREWES, Cr. 8vo. 2s. $6 d$.

Andrewes (Bishop). PRECES PRIVATAE. Translated and edited, with Notes, by F. E. Brightman, M.A., of Pusey House, Oxford. Cr. 8vo. 6s.
Anon. THE WESTMINSTER PRO. BLEMS BOOK. Prose and Verse. Compiled from The Saturday Westminster Gazette Competitions, r904-rgo7. Cr. $8 v 0$. 3s. 6d. net.

VENICE AND HER TREASURES. Illustrated. Round comers. Ficap. 8vo. 5s, net.

Aristotle. THE ETHICS OF. Edited, with an Introduction and Notes, by ЈонN Burnit, M.A. Cheaper issue. Demy $8{ }^{\circ}$. ros. $6 d$. net.

Atkinson (C. T.), M.A., Fellow of Exeter College, Oxford, sometime Demy of Mag. dalen College. A HISTORY OF GER. MANY, from $175_{5}-1815$. Illustrated. Demy 8vo. x2s. $6 d$. net.

Atkinson (T. D.). ENGLISH ARCHI. TECTURE. Illustrated. Ficap. 8vo. $3^{5.6}$. net.

A GLOSSARY OF TERMS USED IN ENGLISH ARCHITECTURE. Illustrated. Second Edition. Fcap. 8vo. 3s. 6d. net.

Atteridge (A, H.). NA POLEON'S BROTHERS. Illustrated. Demy $8 v 0$. I8s. net.

Aves (Ernest). CO-OPERATIVE INDUSTRY. Cr. 8vo. 5s. net.

Bagot (Richard). THE LAKES Or NORTHERN ITALY. Illustrated. Fcap. 8vo. 5s. net. 


\section{General Literature}

Bain (R. Nisbet), THE LAST KING OF POLAND AND HIS CONTEMPORARIES. Illustrated. Demy $8 v 0$. ros. 6d. net.

Balfour (Graham). THE LIFE OF ROBERT LOUIS STEVENSON. Illustrated. Fifth Edition in one Volume. Cr. 8vo. Buckram, 6s.

Baring (The Hon. Maurlee). WITH THE RUSSIANS IN MANCHURIA. Third Edition. Demy 8vo. 7s. 6d. net.

A YEAR IN RUSSIA. Second Edition. Demy 8vo. ros. 6d. net.

RUSSIAN ESSAYS AND STORIES. Second Edition. Cr. 8vo. 5s. net.

LANDMARKS IN RUSSIAN LITERATURE. Cr. 8vo. 6s. net.

Baring-Gould (S.). THE LIFE OF NAPOLEON BONAPARTE. Illustrated. Second Edition. Wide Royal 8vo. ros. $6 d$. net.

THE. TRAGEDY OF THE CESARS A STUdy of THR Charactirs of THB Cesars of the Julian and Claudian Houszs. Illustrated. Seventh Edition. Royal 8vo. ros. $6 d$. net.

A BOOK OF FAIRY TALES. Illustrated. Second Edition. Cr. 8vo. Buckram. 6s. Also Medium 8vo. $6 d$.

OLD ENGLISH FAIRY TALES. Illustrated. Third Edition. Cr. 8vo. Buck. ram. $6 s$.

THE VICAR OF MORWENSTOW. Revised Edition. With a Portrait. Third Edition. Cr. 8vo. 3s. $6 d$.

OLD COUNTRY LIFE. Illustrated. Fifth Edition. Large Cr. 8vo. 6.

A GARLAND OF COUNTRY SONG : English Folk Songs with their Traditional Melodies. Collected and arranged by $S$. BARING-Gould and H. F. SHEPPARD. Demy 4 to. 6s.

SONGS OF THE WEST: Folk Songs of Devon and Cornwall. Collected from the Mouths of the People. By S. BARIng-Gourd M.A., and H. Fleetwood SHePpard, M.A New and Revised Edition, under the musical editorship of CECIL J. SHARP. Large Imperial 8vo. 5s. net.

STRANGE SURVIVALS : SOME ChapteRS IN THE HISTORY OF MAN. Illustrated. Third Edition, Cr, 8vo, 2s, 6d. net.

YORKSHIRE ODDITIES : INCIDENTS and Strange Events. Fifth Edition. Cr. 8vo. 2s. 6d. net.

A BOOK OF CORNWALL. Illustrated. Second Edition. Cr. 8vo. 6s.

A BOOK OF DARTMOOR. Illustrated. Second Edition. Cr. 8vo. 6s.

A BOOK OF DEVON. Illustrated. Third Edition. Cr. $800.6 s$

A BOOK OF NORTH WALES. Illustrated. Cr. 8vo. 6s.

A BOOK OF SOUTH WALES. Illustrated. Cr. $8 v 0$. 6 s.
A BOOK OF BRITTANY. Illustrated, Second Edition. Cr. 8vo. 6s.

A BOOK OF THE RHINE: From Cleve to Mainz. Illustrated. Second Edition. Cr. 8vo. 6s.

A BOOK OF THE RIVIERA. Illus trated. Second Edition. Cr. 8vo. 6s.

A BOOK OF THE PYRENEES. Illustrated. $\mathrm{Cr}$. 8vo. 6s.

Barker (E.), M.A., (Late) Fellow of Merton College, Oxford. THE POLITICAL THOUGHT OF PLATO AND ARISTOTLE. Demy 8vo. ros. 6d. net.

Baron (R. R. N.), M.A. FRENCH PROSE COMPOSITION, Fourth Edition. $C r$. 8vo. 2s. 6d. Key, 3s. net.

Bartholomew (J. G.), F.R.S.E. See Robertson (C. G.).

Bastable (C. F.), LL.D. THE COMMERCE OF NATIONS. Fourth Edition. Cr. 8vo. 2s. $6 d$.

Bastian (H. Charlton), M.A., M.D., F.R.S. THE EVOLUTION OF LIFE, IIIUStrated. Demy 8vo. 7s. 6d. net.

Batson (Mrs. Stephen). A CONCISE HANDBOOK OFGARDEN FLOWERS. Fcap. 8vo. 3s. 6d. net.

THE SUMMER GARDEN OF PLEASURE. Illustrated. Wide Deniy 8vo. 155. net

Beckett (Arthur). THE SPIRIT OF THE DOWNS: Impressions and Reminiscences of the Sussex Downs. Illustrated. Second Edition. Demy 8vo. ros. 6d. net.

Beckford (Peter). THOUGHTS ON HUNTING. Edited by J. Отно PAGET. Illustrated. Second Edition. Demy 8vo. 6s.

Begbie (Harold). MASTER WORKERS. Illustrated. Demy 8vo. 7s. 6d. net.

Behmen (Jacob), DIALOGUES ON THE SUPERSENSUAL LIFE. Edited by Bernard Holland. Fcap. 8vo. 35. 6d.

Bell (Mrs, Apthur G.). THE SKIRTS OF THE GREAT CITY. Illustrated. Second Edition. Cr. 8vo. 6s.

Belloe (H.), M.P. PARIS. Illustrated. Second Edition, Revised. Cr. 8vo. 6s.

HILLS AND THE SEA. Second Edition. Cr. 8vo. 6s.

ON NOTHING AND KINDRED SUBJECTS. Third Edition. Fcap 8vo. ON EVERYTHING. Second Edition. Fcap. 800 . 55 .

MARIE ANTOINETTE. Illustrated. Third Edition. Demy 8vo. 15s. net. THE PYRENEES. Illustrated. Second Edition. Demy 8vo. 7s. $6 d_{\text {a net. }}$

Bellot (H. H. L.), M.A. See Jones (L. A. A) 
Bennett (Joseph). FORTY YEARS OF MUSIC, 1865-rgo5. Illustrated. Demy 8vo. 16s. net.

Bennett (W. H.), M.A. A PRIMER OF THE BIBLE. Fifth Edition. Cr. $8 v 0$. 2s. $6 d$.

Bennett (W. H.) and Adeney, (W. F.). A BIBLICAL INTRODUCTION. With a concise Bibliography. Fifth Edition. $\mathrm{Cr}$. 8vo. 7s. 6d.

Benson (Archbishop). GOD'S BOARD. Communion Addresses. Second Edition. Fcap. 8vo. 3s. 6d. net.

Benson (R. M.). THE WAY OF HOLINESS. An Exposition of Psalm cxix. Analytical and Devotional. $C r .8 v o$. $5 s$.

- Beasusan (Samuel L.). HOME LIFE IN SPAIN. Illustrated. Demy $8 v 0$. ros. $6 d$. net.

Berry (W. Grinton), M.A. FRANCE SINCE WATERLOO. Illustrated. $C r$. 8vo. 6s.

Betham-Edwards (Miss). HOME LIFE IN FRANCE. Illustrated. Fifth Edition. Cr. 8vo. 6s.

Bindley (T. Herbert), B.D. THE OECUMENICAL DOCUMENTS OF THE FAITH. With Introductions and Notes. Second Edition. Cr. 8vo. 6s. net.

Binyon (Laurence). See Blake (William).

Blake (William). ILLUSTRATIONS OE THE BOOK OF JOB. With General Introduction by LAURENCE BINYON. Illus trated. Quarto. 2is. net.

Body (George), D.D. THE SOUL'S PILGRIMAGE: Devotional Readings from the Published and Unpublished writings of George Body, D.D. Selected and arranged by J. H. BurN, D.D., F.R.S.E. Demy r6mo. 2s. $6 d$.

Boulting (W.). TASSO AND HIS TIMES. Illustrated. Demy 8vo. ros. $6 d$. net.

Bovill (W. B. Forster). HUNGARY AND THE HUNGARIANS. Illustrated. Demy 8vo. 7s. 6d. net.

Bowden (E. M.). THE IMITATION OF BUDDHA: Being Quotations from Buddhist Literature for each Day in the Year. Fifth Edition. Cr. 16 mo. 2s. 6d.

Brabant (F. G.), M.A. RAMBLES IN SUSSEX. Illustrated. $C r .8 v 0.6 s$.

Bradley (A. G.). ROUND ABOUT WILTSHIRE. Illustrated. Second Edition. Cr. 8vo. 65.

THE ROMANCE OF NORTHUMBER. LAND. Illustrated. Second Edition. Demy 8v0. 7s. 6 d. net.
Braid (James), Open Champion, rgor, rgos and 1906. ADVANCED GOLF. Illustrated. Fifth Edition. Demy 8vo. ros. 6d. net.

Braid (James) and Others. GREAT GOLFERS IN THE MAKING. Edited by HenRy Leach. Illustrated. Second Edition. Demy 8vo. 7s. 6d. net.

Brailsford (H. N.). MACEDONIA : Irs RACRS AND THEIR FUTURB. Illustrated. Demy 8vo. I2s. $6 d$. net.

Brodrick (Mary) and Morton (A. Ander. son). A CONCISE DICTIONARY OF EGYPTIAN ARCHAOLOGY. A Handbook for Students and Travellers. Illustrated. Cr. 8vo. 3s. $6 d$.

Brown (J. Wood), M.A. THE BUILDERS OF FLORENCE. Illustrated. Demy 4 to. I8s. net.

Browning (Robert). PARACELSUS. Edited with Introduction, Notes, and Bibliography by MARGARET L. LEE and KATHARINe B. Locock. Fcap. 8vo. 3s. 6d. net.

Buckton (A. M.). EAGER HEART: A Mystery Play. Eighth Edition. Cr. 8vo. is. net.

Budge (E. A. Wallis). THE GODS OF THE EGYPTIANS. Illustrated. Two

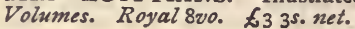

Bull (Paul), Army Chaplain. GOD AND OUR SOLDIERS. Second Edition. Cr. 8vo. 6s.

Bulley (Miss). See Dilke (Lady).

Burns (Robert), THE POEMS. Edited by ANDREW LANG and W. A. Craigie. With Portrait. Third Edition. Wide Demy 8vo, gilt top. $6 s$.

Bussell (F. W.), D.D. C H RISTIAN THEOLOGY AND SOCIAL PROGRESS (The Bampton Lectures of rgo5). Demy 8vo. ros. 6d. net.

Butler (Sir William), Lieut.-General, G.C.B. THE LIGHT OF THE WEST. With some other Wayside Thoughts, r865rgo8. Cr. 8vo. 5s. net.

Butlin (F. M.). AMONG THE DANES. Illustrated. Demy 8vo. 7s. 6d. net.

Cain (Georges), Curator of the Carnavalet Museum, Paris. WALKS IN PARIS. Translated by A. R. Allinson, M.A. Illustrated. Demy 8vo. 7s. 6 d. net.

Cameron (Mary Lovett). OLD ETRURIA AND MODERN TUSCANY. Illustrated. Second Edition. Cr. 8vo. 6s, net.

Carden (Robert W.). THE CITY OF GENOA. Illustrated Derny $8 v 0$. ros. $6 d$. net. 


\section{General Literature}

Carlyle (Thomas). THE FRENCH REVOLUTION. Edited by C. R. L. Fletcher, Fellow of Magdalen College, Oxford. Three Volumes. Cr. 8vo. I8s.

THE LETTERS AND SPEECHES OF OLIVER CROMWELL. With an Introduction by C. H. FiRTH, M.A., and Notes and Appendices by Mrs. S. C. Lomas. Three Volumes. Demy 8vo. 18s. net.

Celano (Brother Thomas of). THE LIVES OF FRANCIS OF ASSISI. Translated by A. G. FerRers Howell. Illustrated. Cr. 8vo. 5s. net.

Chambers (Mrs. Lambert). Lawn Tennis for Ladies. Illustrated. Crown 8vo. 2s. $6 d$. net.

Chandler (Arthur), Bishop of Bloemfontein. ARA CCELI: AN ESSAY IN MYSTICAI Theology. Third Edition. Cr. 8vo. 3s. 6 d. net.

Chesterfield (Lord). THE LETTERS OF THE EARL OF CHESTEREIELD TO HIS SON. Edited, with an Introduction by C. Strachey, with Notes by A. Calthrop. Two Volumes. Cr. 8vo. I2s.

Chesterton (G.K.). CHARLES DICKENS. With two Portraits in Photogravure. Sixth Edition. Cr. 8vo. 6 s.

ALL THINGS CONSIDERED. Fifth Edition. Fcap. 8vo. 5s.

TREMENDOUS TRIFLES. Fourth Edition. Fcap. 8vo. 5s.

Clausen (George), A.R.A., R.W.S. SIX LECTURES ON PAINTING. Illustrated. Third Edition. Large Post. 8v0. 3 s.6d.net.

AIMS AND IDEALS IN ART. Eight Lectures delivered to the Students of the Royal Academy of Arts. Illustrated. Second Edition. Large Post 8vo. 5s. net.

Clutton-Brock (A.) SHELLEY : THE MAN AND THE POET. Illustrated. Demy 8vo. 7s. 6d. net.

Cobb (W. F.), M.A. THE BOOK OF PSALMS : with an Introduction and Notes. Demy 8vo. ros. 6 d. net.

Cockshott (Winifred), St. Hilda's Hall Oxford. THE PILGRIM FATHERS, Therr Church and Colony. Illustrated. Demry 8vo. 7s. 6 d. net.

Collingwood (W. G.), M.A. THE LIFE OF JOHN RUSKIN. With Portrait. Sixth Edition. Cr. 8vo. 2s. 6 d. net.

Colvill (Helen H.). ST. TERESA OF SPAIN. Illustrated. Second Edition. Demy 8vo. 7s. 6d. net.

Condamino (Robert de la). THE UPPEK GARDEN. Fcap. 8vo. 5s. net.

Conrad (Joseph). THE MIRROR OF THE SEA : Memories and Impressions. Third Edition. Cr. 8v0. 6s.
Coolidge (W. A. B.), M.A. THE ALPS. Illustrated. Demy 8vo. 7s. $6 \dot{d}$. net.

Cooper (C.S.),F.R.H.S. See Westell (W.P.) Coulton (G. G.). CHAUCER AND HIS ENGLAND. lilustrated. Second Edition. Demy 8vo. xos. $6 d$. net.

Cowper (William). THE POEMS. Edited with an Introduction and Notes by J. C. BAllex, M.A. Illustrated. Demy 8vo. sos. $6 d$. net.

Crane (Walter), R.W.S. AN ARTIST'S REMINISCENCES. Illustrated. Second Edition. Demy 8vo. I8s. net.

INDIA IMPRESSIONS. Illustrated. Second Edition. Demy 8vo. 7s. 6d. net.

Crispe (T. E.). REMINISCENCES OF A K.C. With 2 Portraits. Second Edition. Demy 8vo. xos. 6 d. net.

Crowley (Ralph H.). THE HYGIENE OF SCHOUL LIFE. Illustrated. $C r$. 8vo. 3s. 6d. net.

Dante (Alighieri). LA COMMEDIA DI DANTE. The Italian Text edited by Paget Toynbee, M.A., D.Litt. Cr.8vo. 6s.

Davey (Richard). THE PAGEANT OF LONDON. Illustrated. In Two Volumes. Demy 8vo. 25s. net.

Davis (H. W. C.), M.A., Fellow and Tutor of Balliol College. ENGLAND UNDER THE NORMANS AND ANGEVINS: ro66-1272. Illustrated. Demy 8vo. ros. 6d. net.

Deans (R. Storry). THE TRIALS OF FIVE QUEENS: KATHARINE OF ARAGON, Anne Boleyn, Mary QueEn of Scots, Marie Antoinette and Caroline of Brunswick. Illustrated. Second Edition. Demy 8vo. ros. 6 d. net.

Dearmer (Mabel). A CHILD'S LIFE OF CHRIST. Illustrated. Large $C r$. 8vo. $6 s$.

D'Este (Margaret). IN THE CANARIES WITH A CAMERA. Illustrated. Cr.8vo. 7s. 6 d. net.

Dickinson (G. L.), M.A., Fellow of King's College, Cambridge. THE GREEK VIEIV OF LIFE. Seventh and Revised Edition. Crown 8vo. 2s. 6 d. net.

Ditchfleld (P. H.), M.A., F.S.A. THE PARISH CLERK. Illustrated. Third Edition. Demy 8vo. 7s. 6d. net.

THE OLD.TIME PARSON. Illustrated. Second Edition. Demy 8vo. 7s. 6d. net.

Douglas (Hugh A.). VENICE ON FOOT With the Itinerary of the Grand Canal. Illustrated. Fcap. 8vo. 5s. net. 
Douglas (James). THE MAN IN THE PULPIT. Cr. 8vo. 2s. 6d. net.

Dowden (J.), D.D., Late Lord Bishop of Edinburgh. FURTHER STUDIES IN THE PRAYER BOOK. Cr. 8vo. $6 s$.

Driver (S. R.), D.D., D.C.L., Regius Professor of Hebrew in the University of Oxford. SERMONS ON SUBJECTS CONNECTED WITH THE OLD TESTAMENT. $C r .8 v 0.6 s$.

Duff (Nora). MATILDA OF TUSCANY. Illustrated. Demy 8vo. sos. $6 d$. net.

Dumas (Alexandre). THE CRIMES OF THE BORGIAS AND OTHERS. With an Introduction by $R$. S. GARN HTT. Illustrated. $C r .8 v 0$. $6 s$.

THE CRIMES OF URBAIN GRAN. DIER AND OTHERS. Illustrated. $C r$. 8vo. $6 s$.

THE CRIMES OF THE MAROUISE DE BRINVILLIERS AND OTHERS. Illustrated. $C r .8 v 0$. $6 s$.

THE CRIMES OF ALI PACHA AND OTHERS. Illustrated. Cr. 8vo. $6 s$.

MY MEMOIRS. Translated by E. M WALLER. With an Introduction by ANDREW LANG. With Frontispieces in Photogravure. In six'Volumes. Cr. $8 v 0$. 6s. each volume. VoL. I. 1802-182x. VoL. IV. 1830-1831. VOL. II. 1822-1825. VOL. V. $183^{1}-1832$. VOL. III. 1826-1830. VOL. VI. $183^{2}-1833$. MY PETS. Newly translated by A. R. Allinson, M.A. Illustrated. Cr. 8vo. $6 s$.

Duncan (David), D.Sc., LL.D. THE LIFE AND LETTERS OF HERBERT SPENCER. Illustrated. Demy 8vo. 15 s.

Dunn-Pattison (R. P.) NAPOLEON'S MARSHALS. Illustrated. Demy $8 v 0$. Second Edition. 12s. 6d. net.

-EDWARD THE BLACK PRINCE. Illustrated. Demy 8vo. 7s. 6d. net.

Durham (The Earl of) A REPORT ON CANADA. With an Introductory Note. Demy 8vo. 4s. 6d. net.

Dutt (W.A.) THE NORFOLK BROADS. Illustrated. Second Edition. Cr. 8vo. 6s. WILD LIFE IN EAST ANGLIA. Illus. trated. Second Edition. Demy 8vo. 7s. 6d. net.

SOME LITERARY ASSOCIATIONS OF EAST ANGLIA. Illustrated. Demy $8 v 0$. Ios. 6d. net.

Edmonds (Major J. E.), R.E. ; D. A. Q.-M, G. See Wood (W. Birkbeck).

Edwardes (Tickner). THE LORE OF THE HONEY BEE. Illustrated. $C r$.

8vo. 6s.
"LIFT-LUCK ON SOUTHERN ROADS. Illustrated. Cr. 8vo. $6 s$.
Egerton (H. E.), M.A. A HISTORY OF BRITISH COLONIAL POLICY. Second Edition, Revised. Demy 8vo. 7s. 6d. net.

Everett-Green (Mary Anne). ELIZA. BETH ; ELECTRESS PALATINE AND QEEEN OF BOHEMIA. Revised by her Niece S. C. Lomas. With a Prefatory Note by A. W. WARD, Litt.D. Demy $8 v 0$. 1os. $6 d$. net.

Fairbrother (W. H.), M.A. THE PHILOSOPHY: OF T. H. GREEN. Second Edition. Cr. 8vo, 3s. 6 d.

Fea (Allan). THE FLIGHT OF THE KING. Illustrated. New and Revised Edition. Demy 8vo. 7s. 6d. net.

SECRET CHAMBERS AND HIDINGPLACES. Illustrated. New and Revised Edition. Demy 8vo. 7s. 6d. net.

JAMES II. AND HIS WIVES. Illustrated. Demy 8vo. ros. 6 d. net.

Fell (E. F. B.). THE FOUNDATIONS OF LIBERTY. Cr. 8vo. 5s. net.

Firth (C. H.), M.A., Regius Professor of Modern History at Oxford. CROM. WELL'S ARMY : A History of the English Soldier during the Civil Wars, the Common. wealth, and the Protectorate. Cr. 8vo. $6 s$.

FitzGerald (Edward). THE RUBAÍYÁT OF OMAR KHAYYÁM. Printed from the Fifth and last Edition. With a Commentary by Mrs. STEPHeN BAtSON, and a Biography of Omar by E. D. Ross. Cr. Evo. 6s.

* Fletcher (B. F. and H. P.). THE ENGLISH HOME. Illustrated. Demy 8vo. I2s. $6 d_{0}$ net.

Fletcher (J. S.). A BOOK OF YORK. SHIRE. Illustrated. Demy 8vo. 7s. $6 d$. net.

Flux (A. W.), M.A., William Dow Professor of Political Economy in M'Gill University, Montreal. ECONOMIC PRINCIPLES. Demy 8vo. 7s. 6d. net.

Foot (Constance M.). INSECT WON-

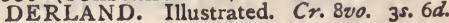
net.

Forel (A.). THE SENSES OF INSECTS. Iranslated by Macleod YearsLEY. Illustrated. Demy 8vo. ros. $6 d$. net.

Fouque (La Motte). SINTRAM AND HIS COMPANIONS. Translated by $A$. C. Farouharson. Illustrated. Demy $8 v 0$. 7s. $6 d$. net. Half White Vellum, ros. $6 d$. net.

Fraser (J. F.). ROUND THE WORLD ON A WHEEL. Illustrated. Fifth Edition. Cr. 8vo. Gs.

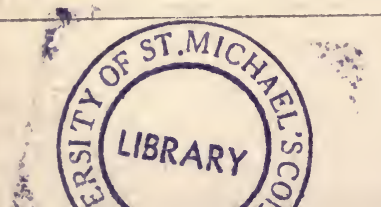


Galton (Sir Francis), F.R.S. : D.C.L., Oxf.; Hon. Sc.D., Camb.; Hon. Fellow Trinity College, Cambridge. MEMORIES OF MY LIFE. Illustrated. Third Edition. Demy 8vo. Ios. 6d. net.

Garnett (Luey M. J.). THE TURKISH PEOPLE: Their Social Life, Religious Beliers and Institutions, and Donestic LiFr. Illustrated. Demy 8vo. Ios. $6 d$. net.

Gibbins (H. de B.), Litt.D., M.A. INDUSTRY IN ENGLAND : HISTORICAL OUTLINES. With 5 Maps. Fifth Edition. Demy 8vo. ros. 6 d.

THE INDUSTRIAL HISTORY OF EN GLA ND. Illustrated. Sixteenth Edition Revised. Cr. 8vo. 3s.

E NGLIS H SOCIAL REFORMERS. Second Edition. Cr. 8vo. 2s. 6d.

See also Hadfield, R.A.

Glbbon (Edward). MEMOIRS OF THE LIFE OF EDWARD GIBBON. Edited by G. BIRKBeCK Hill, LL.D. Cr. 8vo. $6 s$. -THE DECLINE AND FALL OF THE ROMAN EMPIRE. Edited, with Notes, Appendices, and Maps, by J. B. BuRY, M.A., Litt.D., Regius Professor of Modern History at Cambridge. Illustrated. In Seven Volumes. Demy 8vo. Gilt Top. Each ros. 6d. net.

Gibbs (Phillp.) THE ROMANCE OF GEORGE VILLIERS: FIRST DUKE OF BUCKINGHAM, AND SOME MEN AND WOMEN OF THE STUART COURT. Illustrated. Second Edition. Demy 8vo. 15s. net.

Gloag (M. R.) and Wyatt (Kate M.). A BOOK OF ENGLISH GARDENS. Illustrated. Demy 8vo. Ios. 6d. net.

Glover (T. R.), M.A., Fellow and Classical Lecturer of St. John's College, Cambridge. THE CONFLICT OF RELIGIONS IN THE EARLY ROMAN EMPIRE. Third Edition. Demy 8vo. 7s. 6d. net.

Godfrey (Elizabeth). A BOOK OF REMEMBRANCE. Being Lyrical Selections for every day in the Year. Arranged by E. Godfrey. Second Edition. Fcap. 8vo. 2s. 6 d. net.

ENGLISH CHILDREN IN THE OLDEN TIME. Illustrated. Second Edition. Demy 8vo. 7s. 6d. net.

Godley (A. D.), M.A., Fellow of Magdalen College Oxford. OXFORD IN THE EIGHTEENTH CENTURY. Illustrated. Second Edition. Demy 8vo. 7s. 6d. net.

LYRA FRIVOLA. Fourth Edition. Fcap. 8vo. 2s. $6 d$.

VERSES TO ORDER. Second Edition. Fcab. 8vo. 2s. 6d.

SECOND STRINGS. Fcap. 8ve. 2s. $6 d$.
Goll (August). CRIMINAL TYPES IN SHAKESPEARE. Authorised Transla. tion from the Danish by Mrs. Charles WEEkEs. Cr. 8vo. 5s, net.

Gordon (Lina Duff) (Mrs. Aubrey Waterfield). HOME LIFE IN ITALY : LETTERS FROM THE APENNINES. Illustrated. Second Edition. Demy 8vo. ros. 6d. net.

Gostling (Frances M.). THE BRETONS AT HOME. Illustrated. Second Edition. Demy 8vo. ros. 6d. net.

Graham (Harry). A GROUP OF SCOTTISH WOMEN. Illustrated. Second Edition. Demy 8vo. Ios. 6d. net.

Grahame (Kenneth). THE WIND IN THE WILLOWS. Illustrated. Fourth Edition. Cr. 8vo. 6s.

Gwynn (Stephen), M.P. A HOLIDAY IN CONNEMARA. Illustrated. Demy 8vo. ros 6 d. net.

Hall (Cyril). THE YOUNG CARPENTER. Illustrated. Cr. $8 v 0$. 5 s.

Hall (Hammond). THE YOUNG ENGINEER : or MODERN ENGINES AND THEIR MODEls. Illustrated. Second Edition. Cr. 8vo. 5s.

Hall (Mary). A WOMAN'S TREK FROM THE CAPE TO CAIRO. Illustrated. Second Edition. Demy 8vo. I6s. net.

Hamel (Frank). FAMOUS FRENCH SALONS. Illustrated. Third Edition. Demy 8vo. 12s. 6d. net.

Hannay (D.). A SHORT HISTORY OF THE ROYAL NAVY. Vol. I., 1217-1688. Vol. II., 1689-1815. Demy 8vo. Each 7s. 6 d. net.

Hannay (James 0.), M.A. THE SPIRIT AND ORIGIN OF CHRISTIAN MONASTICISM. Cr.8vo. 6 s.

THE WISDOM OF THE DESERT. Fcap. 8vo. 3s. 6d. net.

Harper (Charles G.). THE AUTOCAR ROAD-BOOK. Four Volumes with Maps. Cr. 8vo. Each 7s. 6d. net.

Vol. I.-South of THE Thames.

Vol. II.-NORTh aND SOUTH Wales aNd West Midlands.

Headley (F. W.). DARWINISM AND MODERN SOCIALISM. Second Edition. Cr. 8vo. 5s, net.

Henderson (B. W.), Fellow of Exeter, College, Oxford. THE LIFE AND PRINCIPATE OF THE EMPEROR NERO. Illustrated. New and cheaper issue. Demy 8vo. 7s. 6d. net.

Henderson (M. Sturge). GEORGE MEREDITH : NOVELIST, POET, REFORMER. Illustrated. Second Edition Cr. 8vo. Gs. 
Henderson (T. F.) and Watt (Francis). SCOTLAND OF TO-DAY. Illustrated. Second Edition. Cr. 8vo. 6s.

Henley (W. E.). ENGLISH LYRICS. CHAUCER TO POE, 1340-1849. Second Edition. Cr. 8vo. 2s. 6d. net.

"Heywood (W.). A HISTORY OF PE. RUGIA. Illustrated. Demy $8 v 0$. I2s. $6 d$. net.

Hill (George Franeis). ONE HUNDRED MASTERPIECES OF SCULPTURE. Illustrated. Demy 8vo. Ios. 6 d. net.

Hind (C. Lewis). DAYS IN CORNWALL. Illustrated. Second Edition. Cr. 8vo. 6s.

Hobhouse (L. T.), late Fellow of C.C.C. Oxford. THE THEORY OF KNOWLEDGE. Demy $8 v 0$. ros. $6 d$. net.

Hodgetts (E. A. Brayley). THE COURT OF RUSSIA IN THE NINETEENTH CENTURY. Illustrated. Two volumes. Demy 8vo. 24s. net.

Hodgson (Mrs. W.). HOW TO IDENTIFY OLD CHINESE PORCELAIN. Illustrated. Second Edition. Post 8vo. 6s.

Holdich (Sir T. H.), K.C.I.E., C.B., F.S.A. THE INDIAN BORDERLAND, I8801900. Illustrated. Second Edition. Demy 8vo. ros. 6 d. net.

Holdsworth (W. S.), D.C.L. A HISTORY OF ENGLISH LAW. In Four Volumes. Vols. I., II., III. Demy 8vo. Each ios. $6 d$. net.

Holland (Clive). TYROL AND ITS PEOPLE. Illustrated. Demy 8vo. Ios. $6 d$. net.

Hollway-Calthrop (H. C.), late of Balliol College, Uxford : Bursar of Eton College. PETRARCH : HIS LIFE, WORK, AND TIMES. Illustrated. Demy $8 v 0$. 12s. $6 d$. net.

Horsburgh (E. L. S.), M.A. LORENZO THE MAGNIFICENT: AND FLORENCE IN HER GOLDEN AGR. Illustrated. Second Edition. Demy 8vo. r5s. net.

WATERLOO : with Plans. Second Edition. Cr. 8vo. 5 s.

Hosie (Alexander). MANCHURIA. Illustrated. Second Edition. Demy 8vo. 7s. 6d. net.

Hulton (Samuel F.). THE CLERK OF OXFORD IN FICTION. Illustrated. Demy 8vo. ros. 6d. net.

* Humphreys (John H.). PROPORTIONAL REPRESENTATION. Cr. 8 vo. 3s. 6d. net.

Hutehinson (Horace G.). THE NEW FUREST. Illustrated. Fourth Edition. Cr. 8vo. 6s.

Hutton (Edward). THE CITIES OF
UMBRIA. Illustrated. Third Edition. Cr. 8vo. 6s.

THE CITIES OF SPAIN. Illustrated. Third Edition. Cr. 8vo. 6s.

FLORENCE AND THE CITIES OF NORTHER N T USCAN Y, WITH GENOA. Illustrated. Second Edition. Crown 8vo, 6s.

ENGLISH LOVE POEMS. Edited with an Introduction. Fcap. 8vo. 3s. 6d. net.

COUNTRY WALKS ABOUT FLORENCE. Illustrated. Fcap. 8vo. $5^{\text {s. }}$, net.

IN UNKNOWN TUSCANY With an Appendix by Wrlliam Heywood. Illustrated. Second Edition. Demy 8vo. 7s, 6d. net.

ROME. Illustrated. $\mathrm{Cr} .8 v 0.65$.

Hyett (F. A.) FLORENCE : HeR HISTORY AND ART TO THE FALL OF THE REPUBLIC. Demy 8vo. 7s. 6d. net.

Ibsen (Henrik). BRAND. A Drama. Translated by WILLIAM WiLson. Third Edition. Cr. 8vo. 3s. 6d.

Inge ( W. R.), M.A., Fellow and Tutor of Hertford College, Oxford. CHRISTIAN MYSTICISM. (The Bampton Lectures of 1899.) Demy 8vo. 12s. 6d. net.

Innes (A. D.) M.A. A HISTORY OF THE BRITISH IN INDIA. With Maps and Plans. Cr. 8vo. 6s.

ENGLAND UNDER THE TUDORS. With Maps. Second Edition. Demy $8 v 0$. ros. 6 d. net.

*Innes (Mary). SCHOOLS OF PAINT. IN G. Illustrated. Cr. 8vo. 5s. net.

James (Norman G. B.). THE CHARM OF SWITZERLAND. Cr. 8vo. 5s. net.

Jebb (Camilla). A STAR OF THE SALONS: Julie de Lespinasse. Illustrated. Demy 8vo. Ios. 6 d. net.

Jefiery (Reginald W.), M.A. THE HISTORY OF THE THIRTEEN COLONIES OF NORTH AMERICA, r497-r763. Illustrated. Demy 8vo. 7s. 6d. net.

Jenks (E.), M.A., B.C.L. AN OUTLINE OF ENGLISH LOCAL GOVERNMENT. Second Edition. Revised by R. C. K. ENSOR, M.A. Cr. 8vo. 2s. 6d.

Jennings (Oscar), M.D. EARLY WOOD. CUT INITIALS. Illustrated. Demy $4^{t o .}$ 21s. net.

Jerningham (Charles Edward). THE MAXIMS OF MARMADUKE. Second Edition. Cr. 8vo. 5 s.

Johnston (Sir H. H.), K.C.B. BRITISH CENTRAL AFRICA. Illustrated. Third Edition. Cr. 4to. r8s. net. 


\section{General Literature}

-THE NEGRO IN THE NEW WORLD. Illustrated. Demy 8vo. x6s. net.

Jones (R. Crompton), M.A. POEMS OF THE INNER LIFE. Selected by R. C. Jones. Thirteenth Edition. Fcap 8vo. 2s. $6 d$. net.

Julian (Lady) of Norwich. REVELA. TIONS OF DIVINE LOVE. Edited by Grack Warrack. Third Edition. Cr. 8vo. 3s. 6d.

'Kappa.' LET YOUTH BUT KNOW : A Plea for Reason in Education. Second Edition. Cr. 8vo. 3s. 6d. net.

Keats (John). THE POEMS. Edited with Introduction and Notes by $\mathbf{E}$. de Sélincourt, M.A. With a Frontispiece in Photogravure. Second Edition Revised. Demy 8vo. 7s. 6d. net.

Keble (John). THE CHRISTIAN YEAR. With an Introduction and Notes by W. Lock, D.D., Warden of Keble College. Illustrated. Third Edition. Fcap. 8vo. 35. 6d.; padded morocco. 5 s.

Kempis (Thomas à). THE IMITATION OF CHRIST. With an Introduction by DRAN FARRAR. Illustrated. Third Edition. Fcap 8vo. 3s. 6d.; padded morocco, 5 s.

Also translated by C. Bigg, D.D. Cr. 8vo. 3s. $6 d$.

Kepr (S. Parnell). GEORGE SELWYN AND THE WITS. Illustrated. Demy 8vo. 12s. $6 d$. net.

Kipling (Rudyard). BARRACK-ROOM BALLADS. 94th Thousand. Twentyseventh Edition. Cr. 8vo. 6s. Also Fcap. $8 v 0$, Leather. 5s. net.

THE SEVEN SEAS. 79th Thousand. Fifteenth Edition. Cr. 8vo. 6s. Also Fcap. 8vo, Leather. 5s. net.

THE FIVE NATIONS. 66th Thousand. Sixth Edition. Cr. 8vo. 6s. Also Fcap. 8vo, Leather. 5s. net.

DEPARTMENTAL DITTIES. Eighteenth Edition. Cr. 8vo. 6s. Also Fcap. 8vo, Leather. 5s. net.

Knox (Winifred F.). THE COURT OF A SAINT. Illustrated. Demy $8 v 0$. ros. $6 d$. net.

Lamb (Charles and Mary), THE WORKS. Edited by E. V. LuCAS. Illustrated. In Seven Volumes. Demy 8vo. 7s. 6d. each.

Lane-Poole (Stanley). A HISTORY OF EGYPT IN THE MIDDLE AGES. Illustrated. $C r .8 v o$. $6 s$.

"Lankester (Sir Ray), K.C.B., F.R.S. SCIENCE FROM AN EASY CHAIR. Illustrated. Cr. 8vo. 6s.
Leach (Henry). THE SPIRIT OF THE LINKS. Cr. 8vo. 6s.

Le Braz (Anatole). THE LAND OF PARDONS. Translated by FRANCES M. Gostling. Illustrated. Third Edition. Cr. 8vo. 6s.

Lees (Frederick). A SUMMER IN TOURAINE. Illustrated. Second Edition. Demy 8vo. , ros. 6 d. net.

Lindsay (Lady Mabel). ANNI DOMINI : A Gospel Study. With Maps. Two Volumes. Super Royal 8vo. ros. net.

Llewellyn (Owen) and Raven-Hill (L.). THE SOUTH-BOUND CAR. Illustrated. Cr. 8vo. 6s.

Lock (Walter), D.D., Warden of Keble College. ST. PAUL, THE MASTERBUILDER. Second Edition. Cr. 8vo. 3s. $6 d$.

THE BIBLE AND CHRISTIAN LIFE. Cr. 8vo. 6s.

Lodge (Sip Oliver), F.R.S. THE SUBSTANCE OF FAITH, ALLIED WITH SCIENCE: A Catechism for Parents and Teachers. Tenth Edition. Cr. 8vo. 2s. net. MAN AND THE UNIVERSE: A STUDY OF THE INFLUENCE OF THE ADVANCE IN SCIENTIFIC KNOWLEDGE UPON OUR UNDERSTANDING OF CHRISTIANITY. Seventh Edition. Demy 8vo. 75. 6d. net.

THE SURVIVAL OF MAN. A STUDY IN UNRECOGNISED HUMAN FACULTY. Fourth Edition. Demy 8vo. 75. 6d. net.

Lofthouse (W. F.), M.A. ETHICS AND ATONEMENT." With a Frontispiece. Demy 8vo. 5s. net.

Lorimer (George Horace). LETTERS FROM A SELF-MADE MERCHANT TO HIS SON. Illustrated. Eighteenth Edition. Cr. 8vo. 3s. 6d.

OLD GORGON GRAHAM. Illustrated. Second Edition. Cr. 8vo. 6s.

Lorimer (Norma). BY THE WATERS OF EGYPT. Illustrated. Demy 8vo. I6s. net.

Lucas (E. V.). THE LIFE OF CHARLES LAMB. "Illustrated. Fifth and Revised Edition in One Volume. Demy 8vo. 7s. 6d. net.

A WANDERER IN HOLLAND. Illus. trated. Tenth Edition. Cr. 8vo. 6s.

A WANDERER IN LONDON. Illustrated. Eigluth Edition. Cr. 8vo. 6s. A WANDERER IN PARIS. Illustrated. Fourth Edition. Cr. 8vo. 6s. 
THE OPEN ROAD: A Little Book for Wayfarers. Fifteenth Edition. Fcp. 820 . 5s.; India Paper, 7s. 6 d.

THE' FRIENDLY TOWN : a Little Book for the Urbane. Fifth Edition. Fcap. $8 v 0$. 5s. : India Paper, 75. 6d.

FIRESIDE AND SUNSHINE. Fifth Edition. Fcap. 8ro. 5 s.

CHARACTER AND COMEDY. Fifth Edition. Fcap. 8vo. 5 s.

THE GENTLEST ART. A Choice of Letters by Entertaining Hands. Fifth Edition. Fcap 8vo. 5s.

A SWAN AND HER FRIENDS. Illustrated. Demy 8vo. r2s. 6 d. net.

HER INFINITE VARIETY : A FRMININE Portrait Gallery. Fourth Edition. Fcap. 8vo. 5s.

LISTENER'S LURE: AN OBLIQUE NARRATION. Sixth Edition. Fcap. 8vo. 5 s.

GOOD COMPANY: A Raley of MeN. Second Edition. Fcap. 8vo. $5 s$.

ONE DAY AND ANOTHER. Fourth Edition. Fcas. 8vo. 5s.

OVER BEMERTON'S: AN EASY-GorNG Chronicle, Seventh Edition. Fcap. 8vo. 5s. net.

M. (R.). THE THOUGHTS OF LUCIA HALLIDAY. With some of her Letters. Edited by R. M. Fcap. 8vo. 2s. 6d. net.

Macaulay (Lord). CRITICAL AND HISTORICAL ESSAYS. Edited by F. C. Montagur. MI.A. Three Volumes. Cr. 8 vo. $\quad r 8 \mathrm{~s}$.

McCabe (Joseph) (formerly Very Rev. F. ANTONY, O.S.F.). THE DECAY OF THE CHURCH OF ROME. Second Edition. Demy 8vo. 7s. 6 d. net.

McCullagh (Francis). The Fall of Abd-ul. Hamid. Illustrated. Demy $8 v 0$. ros. $6 d$. net.

MacCunn (Florence A.). MARY STUART. Illustrated. New and Cheaper Edition. Large Cr. 8vo. 6s.

MeDougall (William), M.A. (Oxon., M.B. (Cantab.). AN INTRODUCTION TO SOCIAL PSYCHOLOGY. Second Edition. Cr. 8vo. 5s. net.

'Mdlle. Mori' (Author of). ST. CATHER. INE OF SIENA AND HER TIMES. Illustrated. Second Edition. Demy 8vo. 7s. 6d. net.

Maeterlinck (Maurlee). THE BLUE BIRD: A FaIRY PLAY IN Five ACTs. Translated by Alexander TeIXeIRA DE MatTos. Eighth Edition. Fcap. 8vo. Deckle Edges. 3s. 6d. net. Also Fcap. $8 v 0$. Paper covers, rs. net.

Mahaffy (J. P.), Litt.D. A HISTORY OF THE EGYPT OF THE PTOLEMIES. Illus trated. Cr. 8vo. 6s.

Maitland (F. W.), M.A., L.L.D. ROMAN CANON LAW IN THE CHURCH OF ENGLAND. Royal 8vo. 7s. 6d.
Marett (R. R.), M.A., Fellow and Tutor of Exeter College, Oxford. THE THRES. HOLD OF RELIGION. Cr. $8 v 0.35 .6 \%$ net.

Marriott (Charles). A SPANISH HOLI. DAY. Illustrated. Demy 8vo. 7s. 6d. net.

Marpiott (J. A. R.), M.A. THE LIFE AND TIMES OF LORD FALKLAND. Illustrated. Second Edition. Demy $8 v 0$. 7s. 6d. net.

Maseffeld (John). SEA LIFE IN NEL. SON'S TIME. Illustrated. Cr. 8 vo. 3s. 6 d. net.

A SAILOR'S GARLAND. Selected and Edited. Second Edition. Cr. 8vo. 3s. $6 d$. net.

AN ENGLISH PROSE MISCELLANY. Selected and Edited. Cr. 8vo. 6s.

Masterman (C. F. G.), M.A., M.P. TENNYSON AS A RELIGIOUS TEACHER. Cr. 8vo. 6s.

THE CONDITION OF ENGLAND. Third Edition. Cr. 8vo. 6s.

Mayne (Ethel Colburn). ENCHANTERS OF MEN. Illustrated. Demy 8vo. ros. $6 d$. net.

Meakin (Annette M. B.), Fellow of the Anthropological Institute. WOMAN IN TRANSITION. Cr.8vo. 6s.

GALICIA : The Switzerland of SPaiN. Illustrated. Demy 8vo. I2s. 6d. net.

Medley (D. J.), M.A., Professor of History in the University of Glasgow. ORIGINAL, ILLUSTRATIONS OF ENGLISH CONSTITUTIONAL HISTORY, COMPRISING a Selected Number of thr Chief Charters and Statuths. Cr.8vo. 7s.6d. net.

Methuen.(A. M. S.), M.A. THETRAGEDY OF SOU'H AFRICA. Cr. 8vo. 2s, net. ENGLAND'S RUIN : DISCUSSED IN FOURteen LetTers to a Protectionist. Ninth Edition. Cr. 8vo. 3d. net.

Meynell (Everard). COROT AND HIS FRIENDS. Illustrated. Demy 8vo. ros. $6 d$. net.

Miles (Eustace), M.A. LIFE AFTER LIFE: OR, THE THEORY OF REINCARNATION. Cr. 8vo. 2s. 6d. net.

THE POWER OF CONCENTRATION : How to ACQUIRE IT. Third Edrtion. Cr. 8vo. 3s. 6d. net.

Millais (J. G.). THE LIFE AND LET. TERS OF SIR JOHN EVERETT MILLAIS, President of the Royal Academy. Illustrated. Neze Edition. Demy $8 v 0$. 7s. 6d. net.

Milne (J. G.), M.A. A HISTORY OF EGYPT UNDER ROMAN RULE. Illustrated. Cr. 8vo. 6s. 


\section{General Literature}

Mitton (G. E.), JANE AUSTEN AND HER TIMES. Illustrated. Second and Cheaper Eaition. Large Cr. 8vo. 6s.

Moffat (Mary M.). QUEEN LOUISA OF PRUSSIA. Illustrated. Fourth Edition. Cr. 8vo. 6s.

Money (L. G. Chlozza). RICH ES AND POVERTY. Ninth Edition. Demy $8 v 0$. 5s. net. Also $C$ r. $8 v 0$. rs. net.

MONEY'S FISCAL DICTIONARY, Igro. Demy 8vo. 5s. net.

Moore (T. Sturge). ART AND LIFE. Illustrated. Cr.8vo. 5s. net.

Moorhouse (E. Hallam). NELSON'S LADY HAMILTON. Illustrated. Second Edition. Demy 8vo. 7s. 6d. net.

Morgan (J. H.), M.A. THE HOUSE OF LORDS AND THE CONSTITUTION. With an Introduction by the LORD Chancellor. Cr.8vo. xs. net.

Morton (A. Anderson). See Brodrick (M.).

Norway (A. H.). NAPLES. PAST AND PRHSENT. Illustrated. Third Edition. Cr. 8vo. 6s.

Oman (C. W. C.), M.A., Fellow of All Souls', Oxford. A HISTORY OF THE ART OF WAR IN THE MIDDLE AGES. Illustrated. Demy 8vo. ros. $6 d$. net.

- ENGLAND BEFORE THE CONQUEST. With Maps. Demy $8 v 0$. Ios. 6 d. net.

Oxford (M. N.), of Guy's Hospital. A HANDBOOK OF NURSING. Fifth Edition. Cr. $8 v 0$. 3s. 6 d.

Pakes (W. C. C.). THE SCIENCE OF HYGIENE. Illustrated. Demy 8vo. I5s.

Parker (Eric). THE BOOK OF THE ZOO: BY DAY AND Night. Illustrated. Second Edition. Cr. 8200. 6s.

Parsons (Mrs. C.). THE INCOMPARABLE SIDDONS. Illustrated. Demy 8vo. 12s. 6d. net.

Patmore (K. A.). THE COURT OF LOUIS XIII. Illustrated. Third Edition. Demy 8vo. 10s. 6d. net.

Patterson (A. H.). MAN AND NATURE ON TIUAL WATERS. Illustrated. $C r$. 8vo. 6s.

Peel (Robert), and Minchin (H. C.), M.A. OXFORD. Illustrated. Cr. 8vo. $6 s$.

Petrie (W. M. Flinders), D.C.L., LL.D., Professor of Egyptology at University College. A HISTORY OF EGYPT. Illustrated. In Six Volumes. Cr. 8vo. 6s. each.
Vol. I. From thB EARlizst Kings to XVITh DYNasty. Sixth Edition.

VOL. II. THE XVIITH AND XVIIITH Dynasties. Fourth Edition.

Vol. III. XIXTh to XXXTh Dynasties.

Vol IV. Egypt under the Ptolrmaic Dynasty. J. P. MAHAFFy, Litt.D.

VOL. V. EGYPT UNDER ROMAN RULE. J. G. MiLne, M.A.

Vol. VI. EgYPT in THE MrDdle Ages. Stanley Lane-Poole, M.A.

RELIGION AND CONSCIENCE IN ANCIENT EGYPT. Lectures delivered at University College, London. Illustrated. Cr. 8vo. 2s. $6 d$.

SYRIA AND EGYPT, FROM THE TELL EL AMARNA LETTERS. Cr. $8 v 0$. 2s. $6 d$.

EGYPTIAN TALES. Translated from the Papyri. First Series, Ivth to Xirth Dynasty. Edited by W. M. Flinders Petrie. Illis. trated. Second Edition. Cr. 8vo. 3s.6d.

EGYPTIAN TALES. Translated from the Papyri. Second Series, xvinth to xixth Dynasty. Illustrated. Cr. 8vo. 3s. 6d. EGYPTIAN DECORATIVE ART. A Course of Lectures delivered at the Royal Institution. Illustrated. Cr. 8vo. 3s. 6d.

-Phelps (Ruth S.). SKIES ITALIAN : A LiTTLE BREviary for Travellers iN ITALY. Fcap.8vo. 5s, net.

Phythian (J. Ernest). TREES IN NA. TURE, MYTH, AND ART. Illustrated. Cr. 8vo. 6s.

Podmore (Frank). MODERN SPIRIT. UALISM. Two Volumes. Demy $8 v 0$. 21 s. net.

MESMERISM AND CHRISTIAN SCIENCE: A Short History of Mental Healing. Second Edition. Demy 8vo. ros. 6 d. net.

Pollard (Alfred W.). SHAKESPEARE FOLIOS AND QUARTOS. A Study in the Bibliography of Shakespeare's Plays, 1594-1685. Illustrated. Folio. 2rs. net.

Powell (Arthur E.). FOOD AND HEALTH. Cr. 8vo. 3s. 6 d. net.

Power (J. O'Connor). THE MAKING OF AN ORATOR. Cr. 8vo. 6s.

Price (L. L.), M.A., Fellow of Oriel College, Oxon. A HISTORY OF ENGLISH POLITICAL ECONOMY FROM ADAM SMITH TO ARNOLD TOYNBEE. Sixth Edition. Cr.8vo. 2s. 6 d.

Pullen-Burry (B.). IN A GERMAN COLONY; or, FOUR WEEKS IN NEW Britain. Illustrated. Cr. 8vo. 5s, net.

Pycraft (W. P.). BIRD LIFE. Illustrated. Demy 8vo. ros. 6 d. net. 
Rago (Lonsdale), B.D. Oxon. DANTE AND HIS ITALY. Illustrated. Demy 8vo. r2s. 6 d. net.

-Rappoport (Angelo S.). HOME LIFE IN RUSSIA. Illustrated. Demy 8vo. xos. $6 d$. net.

Raven-Hill (L.). See Llewellyn (Owen).

Rawlings (Gertrude). COINS AND HOW TO KNOW THEM. Illustrated. Second Edition. Cr. 8vo. 5s. net.

Rea (LIlian). THE LIFE AND TIMES OF MARIE MADELEINE COUNTESS OF LA FAYETTE. Illustrated. Dewy 8vo. ros. $6 d$. net.

Read (C. Stanford), M.B. (Lond.), M.R.C.S., L.R.C.P. FADS AND FEEDING. Cr. 8vo. 2s. 6d. net.

Rees (J. D.), C.I.E., M.P. THE REAL INDIA. Second Edition. Demy $8 v 0$. ros. $6 d$. net.

Reich (Emil), Doctor Juris. WOMAN THROUGH' THE AGES. Illustrated. Two Volumes. Demy 8vo. 21s. net.

Reid (Archdall), M.B. The Laws of Here. dity. Demy 8vo. 2is. net.

Richmond (WIIfrid), Chaplain of Lincoln's Inn. THE CREED IN THE EPISTLES. Cr. 8vo. 2s. 6d. net.

Roberts (M. E.). See Channer (C.C.).

Robertson (A.), D.D., Lord Bishop of Exeter. REGNUM DEI. (The Bampton Lectures of rgor.) $A$ New and Cheaper Edition. Demy 8vo. 7s.6d. net.

Robertson (C. Grant), M.A., Fellow of All Souls' College, Oxford. SELECT STATUTES, CASES, AND CONSTITUTIONAL DOCUMENTS, ז660-1832. Demy 8vo. ros. 6d. net.

Robertson (Sir G. S.), K.C.S.I. CHITRAL: The STORY OF A MinOR Siege. Illustrated. Third Edition. Demy 8vo. ros. 6d. net.

Roe (Fred). OLD OAK FURNITURE. Illustrated. Second Edition. Demy 8vo. ros. 6 . net.

Royde-Smith (N. G.). THE PILLOW BOOK: A Garner of Many Moods. Collected. Second Edition. Cr. 8vo. 4s. 6d. net.

POETS OF OUR DAY. Selected, with an Introduction. Fcap. 8vo. $5^{5}$.

Rumbold (The Right Hon. Sir Horace), Bart., G. C. B., G. C. M. G. TH E AUS'TRIAN COURT IN THE NINETEENTH CENTUR Y. Illustrated. Second Edition. Demy 8vo. r8s. net.

Russell (W. Clark). THE LIFE OF ADMIRAL LORD COLLINGWOOD. Illustrated. Fowrth Edition. Cr. 8vo. 6s.
Ryley (M. Beresford). QUEENS OF I'HE RENAISSANCE. Illustrated. Demy $8 v 0$. ros. $6 d$. net.

St. Francis of Assisi. THE LITTLE FLOWERS OF THE GLORIOUS MESSER, AND OF HIS FRIARS. Done into English, with Notes by William Heywood. Illustrated. Demy 8vo. 5s. net.

'Saki' (H. Munro). REGINALD. Second Edition. Fcap. 8vo. 2s. 6d. net.

REGINALD IN RUSSIA. Fcap. $8 v 0$. 2s. $6 d$. net.

Sanders (Lloyd). TH E HOLL A N D HOUSE CIRCLE. Illustrated. Second Edition. Demy 8vo. r2s. 6d. net.

* Scott (Ernest). TERRE NAPOLÊON, AND THE EXPEDITION OF DISCOVERY DESPATCHED TO AUS. TRALIA BY ORDER OF BONAPAR'TE, 1800-1804. Illustrated. Demy $8 v 0$. Ios. $6 d$. net.

Sélincourt (Hugh de). GREAT RALEGH. Illustrated. Demy 8vo. ros. $6 d$. net.

Selnus (Edmund). TOMMY SMITH'S ANIMALS. Illustrated. Eleventh Edition Fcap. 8vo. 2s. 6d.

TOMMY SMI'TH'S OTHER ANIMALS. Illustrated. Fifth Edition. Fcap. 8vo. 2s. $6 d$.

- Shafer (Sara A.). A. WHITE PAPER GARDEN. Illustrated. Demy 8vo. 7s.6d. net.

Shakespeare (William).

THE FOUR FOLIOS, 1623 ; 1632 ; $x 664$; 1685. Each $£_{4} 4^{s}$. net, or a complete set, $£ 12$ 12s. net.

Folios 2,3 and 4 are ready.

THE POEMS OF WILLIAM SHAKESPEARE. With an Introduction and Notes by George Wyndham. Demy 8vo. Buckram, gilt top. ros. $6 d$.

Sharp (A.). VICTORIAN POETS. $C r$. 8vo. 2s. $6 d$.

Sidgwick (Mrs. Alfred). HOME LIFE IN GERMANY. Iilustrated. Second Edition. Demy 8vo. ros. 6d. net.

Sime (John). See Little Books on Art.

Sladen (Douglas). SICILY: The New Winter Resort. Illustrated. Second Edition. Cr. 8vo. 5s. net.

Smith (Adam). THE WEALTH OF NATIONS. Edited with an Introduction and numerous Notes by EDwin CANNAN, M.A. Two Volumes. Demy 8vo. 21s, net.

Smith (Sophia S.). DEAN SWIFT. Illustrated. Demy 8vo. ros. 6 d. net.

Snell (F. J.). A BOOK OF EXMOOR. Illustrated. Cr. 8vo. 6s.

'Stancliffe.' GOLF DO'S AND DONT'S. Second Edition. Fcap. 8vo. Is. 


\section{General Literature}

Stead (Francis H.), M.A. HOW OLD AGE PENSIONS BEGAN TO BE. Illustrated. Demy 8vo. 2s. $6 d$. net.

Stevenson (R. L.). THE LETTERS OF ROBERT LOUIS STEVENSON TO HIS FAMILY AND FRIENDS. Selected and Edited by Sidney Colvin. Eighth Edition. Two Volumes. Cr. 8vo. I2s.

VAILIMA LETTERS. With an Etched Portrait by WILLIAM STRANG. Seventh Edition. Cr. 8vo. Buckram. 6s.

THE LIFE OF R. L. STEVENSON. See Balfour (G.).

Stevenson (M. I.) FROM SARANAC TO THE MARQUESAS. Being Letters written by Mrs. M. I. Stevenson during 1887-88. Cr. 8vo. 6s. net.

LETTERS FROM SAMOA,'x891-95. Edited and arranged by M. C. BALFour. Illustrated. Second Edition. Cr. 8vo. 6s. net.

Store (Vernon F.), M.A., Canon of Win. chester. DEVELOPMENT AND DIVINE PURPOSE. Cr. 8vo. 5s. net.

Streatielld (R. A.). MODERN MUSIC AND MUSICIANS. Illustrated. Second Edition. Demy 8vo. 7s. 6d. net.

Swanton (E. W.). FUNGI AND HOW TO KNOW THEM. Illustrated. $C r .8 v 0$. 6s. net.

-Sykes (Ella C.). PERSIA AND ITS PEOPLE. Illustrated. Demy 8vo. ros. $6 d$. ret.

Symes (J. E.). M.A. THE FRENCH REVOLUTION. Second Edition. Cr. 8v0. 2s. $6 d$.

Tabor (Margaret E.). THE SAINTS IN ART. Illustrated. Fcap. 8vo. 3s. 6d. net.

Taylor (A. E.) THE ELEMENTS OF METAPHYSICS. Second Edition. Demy 8vo. ros. 6 d. net.

Taylor (John W.). THE COMING OF THE SAINTS. Illustrated. Demiy $8 v 0$. 7s. 6 d. net.

Thibaudeau (A. C.). BONAPARTE AND THE CONSULATE. Translated and Edited by G. K. Fortrscue, LL.D. Illustrated. Demy 8vo. ros. 6 d. net.

Thompson (Francis). S E L E C T E D POEMS OF FRANCIS THOMPSON. With a Biographical Note by WILFRID Meynel. With a Portrait in Photogravure. Second Edition. Ficap. 8vo. 5s. net.

Tileston (Mary W.). DAILY STRENGTH FOR DAILY NEEDS. Sixteenth Edition. Medium $16 m o$. 2s. 6 d. net. Also an edition in superior binding, $6 s$.

Toynbee (Paget), M.A., D. Litt. DANTE IN ENGLISH LITERATURE : FROM
CHAUCER TO CARY. Two Volumes. Demy 8vo. 2rs. net.

See also Oxford Biographies.

Tozer (Basil). THE HORSE IN HISTORY. Illustrated. $C r .8 v 0$. 6 s.

Trench (Herbert). DEIRDRE WEDDED AND OTHER POEMS. Second and Revised Edition. Large Post 8vo. 6s.

NEW POEMS. Second Edition. Large Post 8vo. 6s.

APOLLO AND THE SEAMAN. Large Post 8vo. Paper, 1s. 6d. net; cloth, 2s. 6d. ret.

Trevelyan (G. M.), Fellow of Trinity College, Cambridge. ENGLAND UNDER THE STUARTS. With Maps and Plans. Third Edition. Demy 8vo. ros. 6d. net.

Triggs (Inigo H.), A.R.I.B.A. TOWN PLA N N I N G: PAst, PRESENT, AND Possible. Illustrated. Wide Royal 820. r5s. net.

Vaughan (Herbert M.), B.A.(Oxon). THE LAST OF THE ROYAL STUARTS, HENRY STUART, CARDINAL, DUKE OF YORK. Illustrated. Second Edition. Demy 8vo. ras. $6 d$. net.

THE MEDICI POPES (LEO X. AND CLE MENT VII.). Illustrated. Demy $8 v a$. 15 s. net.

THE NAPLES RIVIERA. Illustrated. Second Edition. Cr. 8vo. 6s.

* FLORENCE AND HER TREASURES. Illustrated. Fcap.8vo. 5s. net.

Vernon (Hon. W. Warren), M.A. READ. INGS ON THE INFERNO OF DANTE, With an Introduction by the REv. I) $R$. MOORE Two Volumes. Second Edition. Cr. 8vo. 15s. net.

READINGS ON THE PURGATORIO OF DANTE. With an Introduction by the late DEAN CHURCH. Two Volumes. Third Edition. Cr. 8vo. 15s. net.

READINGS ON THE PARADISO OF DANTE. With an Introduction by the Bishop of Ripon. Two Volunes. Second Edition. Cr. 8vo. I5s, net.

Vineent (J. E.). THROUGH EAST ANGLIA IN A MOTOR CAR. Illus. trated. Cr. 8vo. 6s.

Waddell (Col. L. A.) LL.D., C.B. LHASA AND ITS MYSTERIES. With a Record of the Expedition of $1903^{-1904 . ~ I l l u s t r a t e d . ~}$ Third and Cheaper Edition. Medium $8 v 0$. 7s. 6d. net.

Wagner (Richard). RICHARD WAG. NER'S MUSIC DRAMAS: Interpreta. tions, embodying Wagner's own explanations. By Alich Leighton Cleather and BAsil CRUMr. In Three Volumes. Fcap. 8vo. 2s. 6d. each.

Vol. 1.-The RING of the NiBeIUNG. Thind Edition. 
VOL. II. - PARSIFAL, LOHENGRIN, and The HoLy Grall.

Vol. III.-TRISTAN AND IsOLDE.

Waineman (Paul). A SUMMER TOUR IN FINLAND. Illustrated. Demy $8 v 0$. ros. $6 d$. net.

Walkley (A. B.). DRAMA AND LIFE. Cr. 8vo. 6s.

Waterhouse (Elizabeth). WITH THE SIMPLE-HEARTED : Little Homilies to Women in Country Places. Second Edition. Small Pott 8vo. 2s. net.

COMPANIONS OF THE WAY. Being Selections for Morning and Evening Reading. Chosen and arranged by ELIZABETH WAterhouse. Large Cr. 8vo. 5s. net.

THOUGHTS OF A TERTIARY. Second Edition. Small Pott 8vo. rs. net.

Watt (Francis). See Henderson (T. F.).

Weigall (Arthur E. P.). A GUIDE TO THE AN TIQUITIES OF UPPER EGYPT: From Abydos to the Sudan Frontier. Illustrated. Cr. 8vo. 75. 6d. net.

Welch (Catharine). THE LI T T LE DAUPHIN. Illustrated. Cr. $8 v 0$. 6s.

Wells (J.), M.A., Fellow and Tutor of Wadham College. OXFORD AND OXFORD LIFE. Third Edition. Cr. 8vo. 3s. $6 d$.

A SHORT HISTORY OF ROME. Ninth Edition. With 3 Maps. Cr. $8 v 0$. 3s. 6 d.

Westell (W. Percival). THE YOUNG NATURALIST. Illustrated. Cr. 8vo. $6 s$.

Westell (W. Percival), F.L.S., M.B.O.U., and Cooper (C. S.), F.R.H.S. THE YOUNG BOTANIST. Illustrated. $C r$. 8v0. 3s. 6d. net.

-Wheoler (Ethel R.). FAMOUS BLUE STOCKINGS. Illustrated. Demy $8 v 0$. ros. $6 d$. net.

Whibley (C.). See Henley (W. E.).

White (George F.), Lieut.-Col. A CENTURY OF SPAIN AND PORTUGAL, r788-1898. Demy 8vo. x2s. 6d. net.

Whitley (Miss). See Dilke (Lady).

Wilde (Osear). DE PROFUNDIS. Truelfth Edition. Cr. 8vo. 5s. net.

THE WORKS OF OSCAR WILDE. In Twelve Volwmes. Ficap. 8vo. 55. net each volume.

I. LORD ARthur SAvir.e's CRIME AND the Portrait of Mr. W. H. II. The
Duchess of Padua. III. Poems. Iv. LADY WINDERMERE'S FAN. v. A WOMAN of No Importance. VI. AN Ideal HusBAND. VII. THE IMPORTANCE OF BEING Earnest. vili. A House of POMEgranates. IX. Intentions. $\mathrm{X}$. DE ProFUNDIS AND PRISON LeTters. XI. EsSays. xir. Salome, a Florentine Tragedy, and La Sainte Courtisane.

Williams (H. Noel). THE WOMEN BONAPARTES. The Mother and three Sisters of Napoleon. Illustrated. In Two Volumes. Demy 8vo. 24s. net.

A ROSE OF SAVOY : Marie Adelétde of Savoy, Duchesse de Bourgogne, Mother of Louis $x v$. Illustrated. Second Edition. Demy 8vo. 15s. net.

-THE FASCINATING DUC DE RICHELIEU: LouIS FRANÇOIS ARMAND DU Plessis, Marfehal Dú de Richrlieu. Illustrated. Demy 8vo. 15s. net.

Wood (Sir Evelyn), F.M., V.C., G.C.B., G.C.M.G. FROM MIDSHIPMAN TO FIELD-MARSHAL. Illustrated. Fifth and Cheaper Edition. Demy 8vo. 7s. 6d. net.

THE REVOLT IN HINDUSTAN. $1857-$ 59. Illustrated. Second Edition. Cr. 8vo. 6s.

Wood (W. Birkbeck), M.A., late Scholar of Worcester College, Oxford, and Edmonds (Major J. E.), R.E., D.A.Q.-M.G. A HISTORY OF THE CIVIL WAR IN THE UNITED STATES. With an Introduction by $H$. SPENSER WILKINSON. With 24 Maps and Plans. Second Edition. Demy 8vo. x2s. 6 d. net.

Wordsworth (W.). THE POEMS. With an Introduction and Notes by NoweLL C. Smith, late Fellow of New College, Oxford. In Three Volumes. Demy 8vo. 15s. net.

POEMS BY WILLIAM WORDSWORTH. Selected with an Introduction by STOPFORD A. BRookr. Illustrated. Cr. 8vo. 75. 6d. net.

Wyatt (Kate M.). See Gloag (M. R.).

Wyllie (M. A.). NORWAY AND ITS FJORDS. Illustrated. Second Edition. Cr. 8vo. 6s.

Yeats. (w. B.). A BOOK OF IRISH VERSE. Revised and Enlarged Edition. Cr. 8v0. 35. 6d.

Young (Fllson). See The Complete Series. 


\section{Part II.-A Selection of Series.}

\section{Ancient Cities.}

General Editor, B. C. A. WINDLE, D.Sc., F.R.S.

Cr. 8vo. 4s. 6d. net.

With Illustrations by E. H. NEw, and other Artists.

RRIstoz. By Alfred Harvey, M.B.

Cantrirbury. By J. C. Cox, LL.D., F.S.A.

Chester. By B. C.A. Windle, D.Sc., F.R.S.

Dublin. By S. A. O. Fitzpatrick.
Edinburgh. , By M. G. Williamson, M.A. Lincoln. By E. Mansel Sympson, M.A. ShrewsburY. By T. Auden, M.A., F.S.A. Wells and Glastonburr. By T. S. Holmes.

\section{The Antiquary's Books.}

General Editor, J. CHARLES COX, LL.D., F.S.A. Demy 8vo. 7s. 6d. net.

With Numerous Illustrations.

Arch rozogt and Falsa Antiquities. By R. Munro.

Bells of England, Thr. By Canon J. J. Raven. Second Edition.

Brasses of England, The By Herbert W. Macklin. Second Edition.

Celtic ARt in Pagan and Christian 'Trmas. By J. Romilly Allen.'

Domrsday Inquest, The. By Adolphus Ballard.

English Church Furniture. By J.C. Cox and A. Harvey. Second Edition.

Exglish Costume. From Prehistoric Times to the End of the Eighteenth Century. By George Clinch.

ENGLish Monastic Lirs. By the Right Rev. Abbot Gasquet. Fourth Edition.

English Seals. By J. Harvey Bloom.

Folk-Lore as an Historical SCibnice. By G. L. Gomme.
Gilds and Companies of London, The. By George Unwin.

Manor and Manorial Records, The. By Nathaniel J. Hone.

Medifval Hospitals of Eingland, The. By Rotha Mary Clay.

Old Service Books of The ENGLish Church. By Christopher Wordsworth, M. A., and Henry Littlehales.

Parish Life in Mediaval England. By the Right Rev. Abbott Gasquet. Secont Edition.

- Parish Registers of England, The. By J. C. Cox.

REMAins of the Prehistoric AgE IN England. By B. C. A. Windle. Second Edition.

RoYal Forests of ENGland, $T_{\text {HB. }}$ By J. C. Cox, LL.D.

Shrines of British Saints. By J. C. Wall.

\section{The Arden Shakespeare.}

Demy 8vo. 2s. 6 . net each volume.

An edition of Shakespeare in single Plays. Edited with a full Introduction, Textual Notes, and a Commentary at the foot of the page.

All's Well That Ends Well.

Antony and Cleopatra.

CYMBELINE.

COMEDY OF ERRORS, THE.

HAMLET. Second Edition.

Julius CAESAR.

King HENRY v.

KiNg HeNRY Vi. PT. I.

KING HENRY VI. PT. II.

King HeNRY vi. PT. IIr.

KING LEAR.

KING RICHARD III.

LiFE AND DEATH OF KING JohN, ThE.

Love's Labour's Lost.

МАСветн.
MEASURE FOR MEASURE.

MERChaNT OF VeNice, THE.

MERRY WIVES OF WINDSOR, THE.

Midsummer Night's Drbam, A.

OTHELLO.

Prricles.

ROMEO AND JULIET.

TAMING OF THE SHREW, THR.

TEMPEST, THE.

Timon of Athens.

Titus ANDRONICUS.

Troilus aNd CRESSIDA.

Two Gentlemen of Verona, The. TWELFTH Night. 


\section{Classics of Art. \\ Edited by DR. J. H. W. LAING. \\ With numerous Illustrations. Wide Royal 8vo. Gilt top.}

The ARt of thz Greeks. By H. B. Walters. I2s. 6d. net.

Florentine Sculptors of THe RenaisSANCE. Wilhelm Bode, Ph.D. Translated by Jessie Haynes. r2s. 6 d. net.

*Grorge Romney. By Arthur B. Chamberlain. 12s. 6d. net.

Ghirlandaio. Gerald S. Davies. Second Edition. 10s. 6 d.
Michet.angrio. By Gerald S. Davies. 12s. 6 d. net.

Rubres. By Edward Dillon, M.A. 25s. net. Raphazl. By A. P. Oppé. 12s. 6d. net.

* Titian. By Charles Ricketts. r2s. 6d. net. *Turner's Sketches and Drawings. By A. J. Finberg, 12s. 6d. net.

Velazquez. By A. de Beruete. ros. 6 d. net.

\section{The "Complete" Series. \\ Fully Illustrated. Demy 8vo.}

The Comprete Cook. By Lilian Whitling. 7s. 6 d. net.

The Complete Cricketer. By Albert E. KNIGHT. 75. 6d. net.

The Complete Foxhunter. By Charles Richardson. 12s. 6d. net. Second Edition.

The Complete Golfer. By Harry Vardon. ros. 6d. net. Tenth Edition.

The Complete Hockey-Pla yer. By Eustace E. White. 5s. net. Second Edition.

Thr Complete Lawn Tennis Player. By, A. Wallace Myers. ros. $6 d$. net. Second Edition.
The iComplete Motorist. By Filson Young. r2s. 6d. net. Nerw Edition (Seventh).

The Complete Mountaineer. By G. D. Abraham. 15s. net. Second Edition.

The Complete Oarsman. By R. C. Lehmann, M.P. Ios. $6 d$. net.

The Complete Photographer. By R. Child Bayley. xos. 6d. net. Fourth Edition.

The Complete Rugby Footballer, on the New Zealano System. By D. Gallaher and W. J. Stead. ros. 6 d. net. Second Edition.

The Complete Shot. By G. T. Teasdale Buckell. 12s. 6 d. net. Third Edition.

\section{The Connoisseur's Library.}

With numerous Illustrations. Wide Royal 8vo. Gilt top. 25s. net.

ENGLish Furniture. By F. S. Robinson. Second Edition.

ENGLish Coloured Books. By Martin Hardie.

European Enamezs. By Henry H. Cunynghame, C.B.

Guass. By Edward Dillon.

Golnsmiths' and Silversmiths' Work. By Nelson Dawson. Second Edition.
*Illuminated Manuscripts. By J. A. Herbert.

IVories. By A. Maskell.

Jewrllery. By H. Clifford Smith. Second Edition.

Mezzotints. By Cyril Davenport.

Miniatures. By Dudley Heath.

Porcelain. By Edward Dillon.

Seals. By Walter de Gray Birch. 


\title{
Handbooks of English Church History.
}

\author{
Edited by J. H. BURN, B.D. Crown 8vo. 2s. 6d. net.
}

The Foundations of THE ENGLish ChuRCh. By J. H. Maude.

The Saxon Church and the Norman ConQUest. By C. T. Cruttwell.

The Mediaval Church and the Papact. By A. C. Jennings.
The Reformation Period. By Henty Gee.

The Struggle witm Puritanism. By Bruce Blaxland.

The Church of England in The Eighteenth Century. By Alfred Plummer.

\section{The Illustrated Pocket Library of Plain and Coloured Books.}

Fcap. 8vo. 3s. 6d. net each volume.

\section{WITH COLOURED ILLUSTRATIONS.}

Old Colouran Books. By George Paston. 2s. net.

The Life and Death of John Mytton, EsQ. By Nimrod. Fifth Edition.

The Life of a Sportsman. By Nimrod.

Handley Cross. By R. S. Surtees. Third Edition.

Mr. Sponge's Sportixg Tour. By R. S. Surtees.

JORROCKS' JAUNTS AND JOLLITIES. By R. S. Surtees. Second Edition.

Ask Mamma. By R. S. Surtees.

The Analysis of the Hunting Field. By R. S. Surtees.

The TOUR OF Dr. Syntax IN Search of the Picturesque. By William Combe.

The TOUR OF Dr. Syntax in SEarch or Consolation. By William Combe.

The Third Tour of Dr. Syntax in Search OF A WIfE. By William Combe.

The History of Johnny Quae Genus. By the Author of "The Three Tours."

The ENGLish DanCE of Drath, from the Designs of $T$. Rowlandson, with Metrical Illustrations by the Author of 'Doctor Syntax.' Two Volumes.

\section{WITH PLAIN ILLUSTRATIONS.}

The Grave: A Pocm. By Robert Blair.

ILI.ustrations OF THE BOOK OF JOB. Invented and engraved by William Blake.

Windsor Castle. By W. Harrison Ains. worth.

The TOWer of London. By W. Harrison Ainsworth.
The Dance of Life: A Poem. By the Author of 'Dr. Syntax.'

Lifz in London. By Pierce Egan.

Real Life in London. By an Amateur (Pierce Egan). Two Volumes.

The Life of an Actor. By Pierce Egan.

The Vicar of Wakefield. By Oliver Goldsmith.

The MILITARY ADVENTURES OF JOHNNY Newcombe. By an Officer.

The National Sports of Great Britain. With Descriptions and 50 Coloured Plates by Henry Alken.

The Adventures of a Post Captain. By a Naval Officer.

Gamonia. By Lawrence Rawstone, Esq.

AN ACAdemy for Grown Horsemen. By Geoffrey Gambado, Esq.

Real Life in Ireland. By a Real Paddy.

The Adventures of JoHnNy Newcombe im THE Navy. By Alfred Burton.

The Old English Squire. By John Careless, Esq.

The ENGLish Spy. By Beraard Blackmantle. Twe Volumes. 7s. net.
Frank Fatrlegh. By F. E. Smedley.

HANDY ANDY. By Samuel Lover.

The Compleat Angler. By Izaak Walton and Charles Cotton.

The Pickwick Fayers. By Charles Dickens. 


\section{Leaders of Religion.}

Edited by H. C. BEECHING, M.A., Canon of Westminster. With Portraits. Crown 8vo. 2s. net.

Cardikal Newman. By R. H. Hutton. John Wesley. By J. H. Overton, M.A. Bishor WILBerForce. By G. W. Daniell, M.A.

Cardinal Manning. By A. W. Hutton, M.A.

Charles Simbon. By H. C. G. Moule, D.D. Јонn Knox. By F. MacCunn. Second Edition.

John Howe. By R. F. Horton, D.D.

Thomas Ken. By F. A. Clarke, M.A.

Grorgr Fox, the QuAKer. By T. Hodgkin, D.C.L. Third Edition.
John Keble. By Walter Lock, D.D.

Thomas Chalmers. By Mrs. Oliphant.

Lancelot Andrewes. By R. L. Ottley, D.D. Second Edition.

Augusting of Canterbury. By E. L Cutts, D.D.

William Laud. By W. H. Hutton, M.A. Third Edition.

John Donne. By Augustus Jessop, D.D.

Thomas Cranmer. By A. J. Mason, D.D.

Bishop Latimer. By R. M. Carlyle and A. J. Carlyle, M.A.

Bishor Butler. By W. A. Spooner, M.A.

\section{The Library of Devotion.}

With Introductions and (where necessary) Notes.

Small Pott 8vo, gilt top, cloth, 2s. ; leather, 2s. 6d. net.

The Confessions or St. Augustine. Seventh Edition.

The Imtation of Christ. Fifth Edition. The Christian Year. Fiourth Edition. LYRA INNOCENTIUM. Second Edition.

The Temple. Second Edition. .

A Book of Devotrons. Second Edition.

A Serious Call to a Devout and Holy LIFE. Frourth Edition.

A Guide to Eternity.

The INNBr Way. Second Edition.

ON THE LOVE OF GOD.

The Psalms of David.

LyRa Apostolica.

The Song of Songs.

The Thoughts of Pascal Second Edition.

A Manual of Consolation from the SAINTS AND Fathers.

DeVOTIONS From the APOCRYPha.

The Spiritual Combat.

The Devotions of St. Anstzu.

Bishor Wilson's Sacra Privata.
Grace Abounding to the Chief of SinNERS.

LYRA SACRA: A Book of Sacred Verse. Second Edition.

A Day Book From the Saints aNd Fathers.

A Little Book of Heavenly Wispom. A Selection from the English Mystics.

Light, LIFE, and Love. A Selection from the German Mystics.

AN Introduction to the Devout Lifk.

The Little Flowers of the Glorious MESSER St. Francis AND of his Friars.

Drath AND ImMORTality.

The Spiritual Guidr.

Devotions for Every Day in the Wegk and the Great Fustivals.

Preces Private.

Hore Mystice: A Day Book from the Writings of Mystics of Many Nations. 


\section{Little Books on Art.}

With many Illustrations. Demy 16 mo. Gilt top. 2s. 6d. net.

Each rolume consists of about 200 pages, and contains from 30 to 40 Illustrations, including a Frontispiece in Photogravure.

Albrecht DURer. J. Allen.

Arts of Japan, The. E. Dillon.

Book plates. E. Almack.

Botricell. Mary L. Bloomer.

Burne-Jones. F. de Lisle.

*Christian Symbolism. Mrs. H. Jenner.

Christ in ARt. Mrs. H. Jenner.

Claudro. E. Dillon.

Constable. H. W. Tompkins.

Corot. A. Pollard and E. Birnstingl.

Enamels. Mrs. N. Dawson.

Frederic Leighton. A. Corkran.

George Romney. G. Paston.

GreEK ART. H. B. Walters.

Greuze and Boucher. E. F. Pollard.

\author{
Holbein. Mrs. G. Fortescue. \\ Illuminated Manuscripts. J. W. Bradley. \\ JeWELlery. C. Davenport. \\ Јоhn Hoppner. H. P. K. Skipton. \\ Sir Joshua Reynolds. J. Sime. \\ Millet. N. Peacock. \\ Miniatures. C. Davenport. \\ Our Lady in Art. Mrs. H. Jenner. \\ Raphael. A. R. Dryhurst. Second Edition. \\ Rembrandt. Mrs. E. A. Sharp. \\ TURner. F. Tyrrell-Gill. \\ VANDyck. M. G. Smallwood. \\ Verasquez. W. Wilberforce and A. $\mathbf{R}$ \\ Giibert. \\ Watrs. R. E. D. Sketchley.
}

\section{The Little Galleries. \\ Demy 16 mo. 2s. 6d. net.}

Each volume contains 20 plates in Photogravure, together with a short outline of the life and work of the master to whom the book is devoted.

A Little Gallery of Reynolds.

A Little Gallery of Romney.

A Little Gallery of Hoppner.
A Little Gallery of Millais.

A Little Gallery of English Poets.

\section{The Little Guides.}

With many Illustrations by E. H. NEW and other artists, and from photographs,

Small Pott 8vo, gilt top, cloth, 2s. 6d. net; leather, 3s. 6d. net.

The main features of these Guides are (I) a handy and charming form ; (2) illustrations from photographs and by well-known artists ; (3) good plans and maps ; (4) an adequate but compact presentation of everything that is interesting in the natural features, history, archæology, and architecture of the town or district treated.

\section{Cambridge and its Colleges. A. H. \\ Thompson. Second Edition.}

ENGlish Lakes, The. F. G. Brabant.

Isle of Wight, The. G. Clinch.

Malvery Country, The. B. C. A. Windle.

North Wales. A. T. Story.

OXFORd AND its Collzges. J. Wells. Eighth Edition.
Shakespearz's Country. B. C. A. Windle. Third Edition.

St. Paul's Cathedral. G. Clinch. Westminster Abbey. G. E. Troutbeck. Second Edition.

Buckinghamshire. E. S. Roscoe,

Cheshire. W. M. Gallichan. 
Thr Litrug Guides-continued.

Cornwallo. A. I. Salmon.

Derbyshire. J. C. Cox.

Devon. S. Baring-Gould.

DORSET. F. R. Heath. Second Edition.

Essex. J. C. Cox.

Hampshire. J. C. Cox.

HeRTFORDSHIRE. H. W. Tompkins.

KeNT. G. Clinch.

Kerry. C. P. Crane.

Middlesex. J. B. Firth.

Monmouthshire. G. W. Wade and J. H. Wade.

NoRFOLX. W. A. Dutt.

NORTHAMPTONSHIRE. W. Dry.

"Northumberland. J. E. Morris.

Nottinghamshire. L. Guilford.
OXFORDSHIRE. F. G. Brabant.

Somerset. G. W. and J. H. Wade.

*Staffordshire. C. E. Masefield.

Sufrolk. W. A. Dutt.

SurReY. F. A. H. Lambert.

Sussex. F. G. Brabant. Second Edition.

*Wiltshire. F. R. Heath.

YoRkshire, The EASt Riding. J. E. Morris.

YORKSHIRE, ThE NORTH RIDING. J. E. Morris.

Brittany. S. Baring-Gould.

Normandy. C. Scudamore.

Romr. C. G. Ellaby.

Sicily. F. H. Jackson.

\section{The Little Library.}

With Introductions, Notes, and Photogravure Frontispieces.

Small Pott 8vo. Gilt top. Each Volume, cloth, 1s. 6d. net; leather, 2s. 6d. net.

Anon. A LITTLE BOOK OF ENGLISH LYRICS. Second Edition.

Austen (Jane), PRIDE AND PREJUDICE. Two Volumes.

NORTHANGER ABBEY.

Bacon (Francis). THE ESSAYS OF LORD BACON.

Barham (R. H.). THE INGOLDSBY LEGENDS. T wo Volumes.

Barnet (Mrs. P. A.). A LITTLE BOOK OF ENGLISH PROSE.

Beckford (William). THE HISTORY OF THE CALIPH VATHEK.

Blake (William). SELECTIONS FROM WILLIAM BLAKE.

Borrow (George). LAVENGRO. Two Volumes.

THE ROMANY RYE.

Browning (Robert), SELECTIONS ROBERT BROWNING.

Canning (George). SELECTIONS FROM THE ANTI-JACOBIN : with GEORGE Canning's additional Poems.

Cowley (Abraham). THE ESSAYS OF ABRAHAM COWLEY.

Crabbe (George). SELECTIONS FROM GEORGE CKABBE.

Cralk (Mrs.). JOHN HALIFAX, GENTLEMAN. Two Volumes.
Crashaw (Richard). THE ENGLISH POEMS OF RICHARD CRASHAW.

Dante (Alighieri). THE INFERNO OF DANTE. Translated by H. F. CARY.

THE PURGATORIO OF DANTE. Translated by H. F. CARY.

THE PARADISO OF DANTE. Translated by H. F. CARY.

Darley (George). SELECTIONS FROM THE POEMS OF GEORGE DARLEY.

Deane (A. C.). A LITTLE BOOK OF LIGHT VERSE.

Dickens(Charles). CHRISTMAS BOOKS. Two Volumes.

Ferrier (Susan). MARRIAGE. Two Volumes.

THE INHERITANCE. Two Volumes.

Gaskell (Mrs.). CRANFORD.

Haw thorne (Nathaniel). THE SCARLET LETTER.

Henderson (T. F.). A LITTLE BOOK OF SCOTTISH VERSE.

Keats (John). POEMS.

Kinglake (A. W.). EOTHEN. Second Edition.

Lamb (Charles). ELIA, AND THE LAST ESSAYS OF ELIA.

Locker (F.). LONDON LYRICS.

Longfellow (H. W.). SELECTIONS FROM LONGFELLOW. 


\section{General Literature}

ThE LitTLe LIBrary-continued.

Marvell (Andrew). THE POEMS OF ANDREW MARVELL.

Milton (John). THE MINOR POEMS OF JOHN MILTON.

Moir (D. M.). MANSIE WAUCH.

Nichols (J. B. B.). A LITTLE BOOK OF ENGLISH SONNETS.

Rochefoucauld (La). THE MAXIMS OF LA ROCHEFOUCAULD.

Smith (Horace and James). REJECTED ADDRESSES.

Sterne (Laurence). A SENTIMENTAL JOURNEY.

Tennyson (AlPred, Lord). THE EARLY POEMS OF ALFRED, LORD TENNY. SON.

IN MEMORIAM.

THE PRINCESS.
MAUD.

Thackeray (W. M.). VANITY FAIR. Three Volumes.

PENDENNIS. Three Volumes.

ESMOND.

CHRISTMAS BOOKS.

Vaughan (Henry). THE POEMS OF HENRY VAUGHAN.

Walton (Izaak). THE COMPLEAT ANGLER.

Watephouse (Elizabeth). A LITTLE BOOK OF LIFE AND DEATH. Twelf $t h$ Edition.

Wordsworth (W.). SELECTIONS FROM WORDSWORTH.

Wordsworth (W.) and Coleridge (S. T.) LYRICAL BALLADS.

\section{The Little Quarto Shakespeare.}

Edited by W. J. CRAIG. With Introductions and Notes.

Pott $16 m$ o. In 40 Volumes. Gilt top. Leather, price is. net each volume.

Mahogany Revolving Book Case. ros. net.

\section{Miniature Library.}

\section{Gilt top.}

EUPHRANOR: A Dialogue on Youth. By | Polonius: or Wise Saws and Modern In Edward FitzGerald. Demy $32 m 0$. Leather, 2s. net.

ThE Life of Edward, Lord Herbert of Cherbury. Written by himself. Demy $32 m o$. Leather, $2 s$. net. stances. By Edward FitzGerald. Demy $32 m 0$. Leather, 2s, net.

THE RUBAIyÁt OF OMAR KHAYyAM. By Edward FitzGerald. Fourth Edition. Leather, Is. net.

\section{The New Library of Medicine.}

\section{Edited by C. W. SALEEBY, M.D.; F.R.S.Edin. Demy 8vo.}

Care of the Body, The. By F. Cavanagh. Second Edition. 7s. 6d. net.

Children of THe Nation, The. By the Right Hon. Sir John Gorst. Second Edition. 7s. 6 d. net.

Control of a Scourge, The: or, How Cancer is Curable. By Chas. P. Childe. 7s. 6 d. net.

Diseases of Occupation. By Sir Thomas Oliver. sos. $6 d$. net.

DRINK ProBlem, The, in its Medico-Sociological Aspects, Edited by T. N. Kelynack. 7s. $6 d$. net.

Drugs and the DRUg Habit. By $\mathrm{H}$. Sainsbury.
Functional Nerve Diseases. By A. T Schofield. 7s. 6 d. net.

- Heredity, The Laws of. By Archdall Reid. 2Is. net.

Hygiene of Mind, The. By T. S. Clouston. Fifth Edition. $7 s, 6$ d. net.

Infant Mortality. By George Newman. 75. 6d. net.

Prevention of Tuberculosis (ConsumpTION), ThE. By Arthur Newsholme. ros. $6 d$. net.

Air and Health. By Ronald C. Macfie. 7s. 6d. net. Second Edition. 


\section{The New Library of Music.}

Edited by ERNEST NEWMAN. Illustrated. Demy 8vo. 7s.6d. net.

Hugo Wolf. By Ernest Newman. Illustrated.

HANDrL. By R. A. Streatfeild. Illustrated. Second Edition.

\section{Oxford Biographies.}

lllustrated. Fcap. 8vo. Gilt top. Each volume, cloth, 2s. 6d. net; leather, 3s. $6 d$. net.

Dante Aitighieri. By Paget Tonybee, M.A., D. Litt. Third Edition.

Girolamo Sayonarola By E. L. S. Horsburgh, M.A. Second Edition.

John Howard. By E. C. S. Gibson, D.D., Bishop of Gloucester.

Alfred Tennyson. By A. C. Benson, M.A. Second Edition.

Sir Walter Raleigh. By I. A. Taylor.

Erasmus. By E. F. H. Capey.

The Young Pretendre. By C. S. Terry. Robert BurNs. By T. F. Henderson.

Chatham. By A. S. M'Dowall.

Francis of Assisi. By Anna M. Stoddart.

Canning. By W. Alison Phillips.

BeACONSFIRLD. By Walter Sichel.

Johann Wolfgang Goethe. By H. G. Atkins.

François Fenelon. By Viscount St. Cyres.

\section{Romantic History.}

Edited by MARTIN HUME, M.A. Illustrated. Demy \$ro.

A series of attractive volumes in which the periods and personalities selected are such as afford romantic human interest, in addition to their historical importance.

The First Governess of THE Netheri.ands, Margaret of Austria. Eleanot E. Tremayne. ros. 6 d. net.

[wo English Quenss and Philip. Martin
Hume, M.A. 15s, net.

The Nine Days' Quesn. Richard Davey. With a Preface by Martin Hume, M.A. Ios. $6 d$. net.

\section{Handbooks of Theology.}

The DoCtrine of THE INCARnation. By R. L. Ottley, D.D. Fourth Edition revised. Demey 8vo. x2s. 6d.

A History of Early Christian Doctrine. By J. F. Bethune-Baker, M.A. Demy 8vo. Ios. $6 d$.

AN INTRODUCTION TO THE HISTORY OF ReI.IGION. By F. B. Jevons. M.A. Litt. D. Fourth Edition. Demy 8vo. ros. 6 d.
AN INTRODUCTION TO THE HistoRY OF THE Creeds. By A. E. Bhirn, D.D. Demy 8vo. 205. $6 d$.

Thr Prilosophy of Religion in England and America. By Alfred Caldecott, D.D. Demy 8vo. ros. 6 d.

The XXXIX. Articles of the Church of ENGland. Edited by E. C. S. Gibson, D.D. Sixth Edition. Demy 8vo. 12s. 6d. 


\section{The Westminster Commentaries.}

\section{General Editor, WALTER LOCK, D.D., Warden of Keble College.}

Dean Ireland's Professor of Exegesis in the University of Oxford.

The Acts of the Apostles. Edited by R. B. Rackham, M.A. Demy 8vo. Fourth Edition. ros. 6 d.

The First Epistle of Paul the Apostle to the Corinthians. Edited by H. L. Goudge, M.A. Second Ed. Demy 8vo. 6s. The Book or Exndus. Edited by A. H. M'Neile, B.D. With a Map and 3 Plans. Demy 8vo. ros. $6 d$.

Thx Book of EzeKiel. Edited by H. A. Redpath, M.A., D.Litt. Demy 8vo. xos. 66 .
The Brok of Genesis. Edited with Intro. duction and Notes by S. R. Driver, D.D Seventh Edition. Demy 8vo. ros. $6 d$.

Additions and Corrections in the Seventh Edition of The Book of Genesis. By S. R. Driver, D.D. Demy 8vo. is.

Tив Boок ог Jов. Edited by E. C. S. Gibson, D.D. Second Edition. Demy 8vo. 6s.

Tue Epistue of ST. Jamrs. Edited with In troduction and Notes by $R$. J. Knowling, D.D. Demy 8z'o. 6s.

\section{PART III.-A SELECTION OF WORKS OF Fiction}

Albanesl (E. Marla). SUSANNAH AND ONE OTHER. Fourth Edition. Cr. $8 v 0$. $6 s$.

LOVE AND LOUISA. Second Edition. Cr. 8vo. 6s.

THE BROWN EYES OF MARY. Third Edition. Cr. 8vo. 6s.

I KNOW A MAIDEN. Third Edition. Cr. 8vo. 6s.

THE INVINCIBLE AMELIA: OR, THR Polite AdVRnTURess. Third Edition. Cr. 8vo. 3s. 6d.

- THE GLAD HEART. Cr. 8vo. 6 s.

Allerton (Mark). SUCH AND SUCH THINGS. Cr. 8vo. $6 s$.

Annesley (Maude). THIS DAY'S MAD. NESS. Second Edition. Cr. 8vo. 6s.

Bagot (Rlehard). A ROMAN MYSTERY. Third Edition. Cr.8vo. 6s.

THE PASSPORT. Fourth Edition. Cr. 8 8no. $6 s$.

TEMPTATION. Fifth Edition. Cr. 8 vo.

ANTHONY CUTHBERT. Fowrth Edition. Cr. 8vo. 6s.

LOVE'S PROXY. Cr. 8vo. 6s.

DONNA DIANA. Second Edition. $C r$. 8vo. 6s.

CASTING OF NETS. Twelfth Edition. Cr. 8vo. 6s.

Balley (H. C.). STORM AND TREASURE. Cr. 8vo. 6s.

Ball (Oona H.) (Barbara Burke). THEIR OXFORD YEAR. Illustrated. Cr. 8vo. 6s.
BARBARA GOES TO OXFORD. Illus. trated. Third Edition. Cr. 8vo. 6s.

Baring-Gould (S.). ARMINELL. Fifth Edition. Cr. 8vo. 6s.

URITH. Fifth Edition. Cr. 8vo. 6s.

IN THE ROAR OF THE SEA. Seventh Edition. Cr. 8vo. 6s.

MARGERY OF QUETHER. Third Edition. Cr. 8vo. 6s.

THE QUEEN OF LOVE, Fifth Edition. Cr. 8vo. 6s.

JACQUETTA. Third Edition. Cr. 8vo. 6s. KITTY ALONE. Fifth Edition. Cr. 8vo. $6 s$. NOÉMI. Illustrated. Fourth Edition. $C r$. 8 vo. 6s.

THE BROOM - SQUIRE. Illustrated. Fifth Edition. Cr. 8vo. 6s.

DARTMOOR IDYLLS. Cr. 8vo. $6 s$.

GUAVAS THE TINNER. Illustrated. Second Edition. Cr. 8vo. 6s.

BLADYS OF THE STEWPONEY. Illus. trated. Second Edition. Cr. 8vo. 6s. PABO THE PRIEST. Cr. $8 v 0$. $6 s$.

WINEFRED. Illustrated. Second Edition. Cr. 8vo. 6s.

ROYAL GEORGIE. Illustrated. Cr.8vo. $6 s$. CHRIS OF ALL SORTS. Cr.8vo. $6 s$.

IN DEWISLAND. Second Edition. $C r$. 8vo. $6 s$.

THE FROBISHERS. Cr. 8vo. 6s.

DOMITIA. Illustrated. Second Edition. Cr. 8vo. 6s.

MRS. CURGENVEN OF CURGENVEN. Cr. 8vo. 6s.

Barp (Robert). IN THE MIDST OF ALARMS. Third Edition. Cr. 8vo. 6s. THE COUNTESS TEKIA. Fifth Edition. Cr. 8vo. 68 . 
THE MUTABLE MANY. Third Edition. Cr. 8vo. 6s.

Begbie (Harold). THE CURIOUS AND DIVERTING ADVENTURES OF SIR JOHN SPARROW; OR, THE PROGRESS OF AN OPEN MIND.' Second Edition. Cr. $8 v 0$. $6 s$.

Belloe (H.). EMMANUEL BURDEN, MERCHA NT. Illustrated. Second Edition. Cr. 8vo. 6s.

A CHANGE IN THE CABINET. Third Edition. Cr. 8vo. 6s.

Benson (E. F.). DODO : A DETAIL OF THE DAy. Fifteenth Edition. Cr. 8vo. 6s.

Birmingham (George A.). THE BAD TIMES. Second Edition. Cr. 8vo. $6 s$.

SPANISH GOLD. Fourth Edition. Cr. $8 v 0$. 6s.

THE SEARCH PARTY. Fourth Edition. Cr. 8vo. 6s.

Bowen (Marjorle). I WILL MAINTAIN. Second Edition. Cr. 8vo. $6 s$.

Brotherton(Ralph Harold). AN HONEST MAN. Second Edition. Cr. 8vo. 6s.

Capes (Bernard). WHY DID HE DO IT? Second Eation. Cr. 8vo. 6s.

Castle (Agnes and Egerton). FLOWER $\mathrm{O}^{\prime}$ THE ORANGE, and Other Tales. Third Edition. Cr. 8vo. 6s.

Clifford (Mrs. W. K.). THE GETTING WELL OF DOROTHY. Illustrated. Second Edition. Cr. 8vo. 3s. 6d.

Conrad (Joseph). THE SECRET AGENT : A Simple Tale. Fourth Ed. Cr. 8vo. 6s. A SET OF SIX. Fourth Edition. Cr. 8vo. 6s.

Corelli (Marie). A ROMANCE OF TWO WORLDS. Twenty-Ninth Ed. Cr. 8vo. 6s. VENDETTA. Twenty-Seventh Edition. Cr. 8vo. $6 s$.

THELMA. Fortieth Ed. Cr. 8vo. 6s.

ARDATH: THE STORY OF A DEAD SELF. Nineteenth Edition. $1 C r$. 8vo. $6 s$. THE SOUL OF LILITH. Sixteenth Edition. Cr. 8vo. 6s.

WORMWOOD. Seventeenth Ed. Cr. 8vo. 6s. BARABBAS: A DREAM OF THE WORLD'S TRAGEDY. Forty-Fourth Edition. Cr. 8vo. 6s.

THE SORROWS OF SATAN. Fifty-Fifth Edition. Cr. 8vo. 6s.

THE MASTER CHRISTIAN. Twelfth Edition. I77th Thousand. Cr. 800. 6s.

TEMPORAL POWER: A STUDY IN SUPREMACY. Second Edition. r5oth Thousand. Cr. 8vo. 6s.

GOD'S GOOD MAN : A SIMPLE LOVE STORY. Thirteenth Edition. x50th Thowsand. Cr. 8vo. 6s.

HOLY ORDERS: THE TRAGEDY OF A OUIET LIFE. Second Edition. r2oth Thousand. Crozen 8vo. 6s.

THE MIGHTY ATOM. Twenty-eighth Edition. Cr. 8vo. 6s.
BOY : a Sketch. Eleventh Edition. Cr. 8vo $6 s$.

CAMEOS. Thirteenth Edition. Cr.8vo. 6s. Cotes (Mrs. Everard). See Dunean (Sara Jeannette).

Crockett (S. R.). LOCHINVAR. Illus trated. Third Edition. Cr. 8vo. 6s.

THE STANDARD BEARER. Second Edition. Cr. 8vo. 6s.

Croker (Mrs. B. M.). THE OLD CAN. TONMENT. Cr. 8vo. 6s.

JOHANNA. Second Edition. Cr. 8vo. 6s. THE HAPPY VALLEY. Fourth Edition. Cr. 8vo. 6s.

A NINE DAYS' WONDER. Third Edition. Cr. 8vo. 6s.

PEGGY OF THE BARTONS. Seventh Edition. Cr.8vo. 6s.

ANGEL. Fifth Edition. Cr. 8vo. 6s.

A STATE SECRET. Third Edition. Cr. 8vo. 3s. 6d.

KATHERINE THE ARROGANT. Sixth Edition. Cr. 8vo. 6s.

Cuthell (Edith E.). ONLY A GUARDROOM DOG. Illustrated. Cr. 8vo. $3^{s .6 d}$.

Dawson (Warrington). THE SCAR. Second Edition. Cr. 8ro. 6s.

THE SCOURGE. Cr. 8 vo. $6 s$.

Douglas (Theo.). COUSIN HUGH. Second Edition. Cr. 8vo. 6s.

Doyle (A. Conan). ROUND THE RED LAMP. Eleventh Edition. Cr. 8vo. 6s. Duncan (Sara Jeannette) (Mrs. Everard

A VOYAGE OF CONSOLATION. Illustrated. Third Edition. Cr. 8vo. 6s.

COUSIN CINDERELLA. Second Edition. Cr. 8vo. 6s.

THE BURNT OFFERING. Second Edition. Cr. 8vo. 6s.

*Elliott (Robert). THE IMMORTAL CHARLATAN. Crown 8vo. $6 s$.

Fenn (G. Manville). SYD BELTON; or, The Boy who would not go to Sea. Illustrated. Second Ed. Cr. 8vo. 3s. $6 d$.

Findlater (J.H.). THE GREEN GRAVES OF BALGOWRIE. Fifth Edition. Cr. 8vo. 6s.

THE LADDER TO THE STARS. Second Edition. Cr. 8vo. 6 s.

Findlater (Mary). A NARROW WAY. Third Edition. Cr. 8vo. 6s.

OVER THE HILLS. Second Edition. $\mathrm{Cr}$. 8vo. 6s.

THE ROSE OF JOY. Third Edition. Cr. 8vo. 6s.

A BLIND BIRD'S NEST. Illustrated. Second Edition. Cr. 8vo. 6s.

Francis (M. E.). (Mrs. Francis Blundell). STEPPING WESTWARD. Second Edition Cr. 8vo. 6s. 
MARGERY O' THE MILL. Third Edition. Cr. 8vo. 6s.

HARDY-ON-THE-HILL. Thind Edition. Cr. 8vo. 6s.

GALATEA OF THE WHEATFIELD. Second Edition. Cr. 8vo. 6s.

Fraser (Mrs. Hugh). THE SLAKING OF THE SWOKD. Second Edition. Cr. 8vi. 6s.

GIANNELLA. Second Edition. Cr. 8vo. 6s. IN THE SHADOW OF THE LORD. Third Edition. Cr. $8 v 0$. $6 s$.

Fry (B. and C. B.). A MOTHER'S SON. Fifth Edition. Cr. 8vo. 6s.

Gerard (Louise). THE GOLDEN CENTIPEDE. Second Edition. Cr. 8vo. 6s.

Gibbs (Philip). THE SPIRIT OF RE. VOLT. Second Edition. Cr. 8vo. 6s.

Gissing (George). THE CROWN OF LIFE. Cr. 8vo. $6 s$.

* Glendon (George). THE EMPEROR OF THE AIR. Illustrated. Cr. 8vo. $6 s$.

Hamilton (Cosmo). MRS. SKEFFINGTON. Cr.8vo. 6s.

Harraden (Beatrice). IN VARY I NG MOODS. Fourteenth Edition. Cr. 8vo. 6s. THE SCHOLAR'S DAUGHTER. Fourth Edition. Cr. 8 vo. 6s.

HILDA STRAFFORD and THE REMIT. TANCE MAN. Twelfth Ed. Cr.8vo. $6 s$. INTERPLAY. Fifth Edition. Cr. 8vo. 6s.

Hichens (Robert). THE PROPHET OF BERKELEY SQUARE. Second Edition. Cr. 8vo. $6 s$.

TONGUES OF CONSCIENCE. Third Edition. Cr. 8 vo. 6s.

FELIX. Sixth Edition. Cr. 8vo. $6 s$.

THE WOMAN WITH THE FAN. Seventh Edition. Cr. 8ro. 6s.

BYEWAYS. $C r .8 v 0$. 6 s.

THE GARDEN OF ALLAH. Eighteenth Edition. Cr. 8vo. 6s.

THE BLACK SPANIEL. Cr. 8vo. $6 s$.

THE CALL OF THE BLOOD. Seventh Edition. Cr. 8vo. 6s.

BARBARY SHEEP. Second Edition. Cr. 8vo. 6s.

-Hilliersi(Ashton). THE MASTER-GIRL. nlustrated. Cr.8vo. 6s.

Hope (Anthony). THE GOD IN THE CAR. Eleventh Edition. Cr. 8vo. 6s.

A CHANGE OF AIR. Sixth Edition. $C r$. $8 v 0$. $6 s$.

A MAN OF MARK. Sixth Ed. Cr. 8vo. 6s.

THE CHRONICLES OF COUNT ANTONIO. Sixth Edition. Cr. 8vo. 6s.

PHROSO. Illustrated. Eighth Edition. Cr. 8vo. 6s.

SIMON DALE. Illustrated. Eighth Edition. Cr. 8vo. 6s.

THE KING'S MIRROR. Fowrth Edition. Cr. 8vo. 6s.
QUISANTE. Fourth Edition. Cr. 8vo. $6 s$. THE DOLLY DIALOGUES. $C r .8 v 0.6 s$. A SERVANT OF THE PUBLIC. Illustrated. Fourth Edition. Cr. 8vo. 6s.

TALES OF TWO PEOPLE. Third Edi. tion. Cr. 8vo. 6s.

THE GREAT MISS DRIVER. Fourth Edition. Cr. 8vo. 6s.

Hueffer (Ford Maddox). AN ENGLISH GIRL: A Romance. Second Edition. Cr. 8vo. $6 s$.

MR. APOLlO : A Just Possible Story. Second Edition. Cr. $8 v 0$. $6 s$.

Hutten (Baroness von). THE HALO. Fifth Edition. Cr. 8vo. 6s.

Hyne (C. J. Cuteliffe). MR. HORROCKS, PURSER. Fifth Edition. Cr. $8 v 0$. 6s.

PRINCE RUPERT, THE BUCCANEER. Illustrated. Third Edition. Cr. $8 v 0$. $6 s$.

Jacobs (W. W.). MANY CARGOES. Thirty-first Edition. Cr. 8vo. 3s. 6d. SEA URCHINS. Fiftcenth Edition. $C r$. $8 v 0$. 3s. $6 d$.

A MASTER OF CRAFT. Illustrated. Ninth Edition. Cr. 8 vo. 3s. $6 d$.

LIGHT FREIGHTS. Illustrated. Eighth Edition. Cr. 8 vo. 3 s. $6 d$.

THE SKIPPER'S.WOOING. Ninth Edition. Cr. 8vo. 3 s. $6 d$.

AT SUNWICH PORT. Illustrated. Tenth Edition. Cr. $8 v o$. 3s. $6 d$.

DIALSTONE LANE. Illustrated. Sevent/s Edition. Cr. 8vo. 3 s. $6 d$.

ODD CRAFT. Illustrated. Fourth Edition. Cr. $8 v 0$. 3s. 6 d.

THE LADY OF THE BARGE. Illustrated. Eighth Edition. Cr. 8vo. 3s. 6d.

SALTHAVEN. Illustrated. Second Edition. Cr. 800 . 3. 3. $6 d$.

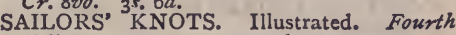
Edition. Cr. 8vo. 3s. $6 d$.

James (Henry). THE SOFT SIDE. Second Edition. Cr. 8vo. 6s.

THE BETTER SORT. Cr. 8vo. $6 s$.

THE GOLDEN BOWL. Third Edition. Cr. 8vo. 6s.

Le Queux (William). THE HUNCHBACK OF WESTMINSTER. Third Edition. Cr. 8vo. 6s.

THE CLOSED BOOK. Third Edition. Cr. 8vo. 6s. Illustrated. Third Edition. Cr. 8vo. 6 s. BEHIND THE THRONE. Third Edition. Cr. 8vo. 6s.

THE CROOKED WAY. Second Edition. Cr. 8vo. 6s.

"Lindsey (Willam). THE SEVERED MANTLE. Cr. 8vo. 6 s.

London (Jack). WHITE FANG. Seventh Edition. Cr. 8vo. 6s. 
Lubbock (Basil). DEEP SEA WARRIORS. Illustrated. Third Edition. $\mathrm{Cr}$. 8vo. $6 s$.

Lueas (St John). THE FIRST ROUND. Cr. 8vo. 6s.

Lyall (Edna). DERRICK VAUGHAN, NOVELIST. 44th Thowsand. Cr. $8 v 0$. $3^{s} .6 d$.

Maartens (Maarten). THE NEW RELIGION: A MODERN Novel. Third Edition. Cr. 8vo. 6s.

BROTHERS ALL; MORE STORIES OF Dutch Peasant Life. Third Edition. Cr. 8vo. 6s.

THE PRICE OF LIS DORIS. Second Edition. Cr. 8vo. 6s.

M'Carthy (Justin H.). THE DUKE'S MOTTO. Fourth Edition. Cr. 8vo. 6s.

Maenaughtan (S.). THE FORTUNE OF CHRISIINA M'NAB. Fifth Edition. Cr. 8vo. 6s.

Malet (Lucas). COLONEL ENDERBY'S WIFE. Fourth Edition. Cr. $8 v 0.6 s$.

A COUNSEL OF PERFECTION. Second Edition. Cr.8vo. 6s.

THE WAGES OF SIN. Sixtenth Edition. Cr. 8vo. 6s.

THE CARISSIMA. Fifth Ed. Cr. 8vo. 6s. THE GATELESS BARRIER. Fifth Edition. Cr. 8vo. 6s.

THE HISTORY OF SIR RICHARD CALMADY. Seventh Edition. Cr. 8vo. 6s.

Mann (Mrs. M. E.). THE PARISH NURSE. Fourth Edition. Cr. 8vo. 6s.

A SHEAF OF CORN. Second Edition. Cr. 820 . 6s.

THE HEART.SMITER. Second Edition. Cr. 8 vo. 6s.

AVENGING CHILDREN. Second Edition. Cr. 8vo. 6s.

Marsh (Richard). THE COWARD BE. HIND THE CURTAIN. Cr. 8vo. $6 s$.

THE SURPRISING HUSBAND. Second Edition. Cr. 8vo. 6s.

A ROYAL INDISCRETION. Seeond Edition. Cr. 8vo. 6s.

LIVE MEN'S SHOES. Second Edition. Cr. 8vo. 6s.

Marshall (Archibald). MANY JUNES. Second Edition. Cr. 8vo. 6s.

THE SQUIRE'S DAUGHTER. Third Edition. Cr. 8vo. 6s.

Mason (A. B. W.). CLEMENTINA. Illustrated. Third Edition. Cr. 8vo. 6s.

Maud (Constance). A DAUGHTER OF FRANCE. Second Edition. Cr. 8vo. 6s.

Maxwell (W. B.). VIVIEN. Ninth Edition. Cr. 8vo. 6s.

THE RAGGED MESSENGER. Third Edition. Cr. $8 v 0$. $6 s$.

FABULOUS FANCIES. Cr. 8vo. $6 s$.
THE GUARDED FLAME. Serenth Edition. Cr. 8vo. 6s.

ODD LENGTHS. Second Ed. Cr. 8vo. 6s. HILL RISE. Fourth Edition. Cr. 8vo. 6s.

THE COUNTESS OF MAYBURY: $\mathrm{BE}^{-}$ TWREN YOU AND I. Fourth Edition. Cr. 8vo. 6s.

Meade (L. T.). DRIFT. Second Edition. Cr. 8vo. 6s.

RESURGAM. Second Edition. Cr. 8vo. 6s. VICTORY. Cr. 8vo. 6s.

A GIRL OF THE PEOPLE. Illustrated. Fourth Edition. Cr. 8vo. 3s.6d.

HEPSY GIPSY. Illustrated. Cr. $8 v 0$. 2s. $6 d$.

THE HONOURABLE MISS: A STORY OF AN OLD-FASHIONED TOWN. Illustrated. Second Edition. Cr. 8vo. 3s. 6 d.

Mitford (Bertram). THE SIGN OF THE SPIDER. Illustrated. Seventh Edition. Cr. 8vo. 3s. $6 d$.

Molesworth (Mrs.). THE RED GRANGE. lllustrated. Second Edition. $C r$. $8 v 0$. $3^{\text {s. } 6 d .}$

Montague (C. E.). A HIND LET LOOSE. Cr. 8vo. 6s.

Montgomery (K. L.). COLONEL KATE. Second Edition. Cr. 8vo. 6s.

Morrison (Arthur). TALES OF MEAN STREETS. Seventh Edition. Cr. 8vo. 6s. A CHILD OF THE JAGO. Fifth Edition. Cr. 8vo. 6s.

THE HOLE IN THE WaLL. Fourth Edition. Cr. 8vo. 6s.

DIVERS VANITIES. $c r .8 v 0$. 6 s.

Nesbit (E.), (Mrs. H. Bland). THE RED HOUSE. Illustrated. Fourth Edition. Cr. 8vo. 6s.

Noble (Edward). LORDS OF THE SEA. Third Edition. Cr. 8vo. 6s.

Ollivant (Alfred). OWD BOB, THE GREY DOG OF KENMUIR. 'With a Frontispiece. Eleverth Ed. Cr. 8vo. 6s.

Oppenheim (E. Phillips). MASTER OF MEN. Fourth Edition. Cr. 8vo. 6s.

Oxenham (John). A WEAVER OF IVEBS. Illustrated. Fourth Ed. Cr. 8vo. 6s. THE GATE OF THE DESERT. Fourth Edition. Cr. 8vo. 6r.

PROFIT AND LOSS. Fourth Edition. Cr. 8vo. 6s.

THE LONG ROAD. Fourth Edition. $C r$. 8vo. 6s.

THE SONG OF HYACINTH, AND OTHER STORIES. Second Edition. Cr. 8vo. 6s.

MY LADY OF SHADOWS. Fowrth Edi. tion. Cr. 8vo. 6s.

Pain (Barry). THE EXILES OF FALOO. Crown 8vo. 6s.

Parker (Gilbert). PIERRE AND HIS PEOPLE. Sixth Edition. Cr. 8wo. 6 s. 
MRS. FALCHION. Fifth Edition. Cr. 8vo. 6s,

THE TRANSLATION OF A SAVAGE. Third Edition. Cr. 8vo. 6s.

THE TRAIL OF THE SWORD. Illustrated. Tenth Edition. Cr. 8vo. 6s.

IVHEN VALMOND CAME TO PONTIAC: The Story of a Lost Napoleon. Sixth Edition. Cr. 8vo. 6s.

AN ADVENTURER OF THE NORTH. The Last Adventures of 'Pretty Pierre.' Finurth Edition. Cr. 8 vo. $6 s$.

THE SEATS OF THE MIGHTY. Illustrated. Sixteenth Edition. Cr. 8vo. 6s.

THE BATTLE OF THE STRONG : a Romance of Two Kingdoms. Illustrated. Sixth Edition. Cr. 8vo. 6s.

$1 \mathrm{HE}$ POMP OF THE LAVILETTES. Third Edition. Cr. 8vo. 3s. 6d.

NORTHERN LIGHTS. Fourth Edition. Cr. 8vo. 6s.

Pasture (Mrs. Henry de la). THE TYRANT. Fourth Edition. Cr. 8vo. 6s. Patterson (J. E.). WATCHERS BY THE SHORE. Third Edition. Cr. 8vo. 6s.

Pemberton (Max). THE FOOTSTEPS OF A THRONE. Illustrated. Third Edition. Cr. 8vo 6s.

I CROWN THEE KING. Illustrated. $C r$. 8vo. $6 s$.

LOVE THE HARVESTER: A STORY OF THE SHIREs. Illustrated. Third Edition. Cr. 8vo. 3s. $6 d$.

THE MYSTERY OF THE GREEN HEART. Second Edition. Cr. 8v0.6s.

Phillpotts(Eden). IYING PROPHETS. Thind Edition. Cr. 8vo. 6s.

CHILDREN OF THE MIST. Fifth Edi. tion. Cr. 8vo. 6s.

THE HUMAN BOY. With a Frontispiece. Seventh Edition. Cr. 8vo. 6s.

SONS OF THE MORNING. Second Edition. Cr. 8vo. 6s.

THE RIVER. Third Edition. Cr. 8vo. 6s.

THE AMERICAN PRISONER. Fourth Edition. Cr. 8vo. 6s.

THE SECRET WOMAN. Fourth Edition. Cr. 8vo. 6s.

KNOCK AT A VENTURE. Third Edition. Cr. 8vo. 6s.

THE PORTREEVE. Fourth Edition. Cr. 8vo. 6s.

THE POACHER'S WIFE. Second Edition. Cr. 8vo. 6 s.

THE STRIKING HOURS. Second Edition. Cr. 8vo. 6s.

THE FOLK AFIELD. Crown 8vo. 6r.

Pickthall (Marmaduke). SAID THE FISHERMAN. Seventh Edition. Cr.8vo. 6s.

'Q' (A. T. Qulller Couch). THE WHITE WOLLF. Second Edition. Cr. 8vo. 6s.

THE MAYOR OF TROY. Fourth Edition. Cr. 8vo. 6s.

MERRY.GARDEN AND OTHER STORIES. Cr. 8vo. 6s.
MAJOR VIGOUREUX. Third Edition. Cr. 8vo. 6s.

Querido (Israel). TOIL OF MEN. Trans lated by F. S. Arnold. Cr. 8vo. 6s.

Rawson (Maud Stepney). THE EN. CHANTED GARDEN. Fourth Edition. Cr. $8 v 0$. 6s.

THE EASY GO LUCKIES : OR, ONE WAY of Living. Second Edition. Cr. 8vo. 6 s. HAPPINESS. Second Edition. Cr. 8vo. 6s.

Rhys (Grace). THE BRIDE. Second Edition. Cr. 8vo. 6s.

Rldge (W. Pett). ERB. Second Edition. Cr. 8vo. 6s.

A SON OF THE STATE. Second Edition. Cr. 8vo. 3s. 6d.

A BREAKER OF LAWS. Cr. 8vo. 3s. $6 d$. MRS. GALER'S BUSINESS. Illustrated. Second Edition. Cr. 8vo..6s.

THE WICKHAMSES. Fourth Edition. Cr. 8vo. 6s.

NAME OF GARLAND. Third Edition. Cr. 8vo. 6s.

SPLENDID BROTHER. Fourth Edition. Cr. 8vo. 6s.

Ritchie (Mrs. David G.). MAN AND THE CASS OCK. Second Edition. Cr. 8vo. 6s.

Roberts (C. G. D.). THE HEART OF THE ANCIENT WOOD. Cr. $8 v 0.35 .6 d$.

Roblns (Ellzabeth). THE CONVERT. Third Edition. Cr. 8 vo. 6s.

Rosenkrantz (Baron Palle). TH E MAGISTRATE'S OWN CASE. Cr. 8vo. 6s.

Russell (W. Clark). MY DANISH SWEETHEART. Illustrated. Fifth Edition. Cr. 8vo. 6s.

HIS ISLAND PRINCESS. Illustrated. Second Edition. Cr. 8vo. 6s.

A BANDONED. Second Edition. Cr. 8vo. 6s. MASTER ROCKAFELLAR'S VOYAGE Illustrated. Fourth Edition. Cr. 8vo. 3s.6d.

Sandys (Sydney). JACK CARSTAIRS OF THE POWER HOUSE. Illustrated. Second Edition. Cr. 8vo. 6s.

Sergeant (Adeline). THE PASSION OF PAUL MARILLIER. Cr.8vo. 6 s.

- Shakespear (Olivia). UNCLE HILARY. Cr. 8vo. 6s.

Sidgwick (Mrs. Alfred). THE KINSMAN. Illustrated. Third Edition. $\mathrm{Cr}$. Bro. $6 s$.

THE SEVERINS. Fourth Edition. Cr. 8vo. 6s.

Stewart (Newton V.). A SON OF THE EMPEROR. : BEING PASSAGES FROM THE Life of Enzio, King of Sardinia and Corsica. Cr. 8vo. 6s.

Swayne (Martin Lutrell). THE BISHOP AND THE LADY. Second Edition. Cr. 8vo. 6s. 
Thurston (E. Temple). MIRAGF. Fourth Edition. Cr. 8vo. 6 s.

Underhill (Evelyn). THE COLUMN OF DUST. Cr. 8vo. 6s.

Vorst (Marie Van). THE SENTIMENTAL ADVENTURES OF JIMMY BULSTRODE. Cr. 8vo. 6s.

IN AMBUSH. Second Edition, Cr. $8 v 0$. $6 s$.

Waineman (Paul). THE WIFE OF NICHOLAS FLEMING. Cr. 8vo. $6 s$.

Watson (H. B. Marriott). TWISTED EGLANTINE. Illustrated. Third Edition. Cr. 8vo. $6 s$.

THE HIGH TOBY. Third Edition. $C r$. $8 v 0$. $6 s$.

A MIDSUMMER DAY'S DREAM. Third Edition. Cr. 8vo. 6s.

THE CASTLE BY THE SEA. Thind Edition. Cr. 8vo. 6s.

THE PRIVATEERS. Illustrated. Second Edition. Cr. 8vo. 6s.

A POPPY SHOW : BeING Divers and DIVERSE TALES. $C r .8 v 0.6 s$.

THE FLOWER OF THE HEART. Third Edition. Cr. 8vo. 6s.

Webling (Peggy). THE STORY OF VIRGINIA PERFECT. Third Edition. Cr. 8vo. 6s.

"THE SPIRIT OF MIRTH. Cr. 8vo. 6s.

Wells (H. G.). THE SEA LADY. $C r$. 8vo. 6s. Also Medium 8vo. 6d.

Weyman (Stanley). UNDER THE RED ROBE. Illustrated. Twenty-Second Edition. Cr. 8vo. 6s.

Whitby (Beatrice). THE RESULT OF AN ACCIDENT. Second Edition. Cr. 8vo. $6 s$.
White (Edmund). THE HEART OF HINDUSTAN. Cr.8vo. 6 s.

White (Perey). LOVE AND THE WISE MEN. Third Edition. Cr. 8vo. 6s.

Williamson (Mrs. C. N.). THE ADVENTURE OF PRINCESS SYLVIA. Second Edition. $\mathrm{Cr}$. $8 v 0$. $6 s$.

THE WOMAN WHO DARED. Cr. $8 v 0$. $6 s$.

THE SEA COULD TELL. Second Edition. Cr. 8vo. 6s.

THE CASTLE OF THE SHADOWS. Third Edition. Cr. 8vo. 6s.

PAPA. Cr. 8vo. 6s.

Williamson (C. N. and A. M.). THE LIGHTNING CONDUCTOR: The Strange Adventures of a Motor Car. Illustrated. Seventeenth Edition. Cr. $8 v 0$. 6s. Also Cr. 8vo. is. net.

THE PRINCESS PASSES : A Romance of a Motor. Illustrated. Ninth Edition. Cr. 8vo. 6s.

MY FRIEND THE CHAUFFEUR. Illustrated. Tenth Edition. Cr. 8vo. 6s.

LADY BETTY ACROSS THE WATER. Tenth Edition. Cr. 8vo. $6 s$.

THE CAR OF DESTINY AND ITS ERRAND IN SPAIN. Illustrated. Fourth Edition. Cr. 8vo. 6s.

THE BOTOR CHAPERON. Illustrated. Fifth Edition. Cr. 8vo. 6s.

SCARLET RUNNER. Illustrated. Third Edition. Cr. 8vo. 6s.

SET IN SILVER. Illustrated. Second Edition. Cr. 8 vo. 6s.

LOR D LOVELAND DISCOVERS AMERICA. Second Edition. Cr. 8vo. $6 s$.

Wyllarde (Dolf). THE PATHWAY OF THE PIONEER (Nous Autres), Fourth Edition. Cr. 8vo. $6 s$.

\section{Books for Boys and Girls.}

\section{Illustrated. Crown 8vo. 3s. 6d.}

The GetTing Well of Dorothy. By Mrs. W. K. Clifford. Second Edition.

ONLY a Guard-RoOM Dog. By Edith E. Cuthell.

Master Rockafellak's Voyage. By W. Clark Russell. Fourth Edition.

Syo Belton: Or, the Boy who would not go to Sea. By G. Manville Fenn. Second Edition.

Thr Red Grange. By Mrs. Molesworth. Second Edition.
A Girl of the Prople. By L. T. Meade. Fourth Edition.

HePSY Gipsy. By L. T. Meade. 2s. $6 d$.

The Honourable Miss. By L. T. Meade. Second Edition.

There was once a Prince. By Mrs. M. E Mann.

Whex ARNold comes Homz. By Mrs. M. X. Mann. 


\section{The Novels of Alezandre Dumas.}

\section{Medium 8vo. Price 6d. Double Volumes, Is.}

Act\&.

The Adventures of Captain Pamphile. AMAURY.

ThE BIRD OF FATE.

The Black Tulip.

The Castle of Eppsteis.

Catherine Blum.

CECrLe.

The Chatelex.

The Chevalier D'Harmental. (Double volume.)

Chicot the Jester.

The Comte de Montgomert.

Conscience.

The Convict's Son.

The Corsican Brothres; and Otho the ARCHER.

Crop-Eared Jacquot.

DOM GORENFLOT.

The fatal Combat.

The Fencing Mastex.

Fernande.

Gabriel LAMBERT.

Grorges.

The Great Massacre.

HenRI de NaVARRE.

Hélène de Chavering.
THE HoROScope.

LOUISE DE LA VAlLik̇RE. (Double volume.)

The MaN IN The Iron Mask. (Double volume.)

Mattre AdaM.

The Mouxh of Helz.

Nanon. (Double volume.)

OLYMPIA.

Pauline; Pascal Bruno; and Bontekor.

Père la Ruine.

The Prince OF ThIEVES.

The Reminiscences or Antony.

ROBIN HOOD.

Samurl Gels.

The SNowbalz and The Sultanetta.

SYLVANDIRE.

The Taking of Calais.

Tales of the Supernatural.

Tales of Strange Adventurz.

TAles of Terror.

The Three Musketerers. (Double volume.) The Tragedy of Nantes.

Twenty Years After. (Double volume.)

The Wild-Duck Shootzr.

The Wolf-Leader.

\section{Methuen's Sixpenny Books.}

Medium 8vo.

Albanesi (B. Maria). LOVE AND LOUISA.

I KNOW A MAIDEN.

Anstey (F.). A BAYARD OF BENGAL. Austen (J.). PRIDE AND PREJUDICE. Bagot (Richard). A ROMAN MYSTERY. CASTING OF NETS.

DONNA DIANA.

Balfour (Andrew). BY STROKE OF
Baring-Gould (S.). FURZE BLOOM.

CHEAP JACK ZITA.

KITTY ALONE.

URITH.

THE BROOM SOUIRE.

IN ITHE ROAR OF THE SEA.

NOÉMI.

A BOOK OF FAIRY TALES. Illustrated LITTLE TU'PENNY.

WINEFRED.

THE FROBISHERS.

THE QUEEN OF LOVE. 
ARMINELI.

BLADYS OF THE STEWPONEY.

Barr (Robert). JENNIE BAXTER.

IN THE MIDST OF ALARMS.

THE COUNTESS TEKLA.

THE MUTABLE MANY.

Benson (E. F.). DODO.

THE VINTAGE.

Bronte (Charlotte). SHIRLEY.

Brownell (C. L.). THE HEART OF JAPAN.

Burton (J. Bloundelle). ACROSS THE SALT SEAS.

Caffyn (Mrs.). ANNE MAULEVERER.

Capes (Bernard). THE LAKE OF WINE.

Clifford (Mrs. W. K.). A FLASH OF SUMMER.

MRS. KEITH'S CRIME.

Corbett (Julian). A BUSINESS IN GREAT WATERS.

Croker (Mrs. B. M.). ANGEL.

A STATE SECRET.

PEGGY OF THE BARTONS.

JOHANNA.

Dante (Alighieri). THE DIVINE COMEDY (Cary).

Doyle (A. Conan). ROUND THE RED LAMP.

Duncan (Sara Jeannette). A VOYAGE OF CONSOLATION.

THOSE DELIGHTFUL AMERICANS.

Eliot (George). THE MILL ON THE FLOSS.

Findlater (Jane H.). THE GREEN GRAVES OF BALGOWRIE.

Gallon (TOM). RICKERBY'S FOLLY.

Gaskell (Mrs.). CRANFORD.

MARY BARTON.

NORTH AND SOUTH.

Gerard (Dorothea), HOLY MATRIMONY.

THE CONQUEST OF LONDON.

MADE OF MONEY.

Gissing (G.). THE TOWN TRAVELLER. THE CROWN OF LIFE.

Glanville (Ernest). THE INCA'S TREASURE.

THE KLOOF BRIDE.
Gleig (Charles), BUNTER'S CRUISE.

Grimm. (The Brothers). GRIMM'S FAIRY TALES.

Hope (Anthony). A MAN OF MARK.

A CHANGE OF AIR.

THE CHRONICLES OF COUNT ANTONIO.

PHROSO.

THE DOLLY DIALOGUES.

Hornung (E. W.). DEAD MEN TELL NO TALES.

Ingraham (J. H.), THE THRONE OF DAVID.

Lo Queux (W.). THE HUNCHBACK OF WESTMINSTER.

Levett-Yeats (S. K.). THE TRAITOR'S WAY.

ORRAIN.

Linton (E. Lynn). THE TRUE HIS TORY OF JOSHUA DAVIDSON.

Lyall (Edna). DERRICK VAUGHAN.

Malet (Lucas). THE CARISSIMA. A COUNSEL OF PERFECTION.

Mann (MrS. M. B.). MRS. PETER HOWARD.

A LOST ESTATE.

THE CEDAR STAR.

ONE ANOTHER'S BURDENS.

THE PATTEN EXPERIMENT.

A WINTER'S TALE.

Marchmont (A. W.). MISER HOAD LEY'S SECRET.

A MOMENT'S ERROR.

Marryat (Captain). PETER SIMPLE. JACOB FAITHFUL.

March (Rlchard). A METAMORPHOSIS. THE TWICKENHAM PEERAGK. THE GODDESS.

THE JOSS.

Mason (A. E. W.). CLEMENTINA.

Mathers (Helen). HONEY.

GRIFF OF GRIFFITHSCOURT.

SAM'S SWEETHEART.

THE FERRYMAN.

Meade (Mrs. L. T.). DRIFT.

Miller (Esther). , LIVING LIES.

Mitford (Bertram). THE SIGN OF THE SPIDER.

Montresor (F. F.). THE ALIEN 


\section{Fiction}

Morrison (Arthur). THE HOLE IN THE WALL.

Nesbit (E.). THE RED HOUSE

Norris (W. E.). HIS GRACE.

GILES INGILBY.

THE CREDIT OF THE COUNTY. LORD LEONARD THE LUCKLESS.

MATTHEW AUSTEN.

CLARISSA FURIOSA.

Oliphant (Mrs.). THE LADY'S WALK. SIR ROBERT'S FORTUNE.

THE PRODIGALS.

THE TWO MARYS.

Oppenheim (E. P.). MASTER OF MEN.

Parker (Gllbert). THE POMP OF THE LAVILETTES.

WHEN VALMOND CAME TO PONTIAC. THE TRAIL OF THE SWORD.

Pemberton (Max). THE FOOTSTEPS OF A THRONE.

I CROWN THEE KING.

Phillpotts (Eden). THE HUMAN BOY. CHILDREN OF THE MIST.

THE POACHER'S WIFE.

THE RIVER.

' $Q$ ' (A. T. Quiller Couch). THE Ridge (W. Pett). A SON OF THE STATE. LOST PROPERTY.

GEORGE and THE GENERAL.
ERB.

Russell (W. Clark). ABANDONED.

A MARRIAGE AT SEA.

MY DANISH SWEETHEART.

HIS ISLAND PRINCESS.

Sergeant (Adeline). THR MASTER Or BEECHWOOD.

BALBARA'S MONEY.

THE YELLOW DIAMOND.

THE LOVE THAT OVERCAME.

Sidgwick (Mrs. Alfred). THE KINS MAN.

Surtees (R. S.). HANDLEY CROSS. MR. SPONGE'S SPORTING TOUR.

ASK MAMMA.

Walford (Mrs. L. B.). MR. SMITH. COUSINS.

THE BABY'S GRANDMOTHER. TROUBLESOME DAUGHTERS.

Wallace (General Lew). BEN-HUR THE FAIR GOD.

Watson (H. B.9Marriott). THE ADVEN. TURERS.

* CAPTAIN FORTUNE.

Weekes (A. B.). PRISONERS OF, WAR. Wells (H. G.). THE SEA LADY.

White (Percy). A PASSIONATE PIL. 





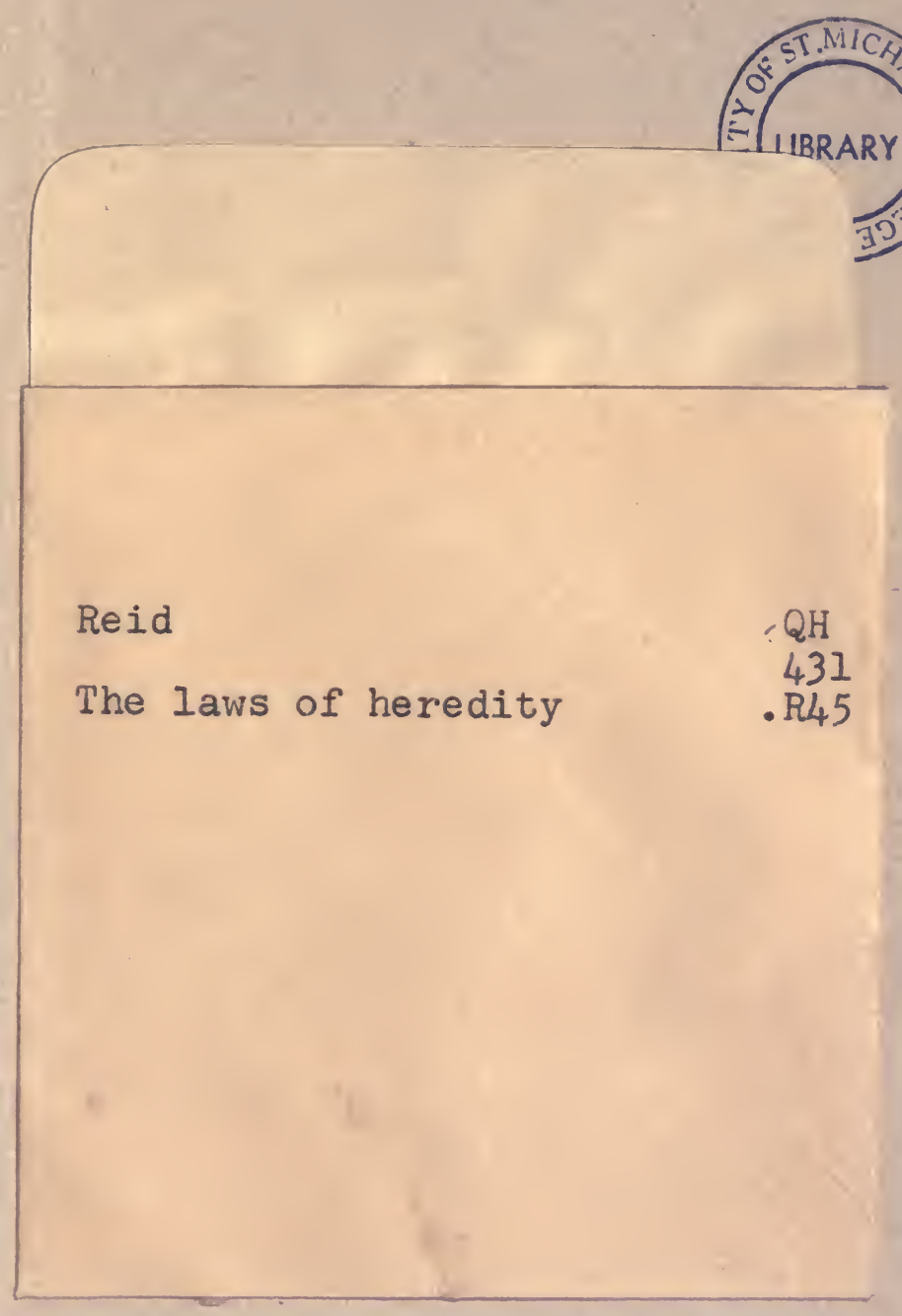


